\title{
New Zealand Building Code Clause G7 Compliance Assessment Tool: Development and Implementation
}

Krystle Stewart 



\section{ABSTRACT}

The New Zealand Building Code Clause G7 (NZBC G7) minimum requirement for natural light is not being met in around half of New Zealand apartments post-construction. The main reasons for this are an acceptable solution that is not effective for apartment buildings and a lack of tools for Territorial Authorities to assess accurately whether an apartment will meet the minimum requirement.

This report outlines the process involved in developing a simplified tool to assess quickly natural light compliance in apartments and preparing it for implementation. The tool was developed through simulation of factors that affect daylight performance at the point in the room specified in the Code: the back of habitable rooms. From these simulations statistical analysis was used to develop mathematical relationships between building features and light levels. These relationships were used to create a tool that specifies whether an apartment would require simulation to prove compliance with NZBC G7.

Calibration measurements were performed, comparing simulated and real measurements in 97 apartments with the predictions of the tool. These demonstrated that the tool provides reliable results, hence determining the accuracy of the predictions provided by the tool. The final step in the research was for potential end-user groups to evaluate the usability and functionality of the tool.

The conclusion of this process is that a tool has been developed that is simple and easy to use, is sufficiently accurate for application by Territorial Authorities as a decision tool and can be easily implemented. 


\section{ACKNOWLEDGEMENTS}

Firstly, I would like to thank my supervisor Michael Donn. Without your help and guidance over the last 3 years, this would not have been possible.

Secondly, I would like to thank Victoria University for the Scholarship I received to assist with this research and Education New Zealand for the Travel Scholarship I received to attend the IBPSA conference BS07 in Beijing and visit Dr Edward Ng at Chinese University of Hong Kong.

I would also like to thank all the people that allowed me access into their homes to take measurements. And those who took the time to participate in the evaluation of the tool, your feedback is greatly appreciated. I would also like to thank those who stood outside on horrible overcast days to assist me in taking light measurements, with a special thank you to Jessica Bennett for dropping everything to help me with most of these with very little notice.

I would also like to thank: $\operatorname{Dr}$ Nokuthaba Sibanda, Victoria University of Wellington Consulting Statistician, for her advice on how to approach the development of my tool; Kit Cuttle for his insight into the development of NZBC G7; David Ragg of the Department of Building and Housing for his help during this research; and Dr Edward Ng for his guidance on developing the research method.

Finally, I would like to thank my family, friends and colleagues for your support throughout my research. 


\section{Contents}

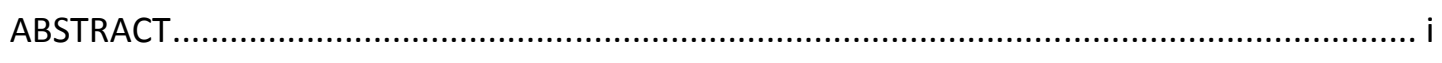

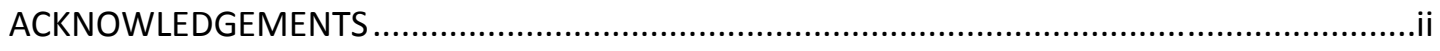

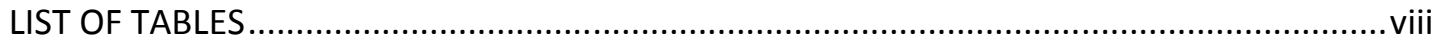

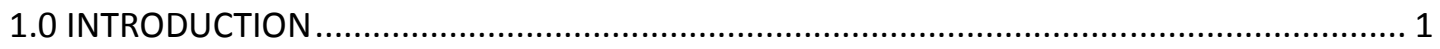

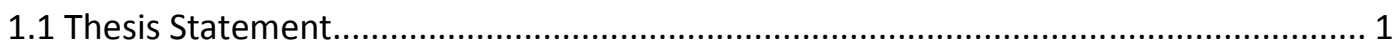

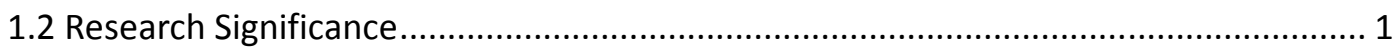

1.3 Scope of Research ........................................................................................ 2

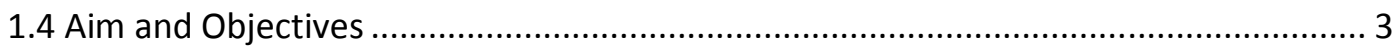

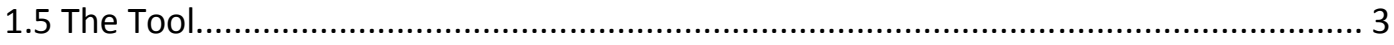

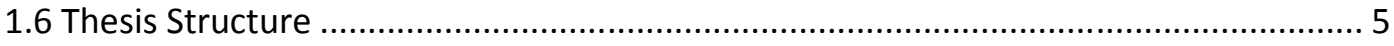

2.0 BACKGROUND ........................................................................................... 7

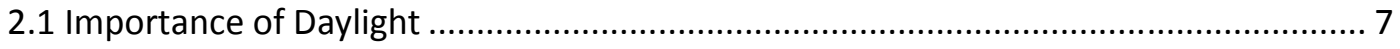

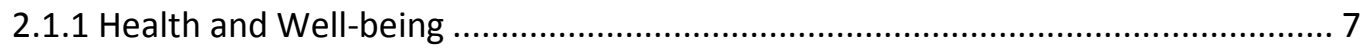

2.1.2 Preference for Daylight ....................................................................... 9

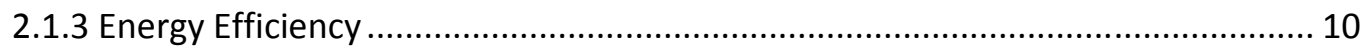

2.2 Previous Research .................................................................................... 10

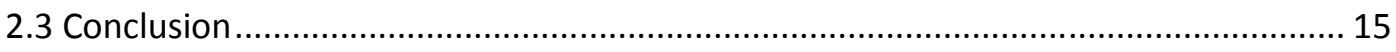

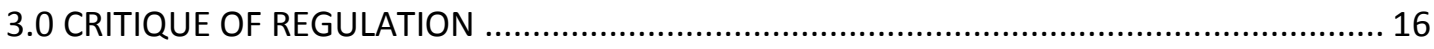

3.1 New Zealand Building Code Clause G7 - Natural Light....................................... 16

3.1.1 Brief History of the New Zealand Building Code......................................... 18

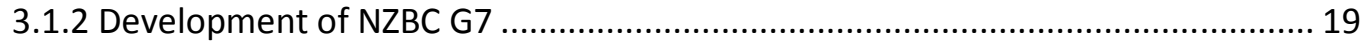

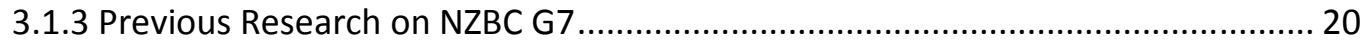

3.2 Comparison of NZBC G7 with International Standards and Recommendations ......... 22

3.2.1 Illuminance-Based Standards and Recommendations .................................... 22

3.2.2 Daylight-Factor Based Standards and Recommendations............................. 28

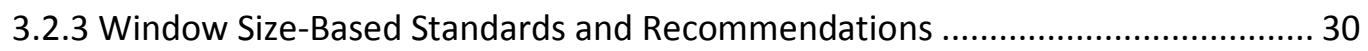

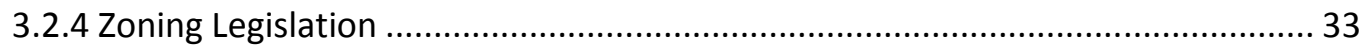

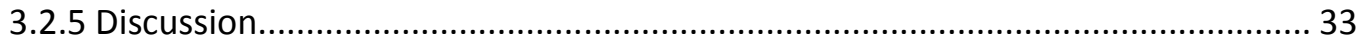

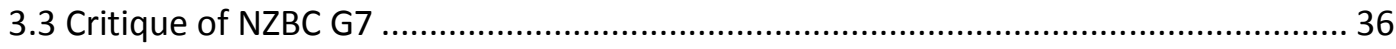

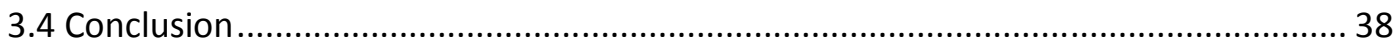

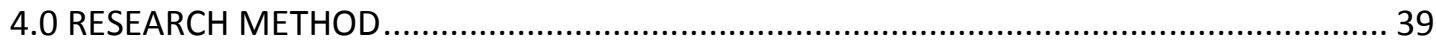

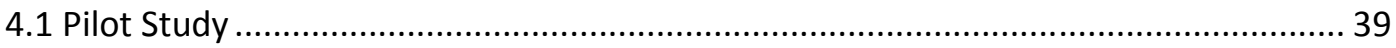




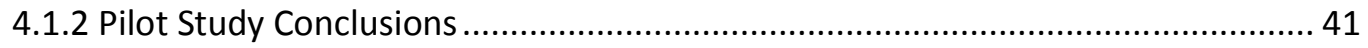

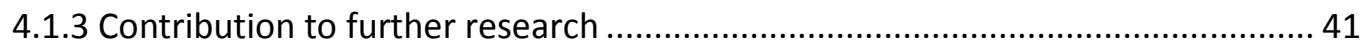

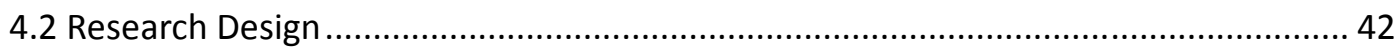

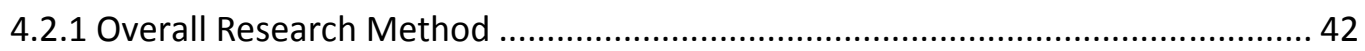

4.2.2 Simulation of Factors that Affect Daylight Performance ..................................... 43

4.2.3 Statistical Analysis Method for Development of the Tool ................................... 47

4.2.4 Method used for Calibration Tests ..................................................................... 48

4.2.5 Method used to Critique the Tool........................................................................ 49

4.2.6 How Research Methods were to fulfill the Criteria of the Tool............................ 50

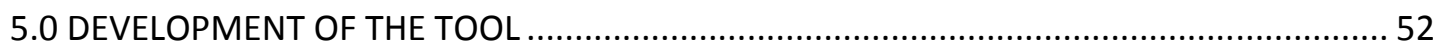

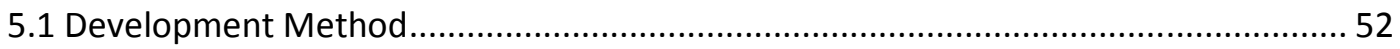

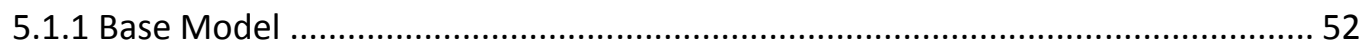

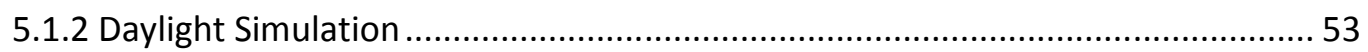

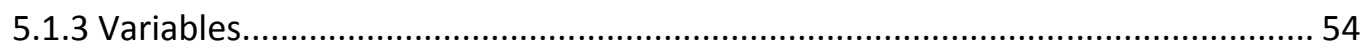

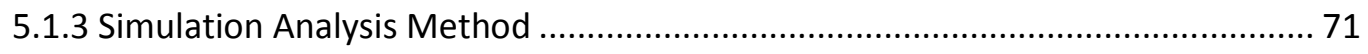

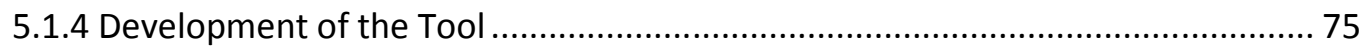

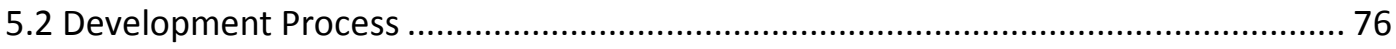

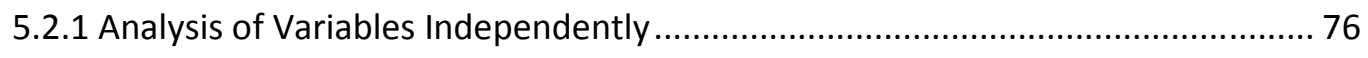

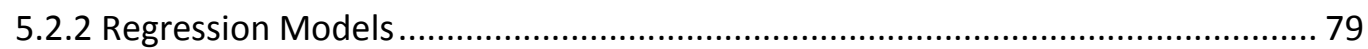

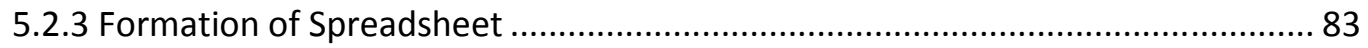

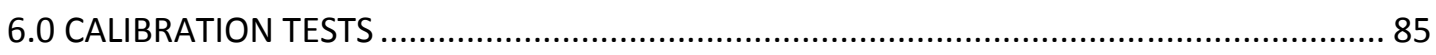

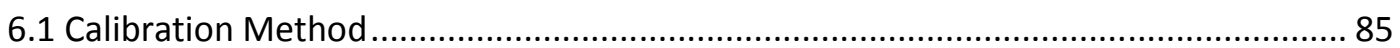

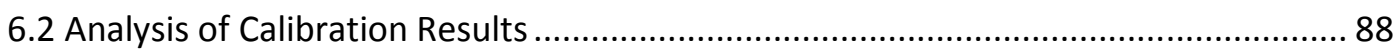

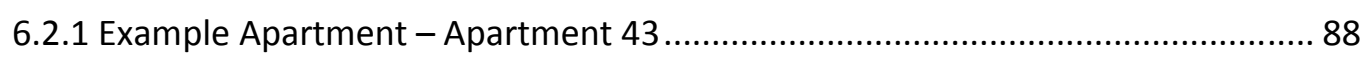

6.2.2 Summary of Calibration Results ......................................................................... 91

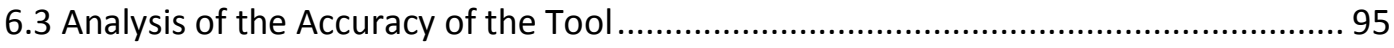

6.3.1 Accuracy of Apartment Outcomes.................................................................... 95

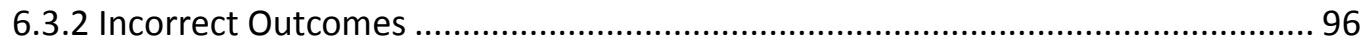

6.3.3 Accuracy of Outcomes for Entire Buildings....................................................... 97

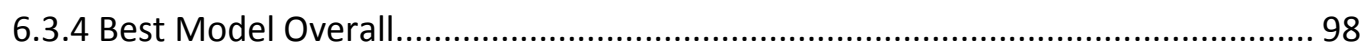

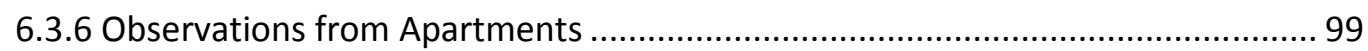

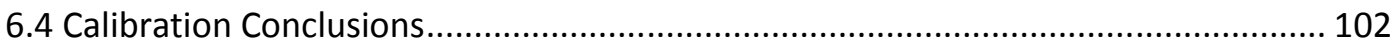

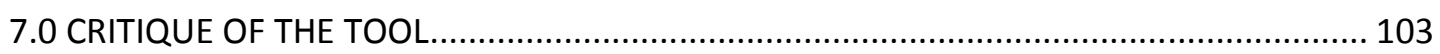

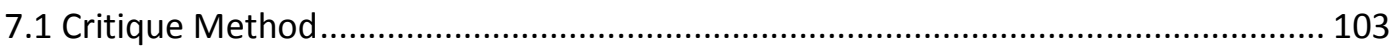




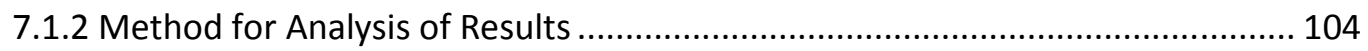

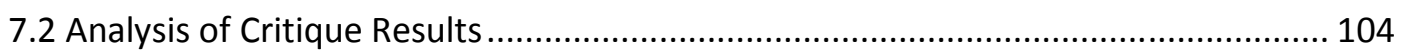

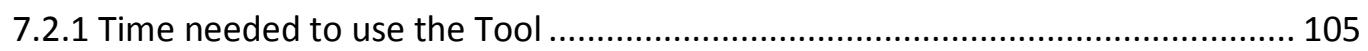

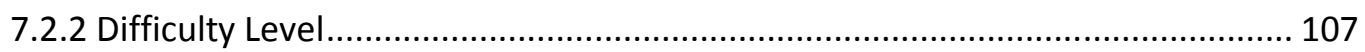

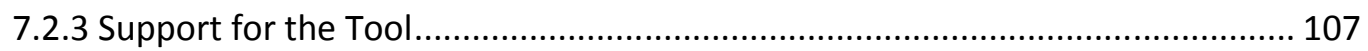

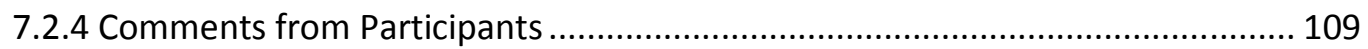

7.3 Changes to Tool as a Result of the Critique ............................................................. 111

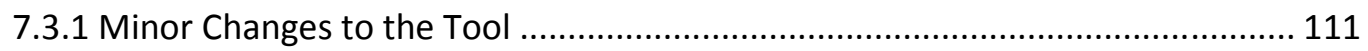

7.3.2 Consideration of Possible Changes to Proposed Building ................................ 113

7.3.3 Consideration of Changes to Adjacent site(s) ................................................... 116

7.3.4 Inclusion of a Reflectance Calculator ................................................................ 118

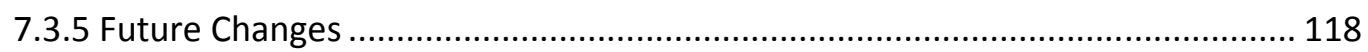

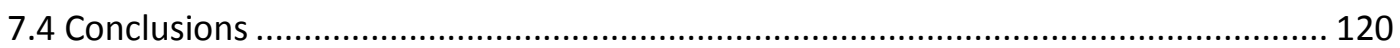

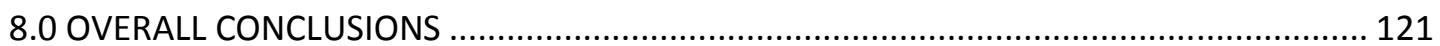

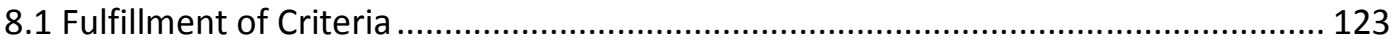

8.1.1 Criterion 1: Simple and Easy to Use................................................................. 123

8.1.2 Criterion 2: Reasonably Accurate................................................................. 123

8.1.3 Criterion 3: Can be easily Implemented........................................................... 124

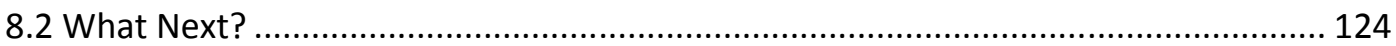

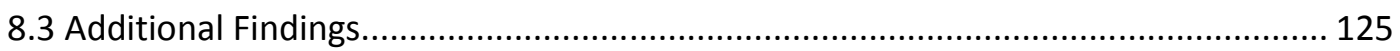

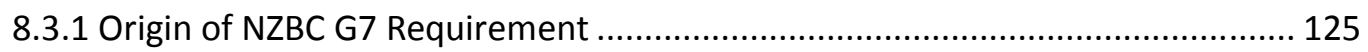

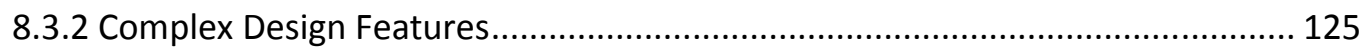

8.3.3 Preservation of Natural Light/View ............................................................. 126

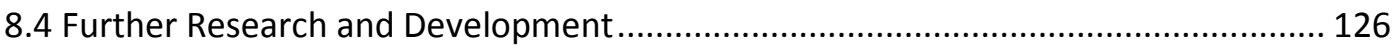

8.4.1 Minimum Requirements for Health and Well-being ......................................... 126

8.4.2 Building Exterior Reflectance Values Database .............................................. 126

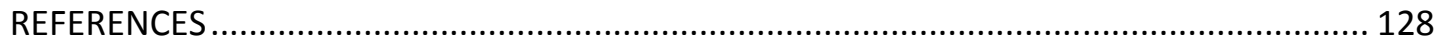

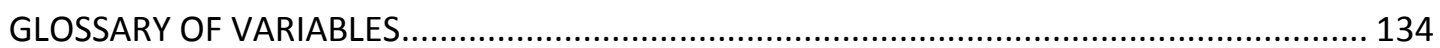

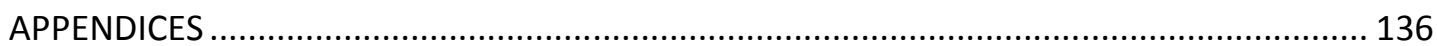




\section{LIST OF FIGURES}

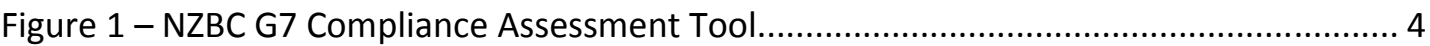

Figure 2 - Decision Process for Daylight Simulation in New Zealand ..................................... 5

Figure 3 - No -sky line as defined by DBH (Department of Building and Housig, 2001, pg 14)

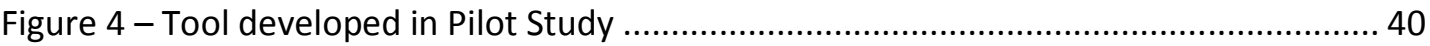

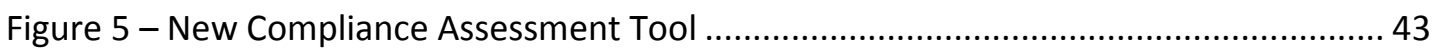

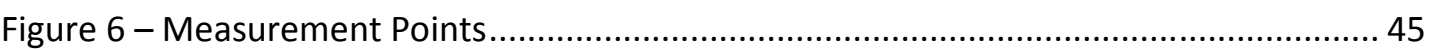

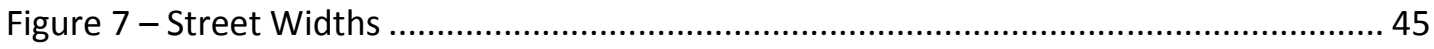

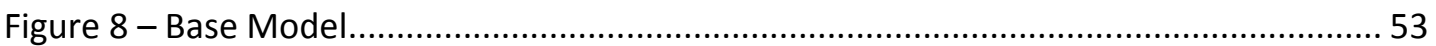

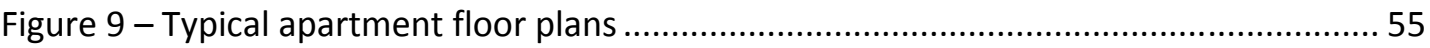

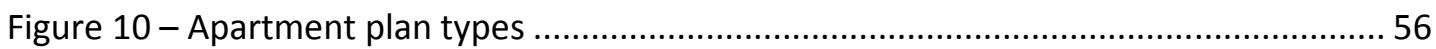

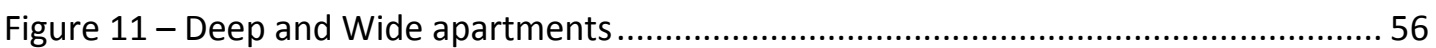

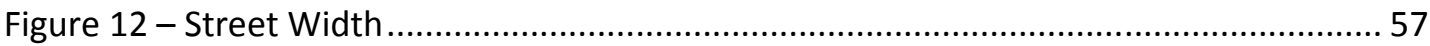

Figure 13 - Estimating average obstruction height (Building Research Energy Conservation

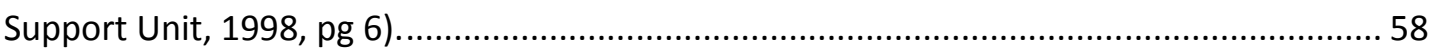

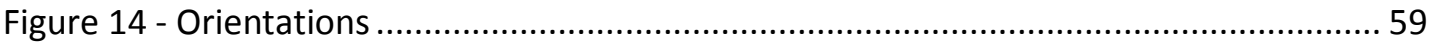

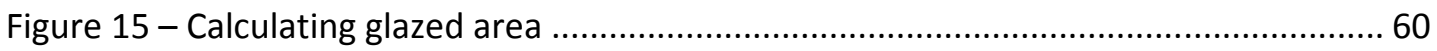

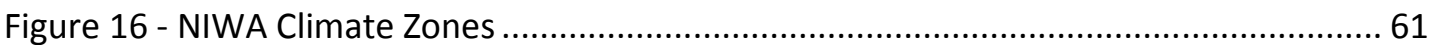

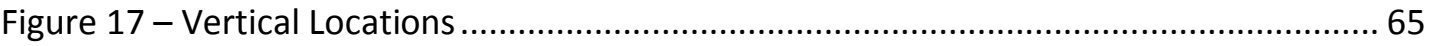

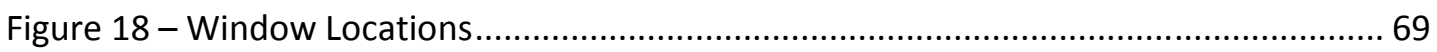

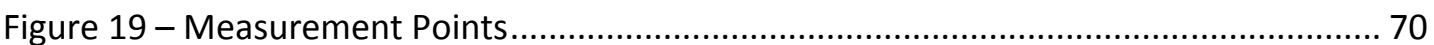

Figure 20 - Apartment Layout ................................................................................... 70

Figure 21 - Line of Best Fit for Linear and Curvilinear Patterns .......................................... 72

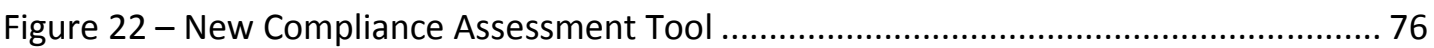

Figure 23 - Individual Relationship between Daylight Performance ad Building Height ...... 77

Figure 24 - Comparison of Predicted Results and Simulated Results .................................. 81

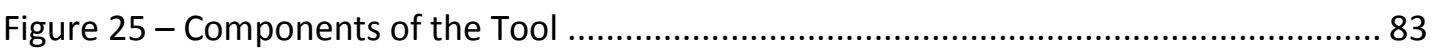

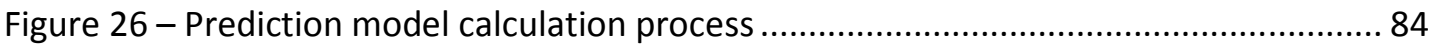

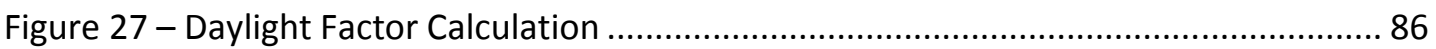

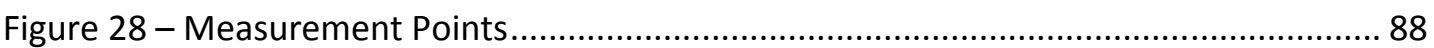

Figure 29 - Illuminance exceeded for $75 \%$ of the Standard Year in Apartment 43 ............... 89

Figure 30 - Application of the tool for Apartment 43 …...................................................... 90

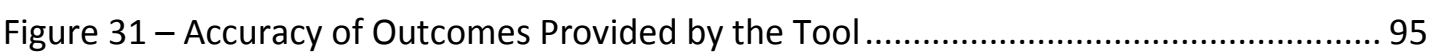

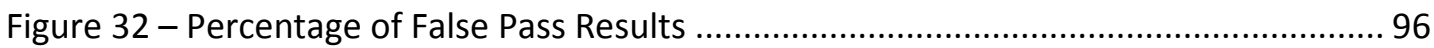

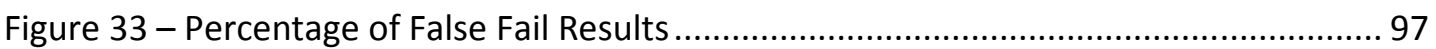

Figure 34 - Accuracies of the Three Prediction Models ..................................................... 98

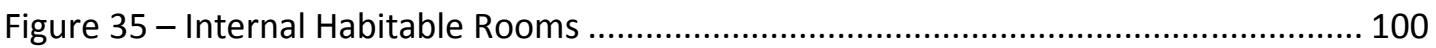

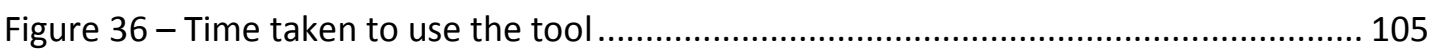

Figure 37 - How users considered the time taken to use the tool..................................... 106

Figure $38-$ How users considered the difficulty of the tool............................................... 107

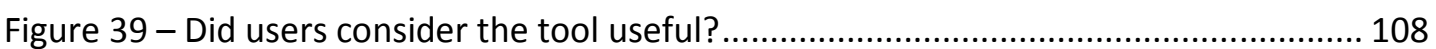

Figure 40 - Did users find the result effective? ................................................................ 108 
Figure 41 - Would users support the implementation of the tool? ................................... 109

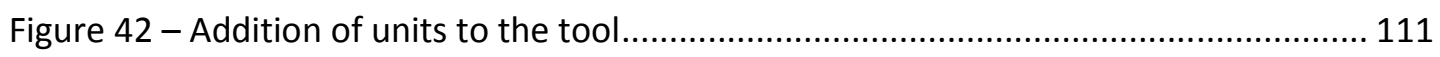

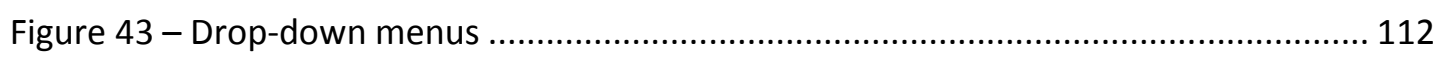

Figure 44 - Tool with Visual Awareness Requirements................................................. 113

Figure 45 - Table of Possible Changes to Proposed Building ............................................. 115

Figure 46 - Table of Possible Changes to Opposite Building ............................................ 117

Figure 47 - Opposite Building Reflectance Calculator ..................................................... 118

Figure 48 - Use of links to definitions and examples ....................................................... 119

Figure 49 - "CompuPan" toilet calculator (Department of Building and Housing, n.d.2) ... 120

Figure 50 - Final Version of Compliance Assessment Tool.............................................. 122 


\section{LIST OF TABLES}

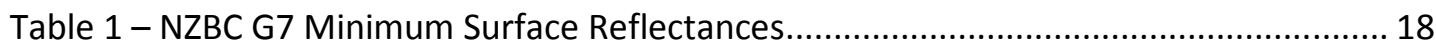

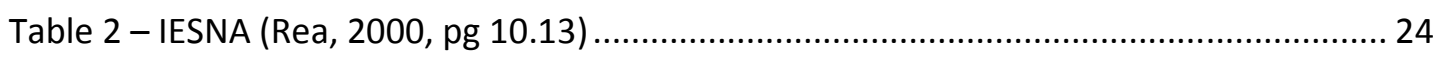

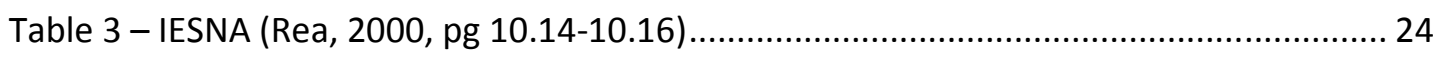

Table 4 - Lettre-circulaire DRT no. 90/11 Recommended Illuminance Levels ....................... 25

Table 5 - BS 8206: Part 2: 1992 Recommended Illuminances (British Standards Institution,

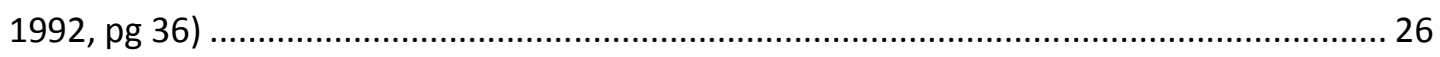

Table 6 - ASNZS 1680.1 Recommended Illuminances (Standard Australia/Standards New

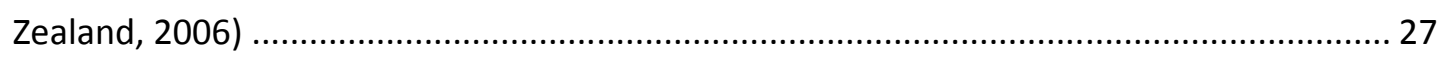

Table 7 - DIN 5034-4 (DIN Deutsches Institut für Normung, 1994, pg 11) Minimum window

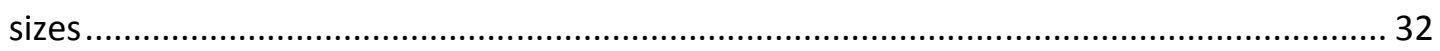

Table 8 - Comparison of NZBC G7 and International Codes and Standards indentified in

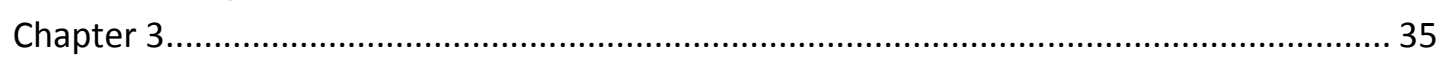

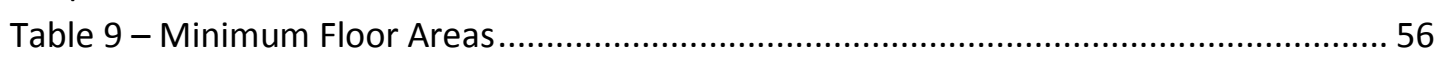

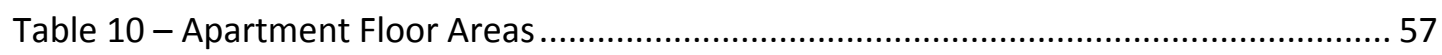

Table 11 - Dimensions of major New Zealand cities (Davies, 1992, pg 65) ............................. 58

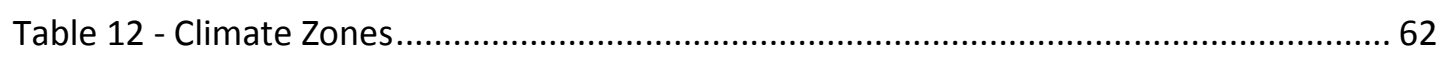

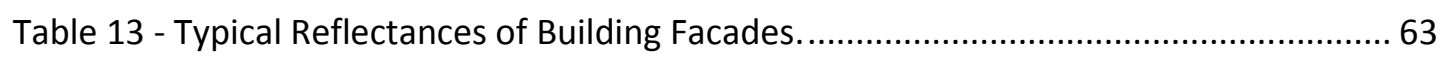

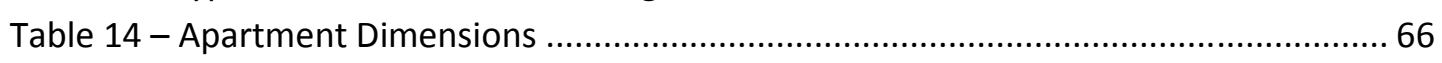

Table 15 - Approximate reflectances of typical building finishes (Standards

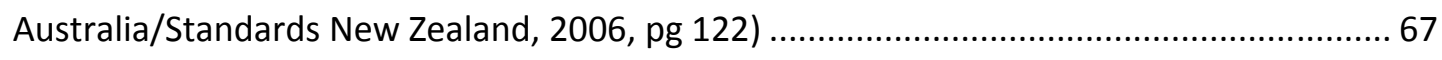

Table 16 - Table used to assess the fit of the relationships .............................................. 73

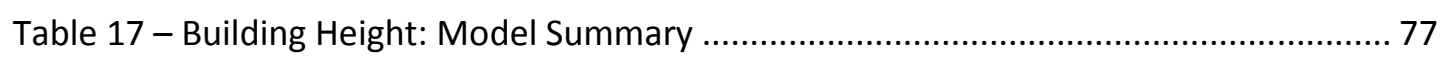

Table 18 - Factors Used to Assess the Strength of Regression Models ................................ 80

Table 19 - External Illuminances from AS/NZS 1680.1:2006 ................................................. 86

Table 20 - Calibration Apartment Matrix for Street Width and Building Height ................... 87

Table 21 - Calculation of Daylight Performance in Calibration Apartments .......................... 89

Table 22 - Prediction Model Results for Apartment 43 ..................................................... 90

Table 23 - Comparison of Calibration Apartments .............................................................. 93

Table 24 - Summary of False Pass and False Fail Apartments ............................................. 94

Table 25 - Variables of the three remaining apartments that received incorrect predictions

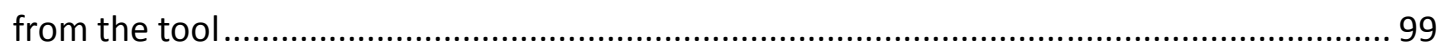

Table 26 - Percentage of apartments with dark, medium and light surfaces for ceilings,

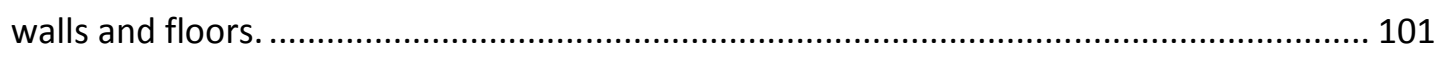

Table 27 - Comparison of the time taken and the number of times users applied the tool

105

Table 28 - Cost Comparison of the Tool vs. Full Simulation 106 


\subsection{INTRODUCTION}

\subsection{Thesis Statement}

New Zealand apartments do not receive adequate natural light because the building code Acceptable Solution is not suitable for apartment buildings and there are currently no tools available that allow Territorial Authorities to accurately assess whether apartments will comply.

The intention of this research was to develop a simple tool that could become the Acceptable Solution for natural light in apartments. This tool was to consider aspects of the building design and environment that are likely to affect daylight in apartments. The output of the tool would be specification of when apartments need simulation to prove compliance, providing Territorial Authorities with the necessary information to determine if an apartment building is likely to receive adequate natural light post-construction.

\subsection{Research Significance}

Around $50 \%$ of the apartments built in New Zealand over the past 10 years fail to meet the minimum natural light level for health and well-being, identified through measurements taken in 97 apartments. With approximately 2,500 apartments built every year (Statistics New Zealand, 2007), this equates to 1,250 apartments built annually that fail to receive adequate natural light.

Inner-city apartments make up $10-20 \%$ of the housing consents given annually in New Zealand (Statistics New Zealand, 1991-2008). With some of New Zealand's Territorial Authorities encouraging the development of inner-city living as a way of addressing urban sprawl; creating sustainable communities; efficiently using infrastructure and public services; and efficiently managing waste (Morrison \& McMurray, 1999), this proportion is expected to grow significantly. However design measures need to be undertaken to ensure the intensification of New Zealand cities does not compromise the health, safety and enjoyment of inner-city dwellers. Natural light is an aspect of health, safety and enjoyment that is at risk of being compromised due to insufficient regulation.

The issue of regulation of natural light in the building code was first highlighted in 2000 (Cuttle, 2000) but very little progress has been made since. With around 2,500 new apartments given building consent in 2007 (Statistics New Zealand, 2007) and growth expected to continue at this rate over the next 5 years (Bayleys Research, 2006), it is clear that the issue needs to be addressed urgently.

The New Zealand Building Code Clause G7 - Natural Light Compliance Documents (NZBC G7) requires adequate natural light to be provided in all habitable spaces to 'safeguard people against illness or loss of amenity due to isolation from natural light and view to the 
outside environment' (Department of Building and Housing, 2001). The performance requirement for NZBC G7 is that a minimum illuminance of 30 lux is provided at floor level for at least 75\% of the standard year (Department of Building and Housing, 2001, pg 3).

A study by Stewart (2006b) identified the reasons behind the failing apartment as an acceptable solution that is not suitable for inner-city apartment developments and insufficient information for Territorial Authorities (TAs) to accurately assess compliance when issuing building consents.

The current acceptable solution, used for most consent applications, requires a glazed area of $10 \%$ of the floor area. This solution was originally adopted because it equates to approximately 33 lux for $75 \%$ of the standard year in typical New Zealand houses (Department of Building and Housing, 2001, pg 13). The acceptable solution does not consider the complex nature of apartments, typically with less glazing and greater obstructions than that of houses.

There is currently a lack for tools for Territorial Authorities to easily assess compliance with NZBC G7 in apartments. Assessing compliance with NZBC G7 currently involves the consent officer making a judgment by looking at the overall design of the building and deciding whether they feel that the building will meet the minimum requirement (Stewart, 2006b). This is not the most robust method of assessment and is a major reason apartment buildings are being built that do not comply with the minimum code requirement.

\subsection{Scope of Research}

Boundaries were defined to ensure this research successfully addressed the need for a more suitable Acceptable Solution and that it provides Territorial Authorities with the necessary information to assess accurately natural light in apartments. The scope of the research has also been defined to ensure the research was able to be executed in the allocated time.

Two performance criteria are used to fulfil the requirements of NZBC G7. The first criterion requires an illuminance of 30 lux to be exceeded at floor level for $75 \%$ of the standard year. The second criterion requires openings to give visual awareness to the outside environment. This research deals specifically with the illuminance criterion of NZBC G7 as this was the area that seemed in need of urgent attention.

This research dealt specifically with apartment buildings. Apartment buildings were identified as the type of housing that the current Acceptable Solution was not assessing accurately (Stewart, 2006a). The current acceptable solution is more likely to still be suitable for all other forms of housing.

The tool produced in this research was to be effective for typical New Zealand apartments. It is not feasible to consider every possible design and environment that may occur, so it was decided to limit this research to situations typically found in New Zealand cities. This will allow a more accurate tool to be produced that can predict the performance in most 
apartments, but may require apartments that do not fit the limits of the tool to use other methods to assess compliance.

\subsection{Aim and Objectives}

The aim of this research was to produce a simple compliance assessment tool that can be included in the NZBC G7 Compliance Documents as an Acceptable Solution for apartment buildings.

To ensure the aim was fulfilled and a remedy was provided for the issues outlined in the problem statement, three key objectives were used. This research was to:

- Produce a suitable method of assessing compliance with NZBC G7 in apartment buildings;

- Provide Territorial Authorities with the necessary information to determine accurately if an apartment complies; and

- Prepare the tool for implementation into everyday practice.

Three criteria were used to ensure the tool fulfils the overall aim and objectives. These criteria were used throughout the research to assess the overall success of the Tool. The tool was to be:

- Simple and easy to use;

- Reasonably accurate; and

- Can be easily implemented.

\subsection{The Tool}

The idea behind this tool was to identify when apartment buildings should be simulated to prove compliance with NZBC G7. The tool was to consider the interaction between building and environmental factors, and daylight performance at the back of habitable rooms (the worst conditions criterion specified in NZBC G7). The tool was to use the method proposed by the International Energy Agency (Ruck, 2000) for simple computer based design tools, where complex calculations are performed but the tools are simple from the user's point of view.

It was assumed that it is not realistic to require daylight simulations for all new apartment buildings due to the extra time and cost involved in producing simulations. With $48 \%$ of the 97 apartments measured exceeding the minimum requirement, it is clear that it is not necessary that all apartments are simulated. The plan was to develop a tool that clearly indentified when an apartment building is at risk of not complying and should therefore have more extensive tests conducted to ensure adequate natural light is provided for health and well-being. 
The tool was developed to assess the 'worst case' for each apartment building. Therefore, the apartment, or apartments, that are expected to have the lowest light levels are assessed using the tool.

Figure 1 shows the format of the tool developed in this research.

\begin{tabular}{|l|l|}
\hline \multicolumn{2}{|c|}{ NZBC G7 Compliance Assessment Tool } \\
\hline \multicolumn{2}{|c|}{ Variable: } \\
\hline Opposite Building Height & \\
\hline Street Width & \\
\hline Glazed Area & \\
\hline Orientation & \\
\hline Location & \\
\hline Reflectance of Opposite Building & \\
\hline Glazing Transmittance & \\
\hline Vertical Location of lowest apartment & \\
\hline \multicolumn{2}{|l}{} \\
\hline Are simulations required to prove compliance: \\
\hline
\end{tabular}

Figure 1 - NZBC G7 Compliance Assessment Tool

Figure 2 shows the New Zealand decision process for daylight simulation in the early design stage with the incorporation of the tool produced by this research at step 3 . This figure is an adaption of Reinhart's (2006) 'decision process for daylight simulation'. 


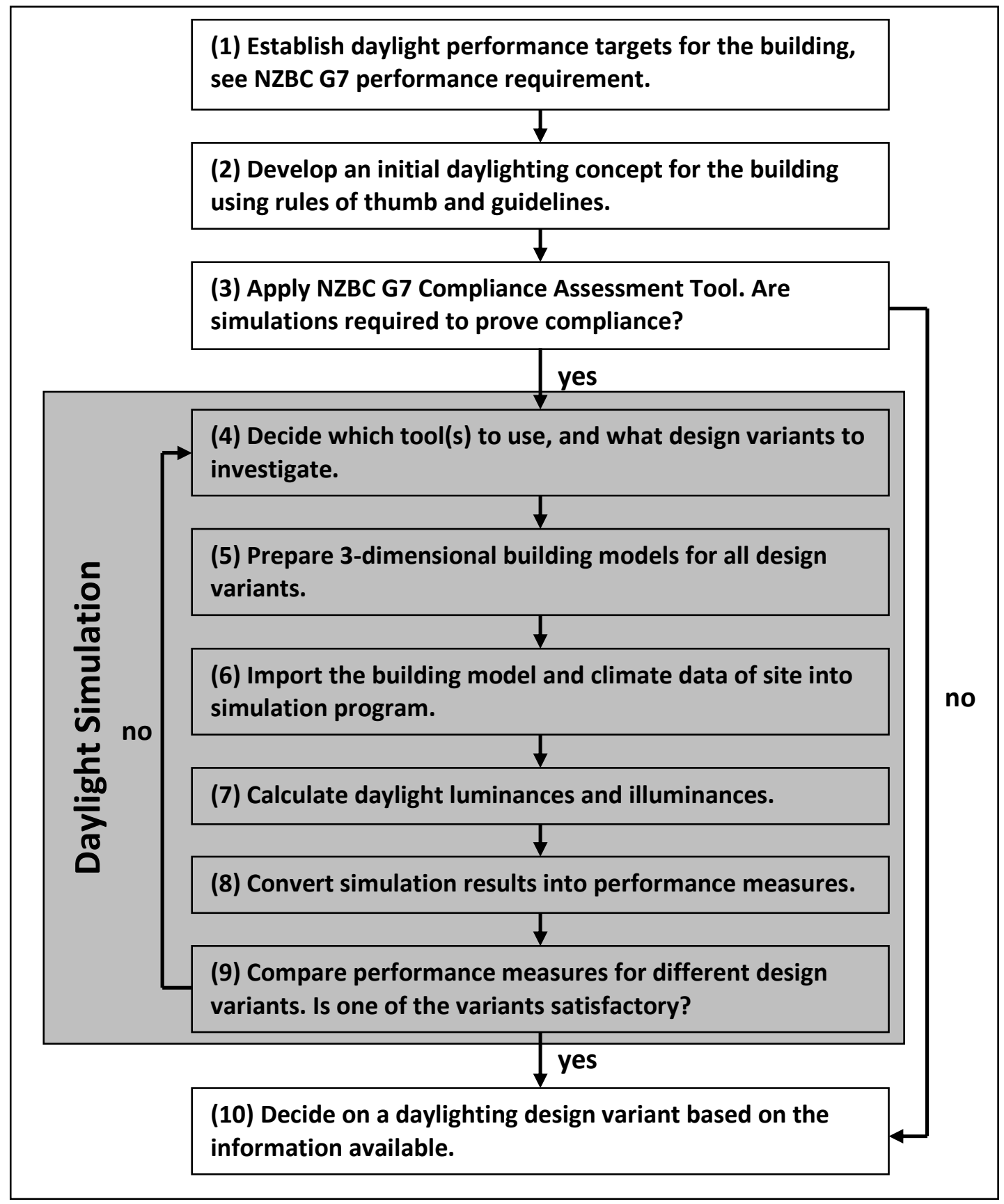

Figure 2 - Decision Process for Daylight Simulation in New Zealand

\subsection{Thesis Structure}

This thesis outlines the research process involved in the development of the compliance assessment tool to fulfil the aim and objectives specified above.

The second chapter provides the overall background information for this research. It includes an investigation into why regulation of natural light is necessary in terms of health, well-being and energy efficiency. This chapter also discusses previous research undertaken in this area to aid the development of a solid research method. 
Chapter three investigates the origins of NZBC G7 and summarises a critique undertaken of the current regulations. The origins of NZBC G7 were explored to become familiar with the requirements and current compliance methods, and to ensure the original intentions of the code were considered in the development of the new assessment method. The critique of the code compares the New Zealand requirements to international standards and recommendations to determine if the code is in line with the original intentions.

The fourth chapter discusses the overall design of this research project. This chapter documents the overall methods used to develop the tool, the calibration tests and the critique of the tool. And discusses how these were intended to satisfy the aim and objectives of this research.

Using the research design defined in Chapter four, the fifth, sixth and seventh chapters outline the development and implementation process for the tool. The fifth chapter discusses the development of the tool, including the simulation method and the process involved in the creation of the tool. The sixth chapter gives a detailed description of the calibration process, comparing the tool against measurements and simulations for real buildings ensuring that the tool provides reasonably accurate results. And the seventh chapter summarises the evaluation of the tool by potential end-users, ensuring the tool is simple and easy to use and can be easily implemented. The overall conclusions are drawn in chapter eight. 


\subsection{BACKGROUND}

Chapter 2 provides the overall background to this research project. Section 2.1 identifies the need to regulate for natural light. Three aspects are discussed in Section 2.1, these are: the effect natural light has on health and well-being; the general preference to be in daylit spaces; and the role daylight plays in the reduction of energy use in dwellings. Section 2.2 discusses the previous research that has been undertaken that relates to daylight performance and regulation.

\subsection{Importance of Daylight}

It is essential when developing regulatory methods that the need for regulation is investigated. This section aims at identifying the key aspects of daylight that need consideration when prescribing regulations. Within this section three topics will be addressed: health and well-being; occupant preference and energy efficiency. These sections were identified throughout the literature, with a number of writers mentioning the relationship between daylight, energy efficiency, occupant health, well-being and a general desire for daylight (Li, Lo, Lam \& Yuen, 1999; Ng, 2005; North Shore City Council, 2002a).

There is a limited amount of information currently available that refers specifically to the importance of daylight in housing. The majority of solid research that has been undertaken in this area has been investigating daylight in offices, school and hospitals. It should be noted that although the majority of research does not specifically consider housing, it typically refers to basic human needs and preference. Therefore, information given in this section can still be considered relevant and further research would need to be undertaken to prove otherwise.

\subsubsection{Health and Well-being}

Health and well-being, when mentioned in this document, follows the World Health Organisation (WHO) definition of health as a state of complete physical, mental and social well-being (World Health Organization, 2008). The three aspects identified in the literature were adequate illumination influencing melatonin production, exposure to Ultraviolet radiation and daylight as a visual stimulus.

Melatonin is a hormone that naturally occurs in the body and is directly linked to the environmental illumination (Farley \& Veitch, 2001). Insufficient exposure to high illuminances (like natural light) causes an increase in the melatonin secretion into the bloodstream (Raw, Aizlewood \& Hamilton, 2001, pg 54). Increased melatonin results in disruption of circadian rhythms, affecting sleeping and waking, and mood states (Raw et al., 2001, pg 54). 
In healthy people, high levels of melatonin can result in (Boyce, Hunter, \& Howlett, 2003; Evans, 2003; Farley \& Veitch, 2001; Raw et al., 2001, pg 54; Ullah and Lin, 2003):

- decreased vigour;

- increased sleepiness;

- confusion;

- fatigue;

- disruption of organ function including the brain, pituitary, thyroid, adrenal and smooth muscles;

- inhibited ovulation;

- modified release of stress hormones like cortisol; and

- is linked to psychiatric disorders such as Seasonal Affective Disorder (SAD).

Ultraviolet radiation is another feature of daylight that is important for health and wellbeing. Ultraviolet radiation is needed to (Evans, 1981, pg 20; Raw et al., 2001, pg 56):

- produce Vitamin D;

- prevent rickets in children;

- prevents osteomalacia in adults (a skeletal disorder);

- keep skin in a healthy condition;

- destroy germs; and

- bring about necessary chemical changes in the body;

In addition to a feeling of well-being, ultraviolet radiation exposure causes a quickening of the pulse rate and metabolism, and a stimulation of energetic activity (Evans, 1981, pg 20).

It is however crucial to understand that the necessary exposure to Ultraviolet radiation can be fulfilled through short daily exposures throughout the year (Raw et al., 2001, pg 56), and that overexposure to ultraviolet radiation has a negative effect including skin cancer and aging. A concern of modern living is that we spend most of the daylight hours inside (Evans, 1981, pg 20), which highlights the need for some ultraviolet radiation to be available in all homes and workplaces.

The final aspect of daylight that contributes to health and well-being is as a visual stimulus. It is a combination of the spectral qualities and the link between daylight and view that influence the psychological health and well-being of humans. In terms of spectral qualities, daylight is unmatched by any artificial light source, with the variation in spectrum throughout the day, year and under different weather conditions (Boyce et al., 2003). Visual stimulation through daylight can (Boyce et al., 2003; Raw et al., 2001, pg 54-55):

- reduce stress

- affect mood; and

- affect emotion;

The effect of being in an unchanged environment leads to: adverse emotional states, psychosomatic and stress symptoms.

From the literature gathered on the effect of daylight on health and well-being, it is clear that daylight is an important part of life. However, a gap was observed in the available 
research. It became apparent that although it is widely acknowledged that exposure to daylight is needed there was little data on exactly how much people need. This observation was supported by Evans (1981) and Raw et al. (2001). Evans (1981) also identified the lack of quantitative data related to daylight in homes.

\subsubsection{Preference for Daylight}

Daylight access is considered a basic right of human beings. This section discusses the information found on occupants' preference for daylight. Aspects discussed are the spectral qualities of daylight and occupants' desire for a daylit environment.

Daylight is often identified as the best light source (Boyce et al., 2003; Li et al., 1999; Ullah \& Lin, 2003). This is due to its 'unequalled qualities of colour rendering' (Ullah \& Lin, 2003, pg 91) and its close match to human visual response (Li et al., 1999). This also ties into the second aspect of occupants' preference; the main reason daylight is a desirable attribute of a space (Ullah and Lin, 2003).

There is a general consensus that people, when give the choice, would opt for a daylit space over an artificially lit space. Wilson and Brotas (2001) identified that the main argument for designing domestic buildings for daylight comes from this preference of the occupants.

Various surveys have been conducted to understand the preference for daylight in both workplaces and domestic dwellings. These found that people prefer to complete visual tasks under daylight (Farley \& Veitch, 2001, pg 9), with between $65 \%$ and $95 \%$ of the people surveyed wanting to work in daylit spaces. More importantly, the surveys investigating domestic dwellings found that a great deal of importance is placed on sunlight (Farley \& Veitch, 2001, pg 9). Interestingly, Ng (2005) observed in his study of Hong Kong residential buildings that it is only when daylight performance is low and substandard that people began to notice and complain.

Occupants prefer daylit spaces because they (Li et al., 1999; Ng, 2005; Wilson \& Brotas, 2001; Ullah \& Lin, 2003):

- $\quad$ are perceived to be healthier;

- allow greater contact with the outside world;

- appear more open;

- provide a more attractive and pleasing atmosphere;

- offer a sense of satisfaction and well-being; and

- are potentially more comfortable for inhabitants.

It has been identified that daylight is a highly desired source of illumination for occupants. Not only do people generally prefer to live and work in daylight, its spectral quality enhances colour discrimination and visual response. 


\subsubsection{Energy Efficiency}

Daylight is an excellent method for reducing energy use in buildings (Ng, 2005; North Shore City Council, 2002a; Wilson \& Brotas, 2001; Ullah \& Lin, 2003). When utilised effectively, it is capable of providing sufficient illumination during daylight hours and can significantly reduce the need for artificial lighting. Energy used for lighting in New Zealand houses makes up approximately $8 \%$ of the total energy use (Isaacs, 2006) with apartments expected to use a higher percentage, meaning that there is a large potential for energy reductions through the use of daylight.

However, there is question as to the validity of this argument in domestic building. The argument lies in the fact the many dwellings are unoccupied during daylight hours and as such the benefits may be small (Wilson \& Brotas, 2001). Further research is required to establish the savings potential, if any, for daylight in domestic building, but it should be noted that good design should not dictate how occupants use their homes. An unsystematic observation based on the work conducted to calibrate the tool developed in this thesis through measurements of New Zealand apartments suggests that a number of people living in apartments are retired or work from home. In these situations there is great potential for reducing their energy use.

\subsection{Previous Research}

A survey of available literature was conducted in the early stage of this research to identify the research methods used by researchers in this field. This process was used to aid the development of a robust research method.

There has been little research conducted in New Zealand, investigating the regulation of natural light in apartment buildings. The majority of previous New Zealand research conducted in this area has been conducted by the author. This research includes two research reports (Stewart, 2006a; Stewart, 2006b) and a conference paper (Stewart \& Donn, 2007).

The first research report was a pilot study for this research (Stewart, 2006a), a detailed summary of the pilot study can be found in Section 4.1, the pilot study was also summarised in a paper presented at the International Building Performance Simulation Association (IBPSA) conference, Building Simulation 2007, in September 2007 (Stewart \& Donn, 2007). The second research report (Stewart, 2006b) investigated daylight and the current apartment stock in Wellington. Part of the research method used in the investigation of Wellington apartments which is relevant to this research was the assessment of daylight levels in apartments. Illuminance levels were taken at various points in six apartments and under an unobstructed overcast sky simultaneously to calculate the daylight factor.

Given the lack of relevant New Zealand research, the research conducted overseas was heavily relied on for guidance. The majority of literature came from two regions: Hong Kong 
and the United Kingdom. The literature from Hong Kong typically discussed regulation of daylight in high-density residential situations and the literature from the United Kingdom typically discussed daylight design/performance and urban planning.

\section{Hong Kong}

Dr Edward Ng of the Chinese University of Hong Kong (CUHK) has conducted a large amount of research in the area of daylight regulation in urban environments. Ng's articles (2001a; 2001b; 2003; \& 2005) provided an understanding of how this research should be formed. Ng's key articles will be summarised in the following paragraphs, identifying the main points that were drawn on for the development of the research method.

$\mathrm{Ng}$ 's first relevant publication (2001a) summarises the development of a design tool for architects, estimating the daylight performance of high-rise residential buildings in the early design stage. In $\mathrm{Ng}^{\prime} \mathrm{s}$ research, he was investigating the suitability of two calculation methods. Validation studies were conducted for the two calculation methods to determine which was most suitable. These tests were conducted by applying both calculations to various situations and then comparing to the results taken in a 'test model'.

Other ideas drawn from Ng's first publication included his use of a 'test model' which was considered to be representative of a typical living room found in Hong Kong ( $\mathrm{Ng}$, 2001a, pg 262 ) and the use of the daylight factor calculation for predicting the daylight performance in the apartments ( $\mathrm{Ng}, 2001 \mathrm{a}, \mathrm{pg} 260)$. Ng also identified the importance of making appropriate assumptions which are considered representative of typical situations ( $\mathrm{Ng}$, 2001a) and to be aware that assumptions limit the variations able to be assessed with the tool, reinforcing the idea that tools such as these should not be used out of context as reliability can no longer be guaranteed ( $\mathrm{Ng}, 2001 \mathrm{a}, \mathrm{pg} 268)$. One of the key advantages to the success of Ng's tool was its speed of use (Ng, 2001a, pg 260); this should be a success indicator in any research investigating the development of simple tools.

$\mathrm{Ng}$ 's next publication was a paper presented at the IBPSA conference, Building Simulation 2001 (Ng, 2001b). It discussed the accuracy of daylight simulation for heavily obstructed buildings in Hong Kong. In this research three residential units, at different heights within a new residential building in Hong Kong, were measured. The measurement process involved illuminance measurements taken in the centre of the windows and on the roof over the period of 10 weeks per window. The data from these measurements was then used to calculate the vertical daylight factor received at each of the windows ( $\mathrm{Ng}, 2001 \mathrm{~b}$ ). Each of the units were then modelled in two lighting simulation programs, Desktop Radiance and Lightscape. Simulations were conducted for various unit heights and reflectances. Radiance was found to be more accurate with lower obstruction angels, less than $35^{\circ}$ ( $\mathrm{Ng}, 2001 \mathrm{~b}$ ), similar to those typically found in New Zealand.

In 2003 Ng's first article regarding the regulation of daylight in apartments was published. This article discussed a study of daylight design and regulation of high-density residential housing in Hong Kong ( $\mathrm{Ng}, 2003)$. A simple tool was developed that calculated the Unobstructed Vision Area (UVA) based on the height of surrounding buildings and a constant. Accuracy tests for building heights between 90 metres and 130 metres were 
conducted, which was considered typical of the situations found in Hong Kong. Ng felt that when forming a regulation tool the onus should rest on the designers to prove that their designs achieve a VDF of no less than the standard and that the method used is robust and proven to be appropriate ( $\mathrm{Ng}, 2003$, pg 136).

Within this article, $\mathrm{Ng}$ gives three requirements for his design and regulatory method $(\mathrm{Ng}$, 2003, pg 135), these were that:

- It must be reasonably accurate for the tasks and conditions at hand;

- It must be easy and straightforward to use; and

- The criteria must be clear that it is enforceable.

These requirements were adapted in the pilot study (Stewart, 2006a) to form the means of assessing the performance of the tool. These were:

- It was to be reasonably accurate;

- It was to be simple and easy to use; and

- Could be easily implemented.

$\mathrm{Ng}$ also identified the primary methods of calculating daylight performance and their ability to cope with urban environments. These were (Ng, 2003, pg 136-137):

- Any three-dimensional method for estimating sky component (SC) could be used. These methods include the use of Waldram diagram, pepper dot diagram, or other suitable stereographic diagrams.

- Modified split flux formula developed by Peter Tregenza could be used. It has been shown that this set of formulae is capable of accurately accounting for conditions where the skyline is complex.

- Computational simulation. To obtain good results, it is important to set the files and configure the variables correctly.

- Measurement using mini-cells (illuminance sensors), scale models and CIE overcast artificial sky. But note the limits of using scale models.

The final article of $\mathrm{Ng}^{\prime} \mathrm{s}$ was published in the Hong Kong Institute of Architects (HKIA) journal as part of a series about the regulation of light, air and healthy living in Hong Kong. This article, Part III: The becoming of PNAP 278 (Ng, 2005), discusses the process involved in the development and implementation of new regulations for natural light and ventilation in Hong Kong. 
The overall research method used by the research team at CUHK is shown in the following table (Ng, 2005):

A review of the existing conditions of the built environment in Hong Kong, identifying problem areas.

A historical review of the current building regulations. Noting their historical basis, developments, assumptions, context of application and as limits.

A territory (Hong Kong) wide user survey to note the performance requirements of local inhabitants, establishing performance criteria acceptable to them.

Based on the performance criteria, develop easy-to use and deem-to-satisfy design rules and guidelines that could easily be applied and evaluated in the day-to-day practice of architecture.

It was noted in the article that with slight adjustments, the methodology could be used for other similar investigations $(\mathrm{Ng}, 2005, \mathrm{pg} 16)$. To add further reliability to this research method, Professor Peter Tregenza, another leading researcher in the field of daylight regulation, was also involved in this research, providing guidance with his vast knowledge of regulation for daylight in the United Kingdom.

Measurements were taken over a period of 6 months during 2000 and 2001 in a number of dense housing estates using the daylight factor method. Ng commented that while the setting up of equipment was straightforward, selecting and obtaining suitable units for measurement, rotating the photocells and waiting for the sky to turn overcast were not predictable (Ng, 2005, pg 17).

The next step in the methodology was to establish an acceptable minimum standard for daylight performance. 12 major high-density housing estates were survey and had computational simulation conducted on 6000 windows within those 12 estates (Ng, 2005). Next, a tool was developed that aimed at achieving simplicity while still being considered reasonable accuracy.

Statistical and parametric methods were used to develop this tool. Computational simulations were used to provide parametric results. This method was chosen as it provided quick and convenient means to gather the necessary data. Design scenarios were constructed to yield data points of the full range of UVA (Ng, 2005).

Dr Danny H. W. Li of the Building Energy Research Group at the City University of Hong Kong was the primary author of two papers useful for this research. The first paper published in 1999, assessed the performance of daylight in a unit in a typical Hong Kong Residential Building using three calculation methods (Li et al., 1999). Li's second article looked at the performance of daylight and energy use in heavily obstructed residential buildings through computer simulation (Li et al., 2006).

Li's first paper involves a discussion on the daylight regulations in Hong Kong and Britain. Identification of the factors of the built environment that affect daylight performance were 
then given. Three calculation methods were then identified and applied to the unit being assessed. To simplify the calculation, a continuous obstruction parallel to the window was used, it was considered a fair representation of the real situations found in Hong Kong. Variations of the obstruction angle were tested to determine the threshold angle to achieve minimum daylight factors in dwellings.

Similar to Li's first article, the building variables that affect daylight were identified and discussed. This research involved the computer simulation of the daylight performance in high-rise residential buildings in Hong Kong. The simulation program used in the study was EnergyPlus. As with his earlier study, Li selected a unit considered representative of typical domestic units in Hong Kong and analysed it with a continuous obstruction parallel to the window representing the heavily obstructed units observed in Hong Kong. The unit was simulated with four different orientations: North, East, South and West. Cumulative frequencies of hourly indoor daylight for each of the orientations were graphed. The percent of the year that the recommended illuminance level of 150 lux was achieved was determined using the graphs.

\section{United Kingdom}

William Allen, of the Building Research Station wrote an article in 1943 that discussed the daylighting of buildings in urban environments (Allen, 1943). This is some of the earlier research in this field and although there have been many technological developments, the underlying methodology of this work is still relevant today. Allen investigated the daylight performance in urban buildings, testing a variety of situations including building form, spacing and height. Each situation was assessed using two calculation methods available at the time: the Waldram diagram and the daylight factor protractor. These calculations were used to identify the relationship between the various factors and provide some recommendations on how to design urban buildings to be well daylit.

An article on daylight in domestic buildings in northern Europe, written by Wilson and Brotas (2001), investigated the effects that energy efficient design of domestic buildings has on the daylight levels. Within this article, the British daylight recommendations are discussed and later used as a means of assessing the performance of the buildings analysed. Computer simulations of illuminance levels in a model building were conducted for various floors and orientations. From these simulations, the data was analysed using regression analysis to give an equation based on the daylight factor formula, which considers the effect of reflected sunlight.

Nabil and Mardaljevic's (2006) paper describes the application of a new method of assessing daylight in buildings. In this paper, a hypothetical building was modelled and simulated using RADIANCE. The 'base case' and two variations were tested to evaluate the Useful Daylight Illuminances method to assess design options. Although this study focused on commercial buildings, the testing method is relevant to this research.

The final piece of literature originates in Singapore; it is similar to the work from Hong Kong in that Singapore is another city where the majority of dwellings are high-density residential 
housing estates. Research conducted by Ullah and Lin (2003) investigated the daylight performance in four housing estates in Singapore. This research involved the simulation of four buildings with three units modelled in each building, and four different orientations. Simplified models were constructed with sufficient detail to obtain indoor and outdoor reflected components. Tables were produced to identify the best performing situations.

\subsection{Conclusion}

From the literature, it is clear that natural light is important for the prevention of illness and for normal human activities, but there is limited knowledge on exactly how much exposure humans need for health and well-being. It is also clear that people generally prefer to live/work in daylit spaces. 'Adequate' natural light increases the potential reductions in energy used for lighting, but there is debate as to the validity of this in dwellings as people are generally at work during daylight hours. Overall, exposure to natural light is an essential part of life and regulation of natural light will ensure that people receive adequate natural light for health and well-being.

This survey of previous research produced a number of projects that aided the development of the research method. Although each project was different, there were often similarities in their approach. One of these similarities was in the use of representative models when simulating urban environment. However, the methodology provided by $\mathrm{Ng}$ (2005) was the most relevant and the thorough documentation of this process was also advantageous. 


\subsection{CRITIQUE OF REGULATION}

Chapter three provides a critique of NZBC G7. Within this critique, the origins of NZBC G7 are explored and the current code is reviewed. In Section 3.1 the origins of the New Zealand Building Code and Clause G7 for Natural Light are explored to become more familiar with the requirements and current compliance methods and to ensure the original intentions of the code were considered in the development of the new assessment method. Within this section, previous research undertaken on NZBC G7 is also discussed. Section 3.2 documents the review of NZBC G7 and how it compares to international codes and standards.

\subsection{New Zealand Building Code Clause G7 - Natural Light}

The New Zealand Building Code is a performance-based code that has been developed to ensure that all new buildings in New Zealand are of an acceptable standard for health, safety and well-being. The code comprises two preliminary clauses and 35 technical clauses. Each technical clause contains (Department of Building and Housing, n.d.1):

1. An Objective - The social objective that completed building work must achieve;

2. A Functional requirement - What the completed building work must do to satisfy the social objective;

3. Performance criteria - Qualitative or quantitative criteria which nominates how far the completed building must go in order to comply.

Compliance with the code is assessed prior to commencement of construction, with building consent being issued if the building meets the code requirements. The building code is enforced for all structures, however, some sections relate to certain types of buildings. This is the case with the code for natural light.

Clause G7 of the New Zealand Building Code is intended to ensure there is adequate natural light in habitable spaces within all types of housing. The objective of Clause G7 is to 'safeguard people from illness or loss of amenity due to isolation from natural light and the outside environment' (Department of Building and Housing, 2001, pg 3). The functional requirement specifies that habitable spaces shall provide adequate openings for natural light and for visual awareness of the outside environment (Department of Building and Housing, 2001, pg 3). The two performance criteria for fulfilling the functional requirement are that (Department of Building and Housing, 2001, pg 3):

- Natural light shall provide an illuminance of no less than 30 lux at floor level for $75 \%$ of the standard year. (G7.3.1)

- Openings to give awareness of the outside shall be transparent and provided in suitable locations. (G7.3.2) 
Definitions (Department of Building and Housing, 2001, pg 9):

- A habitable space is defined as a space used for activities normally associated with domestic living, but excludes any utility areas such as bathrooms and laundries.

- A standard year, for the purpose of determining natural lighting, is the hours between $8 \mathrm{am}$ and $5 \mathrm{pm}$ each day with an allowance being made for daylight saving.

The NZBC G7 Compliance Document gives a Verification Method and an Acceptable Solution. The verification method (G7/VM1) (Department of Building and Housing, 2001, pg 11) specifies the use of one of the Building Research Establishment (BRE) calculation methods for verifying the illuminance requirement. These calculation methods use the daylight factor approach, and the external illuminance level exceeded $75 \%$ of the standard year in Auckland, Wellington, Christchurch or Invercargill (Standards Association of New Zealand, 1984, pg 29-35).

The acceptable solution (G7/AS1) given in the NZBC G7 Compliance Document (Department of Building and Housing, 2001, pg 13) is broken into two parts: vertical windows in external walls; and awareness of the outside environment. Vertical windows in external walls are to have:

a) A window area of no less than $10 \%$ of the floor area;

b) A glazing transmittance of no less than 0.7; and

c) A head height of at least:

i. half the room width for windows on the same side or adjacent sides of a room; and

ii. one quarter the room width for windows on opposite sides of the room.

It is noted in the Compliance Document that $10 \%$ window area to floor area equates to approximately 33 lux at floor level for $75 \%$ of the standard year and that in large rooms where the suggested head height is impractical, a window area in excess of $10 \%$ of the floor area may be needed (Department of Building and Housing, 2001, pg 13). Within the acceptable solution there are three considerations, these relate to obstructions and surface reflectances (Department of Building and Housing, 2001). In situations where natural light entering the building is obstructed, the total window area needs to be increased or high interior surface reflectances should be provided. Where parts of the floor fall beyond the no-sky line, high surface reflectances should be used. 


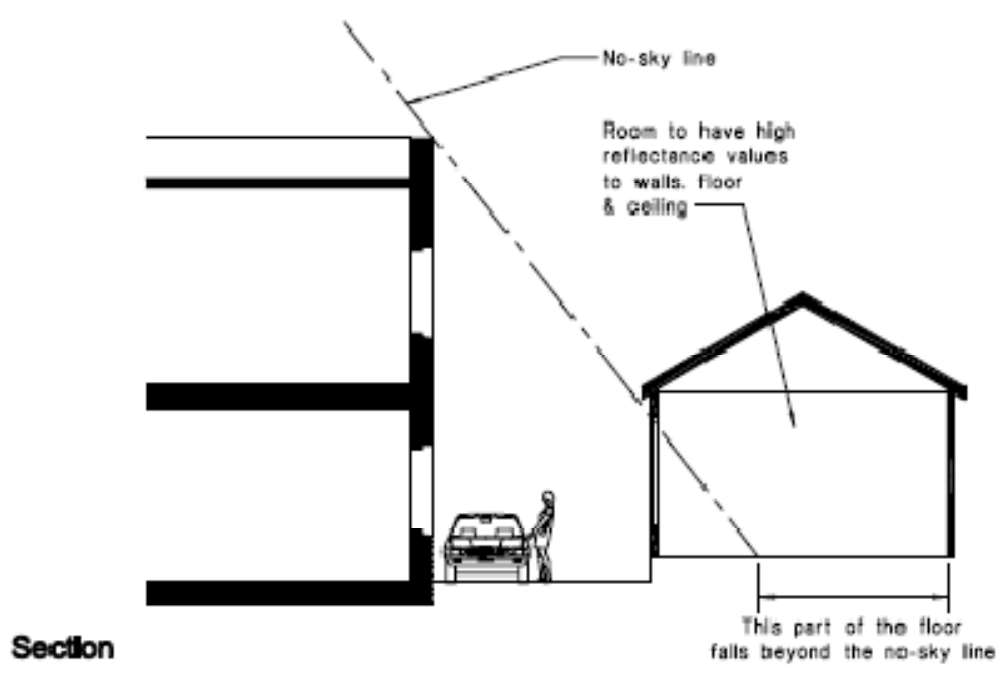

Figure 3 - No -sky line as defined by DBH (Department of Building and Housig, 2001, pg 14)

The minimum surface reflectances are shown below (Department of Building and Housing, 2001, pg 15):

Table 1 - NZBC G7 Minimum Surface Reflectances

\begin{tabular}{|l|c|c|c|}
\hline Reflectance level required & \multicolumn{3}{|c|}{ Minimum surface reflectance } \\
\hline & Ceilings & Walls & Floor \\
\hline Medium reflectance & 0.7 & 0.4 & 0.2 \\
\hline High reflectance & 0.7 & 0.6 & 0.4 \\
\hline
\end{tabular}

The solutions for awareness of the outside environment are (Department of Building and Housing, 2001, pg 16):

1. At least $50 \%$ of the glazed area provided for natural light in habitable spaces shall be clear glazed. The clear glazing shall be located in the zone between the levels $900 \mathrm{~mm}$ and $2000 \mathrm{~mm}$ from floor level.

2. It is acceptable for awareness of the outside to be provided through another space.

As was identified in the Introduction Chapter, this research was dealing specifically with the illuminance requirement (G7.3.1) of Clause G7 and as such, there will be no further mention of the view requirements (G7.3.2).

\subsubsection{Brief History of the New Zealand Building Code}

The first major research on the topic of building controls in New Zealand was a project conducted by the School of Architecture, Victoria University of Wellington, sponsored by the Ministry of Works and Development in 1979 (Archives New Zealand, 2007). The results from this research project found that the lack of uniform records and proliferation of control documents was having an adverse impact on construction cost' (Archives New Zealand, 2007). 
The findings from the 1979 report sparked the Department of Internal Affairs Forum of Building Controls to sanction a major review of the building and planning controls (Archives New Zealand, 2007). In 1983, a 'Review of Planning Building Controls' (Archives New Zealand, 2006, pg 3) was produced by the Office of the Review of Building and Planning Control, identifying the need for: a possible Building Industry Act; a National Building Code; and a Building Industry Council to be formed. In 1986 the Building Industry Commission (BIC) was established. The BIC was formed to 'review the whole spectrum of building controls and to consider a new national building code.' (Archives New Zealand, 2006, pg 4)

A report was presented by the BIC in January of 1990 to Government which identified the proposed reforms, including a draft Building Bill and National Building Code. In December 1991 the Building Act was developed resulting in the BIC being replaced by the Building Industry Authority (BIA) in February 1992. The Building Act was officially implemented in July 1992 (Archives New Zealand, 2006, pg 4).

The Building Regulations 1992 were produced under the Building Act, containing the New Zealand Building Code (Archives New Zealand, 2006, pg 4). It was also the responsibility of the BIA to prepare the 'Approval or Compliance Documents' for establishing compliance with the building code (Archives New Zealand, 2006, pg 4).

The New Zealand Building Code is now managed by the Department of Building and Housing (DBH). The DBH was established in November 2004 and was aimed at 'bringing together various agencies involved with regulatory and compliance issues for the building sector' (Archives New Zealand, 2006, pg 1), of which the Building Industry Authority (BIA) was included.

\subsubsection{Development of NZBC G7}

There is little documentation available on the origin of the natural light performance requirements and means of compliance. A report by Christopher Cuttle (2000) for the BIA identified that he was a consulting adviser to the Board of the Commission, but he was not a party to the Board's discussion and was not a participant in the final drafting. An interview with Cuttle was undertaken to gain an understanding of where the requirements and means of compliance came from and the original intentions of the code.

\section{Intentions}

The outline for the code was that achievable performance measures were suggested. Measurement procedures and verification procedures were to be provided to show that the performance measures were achieved. It was not intended as an advisory document but rather specific requirements that could be measured and verified. The code was to provide minimum levels of satisfaction, where anything below the requirement is considered unsatisfactory. 


\section{Performance requirement}

The suggestions for the performance measures were based on overseas recommendations. Cuttle identified that it was "virtually impossible to find any solid research that said that this is the number above which everything is ok and below which it isn't." American and European recommendations and Cuttle's personal experience were taken into consideration when recommending the performance measures to the Commission.

Recommendations as to the measurement procedure were based on those specified by organisations including the Illuminating Engineering Society (IES) and British Research Establishment (BRE). However, the working plane was adapted to suit the situation being assessed. It was not appropriate to have the working plane at the standard $0.7 \mathrm{~m}$ (for offices) (British Standards Institution, 1992, pg 5), therefore it was suggested that, as all dwellings have a floor, that the measurement plane be floor level. The recommendations were to take the conventional procedures and simply apply them to living situations.

An important finding from the interview was that apartments were considered in the recommendations, however, there has been a lot of development since then where the code requirements have been 'stretched to their absolute limits', which were not envisaged.

\subsubsection{Previous Research on NZBC G7}

This section summarises the previous research on NZBC G7. The first research was conducted by Christopher Cuttle and was an examination of NZBC G7 with a particular focus on the view requirements. The second research was conducted by Auckland Uniservices Ltd providing a review of Auckland apartment stock. The final research was a review of the Wellington apartment stock and attempted to identify shortfalls in the current regulation.

Cuttle's (2000) examination of NZBC G7 provided an assessment focusing on: the original intention of the clause; whether it is serving its intended purpose; and scope for improvement. Cuttle's discussion on the current acceptable solutions highlighted the need for further development in this area. Misapplication and unsatisfactory outcomes were identified as two issues that have arisen in recent years. Stating that it 'requires little imagination to think of situations that would comply but which would not accord with the original intention' (Cuttle, 2000, pg 3). Cuttle identified high-density apartment developments as being of particular concern. One of the major concerns with apartment developments is the use of internal habitable rooms, where the $10 \%$ window area requirement is achieved but this does not correspond to satisfactory view or daylight.

The key findings from Cuttle's report were that (Cuttle, 2000, pg 5-6):

- It seems that Clause G7 is applied to situations that were not envisaged at the time of drafting, leading to approvals being granted that do not accord with the original intention of sufficient light being provided in habitable spaces. 
- NZBC Clause G7 Natural Light is based on the premise that a habitable room should have a window in an external wall, and that the window should provide reasonable levels of daylight illumination and visual amenity, but this is not apparent in the current regulation.

- Compliance with the Clause does not ensure that the solution is in accord with the premise. It is evident that applications that are not in accord with the premise have been granted approval.

- There seems to be no point in requiring designers to devise ways of demonstrating compliance with a clause that does not achieve its intentions.

Living the highlife: A review of apartment living in inner-city Auckland was conducted by Auckland Uniservices Ltd (2004) into the trends and nature of apartment development in Auckland. In this report, the performance of these apartments in terms of the needs of occupants and long term durability and amenity were assessed. As part of this, the natural light performance was assessed and, although the majority of occupants were satisfied with the amount of natural light, 2 out of the 40 apartment occupants were not satisfied with the natural light in their bedrooms (Auckland Uniservies Ltd, 2004, pg 15-16). In the first situation, the room was an internal bedroom, where the natural light was designed to be received through another room (as shown in the diagram below). In this situation, the occupants felt that the bedroom received no light. The second bedroom had a small window that did not allow sufficient light into the room. It can be concluded that overall Auckland apartments are performing well in terms of natural light, however, the 2 occupants that were not satisfied are of concern.

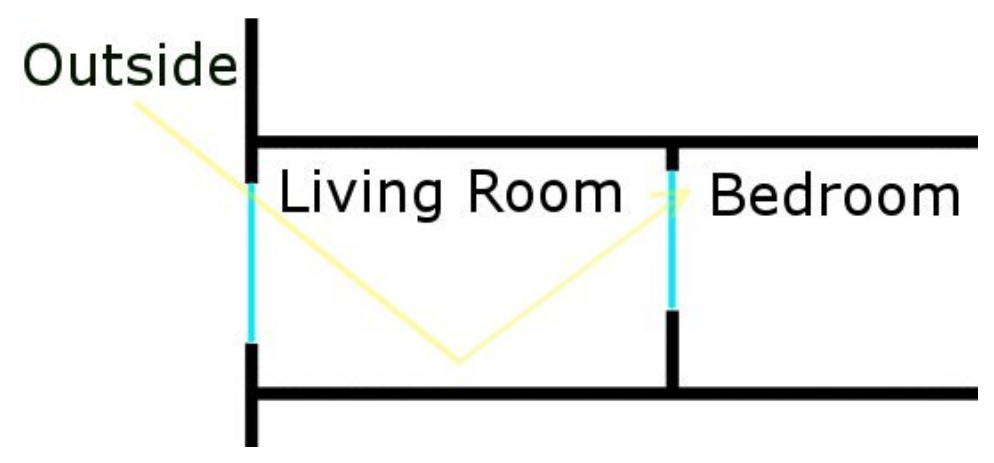

In 2006 an investigation into daylight in the current apartment stock in Wellington (New Zealand's capital) was undertaken (Stewart, 2006b). The findings from the research identified that daylight quality and performance in Wellington apartments is poor. 20 innercity apartment buildings were assessed, looking at the complexity of design for daylight and were assigned ratings of 1 to 5 where 1 meant no daylight design and 5 meant excellent daylighting design. The average rating was 2.55 , which corresponds to adequate glazing but little to no control measures. The research also investigated the quantity of daylight in habitable rooms, identifying that of the five apartments measured only one complied with the minimum code requirement. The final aspect investigated in this research attempted to identify why some apartments are able to receive consent when they do not reach the minimum code requirement post-construction. It was found that many buildings were receiving consent using the acceptable solution of a glazed area $10 \%$ of the floor area, which has proven to not be an adequate assessment method for inner-city apartments. It 
was also mentioned that there is currently a lack of tools available for Territorial Authorities to accurately assess compliance with this clause.

From the previous research on NZBC G7, it was obvious that further research was needed on this topic.

\subsection{Comparison of NZBC G7 with International Standards and Recommendations}

This section provides a review of the international regulations and recommendations. This review was used to evaluate NZBC G7 on an international scale by comparing to other regulations and standards. Daylight regulations can be split into four main types, as given by Boubekri (2004). These types are: Illuminance based; Daylight factor based; Window size based; and Solar Zoning legislations.

\subsubsection{Illuminance-Based Standards and Recommendations}

The first daylight regulation type is illuminance based. The illuminance based requirements measure the amount of light hitting the working plane. The NZBC G7 requirement of 30 lux at floor level fits into this category. The following illuminance recommendations show the minimum illuminance to be provided by daylight in some countries and recommended illuminance levels (artificial and daylight combined) to conduct various tasks.

\section{Dwellings}

USA

The Building Officials and Code Administrators (BOCA) National Building Code for the United States gives an illuminance level that needs to be provided on the vertical plane adjacent to the window in all habitable and occupiable rooms. The code specifies that an illuminance of 250 foot-candles ( 2691 lux) is provided on the vertical plane next to the window to give and average illuminance of 6 foot-candles (64.58 lux) at a working plane height of 30 inches (762 mm) (Boubekri, 2004, pg 58).

The Illuminating Engineering Society of North America (IESNA) produces a Lighting Handbook (Rea, 2000) for industry professionals containing explanations of concepts, techniques and applications. 
Table 2 and Table 3 are drawn from the IESNA Lighting Handbook. They show the recommended illuminance levels for various tasks conducted in dwellings. It should be noted that it is not necessary that these are achieved through natural light alone. 
Table 2 - IESNA (Rea, 2000, pg 10.13)

Orientation and simple visual tasks. Visual tasks are found in public spaces where reading and visual inspection are only occasionally performed. Higher levels are recommended for tasks where visual performance is occasionally important.

\begin{tabular}{|l|l|l|}
\hline A & Public Space & $30 ~ I x$ \\
\hline B & Simple Orientation for short visits & $50 ~ I x$ \\
\hline C & Working spaces where simple visual tasks are performed & $100 \mathrm{Ix}$ \\
\hline
\end{tabular}

Common visual tasks. Visual performance is important. These tasks are found in commercial, industrial and residential applications. Recommended illuminance levels differ because of the characteristics of visual tasks with critical elements of low contrast or small size.

\begin{tabular}{|l|l|l|}
\hline D & Performance of visual tasks of high contrast and large size & $300 \mathrm{Ix}$ \\
\hline $\mathrm{E}$ & $\begin{array}{l}\text { Performance of visual tasks of high contrast and small size, or visual tasks } \\
\text { of low contrast and large size }\end{array}$ & $500 \mathrm{Ix}$ \\
\hline $\mathrm{F}$ & Performance of visual tasks of low contrast and small size & $1000 \mathrm{Ix}$ \\
\hline
\end{tabular}

Table 3 - IESNA (Rea, 2000, pg 10.14-10.16)

\begin{tabular}{|c|c|c|}
\hline Tasks in Residences & Category & Illuminance (lx) \\
\hline General lighting & $\mathrm{B}$ & $50 \mathrm{~lx}$ \\
\hline Conversation, relaxing, and entertaining & A & $30 \mathrm{~lx}$ \\
\hline Passage areas (circulation) & A & $30 \mathrm{~lx}$ \\
\hline \multicolumn{3}{|l|}{ Specific visual tasks } \\
\hline Dining & $\mathrm{B}$ & $50 \mathrm{~lx}$ \\
\hline \multicolumn{3}{|l|}{ Grooming } \\
\hline Makeup and shaving & $\mathrm{D}$ & $300 \mathrm{~lx}$ \\
\hline Dressing evaluation (mirror) & $\mathrm{D}$ & $300 \mathrm{~lx}$ \\
\hline \multicolumn{3}{|l|}{ Handcrafts and hobbies } \\
\hline Ordinary tasks (e.g. crafts) & $\mathrm{D}$ & $300 \mathrm{~lx}$ \\
\hline Difficult tasks (e.g. sewing) & $\mathrm{E}$ & $500 \mathrm{~lx}$ \\
\hline Critical tasks (e.g. workbench) & $\mathrm{F}$ & $1000 \mathrm{~lx}$ \\
\hline Ironing & $\mathrm{D}$ & $300 \mathrm{~lx}$ \\
\hline \multicolumn{3}{|l|}{ Kitchen counter } \\
\hline Critical seeing (e.g. cutting) & $\mathrm{E}$ & $500 \mathrm{Ix}$ \\
\hline General & $\mathrm{D}$ & $300 \mathrm{~lx}$ \\
\hline \multicolumn{3}{|l|}{ Kitchen range } \\
\hline Difficult seeing (e.g. cooking) & $\mathrm{E}$ & $500 \mathrm{Ix}$ \\
\hline \multicolumn{3}{|l|}{ Kitchen sink } \\
\hline Difficult seeing & $E$ & $500 \mathrm{Ix}$ \\
\hline Noncritical (clean up) & $\mathrm{D}$ & $300 \mathrm{~lx}$ \\
\hline Laundry & $\mathrm{D}$ & $300 \mathrm{~lx}$ \\
\hline Music study (piano) & $\mathrm{D}$ & $300 \mathrm{Ix}$ \\
\hline \multicolumn{3}{|l|}{ Reading } \\
\hline In a chair (causal) & $\mathrm{D}$ & $300 \mathrm{~lx}$ \\
\hline In a chair (serious) & $\mathrm{E}$ & $500 \mathrm{Ix}$ \\
\hline In bed (casual) & $\mathrm{D}$ & $300 \mathrm{~lx}$ \\
\hline \multicolumn{3}{|l|}{ At desk } \\
\hline Casual & $\mathrm{D}$ & $300 \mathrm{~lx}$ \\
\hline Serious & $\mathrm{E}$ & $500 \mathrm{Ix}$ \\
\hline Table Games & $\mathrm{D}$ & $300 \operatorname{lux}$ \\
\hline
\end{tabular}




\section{All Buildings}

\section{UK}

The British Standard 8206 provides minimum standards for different types of tasks. Table 5 is adapted from this standard.

\section{NEW ZEALAND/AUSTRALIA}

New Zealand has a joint standard with Australia to specify the minimum acceptable standards for performing various tasks, Table 6 shows the recommended values given by the New Zealand and Australian standard.

\section{Offices/Classrooms}

\section{CANADA}

The Canadian Department of Public Works recommends an average of 200 lux along the perimeter of office spaces at a depth of 3 metres for $80 \%$ of the business hours ( $8 \mathrm{am}-5 \mathrm{pm}$ ) (Boubekri, 2004, pg 58). Although this recommendation is for offices, the use of a percent of the business hours (or standard year) is similar to the NZBC G7 use of $75 \%$ of the standard year to account for the variability of daylight.

\section{FRANCE}

The French Decret no.83-722 and the Lettre-circulaire DRT no. 90/11 provide requirements for lighting in workplaces, the later is concerned with daylight and view. Table 4 shows the recommended illuminance levels given in Lettre-circulaire DRT no. 90/11 (Boubekri, 2004, pg 58).

Table 4 - Lettre-circulaire DRT no. 90/11 Recommended Illuminance Levels

\begin{tabular}{|l|c|c|}
\hline Type of activity & Decreed Level [lux] & $\begin{array}{l}\text { I.A.M } \\
\text { [lux] }\end{array}$ \\
\hline Interior circulation space & 40 & 70 \\
\hline Stairs and storage & 60 & 110 \\
\hline Workplaces, dressing rooms, and bathrooms & 120 & 210 \\
\hline Exterior circulation space & 10 & 20 \\
\hline Exterior spaces where permanent work is performed & 40 & 70 \\
\hline
\end{tabular}

NOTE: 'Decreed' is the French term used for regulation and I.A.M. is the Initial Average Illuminance. 
Table 5 - BS 8206: Part 2: 1992 Recommended Illuminances (British Standards Institution, 1992, pg 36)

\begin{tabular}{|c|c|c|c|c|c|c|c|c|c|c|c|c|}
\hline & 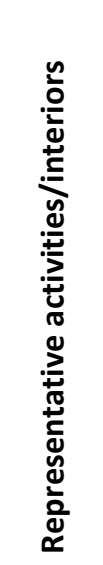 & 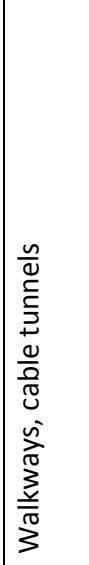 & 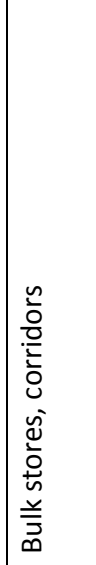 & 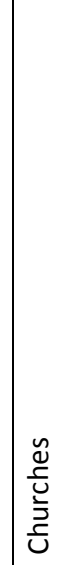 & 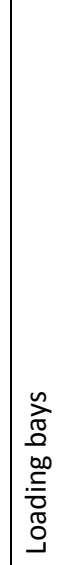 & 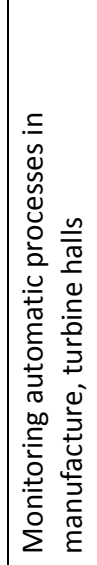 & 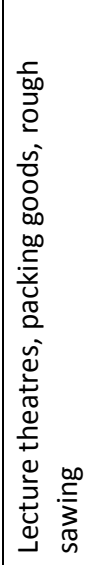 & 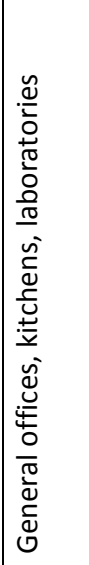 & 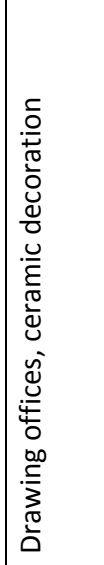 & 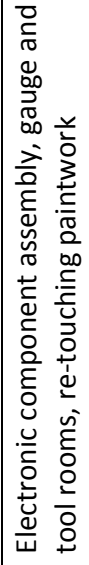 & 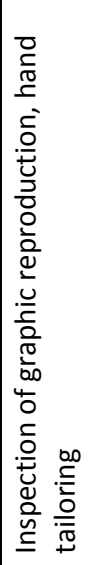 & 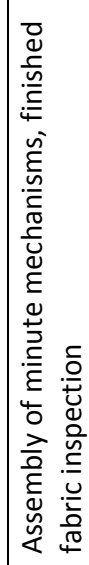 \\
\hline 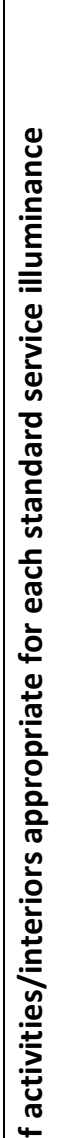 & 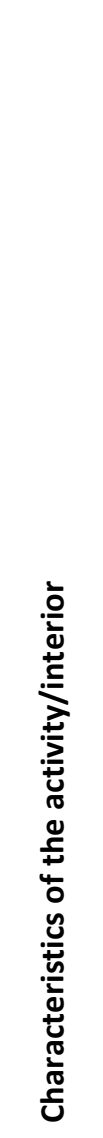 & 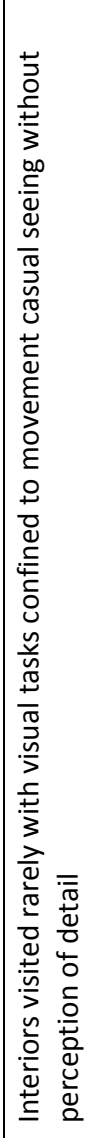 & 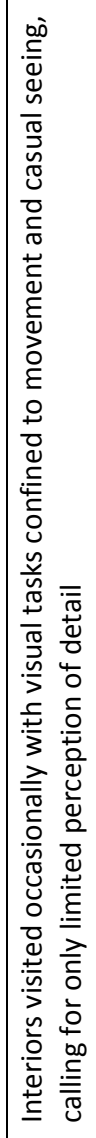 & 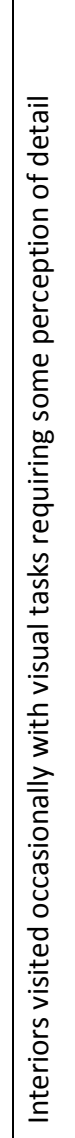 & 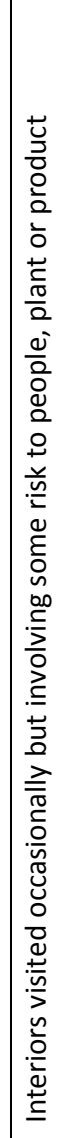 & 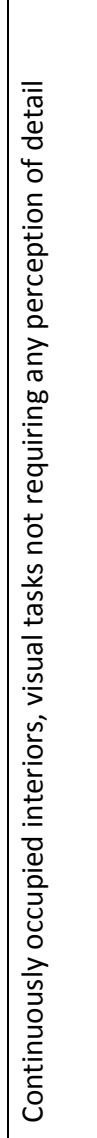 & 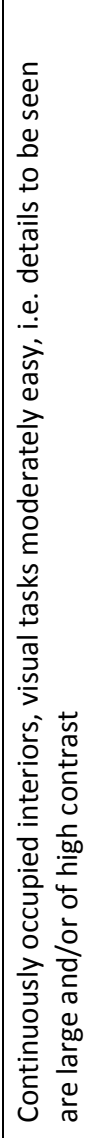 & 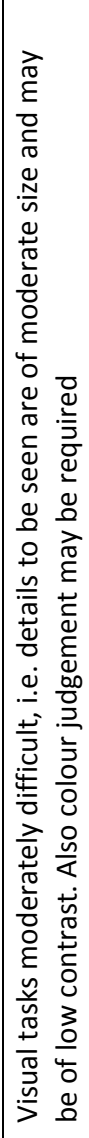 & 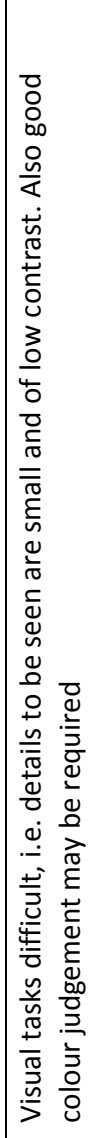 & 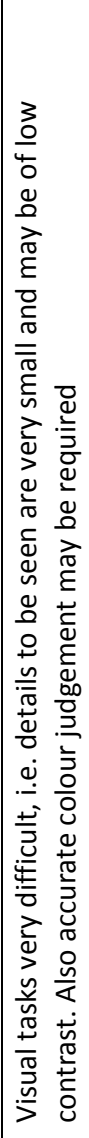 & 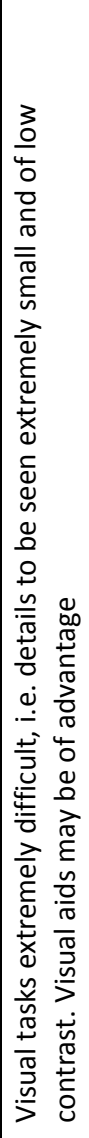 & 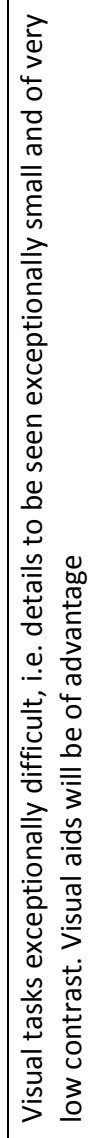 \\
\hline 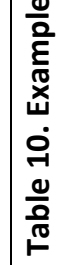 & 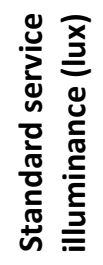 & in & & & In & $\tilde{N}$ & $\bar{m}$ & ถ & 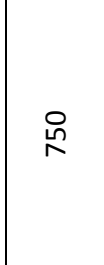 & 우 & $\stackrel{n}{\rightarrow}$ & ָ̊ \\
\hline
\end{tabular}


Table 6 - ASNZS 1680.1 Recommended Illuminances (Standard Australia/Standards New Zealand, 2006)

\begin{tabular}{|c|c|c|c|c|c|c|c|c|c|c|}
\hline 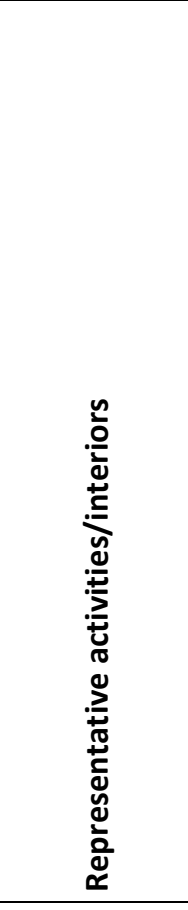 & 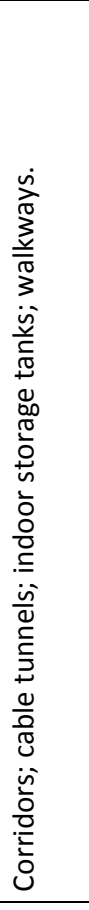 & 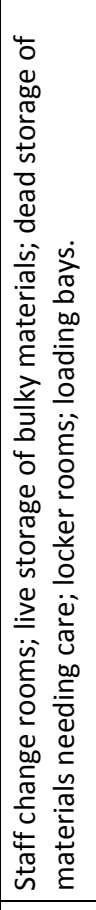 & 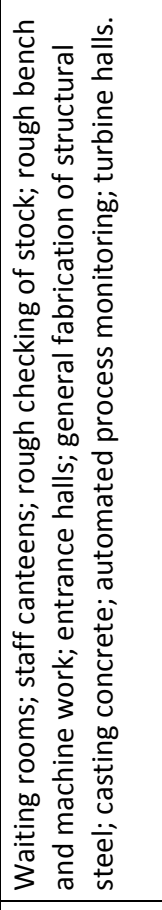 & 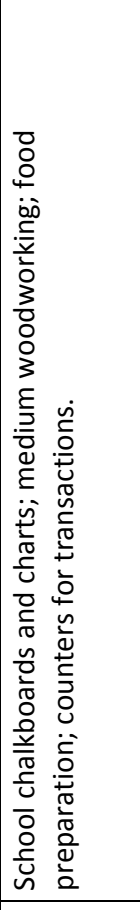 & 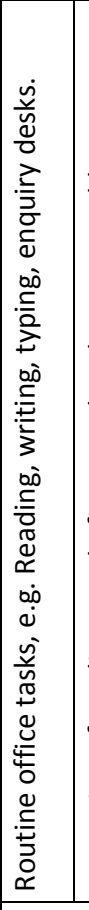 & 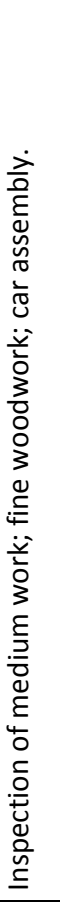 & 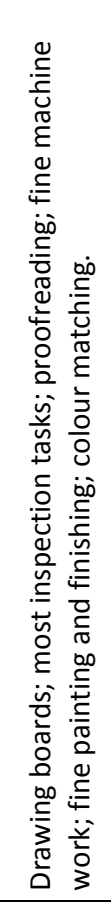 & 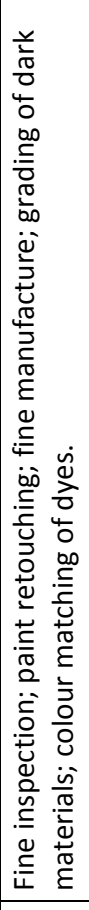 & 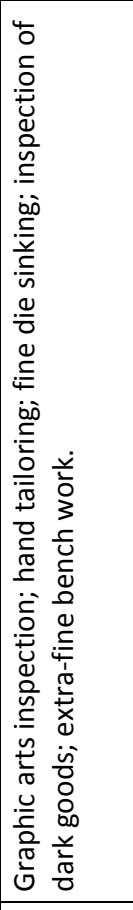 & 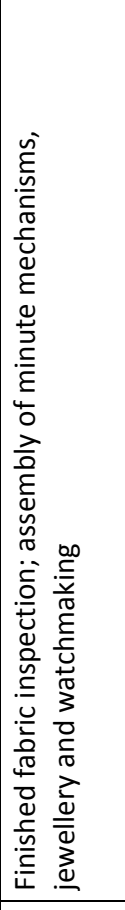 \\
\hline 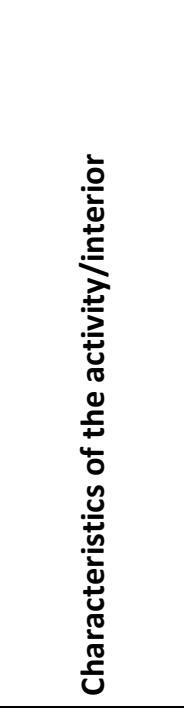 & 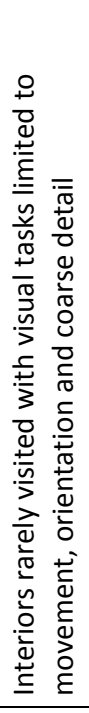 & 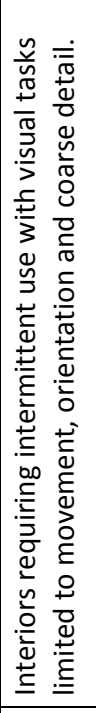 & 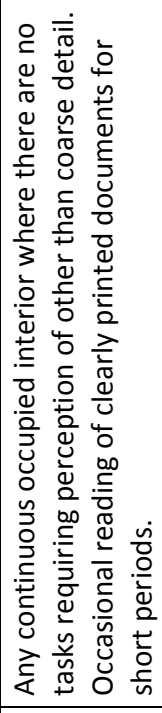 & 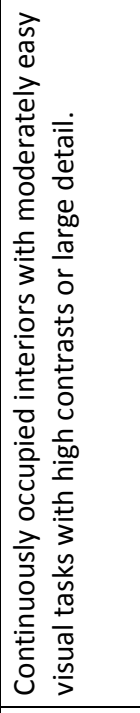 & \multicolumn{2}{|c|}{ 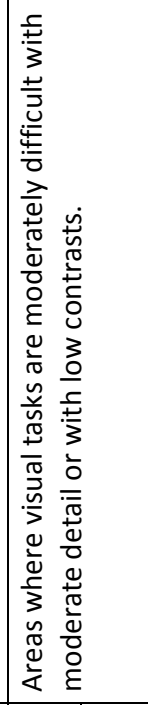 } & 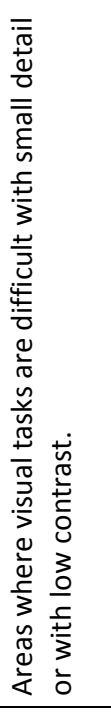 & 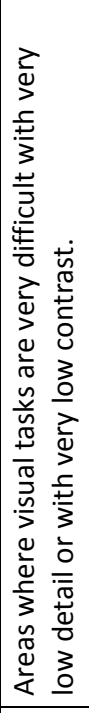 & 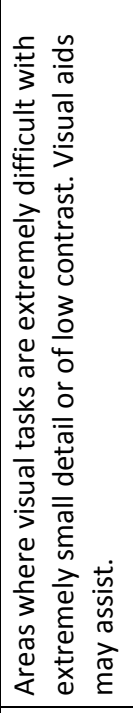 & 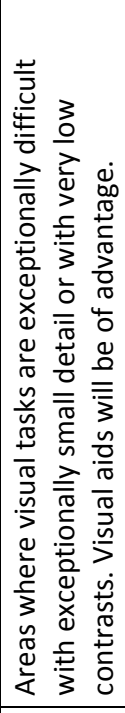 \\
\hline 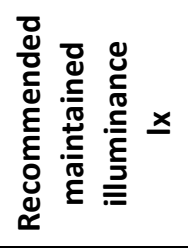 & q & $\triangleright$ & 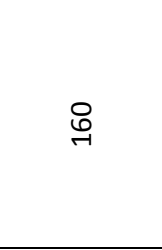 & $\stackrel{\stackrel{+}{\sim}}{\sim}$ & $\underset{m}{\stackrel{N}{*}}$ & ৪্ণ & 8 & ঃి & 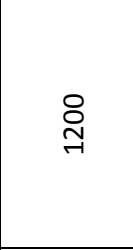 & 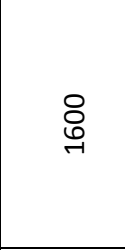 \\
\hline & 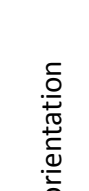 & & $\begin{array}{l}\frac{0}{0} \\
\frac{\xi}{\xi} \\
\bar{n}\end{array}$ & 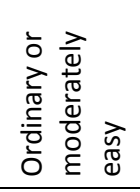 & 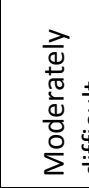 & & 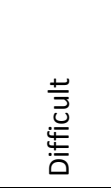 & 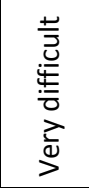 & \multirow[b]{2}{*}{ 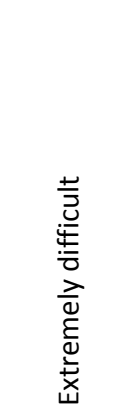 } & \multirow{2}{*}{ 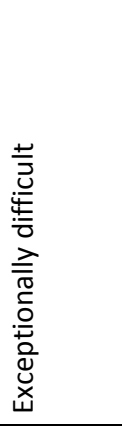 } \\
\hline 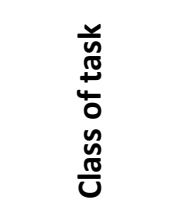 & 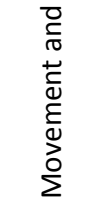 & 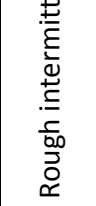 & \multicolumn{6}{|c|}{ 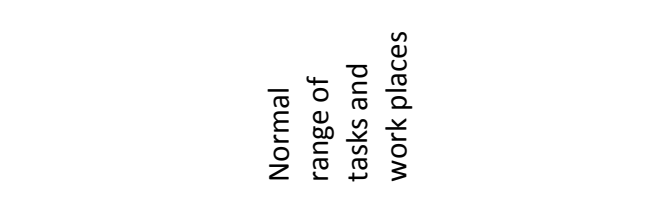 } & & \\
\hline
\end{tabular}




\subsubsection{Daylight-Factor Based Standards and Recommendations}

The second type of daylight regulation specifies the recommended minimum daylight factors for specific spaces. The daylight factors are expressed as a percentage of the daylight available that reaches the working plane. Daylight Factor calculation is discussed in more detail in Section 6.1, including an image of this on page 85.

\section{Dwellings}

\section{GERMANY}

The German standard DIN 5034-1 (DIN Deutsches Institut für Normung, 1999) and 5034-4 (DIN Deutsches Institut für Normung, 1994) give recommended daylight levels in buildings. DIN 5034-1 gives the general recommendations, specifying that a mean daylight factor of at least $0.9 \%$ is considered psychologically satisfactory and a minimum daylight factor of $0.75 \%$ should be provided at the 'least favourable point' in habitable rooms. The 'least favourable point' is measured on a working plane of $0.85 \mathrm{~m}$ and is taken at the midpoint of the room depth at a distance $1 \mathrm{~m}$ from the side walls. These values are also given in 5034-4 and were the basis for the window specifications explained in the next section.

\section{UNITED KINGDOM}

\section{Department of Scientific and Industrial Research}

Post-war construction saw the inclusion of natural light recommendations for UK housing. It was recommended that a daylight factor of $1 \%$ was provided in living rooms, with bedrooms receiving $0.5 \%$ and kitchens, $2 \%$ (Chynoweth, 2005, pg 261). These values were most likely the predecessors for the British Standard for daylight, BS 8206 Part 2.

\section{BS 8206 Part 2}

The British Standard for daylight, BS 8206 Part 2, (British Standards Institution, 1992) gives the minimum average daylight factor values for dwellings. It is recommended that a minimum average daylight factor of $1 \%$ in bedrooms, $1.5 \%$ in living rooms and $2 \%$ in kitchens is provided in all dwellings, even if a predominantly daylit appearance is not required (British Standards Institution, 1992, pg 9). The standard also gives the method for assessing right to light, stating that 'a minimum sky factor of $0.2 \%$ over one-half of the room at a working plane height of $850 \mathrm{~mm}$ is normally considered sufficient.' (British Standards Institution, 1992, pg 14) 


\section{All Buildings}

\section{UNITED KINGDOM}

\section{Right to Light}

A 0.2 percent daylight factor is used in the United Kingdom in 'right to light' assessments. This value has been used since around 1925 and is based on the work of Percy Waldram (Chynoweth, 2005, pg 251). The 0.2 percent daylight factor, often referred to as the "grumble point" is believed to be the threshold level of illuminance below which people will consistently grumble (Chynoweth, 2005, pg 252) and daylight is considered inadequate for any normal purpose (Allen, 1943, pg 85). There is some belief that this value is inadequate by contemporary standards (Chynoweth, 2005, pg 252). There is little solid evidence on the origin of this value and with Waldram referring to this value as an 'assumption' in a paper he wrote in 1925 there has been doubt surrounding the validity of this value in recent years (Chynoweth, 2005, pg 253).

\section{$\underline{B R E}$}

The Building Research Establishment (BRE) published a daylight design guide for architects (Building Research Energy Conservation Support Unit, 1998). Within this publication the following values are given to indicate the daylit appearance of a space (Building Research Energy Conservation Support Unit, 1998, pg 7):

- Less than $2 \%$

- Room looks gloomy under daylight alone

- Full electric lighting often needed during daytime

- Electric lighting dominates daytime appearance

- $2 \%-5 \%$

- Windows give a predominantly daylit appearance but supplementary electric lighting needed

- Usually the optimum range of daylighting for overall energy use

- $5 \%$ or more

- The room is strongly daylit

- Daytime electric lighting rarely needed

- Major thermal problems from large windows

\section{Offices/Classrooms}

\section{FRANCE}

A daylight factor of $1.5 \%$ is recommended for all classrooms in France, as is given in the recommendations for construction by the Ministere de l'Education (Boubekri, 2004, pg 58). 


\subsubsection{Window Size-Based Standards and Recommendations}

The third form of daylight regulation is based on window size. The NZBC G7 acceptable solution of $10 \%$ of the floor area is in this category. This is the most common type of legislation relating to daylight in interiors (Boubekri, 2004, pg 59). Within this section there are two types of sizes given: the glazed area as a percentage of the total floor area; and specific dimensions.

\section{Dwellings}

\section{AUSTRALIA}

The Building Code of Australia (BCA) specifies that all habitable rooms in residential buildings have a glazed area of $10 \%$ of the floor area (Australian Uniform Building Regulations Co-ordinating Council, 1990, pg F-19).

\section{GERMANY}

The German standard DIN 5034-4, (DIN Deutsches Institut für Normung, 1994) as mentioned earlier, gives recommended minimum window sizes in dwellings. The recommended window sizes are based on (DIN Deutsches Institut für Normung, 1994, pg 3):

- $\alpha$ : the angular height of the obstruction above the horizon;

- $h$ : room height, with the relevant window height, $h_{\mathrm{F}}$;

- $\quad b$ : room width; and

- $a$ : room depth.

An example of a window size table is shown in Table 7. There are 77 tables with a range of obstruction angles $\left(0^{\circ}-50^{\circ}\right)$, room heights $(2.4 m-3 m)$, room width $(2 m-8 m)$ and room depth $(3 m-8 m)$ (DIN Deutsches Institut für Normung, 1994, pg 3).

\section{JAPAN}

The Japanese Buildings Code (JBC) stipulates that all habitable rooms of continuous occupancy shall have a window no less than $14 \%\left(1 / 7^{\text {th }}\right)$ of the total floor area (Boubekri, 2004, pg 59).

\section{All Buildings}

\section{HONG KONG}

The Hong Kong Building Regulations, CAP.123 Part IV, gives the minimum lighting and ventilation levels for all rooms used for habitation, offices and kitchens. A glazed area that is $10 \%\left(1 / 10^{\text {th }}\right)$ of the floor area is required for lighting ( $\mathrm{Ng}, 2003 \mathrm{~b} ; 2005$; Li et al., 1999). In recent years there has been discussion on the applicability of this requirement for Hong Kong, as it is based on the laws developed in the UK for low-rise terrace type dwellings, 
with suggestions that it is no longer applicable to high-density cities with varying skylines (Ng, 2003b, pg 127).

\section{UK}

A glazed area of $10 \%$ of the floor area was used in the UK up until 1985 for the provision of daylight in dwellings. However, in 1985 that was removed, with the focus solely on assuring other's enjoyment to light is not compromised ( $\mathrm{Ng}, 2003 \mathrm{a})$. Recently there have been moves to reinstate a requirement for adequate daylight in dwellings, but this issue is currently unresolved (Wilson \& Brotas, 2001, pg 27).

\section{USA}

The US Building Officials and Code Administrators (BOCA) National Building Code also specifies a minimum glazed area of no less than $8 \%$ of the floor area for all rooms intended for human occupancy (Boubekri, 2004, pg 60). 
Table 7 - DIN 5034-4 (DIN Deutsches Institut für Normung, 1994, pg 11) Minimum window sizes

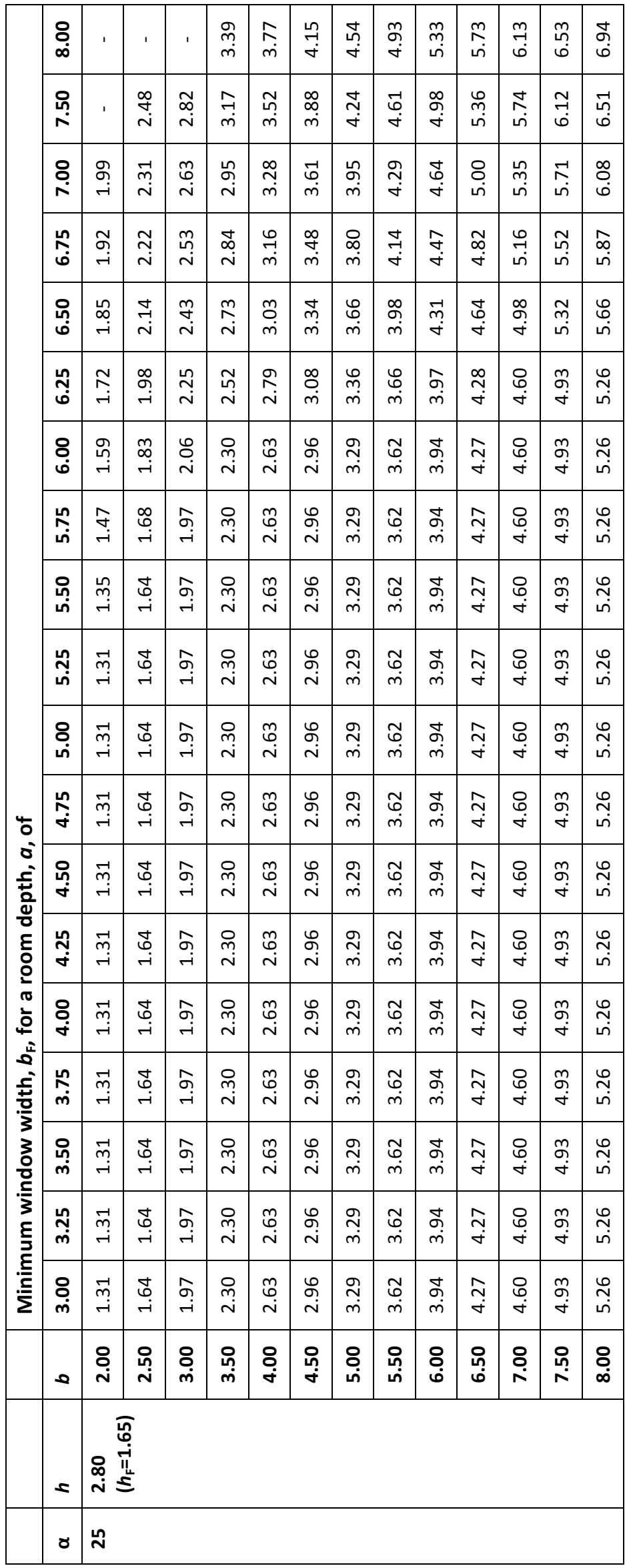




\subsubsection{Zoning Legislation}

The final type of daylight regulation is solar zoning legislation. This refers to the limiting of building massing to protect solar access. This form of regulation is typically controlled by planning departments of each city rather than at a national level (Boubekri, 2004, pg 59; Wilson \& Brotas, 2001, pg 28). This is also the case in New Zealand, where it is the Territorial Authorities responsibility to preserve natural light within their area. Two of New Zealand's major urban centres, Auckland and Wellington, both have legislation in place to preserve daylight and sunlight within the city. Both councils have District Plans that limit building heights and solar access in public areas (Auckland City Council, 2007a, pg 3-14; Wellington City Council, 2000, pg 12/5-12/6, 13/11, Map 32).

\section{HONG KONG}

In Hong Kong windows are required to face onto a street which is no less than 15 feet (4.5 metres) wide or face into a space with a Rectangular horizontal plane (RHP) of no less than $21 \mathrm{~m}^{2}$. A maximum incline angle to limit the height of obstructions is $71.5^{\circ}$ for habitable spaces, which equates to a ratio of 1:3 (street width to building height) ( $\mathrm{Ng}, 2004$, pg 24; Li et al., 1999, pg 213-214).

\section{PORTUGAL}

In Portugal, a street width to building height ratio of 1:1 is used to preserve daylight access at street level (Wilson \& Brotas, 2001, pg 28).

UK

A vertical sky component (a component of vertical daylight factor) of $27 \%$ is recommended as an acceptable level of light to receive at street level. This value is based on a $12 \mathrm{~m}$ wide street for an average terraced house. It can be difficult to achieve this in urban environments (Boubekri, 2004, pg 58-59).

\subsubsection{Discussion}

Following the review of overseas legislation it became clear that there are a number of different recommendations and techniques being used. This section compares these different recommendations and discuss the issues raised by some researches on the regulations of daylight.

Some researchers have suggested there is still a great deal of uncertainty surrounding the regulation of daylight. Boubekri (2004) stated that 'daylight legislation is still lagging behind,' with some problems due to the field of lighting and others related to the nature of daylight as a light source. Boubekri also highlighted the lack of consensus on how the visual performance standards ought to be expressed. Another issue highlighted by Boubekri was the development of standards that consider both quality and quantity of daylight. 
Allen (1943) highlighted the issue of devising performance measures that are capable of considering the variability of daylight throughout the day and year. Allen discouraged the use of specific intensities in regulation of daylight (i.e. Illuminance-based standards), suggesting the method should be to calculate the proportion light available outdoors that is provided at given points indoors, also known as the daylight factor. Chynoweth also supports the use of daylight factor values in legislations, identifying that this method 'reflects the capacity of the human eye to adapt to changing levels of sky luminance due to seasonal variations and weather conditions,' (Chynoweth, 2005, pg 254). Suggesting that this corresponds to the same perceived illumination regardless of the actual illuminance levels in the space. And based on this idea, illuminance values should not be used to determine the threshold level of adequate illumination (Chynoweth, 2005, pg 254).

The difficulty in distinguishing a difference between 'minimum' and 'reasonable' standards was another issue raised. Allen (1943) commented that the daylight provided is often not enough for any practical purpose and that the standards for daylight are often not achievable in dense development in cities. While guiding the development of daylight regulations in Hong Kong, Tregenza highlighted the importance of separating 'minimum' and 'reasonable' standards when developing regulations for urban environments ( $\mathrm{Ng}, 2005$, pg 16). This aspect becomes clear in Table 8 where there are two distinct groups on of standards: the 'minimum' standards like NZBC G7, BOCA, the Right to Light value and the German standard DIN 5034-1; and the 'reasonable' standards BS 8206.2 and the BRE Desktop guide to daylighting.

Many of the regulations in use today have come from the regulations developed in the UK in the late nineteenth century. These laws were originally developed for low-rise terrace type dwellings ( $\mathrm{Ng}, 2003 \mathrm{~b}, \mathrm{pg}$ 127). These laws did not take into account the complex skylines that exist in modern cities and as such are no longer able to accurately predict the performance of daylight in inner-city dwellings. Ng suggested that new laws and design methods must be developed to cope with contemporary design ( $\mathrm{Ng}, 2003 \mathrm{~b}, \mathrm{pg} 128$ ).

There was also a suggestion that some regulations are based on ventilation considerations rather than daylight performance, Li (1999) suggested that this was the case in both Hong Kong and the earlier British daylight requirements.

Table 8 shows a comparison of the different international regulations. Within this table, the recommended values for illuminance, daylight factor and window area are compared. To relate the different values easily the NZBC value has been converted to a daylight factor using the external illuminance levels exceeded for $75 \%$ of the standard year in Auckland, Wellington, Christchurch and Invercargill (Standards Australia/Standards New Zealand, 2006, pg 61). Using these values the international daylight factors were also converted to illuminance levels to compare with the NZBC G7 requirement. The purpose of Table 8 is to see whether NZBC G7 is in line with the various daylight codes and standards internationally. 
Table 8 - Comparison of NZBC G7 and International Codes and Standards indentified in Chapter 3.

\begin{tabular}{|c|c|c|c|c|c|c|c|c|c|c|c|}
\hline$\underbrace{y}_{0}$ & $\begin{array}{l}3 \\
3 \\
0 \\
0 \\
0 \\
\frac{\pi}{0} \\
0\end{array}$ & & $\frac{x}{3}$ & ठें & & & ' & ' & & & \\
\hline \multirow{3}{*}{ 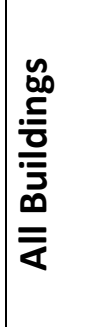 } & \multirow{2}{*}{ 음 } & & ' & ' & ํํ & $\stackrel{n}{\stackrel{n}{n} \underline{x}}$ & O을 & 㶽 & 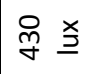 & ' & \\
\hline & & & , & ' & ঐे & 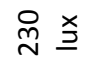 & 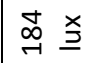 & 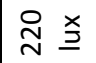 & $\underset{\exists}{\stackrel{ }{\Xi}}$ & ' & \\
\hline & 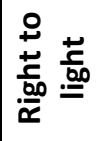 & & ' & ' & خें & $\frac{\underset{x}{a}}{\underset{N}{N}}$ & $\begin{array}{l}\frac{x}{\partial} \\
\underset{\sigma}{\sigma} \\
\stackrel{x}{\rightarrow} \\
\rightarrow\end{array}$ & $\frac{\stackrel{x}{I}}{\stackrel{N}{N}}$ & $\begin{array}{l}\frac{x}{3} \\
\stackrel{N}{A}\end{array}$ & 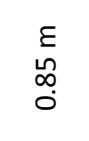 & \\
\hline \multirow{11}{*}{ 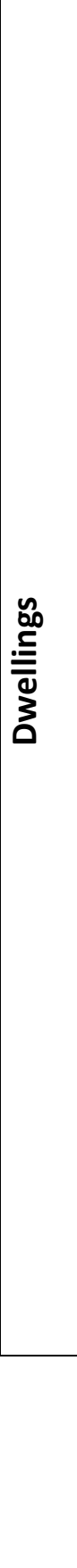 } & 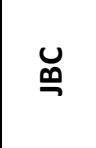 & 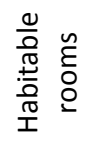 & ' & ' & & & ' & ' & ' & ' & ১े \\
\hline & ఫ్థ & 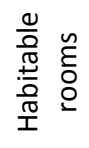 & ' & ' & & & ' & ' & ' & ' & すి \\
\hline & 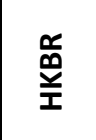 & 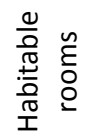 & ' & ' & & & ' & ' & ' & ' & ठें \\
\hline & \multirow{2}{*}{ 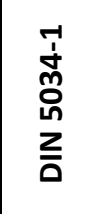 } & \multirow{2}{*}{ 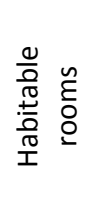 } & ' & ' & 总 & $\stackrel{\stackrel{\sim}{\oplus}}{\stackrel{x}{=}}$ & $\underset{\dot{0}}{+} \stackrel{x}{=}$ & $\underset{\infty}{\stackrel{n}{\mathcal{D}}} \underset{\underline{X}}{\underline{X}}$ & 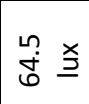 & $\stackrel{\substack{\infty \\
0}}{0} \varepsilon$ & \\
\hline & & & ' & ' & ذั. & 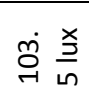 & $\underset{\infty}{\infty} \stackrel{x}{\underline{\infty}}$ & g $\stackrel{x}{\underline{x}}$ & $\stackrel{+}{\stackrel{x}{i}}$ & 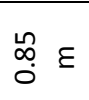 & \\
\hline & \multirow{3}{*}{ 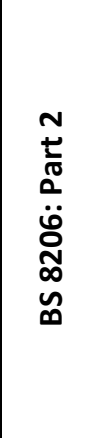 } & 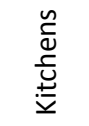 & ' & ' & ৯े & $\begin{array}{l}\frac{x}{\partial} \\
\stackrel{y}{N} \\
\text { N }\end{array}$ & 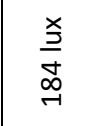 & 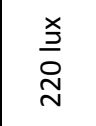 & 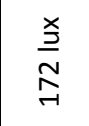 & ' & \\
\hline & & 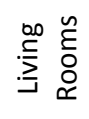 & ' & ' & 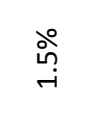 & 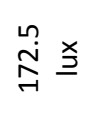 & $\stackrel{\infty}{\stackrel{m}{\rightarrow}} \underline{\underline{x}}$ & 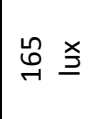 & $\underset{\exists}{\stackrel{్}{(x}}$ & ' & \\
\hline & & 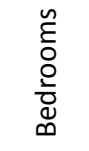 & ' & ' & ঐे & 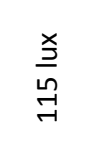 & $\frac{\frac{x}{\Xi}}{\sigma}$ & 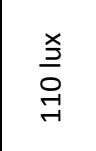 & $\frac{\sqrt[x]{3}}{0}$ & ' & \\
\hline & ఫั & 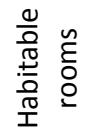 & 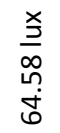 & ' & & & ' & ' & ' & $\begin{array}{l}\varepsilon \\
\stackrel{\varepsilon}{0} \\
\stackrel{0}{0}\end{array}$ & ১̊ \\
\hline & \multirow[t]{2}{*}{$\begin{array}{l}\text { O } \\
\text { N } \\
\text { Z }\end{array}$} & 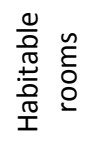 & $\frac{\underline{x}}{\underline{\underline{y}}}$ & 웃 & & $\begin{array}{l}\stackrel{\circ}{0} \\
\stackrel{0}{0}\end{array}$ & $\begin{array}{l}\stackrel{े}{\text { }} \\
\text { o }\end{array}$ & \ْ & $\stackrel{\text { ๖े }}{\text { mे }}$ & 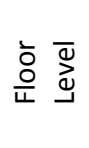 & ذ్తి \\
\hline & & & 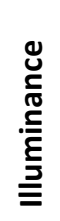 & 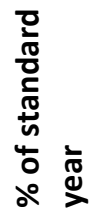 & 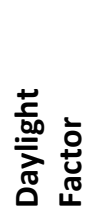 & 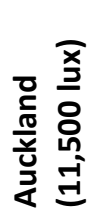 & 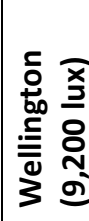 & 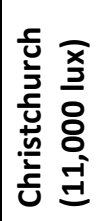 & 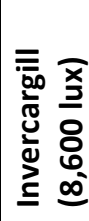 & 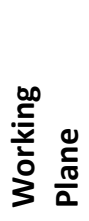 & $\begin{array}{l}\mathbb{8} \\
\frac{d}{4} \\
3 \\
0 \\
0 \\
\frac{1}{0} \\
3\end{array}$ \\
\hline
\end{tabular}


It can be seen, from Table 8 , that the New Zealand requirement is clearly an absolute minimum requirement. When comparing the illuminance requirement (G7.3.1) to the illuminance requirements of the BOCA, the New Zealand requirement is considerably lower. The BOCA requirement is for $100 \%$ of the time, therefore the illuminance should never drop below 64.58 lux during daylight hours. When the illuminance values calculated using the recommended daylight factors were compared, it was again clear that most recommendations were considerably higher than 30 lux. The only standard lower than the New Zealand requirement is the UK's right to light requirement. As the $0.2 \%$ sky factor of the right to light standard is recognised as the point below which people complain about inadequate light, the New Zealand requirement of 30 lux for $75 \%$ of the year can be considered satisfactory.

The stipulation of the working plane in NZBC G7 differs greatly from others internationally with all other standards specifying a working plane height of approximately desk height as is the standard for measuring light levels in offices where there is less flexibility than in houses. There is a consensus on the use of a glazed area of $10 \%$ of the floor area, as is used in the Hong Kong Building Regulations and Australian Building Code, with similar values of $8 \%$ and $14 \%$ used in the US and Japan respectively.

From this discussion it can be seen that the New Zealand requirement is in some respects similar to international standards and regulations, but by no means ensures good quality daylight.

\subsection{Critique of NZBC G7}

This section will critique the current regulations based on the information gathered in the previous sections. The aspects considered in this critique were based on those given by $\mathrm{Ng}$ in his critique of the Hong Kong and British daylight regulations (Ng, 2003a).

\section{Are the laws adequate?}

Based on the comparison between the NZBC G7 performance requirement, of 30 lux for $75 \%$ of the standard year, and the various international standards it can be concluded that the current regulation is adequate. The intention of the New Zealand code was to provide an absolute minimum requirement that would totally reject unsatisfactory proposals. The only international standard for this threshold value was the UK right to light daylight factor value. The New Zealand code requirement is greater than this $0.2 \%$, with the lowest daylight factor observed in Auckland of $0.26 \%$.

\section{Are they up-to-date?}

There have been no major advancements internationally on the regulation of daylight since the implementation of the New Zealand regulations. As such, the NZBC G7 performance requirement can be considered up-to-date. However, as has been mentioned earlier in this chapter, there is a lack of quantifiable data available on the minimum daylight requirements for health and well-being. If research is undertaken on this topic in the future, revision of the code might be needed. 


\section{Are they too restrictive and therefore should be relaxed? Or are they too lax and thus should be tightened up?}

The survey of daylight performance in Wellington Apartments (Stewart, 2006b) identified that in a number of situations, it is difficult for inner-city apartments to comply with the current regulation. The survey of Auckland apartments (Auckland Uniservices Ltd, 2004) found that the majority of people were satisfied with the daylight in their apartments. These two surveys indicate that the current regulation is neither too restrictive nor too lax and can be considered appropriate for ensuring occupants' health and well-being.

\section{Should new laws be implemented?}

Based on the first three questions, new laws do not need to be implemented at this stage. However, the current means of compliance (Acceptable Solution G7/AS1) does not correctly identify apartments that do and do not meet the performance requirement of G7, therefore a new means of compliance is needed to ensure apartments meet the performance requirement of NZBC G7.

\section{Why should it be $10 \%$ window to floor area for light?}

The $10 \%$ of the floor area recommendation is commonly used for ensuring adequate daylight in dwellings. However it has been shown that this is not an acceptable solution for assessing apartments in New Zealand. As has been mentioned by Ng (2003b) and Allen (1943), the development of inner city dwellings presents a number of complexities in the assessment of daylight performance. The current acceptable solution does not take into consideration these complexities and has been shown to not be an effective solution for ensuring compliance in apartments (Stewart, 2006b).

\section{What kind of method should be in place?}

Measuring compliance of daylight in urban dwellings requires a more complex assessment method than is currently being used. Aspects of the environment and building design need to be considered when designing buildings in cities. However, it is important that new solutions still fit with the intention of the code and the performance requirement of NZBC G7.

\section{How should one justify the way the prescribed plane should be calculated?}

Questions have been raised relating to the definition of the measurement plane. There are a number of different methods for defining the measurement plane, including the German DIN 5034.1 (DIN Deutsches Institut für Normung, 1999) least favourable points and the average daylight factor (British Standards Institution, 1992). The measurement plane given for NZBC G7 states that 'an illuminance of no less than 30 lux at floor level' is to be provided. This is a minimum requirement and no specific measurement points are given, it would be fair to interpret this as no point in the room drops below 30 lux for more than $25 \%$ of the standard year. This was the interpretation intended when the code was originally developed. The specification of a measurement plan at floor level differs from international convention, but is a logical way to address the issue of task identification in dwellings. 


\section{And where do all these numbers and requirements come from?}

As has been described earlier in this chapter, the values are based on international standards at the time of developing the code and the experience of an advisor for the natural light code requirement.

\section{What is the scientific basis behind them?}

The specific source of $\mathbf{3 0}$ lux is loosely based on scientific standards, but as there are no universal requirements for the minimum daylight needed for health and well-being in housing, it is difficult to provide a scientifically based recommendation until further research has been conducted in this area.

\subsection{Conclusion}

This critique has identified that the current code requirement is adequate but the acceptable solution is not effectively ensuing adequate daylight in urban dwellings. It is clear that methods need to be implemented to improve the quality of daylight in inner-city apartments in New Zealand. In Ng's assessment of the Hong Kong building regulations the following lessons were learnt ( $\mathrm{Ng}, 2004, \mathrm{pg} 26)$ :

- Changes in circumstances always require revision of legislation to be made.

- It has always been the poor who require the protection of building laws.

- One must remember that our children will live in a world we define for them. It is for them that we must try to model a better living environment.

These lessons are relevant to the situation in New Zealand where new compliance methods are needed for inner city dwellings to ensure that future apartment developments achieve adequate daylight. 


\subsection{RESEARCH METHOD}

Chapter 4 outlines the overall research design used to develop the compliance assessment tool. Section 4.1 is a summary of the pilot study conducted prior to this research and identifies how it influenced the research method. Section 4.2 discusses the research method. Within Section 4.2 six aspects were discussed, the overall method, the use of simulation for parametric data, the analysis processes used to develop the tool, the calibration process, the critique of the tool and how the criteria were to be assessed using these research methods.

\subsection{Pilot Study}

A pilot study was conducted in 2006 as a Building Science (honours) research project, forming a platform for further research in this area (Stewart, 2006a). The pilot study investigated whether it would be feasible to develop a tool for the New Zealand Building Code natural light requirement. The focus of the study was to develop and trial a research method rather than the development of a complete tool.

The research method used in the pilot study involved simulation of aspects of the environment that affect daylight in apartment buildings (aspects are given in Stewart \& Donn, 2007). The simulation results were then used to establish the degree to which the aspects of the environment affected daylight levels using a basic categorisation method. From the categorisation, a tool was developed as a simple spreadsheet calculation to specify whether the apartment building required simulation to prove compliance with the building code. The final aspect of the research method was calibration of the tool with real situations.

The data provided by the simulations were analysed using a categorisation method to develop the tool. Advice was sought from a consulting statistician in the beginning stages of the pilot study to establish the data requirements for reliable statistical relationships. It was determined that it would not be feasible in the time allocated for this research to obtain the data required to establish these statistical relationships. As a result of this consultation, a categorisation method was adopted for this stage of the research. The categorisation method involved the variables being assigned categories and weightings based on the average variations observed from the simulations.

An example of the tool is shown in Figure 4, the tool has been filled in for a case used in the pilot study. 
Figure 4 - Tool developed in Pilot Study

\section{NZBC G7 - Decision Tool}

\begin{tabular}{|c|c|c|c|}
\hline Apartment Type & Wide & Deep & \\
\hline $\begin{array}{l}\text { What is the typical apartment type for apartments } \\
\text { situated on the lower floors? }\end{array}$ & & 口 & \\
\hline Street Width & $8 m-14 m$ & $15 m-27 m$ & $\begin{array}{l}28 m \text { or } \\
\text { Greater }\end{array}$ \\
\hline $\begin{array}{l}\text { The proposed building is situated on a street with } \\
\text { a width of }\end{array}$ & 口 & & \\
\hline
\end{tabular}

\begin{tabular}{|l|c|c|}
\hline Building Heights & 26m or Less & $\mathbf{2 7 m}-\mathbf{4 2 m}$ \\
\hline $\begin{array}{l}\text { The proposed building is situated in a Height Limit } \\
\text { area of }\end{array}$ & $\square$ & \\
\hline The Height of the proposed building is & & \\
\hline The Maximum Height of the Adjacent Buildings is & \\
\hline & & Vo \\
\hline Internal Room & & Yes \\
\hline $\begin{array}{l}\text { Do any apartments in the proposed building } \\
\text { contain internal rooms? }\end{array}$ & & 口 \\
\hline
\end{tabular}

\begin{tabular}{|c|c|c|c|}
\hline \multirow[t]{2}{*}{ Site Variables } & \multirow[b]{2}{*}{ Upper North Is } & \multirow[b]{2}{*}{$\begin{array}{l}\text { Lower North } \\
\text { Is }\end{array}$} & \multirow[b]{2}{*}{ South Is } \\
\hline & & & \\
\hline \multirow[t]{2}{*}{ Where in New Zealand is the site situated? } & & 田 & \\
\hline & North & East & South \\
\hline What direction is the main façade orientated? & $\square$ & & \\
\hline Glazed Area & $\begin{array}{l}10 \% \text { of Floor } \\
\text { Area }\end{array}$ & \multicolumn{2}{|c|}{$\begin{array}{l}\text { Between } 10 \% \text { and Full } \\
\text { Façade }\end{array}$} \\
\hline $\begin{array}{l}\text { What is the area of the main façade that is } \\
\text { glazed? }\end{array}$ & & \multicolumn{2}{|c|}{$\square$} \\
\hline \multicolumn{4}{|l|}{ Qualities of Adjacent Buildings } \\
\hline & Light & Medium & Dark \\
\hline \multirow[t]{2}{*}{ What is the main colour of adjacent buildings? } & 曰 & & \\
\hline & Fully Glazed & Medium & Stone \\
\hline $\begin{array}{l}\text { What is the façade of the adjacent building } \\
\text { constructed of? }\end{array}$ & & ஏ & \\
\hline
\end{tabular}

Are Simulations Required: 


\subsubsection{Pilot Study Conclusions}

The result of the pilot study was a basic tool that was capable of identifying if an apartment building should be simulated to prove compliance with the code. The success of the tool was assessed using three criteria.

The first criterion specified that the tool was to be simple and easy to use. It was felt that as the user was not required to perform any calculations this criterion was fulfilled, however end-users of the tool were not consulted to determine if they found it simple and easy to use.

The second criterion specified that the tool was to be sufficiently accurate. Based on the findings of the calibration tests it was concluded that the tool did provide reasonably accurate results. However, before the tool is reliable enough for implementation, a more robust calculation method needed to be developed. And a more extensive set of calibration tests were also required to prove the accuracy of the tool for a wider range of conditions.

The third criterion specified that the tool could be easily implemented. This criterion was not specifically addressed in the pilot study although it is partially fulfilled provided the first two criteria are fulfilled. To fulfil this criterion it is essential that end-users are consulted during the development of the tool.

Overall this research found that it is possible to develop a tool for assessing compliance with NZBC G7 Performance Requirements. Although a tool was produced, it was concluded that further development was required to fulfil the specified criteria and prepare the tool for implementation.

\subsubsection{Contribution to further research}

The pilot study was intended as a platform for further research and development of the tool. The research method used in the pilot study was found to be appropriate for achieving the desired results. This study also found that further research on this topic was feasible and essential in improving compliance for daylight in New Zealand apartments.

The lessons learnt from the pilot study assisted in the development of a stronger, more robust research method for this next stage of research. The improvements that were highlighted by the pilot study were that: more data was needed; a stronger mathematical basis needed to be used; more extensive calibration tests and a critique of the tool were needed.

There were a number of aspects of the research method drawn from the pilot study and utilised in this next stage of the research. These included the overall process; formatting of the tool; and the calibration process. The overall process of simulation to gather data to form the tool was used successfully in the pilot study and was therefore utilised in this next stage of the research. The use of a spreadsheet in the pilot study was found to be an 
excellent method of calculating the result of the tool, it provided a quick result with minimal input from the user. The use of a spreadsheet also removed potential user-errors from the calculation process. The calibration process tested in the pilot study was found to work well and has been adapted to a larger data set.

\subsection{Research Design}

\subsubsection{Overall Research Method}

The tool was to be developed so that not all apartment projects had to go through the costly and time consuming process of full compliance simulation. However, it is reasonable to expect that some may require simulation, which would ultimately pass. A sufficient level of accuracy for this tool was $90 \%$, meaning that 1 in 10 apartment building would require simulation when it wasn't necessarily needed. With around 12 apartment buildings built annually in Auckland and Wellington (Bayleys Research, 2006; 2008), means approximately one apartment building would require simulation unnecessarily every year.

The plan for this research project (Stewart \& Donn, 2007) was to develop a tool based on the mathematical relationship between daylight at the back of the apartment and factors of the design and surroundings that affect daylight performance. The results from daylight simulation of these factors were analysed to see if patterns exist, that could describe the relationship between these factors and daylight performance. From this analysis a tool could be developed to assess daylight performance in apartments compared to the minimum code requirement. The tool was tested against real apartments and evaluated by potential end-users to ensure the tool is simple and easy to use, reasonably accurate and can be easily implemented.

Simulations were conducted to predict the daylight performance at the back of apartments. Over 600 simulations were produced for typical variations of the building design and the surrounding environment that affect daylight performance. The simulation results were used as the parametric data to establish relationships between daylight performance and the simulated factors.

The parametric data from the simulations was analysed in a statistical analysis program to identify the relationships between daylight performance and the factors of the building design and surroundings. As a result of the analysis, an equation was produced that describes this relationship.

The equation was converted into a simple tool that allows complex calculations to be performed but is simple from the user's point of view. This fits into the category of 'simple computer based design tools' identified by the International Energy Agency (Ruck, 2000). The tool was intended to identify when apartment buildings should be simulated to prove compliance with NZBC G7 from the early design stage, based on the worst apartment(s) in the building. Figure 5 shows the format of the tool.

The user enters the relevant numbers or information in the column to the right and a result is given in yellow cell. An example would be if the opposite building was 21 metres high, 
then ' 21 ' would be entered in the cell to the right of 'Opposite Building Height', the rest of the information would be entered in the same manner and then an answer is automatically provided in the yellow cell.

\begin{tabular}{|c|c|}
\hline \multicolumn{2}{|c|}{ NZBC G7 Compliance Assessment Tool } \\
\hline \multicolumn{2}{|l|}{ Variable: } \\
\hline \multicolumn{2}{|l|}{ Opposite Building Height } \\
\hline \multicolumn{2}{|l|}{ Street Width } \\
\hline \multicolumn{2}{|l|}{ Glazed Area } \\
\hline \multicolumn{2}{|l|}{ Orientation } \\
\hline \multicolumn{2}{|l|}{ Location } \\
\hline \multicolumn{2}{|l|}{ Reflectance of Opposite Building } \\
\hline \multicolumn{2}{|l|}{ Glazing Transmittance } \\
\hline \multicolumn{2}{|l|}{ Vertical Location of lowest apartment } \\
\hline Are simulations required to prove compliance: & ? \\
\hline
\end{tabular}

Figure 5 - New Compliance Assessment Tool

The result from the tool was compared to measurements taken in real apartments to determine if the tool provides correct results. 97 apartments that represent the range of typical situations found in New Zealand were measured. The results were compared to the building code requirement to determine if the apartment passed or not, post-construction. The tool was then applied to each apartment and the result was compared to the measurement results to determine if the correct result was given. This process was used to determine if the tool provided reasonably accurate results.

A critique of the tool was to be undertaken to assess the usability of the tool. Representatives from architecture and engineering firms and regulatory bodies were invited to participate in the evaluation of the tool. Participants were asked to test out the tool and answer a short questionnaire about the tool. The purpose of this was to ensure that the tool is simple and easy to use and, by involving potential end-users in the development of the tool, make the implementation process easier.

\subsubsection{Simulation of Factors that Affect Daylight Performance}

Daylight simulations were used to provide an understanding of the daylight performance in different apartments. Typical variations of the building design and surroundings were simulated to determine the daylight performance at the back of the apartment in each situation. The simulation process played a crucial role in the development of the tool as it provided the necessary information to establish the relationships between the different factors and daylight performance. 
The use of simulation to gather data about daylight performance in inner-city apartments was used successfully in the pilot study so was used again in this research. This decision was supported by Ng (2001a; 2001b; 2003b; 2005), Li et al. (1999; 2006), Allen (1943), Wilson and Brotas (2001) and Ullah and Lin (2003) who all used daylight simulation (computational and/or physical models) in their research as a data gathering tool.

The other methods that could have been used were: to measure actual apartments and use that information to develop the tool; or to validate a tool developed by other researchers for the New Zealand situation. The first method, used by Ng (2005), was eliminated because it can be time consuming and access to the number of apartments needed to achieve an adequate sample size was not feasible. It is also difficult to determine which of the many factors that vary from one apartment to the next is the cause of the change of light level, one could expect that what one does with a simulation of 600 cases might require 10 times that number of cases to isolate effect of the same parameters. The second method of adapting an existing method to New Zealand situations, as used by Ng (2001), did not fit with the aim of this research.

From the literature the two main methods of simulating daylight performance were through computer models/daylight simulation programs and scale models constructed and tested in an artificial sky simulator. The method used in this research was computer modelling and simulation. The reasons for selecting this method were:

- the ability to gather a large range of data in the available time; and

- ease of making changes.

A base model was developed to represent a typical New Zealand inner-city apartment building. The model was systematically altered to assess the effect that different factors have on daylight performance. This method was also used by Ng (2001a; 2001b; 2003b; 2005), Li et al. (1999; 2006), Allen (1943), Wilson \& Brotas (2001) and Ullah \& Lin (2003) to assess the variations in daylight performance due to different factors.

In the base model, one key measurement point was set for consistency throughout this research. The measurement point was based on the specification given by the Australian Department of Employment and Industrial Relations (1983, pg vii), stating that the lowest value of daylight available in a room (at desk height) is along the centre line of the room at a distance of $600 \mathrm{~mm}$ from the rear wall in rooms with windows in one wall.' This may not be the lowest illuminance point exactly for a working plane at floor level but would most likely be the lowest point situated in usable space. Two additional measurement points were included to check for consistency in results as all three points should have similar results. Figure 6 shows the three measurement points used throughout this research. 


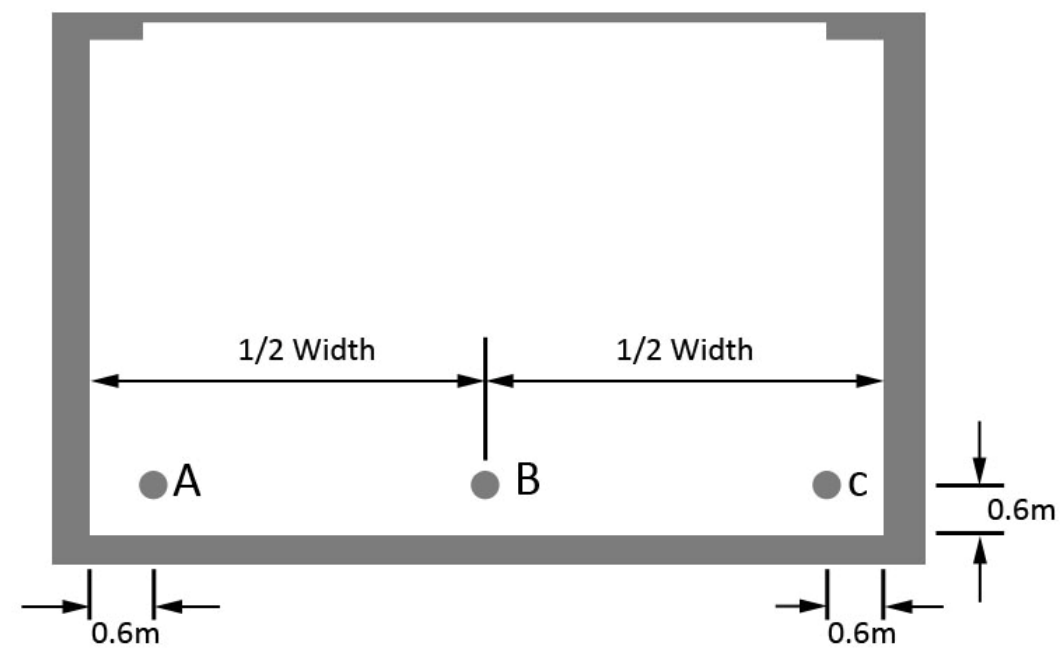

Figure 6 - Measurement Points

Variations of factors that affect daylight performance at the back of an apartment were systematically applied to the base model to test the widest range that could be expected in New Zealand. The minimum number of variations per factor needed to reliably establish statistical patterns, as recommended by Roscoe (1975), is 5 . Therefore a minimum of 5 variations for each factor were selected, ranging from best case to worst case and at intervals between the two. An example of these variations is shown in Figure 7, where 7 different street widths were assessed ranging from best case (30 metres) to worst case (5 metres), and with 5 variations between.

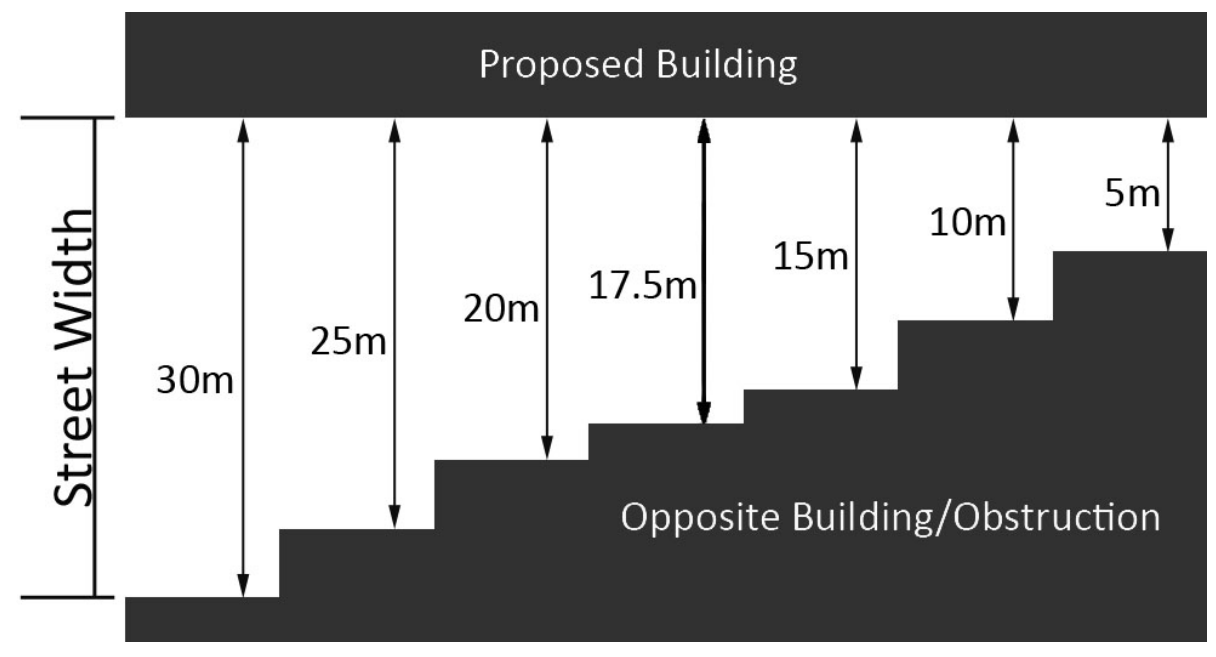

Figure 7 - Street Widths

As this research was also interested in determining the interaction between multiple variables and daylight performance, variations were applied for combinations of factors. This involved two factors being altered in the base model per simulation. This resulted in over 600 variations being simulated to represent the full range that could be expected in New Zealand cities.

To investigate the daylight performance in inner-city apartments, a range of variations of the building design and surrounding environment were identified for simulation. The 
variations to be tested in the simulations were identified by previous researchers and in the pilot study. The variations were then separated into three categories, these categories were: primary variables; secondary variables and assumed variables. This process was used in the pilot study and was found to be effective at keeping the simulations at a manageable scale for the research project.

The primary variables were those identified through the literature and the pilot study as being factors of the environment and apartment design that have a large effect on daylight performance. The secondary variables were identified as having an effect on daylight performance but were not as important as the primary variables. The assumed variables were aspects of the apartment and environment that either had little variation for innercity apartments or were difficult for users to specify at the early design stage. The followings lists show the variables that were considered to have an effect on daylight performance, more detailed explanations can be found in Chapter 5 and the Glossary on pages 129-130.

The primary variables were:

- Apartment Type;

- Building Height;

- Geographical Location;

- Glazed Area;

- Orientation;

- Reflectance; and

- Street Width.

The secondary variables were:

- Ceiling height;

- Glazing Transmittance;

- Proposed Building Height; and

- Vertical Location.

The assumed variables were:

- Apartment Dimensions;

- Apartment Layout;

- Building Form;

- Furniture;

- Ground Reflectance;

- Internal Reflectances;

- Shading Factors; and

- Window Position.

These assumptions were made to limit the scale of the project and to gain accurate results for the factors that were most important. The decision to use assumptions was supported by the research of Ng (2001a; 2003b), Li et al. (1999; 2006) and Wilson and Brotas (2001), where assumptions were made to represent typical situations in each of the research 
projects. The assumptions were made for two main reasons, the first being that for some factors (e.g. Internal Reflectance) insufficient information is available in the early design stage. And to limit the number of variables needing simulation and analysis, some factors (e.g. Furniture) that have only small effects on daylight performance were assumed based on common practice.

If all variables had at least 5 variations simulated it is estimated that around 2000 simulations would have been needed. In addition to this, some variables, like apartment dimensions and building form, would have required considerably more to accurately predict the performance for the range of typical situations. With the simulation process taking around 45 minutes per variation, it is estimated that it would have taken 26 weeks to produce the data if all variables were considered. This was not feasible for this research and the additional time would outweigh the added value to the tool, which was expected to be minimal.

It is estimated that the use of assumptions could account for an error of 5-6\% at the back of the room, based on the error for the calculation method in Ng's (2001b) study of the accuracy of daylight simulation and calculation methods in Hong Kong. This is a small error and would only become an issue for apartments that barely reached the minimum requirement. Because of the assumptions, these apartments may require simulation even though they just pass with 30 lux exceeded $80 \%$ of the year. To check the validity of some of the assumptions, like internal reflectance and shadings, notes were taken in the calibration apartments to check that the assumptions were fair and did not affect the accuracy of the predictions.

\subsubsection{Statistical Analysis Method for Development of the Tool}

The data provided by the simulation was analysed to establish patterns that represent the relationship between daylight and the variables tested. As a result of the analysis, an equation was able to be produced that assesses the daylight performance $0.6 \mathrm{~m}$ from the rear wall (the standard measurement point for this research). The use of data analysis to establish an equation (or prediction model) to assess daylight performance has been used by Ng (2001a; 2003b; 2005) and Wilson and Brotas (2001).

Discussions with a consulting statistician identified that multiple regression analysis would be the best method to achieve the desired outcome from this research. This decision was supported by Cohen, Cohen, West and Aiken (2003) and Roscoe (1975), where the suitability of regression analysis methods for developing prediction equations was identified. Cohen stated that 'multiple regression/correlation can be used in practical prediction problems where the goal is to forecast an outcome based on data that were collected earlier,' (Cohen et al., 2003, pg 3) which perfectly describes the intention of this stage of the research. 
The equation for the relationship between daylight performance and the factors of the building design and surroundings was based on the standard regression equation for multiple variables. The following equation shows the general format of the equation to be produced in this research:

$$
D A=a+b B H+c S W+d G A+e O \ldots
$$

Where:

- $D A$ is the daylight performance (daylight autonomy value), as a percent of the year that 30 lux is exceeded (\%);

- $B H$ is the height of the opposite building $(\mathrm{m})$;

- $S W$ (street width) is the distance between the proposed building and obstruction (opposite building) (m);

- $G \boldsymbol{A}$ is the percent of glazed area compared to of the floor area $\left(\mathrm{m}^{2}\right)$; and

- Oris the orientation of the apartment.

The constant (a) and the coefficients (b, c, d and e) are found through the analysis of the simulation data. The statistical analysis program, SPSS (2007), provides these values.

The prediction model was then converted into a computer based tool, as per the pilot study, to allow complex calculations to be undertaken with minimal effort required by the users. As a prototype, the tool was Excel based, but future developments may see this converted into a web-based tool.

\subsubsection{Method used for Calibration Tests}

Accuracy of the tool was one of the main criteria, by ensuring the tool was able to reliably predict whether apartments would require simulation to prove compliance. The method for assessing the accuracy of the tool was drawn from Ng's (2001b) research investigation of the accuracy of daylight simulation for Hong Kong residential buildings. In $\mathrm{Ng}^{\prime} \mathrm{s}$ investigation, residential units (apartments) were measured at different heights within a new residential building to determine the vertical daylight factor for each of the windows. These results were then compared to the results from the simulation of the windows in two different simulation programs to determine their accuracy.

This method of comparing predicted performance to reality was used in this research also. As with the pilot study, daylight levels in apartments were measured and the results were compared to the result given by the tool. The final results from the measurements identified whether an apartment complied with the minimum building code requirement post-construction. From the data gathered during the onsite measurements and from the original building plans, the appropriate information was entered into the tool to determine if the building being assessed should have had daylight simulations conducted. 
An outcome of YES, meaning the building would likely not comply with the minimum code requirement, would be considered as accurate if the measurements found that the apartment measured did not comply with the minimum requirement post-construction. Likewise, if the outcome of the tool was NO, meaning that the apartment would definitely meet the minimum code requirement, the result would be considered accurate if the apartment measurements exceeded the minimum requirement post-construction.

From the literature survey, there was a clear consensus on the method of assessing daylight performance in buildings. The daylight factor method was used by Ng (2001a; 2005), Li et al. (1999), Allen (1943), Littlefair (2001), Fontonynont (1999) and Wilson and Brotas (2001) for onsite measurements and simulation of daylight in buildings. Within the literature there were variations of the daylight factor: vertical daylight factor, the daylight factor formula and the average daylight factor. These are all based on the fundamental principle of the percentage of available daylight that reaches a certain point or specified plane. In this research it is the percentage of the daylight available that reaches each horizontal measurement point.

This method was used in the pilot study (Stewart, 2006a) and in Stewart's (2006b) assessment of Daylight in Wellington Apartments. These two research projects found that the daylight factor assessment method was applicable to the New Zealand environment and was effective at providing the necessary data to assess compliance in a variety of New Zealand apartments.

The number of calibration tests conducted was based on the basic rule of thumb for assessing the reliability of prediction equations to predict reality (Roscoe, 1975, pg 184). The rule of thumb specifies a sample size for multivariable research of at least 10 times the number of variables. The final number of variables (primary and secondary) was 8 , therefore requiring a minimum of 80 apartments.

In each apartment, measurements were taken at the same three measurement points used in the simulations, in the centre and $0.6 \mathrm{~m}$ from the side walls, $0.6 \mathrm{~m}$ from the rear wall. However, most of the apartments measured were inhabited which meant that shading from furniture became an issue. It was not possible to move the furniture so measures were undertaken to ensure that apartments were not given fail results incorrectly because of shading from furniture. The measurements taken at the three points should all be similar, but in situations where one of the points was significantly lower than the other two because it was shaded by furniture it was not included in the calibration results.

\subsubsection{Method used to Critique the Tool}

A critique (or evaluation) was undertaken of the usability of the tool. Potential end-users of the tool were invited to participate in the critique. The targeted end-users were designers/consultants and regulators. The recommended number of participants was 3-4 per group to identify $98 \%$ of the usability problems (Nielson, 2000). 
Architecture and engineering firms within New Zealand were approached and invited to participate in the critique. Firms with experience in the design of apartment buildings were selected from throughout New Zealand. Following implementation of the tool, architects and consultants would be required to assess proposed apartment buildings, ideally in the early design phase, to establish whether it would be necessary to provide simulations with the building consent application. An assessment using the tool is intended to be simple and easy for the architects/consultants to perform and would require very little time and resources.

The Building Controls departments of the main urban Territorial Authorities (TAs) and regulatory bodies were invited to participate in the evaluation. The TAs invited to participate in the evaluation were City Councils that deal with apartments and urban developments on a regular basis. It was essential that TAs were involved in the critique as they will be responsible for enforcing the tool's use. TAs were intended to benefit from the implementation of this tool and as such, it was important they were given the opportunity to suggest modifications to tailor the tool to their requirements.

The evaluation participants were asked to apply the tool to an apartment development. Following the application of the tool, the participants were asked to fill out a short questionnaire. The questionnaire was aimed at gaining the necessary information to assess usability of the tool. From the questionnaire a number of conclusions were able to be drawn as to the time involved, ease of use and suitability of the tool. Another key aim of the questionnaire was to give potential end-users a chance to suggest changes that would make the tool easier to use and more suitable for them.

The survey results were analysed and suggestions from participants were considered. Where possible, variations were made to the tool from the suggestions given by participants.

\subsubsection{How Research Methods were to fulfill the Criteria of the Tool}

As was identified in Chapter 1, the aim of this research was to produce a simple compliance assessment tool that can be included in the NZBC G7 Compliance Documents as an acceptable solution for apartment buildings. The three success indicators and key objectives of the tool were that it was to be:

- simple and easy to use;

- reasonably accurate; and

- could be easily implemented.

The methods used to fulfil these criteria are as follows.

\section{Criterion 1: Simple and easy to use.}

This criterion was to be fulfilled though the careful consideration of simplicity and usability throughout the development process. The tool was designed so there is minimum impact 
on users' time. The formatting of the tool was to be easy to follow and understand, even for first time users.

This criterion was assessed using the information provided from the questionnaire completed by potential end-users. Potential end-users provided an understanding of the time involved in applying the tool to a project and the difficulty level of the tool. With 3-4 participants form each group, $98 \%$ of the usability errors can be identified (Nielson, 2000). The results of the questionnaire allowed conclusions to be drawn as to the ease and simplicity of use.

\section{Criterion 2: Reasonably accurate.}

This criterion was fulfilled through the development of a robust research method that documents individual stages of the development of the tool and is based on previous research adding to the reliability of the tool.

The main method used to assess accuracy was the calibration tests. These tests were developed with the sole aim of determining how well the tool assesses real situations. The calibration stage will produce a percentage of cases that had the correct result, which can then be used to determine if the tool is 'reasonably accurate', meaning that less than $10 \%$ of the building can be expected to receive incorrect results from the tool.

\section{Criterion 3: Easily implemented.}

The third criterion is not as simple to assess. However, it needed to be considered at every stage of this research. Key factors that will ensure the tool can be easily implemented are the fulfilment of the first two criteria. By developing a tool that is simple and easy to use, less resistance would be expected by potential end-users. Accuracy is also essential in successfully implementing the tool; when a strong research method is used and accuracy is proven, then the argument for implementation is strong. The most important aspect of this research used to fulfil this criterion was the involvement of potential end-users. Involving potential end-users in the development phase allows them to voice concerns and tailor the tool to better suit their specific needs. Ideally this would also make the implementation process smoother. 


\subsection{DEVELOPMENT OF THE TOOL}

Chapter 5 discusses the development of the tool. Within this chapter, the detailed research methods used to produce the simulations and analysis process are outlined in Section 5.1. A summary of the analysis of the simulations is given and the equations developed from this analysis are described in Section 5.2. The formatting of the equation into an Excel spreadsheet is also described in Section 5.2.

\subsection{Development Method}

The method used to gather the data for the prediction equation was through simulation of factors that affect daylight performance. A base model was simulated that represents typical situations found in New Zealand. Variations of the building and surrounding environment were simulated to determine the effect they have on daylight performance.

Regression analysis was used to develop a prediction equation using the data provided by the simulations. The prediction equation is used to calculate the percentage of the year in which the worst apartment in an apartment building will exceed the minimum of 30 lux of natural light. This equation was then formatted into an Excel spreadsheet to calculate whether apartments would require simulations to prove compliance.

\subsubsection{Base Model}

It was not realistic to simulate every possible situation that could occur. Therefore, methods were adopted that assess the range of typical situations. A base model was used to assess typical situations and allows the effects of each variable to be isolated.

The pilot study used models of three sites that represented the range found in Wellington City. The decision to use models of real situations rather than a standard city model was based on Davies's (1992) investigation into the use of a standard city model in wind tunnel modelling. Davies (1992) found that it was not possible to develop a standard city model to represent New Zealand cities. A concern with this method, as a result of the pilot study, was the inability to isolate the effect each variable or combination of variables has on daylight performance. It is essential in the analysis and development of the prediction model that these effects can be isolated.

A survey of the methods used by other researchers in this field found that although standard models are not suitable for wind studies, they can be used effectively in daylight research. Ng (2001a), Li et al. (1999; 2006), Allen (1943) and Nabil and Mardaljevic (2006) used base (or test) models to represent a typical building. In each of these cases, the base model was assessed and then had a number of variations applied to determine the difference in daylight performance as a result of the variations. 
A base model was developed following the methods used by the aforementioned researchers. In this base model a combination of typical situations and mid-point values for the variables were used. Base model values for variables including glazing transmission and vertical location of apartments were based on the most common values observed in New Zealand apartments. Variables including street width and geographical location for the base model were based on the mid-point values being assessed.

The base model was developed to represent the poorest performing apartment in a typical apartment building. A number of factors were assumed in the development of the base model (assumed variables), this is a crucial step in ensuring the most accurate result possible is obtained from the simulations, but can result in errors of around $5 \%(\mathrm{Ng}, 2001 \mathrm{~b})$.

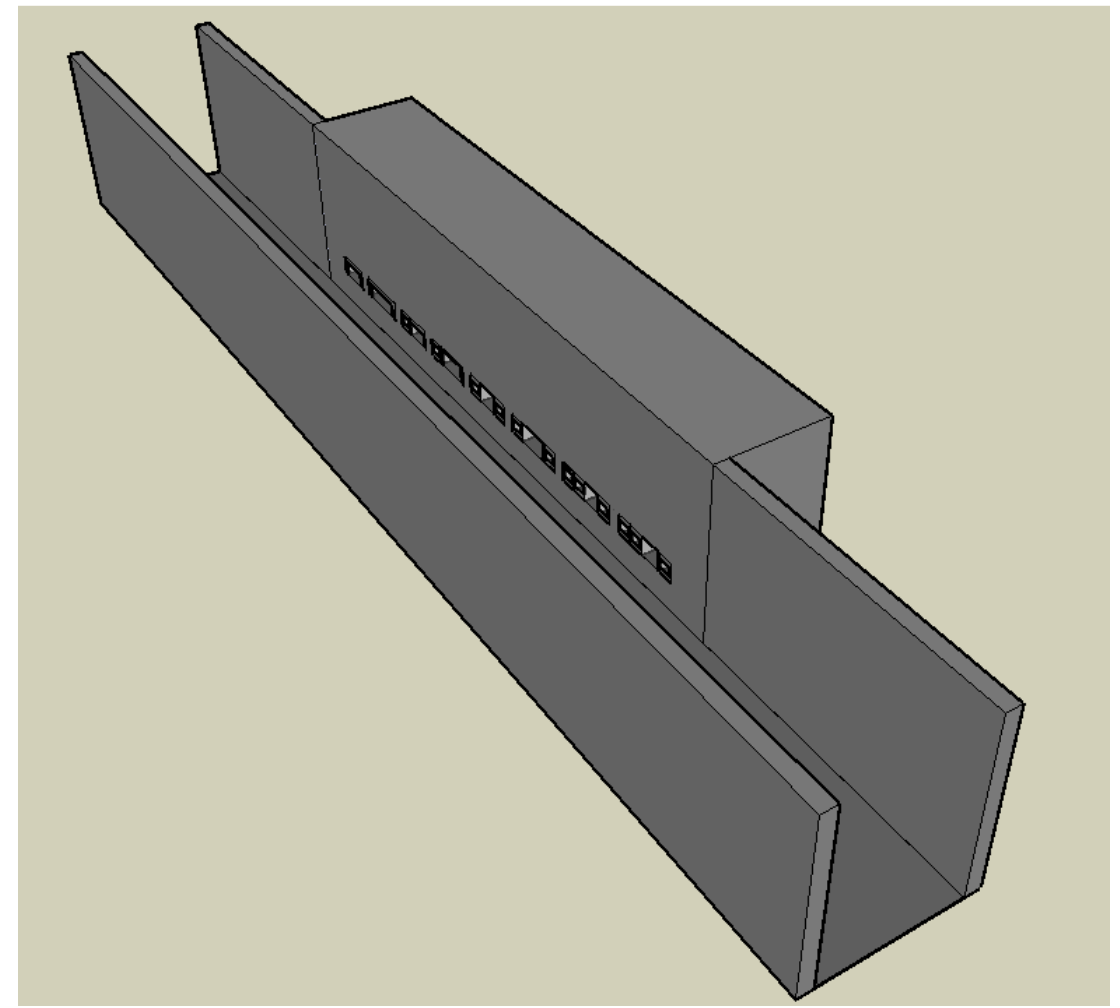

Figure 8 - Base Model

\subsubsection{Daylight Simulation}

An investigation was performed into the daylight simulation technologies and programs available. The two primary methods of simulation are raytracing and radiosity. Of these two methods, raytracing was identified as the method that provides the most reliable results for complex situations like daylight in urban environments. The industry standard raytracer is RADIANCE (Lawrence Berkeley National Laboratory), so a list of dynamic RADIANCE-based simulation programs was gathered to decide which program was most suitable for this research. From this list, DAYSIM was selected as the simulation program to be used because previous use had found it to be relatively easy to use and reliable. DAYSIM was used in the pilot study and was found to provide results that could easily be used in this 
research. A more detailed explanation of these technologies and programs can be found in Appendix 1.

DAYSIM is a RADIANCE-based daylight simulation tool developed by the Lighting Group of the National Research Council Canada and the Solar Building Design Group of the Fraunhofer Institute for Solar Energy Systems (National Research Council Canada, 2006). DAYSIM uses daylight coefficients and the Perez sky luminance model to simulate the daylight performance throughout the year (Reinhart \& Walkenhorst, 2001). Within one run, DAYSIM calculates the annual indoor illuminance levels for intervals as small as 5 minutes and for the range of different sky condition based on climate data. Development of tools like DAYSIM has meant it is now feasible for annual simulations of daylight to be regularly conducted.

The output provided by DAYSIM gives a variety of useful information including: Daylight Factors, Daylight Autonomy, Useful Daylight Illuminances and Annual light exposure (Reinhart, Mardaljevic \& Rogers, 2006). The key feature of the output that assisted this study is the Daylight Autonomy information. The Daylight Autonomy function allows the user to determine the percentage of annual daylight hours in which a specified illuminance is exceeded. In this study, the illuminance value was set at $\mathbf{3 0 ~ l u x , ~ a s ~ i s ~ s p e c i f i e d ~ i n ~ t h e ~}$ building code, and the percentage values were able to be compared to the minimum building code percentage of $75 \%$ of the standard year.

\subsubsection{Variables}

The variables that affect daylight performance in urban environments, as were introduced earlier, are discussed in more detail in this section (for further clarification on these features please refer to the Glossary on page 129). As identified in the Chapter 4, the variables have been separated into three groups: primary variables; secondary variables; and assumed variables. Where the primary variables are the factors of the environment and apartment design that have a large effect on daylight performance. The secondary variables are the factors that have an effect on daylight performance but were not as important as the primary variables. And the assumed variables are the aspects of the apartment and environment that either had little variation in the urban environment or were difficult to specify at the early design stage.

\section{Primary Factors}

\section{APARTMENT TYPE}

The apartment type can affect the daylight distribution, potential glazed area and the spatial arrangement. Three aspects of design were considered when developing the range of apartment types to be tested. These were: the number of habitable rooms; floor area; and configuration (plan type).

The number of habitable rooms per dwelling is a method used to categorise different apartment types. This method was used by the Auckland (2007b), North Shore (2002b, pg 
66) and Waitakere (2005) City Councils, the New South Wales Planning Department (2002) and a report on the minimum standards for Auckland apartments (Clinton Bird Urban Design Limited, 2005).

The standard apartment types are:

- Studio;

- 1 bedroom;

- 2 bedrooms; and

- $3+$ bedrooms.

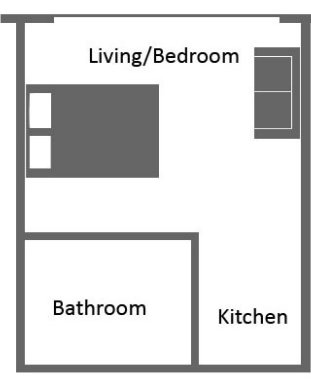

Studio

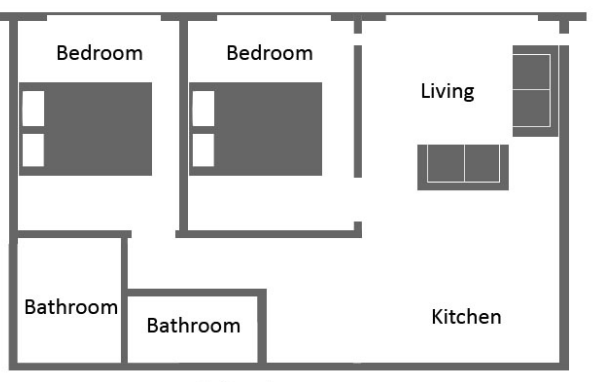

2 Bedroom

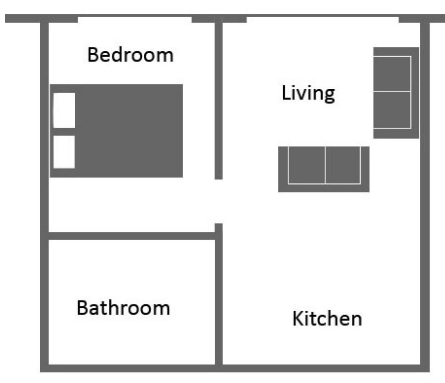

1 Bedroom

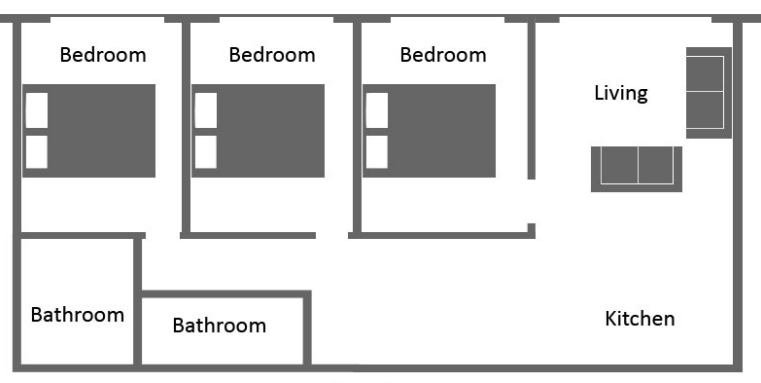

3 Bedroom

Figure 9 - Typical apartment floor plans

Floor area was found to be a factor of apartment design that affects the distribution of light, as mentioned by $\mathrm{Ng}$ (2001a, pg 259) and Li et al. (1999, pg 215; 2006, pg 1344). The Auckland (2007b) and Waitakere (2005) City Councils, the New South Wales Planning Department (2002) and a report on the minimum standards for Auckland apartments (Clinton Bird Urban Design Limited, 2005) also give the minimum gross floor areas. The following table shows the values given by each of these sources, including the comparison with international minimum floor areas given in the minimum standards report (Clinton Bird Urban Design Limited, 2005). 
Table 9 - Minimum Floor Areas

\begin{tabular}{|l|c|c|c|c|}
\hline & Studio & 1 bedroom & 2 bedrooms & 3+ bedrooms \\
\hline Auckland City Council & $35 \mathrm{~m}^{2}$ & $45 \mathrm{~m}^{2}$ & $70 \mathrm{~m}^{2}$ & $90 \mathrm{~m}^{2}$ \\
\hline Waitakere City Council & $40 \mathrm{~m}^{2}$ & $50 \mathrm{~m}^{2}$ & $70 \mathrm{~m}^{2}$ & $90 \mathrm{~m}^{2}$ \\
\hline NSW Planning Department & $38.5 \mathrm{~m}^{2}$ & $50 \mathrm{~m}^{2}$ & $80 \mathrm{~m}^{2}$ & $124 \mathrm{~m}^{2}$ \\
\hline Sydney & $40 \mathrm{~m}^{2}$ & $55 \mathrm{~m}^{2}$ & $80 \mathrm{~m}^{2}$ & $100 \mathrm{~m}^{2}$ \\
\hline Toronto & $\mathrm{N} / \mathrm{A}$ & $48 \mathrm{~m}^{2}$ & $65 \mathrm{~m}^{2}$ & $74 \mathrm{~m}^{2}$ \\
\hline Illinois & $27 \mathrm{~m}^{2}$ & $50 \mathrm{~m}^{2}$ & $63 \mathrm{~m}^{2}$ & $81 \mathrm{~m}^{2}$ \\
\hline Dublin & $\mathrm{N} / \mathrm{A}$ & $38 \mathrm{~m}^{2}$ & $55 \mathrm{~m}^{2}$ & $70 \mathrm{~m}^{2}$ \\
\hline Vienna & $32 \mathrm{~m}^{2}$ & $32 \mathrm{~m}^{2}$ & $50 \mathrm{~m}^{2}$ & $60 \mathrm{~m}^{2}$ \\
\hline
\end{tabular}

There are three generic plan types, given by Sherwood (1978) that can be used to describe apartments internationally. A study by Whyte (1996) established that these generic plan types can be used to describe the typical apartments found in Wellington. The three plan types are: Single Orientation, Double Orientation - Open End and Double Orientation - 90 as shown below.

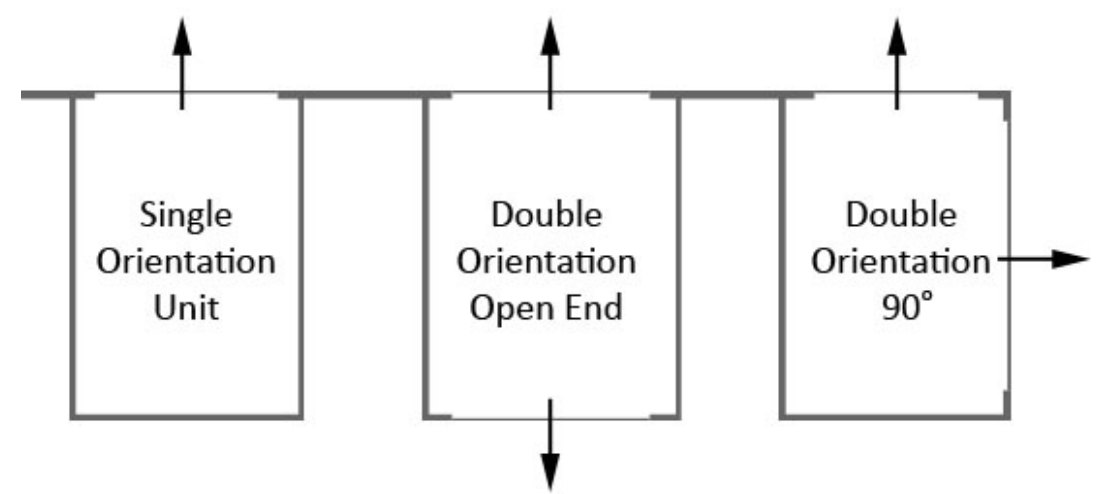

Figure 10 - Apartment plan types

Whytes' (1996) research also found that $80 \%$ of the apartments found in Wellington are single orientation units. Single orientation units can be separated further into two types, Deep and Wide, these are shown in Figure 11.

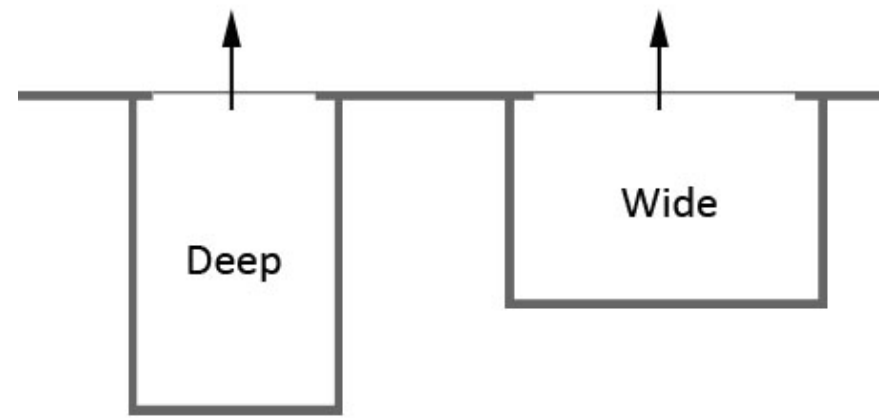

Figure 11 - Deep and Wide apartments

In this research 16 apartment types were simulated. This is based on a deep and a wide version of the four standard apartment type (Studio, 1 Bedroom, 2 Bedroom and 3+ Bedroom), with two different floor areas (small and large) per apartment type. 
The floor areas were determined using the Waitakere City Council minimum floor areas for a small apartment and the large floor areas were determined by surveying real estate advertisements to determine an average maximum floor area, the 16 apartment types simulated are shown in Table 10.

Table 10 - Apartment Floor Areas

\begin{tabular}{|l|c|c|c|c|}
\hline & Studio & 1 bedroom & 2 bedroom & 3+ bedroom \\
\hline Wide - Small & $40 \mathrm{~m}^{2}$ & $50 \mathrm{~m}^{2}$ & $70 \mathrm{~m}^{2}$ & $95 \mathrm{~m}^{2}$ \\
\hline Wide - Large & $60 \mathrm{~m}^{2}$ & $70 \mathrm{~m}^{2}$ & $95 \mathrm{~m}^{2}$ & $110 \mathrm{~m}^{2}$ \\
\hline Deep - Small & $40 \mathrm{~m}^{2}$ & $50 \mathrm{~m}^{2}$ & $70 \mathrm{~m}^{2}$ & $95 \mathrm{~m}^{2}$ \\
\hline Deep - Large & $60 \mathrm{~m}^{2}$ & $70 \mathrm{~m}^{2}$ & $95 \mathrm{~m}^{2}$ & $110 \mathrm{~m}^{2}$ \\
\hline
\end{tabular}

In the simulation of multiple variations with apartment type, daylight performance and the other variables, only the eight deep apartments were simulated. The deep apartments were selected as these typically received lower illuminances at the back of the space than wide apartments as was determined in the pilot study for this research (Stewart, 2006a, pg 40). A small, deep 1 bedroom apartment was used as the 'base' apartment type.

\section{STREET WIDTH}

As was identified by Ng (2001a; 2001b; 2003b; 2005), Li et al. (1999; 2006), Allen (1943), Wilson and Brotas (2001), Walsh (1961), CIBSE (1999) and the IEA (Ruck, 2000), the obstruction angle is one of the most important factors in daylight performance in urban environments. The two components of the obstruction angle are obstruction height and proximity. In this research, these two components were assessed separately and proximity is referred to as the street width, but could easily apply to any other space separating buildings.

The street width $(\mathrm{m})$ is defined as the distance from the façade of the proposed building to the façade of the building directly opposite, as shown in Figure 12.

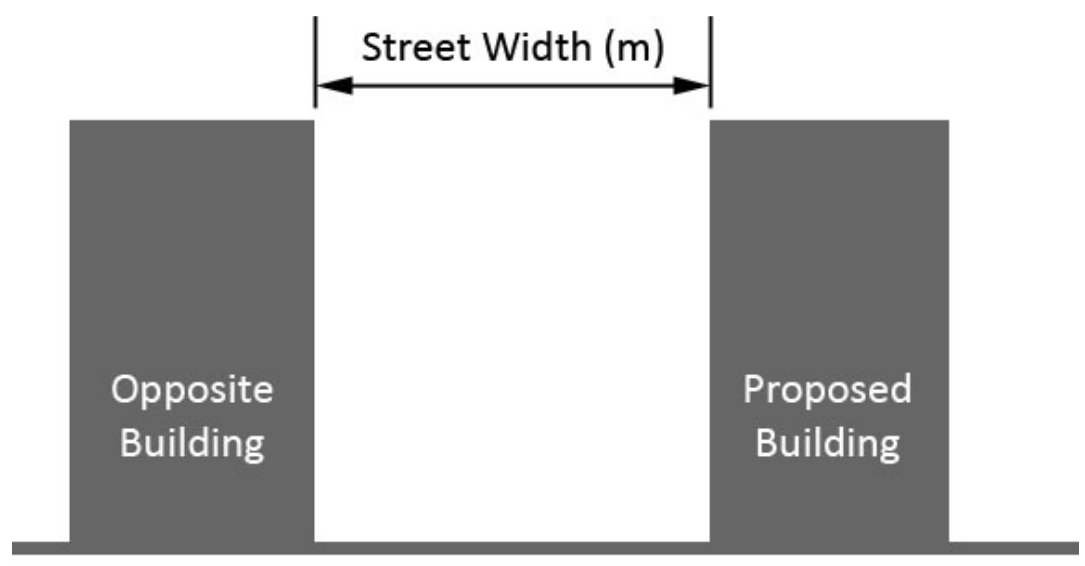

Figure 12 - Street Width

Davies' (1992) wind tunnel study investigated the typical dimensions of major New Zealand cities. Table 11 shows the findings from this survey. 
Table 11 - Dimensions of major New Zealand cities (Davies, 1992, pg 65)

\begin{tabular}{|l|c|}
\hline City & Street Width (m) \\
\hline Auckland & $15-30$ \\
\hline Wellington & $15-25$ \\
\hline Christchurch & $20-25$ \\
\hline Dunedin & $20-25$ \\
\hline
\end{tabular}

A survey of Wellington City plans generally confirmed Davies' findings However, some streets in the major residential area were found to be as narrow as 8 metres. To account for the narrow alleys and buildings stepped back from boundary lines, the minimum street width (worst case) simulated was 5 metres. Using Davies' street widths, a maximum typical street width for a New Zealand city of 30 metres was selected. Therefore, the range of street widths tested was 5-30 metres. Seven street widths were simulated to provide enough variations to determine a statistical relationship between daylight performance and street width. The simulated street widths were 5, 10, 15, 17.5, 20, 25 and 30 metres.

In the simulation of interactions, only five street widths were simulated. These were 5, 10, $17.5,25$ and 30 metres. The street width used for the base model was 17.5 metres as this was the mid-point.

\section{BUILDING HEIGHT}

The second component of obstruction angle is the height of the obstruction. This is defined as the mean height above sea level of the building directly opposite the proposed building. In urban environments, the height of obstructions can be difficult to determine. The rule of thumb for estimating obstruction height for complex skylines, given by the Building Research Energy Conservation Support Unit's 'Desktop guide to daylighting' (1998, pg 6) and Walsh's 'Science of Daylight' (1961, pg 82), will be used in this research for determining obstruction height. Mean sea level was used because this is how territorial authorities in New Zealand define building heights in district plans.

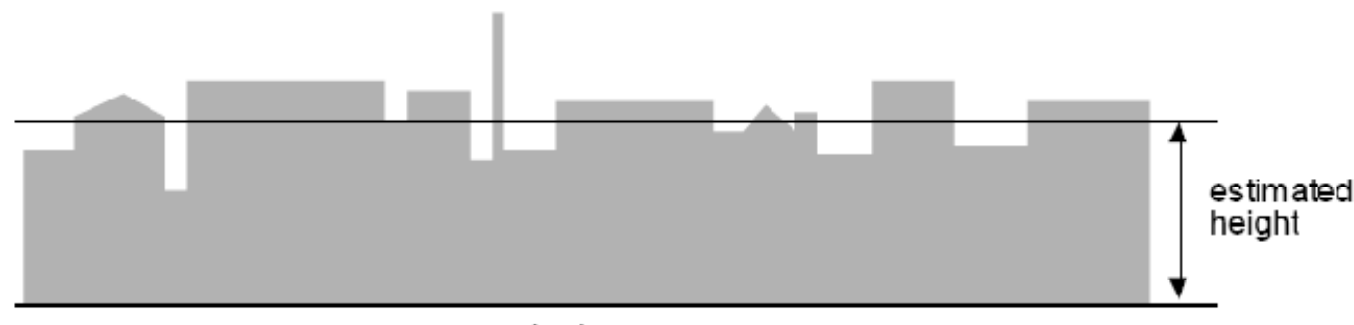

horizon

Figure 13 - Estimating average obstruction height (Building Research Energy Conservation Support Unit, 1998, pg 6).

The minimum building height simulated was 0 metres, to account for situations where there is no obstruction. The maximum building height simulated was 90 metres. The maximum height in the Wellington City District Plan (2000, Map 32) is 95 metres, but this is 
a relatively small area and it is unlikely that all buildings in this area will be built to 95 metres. A maximum of 90 metres was more common and was considered practical using the average obstruction height method. Seven building heights were assessed to determine the interaction between building height and daylight performance.

These were: $0,10.2,18.6,24,35.4,60$ and 90 metres. The variations in building height were determined using the Wellington City District Plan height limits. The heights tested for the interaction between multiple variables and daylight performance were: 10.2, 18.6, 24, 60 and 90 metres. The 'base' obstruction height was 24 metres.

\section{ORIENTATION}

As was identified by Li et al. (1999; 2006), Ullah and Lin (2003), Evans (1981), Nabil and Mardaljevic (2006), CIBSE (1999) and the IEA (Ruck, 2000), orientation is a factor of the environment that affects daylight performance. Orientation is important in both suburban and urban environments as it accounts for the light provided by sunlight directly and reflected into urban canyons.

Seven orientations were simulated, the four primary orientations (North, South, East and West) and three secondary orientations (North-east, North-west and South-east). It was assumed that as the daylight available for South-east and South-west is primarily skylight, the difference between these variations would be minimal and as such only one of these orientations needed to be simulated.

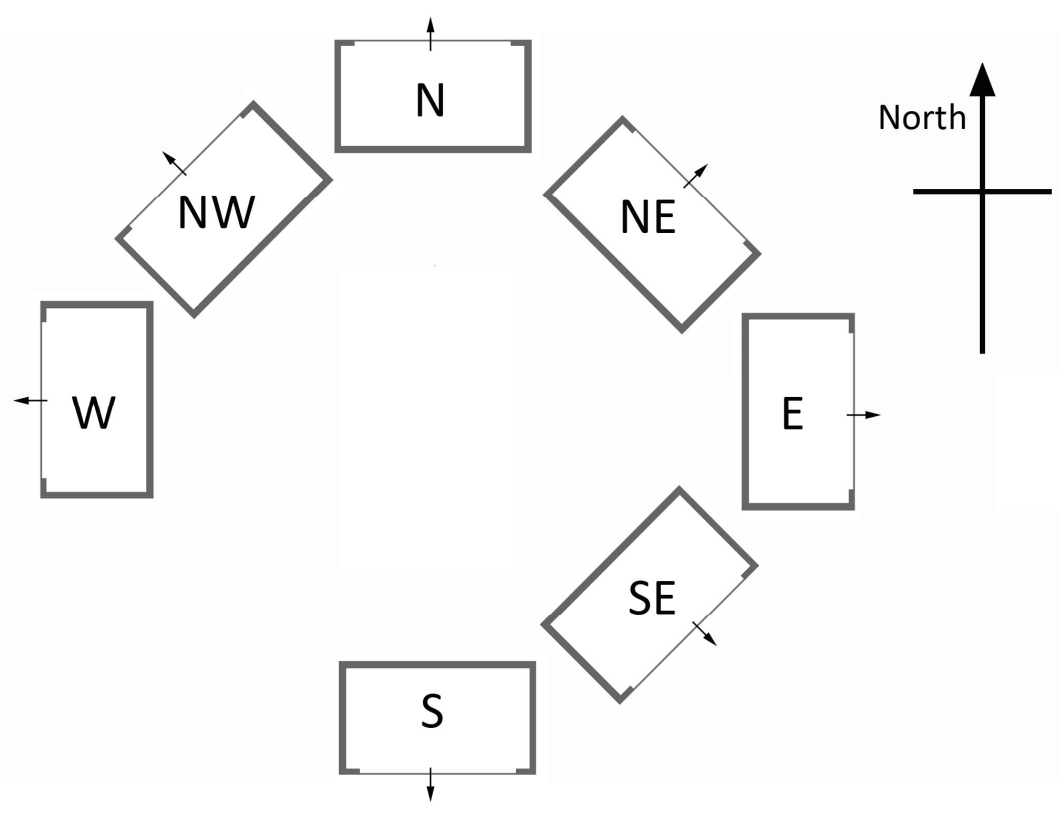

Figure 14 - Orientations

For the simulation of interactions with multiple variables, only the four primary orientations were tested. The orientation used in the 'base model' was North. 


\section{GLAZED AREA}

Glazing area affects the amount of the available daylight that reaches the interior and was identified by Ng (2001a), Li et al. (1999; 2006), CIBSE (1999) and the IEA (Ruck, 2000) as a major factor affecting daylight performance in inner city apartments. The two methods of describing glazed area are window-to-wall ratio and the glazed area as a percentage of the floor area. The latter will be used in this research as this is the method used in the New Zealand Building Code (Department of Building and Housing, 2001).

The minimum glazed area assessed in this research was $10 \%$ of the floor area because this is the minimum acceptable floor area given in the New Zealand Building Code (Department of Building and Housing, 2001). The 'base' glazed area used was $20 \%$, this is the minimum glazed area for apartments in the Auckland region as given by the Auckland (2007b) and North Shore (2002b) City Councils and the minimum standards for apartments in Auckland (Clinton Bird Urban Design Ltd, 2005). The largest glazed area was the exterior wall fully glazed, this was calculated as $40 \%$ of the floor area. The variations of glazed area were $10 \%$, $20 \%, 30 \%$ and $40 \%$, the method of calculating the glazed area as a percentage of floor area is shown in the following figure.

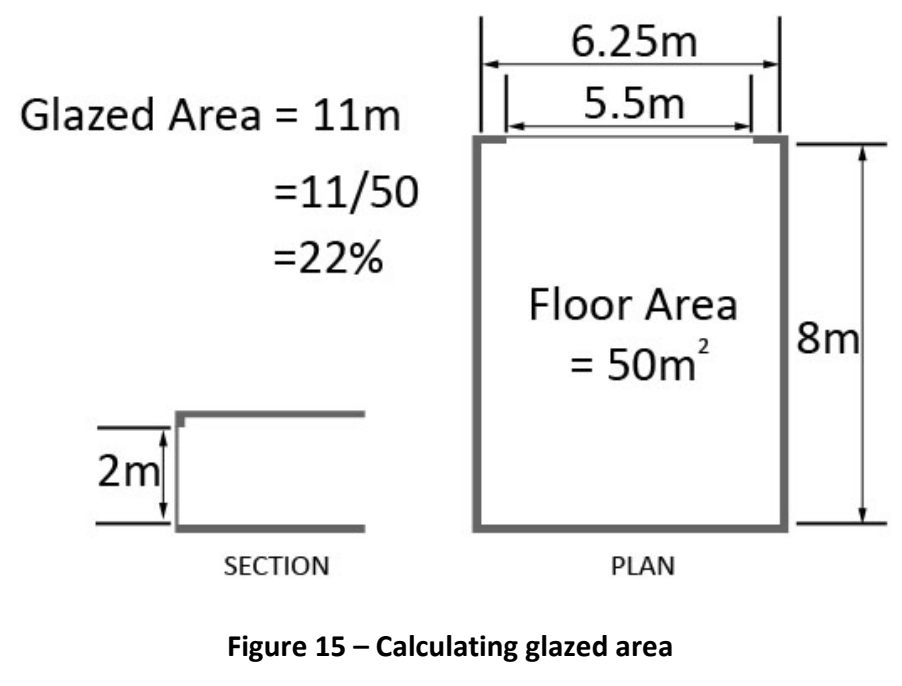

\section{GEOGRAPHICAL LOCATION}

The daylight performance in different locations is affected by a combination of daylight availability (solar radiation) and sun angles (latitude). The aspect of latitude was identified by Wilson and Brotas (2001), Littlefair (2001) and the IEA (Ruck, 2000). Due to the variation in climate throughout New Zealand, the range of solar radiation zones needs to be explored. The simulation process uses standard weather files to calculate the available daylight in different climates. (Reinhart, 2006) Weather files were obtained for the 16 National Institute of Water and Atmospheric Research (NIWA) climate zones shown in Figure 16. 


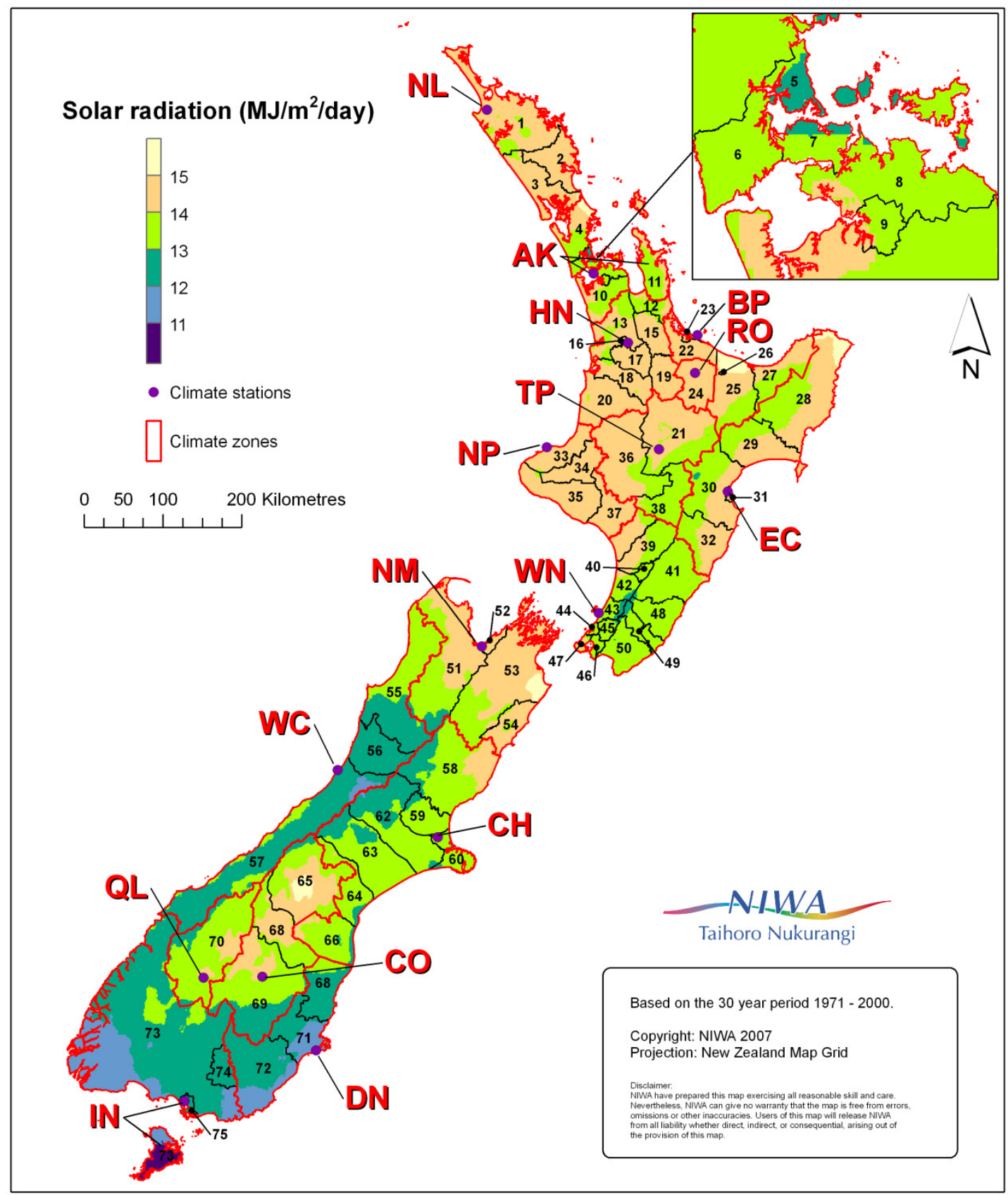

Figure 16 - NIWA Climate Zones

The climate zones were simplified to reduce the number of simulations required. A comparison was conducted to identify climate zones with similar latitudes in the same solar radiation zones. From Table 12, five climate zones were able to be combined with another zone to bring the total number of zones down to 11 . The merged zones were:

- Hamilton, Bay of Plenty and Rotorua;

- New Plymouth, Taupo and East Coast; and

- Queenstown Lakes and Central Otago. 
Table 12 - Climate Zones

\begin{tabular}{|l|c|c|}
\hline Climate Zone & $\begin{array}{c}\text { Solar Radiation } \\
\text { (MJ/m } \mathbf{2} / \text { day) }\end{array}$ & Latitude \\
\hline Northland (NL) & $14-15$ & -35.13 \\
\hline Auckland (AK) & $12-15$ & -37.01 \\
\hline Hamilton (HN) & $14-15$ & $-37-78$ \\
\hline Bay of Plenty (BP) & $14-15$ & -38.11 \\
\hline Rotorua (RO) & $14-15$ & -38.20 \\
\hline Taupo (TP) & $14-15$ & -38.99 \\
\hline New Plymouth (NP) & $14-15$ & -39.01 \\
\hline East Coast (EC) & $14-15$ & -39.46 \\
\hline Wellington (WN) & $13-14$ & -40.91 \\
\hline Nelson/Marlborough (NM) & $14-15$ & -41.30 \\
\hline West Coast (WC) & $12-13$ & -42.72 \\
\hline Christchurch (CH) & $13-14$ & -43.48 \\
\hline Queenstown Lakes (QL) & $13-15$ & -45.02 \\
\hline Central Otago (CO) & $12-15$ & -46.42 \\
\hline Dunedin (DN) & & \\
\hline Invercargill (IN) & & \\
\hline & & \\
\hline & & \\
\hline & & \\
\hline & & \\
\hline & & \\
\hline & & \\
\hline & & \\
\hline
\end{tabular}

For the simulation of interactions between variables, the climates simulated were reduced further. Three climates, Auckland, Wellington and Dunedin, were simulated for the interactions. These three were selected because they are large urban centres situated at either end of New Zealand (Auckland and Dunedin) and at the approximate mid-point (Wellington). Wellington was used as the 'base' climate for this research. Table 12 indicates that higher latitudes typically have higher solar radiation values, meaning that apartment located at the top of the North Island are expected to have higher daylight levels than an apartment located at the bottom of the South Island.

\section{REFLECTANCE}

As was identified by Ng (2001a; 2001b; 2003; 2005), Li et al. (1999; 2006), Wilson and Brotas (2001), Paix (1982) and Littlefair (2001), the reflectance of the obstruction is one of 
the most important factors of daylight in urban environments. The reflectance of the obstruction gives the percentage of light reflected into urban canyons. Two factors are taken into consideration when specifying surface reflectance, the colour and the construction material. The full range of reflectance was simulated, $0 \%$ to $90 \%$. The maximum was selected as $90 \%$ but in typical urban situations the reflectances would range between $10 \%$ and $40 \%$ as shown in Table 13 .

Table 13 - Typical Reflectances of Building Facades.

\begin{tabular}{|l|c|c|}
\hline $\begin{array}{l}\text { Wall Material (Standards } \\
\text { Australian/ Standards New } \\
\text { Zealand, 2006, pg 122) }\end{array}$ & $\begin{array}{c}\text { Percentage of Wall } \\
\text { that is Glazing }\end{array}$ & $\begin{array}{c}\text { Reflectance Value } \\
\text { (\%) }\end{array}$ \\
\hline White Glazed Tiles & $0 \%$ & 80 \\
\hline $\begin{array}{l}\text { Portland cement Smooth finish/ } \\
\text { Light Grey Concrete Block }\end{array}$ & $5 \%$ & 38.5 \\
\hline $\begin{array}{l}\text { Portland cement Smooth finish/ } \\
\text { Light Grey Concrete Block }\end{array}$ & $20 \%$ & 33.3 \\
\hline $\begin{array}{l}\text { Portland cement Smooth finish/ } \\
\text { Light Grey Concrete Block }\end{array}$ & $60 \%$ & 20.8 \\
\hline $\begin{array}{l}\text { Portland cement Rough finish/ } \\
\text { Light Grey Concrete }\end{array}$ & $5 \%$ & 24.0 \\
\hline $\begin{array}{l}\text { Portland cement Rough finish/ } \\
\text { Light Grey Concrete }\end{array}$ & $20 \%$ & 21.4 \\
\hline $\begin{array}{l}\text { Portland cement Rough finish/ } \\
\text { Light Grey Concrete }\end{array}$ & $60 \%$ & 14.8 \\
\hline Dark Concrete & $5 \%$ & 14.6 \\
\hline Dark Concrete & $60 \%$ & 13.5 \\
\hline Dark Concrete & $95 \%$ & 10.8 \\
\hline Glazed Façade & & $25 \%$ \\
\hline
\end{tabular}

As is shown in Table 13, practical applications typically require a combination of the main construction material, colour and glazing. For the simulation of interactions, only five reflectances were simulated, these being $0 \%, 20 \%, 50 \%, 70 \%$ and $90 \%$. The 'base' value simulated was $50 \%$ reflectance.

\section{Secondary Factors}

\section{CEILING HEIGHT}

The floor-to-ceiling height was identified by Ng (2001a) and the IEA (Ruck, 2000) as a factor that affects daylight distribution in apartments. Ceiling height is a secondary factor as there is very little variation between the different ceiling heights and most apartments on lower floors have the same ceiling height.

Five ceiling heights were simulated. These were based on the standard floor to ceiling heights given in the Auckland (2007b) and North Shore (2002b) City Council guidelines. The minimum ceiling height given by the Auckland City Council (2007b) is 2.4 metres. The North Shore City Council (2002b) specifies that apartments on the ground floor should have a 
floor to ceiling height of 3.3 metres, and retail spaces on the ground should have a ceiling height of 4 metres. Apartments on the first floor of mixed-used apartment complexes should have a floor to ceiling height of 3.3 metres. The recommended floor to ceiling height for all other floors is 2.7 metres. Therefore the ceiling heights assessed were 2.4, 2.7, 3, 3.3 and 4 metres. The 'base' ceiling height used was 2.7 metres.

\section{PROPOSED BUILDING HEIGHT}

The height of the proposed building was identified by Ng (2001b; 2003) as a factor that affects daylight performance. Proposed building height was a primary factor in the pilot study but was found to only have a small effect on daylight performance in New Zealand (Stewart, 2006a), therefore it was downgraded to a secondary factor for this research. Five variations of proposed building height were simulated. These were based on the height used for obstructions, but the maximum height tested was just 60 metres. The heights simulated were 10.2, 18.6, 24, 35.4 and 60 metres. The proposed building height used in the base simulations was 24 metres.

\section{GLAZING TRANSMITTANCE}

Glazing transmittance (or type) was identified by Ng (2001a), Li et al. (1999; 2006), Evans (1981), Nabil and Mardaljevic (2006) and the IEA (Ruck, 2000) as a major factor in daylight performance in apartments. Glazing transmittance refers to the percentage of the daylight available that is transmitted through the glazing. However, most apartments use standard glazing types which allows this factor to be a secondary factor.

The glazing transmittance values were adapted from the range of glazing types commercially available. Metro Glass (2006) provides an online catalogue which provides the technical data for the range of typical glazing types. Standard clear single glazing has a visible light transmittance between $86 \%$ and $89 \%$ depending on thickness. This was rounded to $90 \%$ and used as the maximum transmittance value. The minimum transmittance value was taken from the Metro Glass Stopsol Supersilver Blue glazing type, which has a visible light transmittance of $42 \%$. This was rounded down to $40 \%$ to give the minimum transmittance simulated. The glazing transmittance variations simulated were $40 \%, 60 \%, 70 \%, 80 \%$ and $90 \%$. The glazing transmittance used for the base model was $86 \%$ as it was considered to be the glazing type used in most apartment buildings, clear single glazing.

\section{VERTICAL LOCATION}

The vertical location of an apartment within a building is important in the prediction of daylight in apartments, as identified by Ng (2001b; 2003), and Ullah and Lin (2003). Apartments on lower floors generally receive less daylight as their primary source of daylight is that reflected from surrounding obstructions. Therefore, in this research, the vertical location of the lowest apartment will be considered the worst case. 
The majority of inner city apartment buildings in New Zealand have retail spaces located on the ground floor with the lowest floor of apartments being the first floor. In some apartment complexes, the lowest floor of apartments is Ground floor (North Shore City Council, 2002b. pg 93). In some situations, the lower floors of the apartment building are taken up by car parks, making the lowest floor of apartments between 3 and 5 storeys above ground level. The vertical locations that were simulated are shown below in Figure 17. As the most common location of the lowest floor of apartments is $1^{\text {st }}$ floor, this will be used in the base model.

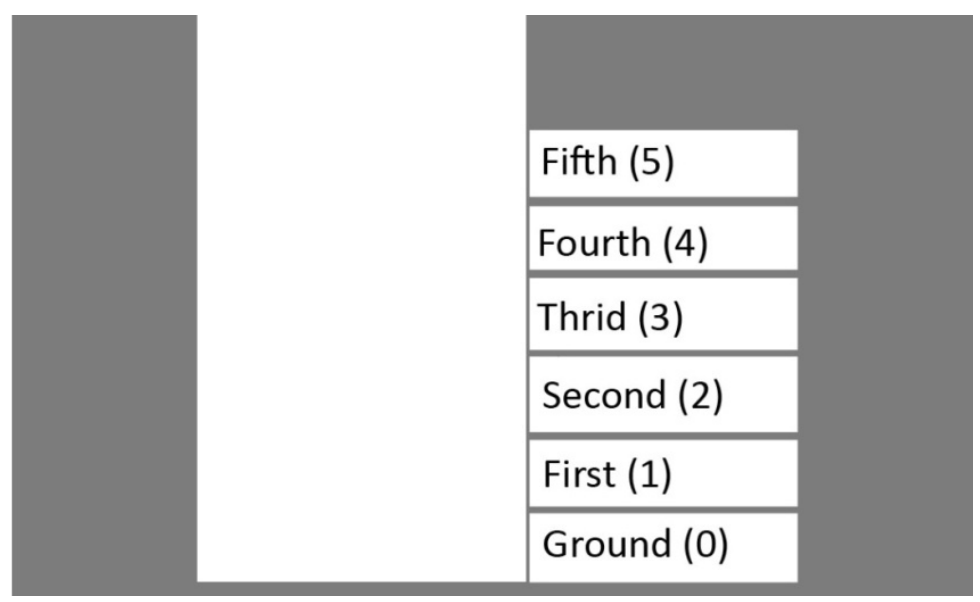

Figure 17 - Vertical Locations

\section{Assumptions}

As was described in Chapter 4, assumptions were needed to limit the scale of the project and provide more accurate results for the most important factors of the building design and surroundings that affect daylight performance. The assumed variables were selected either because there is not sufficient information available in the early design stage (like Internal Reflectances and the presence or absence of furniture) or it was not practical to simulate the number of variations needed to accurately predict the variable (like building form). Practical assumptions were made based on the common variations observed in real situations. An error of 5-6\% (Ng, 2001b) could be expected at the back of the apartments because of these assumptions.

\section{APARTMENT DIMENSIONS}

Although apartment dimensions is a factor that affects daylight performance in apartments, as specified by Ng (2001a), Evans (1981), CIBSE (1999) and the IEA (Ruck, 2000), it was an assumed factor for this research. Specific apartment dimensions are not always available in the early design stage when this tool could be applied. To test the wide range of apartment dimensions observed in New Zealand, an additional 224 simulations would be needed. It was decided that due to the excessive number of simulations required, specific apartment dimensions would not be included as a variable in this research. However, the range of dimensional proportions of apartments were assessed under apartment type, where wide 
and deep apartments give a reasonable representation of the range of possible light penetration mechanisms.

The assumptions for apartment dimensions are based on recommendations of maximum dimensions and realistic combinations of depth and width derived from the floor area. The North Shore City Council (2002b) recommends that single aspect apartments should be no more than 8 metres deep. The room depths were formed around this guideline. However, it was not possible to have a deep apartment with a floor area of $70 \mathrm{~m}^{2}$ or more with a depth of $8 \mathrm{~m}$ or less, so dimensions were devised to have a realistic depth for each apartment. Table 14 shows the dimensions of the eight apartment types.

Table 14 - Apartment Dimensions

\begin{tabular}{|l|c|c|}
\hline & $\begin{array}{c}\text { Depth (Deep Apartment) } \\
\text { Width (Wide Apartment) }\end{array}$ & $\begin{array}{c}\text { Width (Deep Apartment) } \\
\text { Depth (Wide Apartment) }\end{array}$ \\
\hline Small Studio & $8 \mathrm{~m}$ & $5 \mathrm{~m}$ \\
\hline Large Studio & $8 \mathrm{~m}$ & $7.5 \mathrm{~m}$ \\
\hline Small 1 Bedroom & $8 \mathrm{~m}$ & $6.25 \mathrm{~m}$ \\
\hline Large 1 Bedroom & $8.75 \mathrm{~m}$ & $8 \mathrm{~m}$ \\
\hline Small 2 Bedroom & $8.75 \mathrm{~m}$ & $8 \mathrm{~m}$ \\
\hline Large 2 Bedroom & $10 \mathrm{~m}$ & $9.5 \mathrm{~m}$ \\
\hline Small 3 Bedroom & $10 \mathrm{~m}$ & $9.5 \mathrm{~m}$ \\
\hline Large 3 Bedroom & $11 \mathrm{~m}$ & $10 \mathrm{~m}$ \\
\hline
\end{tabular}

This assumption will have a small effect on the overall accuracy of the tool. Apartments that are deeper than the apartments tested will have lower values than those given by the tool, but because these were based on the maximum recommended depth it is unlikely that apartments would be deeper than these values. This assumption will also result in lower predictions for apartments that are not as deep as the apartment simulated but it is not expected to make a significant difference as this tool is testing for worst case scenarios.

\section{INTERIOR REFLECTANCE}

Similar to apartment dimensions, interior reflectance has been identified as a factor that affects daylight performance in buildings but is often not known in the early design stage. In the early stage of lighting design, standard reflectance values are often used. Standard reflectance values are provided by lighting design standards, including: AS/NZS 1680.1:2006 (Standards Australia/Standards New Zealand, 2006), BS 8206 (British Standards Institution, 1992), DIN 5034-3 (DIN Deutsches Institut für Normung, 1994) and the IESNA Lighting Handbook (Rea, 2000).

Using the New Zealand Standard for interior lighting, values were selected that represent the typical situations found in New Zealand apartments. Table 15 shows the approximate reflectance values for typical building finishes. A standard value was selected for a ceiling, walls and a floor. 
Table 15 - Approximate reflectances of typical building finishes (Standards Australia/Standards New Zealand, 2006, pg 122)

\begin{tabular}{|c|c|c|}
\hline Building surface & Reflectance & Material or finish \\
\hline \multirow{4}{*}{ Ceilings } & 0.8 & White water-based paint on plain plasterboard \\
\hline & 0.7 & White water-based paint on acoustic tile \\
\hline & 0.6 & White water-based paint on no-fines concrete \\
\hline & 0.5 & White water-based paint on wood-wool slab \\
\hline \multirow{8}{*}{ Walls } & 0.8 & White water-based paint on plain plasterboard; Tiles: white glazed \\
\hline & 0.4 & $\begin{array}{l}\text { White fibre cement; Brick: concrete, light grey; Portland cement, } \\
\text { smooth }\end{array}$ \\
\hline & 0.35 & Stainless steel \\
\hline & 0.3 & Brick: common \\
\hline & 0.25 & $\begin{array}{l}\text { Concrete, light grey; Portland cement, rough (as board market); } \\
\text { Brick: red; Timber panelling: teak, medium oak; mahogany, gaboon }\end{array}$ \\
\hline & 0.2 & Timber panelling: teak, medium oak; Brick: concrete, dark grey \\
\hline & 0.15 & Brick: dark hard-fired \\
\hline & 0.05 & Chalkboard, painted black (new) \\
\hline \multirow{6}{*}{$\begin{array}{l}\text { Floors and } \\
\text { furniture }\end{array}$} & 0.8 & Paper, white \\
\hline & 0.45 & Cement screed; PVC tiles: cream; Carpet: light grey, middle bluff \\
\hline & 0.35 & Timber: light \\
\hline & 0.25 & $\begin{array}{l}\text { Timber: medium; PVC tiles: brown and cream marbled; Carpet: } \\
\text { turquoise, sage green }\end{array}$ \\
\hline & 0.2 & Timber: dark; Tiles: cork, polished \\
\hline & 0.1 & $\begin{array}{l}\text { Quarry tiles: red, heather brown; Carpet: 'low maintenance'; PVC } \\
\text { tiles: dark brown; Timber: very dark }\end{array}$ \\
\hline
\end{tabular}

The value for ceilings was assumed to be $0.8(80 \%)$, for white water-based paint on plain plasterboard. The value for walls was assumed to be 0.7 (70\%) this was adapted from the value for white water-based paint on plain plasterboard as walls in apartments were typically off-white. This also meant that the wall reflectance value was closer to the 'high reflectance' value of 0.6 given in NZBC G7 Compliance Documents (Department of Building and Housing, 2001). The value for floors was assumed to be 0.25 (25\%), for medium-dark colour carpets. These assumptions were checked against a survey of the surface reflectances of the calibration apartments.

Variation of interior surfaces that differ from that of the tool will result in incorrect predictions of up to $15 \%$ Daylight Autonomy (DA). This estimated error was found using the simulated results of the base model with assumed surface reflectances and then with dark walls and floor as dark as possible ( 0.2 and 0.2 respectively). In the unlikely event that walls are painted in this manner, there is a case to be made that the tool should be limited to cases that have standard reflectances. This is a feature of the design that may change over time (e.g. people deciding to paint their walls dark). However, this tool was intended for standard apartments and cannot be responsible for all situations. It seems sensible to build a design tool in this situation around common practice, not the worst imaginable situation, so because most apartments have these relatively high reflectances, this seems a reasonable assumption. 


\section{GROUND REFLECTANCE}

Ground reflectance was a factor identified by Ng (2001a) and Littlefair (2001) that affects daylight performance. However this value was also assumed. It is acceptable to assume this value because there is little variation in ground reflectances documented in the international literature. In urban environments the ground reflected component plays a minor role in the overall daylight performance.

$\mathrm{Ng}$ (2001a, pg 262) identified that ground reflectance is generally assumed to be 0.2 . Compared to the Standards New Zealand (2006) material reflectances this corresponds to a dark grey brick and compared to the British Standard (1992) material reflectances, this corresponds to granite. The common materials used on New Zealand roads are Asphalt and Bitumen but neither of the standards gave a reflectance for these. Both these materials are dark, stone based materials, therefore the use of the reflectance value of 0.2 for dark grey brick and granite (both dark, stone based materials) is a reasonable assumption for the typical reflectance of New Zealand roads. Variations of ground reflectance are minimal and would have a negligible effect on the prediction.

\section{WINDOW POSITION}

Window position within the exterior wall was identified by Ng (2001a) and the IEA (Ruck, 2000) as a factor that affects daylight distribution. However, this is another factor that is typically not known at the early design stage. Also, from observations of Wellington Apartments (Stewart, 2006b), it was noticed that the majority of apartment living rooms had windows that were around floor to ceiling height and positioned in the centre of the exterior wall. The window positions in bedrooms were not as easy to generalise as some were floor to ceiling, similar to living rooms but many other configurations were observed. For most bedrooms though, the windows were centred horizontally in the external wall.

Using these observations, assumptions were made about the window location. All windows were assumed to be centred horizontally within the exterior wall. Living rooms had windows around floor to ceiling height (varying depending on the specified glazed area). Bedrooms had windows to satisfy the view requirements specified in the building code (Department of Building and Housing, 2001, pg 16). Figure 18 shows the assumed window locations and sizes for the base model. This assumption may result in slightly lower illuminances than if the windows were located higher in the exterior wall. 


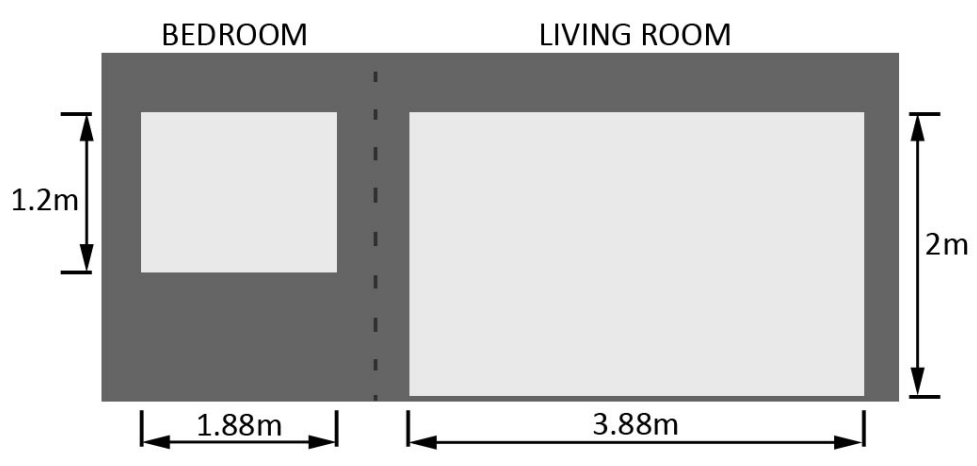

Figure 18 - Window Locations

\section{SHADING FACTORS}

Shading factors are widely recognised as a factor that affects the penetration of daylight in buildings. Shading factors include overhangs, side-fins and balconies. However, as with many of the other assumed factors, the size and form of shading devices is often not known in the early design stage. It is also a complex design feature and would require a large number of variations to be simulated and would add to the complexity of the tool. It was decided to assume that no shading factors were present.

This was a variable that would greatly contribute to the overall errors from assumptions if shading devices were present. Shading factors would cause lower daylight levels at the back of the apartment than was simulated. To ensure that this assumption did not impact greatly on the overall accuracy of the tool, calibration apartments with and without shadings were measured and compared to ensure that apartments with shading devices were not receiving incorrect pass results because of this assumption.

\section{MEASUREMENT POINTS}

The tool was aimed at determining whether the areas of the room with the lowest illuminance exceeded the minimum code requirement. Specific points needed to be selected that represented the lowest illuminance points. These points were identified in Chapter 4 as the standard measurement points for this research.

To account for possible changes and to provide extra data for possible further research, other points within the room were also simulated, however these will not be studied for the development of the tool. Figure 19 shows the simulation points measured, with point $B$ being the focal measurement point for this study. Points $A$ and $C$ were used to ensure consistency in the results as these points should all have similar results. 


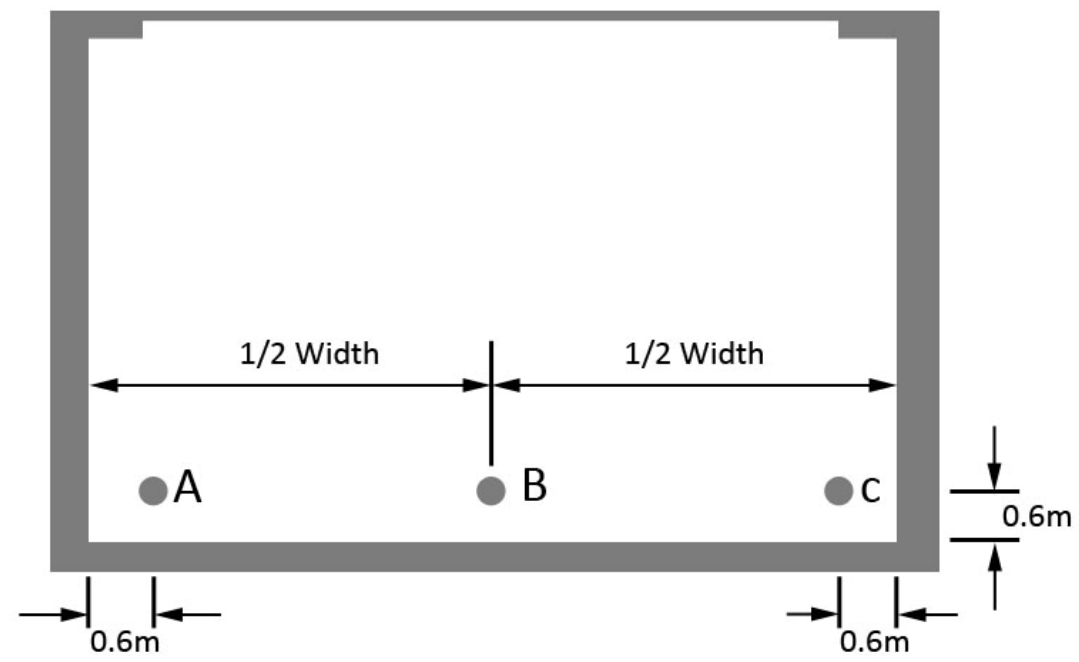

Figure 19-Measurement Points

\section{LAYOUT}

The spatial configuration of the apartment is guided by the need for natural light. NZBC G7 requires natural light in habitable spaces, so these are typically located next to windows, meaning that the deeper, darker areas are used by spaces that do not require natural light. Although it is not a major factor affecting daylight performance, it is still important to replicate as closely as possible the situations typically found in New Zealand apartments. The North Shore City Council Good Solutions Guide for Apartments (2002b) provides some general layouts to achieve satisfactory daylight access, natural ventilation and aspect/view. These layouts were supported by the findings of Whyte (1996), where it was identified that utility spaces are typically located at the rear in Wellington apartments. Figure 20 shows the standard layout given in the Good Solutions Guide for Apartments for single aspect apartments.

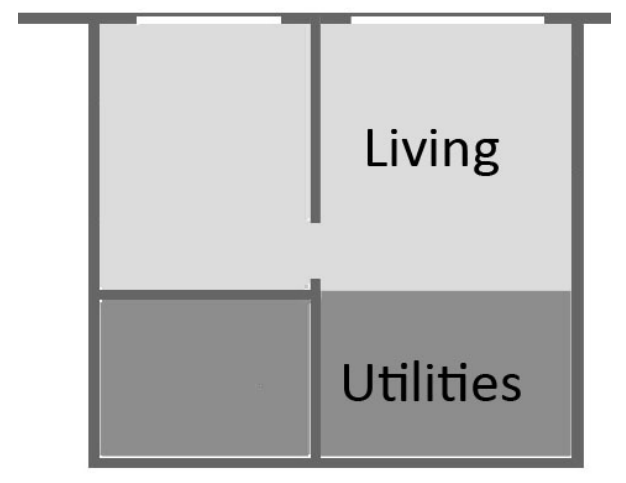

Figure 20 - Apartment Layout

\section{FURNITURE}

Apartment fit-out and furnishings have not been modelled. The introduction of internal obstructions such as beds, couches and tables will have an effect on the performance of daylight in the space. Furnishing and fit-out will affect the reflectance value of the space as 
porous materials would reflect considerably less light. Fit-out and furnishings also create obstructions, reducing daylight at the rear of the space. However predicting precise layout and spectral qualities of furnishings is difficult (Standards Australia/Standards New Zealand, 2006, pg 91) and can differ between tenants. Because this tool is only providing code practice results, and the code does not require the room to be furnished, it was assumed that there are no furnishings in the space.

\section{BUILDING FORM}

Building form is a major factor of daylight access into an apartment, as was identified by Allen (1943), CIBSE (1999) and the IEA (Ruck, 2000). Building form includes atria, light-wells and all variations of building plan. Due to the large variety of possibly variations it is not possible to define 'typical forms' other than the basic rectangular form commonly used in New Zealand cities. Building form is often site specific, where the shape and boundary types define the way in which the site can be used. The base model for this research represents a standard block found in the residential area of any New Zealand city. Therefore, the site was assumed to be a simple rectangular site with one boundary facing onto the street. In the calibration tests, some atria and light-wells were measured to determine how well these design features perform.

\subsubsection{Simulation Analysis Method}

Patterns in the parametric data provided by the simulations were used to develop an equation that can predict the daylight performance $0.6 \mathrm{~m}$ from the back of an apartment building (the standard measurement point used in this research). Regression analysis was used to create the equation. The following equation shows the form the equation was to take, where a, b, c, d, e, etc are the products of the analysis.

$$
D A=a+b B H+c S W+d G A+e O r \ldots
$$

- $D A$ is the daylight autonomy value, as a percentage of the year that 30 lux is exceeded;

- $B H$ is the height of the opposite building;

- $S W$ is the distance between the proposed building and obstruction (opposite building);

- $G A$ is the percentage of glazed area compared to of the floor area; and

- $O r$ is the orientation of the apartment.

SPSS (2007) is a statistical analysis program that provides these values. To find a, b, c, d etc two types of analysis needed to be performed using SPSS. The first was the analysis of each variable independently to determine the type of relationship each variable had with daylight performance (daylight autonomy) to aid the second stage of analysis. The second 
was the analysis of the relationship between all variables and daylight performance (DA), this was the analysis that provided the values to be added to the equation.

\section{Method of Analysis for Variables Independently}

Each of the 11 variables were independently analysed to determine the type of relationship they had with daylight performance. There are two main types of relationships (or models), linear and curvilinear, based on the data patterns. This was an essential step in the development process because the results influenced the method used for the combination of variables.

The simulation results for each variation were plotted and the line of best fit was found, whether it was linear (red) or curvilinear (blue) as shown in Figure 21.

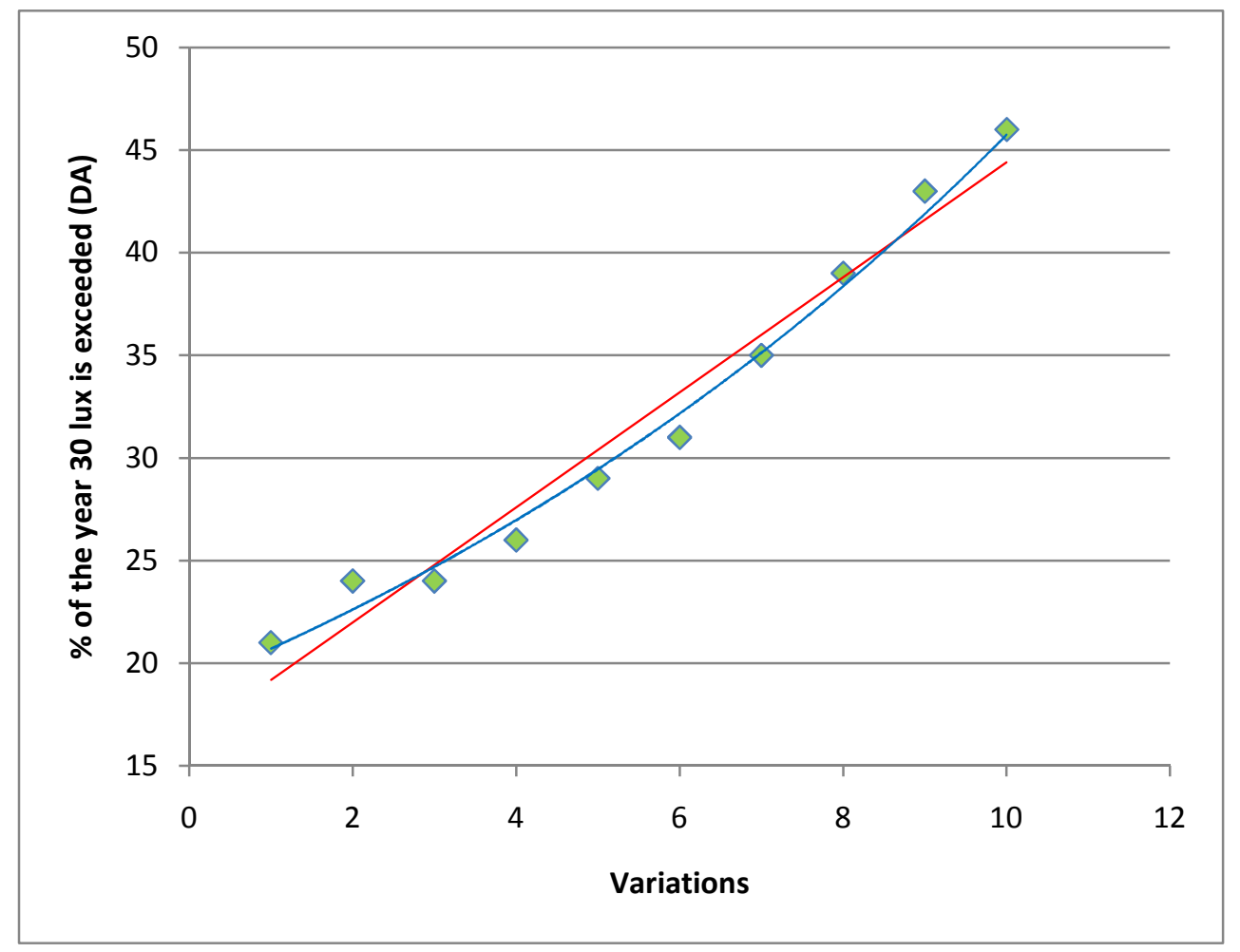

Figure 21 - Line of Best Fit for Linear and Curvilinear Patterns

SPSS also provides a table that describes how well the linear model and the 10 curvilinear models fit the simulation data. From this table the model with the best fit was selected and an equation for that variable was created. The information used to identify good fit of a model was the R Square values and the Significance values (Sig.).

The R Square value describes the percentage of variations explained by that model, where 0 indicates none of the situations were explained by the model (no relationship) and 1 means that all variations were explained by the model (perfect relationship) (Cohen et al., 2003, pg 70). 
The Significance level (Sig.) is used to indicate whether the variation in results is due to chance or because of the variable being assessed, where 0 indicates that the variation is definitely because of the variable (perfect relationship) and 1 indicates that it is purely by chance (no relationship) (Cohen et al., 2003, pg 15).

The minimum acceptable $\mathrm{R}$ Square value for this research is 0.7 , as recommended by Roscoe $(1975, \mathrm{pg} 101)$ and the maximum Significance level is 0.05 , as recommended by Cohen et al. (2003, pg 15). The use of these values would not have any effect on the accuracy of the results as they are only used to eliminate the models that would not provide reliable results.

Table 16 - Table used to assess the fit of the relationships

\begin{tabular}{|c|c|c|c|c|c|c|c|c|c|}
\hline \multirow[b]{2}{*}{ Equation } & \multicolumn{4}{|c|}{ Model Summary } & \multicolumn{5}{|c|}{ Parameter Estimates } \\
\hline & R Square & $\mathrm{F}$ & df1 & df2 & Sig. & Constant & $\mathrm{b} 1$ & b2 & b3 \\
\hline Linear & .665 & 9.926 & $\overline{1}$ & $\overline{5}$ & .025 & 61.143 & -.848 & & \\
\hline Logarithmic & .882 & 37.455 & 1 & 5 & .002 & 47.310 & -7.689 & & \\
\hline Inverse & .746 & 14.665 & 1 & 5 & .012 & 21.663 & .074 & & \\
\hline Quadratic & .932 & 27.573 & 2 & 4 & .005 & 83.984 & -2.735 & .021 & \\
\hline Cubic & .976 & 40.124 & 3 & 3 & .006 & 91.679 & -4.190 & .067 & .000 \\
\hline Compound & .957 & 110.479 & 1 & 5 & .000 & 78.231 & .956 & & \\
\hline Power & .450 & 4.083 & 1 & 5 & .099 & 27.231 & -.242 & & \\
\hline S & .283 & 1.973 & 1 & 5 & .219 & 2.542 & .002 & & \\
\hline Growth & .957 & 110.479 & 1 & 5 & .000 & 4.360 & -.045 & & \\
\hline Exponential & .957 & 110.479 & 1 & 5 & .000 & 78.231 & -.045 & & \\
\hline
\end{tabular}

The independent varlable Is Building height.

For the next stage of analysis, equations were needed for each of the variables. For the following linear equation, $\mathrm{a}$ and $\mathrm{b}$ can be drawn from Table 16, where $\mathrm{a}$ is the Constant and $\mathrm{b}$ is $b 1$. Each model has a specific equation type which is needed for the next stage of analysis.

\section{$D A=a \times b B H$}

- $D A$ is the Daylight Autonomy value (percentage of the standard year that 30lux is exceeded); and

- $\boldsymbol{B H}$ is the height of the opposite building (or obstruction).

\section{Method of Analysis for All Variables Combined}

The next stage of analysis was the development of the equation (prediction model) for the relationship between all variables and the daylight performance. Because both linear and curvilinear models were found in the analysis of the variables independently, Linear and Nonlinear analysis was needed to find the best prediction model for this relationship. 


\section{LINEAR}

The linear analysis was used to produce an equation for all variables assuming that all variables had a linear relationship with daylight performance as this was the simplest equation however this was not expected to yield the most reliable results. The prediction model produced by linear analysis takes the following form, where $a, b, c$, d etc are the product of this analysis.

$$
D A=a+b B H+c S W+d G A+e O r \ldots
$$

Where:

- $D A$ is the daylight autonomy value, as a percentage of the standard year in which 30 lux is exceeded;

- $B H$ is the height of the opposite building;

- $S W$ (street width) is the distance between the proposed building and obstruction (opposite building);

- $G A$ is the percent of glazed area compared to of the floor area; and

- Or is the orientation of the apartment.

SPSS computes the relationship between all variables and daylight performance, producing parameters for the equation (to replace $a, b, c, d$, and e in the equation) and an assessment of the strength of the prediction model. As with the analysis of variables independently, $R$ Square and Significance values were used to assess the strength of the model. The same minimum $R$ Square and maximum Significance values of 0.7 and 0.05 were used respectively. In addition to the $\mathrm{R}$ Square and Significant values for the model, a Significance value is also provided for each variable to indicate how well the variables fit into the overall model.

\section{CURVILINEAR}

Through the independent analysis of each variable it was found that some of the variables had curvilinear relationships with daylight performance. This required the more complex nonlinear analysis to be undertaken. Fundamentally, nonlinear analysis is the same as linear analysis, but rather than using a pre-defined equation, the equation to be assessed needs to be defined. This allows SPSS to analyse the curvilinear relationships of different variables.

To define the equation, the independent equations produced in the first stage of analysis were used. Overall the equation was to take the same form as the linear equation, but curvilinear components were inserted for particular variables when needed. The following equation shows how an exponential component would replace the linear component for Building Height and Glazed Area. 


$$
D A=a+\exp ^{(b B H)}+c S W+\exp ^{(d G A)}+e O r \ldots
$$

Where:

- $D A$ is the daylight autonomy value, as a percentage of the year in which 30 lux is exceeded;

- $B H$ is the height of the opposite building;

- $S W$ (street width) is the distance between the proposed building and obstruction (opposite building);

- $G A$ is the percent of glazed area compared to of the floor area; and

- Or is the orientation of the apartment.

SPSS then computes the parameters to replace $a, b, c$, d etc and the assessment of how well this prediction model fits the simulation data. The $\mathrm{R}$ Square value is used to assess the strength of the overall model again, but a Significance value is not provided for nonlinear analysis. To check how well the model fits each variable, estimated errors are provided.

To determine which of the linear and nonlinear models provided the best predictions, the equations were used to predict the results for each of the simulated cases. The predicted results were compared to the simulated results to determine how well the equation fits the simulation data. The $\mathrm{R}$ Square values and the accuracy of predictions against simulated results were used to select the best equation for use in the tool.

\subsubsection{Development of the Tool}

The tool developed in the pilot study was used as the model for further developments. However, the development of an equation allowed for more complex calculations to be undertaken with no extra input required by users of the tool. The result provided by the tool was to be maintained. The input style has moved from a 'selecting of categories' to entering the specific information This gives more accurate results but may require extra time in the application of the tool.

Figure 22 shows the how calculation process is formatted in the tool, where the cells framed in grey are hidden from the user. 


\begin{tabular}{|c|c|c|c|}
\hline \multicolumn{2}{|c|}{ NZBC G7 Compliance Assessment Tool } & & \\
\hline \multicolumn{4}{|l|}{ Variable: } \\
\hline Opposite Building Height & & $b B H$ & \\
\hline Street Width & & $c S W$ & \\
\hline Glazed Area & & $d G A$ & \\
\hline Orientation & & $e O$ & \\
\hline Location & & $f L$ & \\
\hline Reflectance of Opposite Building & & $g R$ & \\
\hline Glazing Transmittance & & $h G T$ & \\
\hline \multirow{2}{*}{\multicolumn{2}{|c|}{ Vertical Location of lowest apartment }} & $i V L$ & \\
\hline & & SUM & $\begin{array}{c}a+S U \\
M\end{array}$ \\
\hline Are simulations required to prove compliance: & No & & \\
\hline
\end{tabular}

Figure 22 - New Compliance Assessment Tool

The format of the tool assumes that users have experience with it. Therefore it is essential that an accompanying document is provided which explains its application and examples for first time users and more complex situations.

\subsection{Development Process}

\subsubsection{Analysis of Variables Independently}

This section provides a summary of the analysis of the relationship between daylight performance and each of the variables independently. First, an example is provided showing how the variables were analysed. Then a summary of each of the relationship types is then given, with the relevant variables identified.

\section{Analysis Example - Building Height}

Using SPSS, the simulation results for the 7 variations of Building Height were plotted against the daylight autonomy values to find the line of best fit. 11 different lines (models) were tested, including linear, logarithmic and exponential, to find which line represented the relationship between daylight performance and the variable being tested, building height in this case. Figure 23 shows the results for building height, with the linear model and the best of the curvilinear models which was exponential. 


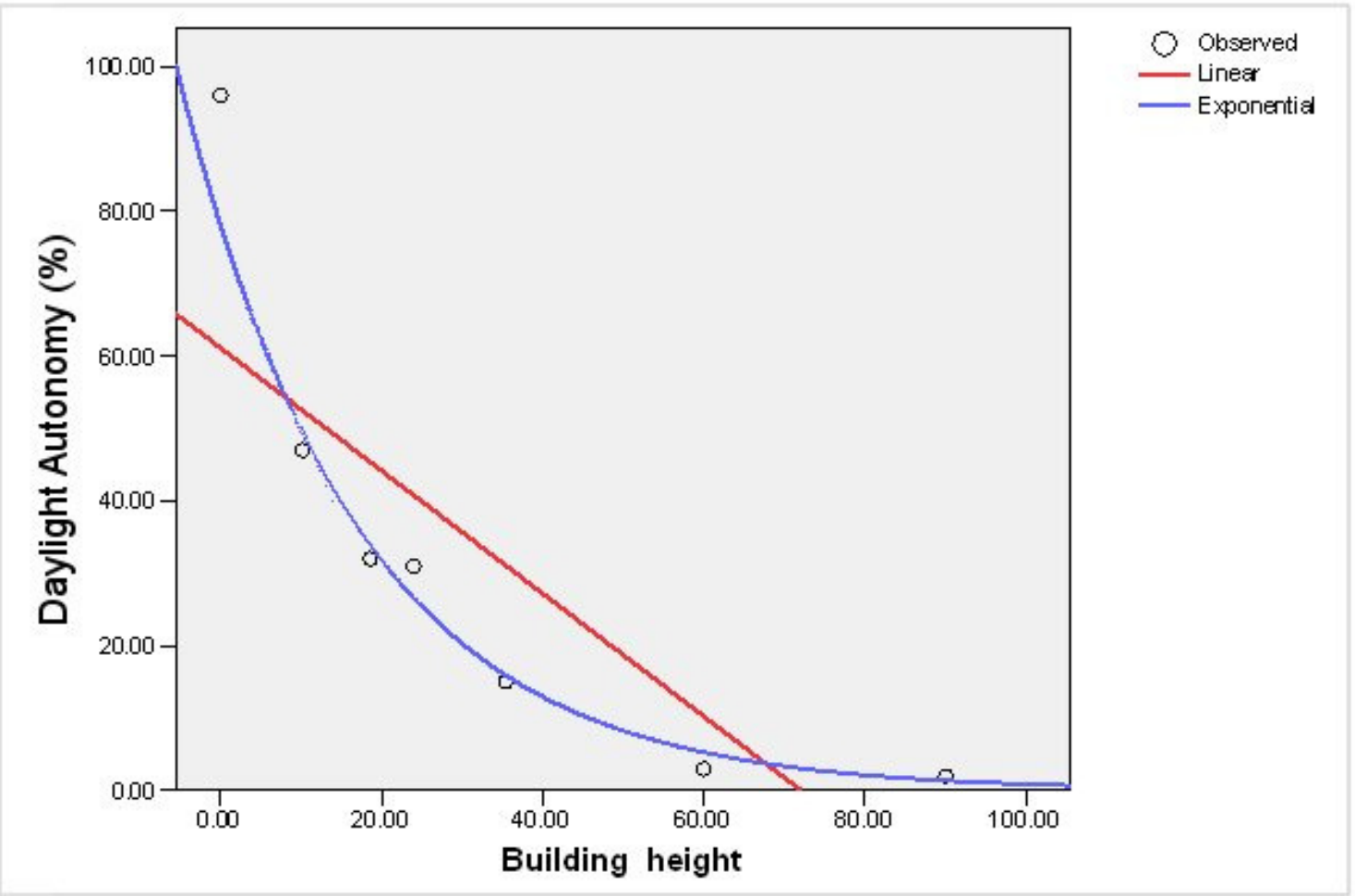

Figure 23 - Individual Relationship between Daylight Performance ad Building Height

From this graph, it was decided whether the relationship was linear or curvilinear. For building height it can be seen that the relationship is clearly curvilinear. To check this conclusion, SPSS provides statistics about the fit of each model. The statistics were assessed to establish which of the 11 models provided the best representation of the relationship between daylight performance (DA) and building height in this case. From Table 17 it was identified that the exponential model provided the best representation as the $\mathrm{R}$ Square value is close to 1 and is over the minimum of 0.7 , and the model has a significance level of 0 which suggests a almost perfect relationship. Note that compound and logistic models provide the same results as the exponential model but the exponential model was selected because it had the simplest equation - following the general scientific principle normally referred to as Occam's Razor.

Table 17 - Building Height: Model Summary

\begin{tabular}{|c|c|c|c|c|c|c|c|c|c|}
\hline \multirow[b]{2}{*}{ Equation } & \multicolumn{5}{|c|}{ Model Summary } & \multicolumn{4}{|c|}{ Parameter Estimates } \\
\hline & R Square & $\mathrm{F}$ & df1 & df2 & Sig. & Constant & b1 & b2 & b3 \\
\hline Linear & .665 & 9.926 & 1 & 5 & .025 & 61.143 & -.848 & & \\
\hline Logarithmic & .882 & 37.455 & 1 & 5 & .002 & 47.310 & -7.689 & & \\
\hline Inverse & .746 & 14.665 & 1 & 5 & .012 & 21.663 & .074 & & \\
\hline Quadratic & .932 & 27.573 & 2 & 4 & .005 & 83.984 & -2.735 & .021 & \\
\hline Cubic & .976 & 40.124 & 3 & 3 & .006 & 91.679 & -4.190 & .067 & .000 \\
\hline Compound & .957 & 110.479 & 1 & 5 & .000 & 78.231 & .956 & & \\
\hline Power & .450 & 4.083 & 1 & 5 & .099 & 27.231 & -.242 & & \\
\hline$S$ & .283 & 1.973 & 1 & 5 & .219 & 2.542 & .002 & & \\
\hline Growth & .957 & 110.479 & 1 & 5 & .000 & 4.360 & -.045 & & \\
\hline Exponential & .957 & 110.479 & 1 & 5 & .000 & 78.231 & -.045 & & \\
\hline Logistic & .957 & 110.479 & 1 & 5 & .000 & .013 & 1.046 & & \\
\hline
\end{tabular}

The independent variable is Building_height. 
To provide the equations for an overall curvilinear equation in the second stage of analysis, the equations for each variable independently were needed. This information is also provided in Table 17. The equation for building height was an exponential equations as shown below, where $a$ and $b$ can be found using the table and inserted into the equation.

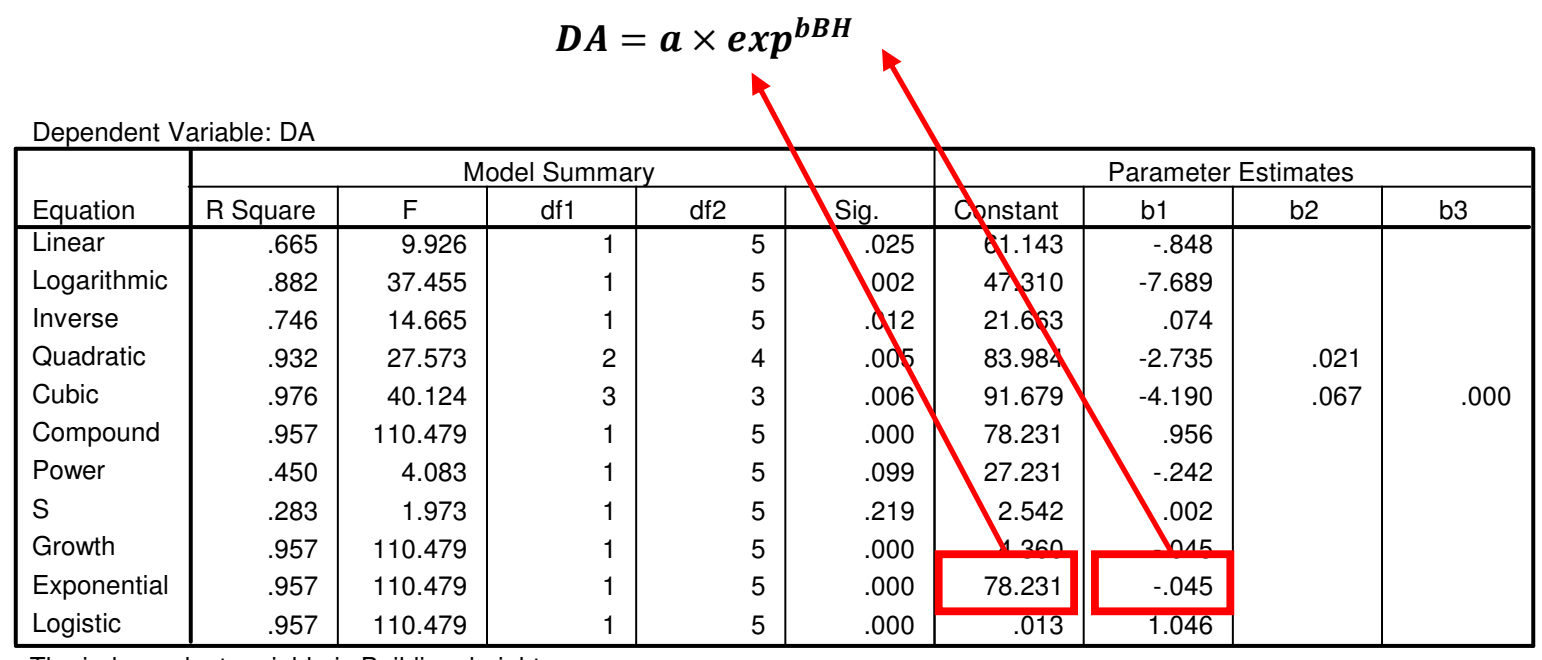

The independent variable is Building_height.

This was done for all 11 variables. The full analysis for each variable can be found in Appendix 2.

\section{Linear Variables}

Three of the 11 variables had clear linear relationships with daylight performance. These variables were: Street Width, Geographical Location and Glazing Transmittance. In addition to these three variables, two of the variables with curvilinear relationships were also able to be expressed as linear models to simplify the final equation if needed. These variables were: Glazed Area and Reflectance.

All three of the variables with linear relationships, and reflectance, had very strong linear relationships, with $\mathrm{R}$ Square values greater than 0.9 and Significance values less than 0.01 . This meant that these variables can be predicted with a reasonable level of accuracy in the final prediction models.

\section{Curvilinear Variables}

Five of the 11 variables have curvilinear relationships with daylight performance (DA). These variables were Opposite Building Height (the example case), Glazed Area, Reflectance, Proposed Building Height and Vertical Location. Of these five curvilinear variables, two had exponential relationships with daylight performance (Opposite Building Height and Glazed Area), one had a quadratic relationship (Reflectance), one had an inverse relationship (Proposed Building Height) and one had a cubic relationship (Vertical Location).

Four of these five variables had very strong relationships with their respective models, with R Square values greater than 0.9 and Significance values less than 0.01 . This indicates that if 
a nonlinear equation was used, reliable predictions could be obtained for these variables. The one variable that was not considered to have a very strong relationship was Vertical Location, which had a near perfect $R$ Square value of 0.999 , but had a high significance level of 0.037 . This was still within the acceptable range.

\section{No Significant relationship}

Three of the variables were found to have no significant relationship with daylight performance. These variables were: Apartment Type, Orientation and Ceiling Height.

Apartment Type was a variable that could not be expressed linearly and therefore could not be analysed using regression analysis. If large variations between different apartment types were observed in this variable, then an equation would have been needed for each different apartment type. Most apartments were within a $10 \%$ range which meant that there was not a great deal of variation between different Apartment Types. As such, it was decided to exclude this variable from further analysis as the time involved in creating 16 different equations would have been significant for such a small range of variations. Because of this small range in variations it was expected that this decision would not affect the overall accuracy of the tool.

The best models for Orientation were cubic or quadratic, where both had an acceptable $\mathrm{R}$ Square over 0.7 , but the significance values were well over the maximum acceptable value of 0.05 . Despite this variable not achieving the acceptable significance values, it was decided to continue with this variable as it was identified in the research method to have a large effect on daylight performance and had an R Square well within the acceptable range.

The best model for Ceiling Height was cubic, which had an acceptable R Square but the Significance value was greater than the maximum acceptable value of 0.05 . It was decided to exclude Ceiling Height from further analysis. This is expected to have a negligible effect on the results because there was very little variation in the results for Ceiling Height, with a range of just $5 \%$ DA.

\subsubsection{Regression Models}

To develop an equation that considered the relationship between all variables and daylight performance (DA), two types of regression analysis were performed, Linear and Nonlinear. Four different linear models were developed using SPSS, based on different combinations of variables. Three different nonlinear models were developed using SPSS, based on the relationships of the each variable independently found in part 1. The purpose of testing different models was to find the most reliable equation for the tool. The full analysis of these models can be found in Appendix 3. 


\section{Example of Analysis of a Regression Model}

To analyse the strength of the different models, SPSS provides an R Square value, Standard Error and Significance value for the whole model and a Significance value and standard error for each variable. The coefficients for the equations ( $a, b, c$, etc) are also given by SPSS. The equation for the example case is shown below, with the substituted coefficient values also shown. Table 18 shows the factors used to assess the strength of the different equations. As can be seen, the example case has an acceptable R Square (greater than 0.7) and Significance levels (less than 0.05), but Vertical Location and Glazing Transmittance are not predicted as well as the other variables.

$$
\begin{gathered}
D A=a+b B H+c S W+d O r+e G A+f L+g R+h V L+i G T \\
D A=-56-0.48 B H+1.9 S W+0.0260 r+1.3 G A-1.4 L+0.25 R+4.2 V L \\
+0.36 G T
\end{gathered}
$$

\begin{tabular}{|c|c|c|c|c|c|}
\hline \multirow{2}{*}{ Overall Equation } & \multirow{2}{*}{$\begin{array}{c}\text { R Square } \\
0.793\end{array}$} & \multirow{2}{*}{$\begin{array}{c}\begin{array}{c}\text { Overall } \\
\text { Significance Level }\end{array} \\
0.000\end{array}$} & \multirow{2}{*}{$\begin{array}{c}\begin{array}{c}\text { Standard } \\
\text { Error }\end{array} \\
\pm 8.520 \\
\end{array}$} & \multicolumn{2}{|c|}{ Variables } \\
\hline & & & & \multirow{2}{*}{$\begin{array}{c}\text { Significance } \\
\text { Level }\end{array}$} & \multirow{2}{*}{$\begin{array}{c}\text { Standard } \\
\text { Error }\end{array}$} \\
\hline & & & & & \\
\hline Constant (a) & & & & 0.000 & $\pm 24 \%$ \\
\hline Building Height (b) & & & & 0.000 & $\pm 5 \%$ \\
\hline Street Width (c) & & & & 0.000 & $\pm 4.7 \%$ \\
\hline Orientation (d) & & & & 0.006 & $\pm 38 \%$ \\
\hline Glazed Area (e) & & & & 0.000 & $\pm 6.3 \%$ \\
\hline Location (f) & & & & 0.000 & $\pm 19 \%$ \\
\hline Reflectance (g) & & & & 0.000 & $\pm 9.5 \%$ \\
\hline $\begin{array}{l}\text { Vertical Location } \\
\text { (h) }\end{array}$ & & & & 0.022 & $\pm 43 \%$ \\
\hline $\begin{array}{l}\text { Glazing } \\
\text { Transmittance (i) }\end{array}$ & & & & 0.017 & $\pm 42 \%$ \\
\hline
\end{tabular}

Table 18 - Factors Used to Assess the Strength of Regression Models

As an extra measure of assessing the strength of the models, the simulation results were graphed against the predicted results. Figure 24 shows the comparison of simulated results ( $x$-axis) and predicted results ( $y$-axis) for the example equation. It can be seen that generally the results are reasonably similar as they typically follow the desired trend-line shown in black. However, some of the apartments with the higher daylight autonomy values receive under-predictions from the equation (those circled in red). 


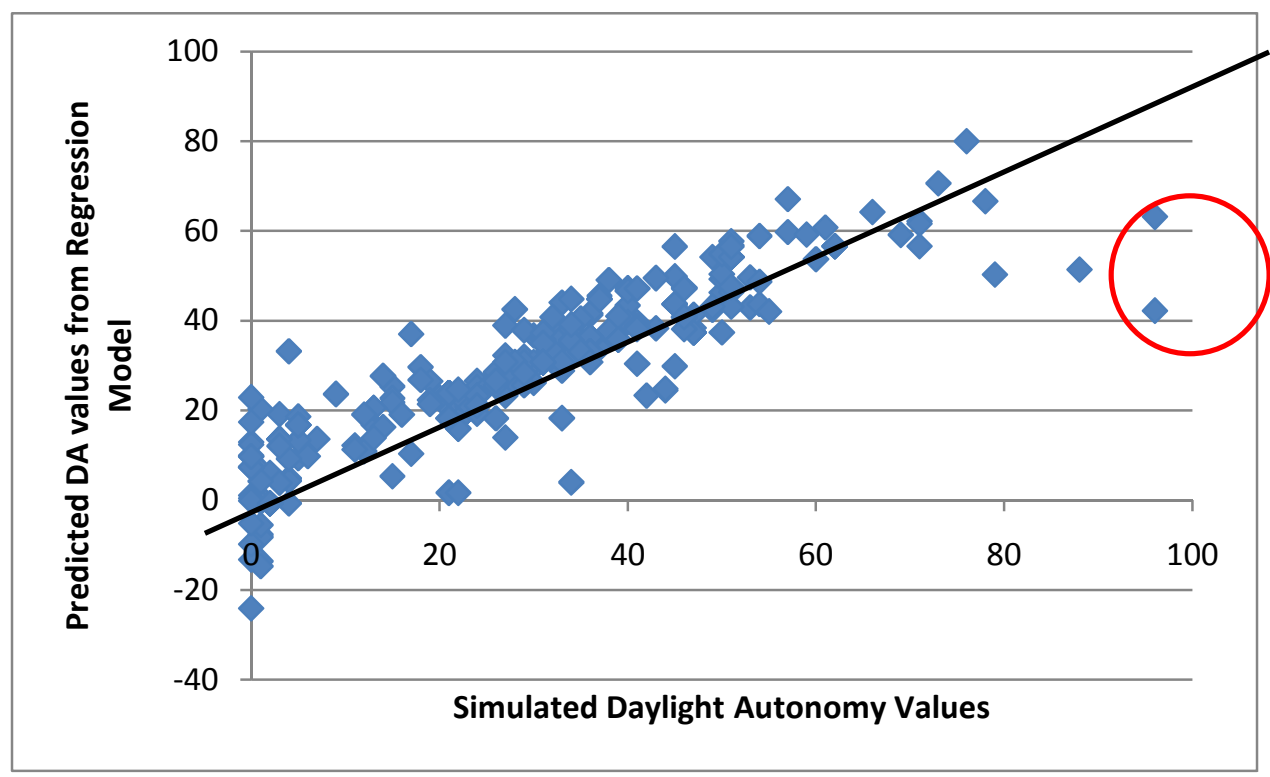

Figure 24 - Comparison of Predicted Results and Simulated Results

Each of the models were then compared using the R Square and Significance values, and the accuracy of the prediction results against the simulated results to determine which model provided the most reliable predictions.

\section{Summary of Linear Models}

From the comparison of the four linear models, the strongest overall model was between Model A (all variables) and Model B (all variables except proposed building height, this was the example case shown). Model A had the highest $R$ Square value, but Model $B$ had the lowest Standard Error. The coefficients were needed to determine which of these two models was strongest.

Model A, with all variables, had some high Standard errors, especially for the constant, proposed building height and glazing transmittance. The Significance levels for Model A were generally acceptable. However, 2 of the coefficients had unacceptably high levels, in particular proposed building height.

Model B had lower standard errors, particularly for the constant and glazing transmittance. Noticeable improvements were seen for the Significance levels, the constant went from 0.013 to 0 and glazing transmittance went from an unacceptable 0.134 to 0.017 , which is well within the acceptable range.

From the comparison of the predicted results against the simulated results, there was very little variation between all four equations. Because of the improved significance levels, it was concluded that the strongest model was Model B. 


\section{Summary of Nonlinear Models}

From the comparison between predicted results and simulated results it was difficult to determine whether nonlinear Model A (complex nonlinear equation) or B (simpler nonlinear equation) should be used to represent the relationship between the various factors and daylight performance. Statistically, the strongest overall model was between Models A and B. Model A had the highest R Square value and lowest Standard Error, but had some extremely high errors for variables. Model B had considerably lower errors for variables but also had a lower $\mathrm{R}$ Square value. It seemed that model B would most likely yield the best results. However, due to the difficulty in determining the differences in the predictions of Models A and B, both models were selected for further study.

\section{Summary of Models Overall}

From the analysis of four linear models and three nonlinear models, three were identified as being the best equations to predict the relationship between daylight performance and the variables tested. Each of these three models had strengths and weaknesses. No purely objective statistical fit information could be used to determine which model was conclusively better. It was decided that each of these three equations would be tested in the calibration tests to determine which model provided the best results for real situations. The three equations are shown below, with estimated errors based on the statistic provided by SPSS.

\section{LINEAR MODEL}

$$
\begin{gathered}
D A=-56-0.48 B H+1.9 S W+0.0260 r+1.3 G A-1.4 L+0.25 R+4.2 V L \\
+0.36 G T
\end{gathered}
$$

Standard Error of $\pm 8.5 \%$ DA

\section{MODEL 2A (NONLINEAR MODEL A)}

$$
\begin{gathered}
D A=-90+78 \exp (-0.05 B H)+1.9 S W+0.0360 r+17 \exp (0.032 G A)-1.4 L \\
+0.18 R+0.001 R^{2}+2.2 V L-0.31 V L^{2}+0.18 V L^{3}+0.27 G T
\end{gathered}
$$

Standard Error of $\pm 6.7 \%$ DA

\section{MODEL 2B (NONLINEAR MODEL B)}

$$
\begin{gathered}
D A=16 \times E X P(-0.047 B H) \times E X P(0.04 G A) \times E X P(0.01 R) \times E X P(0.176 V L) \\
+1.8 S W-1.5 L+0.0360 r-0.21 G T
\end{gathered}
$$




\subsubsection{Formation of Spreadsheet}

An Excel spreadsheet was used as the platform for applying the tool as it is an excellent way to conduct calculations with minimal effort required by the users. The spreadsheet is comprised of three components: the user inputs; the prediction calculation; and the specification of the result. These sections are shown below in Figure 25.

\begin{tabular}{|c|c|c|c|}
\hline & er Input & \multicolumn{2}{|c|}{ Prediction Calculation } \\
\hline \multicolumn{4}{|c|}{ NZBC G7 Compliance Assessment Thol } \\
\hline \multicolumn{4}{|l|}{ Variable: } \\
\hline Opposite Building Height & 15 & -7.155 & \\
\hline Street Width & 20 & 37.46 & \\
\hline Glazed Area & 26 & 33.566 & \\
\hline Orientation & West & 2.34 & \\
\hline Location & Wellington & -8.352 & \\
\hline Reflectance of Opposite Building & 13.5 & 3.3885 & \\
\hline Glazing Transmittance & 87 & 31.32 & \\
\hline \multirow[t]{2}{*}{ Vertical Location of lowest apartment } & 7 & 29.316 & \\
\hline & & 121.8835 & 100.7 \\
\hline Are simulations required to prove compliance: & No & & \\
\hline & & & \\
\hline
\end{tabular}

Figure 25 - Components of the Tool

The three prediction models identified in Section 5.2.2 for further assessment were used in the tool until it was confirmed which model is most reliable from the calibration process in Chapter 6 . The prediction models were calculated in stages, as is shown in Figure 26, where each variable is calculated separately and then the interaction between the variables is applied. 


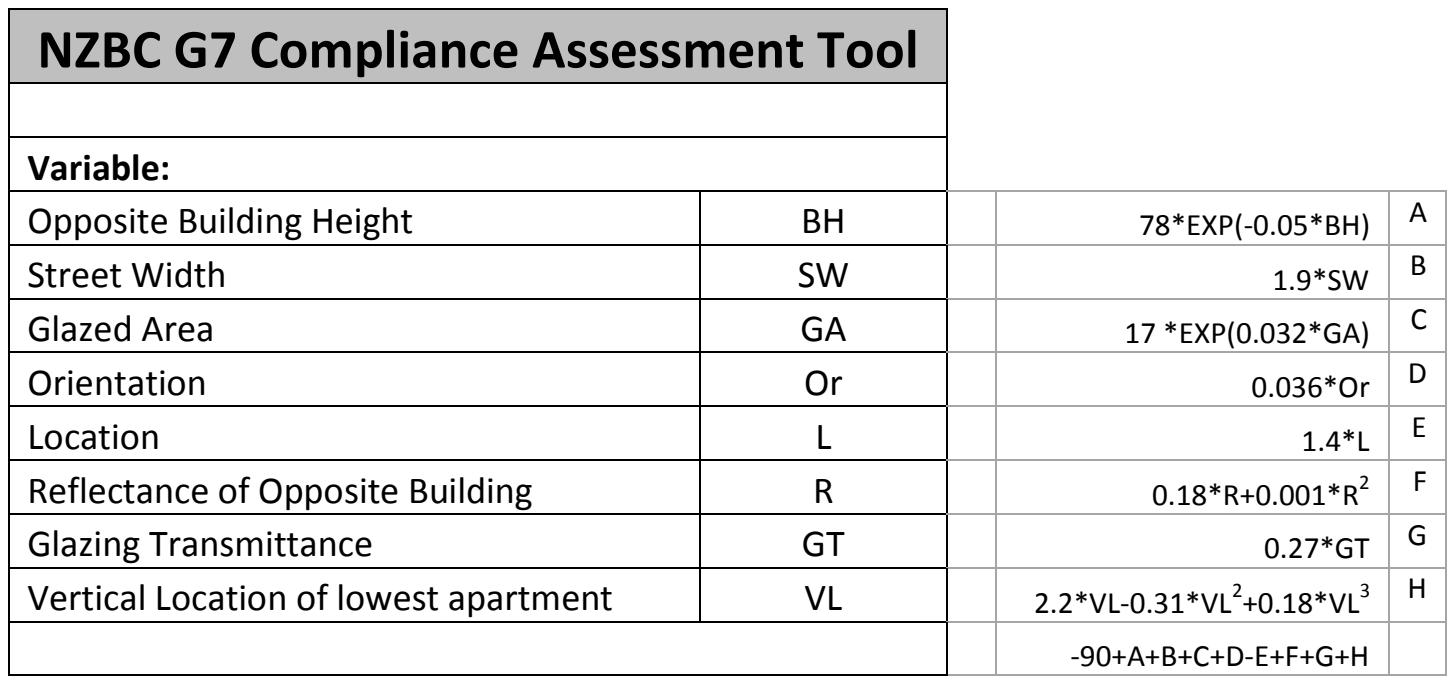

Figure 26 - Prediction model calculation process

The result provided by the prediction model is then compared to the threshold value of $75 \%$ DA. If the prediction is less than $75 \%$ DA, then a result of ' $Y E S$ ' is given. If the prediction is greater than or equal to $75 \% \mathrm{DA}$, then a result of ' $\mathrm{NO}$ ' is given. It was intended that all calculations are hidden from users to make the format of the tool easier to follow. All the user is required to do is enter the relevant information next to the variables and they are automatically provided with a 'YES' or 'NO'.

This has shown that through the use of a test platform, the idea of an equation based tool to calculate daylight performance is achievable. Future development may see this tool use other platforms, e.g. Web-based, but to test the concept Excel has proven that the equation can be formatted into a simple and easy to use tool, based on the International Energy Agency (Ruck, 2000) concept of simple computer based tools.

This platform also allows all three models to be used if this were found to be the most reliable method. This could be done by selecting the most accurate model for the range of variables in each separate case. This would mean the tool would consider the variables and select the most effective model. 


\subsection{CALIBRATION TESTS}

Calibration tests were undertaken to assess the accuracy of the tool, one of the main criteria for the acceptability of tool. They were also used to determine which prediction equation provides the most reliable results for real situations. In this chapter, the methods used to calibrate the tool are described and the results from the calibration tests are summarised, determining whether the tool was reasonably accurate. In Section 6.1 the method is discussed, including the measurement process for 97 calibration apartments and the method of comparing results. Section 6.2 is a summary of the calibration results, including the selection of the most accurate of the three prediction equations identified in Chapter 5 and includes additional observations from the calibration process.

\subsection{Calibration Method}

The calibration process was conducted to establish the reliability of the result provided by the tool. A general rule of thumb for multi-variable research is that a minimum sample size of ten times the number of variables is needed to test accuracy (Roscoe, 1975, pg 184). Following the analysis and development of the prediction model, there were 8 variables. 97 apartments were measured, which is greater than the minimum sample size of 80 .

Following the measurement procedure outlined by Fontoynont (1999) in Daylight Performance of Buildings, daylight factor measurements were taken in 97 apartments from three New Zealand cities. The use of daylight factor calculations to assess daylight performance in buildings is one of the most frequently used methods and has been used by a number of researchers including Ng (2001a; 2005), Li et al. (1999), Allen (1943), Littlefair (2001) and Wilson and Brotas(2001). A daylight factor assessment involves the simultaneous measurement of internal and external illuminances under overcast sky conditions $^{1}$ (Fontoynont, 1999, pg 4). The results were then calculated to determine the percentage of the available illuminance (unobstructed external illuminance) which is received at a given point in the room, as shown in Figure 27.

\footnotetext{
${ }^{1}$ It is not possible to get a perfectly uniform overcast sky in reality, so overcast days with full cloud cover were selected for measurement as they are the closest conditions to perfect uniformity of the sky.
} 


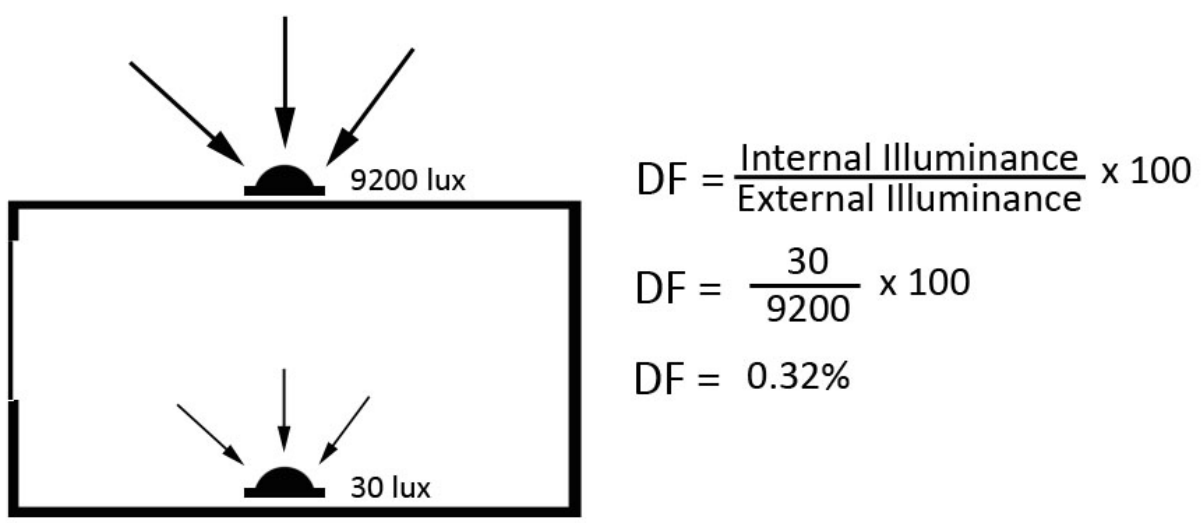

Figure 27 - Daylight Factor Calculation

To obtain results that were comparable to the result provided by the tool, a further calculation step was required. Using the external illuminance levels exceeded for $75 \%$ of the standard year, as given in the New Zealand Standard for interior lighting (2006, pg 61), the internal illuminance exceeded for $75 \%$ of the standard year can be determined for each measurement point.

Table 19 - External Illuminances from AS/NZS 1680.1:2006

\begin{tabular}{|l|l|l|l|l|l|l|l|}
\hline \multicolumn{7}{|c|}{ External skylight illuminance in klux for percentage of working hours. } \\
\hline & $90 \%$ & $85 \%$ & $80 \%$ & $\mathbf{7 5 \%}$ & $70 \%$ & $65 \%$ & $60 \%$ \\
\hline Auckland & 6.4 & 7.9 & 9.9 & $\mathbf{1 1 . 5}$ & 13.1 & 14.5 & 16.3 \\
\hline Christchurch & 5.1 & 7.0 & 9.3 & $\mathbf{1 1 . 0}$ & 12.6 & 13.9 & 15.6 \\
\hline Wellington & 5.1 & 6.3 & 7.8 & $\mathbf{9 . 2}$ & 10.6 & 11.9 & 13.2 \\
\hline Invercargill & 4.1 & 5.4 & 7.1 & $\mathbf{8 . 6}$ & 10.1 & 11.5 & 13.0 \\
\hline
\end{tabular}

The tool was also applied to each of the 97 apartments. Information for the tool was gathered through observations when conducting measurements and from building plans.

The results were then separated into two categories: Pass or Fail. This was done for both the results from the tool and the results from the actual apartments. A Pass result for the apartment measurements was 30 lux or greater and for the results from the tool it was $75 \%$ (DA) or greater. Therefore a Fail result was less than 30 lux for apartment measurements and less than $75 \%(D A)$ for the tool.

The category given for the tool was then compared to the category given for the real apartments to determine if the results were the same.

From this process, a result was provided that indicated the level of accuracy of the compliance assessment tool. The target accuracy for this tool is $90 \%$, this would mean that 1 in 10 buildings would require simulation when it wasn't necessary to prove compliance or approximately 1 building every year. Of the incorrect outcomes, $100 \%$ were to be incorrect fail results, as incorrect pass results would mean some buildings could avoid simulation when it was needed to prove compliance. 


\section{Selection of Apartments}

23 apartment buildings from New Zealand's main urban centres (Auckland, Wellington and Christchurch) were selected to represent the range of typical apartment developments. The majority of these apartments were situated in Wellington due to ease of access. The apartments in Auckland and Christchurch were included to ensure the results were not biased. Christchurch was selected as the southern location rather than Dunedin (as used in the simulations) because skylight illuminance levels were given in AS/NZS 1680:2006 for Christchurch but not Dunedin as shown in Table 19.

A major criterion for the selection of the apartment buildings was that they were to be less than 10 years old. This was to ensure that they embodied the characteristics typical of buildings currently being built. Aspects from the tool including street width, building height, glazed area, orientation and reflectance of the opposite building were recorded for all apartment buildings in Wellington and from this, the range of apartments was selected to represent a wide range of different cases.

700 tenants within 23 apartment buildings were invited, by letter, to participate in the research. From around 100 responses received, 97 were measured, with omissions due to apartments being too similar to each other or timing problems. A matrix for the variables was produced to ensure that an acceptable range had been assessed, an example of the matrix is shown below in Table 20, where the number of cases within each range is given. From Table 20 it can be seen that most combinations were tested for at least 1 apartment.

Table 20 - Calibration Apartment Matrix for Street Width and Building Height

\begin{tabular}{|c|c|c|c|c|c|c|c|c|}
\hline & \multicolumn{7}{|c|}{ Street width $(\mathrm{m})$} \\
\hline & & $<5$ & $5-9$ & $10-14$ & $15-19$ & $20-24$ & $25-30$ & $>30$ \\
\hline \multirow{5}{*}{ 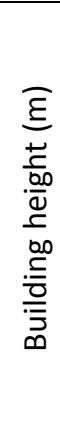 } & $<10$ & $\begin{array}{c}8 \\
\text { apartments }\end{array}$ & $\begin{array}{c}2 \\
\text { apartments }\end{array}$ & $\begin{array}{c}1 \\
\text { apartment }\end{array}$ & $\begin{array}{c}2 \\
\text { apartments }\end{array}$ & $\begin{array}{c}1 \\
\text { apartment }\end{array}$ & $\begin{array}{c}0 \\
\text { apartments }\end{array}$ & $\begin{array}{c}1 \\
\text { apartment } \\
\end{array}$ \\
\hline & $\begin{array}{l}10- \\
19\end{array}$ & $\begin{array}{c}2 \\
\text { apartments }\end{array}$ & $\begin{array}{c}5 \\
\text { apartments }\end{array}$ & $\begin{array}{c}3 \\
\text { apartments }\end{array}$ & $\begin{array}{c}4 \\
\text { apartments }\end{array}$ & $\begin{array}{c}0 \\
\text { apartments }\end{array}$ & $\begin{array}{c}3 \\
\text { apartments }\end{array}$ & $\begin{array}{c}8 \\
\text { apartments }\end{array}$ \\
\hline & $\begin{array}{l}20- \\
29\end{array}$ & $\begin{array}{c}5 \\
\text { apartments }\end{array}$ & $\begin{array}{c}4 \\
\text { apartments }\end{array}$ & $\begin{array}{c}2 \\
\text { apartments }\end{array}$ & $\begin{array}{c}1 \\
\text { apartment }\end{array}$ & $\begin{array}{c}3 \\
\text { apartments }\end{array}$ & $\begin{array}{c}0 \\
\text { apartments }\end{array}$ & $\begin{array}{c}6 \\
\text { apartments }\end{array}$ \\
\hline & $\begin{array}{l}30- \\
39 \\
\end{array}$ & $\begin{array}{c}8 \\
\text { apartments }\end{array}$ & $\begin{array}{c}1 \\
\text { apartment }\end{array}$ & $\begin{array}{c}0 \\
\text { apartments }\end{array}$ & $\begin{array}{c}1 \\
\text { apartment }\end{array}$ & $\begin{array}{c}0 \\
\text { apartments }\end{array}$ & $\begin{array}{c}3 \\
\text { apartments }\end{array}$ & $\begin{array}{c}4 \\
\text { apartments }\end{array}$ \\
\hline & $>40$ & $\begin{array}{c}0 \\
\text { apartments }\end{array}$ & $\begin{array}{c}6 \\
\text { apartments }\end{array}$ & $\begin{array}{c}0 \\
\text { apartments }\end{array}$ & $\begin{array}{c}2 \\
\text { apartments }\end{array}$ & $\begin{array}{c}0 \\
\text { apartments }\end{array}$ & $\begin{array}{c}0 \\
\text { apartments }\end{array}$ & $\begin{array}{c}3 \\
\text { apartments }\end{array}$ \\
\hline
\end{tabular}

\section{Measurements}

The measurements were taken on overcast days, as close to uniform as possible, during June and July 2008. Measurements were taken in the living room and at least one bedroom, where possible. It was not feasible to measure apartments without furniture, as was tested in the simulations, because most of the apartments were occupied and furniture was not able to be moved. As was described in Chapter 4, measurement points with significantly lower results than the other two points were eliminated due to shading from furniture. 
In each habitable space three measurements were taken. These measurement points were the same as those used in the simulation, as shown below in Figure 28, to ensure consistency.

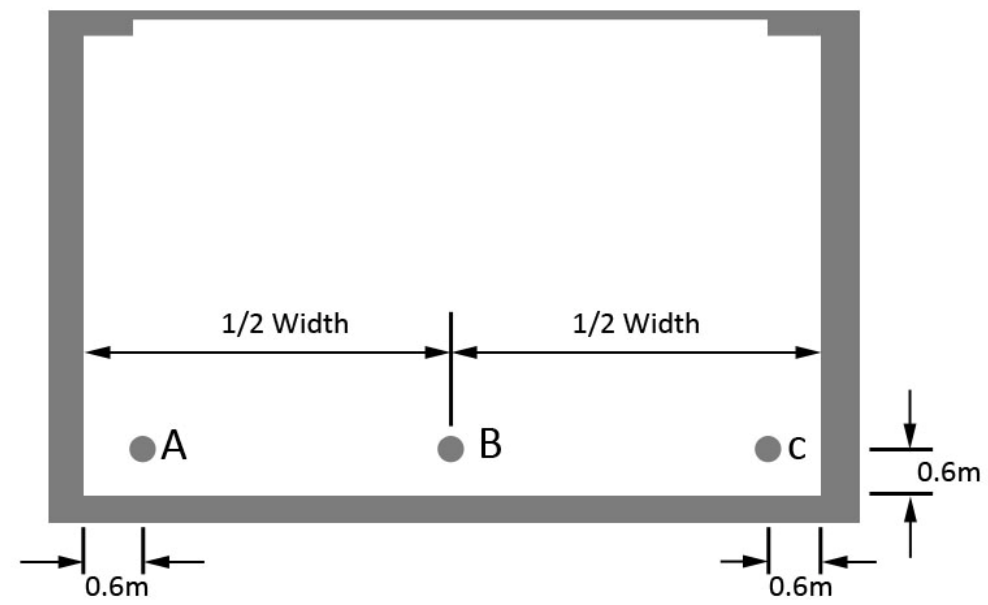

Figure 28 - Measurement Points

Measurements were taken with an illuminance meter placed on the floor at each measurement point. Simultaneously, illuminance levels were taken outside under an unobstructed overcast sky nearby. To ensure consistency of results, the same two illuminance meters were used for all measurements and the internal illuminance meter was calibrated against the external meter to ensure the results were reliable.

\subsection{Analysis of Calibration Results}

This section gives a summary of the calibration results for the 97 calibration apartments. An example case is given to show how each case was analysed. This example is an apartment that only just fails to meet the minimum requirement. The results for all calibration apartments can be found in Appendix 4, with analysis of all borderline apartments (within 5 lux of the threshold value) given in Appendix 5 and analysis of all incorrect cases (both incorrect pass and incorrect fail results) given in Appendix 6.

\subsubsection{Example Apartment - Apartment 43}

The illuminance level exceeded for $75 \%$ of the standard year at each measurement point was calculated using the daylight factors and the sky illuminance exceeded for $75 \%$ of the standard year. Firstly, the measured internal illuminance was divided by the measured external illuminance to get the daylight factor at each point. Then the external sky illuminance for $75 \%$ of the standard year was multiplied by the daylight factor to get the internal illuminance exceeded for $75 \%$ of the standard year. In this case the external sky illuminance was 9200 lux (9.2 klux), this process is shown below in Table 21. 
Table 21 - Calculation of Daylight Performance in Calibration Apartments

\begin{tabular}{|l|c|c|c|c|}
\hline & $\begin{array}{c}\text { Measured } \\
\text { Internal Lux }\end{array}$ & $\begin{array}{c}\text { Measured } \\
\text { External Lux }\end{array}$ & $\begin{array}{c}\text { Daylight } \\
\text { Factor }\end{array}$ & $\begin{array}{c}\text { Internal Illuminance } \\
\text { Exceeded 75\% of the Year }\end{array}$ \\
\hline P1 & 285 & 9570 & $0.3 \%$ & $\mathbf{2 7 . 6}$ \\
\hline P2 & 40.5 & 9700 & $0.4 \%$ & $\mathbf{3 6 . 8}$ \\
\hline
\end{tabular}

Using the results from Table 21, a graph was produced comparing the result to the minimum requirement of 30 lux for $75 \%$ of the standard year to determine if the apartment passed or failed. This process is shown below in Figure 29 for the example apartment, note that only two measurement points are shown because P3 was eliminated due to shading from furniture. From Figure 29 it can be seen that Apartment 43 failed to meet the minimum requirement of 30 lux at one of the measurement points which meant the apartment was given a fail result overall.

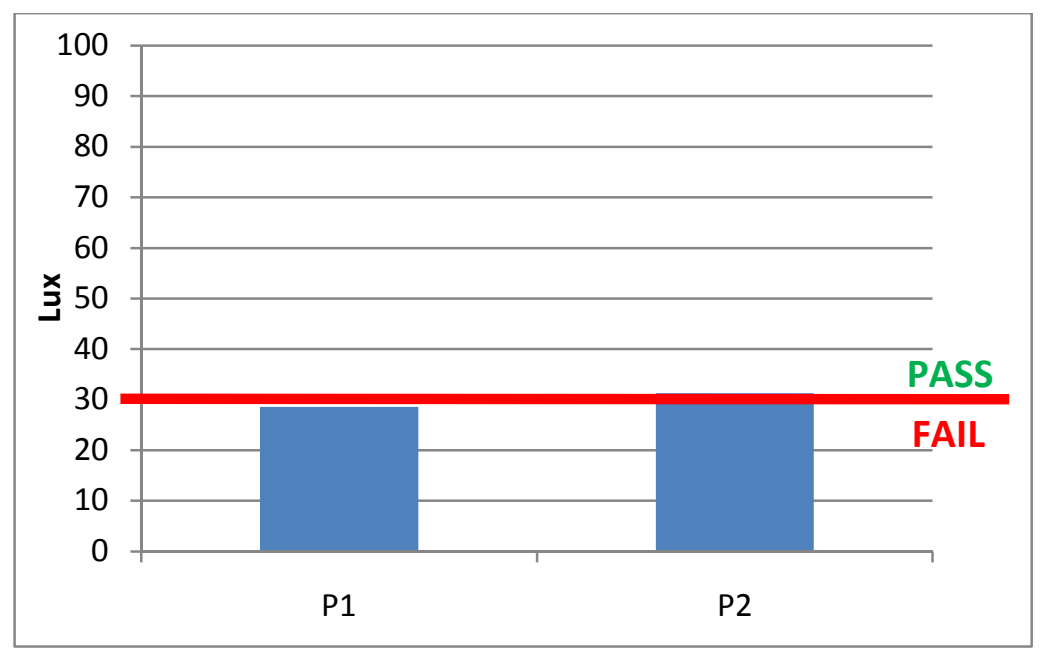

Figure 29 - Illuminance exceeded for $\mathbf{7 5 \%}$ of the Standard Year in Apartment 43

The tool was then applied to the apartment using information gathered during the measurement process and from building plans. Figure 30 shows the application of the tool for the example apartment. It can be seen in Figure 30 that the tool stated that Apartment 43 would require simulations to prove compliance with NZBC G7 (a fail result). 


\begin{tabular}{|l|c|}
\hline \multicolumn{2}{|c|}{ NZBC G7 Compliance Assessment Tool } \\
\hline \multicolumn{2}{|l|}{ Variable: } \\
\hline Opposite Building Height & 18 \\
\hline Street Width & 16 \\
\hline Glazed Area & 17.5 \\
\hline Orientation & North \\
\hline Location & Wellington \\
\hline Reflectance of Opposite Building & 10 \\
\hline Glazing Transmittance & 87 \\
\hline Vertical Location of lowest apartment & 6 \\
\hline \multicolumn{2}{|}{} \\
\hline Are simulations required to prove compliance: \\
\hline
\end{tabular}

Figure 30 - Application of the tool for Apartment 43

As a final equation for the tool had not been decided at this stage, all three equations were applied. Table 22 shows the results provided by the three best equations.

Table 22 - Prediction Model Results for Apartment 43

\begin{tabular}{|c|c|c|c|}
\hline Measurements & Linear Model & Model 2A & Model 2B \\
\hline Fail & $73 \%$ & $59 \%$ & $46 \%$ \\
\hline
\end{tabular}

The correct result was provided for Apartment 43. The tool stated the apartment would require simulation and the measurements in the apartment confirmed that this apartment did fail to meet the minimum requirement. The correct result was also provided by all three of the equations.

Some of the apartments had unreliable results provided by the measurements in the apartments due to shading from furniture for more than one measurement point. The method specified early for dealing with measurement points shaded by furniture did not consider apartments with multiple shaded points. In situations where the tool gives inaccurate results for some apartments and the measurement points were known to be shaded, the two shaded points were not included in the assessment of the accuracy of the tool. For example, apartment 39 had one measurement point that was just under the threshold, with two of the points known to be shaded by furniture. The tool identified that this apartment would comply with NZBC G7, if the shaded measurement points were considered this was an incorrect outcome, but measurement point 1 indicated that this apartment was well over the minimum requirement and confirmed the findings of the tool. 


\subsubsection{Summary of Calibration Results}

All 97 apartments were assessed using the method shown in Section 6.2.1. This section summarises the results from the calibration tests.

From the calibration tests, 51 of the 97 apartments were found to comply with the minimum daylight requirements of NZBC Clause G7, meaning that 46 of the apartments failed to meet the requirement that a minimum of 30 lux is available at floor level for $75 \%$ of the standard year.

In 72 out of the 97 cases, all three of the equations correctly determined whether simulations were required or not, and a further 13 received correct outcomes from the tool from at least one of the equations.

By formatting the tool so that all three equations can be used, a more accurate tool can be developed. This process involved analysing the correct/incorrect predictions for each equation in relation to the variables being assessed to determine when the models gave correct/incorrect predictions. Then, the tool was set to use the most effective equation for the combination of variables. For example, if apartments with opposite buildings greater than $50 \mathrm{~m}$ received incorrect predictions from say the Linear Model and Model $2 \mathrm{~b}$, then Model $2 \mathrm{a}$ would be used to assess this building. This method meant that now, 85 apartments were correctly assessed by the tool, which equates to an accuracy of $88 \%$.

Of the 12 incorrect outcomes 7 were false pass results, where the tool said simulations were not required but the apartment measurements indicated the apartment failed, and 5 were false fail results, where the tool said simulations were needed when the apartment passed. With the incorporation of the standard errors, identified in Section 5.2.2, two of the 8 false pass results would receive the correct outcome from the tool of YES (simulations are needed) for at least one of the equations. However, this would also result in three of the 84 apartments that received correct outcomes from the tool no longer having the correct outcome. Table 23 shows the measured results for each of the 97 apartments within the 23 buildings assessed and the predicted results for each of the three prediction equations. From this table it can be seen that 5 out of the 23 apartment buildings would pass and should not require simulation for any of the apartments to prove compliance. However, these 5 buildings only had a small number of apartments meaning that they may be found to fail also if more apartments were measured. Another point indentified in Table 23 is that care needs to be taken when selecting the apartments to be assessed by the tool, if the incorrect apartments are selected, this could result in the building being given a pass result when in reality it fails. An example of this is building 5 , if apartments $60,70,73,80$, 90 or 94 were selected and assessed by the tool then this building would receive a pass result when in reality it clearly fails.

Table 24 provides a summary of the false pass and false fail apartments. This summary identified that one of the reasons cited for 7 out of the 12 incorrect assessments for apartments was vertical locations greater than the $7^{\text {th }}$ floor. For many of these cases, the combinations of variables should result in the apartment passing; a good example of this 
was apartment 35 , where there was no obstruction and it was located on the $11^{\text {th }}$ floor. It is possible that furniture may have resulted in lower daylight performance in these apartments, but it is clear that vertical location of the apartments was a key factor in all the false pass results. A consensus was unable to be found for the false fail apartments as they all had different combinations of variables. 
Table 23 - Comparison of Calibration Apartments

\begin{tabular}{|c|c|c|c|c|c|c|c|c|c|c|c|c|c|}
\hline \multicolumn{2}{|c|}{$\begin{array}{l}\text { Apartment } \\
\text { Buildings }\end{array}$} & \multicolumn{2}{|c|}{$\begin{array}{l}\text { Individual } \\
\text { Apartments }\end{array}$} & $\begin{array}{l}\text { Linear } \\
\text { Model }\end{array}$ & $\begin{array}{c}\text { Model } \\
\text { A }\end{array}$ & $\begin{array}{c}\text { Model } \\
\text { B }\end{array}$ & \multicolumn{2}{|c|}{$\begin{array}{l}\text { Apartment } \\
\text { Buildings }\end{array}$} & \multicolumn{2}{|c|}{$\begin{array}{l}\text { Individual } \\
\text { Apartments }\end{array}$} & $\begin{array}{l}\text { Linear } \\
\text { Model }\end{array}$ & $\begin{array}{c}\text { Model } \\
\text { A }\end{array}$ & $\begin{array}{c}\text { Model } \\
\text { B }\end{array}$ \\
\hline \multirow{7}{*}{ B 1} & \multirow{7}{*}{ Fail } & A 1 & Fail & Fail & Fail & Fail & \multirow{3}{*}{ B 10} & \multirow{3}{*}{ Fail } & A 18 & Fail & Fail & Fail & Fail \\
\hline & & $\mathrm{A} 2$ & Fail & Fail & Fail & Fail & & & A 19 & Fail & Fail & Fail & Fail \\
\hline & & A 9 & Fail & Fail & Fail & Fail & & & A 37 & Fail & Fail & Fail & Fail \\
\hline & & A 12 & Fail & Fail & Fail & Fail & \multirow{2}{*}{ B 11} & \multirow{2}{*}{ Fail } & A 20 & Fail & Pass & Fail & Fail \\
\hline & & A 34 & Fail & Fail & Fail & Fail & & & A 30 & Fail & Fail & Fail & Fail \\
\hline & & A 39 & Pass & Pass & Pass & Pass & \multirow{2}{*}{ B 12} & \multirow{2}{*}{ Fail } & A 24 & Fail & Fail & Fail & Fail \\
\hline & & A 54 & Pass & Pass & Pass & Pass & & & A 27 & Fail & Fail & Fail & Fail \\
\hline B 2 & Fail & A 3 & Fail & Fail & Fail & Fail & \multirow{2}{*}{ В 13} & \multirow{2}{*}{ Fail } & A25 & Fail & Fail & Fail & Fail \\
\hline B 3 & Fail & A 4 & Fail & Fail & Fail & Fail & & & A 62 & Pass & Pass & Pass & Pass \\
\hline D & rall & A 8 & Fail & Fail & Fail & Fail & & & A 28 & Fail & Pass & Pass & Pass \\
\hline & & A 5 & Fail & Fail & Fail & Fail & & & A 35 & Fail & Pass & Pass & Pass \\
\hline & & A 32 & Fail & Fail & Fail & Fail & D 14 & rall & A 40 & Fail & Pass & Pass & Pass \\
\hline & & A 55 & Pass & Pass & Pass & Pass & & & A 50 & Pass & Pass & Pass & Pass \\
\hline B 4 & Fail & A 68 & Pass & Fail & Fail & Fail & & & A 31 & Fail & Pass & Pass & Pass \\
\hline 04 & ail & A 69 & Pass & Pass & Pass & Pass & В 15 & Fail & A 47 & Pass & Pass & Pass & Fail \\
\hline & & A 76 & Pass & Pass & Pass & Pass & & & A 88 & Pass & Pass & Pass & Pass \\
\hline & & A 86 & Pass & Pass & Pass & Pass & B 16 & Fail & A 36 & Fail & Fail & Fail & Fail \\
\hline & & A 92 & Pass & Pass & Pass & Pass & B 17 & Fail & A 38 & Fail & Fail & Fail & Fail \\
\hline & & A 6 & Fail & Fail & Fail & Fail & 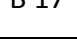 & rall & A 72 & Pass & Pass & Pass & Pass \\
\hline & & A 11 & Fail & Fail & Fail & Fail & & & A 45 & Fail & Pass & Pass & Fail \\
\hline & & A 21 & Fail & Fail & Fail & Fail & & & A 63 & Pass & Pass & Pass & Pass \\
\hline & & A 29 & Fail & Fail & Fail & Fail & B 18 & Fail & A 64 & Pass & Pass & Pass & Pass \\
\hline & & A 33 & Fail & Fail & Pass & Fail & $D 10$ & rdII & A 79 & Pass & Pass & Pass & Pass \\
\hline B 5 & Fail & A 60 & Pass & Pass & Pass & Pass & & & A 81 & Pass & Pass & Pass & Pass \\
\hline D J & rall & A 70 & Pass & Pass & Pass & Pass & & & A 91 & Pass & Pass & Pass & Pass \\
\hline & & A 73 & Pass & Pass & Fail & Fail & & & A 48 & Pass & Fail & Pass & Fail \\
\hline & & A 80 & Pass & Pass & Pass & Fail & & & A 53 & Pass & Pass & Fail & Fail \\
\hline & & A 90 & Pass & Pass & Pass & Pass & В 19 & Pass & A 57 & Pass & Pass & Pass & Pass \\
\hline & & A 94 & Pass & Pass & Pass & Pass & & & A 59 & Pass & Pass & Pass & Pass \\
\hline & & A 95 & Pass & Pass & Pass & Pass & & & A 65 & Pass & Pass & Fail & Fail \\
\hline & & A 7 & Fail & Fail & Fail & Fail & & & A 58 & Pass & Pass & Pass & Pass \\
\hline & & A 15 & Fail & Fail & Fail & Fail & B 20 & Pass & A 71 & Pass & Pass & Pass & Pass \\
\hline & & A 41 & Fail & Pass & Pass & Fail & & & A 84 & Pass & Pass & Pass & Pass \\
\hline B 6 & Fail & A 43 & Fail & Fail & Fail & Fail & & & A 74 & Pass & Pass & Pass & Pass \\
\hline & & A 46 & Fail & Pass & Fail & Fail & & & A 78 & Pass & Pass & Pass & Pass \\
\hline & & A 49 & Pass & Pass & Pass & Pass & B 21 & Pass & A 85 & Pass & Pass & Pass & Pass \\
\hline & & A 66 & Pass & Fail & Fail & Fail & & & A 89 & Pass & Pass & Pass & Pass \\
\hline & & A 10 & Fail & Fail & Fail & Fail & & & A 96 & Pass & Pass & Pass & Pass \\
\hline & & A 16 & Fail & Fail & Fail & Fail & R 27 & Pacc & A 77 & Pass & Pass & Pass & Pass \\
\hline & & A 23 & Fail & Fail & Fail & Fail & $D \angle L$ & rass & A 93 & Pass & Pass & Pass & Pass \\
\hline B 7 & Fail & A 51 & Pass & Fail & Fail & Fail & B 23 & Pass & A 83 & Pass & Pass & Pass & Pass \\
\hline & & A 52 & Pass & Pass & Pass & Pass & $D<D$ & rass & A 87 & Pass & Pass & Pass & Pass \\
\hline & & A 56 & Pass & Fail & Fail & Fail & & & & & & & \\
\hline & & A 82 & Pass & Pass & Pass & Pass & & & & & & & \\
\hline & & A 13 & Fail & Fail & Fail & Fail & & & & & & & \\
\hline & & A 14 & Fail & Pass & Pass & Pass & & & & & & & \\
\hline & & A 22 & Fail & Pass & Pass & Pass & & & & & & & \\
\hline B 8 & Fail & A 42 & Fail & Pass & Pass & Fail & & & & & & & \\
\hline & & A 44 & Fail & Pass & Pass & Pass & & & & & & & \\
\hline & & A 61 & Pass & Pass & Pass & Pass & & & & & & & \\
\hline & & A 97 & Pass & Pass & Pass & Pass & & & & & & & \\
\hline & & A 17 & Fail & Fail & Fail & Fail & & & & & & & \\
\hline B 9 & Fail & A 26 & Fail & Fail & Fail & Fail & & & & & & & \\
\hline D 5 & rdII & A 67 & Pass & Pass & Pass & Pass & & & & & & & \\
\hline & & A 75 & Pass & Fail & Fail & Fail & & & & & & & \\
\hline
\end{tabular}


Table 24 - Summary of False Pass and False Fail Apartments

\begin{tabular}{|c|c|c|c|c|c|c|c|c|c|c|c|c|c|}
\hline \multicolumn{2}{|c|}{ 藏 } & 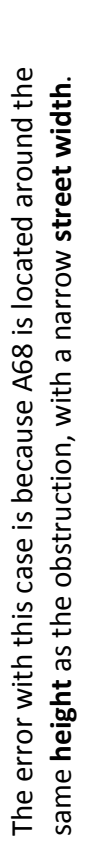 & 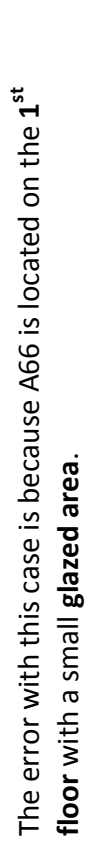 & 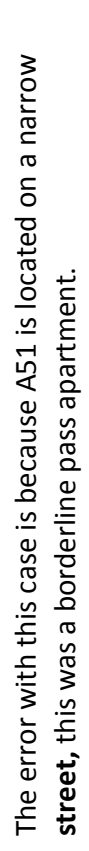 & 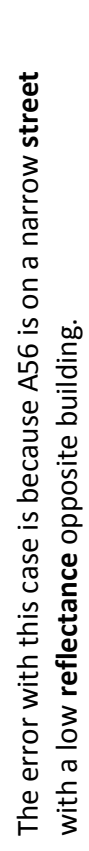 & 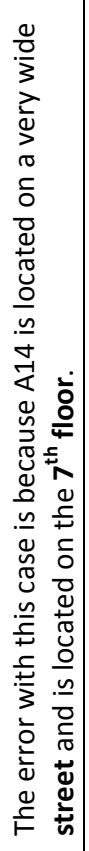 & 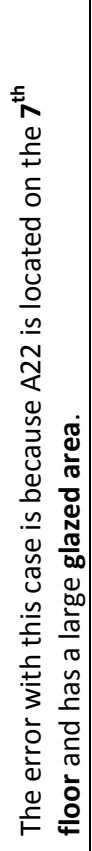 & 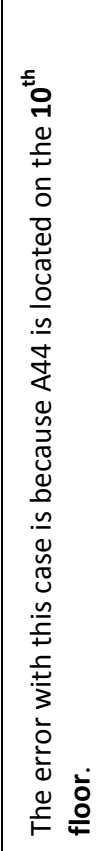 & 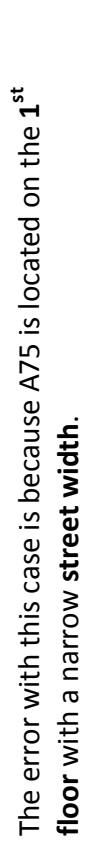 & 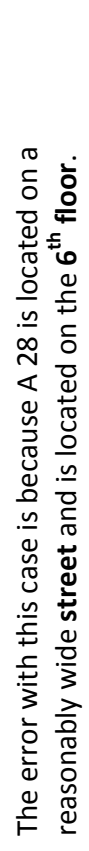 & 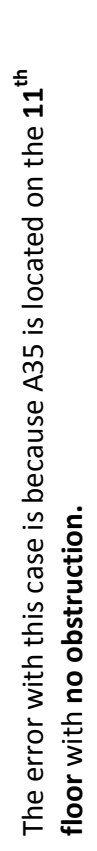 & 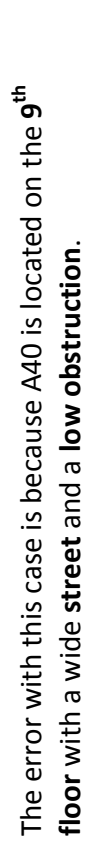 & 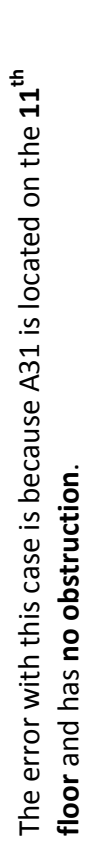 \\
\hline \multirow{8}{*}{$\begin{array}{l}\frac{\tilde{u}}{0} \\
\frac{0}{0 \pi} \\
\frac{0}{\pi} \\
>\end{array}$} & 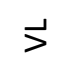 & $\infty$ & $\rightarrow$ & $m$ & 0 & $r$ & $r$ & 우 & -1 & $\infty$ & $\exists$ & $a$ & $\exists$ \\
\hline & 厄 & $\hat{\infty}$ & $\hat{\infty}$ & $\widehat{\infty}$ & $\widehat{\infty}$ & ㅇ & $\hat{\infty}$ & $\hat{\infty}$ & $\widehat{\infty}$ & $\hat{\infty}$ & $\hat{\infty}$ & $\hat{\infty}$ & $\hat{\infty}$ \\
\hline & $\stackrel{4}{\mathscr{\Phi}}$ & $\stackrel{\llcorner}{\sim}$ & ㅇ & $m$ & ᄀ & $\vec{\sim}$ & $\vec{N}$ & $\vec{N}$ & $\begin{array}{l}\infty \\
\underset{\sim}{J}\end{array}$ & $\begin{array}{l}\infty \\
\stackrel{\sim}{0}\end{array}$ & 0 & $\stackrel{\llcorner}{\sim}$ & in \\
\hline & ـ & 3 & 3 & 3 & 3 & 3 & 3 & 3 & 3 & u & u & $u$ & $\varangle$ \\
\hline & ঠ & س & $n$ & $z$ & $z$ & $\backsim$ & $z$ & $n$ & ш & 3 & $n$ & $z$ & $\sim$ \\
\hline & ৫্ত & 으 & $\approx$ & $\stackrel{m}{\sim}$ & $\vec{N}$ & $\vec{N}$ & मे & $\stackrel{\llcorner}{m}$ & $\approx$ & $\stackrel{\bullet}{\sim}$ & $\stackrel{\sim}{N}$ & $\stackrel{\mathscr{N}}{ }$ & $\vec{m}$ \\
\hline & 3 & $m$ & $\stackrel{\Perp}{\sim}$ & n & n & $\forall$ & $m$ & $m$ & $a$ & $\stackrel{d}{\sim}$ & 0 & $\stackrel{\infty}{\sim}$ & $a$ \\
\hline & I & $\stackrel{\sim}{m}$ & $\stackrel{9}{-}$ & i & i & $\vec{J}$ & $\approx$ & $\approx$ & $\underset{-1}{\mathscr{1}}$ & $\stackrel{\mathscr{N}}{ }$ & 0 & $\stackrel{\eta}{-}$ & 0 \\
\hline \multicolumn{2}{|c|}{$\stackrel{\overline{\mathrm{d}}}{\mathrm{O}} \infty^{\circ}$} & 丞 & 耑 & 产 & $\begin{array}{l}\overline{\bar{\sigma}} \\
\text { एँ }\end{array}$ & 亗 & $\begin{array}{l}\tilde{y} \\
\tilde{c} \\
0\end{array}$ & 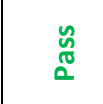 & 产 & $\begin{array}{l}\breve{u} \\
\tilde{c} \\
0\end{array}$ & $\begin{array}{c}\tilde{y} \\
\text { ⿷匚⿱ }\end{array}$ & $\begin{array}{l}\tilde{y} \\
\tilde{0}\end{array}$ & $\begin{array}{l}\tilde{u} \\
\tilde{c}\end{array}$ \\
\hline \multicolumn{2}{|c|}{$\begin{array}{l}\overline{\mathrm{d}} \\
\overline{\mathrm{o}}\end{array}$} & $\overline{\bar{\sigma}}$ & $\overline{\bar{\sigma}}$ & 趂 & 覀 & $\begin{array}{l}\tilde{y} \\
\tilde{\delta}\end{array}$ & 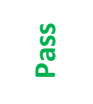 & ⿸厃㔾 & 产 & $\begin{array}{l}\tilde{y} \\
\tilde{\delta}\end{array}$ & $\begin{array}{l}\tilde{y} \\
\tilde{c} \\
\tilde{c}\end{array}$ & $\begin{array}{l}\mathscr{u} \\
\tilde{\sigma}\end{array}$ & $\begin{array}{l}\tilde{u} \\
\tilde{0} \\
\tilde{\Xi}\end{array}$ \\
\hline \multicolumn{2}{|c|}{ 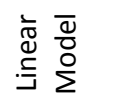 } & 产 & 就 & $\begin{array}{l}\overline{\bar{\sigma}} \\
\overline{\mathrm{L}}\end{array}$ & $\overline{\bar{\pi}}$ & 㟧 & 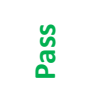 & $\begin{array}{l}\tilde{y} \\
\tilde{\Xi}\end{array}$ & 覀 & $\begin{array}{l}\tilde{y} \\
\tilde{\delta} \\
\tilde{0}\end{array}$ & $\begin{array}{l}\tilde{u} \\
\tilde{0} \\
\tilde{\sigma}\end{array}$ & $\begin{array}{l}\mathscr{y} \\
\tilde{c} \\
\tilde{c}\end{array}$ & $\begin{array}{c}\tilde{u} \\
\tilde{\Xi} \\
\tilde{c}\end{array}$ \\
\hline \multirow{2}{*}{\multicolumn{2}{|c|}{ 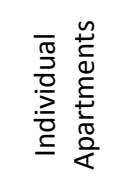 }} & $\begin{array}{l}\tilde{y} \\
\tilde{0} \\
\tilde{c}\end{array}$ & $\begin{array}{l}\mathscr{y} \\
\tilde{c}\end{array}$ & $\begin{array}{l}\tilde{y} \\
\tilde{c} \\
\tilde{c}\end{array}$ & $\begin{array}{l}\tilde{y} \\
\tilde{c} \\
\tilde{c}\end{array}$ & $\overline{\overline{\bar{\kappa}}}$ & $\overline{\overline{\tilde{\sigma}}}$ & $\overline{\overline{\bar{\sigma}}}$ & $\begin{array}{l}\tilde{y} \\
\tilde{c} \\
\tilde{c}\end{array}$ & $\overline{\overline{\tilde{N}}}$ & $\overline{\bar{\varpi}}$ & $\overline{\overline{\bar{\sigma}}}$ & $\overline{\overline{\widetilde{\sigma}}}$ \\
\hline & & $\begin{array}{l}\infty \\
0 \\
\ll\end{array}$ & $\begin{array}{l}8 \\
\&\end{array}$ & $\stackrel{i n}{i n}$ & $\stackrel{\substack{n \\
\ll}}{<}$ & $\underset{⿱ 亠 乂}{\mathbb{Z}}$ & $\underset{\mathbb{Z}}{\mathbb{Z}}$ & $\underset{⿱}{\&}$ & $\stackrel{\substack{n \\
<}}{4}$ & $\stackrel{\infty}{\underset{\&}{\ll}}$ & $\stackrel{\substack{n \\
\ll}}{\ll}$ & $\frac{g}{4}$ & $\stackrel{-1}{m}$ \\
\hline \multirow{2}{*}{\multicolumn{2}{|c|}{ 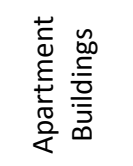 }} & $\overline{\overline{\tilde{\omega}}}$ & $\overline{\overline{\bar{\sigma}}}$ & \multicolumn{2}{|c|}{$\frac{\overline{\bar{\pi}}}{\bar{L}}$} & \multicolumn{4}{|c|}{$\frac{\overline{\bar{\sigma}}}{\stackrel{\bar{W}}{4}}$} & \multicolumn{3}{|c|}{$\overline{\overline{\tilde{\sigma}}}$} & $\overline{\bar{\sigma}}$ \\
\hline & & $\stackrel{\nabla}{\infty}$ & $\infty$ & \multicolumn{2}{|c|}{$\hat{\infty}$} & \multicolumn{4}{|c|}{$\begin{array}{l}\infty \\
\infty\end{array}$} & \multicolumn{3}{|c|}{$\underset{\infty}{\stackrel{J}{D}}$} & $\stackrel{-1}{\infty}$ \\
\hline
\end{tabular}




\subsection{Analysis of the Accuracy of the Tool}

The results from all 97 calibration apartments were used to assess the accuracy of the tool. There were four aspects of the results that were analysed further. The first of these was the accuracy of the tool when predicting the outcome of each apartment independently. The second analysed the false pass and false fail results. The third was the accuracy of predicting the outcome of the overall building. And the final aspect was an assessment of the prediction equations to determine which equation provided the best results.

\subsubsection{Accuracy of Apartment Outcomes}

The calibration results were assessed to determine the accuracy of the outcomes, Figure 31 shows the accuracy of the three prediction equations and the combination of these models. For the combined model, the result is considered correct when at least one of the prediction equations provided the correct outcome.

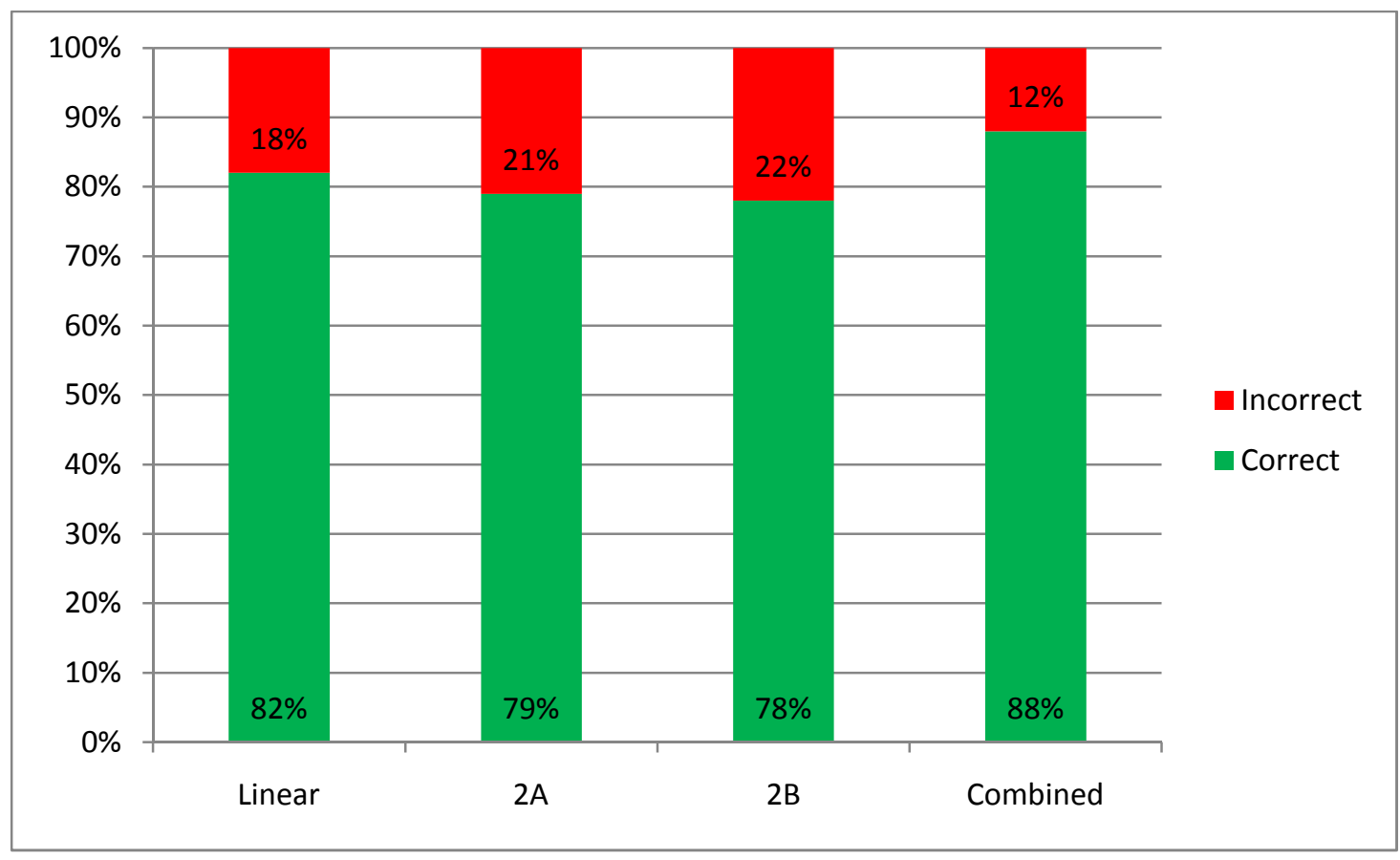

Figure 31 - Accuracy of Outcomes Provided by the Tool

The results from the calibration tests shown in Figure 31, found that the accuracy ranged between $78 \%$ and $82 \%$ for the prediction equations. This equates to around 1 out of every 5 apartments receiving an incorrect result. With around 12 apartment buildings given consent every year in Auckland and Wellington this would mean 5 buildings could be expect to receive incorrect results every 2 years. If all three models were used and at least one of these provided an accurate result, there is an improvement in the accuracy, with $88 \%$ of the cases receiving correct outcomes. As was discussed in Section 5.2.3, this could be done by selecting the model that provides the most reliable outcome for the combination of variables. This would reduce the number of incorrect assessments of buildings to just 3 
every 2 years, this is more than the goal of around 2 buildings every 2 years ( $10 \%$ of the cases).

\subsubsection{Incorrect Outcomes}

\section{False Pass}

To better understand the incorrect outcomes, they were separated into two types, false pass and false fail. A false pass result is where the measurements from the apartment provided a fail result but the tool stated that the apartment would not require simulation. The goal for this research was to have no false pass results. From the calibration results it was identified that there were 7 false pass results if the combined model was used. Figure 32 shows the percentage of false pass results.

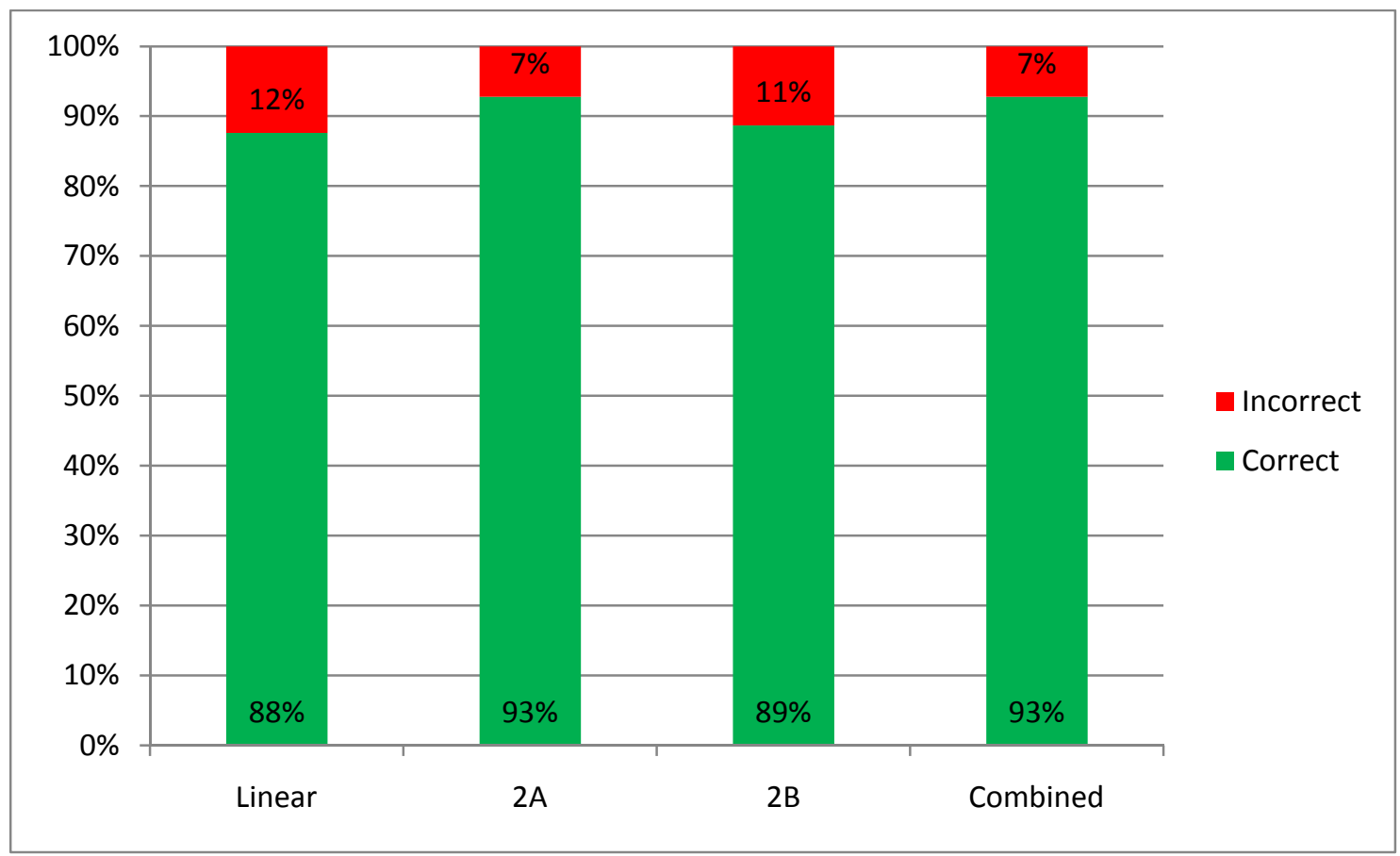

Figure 32 - Percentage of False Pass Results

From Table 24 shown earlier, the variable that had the greatest effect on false pass results was vertical location. All 7 of the false pass apartments were located on the $7^{\text {th }}$ floor or higher. In the early stage of this research it was decided to only simulate up to the $5^{\text {th }}$ floor for the lowest floor of apartments. These false pass results have shown that if the lowest floor of apartments were located on the $7^{\text {th }}$ floor or higher than the tool could not provide reliable results. This means that if apartments were to be built on top of a parking building higher than 7 floors then the tool could not reliably assess this building. To avoid misapplication of the tool the vertical location variable should be limited to the $5^{\text {th }}$ floor or lower.

The calibration results found that the false pass results achieved the desired $0 \%$, provided vertical locations of the $5^{\text {th }}$ floor or less were used. 


\section{False Fail}

The second type of incorrect outcome was a false fail result. A false fail result is where the measurements from the apartment provided a pass result but the prediction equations stated that the apartment would require simulation. False fail results would require apartments to be simulated when simulations were not necessary to prove compliance, adding extra time and costs to the project. However, this is not as important as eliminating false pass results. The goal for this research was to have less than $10 \%$, or 1 building every year, require simulation unnecessarily. Figure 33 shows the false fail result as a percentage of the overall results.

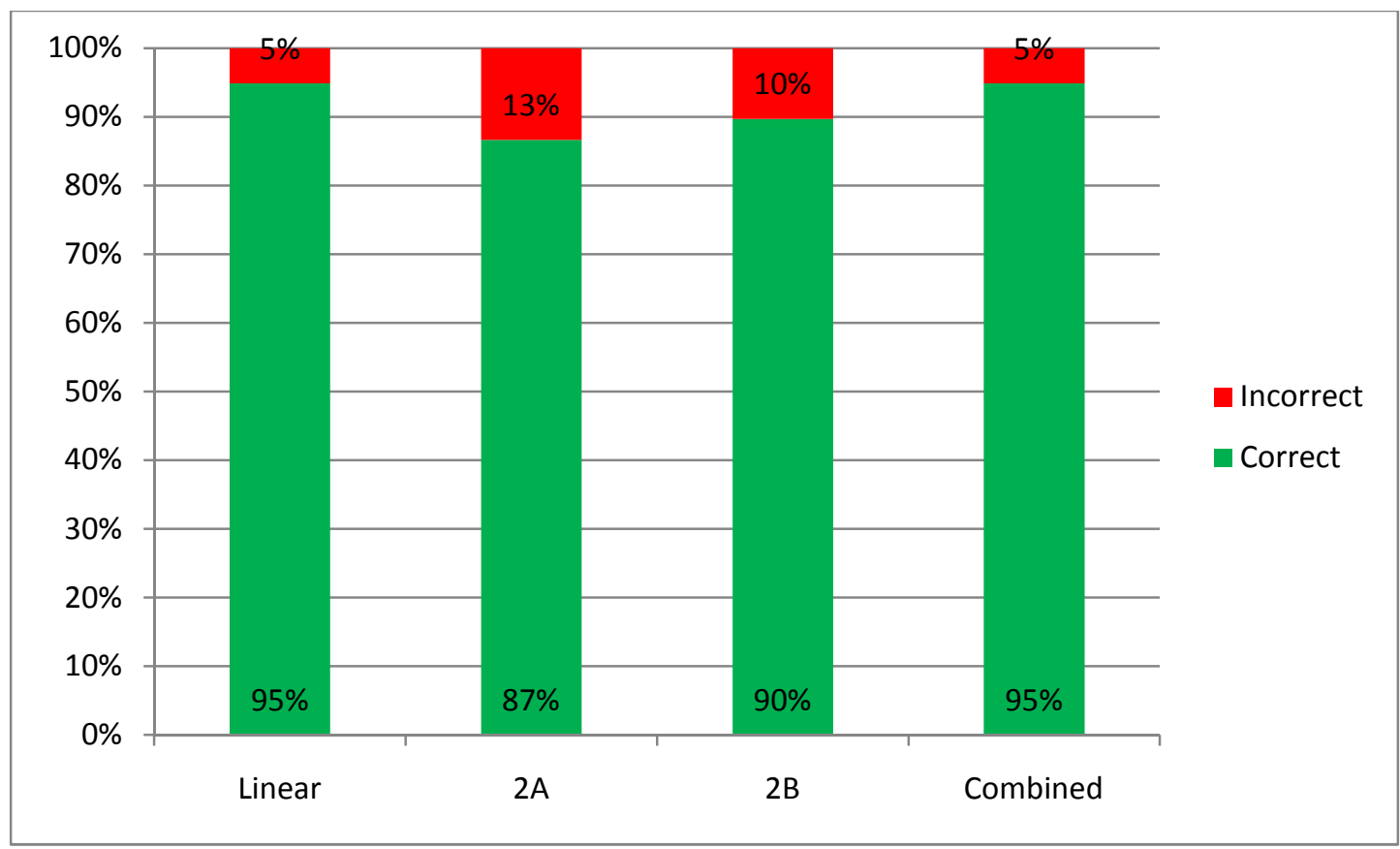

Figure 33 - Percentage of False Fail Results

The calibration results found that the false fail outcomes reached the goal of $10 \%$ or less, with the exception of Model 2A. If, as suggested earlier, the combined model was to be used, then the false fail predictions would be $5 \%$. False fail results of $5 \%$ mean just over 1 building every 2 years requiring simulation when it wasn't needed to prove compliance. This is an acceptable error rate.

Variables of false fail results were compared but there was no clear cause for the false fail results.

\subsubsection{Accuracy of Outcomes for Entire Buildings}

The results were also analysed in terms of the whole building. 97 apartments were measured within 23 buildings. As the tool was intended to specify when a building required simulation, each calibration building was assessed. A correct outcome for a failing building was where at least one of the failing apartments received the correct outcome and a 
correct outcome for a passing building is where all apartments received pass results. This assessment found that all buildings received the correct outcome provided the vertical locations were limited to the $5^{\text {th }}$ floor or lower.

\subsubsection{Best Model Overall}

As a decision was unable to be made as to which model provided the best result during the development stage of the tool, it was decided to continue with all three through the calibration stage and assesses which model provided the best results for real situations. Figure 34 compares the accuracy of the three prediction equations. The 7 false pass results that were eliminated earlier have been included in these results.

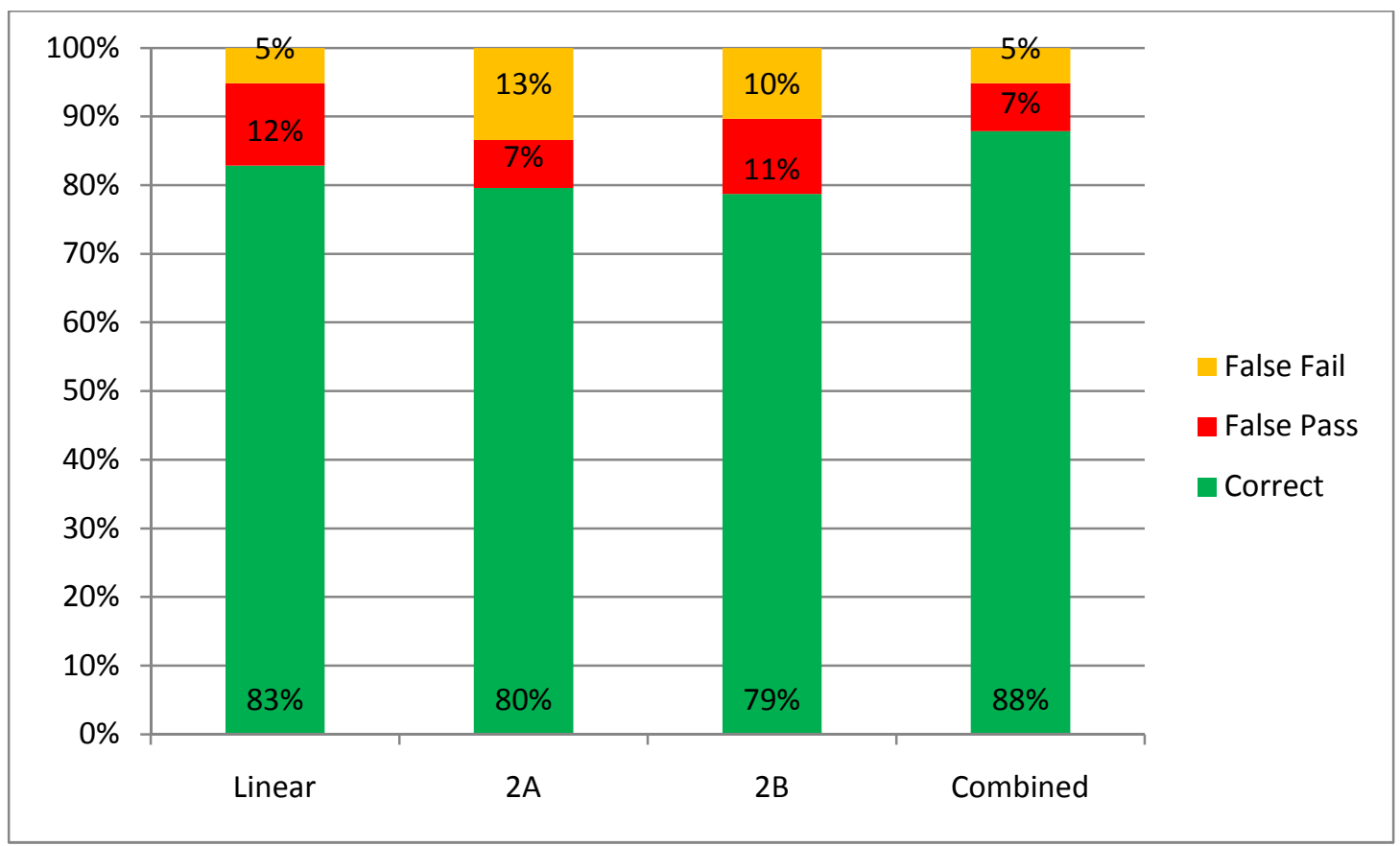

Figure 34 - Accuracies of the Three Prediction Models

From Figure 34, the linear model had the most accurate results, but also had the highest percentage of false pass results with 3 buildings every 2 years expected to receiving false pass results and avoiding simulation when it is necessary to prove compliance. Model $2 \mathrm{~A}$ had the second highest accuracy as shown in Figure 34. Model 2A had the lowest false pass result, but also had the highest false fail result, requiring 3 buildings every 2 years to have simulations conducted to prove compliance when they were not necessary. Model 2B had the lowest accuracy as shown in Figure 34. Model 2B had a relatively even split between false pass and false fail results.

The variables for the incorrect results in each model were compared to determine if any variables were affecting the results. Vertical location consistently came up for all three models, confirming that this variable needs to be limited to vertical locations of the lowest apartment of the fifth floor or lower. No other significant errors could be found in the models. Tables used to compare the incorrect outcomes and graphs for all variables can be found in Appendix 8. 


\section{Combination of Models}

None of the models stood out as being noticeably better than the other two. Figure 34 identified that the best results were provided when the strengths of each of the models were used to provide a result, where the most accurate model was applied based on the variables.

If a combined model was used and apartment buildings with the lowest floor of apartments higher than the $5^{\text {th }}$ floor were removed from the assessment, only 3 incorrect outcomes were given, all were false fail results. This gives the tool an accuracy of $97 \%$, with no buildings likely to receive a false pass result and just over 1 building every 2 years likely to receive a false fail result.

Table 25 shows the variable inputs for the 3 incorrect outcomes. The purpose of this table is to display any commonality between the three incorrect apartments. The aim was to see if any variables were similar in order to highlight where further investigation might be needed. Ideally, Table 25 would show that all three apartments had more than one variable in common, eg all had opposite building heights of around $20 \mathrm{~m}$ and were all North facing. However, it is clear in Table 25 that there is a lot of variation in the results meaning that no further similarities can be drawn between these incorrect predictions.

Table 25 - Variables of the three remaining apartments that received incorrect predictions from the tool

\begin{tabular}{|l|c|c|c|}
\hline & $\begin{array}{c}\text { Apartment } \\
\mathbf{5 1}\end{array}$ & $\begin{array}{c}\text { Apartment } \\
\mathbf{6 6}\end{array}$ & $\begin{array}{c}\text { Apartment } \\
\mathbf{7 5}\end{array}$ \\
\hline Building Height & $20 \mathrm{~m}$ & $19 \mathrm{~m}$ & $16 \mathrm{~m}$ \\
\hline Street Width & $5 \mathrm{~m}$ & $25 \mathrm{~m}$ & $9 \mathrm{~m}$ \\
\hline Glazed Area & $23 \%$ & $12 \%$ & $22 \%$ \\
\hline Orientation & North & South & East \\
\hline Reflectance & $33 \%$ & $20 \%$ & $15 \%$ \\
\hline Vertical Location & $3^{\text {rd }}$ Floor & $1^{\text {st }}$ Floor & $1^{\text {st }}$ Floor \\
\hline
\end{tabular}

\subsubsection{Observations from Apartments}

This section discusses the various observations and findings from the measurement of the calibration apartments. The keys findings discussed are: the overall pass/fail results; the effect of complex design features like atria, light-wells and internal habitable rooms; assessment of assumptions including internal surface reflectances and shading devices; and comments made by a number of residents about the protection of their natural light/view.

\section{Apartment measurements}

The measurements taken in the apartments found that $49 \%$ of the habitable rooms exceeded the minimum requirement, meaning that $51 \%$ failed to meet the requirement. $13 \%$ of the apartments were borderline failing (within 5 lux of passing), where it was possible that furniture may have caused the fail result in some of these cases. As the range of apartments measured is a fair representation of New Zealand apartments, it is fair to 
conclude that half the apartments in Auckland, Wellington and Christchurch do not comply with the minimum requirement for natural light.

\section{Atria and Light-wells}

Six buildings were assessed that used an atrium or light-well to provide natural light to habitable spaces. Four rooms were measured where the only source of natural light was from the atrium. All four of these spaces failed to meet the minimum requirement at the back of the room. However, measurements directly beside the window did exceed the minimum requirement in three of the four cases. Of these 11 rooms that used light-wells to gain access to natural light, 8 failed to meet the minimum requirement.

Atria and light-wells are considered complex daylight design features and as such, compliance should not be assessed using the tool or the current Acceptable Solution. As a result of the poor performance of the four spaces measured, it is recommended that ALL atria should be simulated.

\section{Internal Rooms}

Of the 97 apartments measured, 6 had internal habitable rooms, similar to Figure 35 . There has been a question surrounding these rooms and whether they fulfil the requirements of NZBC G7. From the measurements taken in these rooms, it was found that none exceeded the minimum requirement at any point within the room. Measurements were taken beside the window and at the three measurement points used throughout this research. The best internal room had an illuminance of 26 lux exceeded for $75 \%$ of the standard year directly beside the window and 10 lux exceeded for $75 \%$ of the standard year at the back of the room. The worst internal room had just 2 lux for $75 \%$ of the standard year directly beside the window and less than 1 lux at the back of the room. It is for this reason that it is recommended that $\underline{A L L}$ internal habitable rooms should require simulation to prove they comply with NZBC G7.

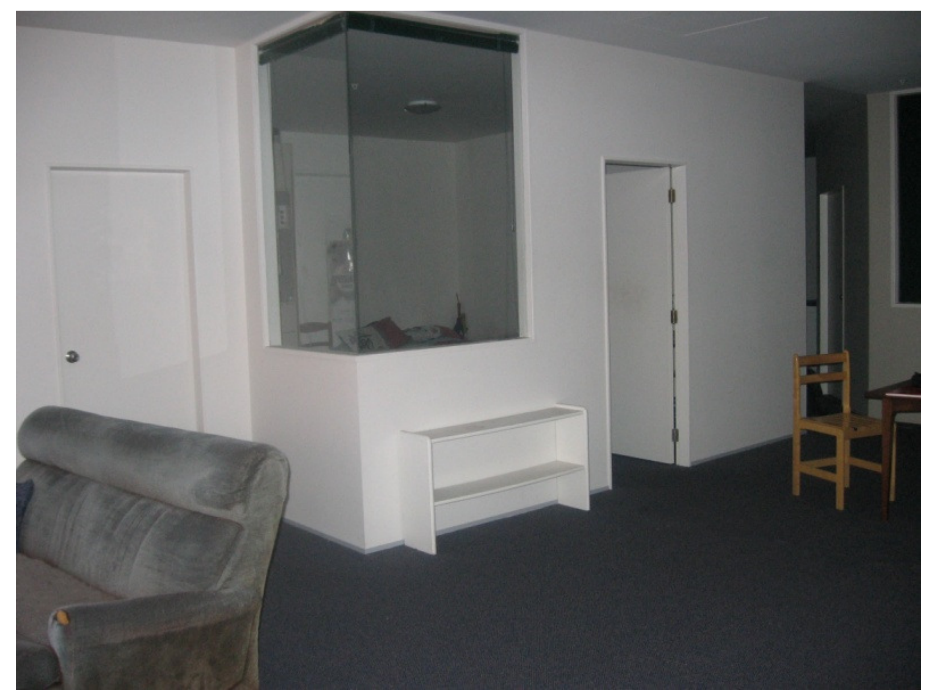

Figure 35 - Internal Habitable Rooms 


\section{Internal Reflectances}

As was discussed in Section 5.1.2 (pg 66), internal surface reflectance values were assumed to be $80 \%$ for ceiling, $70 \%$ for walls and $25 \%$ for floors, these values represented light coloured walls and ceilings, and medium-dark coloured floors.

During the measurement of the calibration apartments, a quick review of internal surfaces was undertaken, where walls, ceiling and floors were noted down as being either dark, medium or light. The results of this review, as shown in Error! Reference source not found., where then compared to the original assumption, that typical apartments have light coloured walls and ceilings with medium-dark coloured floors, to check that this was a valid assumption and was a fair representation of the typical surface reflectance found in New Zealand Apartments.

Table 26 - Percentage of apartments with dark, medium and light surfaces for ceilings, walls and floors.

\begin{tabular}{|l|r|r|r|}
\hline & \multicolumn{1}{|l|}{ Ceiling } & \multicolumn{1}{l|}{ Walls } & \multicolumn{1}{l|}{ Floor } \\
\hline Dark & $0 \%$ & $3 \%$ & $30 \%$ \\
\hline Medium & $1 \%$ & $14 \%$ & $70 \%$ \\
\hline Light & $99 \%$ & $82 \%$ & $0 \%$ \\
\hline
\end{tabular}

Error! Reference source not found. shows that almost all (99\%) of the calibration apartments had light (white) coloured ceiling, most (82\%) of the apartments had light coloured walls and all floors were either medium or dark coloured, with the majority (70\%) being medium coloured. Therefore, it can be concluded that the assumption that a typical apartment had light coloured walls and ceilings and medium-dark coloured floors was fair and representative.

\section{Shadings Devices}

Another assumption made in the early stage of this research was that the models used in the simulations would not have any shading devices like balconies and overhangs, meaning that these were not considered in the prediction of performance in apartments. During the measurement of the apartments, notes were taken regarding the presence of any types of shading device. Forty out of the 97 had shading devices and 57 did not. The apartments with shading devices were cross-referenced against the incorrect results, the false pass result in particular, to ensure that this assumption did not over-estimate the daylight performance in apartments where shading devices were present. It was found that 4 out of the 7 false pass results had shading devices. It is possible that if shading devices had been considered in the calculation then these false pass results may not have been given.

\section{Preservation of Natural Light and View}

While measuring illuminance levels in the apartments, a number of residents mentioned that they are currently happy with the exposure to natural light and views, however they were concerned that future building developments on surrounding sites may obstruct their views and reduce the amount of daylight they receive. In one situation, an apartment had 
views of Wellington Harbour and plenty of natural light, but recently a large building had been built on the adjacent site. The residents of this apartment now have a view of a concrete wall approximately 3 metres from their balcony and the space requires artificial light throughout the day. To ensure situations like this do not continue to happen, two methods could be adopted. The first is to use the UK's 'Right to Light' laws requiring all new developments to prove they will not negatively affect neighbouring buildings (British Standards Institution, 1992). The alternative would be to include 'worst case scenario' tests into the compliance assessment tool to investigate the effects that changes to the surrounding buildings would have.

\subsection{Calibration Conclusions}

Overall, the accuracy of the tool achieved the target accuracy of $90 \%$, specified for 'reasonable accuracy'. It can be expected that no buildings would receive a false pass result if limits are placed on vertical location. And 1 building every 2 years could be expected to receive a false fail result, requiring simulation when it wasn't necessary to prove compliance.

As there was still no prediction model that was clearly better than the other two, it was decided that a combination of these models was most reliable. The combination of models provided the best accuracy overall of $97 \%$, because it uses the most reliable equation for the combination of variables in each case.

Also, complex design features like atria, light-wells and internal habitable rooms required specific consideration in the tool. 


\subsection{CRITIQUE OF THE TOOL}

Chapter 7 discusses the critique (or evaluation) of the tool undertaken to assess the usability and functionality of the tool. Section 7.1 outlines the methods used to conduct the critique and how it contributes to this research. Section 7.2 summarises the findings from the surveys. And Section 7.3 tests some possible changes to the tool as a result of the evaluation process.

\subsection{Critique Method}

The potential end-users of the tool were invited to participate in a critique (or evaluation) with the aim of assessing the usability of the tool. Two types of 'potential end-users' were identified: designers (architects and engineers); and regulators (building control and regulatory bodies).

If end-users agreed to participate, they were sent an evaluation pack which contained the tool, instructions for how to apply the tool and a questionnaire to complete after applying the tool. They were asked to apply the tool to a recent apartment building project or hypothetical situation to gain an understanding of how the tool works and then fill out the questionnaire.

The questionnaire was formed to determine if the tool is easy to use and was not time consuming, compared to full simulation, and also if end-users would generally support the implementation of the tool. The questionnaire also gave potential end-users the chance to give suggestions for aspects of the tool that may be altered to make the tool easier to use. A copy of the questionnaire can be found in Appendix 10.

It is recommended by Nielson (2000) that if two different groups are participating in usability tests then 3-4 users are sufficient per group and the 8 users will successfully be able to identify around $98 \%$ of usability problems.

\subsubsection{Selection of Participants}

16 architecture/engineering firms in New Zealand were approached, via email and phone, and asked to participate in the evaluation. These firms were selected using the New Zealand Institute of Architects (NZIA) architecturenz.net (2005) directory of registered NZIA practices. From this search, a list of larger firms (more than 5 people) was developed. From this list, a search was conducted using their websites provided on the NZIA directory to determine which of these firms have designed apartment buildings. These were the 16 firms approached. 
Representatives from six regulatory bodies were invited to participate in the evaluation. The regulatory bodies were selected as those that would deal with consents for apartment buildings on a regular basis. Four City Councils were selected from the 16 throughout New Zealand. These councils were selected because of their higher number of consents for apartment buildings over the last five years (Statistics New Zealand, 1991-2008) and their variations in geographical location. Two regulatory advisors were also invited to participate in the evaluation. Although they are not necessarily 'potential end-users', their knowledge in the use of tools like this for regulation was invaluable.

\subsubsection{Method for Analysis of Results}

There were two forms of analysis used to interpret the results from the questionnaire. The first was through graphs for the questions having 'yes' or 'no' answers or ranking. The second is through a discussion of suggestions given on improvements to the tool.

Graphs were produced to easily compare peoples' reactions to the tool. The graphs allowed conclusions to be drawn about how users generally found the tool.

Where written suggestions were given regarding the usability, each suggestion was reviewed. In the review, ideas were given on how this could be applied to the tool and where feasible, modifications were made to the tool.

\subsection{Analysis of Critique Results}

Of the 16 architecture/engineering firms that were approached and invited to participate in this critique, four people agreed to participate in the evaluation. Reasons for the low response rate were the time involved in participating (approximately 1 hour) and people were often too busy. However, based on Neilson's (2000) recommendations, four participants is sufficient to identify any usability problems.

Of the six regulatory bodies (city councils and regulatory advisors), four people agreed to participate in the evaluation. Although this appears to be a reasonable response rate, only one of the four city councils approached assisted in this study, meaning that two representatives participated in this critique from the one council. The reasons for this response from the councils were that people were too busy and administrative/communication difficulties when dealing with a large organisation like city councils. The two regulatory advisors approached agreed to participate in this evaluation.

Therefore, a total of eight people agreed to evaluated the tool. The eight participants comprised of four architects/engineers, two building controls officers (from one city council) and two regulatory advisors. 


\subsubsection{Time needed to use the Tool}

Participants were asked to estimate how long it took to assess a project using the tool, how they considered this time (too long-too short) and how many times they applied the tool. These questions were intended to help determine whether the first criterion, that the tool was to be simple and easy to use, was fulfilled.

Users applied the tool between 1 and 5 times, before answering the questionnaire, with the tool being applied a total of 19 times. It was thought that the number of applications may have influenced the time taken to apply the tool, but it was found that there was no relationship between the time taken to apply the tool and the number times it was applied as shown below in Table 27.

Table 27 - Comparison of the time taken and the number of times users applied the tool

\begin{tabular}{|l|c|c|}
\hline & Time taken to apply tool & Number of time tool was applied \\
\hline Participant 1 & 60 mins & 5 \\
\hline Participant 3 & 15 mins & 5 \\
\hline Participant 5 & $2-3$ mins & 3 \\
\hline Participant 7 & 30 mins & 3 \\
\hline Participant 2 & 45 mins & 1 \\
\hline Participant 4 & 2 mins & 1 \\
\hline Participant 8 & 10 mins & 1 \\
\hline
\end{tabular}

Five out of the eight respondents said that the tool takes less than 20 minutes to complete, as shown below in Figure 36. This indicates that using the tool would not have a large impact on users' time, but as some of the results were between 30 minutes and 1 hour some issues need to be resolved in the instructions so that all applications take less than 20 minutes.

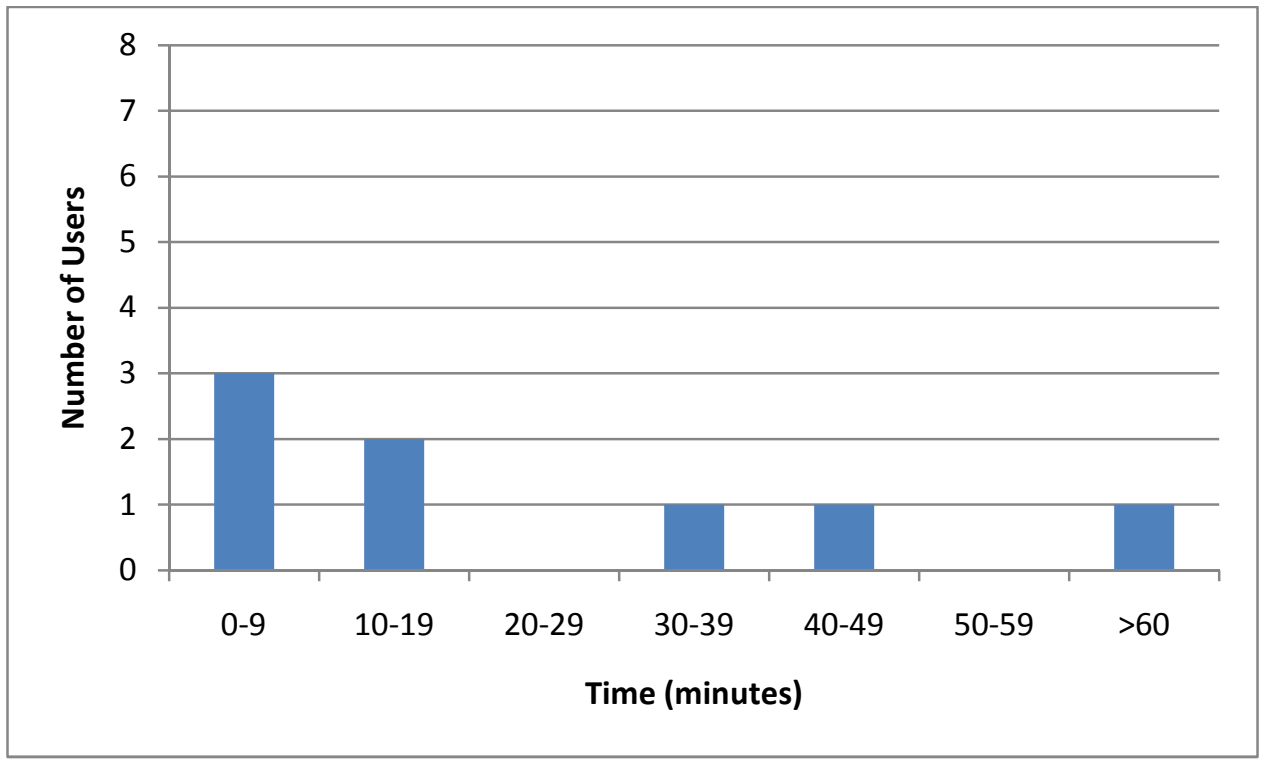

Figure 36 - Time taken to use the tool 
With the tool typically taking less than 20 minutes to use, it would save time and money on projects that were expected to perform well. Table 28 compares the estimated costs (time and financial) for applying the tool and performing a full simulation.

Table 28 - Cost Comparison of the Tool vs. Full Simulation

\begin{tabular}{|l|c|c|}
\hline & Estimated Time & Cost $\mathbf{\text { \$ based on } \$ 1 5 0 \text { Charge out rate) }}$ \\
\hline G7 Tool & $20 \mathrm{mins}$ & $\$ 50$ \\
\hline Full Simulation & $16 \mathrm{hrs}$ & $\$ 2400$ \\
\hline
\end{tabular}

Users were asked to rate how they considered the time taken to apply the tool, from Too Long - Too Short. Six out of the 8 participants thought the time taken to apply the tool was 'Just Right', as shown in Figure 37. The participant that thought the tool took a little bit too long (between Too Long and Just Right) was a representative from the City Council and estimated it took 30 minutes to assess a project using the tool. The participant that thought the time taken to assess a project using the tool was a little too short (between Just Right and Too Short) was from a designer and took 60 minutes to apply the tool. The response from this participant was very interesting. However, in support of this person's response to the question, they stated that 'we (architects) would be happy to spend a little extra time inputting figures if the tool was more accurate for higher performing apartments'.

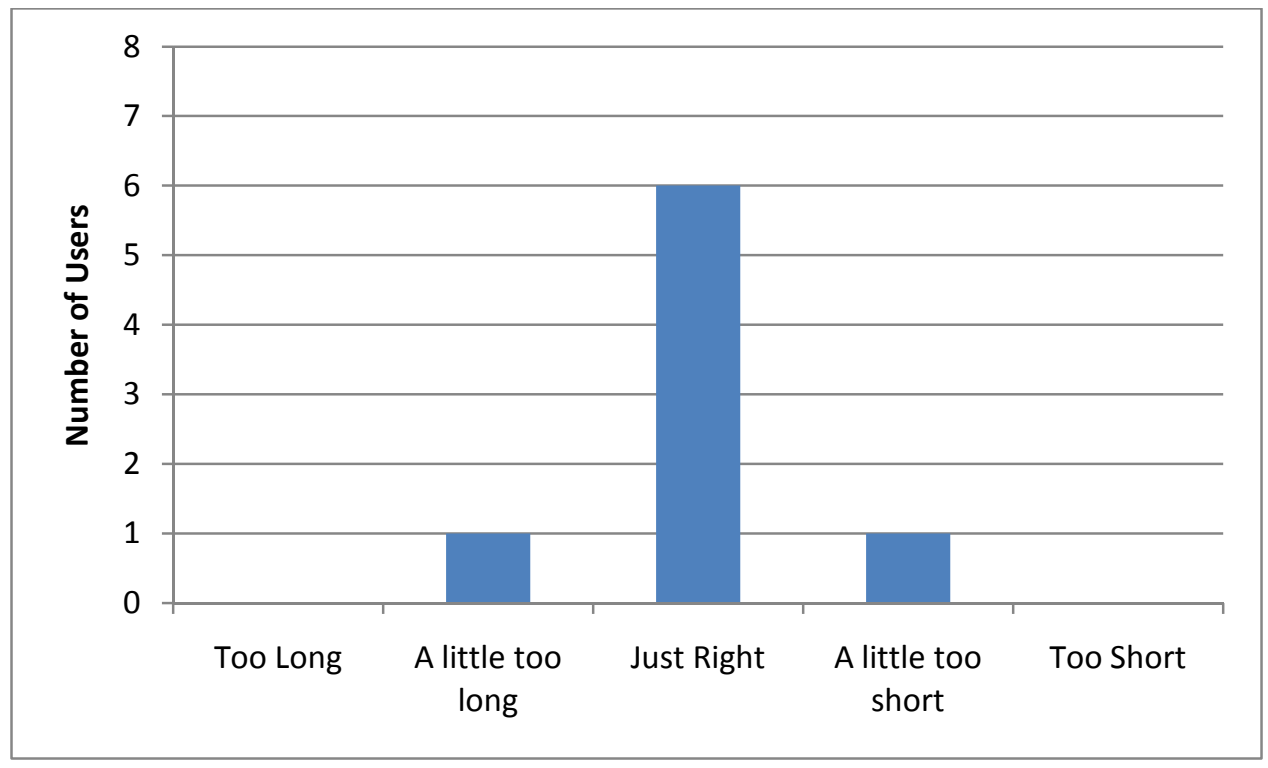

Figure 37 - How users considered the time taken to use the tool

Generally, most participants were happy with the time required to assess an apartment building using the tool. All respondents that provided times less than 30 minutes in question 1 were satisfied with the time taken. Interestingly, the participant that estimated it took 45 minutes to assess a building using the tool thought the time it takes was about right. 


\subsubsection{Difficulty Level}

To determine if users found the tool simple and easy to use, the first criteria for the tool, participants were asked how difficult they found the tool to be. All the participants found the tool to be between a little too hard and a little too easy, as can be seen in Figure 38 . The two respondents that found the tool to be a little too hard were both from architecture/engineering firms. Two of the three respondents that found the tool to be a little too easy were from regulatory bodies. The participant that estimated the longest time to assess a project using the tool and found that time to be a little too short also found the tool to be a little too easy.

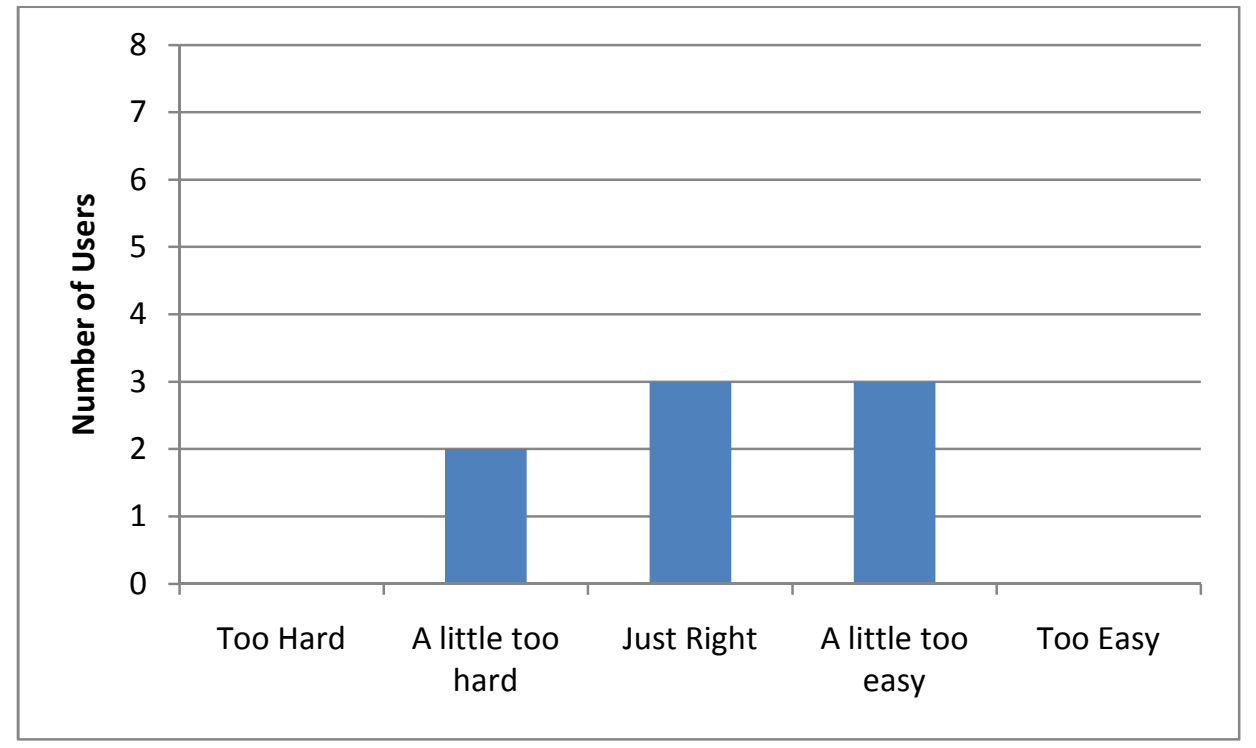

Figure 38- How users considered the difficulty of the tool

As there was no predominant answer given by participants, no generalisations can be drawn for the ease and simplicity of use. However, no users found the tool to be too hard or too easy so it can be concluded that participants were reasonably happy with the tool and thought the tool was about right in terms of complexity. Small changes made to the tool using the information provided in the questionnaire and further comments provided by participants would most likely improve the participants' impressions on the difficulty of the tool.

\subsubsection{Support for the Tool}

Participants were asked three yes/no questions relating to the tool that would indicate how people generally felt about the tool and would help fulfil criterion three, that the tool could be easily implemented. The questions asked whether participants considered the tool to be useful and the result to be effective, and whether they would support the implementation of the tool.

Seven out of the 8 participant found the tool to be useful, the result to be effective and would support the implementation of the tool, as can be seen in Figure 39, Figure 40 and 
Figure 41. The one participant who did not find the tool useful, the result effective and would not support the implementation of the tool was from an architecture firm. Later comments suggested that this was because the firm already works closely with engineers and natural light compliance issues would be dealt with by the engineers rather than the architects. This also indicated that some firms misunderstood the purpose of the tool, meaning that a more thorough explanation of the tool and its objectives is needed.

(Note: Supporting the implementation was not meant as any form of endorsement but rather asking people whether they thought it was a good idea to implement the tool.)

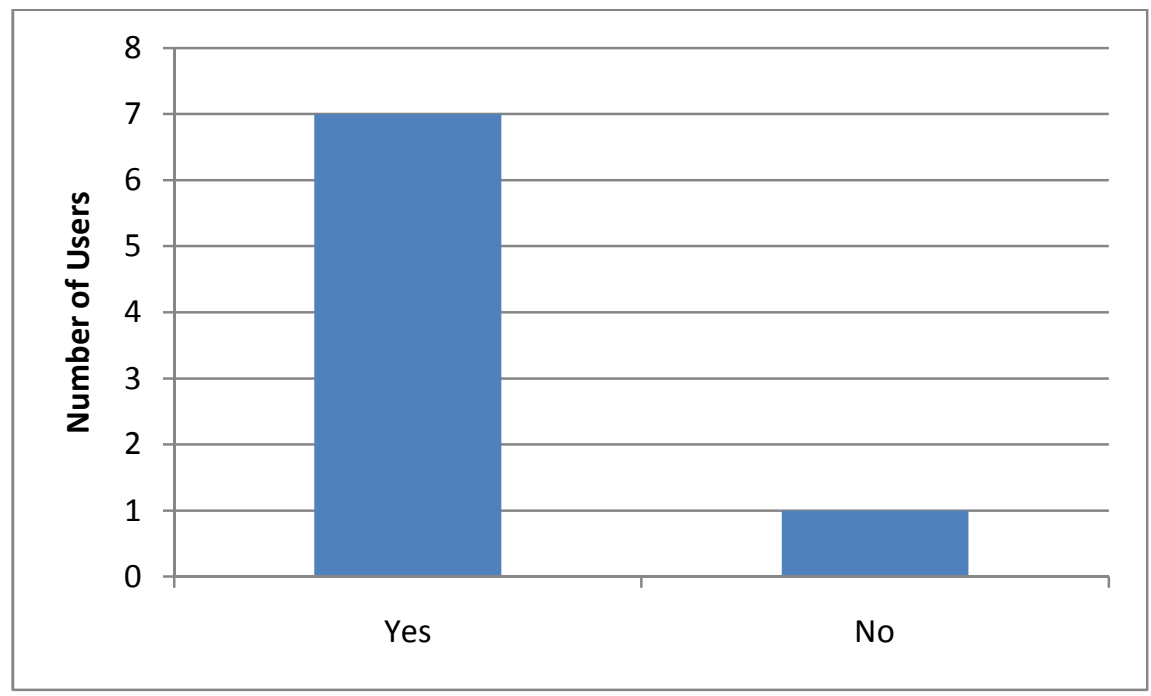

Figure 39 - Did users consider the tool useful?

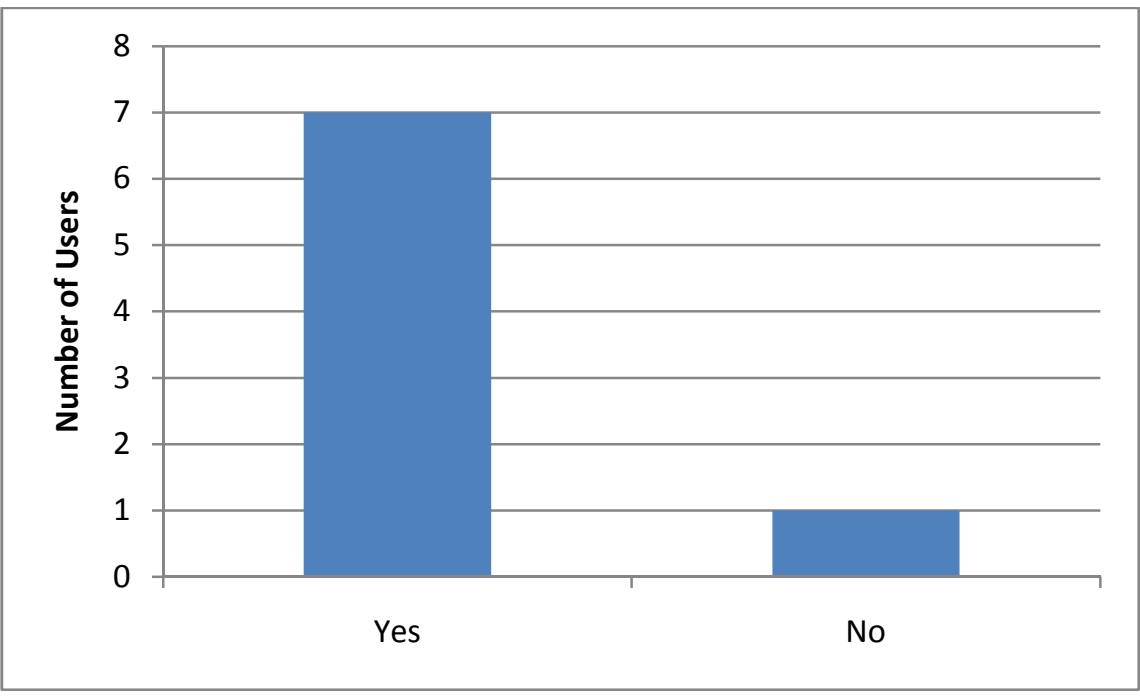

Figure 40 - Did users find the result effective? 


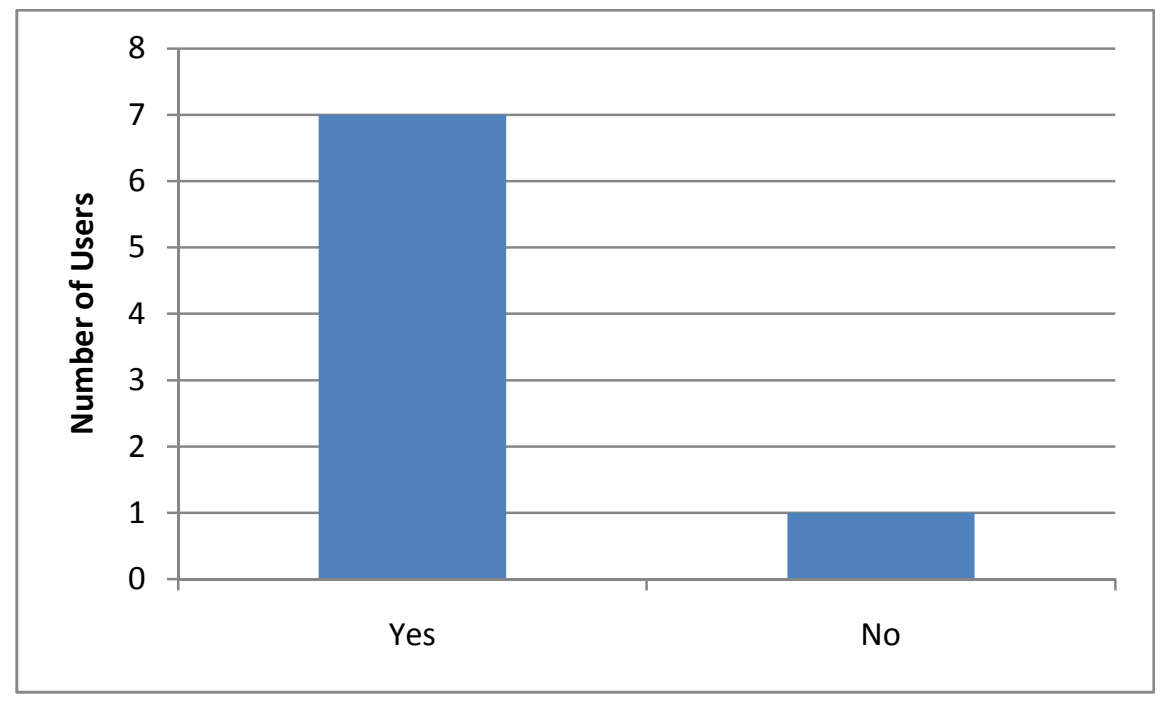

Figure 41 - Would users support the implementation of the tool?

It can be concluded that generally participants found the tool to be useful, the result to be effective and would support the implementation of the tool. This result means that the implementation will be easier as end-users would not oppose the implementation of the tool.

\subsubsection{Comments from Participants}

This section outlines some of the general comments and concerns provided by the participants. These are indicative of the types of issues that will likely be raised in the implementation process.

Two of the four architecture/engineering firms questioned the application of the tool for complex situations, identifying different cases where it was not clear how they should apply the tool. Some examples are situations where the opposite building is not parallel to the proposed building and where the opposite building has multiple reflectances on the façade. Many of these situations can easily be assessed using the tool, but this highlighted the need for thorough instructions to describe how the tool should be applied for typical situations and examples of complex situations. However, it was not the intention of the tool to provide results for every possible situation, which means that some cases will not be able to be accurately assessed by the tool. In these cases, simulation would be required.

Two of the participants from architecture firms suggested that the tool should be more technical. One participant suggested that 'more building inputs were required so the tool is reliable in various scenarios'. The other participant felt that the tool 'needs to be more accurate, and not to give an indication only'. It was taken from these comments that these participants wanted a tool that essentially replaced the need for simulations i.e. gave predictions of illuminance levels, however this was not the intention of this research. The tool does provide accurate and reliable results for a number of different scenarios. 
Providing exact predictions of daylight performance is extremely complex and is not realistic for this type of tool and for the time constraints of this research. There are already a number of tools that accurately predict daylight performance, including daylight simulation, therefore there was no point in producing a tool that provides the same information as simulation. It is clear that the Architecture firms did not understand the intentions of the tool, where the idea behind the tool was that it was to determine if simulation was needed rather than to replace simulations. To address this, more upfront information about the intentions of the tool need to be provided.

It was not feasible to produce the number of simulations required to accurately assess the daylight performance in all situations. Over 600 simulations were produced to develop this tool, but it is estimated that at least 5 times as many would be needed to accurately assess all variables that affect daylight performance and would still not include all the possible situations. Including more building inputs would also mean that the tool could not be applied in the early design stage, as was intended, as the information would not be available until the later design stages.

It was also highlighted that surrounding buildings are currently not taken into considerations in building consent applications and that designers are reluctant to provide this type of information. However surrounding buildings have a large effect on the daylight available in urban environmental and need to be considered if daylight performance is to be accurately predicted in cities.

One representative from a city council highlighted that support from the Department of Building and Housing (DBH) is vital to getting the tool implemented and used in everyday practice, stating that 'TAs are unlikely to adopt this unless given a directive from the $\mathrm{DBH}^{\text {'. }}$ It was intended that the DBH would be involved in the implementation process, but this survey response has confirmed that without DBH support there will be a limited future for the tool.

One participant felt that it was the engineer's responsibility to be dealing with issues of compliance and that assessing apartment buildings using the tool is not necessary for an architecture firm as engineers are already actively involved in the early design stages. The main reason this tool was developed was to equip Consenting Officers from Territorial Authorities with the tools to reliably assess daylight performance in apartments. If an architect or engineer uses the tool to check whether his/her building would comply earlier, then it could save them time and money in the long run, but no stipulation is placed on who must use the tool and when it must be used prior to the consent process.

Overall, the comments can be dealt with relatively easily. The most important comment was the comment relating to DBH support and highlighted that gaining their support is an essential step in the implementation of the tool. 


\subsection{Changes to Tool as a Result of the Critique}

\subsubsection{Minor Changes to the Tool}

\section{Adding Units to the Tool}

One participant indicated that showing the units for each of the variables would make the tool easier to understand. This was a very minor change that may make it easier for some users to understand so it is worth including in the tool. Figure 42 shows the addition of units to the tool.

\begin{tabular}{|l|l|l|}
\hline \multicolumn{2}{|c|}{ NZBC G7 Compliance Assessment Tool } \\
\hline Variable: & \\
\hline Opposite Building Height & & $\mathrm{m}$ \\
\hline Street Width & $\mathrm{m}$ \\
\hline Glazed Area & & $\%$ \\
\hline Orientation & & \\
\hline Location & & $\%$ \\
\hline Reflectance of Opposite Building & $\%$ \\
\hline Glazing Transmittance & & \\
\hline Vertical Location of lowest apartment & \\
\hline \multicolumn{2}{|c|}{ YES } \\
\hline Are simulations required to prove compliance: & & \\
\hline
\end{tabular}

Figure 42 - Addition of units to the tool

Also, by noting the units, this will remove any complication regarding the input of variables. This is important for opposite building height and street width which are to be measured in metres, if unit aren't given there is a chance some users may assume the values were to be in millimetres, likewise for reflectance and glazing transmittance which could also be given as factors.

\section{More Interactive Interface}

Three of the participants recommended that a more interactive interface was needed rather than the simple spreadsheet. The participants recommended features like dropdown menus and yes/no boxes (for complex features like internal rooms and atria) to simplify the application process and remove the possibility of errors from incorrect spelling. Only the use of drop-down menus is able to be added in the current format of the tool, see Figure 43. 


\begin{tabular}{|l|l|l|}
\hline \multicolumn{2}{|c|}{ NZBC G7 Compliance Assessment Tool } \\
\hline Variable: & & \\
\hline Opposite Building Height & & $\mathrm{m}$ \\
\hline Street Width & & $\mathrm{m}$ \\
\hline Glazed Area & & $\%$ \\
\hline Orientation & & \\
\hline Location & \begin{tabular}{l} 
Northland \\
\hline Reflectance of Opposite Building \\
Auckland \\
Hamilton \\
Bay of Plenty \\
Rotorua \\
Taupo \\
New Plymouth \\
East Coast
\end{tabular} \\
\hline Vertical Location of lowest apartment \\
\hline \multicolumn{1}{|c|}{ YES } \\
\hline Are simulations required to prove compliance:
\end{tabular}

Figure 43 - Drop-down menus

Adding drop-down menus for Orientation, Location and Vertical Location were relatively easy to apply to the tool and will make the application process simpler. These will allow users to avoid errors due to incorrect spelling and also limits the users to the variations that the tool is able to accurately predict. An example of this is for Vertical Location, where it was identified in Chapter 6 that only buildings with the lowest floor of apartments located on the $5^{\text {th }}$ floor and lower can be accurately assessed by the tool, the options would be limited to $0,1,2,3,4$ and 5 .

\section{Including Visual Awareness}

It was suggested by one of the council representative that Visual Awareness needs to be considered in the tool. The Acceptable Solution for NZBC G7 (G7/AS1) specifies two aspects that need to be met in order to comply with the visual awareness requirement of NZBC G7. These are (Department of Building and Housing, 2001, pg 16):

- At least $50 \%$ of the glazed area provided for natural light in habitable spaces shall be clear glazing. The clear glazing shall be located in the zone between the levels $900 \mathrm{~mm}$ and $2000 \mathrm{~mm}$ from floor level.

- It is acceptable for awareness of the outside to be provided through another space.

Only the first aspect needs to be added to the tool. Given that these are simple requirements, these can easily be added to the tool with no testing required. An additional section can be added to the tool as shown below in Figure 44 to assess compliance with the visual awareness requirements. 


\begin{tabular}{|c|c|c|}
\hline \multicolumn{3}{|c|}{ NZBC G7 Compliance Assessment Tool } \\
\hline \multicolumn{3}{|l|}{ Illuminance Requirements } \\
\hline \multicolumn{3}{|l|}{ Variable: } \\
\hline Opposite Building Height & & $\mathrm{m}$ \\
\hline Street Width & & $\mathrm{m}$ \\
\hline Glazed Area & & $\%$ \\
\hline \multicolumn{3}{|l|}{ Orientation } \\
\hline \multicolumn{3}{|l|}{ Location } \\
\hline Reflectance of Opposite Building & & $\%$ \\
\hline Glazing Transmittance & & $\%$ \\
\hline \multicolumn{3}{|l|}{ Vertical Location of lowest apartment } \\
\hline Are simulations required to prove compliance: & \multicolumn{2}{|l|}{ YES } \\
\hline \multicolumn{3}{|l|}{ Visual Awareness Requirements } \\
\hline Percent of Clear Glazing & & $\%$ \\
\hline Clear Glazing Maximum Height & & $\mathrm{m}$ \\
\hline Clear Glazing Minimum Height & & $\mathrm{m}$ \\
\hline $\begin{array}{l}\text { Does this apartment meet the visual awareness } \\
\text { requirements: }\end{array}$ & FAIL & \\
\hline
\end{tabular}

Figure 44 - Tool with Visual Awareness Requirements

The addition of these visual awareness requirements could be used, but would make the tool more complex - a conflict with the original goal. It may be that in a web-based tool such an addition would be less problematic.

\subsubsection{Consideration of Possible Changes to Proposed Building}

It was suggested that possible changes to the proposed building could be included with the tool.

Figure 45 shows how this could be done. Using the prediction model, variations in street width, glazed area, glazing transmittance and the location of the lowest apartment were used in the place of the values entered in the tool. This would allow users to see if the worst apartment would pass or fail with different conditions. This table also updates with every change to the original 8 variables except the one being varied. 
To get Figure 45, the user would enter the standard numbers in the tool and then click on a table to be shown the results. The cells shown in red indicate the cases where the building would not pass and the cells shown in green indicate the cases that would pass. For example, if the proposed building had a glazed area of $50 \%$ of the floor area but the designer was thinking about reducing this, they could refer to Figure 45 and find out that the building will still pass provided they have a glazed area of at least $30 \%$ for the floor area. 


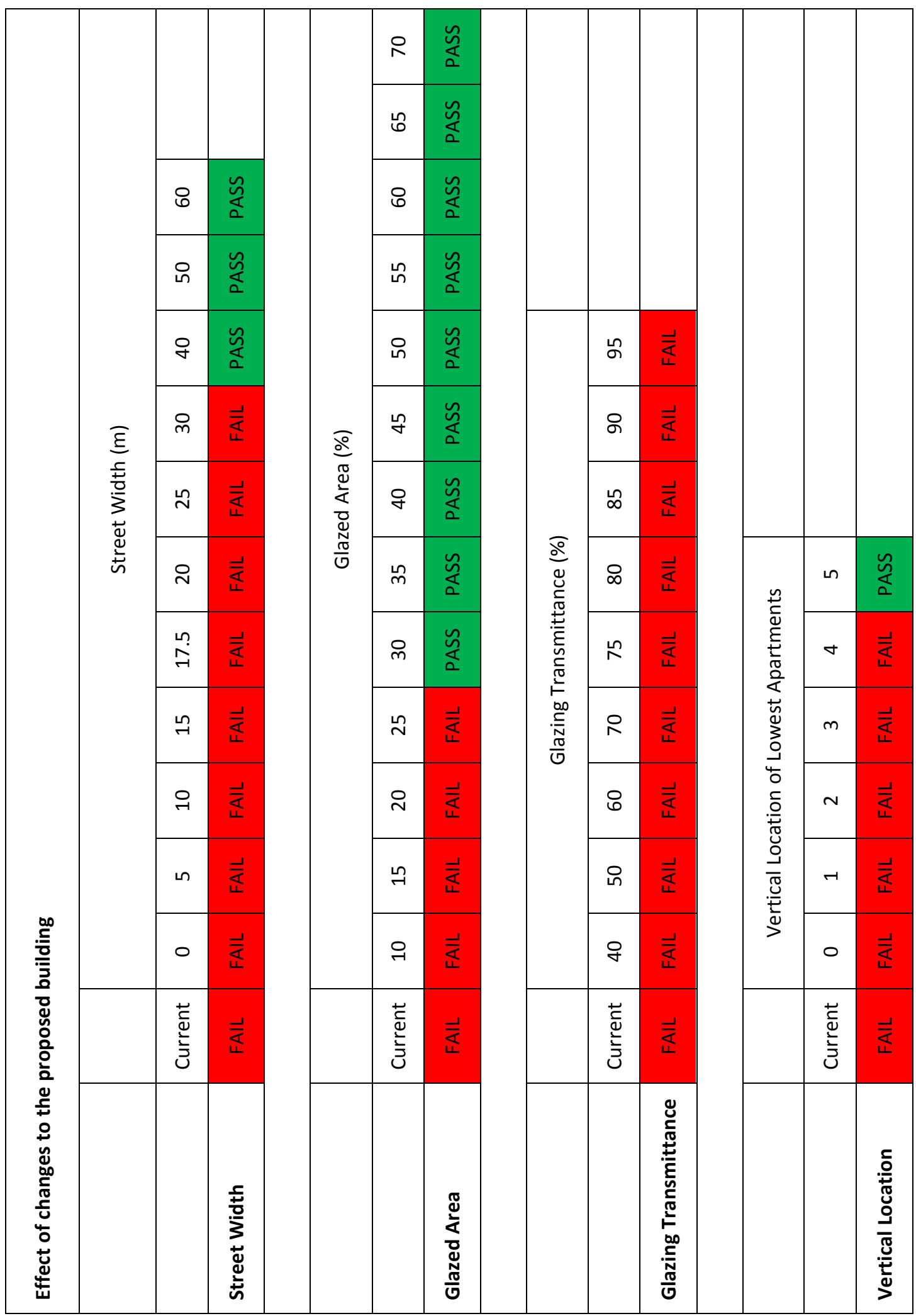

Figure 45 - Table of Possible Changes to Proposed Building

The solution to this could be confusing in the form of a spreadsheet. It would be better suited to a web-based tool. 


\subsubsection{Consideration of Changes to Adjacent site(s)}

Similar to the changes to proposed building, it was suggested that changes on adjacent sites (opposite buildings) are also included in the assessment. Using the same format as the changes to the proposed building, variations of the two factors of the opposite building can be assessed. This could be an important feature to ensure that changes to the surrounding buildings will not significantly impact on the proposed building 


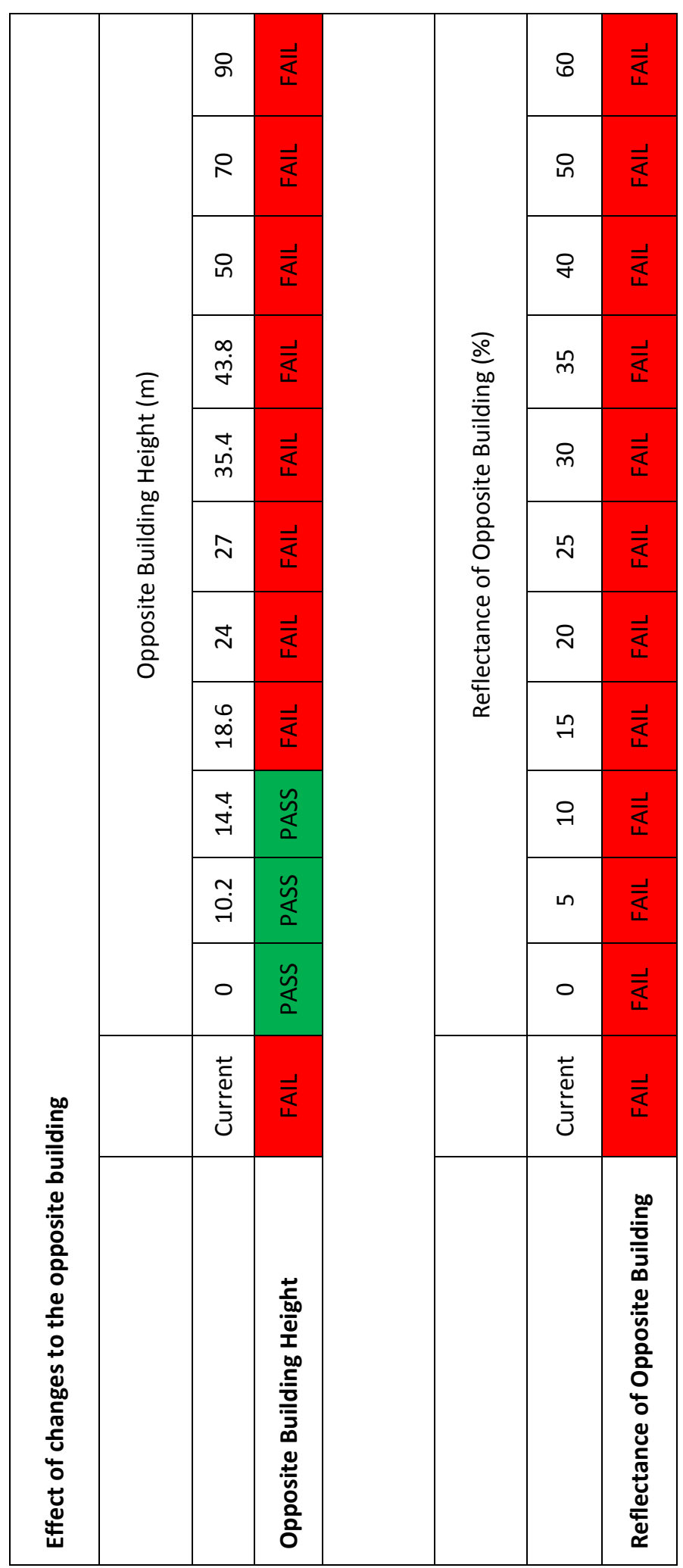

Figure 46 - Table of Possible Changes to Opposite Building

As with the features of the proposed building, this feature would be better suited to a webbased version of the tool. 


\subsubsection{Inclusion of a Reflectance Calculator}

As one of the users found it difficult to determine the reflectance of the opposite building, the following reflectance calculator was created. It works out the reflectance based on the reflectance of the walls and glazing and the proportion of the opposite face that is glazed. This is only an estimate tool and use of the actual reflectance would be preferable.

\begin{tabular}{|c|c|c|c|}
\hline $\bar{\lambda}$ & A & B & $\mathrm{C}$ \\
\hline $\mathrm{L}$ & Building Reflectance Calculator & & \\
\hline \multicolumn{4}{|l|}{2} \\
\hline 3 & Opposite building material & Portland Cement Smooth Finish & 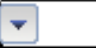 \\
\hline+ & Height of opposite building & \multirow{4}{*}{$\begin{array}{l}\text { Portland Cement Smooth Finish } \\
\text { Light Grey Concrete Blocks } \\
\text { Portland Cement Rough Finish } \\
\text { Light Grey Concrete } \\
\text { Dark Concrete } \\
\text { Glazed Facade }\end{array}$} & $m$ \\
\hline ; & Width of opposite building & & $\mathrm{m}$ \\
\hline j & Height of Typical Window & & $\mathrm{m}$ \\
\hline 7 & Width of Typcial Window & & $m$ \\
\hline 3 & Number of windows & 1 & \\
\hline 3 & Glazing type of opposite building & Clear Glazing & \\
\hline \multicolumn{4}{|l|}{0} \\
\hline 1 & REFLECTANCE OF OPPOSITE BUILDING & 38.4 & $\%$ \\
\hline
\end{tabular}

Figure 47 - Opposite Building Reflectance Calculator

This calculator would make it easier to determine the reflectance of the opposite building, but will also add additional time to the application of the tool. The reflectance values for the building materials are currently drawn from the Australian and New Zealand Standard which are only estimates and there is not a broad range of options. To improve this calculator, measurements of actual building façade materials in New Zealand cities are needed so that a full database of material can be built up. A solution is that use of this calculator is optional when the actual reflectance of the opposite building is not available.

\subsubsection{Future Changes}

\section{Clearer Instructions}

Clearer instructions that include a thorough list of assumptions, diagrams and examples of complex situations are needed to avoid confusion. Participants were sent an 'accompanying document' (see Appendix 9)with brief instruction on how to apply the tool, but it appears users would prefer this type of information in the tool itself. An effective way of including this information in the web-based tool would be to provide links to the additional information, similar to Figure 48, but for the Excel based tool this information is better suited in the 'accompanying document' form to maintain the visual simplicity of the tool. 


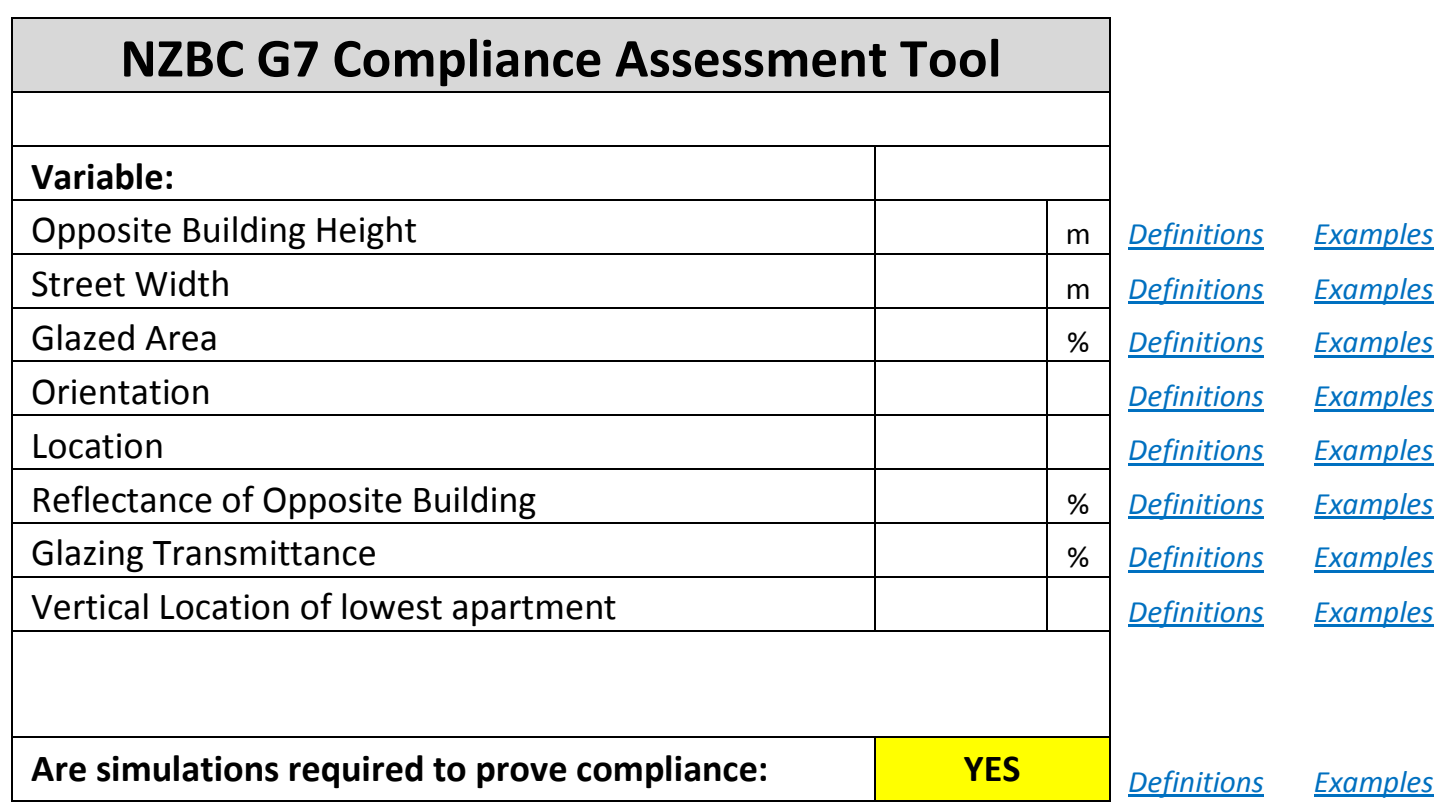

Figure 48 - Use of links to definitions and examples

The linking to further information appears to be an effective method of providing addition information to unfamiliar users. However, it is difficult to execute this in the Excel spreadsheet. If the tool is developed into a web-based tool it is relatively easy to incorporate these links into the final product. This would also allow for examples to be added assisting users with complex situations when needed.

\section{Web-based Tool}

Four of the eight participants felt that the tool would be better as a web-based tool. This would be the best method of allowing users to access the tool when needed. A web-based tool would also make it easier to add to or modify the tool and supporting information if/when needed. One participant suggested that an automated report for council is produced using the web-based tool. This would be a simple inclusion to the web-based version of the tool.

The intention of this research was to build a tool as proof-of-concept, a customisable platform for a feasibility study, not a complete, working tool. If the tool is implemented, this should be done prior to implementation. Also, if it is implemented it would most likely be added to the Department of Building and Housing website (www.dbh.govt.nz) and it would need to conform to the style and formatting of their website, in the hands of a competent web-designer, this would be simple. Figure 49 is an example of a similar type of tool that has been included on the DBH website. 
Calculate the number of toilet pans, basins and urinals here.

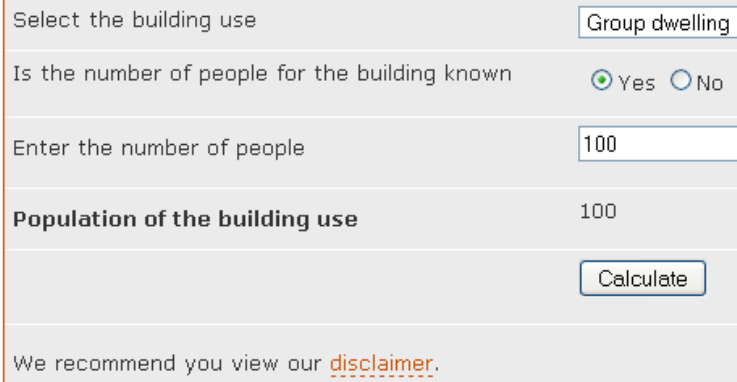

Figure 49 - “CompuPan” toilet calculator (Department of Building and Housing, n.d.2)

\subsection{Conclusions}

The tool was evaluated by 8 potential end-users of the tool, 4 architects/engineers and 4 representatives from regulatory bodies.

The critique found that the tool did take considerably less time than simulation, users generally found the tool easy to use and found the tool to be useful.

The participants found the tool to be about right in terms of complexity and the time taken to apply the tool, with the tool typically taking less than 20 minutes to assess a project. Most of the participants found the tool to be useful and the result to be effective and 7 out of 8 participants supported the implementation of the tool.

Some minor changes were suggested to the tool to make it easier to use, like including units. Some of these have been incorporated into the spreadsheet version of the tool enclosed in the accompanying CD. Further changes could be included if the tool became a web-based tool.

Some additional features were suggested that could be included with the tool to provide users with more information to assist the design process and make the use of the tool easier, like a table that indicates the effect changes will have on the results. Some suggestions were made that were not carried through at this stage of the research but would need to be done prior to the implementation of the tool, including a thorough list of instructions and assumptions and converting the tool into a web-based tool. 


\subsection{OVERALL CONCLUSIONS}

The intention of this research was to develop a simple tool that could become the Acceptable Solution for natural light in apartments, preventing more apartments that do not meet the minimum natural light requirement from being built. This report has shown the development of a tool that fulfils this intention.

The overall aim of this research was to produce a simple compliance assessment tool that could be included in the NZBC G7 Compliance Documents as an Acceptable Solution for apartment buildings. This tool was intended to provide a better Acceptable Solution for predicting natural light compliance in apartment buildings and to provide Territorial Authorities with adequate information to assess compliance accurately. Also, this research was to prepare the tool for implementation into everyday practice. Three criteria were used to ensure the tool fulfilled the aim, the tool was to be: simple and easy to use; sufficiently accurate and could be easily implemented.

Through this research a tool has been developed that fulfils the original aims. The tool developed can be included in the building code as an acceptable solution for assessing natural light in apartment buildings. The tool, if implemented carefully, could be designed to have a $97 \%$ confidence which would mean that the $50 \%$ of buildings that are approved when they do not provide adequate daylight would not be able to be built. And an evaluation of the tool by regulatory bodies found the tool to be very useful for assessing compliance of apartments.

The tool was developed by simulating factors of the external environment and building design (variables) that affect daylight performance at the back of the apartment. The simulation results were statistically analysed to establish relationships between the variables. The statistical analysis resulted in three equations that could be used to predict daylight performance at the back of the room. The equations were then formatted into an Excel spreadsheet to allow the users to determine if an apartment building would require simulation to prove compliance with NZBC G7, this was intended to a user-friendly interface for the equation developed in this research. The final interface of the tool is shown below in Figure 50. 


\begin{tabular}{|l|l|l|}
\hline \multicolumn{2}{|c|}{ NZBC G7 Compliance Assessment Tool } \\
\hline Variable: & & $\mathrm{m}$ \\
\hline Opposite Building Height & & $\mathrm{m}$ \\
\hline Street Width & & $\%$ \\
\hline Glazed Area & & \\
\hline Orientation & & \\
\hline Location & & $\%$ \\
\hline Reflectance of Opposite Building & $\%$ \\
\hline Glazing Transmittance & & \\
\hline Vertical Location of lowest apartment & \\
\hline \multicolumn{2}{|c|}{ YES } \\
\hline Are simulations required to prove compliance: & & \\
\hline
\end{tabular}

Figure 50 - Final Version of Compliance Assessment Tool

The tool was calibrated against real measurements taken in 97 apartments in Auckland, Wellington and Christchurch. The key finding was that around 1 apartment every 2 years would receive an incorrect result that meant simulations are required when it wasn't necessary to prove compliance and no buildings were give false pass results, based on assumed construction of 12 apartment buildings per year.

The tool was also evaluated by 8 potential end-users: 4 representatives from architecture/engineering firms and 4 representatives involved in the regulation process. The results from the evaluation confirmed that the tool was easy to use and had a minimal impact on users' time, with 7 out of the 8 participants supporting the implementation of the tool. A number of useful additions to the tool were also suggested to make it easier to use and provide additional information to help improve the design process.

In the development of the tool a number of assumptions were made that may result in errors up to 5-6\%. Assumptions that may have had a large effect on the overall result were internal reflectance and shading factors. The assumed values for internal reflectance were found to be valid with most of the apartments measured having the assumed surface reflectance values. Shading factors were assessed in the calibration apartments, half of the apartments had shading devices and it was found that this did not cause any incorrect assessments. A safety margin of $5 \%$ could be added to the tool to eliminate any errors. However the tool does provide sufficiently accurate assessments, so this is not necessary. 


\subsection{Fulfillment of Criteria}

Throughout this research three criteria have been used to ensure a tool was created that fulfilled the original aim and objectives of this research.

\subsubsection{Criterion 1: Simple and Easy to Use.}

The first criterion, that the tool was to be simple and easy to use, was fulfilled through the careful consideration of simplicity and usability throughout the development process, it was designed to have a minimal impact on users' time and was to be easy to follow and understand. The criterion was assessed through a questionnaire completed by potential end-users who tested the tool.

The tool was considered by these end-users to be simple and easy to use. Users typically took less than 20 minutes to apply the tool, which would result in the tool having little impact on users' time. Users who tested the tool also found the tool to be 'about right' in terms of difficulty, but as first time users found it a little difficult to follow at times. More thorough instructions are needed to cut the application time to less than 20 minutes for ALL users and to make it easier for first time users to understand.

Overall, this criterion was fulfilled, a simple and easy to use tool was developed, but some additional definitions and examples are needed if this tool is to be implemented.

\subsubsection{Criterion 2: Reasonably Accurate.}

The second criterion, that the tool was to be sufficiently accurate, was fulfilled through the use of a robust research method and previous research to influence the development of the tool. The criterion was assessed through the calibration of the tool against illuminance measurements from 97 apartments that represented the range of apartments typically found in New Zealand cities.

The tool was considered to be sufficiently accurate. The calibration tests found that if appropriate limits were placed on the tool, then the tool accurately assesses compliance in $97 \%$ of the apartments. The desired accuracy was $90 \%$ or greater was given in Chapter 4 , meaning that around 1 building every year would receive an incorrect outcome. The accuracy of the calibration tests found that the tool exceeded the desired $90 \%$ accuracy.

Criterion 2 was fulfilled, the tool has been proven to be sufficiently accurate for typical situations found in New Zealand cities. 


\subsubsection{Criterion 3: Can be easily Implemented.}

The third criterion, that the tool could be easily implemented, was more complex to assess than the previous two criteria. The two key factors used to ensure that the implementation process is as easy as possible were the fulfilment of the first two criteria and involvement of potential end-users in the development of the tool. This was assessed through the evaluation by potential end-users.

The first two criteria have been met, the tool was found to be simple and easy to use and reasonably accurate. The evaluation process allowed users to participate in the development of the tool prior to implementation which was intended to make the implementation process easier. Seven out of the 8 participants in the evaluation found the tool to be useful, effective and would support the implementation of the tool. However, one of these seven participants highlighted the need to have Department of Building and Housing support to get this tool implemented.

Criterion 3 was fulfilled, the tool is simple and easy to use, reasonably accurate, and potential end-users were involved in the development of the tool. Given that most of the participants in the evaluation would support the implementation of the tool, implementation should be reasonably smooth.

\subsection{What Next?}

Now that a tool has been developed that fulfils the original aims and intentions set out at the beginning of this research the next step is to have the tool implemented and used in everyday practice. Three steps need to be undertaken to ensure the successful implementation of the tool. These are: gaining support of Department of Building and Housing; conversion of the tool into a web-based tool; and a trial period to fine tune the tool and accompanying information.

The ideal outcome from this process would be that the DBH would agree to include this tool as part of the NZBC G7 Compliance Documents and it would be made available on their website. This is an essential step in getting the tool implemented into everyday practice.

Additional tests for vertical location should be conducted to allow the full range of options to be included in the tool. Heights above the $5^{\text {th }}$ floor will need to be simulated and assessed using SPSS to obtain a new equation for the tool that considers higher vertical locations.

With the support of the $\mathrm{DBH}$, the tool would then be developed into a web-based tool to make it more accessible. This would also allow additions to be made easily throughout the life of the tool. To accompany the tool, detailed definitions, assumptions and examples need to be included as links on the web-based tool. This process should not take more than a month with the assistance of an experienced web-designer. 
It is recommended that a trial period of 6 months is undertaken prior to the full implementation of the tool. The purpose of the trial period would be for users to get familiar with the tool and provide additional suggestions before it is to be used in everyday practice. Another advantage of this would be that complex examples could be added to the tool based on situations that users have experienced. This would result in examples being provided for many of the complex situations that were likely to come up in practice.

At the end of the trial period, revisions of the tool would be made, if needed, and the tool would become a part of everyday practice.

\subsection{Additional Findings}

During this research some additional findings were discovered, these were: that NZBC G7 is only loosely based on scientific standards; complex design features like atria, light-wells and internal rooms should always be simulated; and natural light and view preservation needs to be considered in New Zealand cities.

\subsubsection{Origin of NZBC G7 Requirement}

As was discussed in Chapter 3, the building code requirement for natural light is based on American and European recommendations and the personal experience of a consulting advisor. No detailed research was available at the time the code was established that identified the threshold value for health and safety. The building code requirement was developed using the best information available at the time, but no further advancements have been made in this area, so if research is conducted in the future that establishes a different threshold value, revision of this requirement may be needed. If the 30 lux threshold was revised, then the calculations for the design tool would need to be repeated. It is anticipated that this would be relatively simple.

\subsubsection{Complex Design Features}

Within the calibration process detailed in Chapter 6, apartments that relied on light provided by an atrium or light-well were assessed for daylight illumination, as well as internal rooms that relied on 'borrowed light'. These apartments were shown to perform poorly. Four rooms were measured off an atrium and none of these rooms received adequate natural light at the back of the room. Eleven rooms were measured that used a light-well to receive natural light; 8 of the 11 failed to meet the minimum requirement at the back of the room. Six internal habitable rooms were measured and none of these received adequate natural light at any point throughout the room. Because of the complex nature of these design features it has been concluded that ALL atria, light-wells and internal habitable rooms should be simulated to prove they comply with the minimum building code requirement. 


\subsubsection{Preservation of Natural Light/View}

While measuring apartments, a number of residents voiced their concerns about changes to surrounding sites that may affect the natural light and views in their apartments as was outlined in Chapter 6. This is a complex issue that has received a great deal of debate internationally. This is an issue that needs to be investigated. The suggested remedies are the implementation of a 'right to light' law similar to the UK (British Standards Institution, 1992) or to require that changes to surrounding buildings are considered in the design of new apartment buildings.

\subsection{Further Research and Development}

Two research and development questions have arisen as a direct result of this research. The first was the gap in understanding internationally of the minimum natural light levels required in housing for health and well-being. The second was that a database of New Zealand building material reflectance values is needed.

\subsubsection{Minimum Requirements for Health and Well-being}

It was discovered through the evaluation of the current regulation and international standards/regulations that there is a gap in the literature regarding the levels of natural light required for health and well-being. It is known that as humans we need exposure to natural light to prevent high levels of melatonin and receive adequate ultraviolet radiation in particular. But there is no consensus on exactly how much is needed. This was reflected in the wide variation in international regulations and standards. This finding was supported by other researchers, with Boubekri (2004, pg 57) stating that 'daylight legislation is still lagging behind.'

\subsubsection{Building Exterior Reflectance Values Database}

While developing the calculator of reflectances of buildings opposite, it became apparent that a database was needed of the typical reflectance of building materials found in New Zealand. This would make the calculation of the reflectance of the opposite building for the tool developed in this research much easier, but would also add to the accuracy of simulations. The Australian and New Zealand Standard for Interior Lighting (Standards Australia/Standards New Zealand, 2006) provides a table of approximate reflectance for typical building finishes, but mainly provides material used on the interior and only provides a small range of the materials and reflectances found in New Zealand cities. As the reflectance of surrounding building significantly affects the light available on lower floors in cities this would be a useful tool to the New Zealand building industry. 
This research has proven that a tool can be developed to accurately assess compliance with NZBC G7. This tool is simple and easy to use and does not have a significant impact on users' time. This tool can become the NZBC G7 Acceptable Solution for apartment buildings and will ensure that Territorial Authorities are provided with sufficient information to determine whether an apartment building meets the minimum code requirement. This tool by no means ensures good quality daylight design, but it does mean that all apartment dwellers will have access to adequate natural light for health and well-being in their homes. 


\section{REFERENCES}

Allen, W. (1943). Daylighting of Buildings in Urban Districts. Journal of the Royal Institute of British Architects, February 1943, 85-87.

Archives New Zealand. (2006). Appraisal Report for Department of Building and Housing. Last accessed $7^{\text {th }}$ June 2008, from: www.archives.govt.nz/docs/pdfs/appraisalreports/AppraisalReport70.pdf

Archives New Zealand. (2007). Building Industry Authority 1992-2004: Administrative History. Last accessed $7^{\text {th }}$ June 2008, from: archway.archives.govt.nz/ViewFullAgencyHistory.do

Auckland City Council. (2007a). District Plan - Central area section. Last accessed $26^{\text {th }}$ June 2008: http://www.aucklandcity.govt.nz/council/documents/central/default.asp

Auckland City Council. (2007b). Appendix 12 - Minimum Residential Apartment Standards. [PDF] Last accessed 26 June 2008: http://www.aucklandcity.govt.nz/council/documents/central/pdfs/appendix12.pdf

Auckland Uniservies Ltd. (2004, April). Living the Highlife? A review of apartment living in inner city Auckland. Report prepared for Building Industry Authority.

Australian Uniform Building Regulations Co-ordinating Council. (1990). Building Code of Australia: 1990. Canberra: Australian Uniform Building Regulations Co-ordinating Council

Bayleys Research. (2006). Wellington CBD Apartments Report 2006 [PDF]. Last accessed June $26^{\text {th }} 2008$, from: http://www.bayleys.co.nz/Research/Residential/WELLINGTON_CBD_APARTMENTS.htm

Bayleys Research. (2007). Wellington Residential Report October 2007 [PDF]. Last accessed June $7^{\text {th }} 2008$, from: www.bayleys.co.nz/Research/Residential/ WELLINGTON_RESIDENTIAL_REPORT_OCTOBER_2007.htm

Bayleys Research. (2008). Auckland CBD Apartments Report 2008 [PDF]. Last accessed June $26^{\text {th }} 2008$, from: http://www.bayleys.co.nz/Research/Residential/AUCKLAND_CBD_APARTMENTS_2008.htm

Boubekri, M. (2004). A Overview of The Current State of Daylight Legislation. Journal of the Human-Environmental System, 7 (2), 57-63.

Boyce, P., Hunter, C. and Howlett, O. (2003). The Benefits of Daylight through Windows [Electronic Version]. Last accessed $26^{\text {th }}$ June 2008:

http://www.Irc.rpi.edu/programs/daylighting/pdf/DaylightBenefits.pdf 
British Standards Institution. (1992). Lighting for buildings - Part 2: Code of practice for daylighting (BS 8206:Part 2:1992). London: British Standards Institution.

Building Research Energy Conservation Support Unit. (1998). Good Practice Guide 245: Desktop guide to daylighting - for architects [Electronic Version]. Last accessed $26^{\text {th }}$ June 2008: http://www.cibse.org/pdfs/GPG245.pdf

Chartered Institute of Building Service Engineers. (1999). Daylight and window design Lighting Guide (LG10:1999). London: Chartered Institute of Building Service Engineers.

Chynoweth, P. (2005). Progressing the right to light debate - Part 2: the grumble point revisited. Structural Survey, 23 (4), 251-264.

Clinton Bird Urban Design Limited. (2005). Minimum standards for residential apartments in central Auckland. Auckland: Author.

Cohen, J., Cohen, P., West, S.G. and Aiken, L.S. (2003). Applied Multiple Regression/Correlation Analysis for the Behavioural Sciences ( $3^{\text {rd }}$ edition). New Jersey: Lawrence Erlbaum Associates

Cuttle, C. (2000, March). NZBC Clause G7: NATURAL LIGHT. Report prepared for Building Industry Authority.

Davies, K. (1992). Wind tunnel modelling of pedestrian wind environment: modelling the built environment. Report Submitted [to the Victoria University of Wellington] in fulfilment of an MBSc.

Department of Building and Housing. (2001). Compliance Document for New Zealand Building Code - Clause G7 - Natural Light [PDF]. Last accessed June $7^{\text {th }} 2008$, from: www.dbh.govt.nz/UserFiles/File/Publications/Building/Compliance-documents/clauseG7.pdf

Department of Building and Housing (n.d.1). About the Building Code. Last accessed June $20^{\text {th }} 2008$, from: http://www.dbh.govt.nz/bcr-about-the-building-code

Department of Building and Housing (n.d.2). Calculator for toilet pans, basins and urinals. Last accessed $29^{\text {th }}$ September 2008, from: http://www.dbh.govt.nz/calculator-for-toilet-pans

Department of Employment and Industrial Relations - Working Environment Branch (Australia). (1983). Daylight at Work. Canberra: Australian Government Publishing Service.

DIN Deutsches Institut für Normung (German Standards Institute). (1999). Daylight in Interiors - Part 1: General Requirements (DIN 5034-1) [English Version]. Berlin: DIN Deutsches Institut für Normung. 
DIN Deutsches Institut für Normung (German Standards Institute). (1994). Daylight in Interiors - Part 3: Methods of Calculation (DIN 5034-3) [English Version]. Berlin: DIN Deutsches Institut für Normung.

DIN Deutsches Institut für Normung (German Standards Institute). (1994). Daylight in Interiors -Part 4: Simplified method for determining window sizes in dwellings (DIN 5034-4) [English Version]. Berlin: DIN Deutsches Institut für Normung.

Evans, B.H. (1981). Daylight in Architecture. New York: McGraw-Hill.

Evans, G.W. (2003). The Built Environment and Mental Health. Journal of Urban Health: Bulletin of the New York Academy of Medicine, 80 (4), 536-555.

Farley, K.M.J. and Veitch, J.A. (2001). A Room with a View: A review of the effects of windows on work and well-being [Electronic Version]. Last accessed $26^{\text {th }}$ June 2008 : http://irc.nrc-cnrc.gc.ca/pubs/fulltext/rr/rr136/rr136.pdf

Fontoynont, M. (Ed.). (1999). Daylight Performance of Buildings. London: James \& James.

International Commission on Illumination (CIE) E-3.2. (1970). Daylight: International recommendations for the calculation of natural daylight. Paris: Bureau Central.

Isaacs, N. (Ed). (2006). Energy Use in New Zealand Households: Report on the Year 10 Analysis for the Household Energy End-use Project [HEEP] Executive Summary [Electronic Version]. Last accessed $9^{\text {th }}$ October 2008: http://www.branz.co.nzcms_show_download.php?id=97.

Lawrence Berkeley National Laboratory. RADIANCE. Last accessed $5^{\text {th }}$ October 2008: http://radsite.lbl.gov/radiance/

Li, D.H.W., Lo, S.M., Lam, J.C. and Yuen, R.K.K. (1999). Daylighting performance in residential buildings. Architectural Science Review, 42, 213-219.

Li, D.H.W., Wong, S.L., Tsang, C.L. and Cheung, G.H.W. (2006). A study of the daylighting performance and energy use in heavily obstructed residential buildings via computer simulation techniques. Energy and Building, 38, 1343-1348.

Littlefair, P. (2001). Daylight, sunlight and solar gain in the urban environment. Solar Energy, 70 (3), 177-185.

Lynes, J.A. (1968). Principles of Natural Light. London: Applied Science Publishers.

Metropolitan Glass and Glazing Ltd. (2006). Catalogue and Reference Guide (6 $6^{\text {th }}$ edition) [Electronic Reference]. Last accessed 19 July 2008:

http://www.glasstech.co.nz/008_glass.aspx

Morrison, P.S. and McMurray, S. (1999). The Inner-city Apartment versus the Suburb: Housing Sub-markets in a New Zealand City. Urban Studies, 36 (2), 377-397. 
Nabil, A. and Mardaljevic, J. (2006). Useful daylight illuminances: A replacement for daylight factors. Energy and Buildings, 38, 905-913.

National Research Council Canada: Institute for Research in Construction. (2006). DAYSIM. Last accessed 19 July 2008: http://irc.nrc-cnrc.gc.ca/ie/lighting/daylight/daysim_e.html

New South Wales Department of Planning. (2002). Residential Flat Design Code - Part 3 Building Design [Electronic Version]. Last accessed 19 July 2008: http://www.planning.nsw.gov.au/programservices/pdf/designcode/03_part03_a.pdf

New Zealand Institute of Architects. (2005). Last accessed 14 August 2008: http://www.architecturenz.net/default.aspx

Ng, E. (2001a). A simplified daylighting design tool for high-density urban residential buildings. Lighting Research and Technology, 33 (4), 259-272.

$\mathrm{Ng}$, E. (2001b). A study on the accuracy of daylighting simulation of heavily obstructed buildings in Hong Kong. In Building Simulation 2001, Proceedings of the $7^{\text {th }}$ International Building Performance Simulation Association Conference (pp. 12151222). Last accessed $26^{\text {th }}$ June 2008: http://www.ibpsa.org/proceedings/BS2001/BS01_1215_1222.pdf

Ng, E. (2003a). Regulate for Light, Air and Healthy Living - Part I: Regulating the provision of natural light and ventilation of buildings in the UK. Hong Kong Institute of Architects Journal, 36 (3), 27-34.

$\mathrm{Ng}$, E. (2003b). Studies on daylight design and regulation of high-density residential housing in Hong Kong. Lighting Research and Technology, 35 (2), 127-139

Ng, E. (2004). Regulate for Light, Air and Healthy Living - Part II: Regulating the provision of natural light and ventilation of buildings in Hong Kong. Hong Kong Institute of Architects Journal, 37 (1), 14-27.

$\mathrm{Ng}$, E. (2005). Regulate for Light, Air and Healthy Living - Part III: The becoming of PNAP 278. Hong Kong Institute of Architects Journal, 44 (4), 14-25.

Nielson, J. (2000). Usable Information Technology: Why you only need to test with 5 users. Last accessed $13^{\text {th }}$ October 2008: http://www. useit.com/alertbox/20000319.html

North Shore City Council. (2002a). Good Solutions Guide for Apartments - Introduction [Electronic Version]. Last accessed $26^{\text {th }}$ June 2008:

http://www.northshorecity.govt.nz/PDFs/Urban-design/Apartment-guides/Introduction.pdf

North Shore City Council. (2002b). Good Solutions Guide for Apartments - Section C: The Apartment [Electronic Version]. Last accessed 19 July 2008: http://www.northshorecity.govt.nz/PDFs/Urban-design/Apartment-guides/Part-twosection-c.pdf

Paix, D. (1982). The design of buildings for daylighting ( $2^{\text {nd }}$ ed.). Canberra: Australian Government Publishing Service. 
Raw, G.J., Aizlewood, C.E. and Hamilton, R.M. (2001). Building regulation, health and safety: A report concerning the influence on the health and safety of building users of fabric and services controlled or controllable by regulation of buildings. London: Building Research Establishment.

Rea, M.S. (Ed.). (2000). The IESNA lighting handbook: Reference \& application ( $9^{\text {th }}$ ed.). New York: Illuminating Engineering Society of North America.

Reinhart, C. F. (2006). Tutorial on the use of Daysim Simulations for Sustainable Design [Electronic Reference]. Last accessed $5^{\text {th }}$ October 2008: http://irc.nrc-cnrc.gc.ca/ie/lighting/daylight/daysim/docs/DaysimTutorial.pdf

Reinhart, C.F. and Herkel, S. (2000). The simulation of annual daylight illuminance distributions - a state-of-the-art comparison of six RADIANCE-based methods. Energy and Buildings, 32, 167-187.

Reinhart, C.F. and Walkenhorst, O. (2001). Validation of dynamic RADIANCE-based daylight simulations for a test office with external blinds. Energy and Buildings, 33, 683-697.

Reinhart, C.F., Mardaljevic, J. and Rogers, Z. (2006, July). Dynamic Daylight Performance Metrics for Sustainable Building Design. LEUKOS, 3 (1), 7-31.

Roscoe, J. T. (1975). Fundamental Research Statistics for the Behavioural Sciences $\left(2^{\text {nd }}\right.$ edition). New York: Holt, Rinehart and Winston, Inc.

Ruck, N. (Ed.). (2000). Daylight in Buildings: A source book on daylighting systems and components. Berkeley, California: Lawrence Berkeley National Laboratory.

Sherwood, R. (1978). Modern Housing Prototypes. Cambridge: Harvard University Press.

SPSS Inc. (2007). SPSS Statistics. Last accessed 19 July 2008: http://www.spss.com/statistics/

Standards Association of New Zealand. (1984). Code of practice for interior lighting design (NZS 6703:1984). Wellington: Standards Association of New Zealand.

Standards Australia/Standards New Zealand. (2006). Interior and workplace lighting - Part 1: General principles and recommendations (AS/NZS 1680.1:2006). Wellington: Standards New Zealand.

Statistics New Zealand. (1991-2008). Building consent data. Last accessed 21 May 2008: from INFOS database.

Statistics New Zealand. (2008, February). Building Consents Issued: January 2008 [PDF]. Last accessed June $7^{\text {th }} 2008$, from: www.stats.govt.nz/products-and-services/info-releases/building-consents-issued.htm

Stewart, K. (2006a). Daylight Simulations for Code Compliance: Creating a Decision Tool. Report Submitted [to the Victoria University of Wellington] in partial fulfilment of BBSc 401 - Research Methods. 
Stewart, K. (2006b). Daylight in Wellington Apartments. Report Submitted [to the Victoria University of Wellington] in partial fulfilment of BBSc 431 - Lighting in Buildings.

Stewart, K. and Donn, M. (2007). Daylight simulation for code compliance: creating a decision tool. In Building Simulation 2007, Proceedings of the $10^{\text {th }}$ International Building Performance Simulation Association Conference (pp. 1189-1196). Last accessed $26^{\text {th }}$ June 2008: www.ibspa.org/proceedings/BS2007/p746_final.pdf

Ullah, M.B. and Lin, L.W. (2003). Daylight distribution in the living rooms of four types of public housing building in Singapore. Lighting Research and Technology, 35 (2), 91100.

Unknown. (2006, December). Appraisal Report for Department of Building and Housing [PDF]. Last accessed $7^{\text {th }}$ June 2008, from: www.archives.govt.nz/docs/pdfs/appraisalreports/AppraisalReport70.pdf

U.S. Department of Energy, Energy Efficiency and Renewable Energy. (2007). Building Energy Software Tools Directory. Last accessed $5^{\text {th }}$ October 2008: http://apps1.eere.energy.gov/buildings/tool_directory/

Waitakere City Council. (2005). Proposed District Plan Change 18 - City Wide Rule 1 General Apartment Design. [PDF] Last accessed 19 July 2008:

http://www.waitakere.govt.nz/Abtcnl/pp/districtplan/dplanchanges/gaaa/citywiderule1ge naptmntdsgn.pdf

Walkenhorst, J.L., Luther, J., Reinhart, C. and Timmer, J. (2002). Dynamic annual daylight simulations based on one-hour and on-minute means of irradiance data. Solar Energy, 72 (5), 385-395.

Walsh, J.W.T. (1961). The science of daylight. London: Macdonald \& co.

Wellington City Council. (2000). Wellington City district plan: Te kaupapa whenua o Poneke. Wellington: Wellington City Council.

Whyte, D. (1996). Inner city apartments in Wellington post 1990. Wellington: Report submitted [to Victoria University of Wellington] in partial fulfilment of the requirement of a Bachelor of Architecture degree.

Wilson, M.P. and Brotas, L. (2001, June). Daylight and Domestic Buildings. In Proceedings of XIth National Conference on Lighting (Bulgaria), Varna, Bulgaria (pp. 27-32). [PDF] Last accessed $26^{\text {th }}$ June 2008:

http://www.learn.londonmet.ac.uk/about/doc/brotas_bulgaria2001.pdf

World Health Organization. (2008) Frequently asked questions. Last accessed 17 June 2008: http://www.who.int/suggestions/faq/en/index.html 


\section{GLOSSARY OF VARIABLES}

Building Form (Building Variable) refers to the overall shape of the building in plan, for example the cruciform plans often used in Hong Kong residential estates, or the use of an atrium or light-well for daylight.

Ceiling Height (Apartment Variable) refers to the distance from the floor to the ceiling. This factor effects reflected daylight at the rear of spaces. Ceiling height also influences the placement and area of windows.

Depth (Apartment Variable) is the from the exterior wall to the back wall of the space. Apartment depth affects the distribution of daylight in the space, the deeper the apartment, the less daylight is available at the back of the room.

Floor Area (Apartment Variable) is a factor of the depth and width of the apartments. It was suggested by Li et al. (1999) that the larger the surface area (floor area), the lower the average daylight factor.

Glazed Area (Apartment Variable), there are two different methods for specifying this in apartments: the window-to-wall ratio; and as a percentage of the floor area. The percent of the floor area is the more widely used method.

Glazing Type (Apartment Variable), also referred to as transmittance, the glazing type refers to the amount of light transmitted through the glass, as a fraction or percentage of the light available.

Ground Reflectance (External Variable) is included in Average Daylight Factor calculations as part of the Externally Reflected Component (ERC). This is the amount of light that hit the ground that is reflected as a percentage or fraction.

Height of the proposed building (Building Variable), this is the mean height above sea level of the proposed building. Independently, this factor means little to the daylight performance, but in urban environments, where the primary source of light is reflected light from building facades, this factor becomes relevant.

Latitude (External Variable) is a factor in assessing daylight availability. The IEA sourcebook (Ruck, 2000) highlights that high latitudes have distinct seasonal variations, i.e. low daylight levels in winter because of sun angles which also causes less daylight hours. This is highly relative to New Zealand as the latitudes range from 35.07South (Kaitaia) to 46.37South (Bluff) and are therefore considered 'high latitudes'.

Obstruction Angle (External Variable) is a factor of Opposite Building Height and Street Width. This factor represents the proportion of the sky that is obstructed and consequently unobstructed. This is one of the most important factors of daylight performance in urban environments identified in the literature. 
Opposite Building Height (External Variable) is the mean height about sea level of the opposite building(s).

Orientation (Building Variable) refers to the direction of the façade (eg, North, East, South and West) and effects the direct light available for a space. This factor, in combination with the surface reflectance of the buildings, plays a major role in daylight reaching the lower floors in urban environments.

Shadings (Building Variable) include overhangs, side-fins and balconies, which block direct sun from penetrating the space but still allow diffuse daylight.

Street Width (External Variable) is the distance from the façade of the proposed building to the façade of the building directly opposite.

Surface Reflectance (Apartment Variable), this is the percent of light reflected off each of the interior surfaces, (walls, floor and ceiling) affecting the distribution of light in the space. These values are often assumed in the design stages.

Surface Reflectance of Opposite Building(s) (External Variable) refers to the amount of light reflected off the obstructing buildings. The surface reflectance of the obstruction is especially important in urban environments as it becomes the main light source for the lower floors. The reflectance is a combination of the colour and construction material of the opposite building(s). Reflectance is given as a percentage (or factor) of the light that hit the surface that is reflected, in urban environments this typically ranges between $20 \%$ (0.2) for a dark building and $50 \%$ (0.5) for a white building.

Vertical Location (Building Variable) of the apartment refers to the floor level that the apartment is located on within the building (eg. Ground, $1^{\text {st }}$ Floor, $2^{\text {nd }}$ Floor, etc.), the apartments on the lower floors perform worse than higher floors, as a result of the lower floors relying on light reflected off surrounding buildings.

Width (Apartment Variable), the distance from one wall to the opposite wall, across the space. As with apartment depth, apartment width affects the distribution of daylight in the space.

Window Location (Apartment Variable), this is the position of the window on the exterior wall. Windows located near the top of the exterior wall will allow daylight to penetrate deeper into the space. 


\section{Appendices}

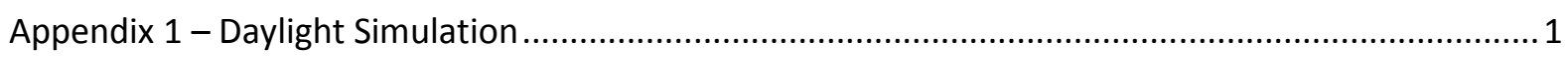

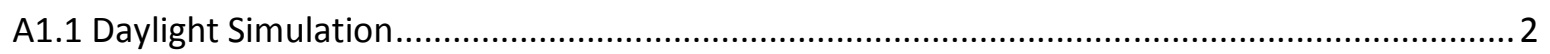

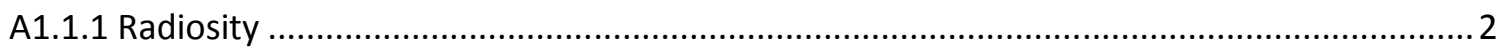

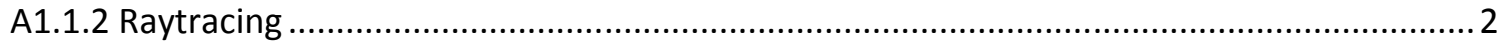

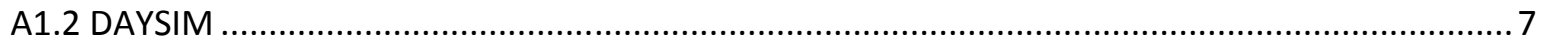

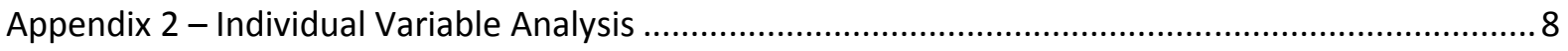

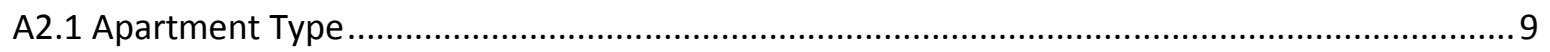

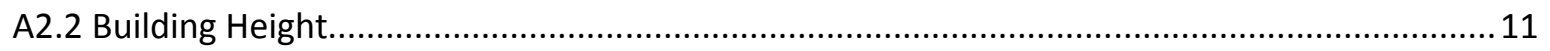

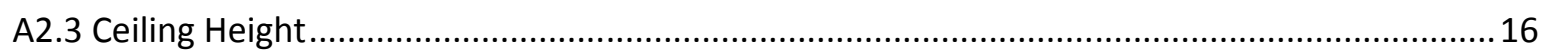

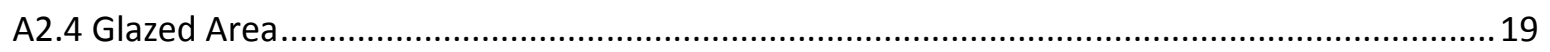

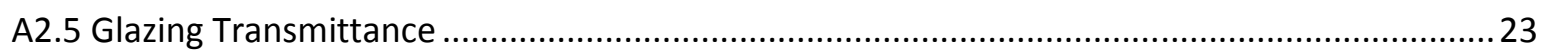

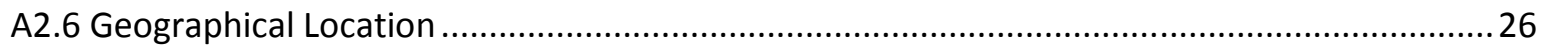

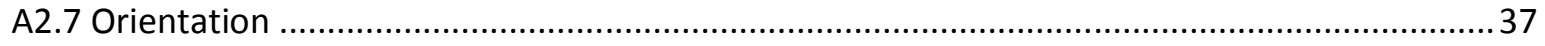

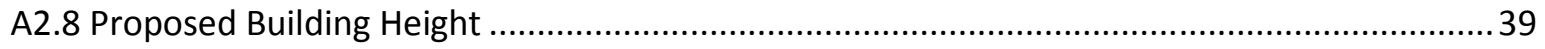

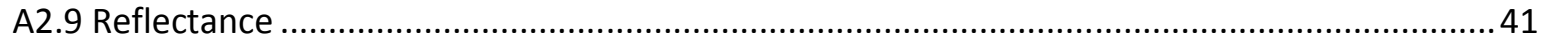

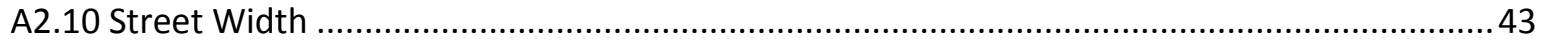

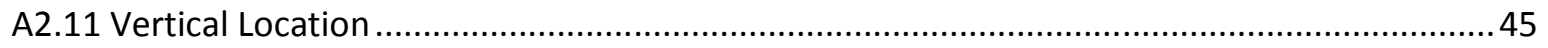

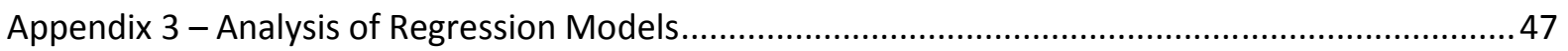

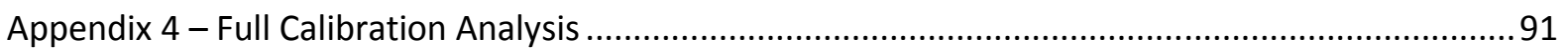

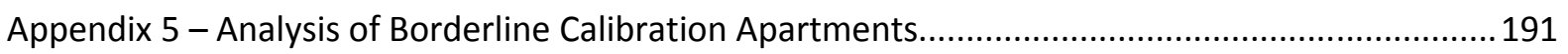

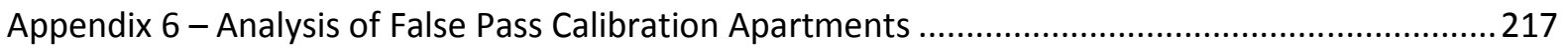

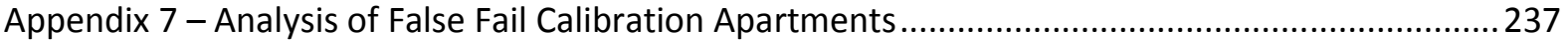

Appendix 8 - Analysis of Overall Calibration Results for Models ......................................................259

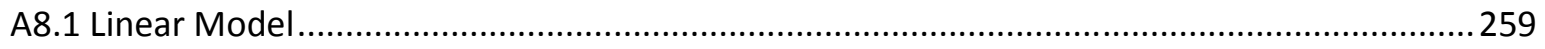

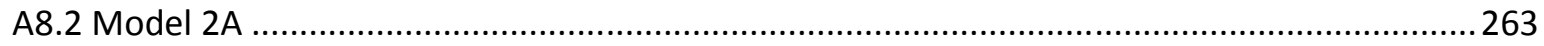

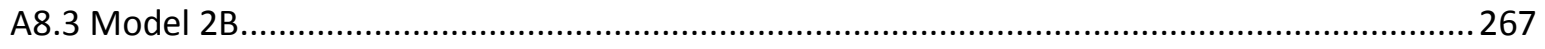

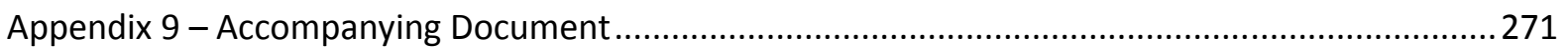

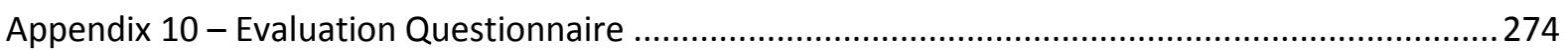




\section{Appendix 1 - Daylight Simulation}

Appendix 1 summaries a study undertaken to determine which simulation type and program would be most suitable for this research. Within this study the two types of simulation were explored, Radiosity and Raytracing. A list of Daylight Simulation programs is given, with the necessary information to select an appropriate program. A more detailed description of the selected simulation program DAYSIM is also given.

The information in this appendix will be useful background information for the tool. With the tool specifying when simulation is needed, it is important to equip people with the information to select the right simulation program. 


\section{A1.1 Daylight Simulation}

Computational daylight simulation is a tool that is replacing many of the traditional complex methods of predicating daylight performance. Architectural practitioners are relying more on computers throughout the design process, which many daylight simulation tools can easily tap into (Ruck, 2000). Daylight simulation allows complex calculation algorithms and lighting simulation techniques to be undertaken without the need for specialist knowledge and extensive training (Ruck, 2000), however this is always an advantage.

The two primary calculation methods used in lighting simulation are: Radiosity and Raytracing. These methods will be described in the following sections, providing advantages and disadvantages of using each method. These factors played a major role in the selection of the calculation method to be used in this research.

\section{A1.1.1 Radiosity}

In a radiosity based simulation, the surfaces in a room are divided into light reflecting elements, where the number of elements affects the number of calculations which affect the overall simulation time (CIBSE, 1999). The calculation involves the reflections between elements to determine the illuminance and luminance values (Ruck, 2000).

The main advantage of the radiosity method over other methods is that simulations do not required fixed viewpoints, allowing users to perform one simulation for the space and select different views without having to perform additional calculations (Ruck, 2000).

The major disadvantage to the radiosity method is that all surfaces are assumed to be perfect diffusers (Ruck, 2000), which in reality they are not. Radiosity calculations also cannot support complex models due to the amount of memory required (CIBSE, 1999).

\section{A1.1.2 Raytracing}

The raytracing technique calculates the illuminance or luminance levels by tracing the path of the light rays from a set viewpoint. There are two types of raytracing, these are backward and forwards raytracing. Backwards raytracing, the most common, projects rays from the centre of the view plane (or measurement point) back to the light source (Ruck, 2000). The less common raytracing technique is forward raytracing, this is fundamentally the same as backwards raytracing, but traces the light rays from the light source to the centre of the view plane (Ruck, 2000).

The main advantage of raytracing methods is the tested reliability and accuracy for a variety of different lighting systems (Ruck, 2000; CIBSE, 1999). Raytracing is also able to simulate specular and partly specular materials and can cope with complex geometry (Ruck, 2000). 
The major disadvantage of raytracing methods is that simulations and modelling is extremely complicated and time-consuming (Ruck, 2000). However, technological developments in computer hardware and simulation programs are reducing the time and difficulty involved in these simulations.

The calculation method selected for this research was raytracing. From the literature it was found that generally raytracers give more reliable results for complex daylight applications. Due to the nature of this research, it is crucial that accurate results are obtained from the simulations. From Table 2 it can be seen that there are a number of programs available that use raytracing. The most commonly used raytracer is RADIANCE (Lawrence Berkeley National Laboratory), as was identified by Reinhart.

In the selection of the simulation tool for this research, an important consideration was the ability to calculate annual daylight performance and the ability to consider both direct and diffuse daylight. Reinhart and Herkel (2000) identified that in order to analyse annual building performance, it is essential that all sky conditions at the site over the course of the year are considered.

The following table shows six methods available for calculating annual daylight performance using RADIANCE-based calculation methods. The table summarises the findings of Reinhart and Herkel (2000) comparing the six RADIANCE-based methods. The information from Table 2 and Table 1 were used to support the continued use of DAYSIM as the daylight simulation tool, as was used in the pilot study.

Table 1 - RADIANCE-based tools (Reinhart and Herkel, 2000)

\begin{tabular}{|c|c|}
\hline Tool & Advantages/Disadvantages \\
\hline $\begin{array}{l}\text { Complete year-runs } \\
\text { with RADIANCE }\end{array}$ & $\begin{array}{l}\text { - Best for academic purposes due to lengthy simulation times of } \\
\text { between } 12 \text { and } 80 \text { days. } \\
\text { Not suitable for short time-steps of weather data (1 hour intervals } \\
\text { minimum). }\end{array}$ \\
\hline Daylight Factor & $\begin{array}{l}\text { Provides satisfactory results for diffuse daylight and simple building } \\
\text { geometries. } \\
\text { Reliable external illuminances are essential to obtain acceptable } \\
\text { results. } \\
\text { - The use of the CIE overcast sky limits accuracy, as it tends to } \\
\text { underestimate real horizontal illuminances. }\end{array}$ \\
\hline ADELINE & $\begin{array}{l}\text { - Underestimation of external illuminances is again an issue with } \\
\text { ADELINE due to the use of the CIE overcast sky. } \\
\text { - Simulation times are high compared to some of the other methods, } \\
\text { taking around } 25 \text { hours to complete. }\end{array}$ \\
\hline $\begin{array}{l}\text { Classified weather } \\
\text { data (Herkel and } \\
\text { Pasquay) }\end{array}$ & $\begin{array}{l}\text { - Some accuracy issues with illuminances greater than } 1000 \mathrm{~lx} . \\
\text { - } \quad \text { Simulation times are also high, taking around } 20 \text { hours to complete. }\end{array}$ \\
\hline Daylight coefficients & $\begin{array}{l}\text { Daylight coefficient methods (ESP-r and DAYSIM) are the most efficient and } \\
\text { accurate for predicting hourly mean indoor illuminances and annual } \\
\text { cumulative indoor illuminance distribution. }\end{array}$ \\
\hline ESP-r & $\begin{array}{l}\text { - ESP-r needs to consider ground reflectances as they significantly } \\
\text { contribute to more advanced building geometry. }\end{array}$ \\
\hline DAYSIM & $\begin{array}{l}\text { - Very efficient method of calculating diffuse daylight, taking around } 8 \\
\text { mins to complete. } \\
\text { - Accurate to within } 3 \% \text { of the reference case. }\end{array}$ \\
\hline
\end{tabular}


Table 2 - Lighting Simulation Programs Available (U.S. Department of Energy)

\begin{tabular}{|c|c|c|c|c|c|c|}
\hline 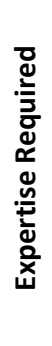 & 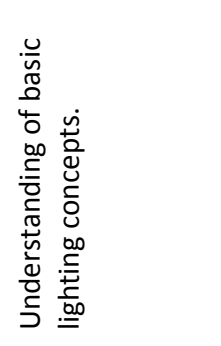 & 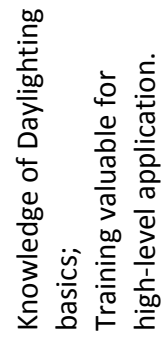 & 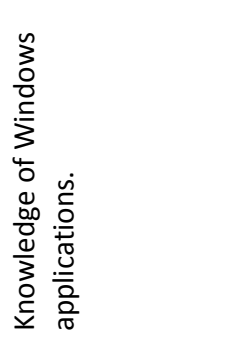 & $\begin{array}{l}\text { İ } \\
\text { ò }\end{array}$ & 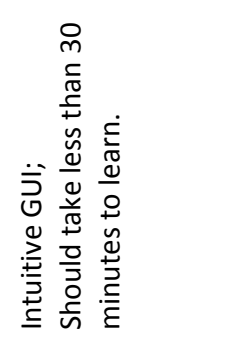 & 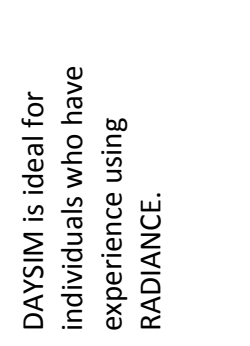 \\
\hline 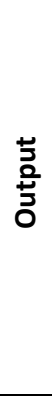 & 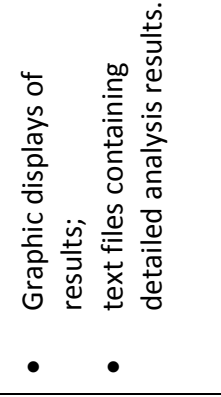 & 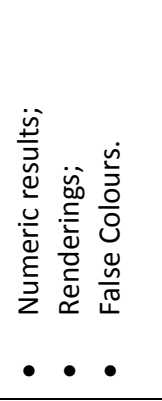 & 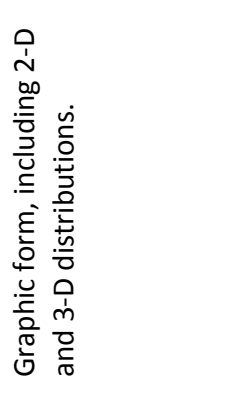 & 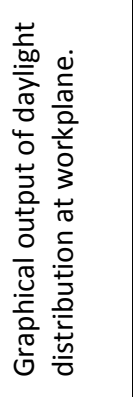 & 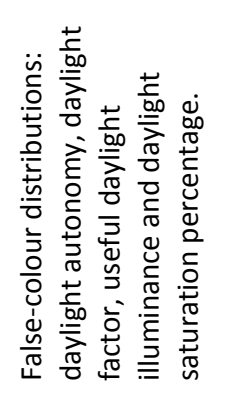 & 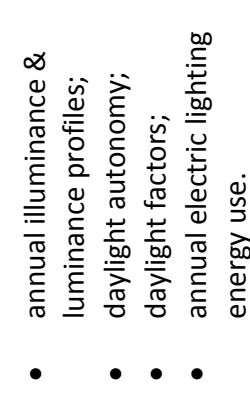 \\
\hline 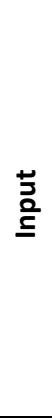 & 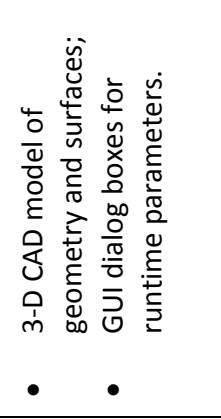 & 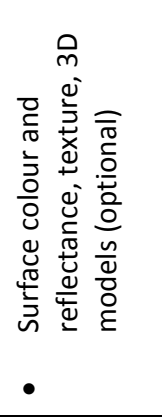 & 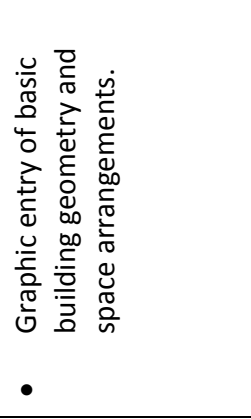 & 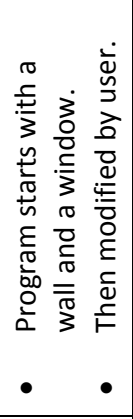 & 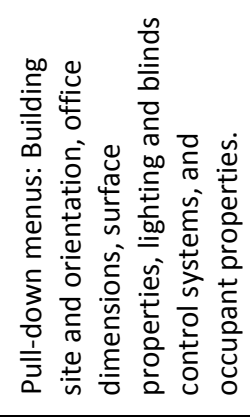 & 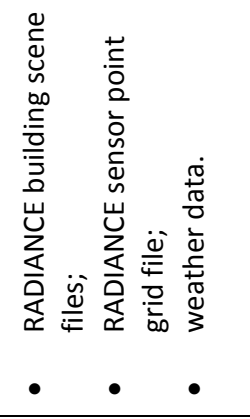 \\
\hline & 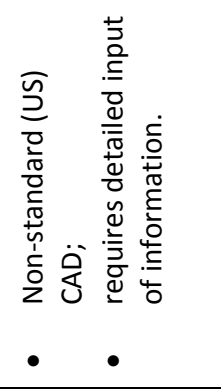 & ' & 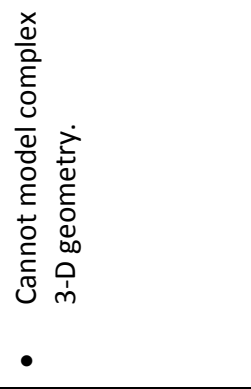 & 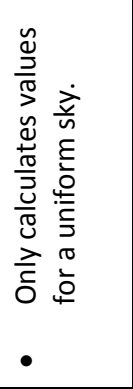 & 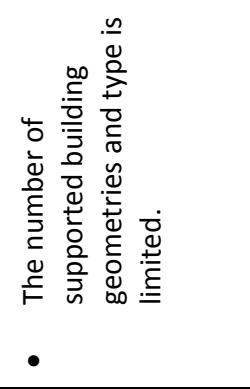 & 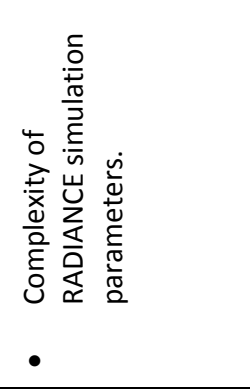 \\
\hline & 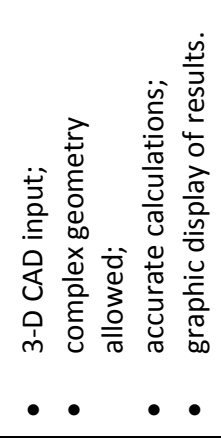 & 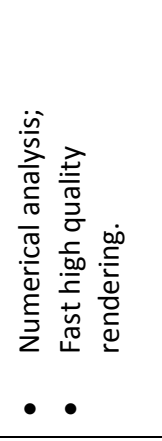 & 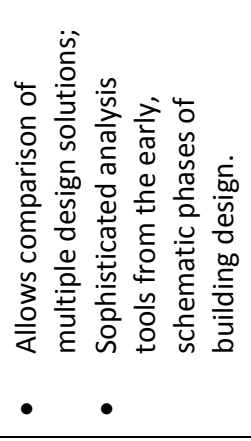 & 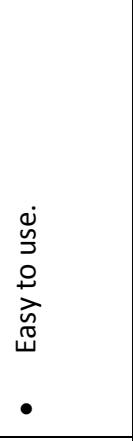 & 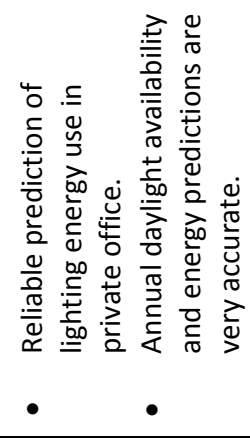 & 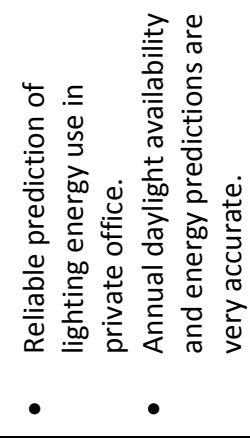 \\
\hline & 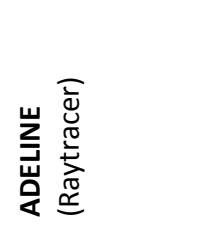 & 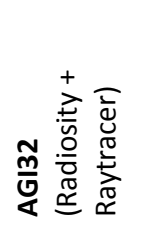 & 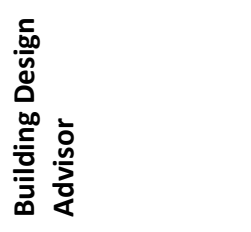 & 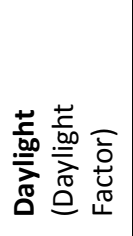 & 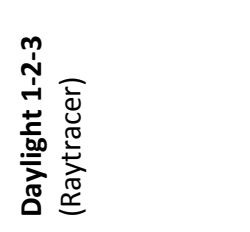 & 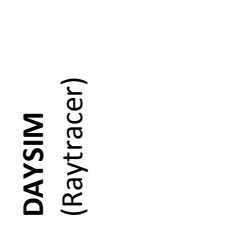 \\
\hline
\end{tabular}




\begin{tabular}{|c|c|c|c|c|c|}
\hline 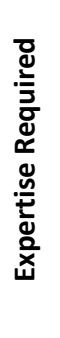 & 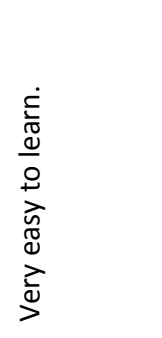 & 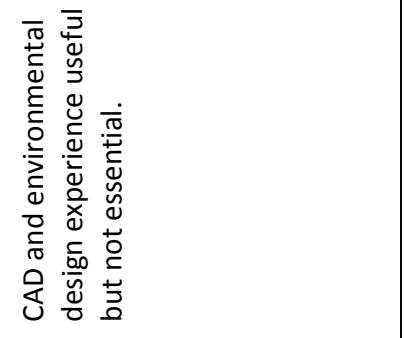 & 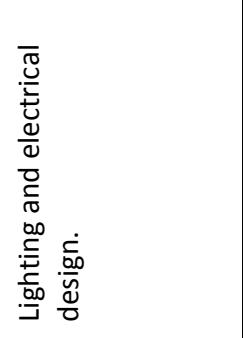 & 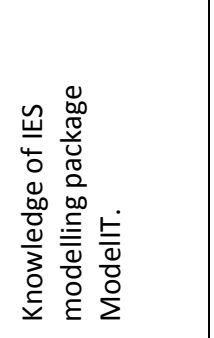 & 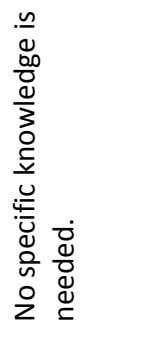 \\
\hline 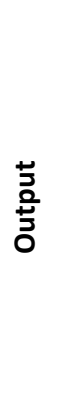 & 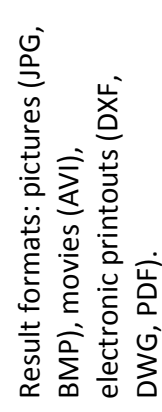 & 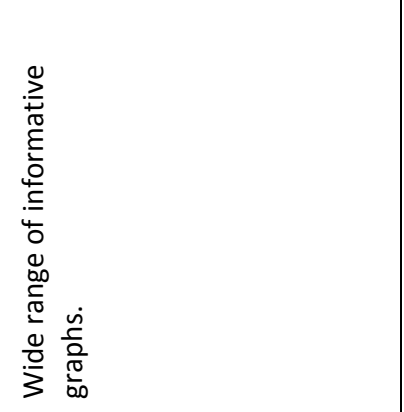 & 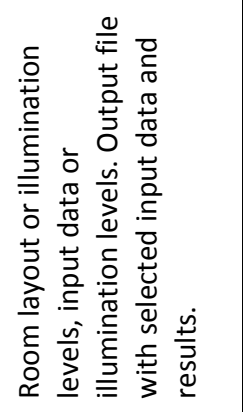 & 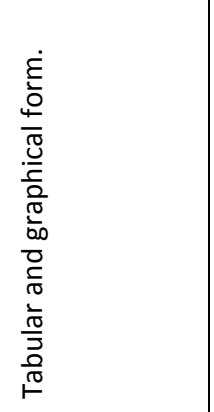 & 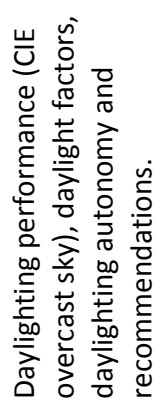 \\
\hline 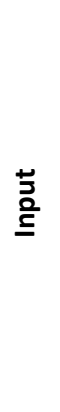 & 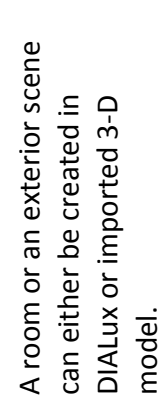 & 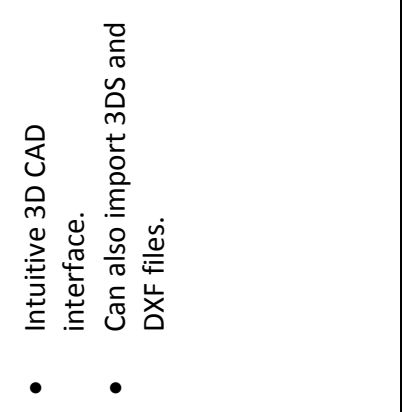 & 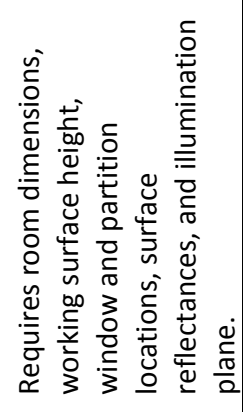 & 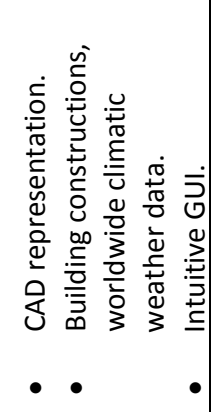 & 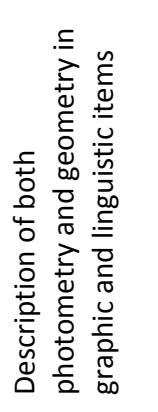 \\
\hline 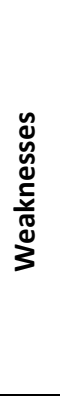 & ' & 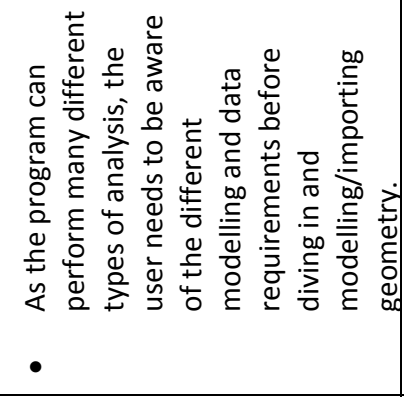 & 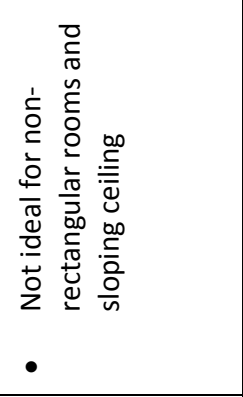 & 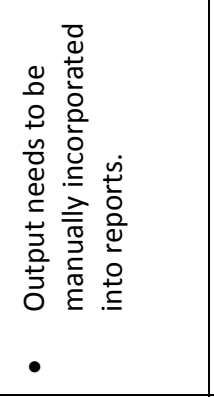 & 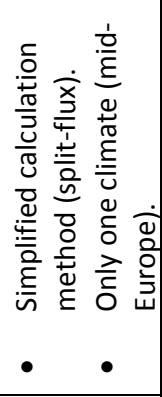 \\
\hline 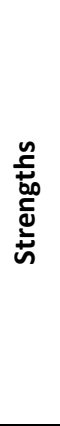 & 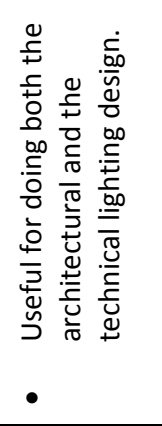 & 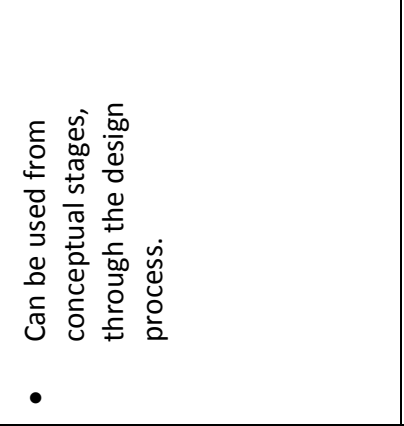 & 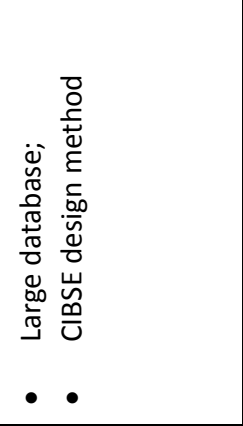 & 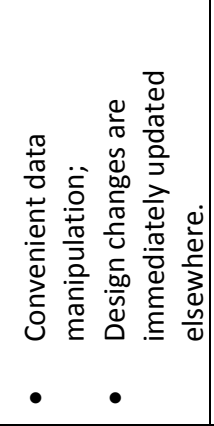 & 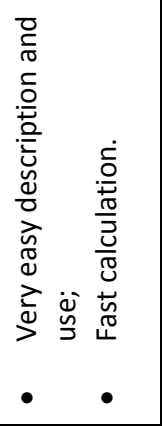 \\
\hline & 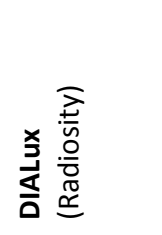 & 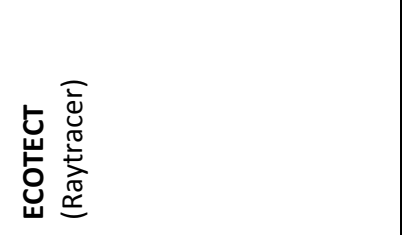 & $\overbrace{\mathcal{U}}^{\widetilde{U}}$ & 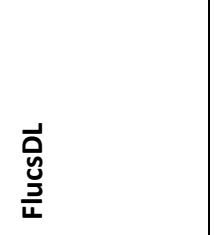 & 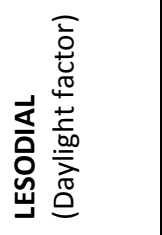 \\
\hline
\end{tabular}




\begin{tabular}{|c|c|c|c|c|c|c|}
\hline 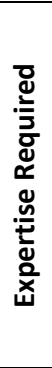 & 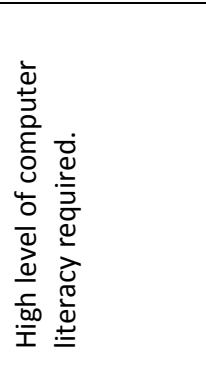 & 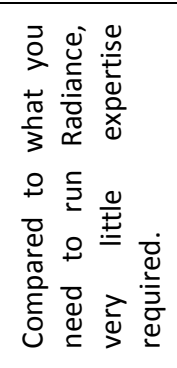 & 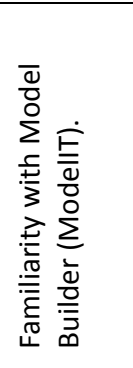 & 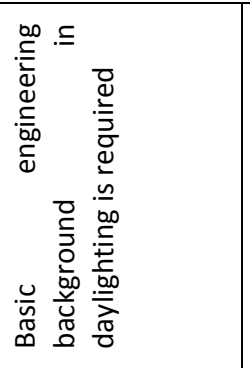 & 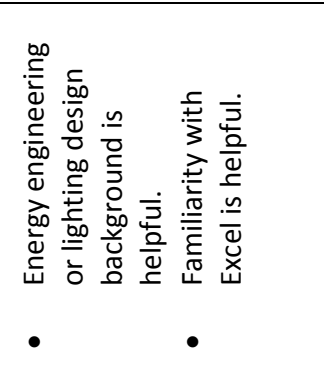 & 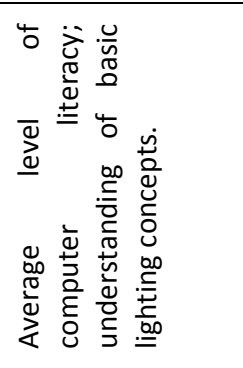 \\
\hline $\begin{array}{l}\text { 蒙 } \\
\text { ț }\end{array}$ & 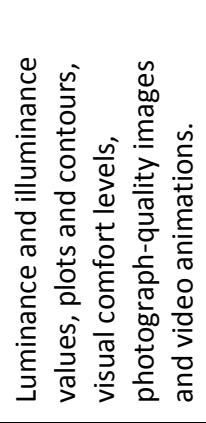 & 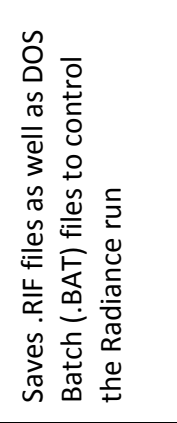 & 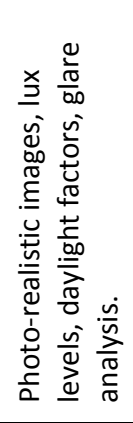 & 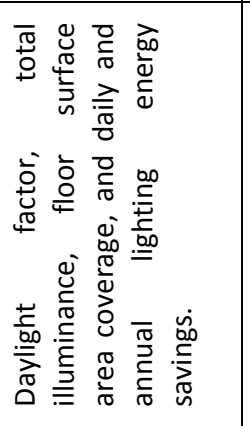 & 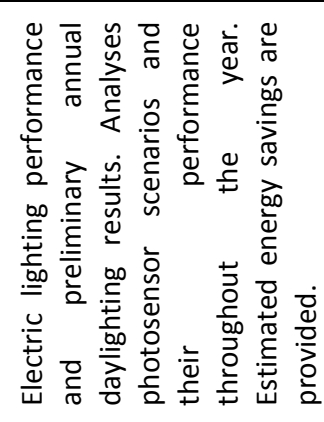 & 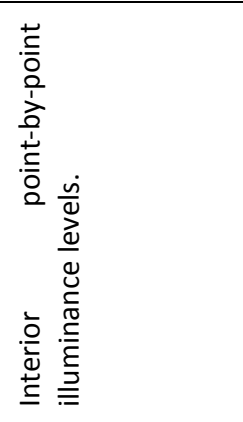 \\
\hline $\begin{array}{l}\underline{\underline{\partial}} \\
\underline{\underline{\underline{a}}}\end{array}$ & 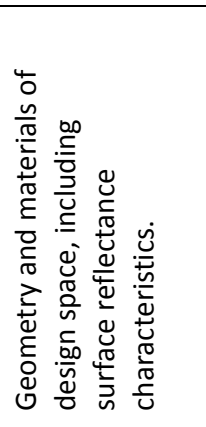 & 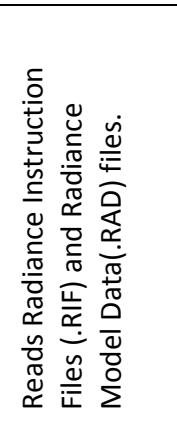 & 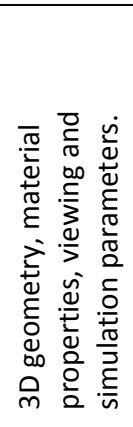 & 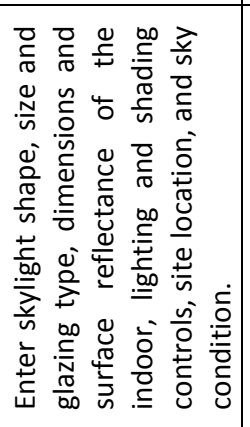 & 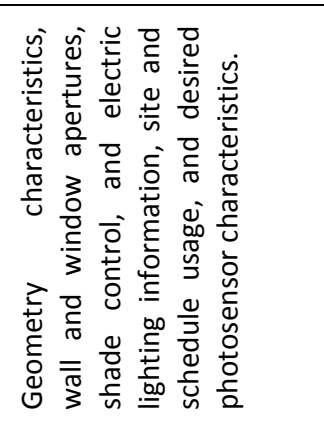 & 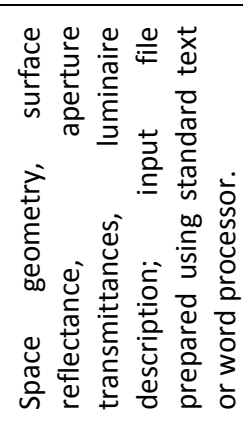 \\
\hline 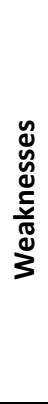 & 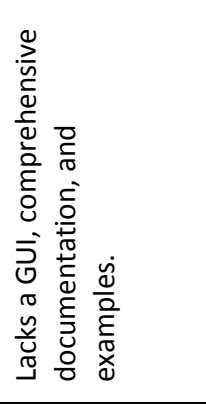 & & & 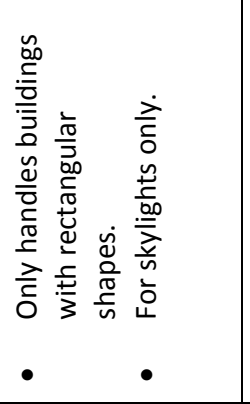 & 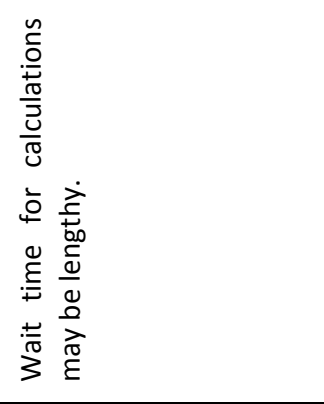 & 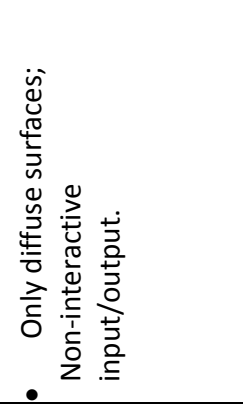 \\
\hline 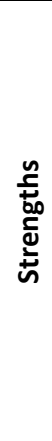 & 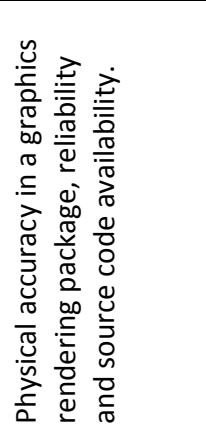 & 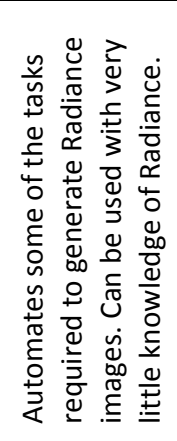 & 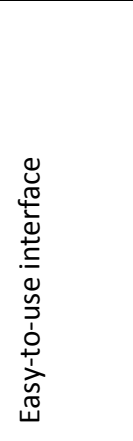 & 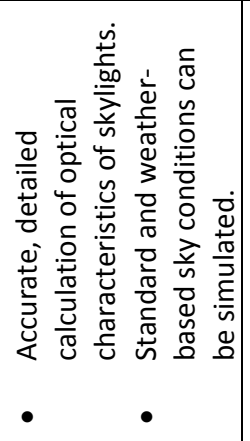 & 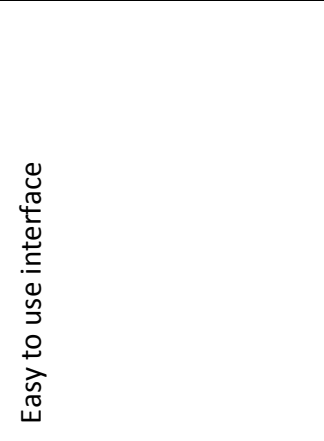 & 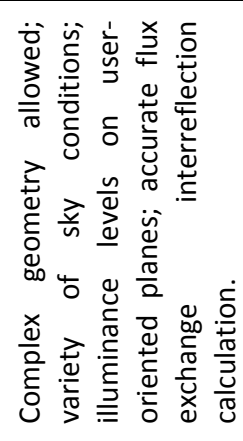 \\
\hline & 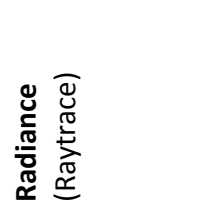 & 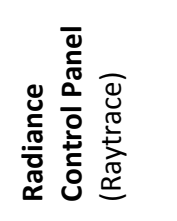 & 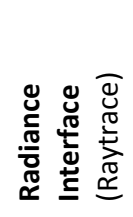 & 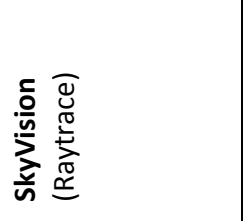 & 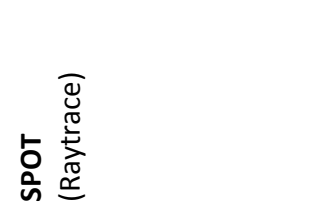 & 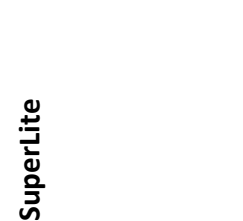 \\
\hline
\end{tabular}




\section{A1.2 DAYSIM}

DAYSIM is a RADIANCE-based daylight simulation tool developed by the Lighting Group of the National Research Council Canada and the Solar Building Design Group of the Fraunhofer Institute for Solar Energy Systems (National Research Council Canada, 2006). DAYSIM was primarily developed to predict the energy use in offices with daylight and electric lighting installations, but can essentially be used for any daylighting applications. It incorporates sophisticated user behaviour algorithms to predict how occupants would use artificial lighting and shading devices.

DAYSIM uses daylight coefficients and the Perez sky luminance model to simulate the daylight performance throughout the year (Reinhart \& Walkenhorst, 2001). Within one run, DAYSIM calculates the annual indoor illuminance levels for intervals as small as 5 mins and for the range of different sky condition based on a climate data. This method is commonly known as 'dynamic daylight simulation' (Reinhart \& Walkenhorst, 2001) and is a recent development in daylight simulation tools. Development of tool like DAYSIM has meant it is now feasible for daylight simulations to be regularly conducted in practice.

DAYSIM uses the annual climate files (*.wea or *.epw) to calculate daylight coefficients for direct and diffuse daylight at different times of the day and year. This calculation takes into consideration: date and time, geographical site, irradiance data and sky luminous distribution (Reinhart, 2006). A RADIANCE calculation is performed for sky conditions of each of the time steps to determine the illuminance for each specified measurement point. A RADIANCE calculation considers aspects of the building including: building geometry, optical properties of material surfaces, status of artificial lighting and shading devices, surrounding landscape and ground reflectance (Reinhart, 2006).

The output provided by DAYSIM gives a variety of useful information including: Daylight Factors, Daylight Autonomy, Useful Daylight Illuminances and Annual light exposure (Reinhart, Mardaljevic \& Rogers, 2006). A key feature of the output that assisted this study is the Daylight Autonomy information. The Daylight Autonomy function allows the user to determine the percent of daylight hours that a specified illuminance is exceeded. In this study, the illuminance value was set at 30 lux, as is specified in the building code, and the percentage values were able to be compared to the minimum building code percentage of $75 \%$ of the standard year.

The simulation engine used by DAYSIM, RADIANCE, has been referred to as an industry-standard for advanced daylight simulations (Reinhart, 2006). This results from adequate validation of the simulation results for a wide range of situations. This has meant that RADIANCE calculations are used by a number of daylight tools to perform global illumination calculations for single sky conditions (Reinhart \& Herkel, 2000).

A comparison of tools for simulating annual daylight illuminance distributions found that DAYSIM performed highly in terms of simulation time and accuracy when compared to other dynamic simulation methods (Reinhart \& Herkel, 2000). The accuracy of DAYSIM has been tested in a study of a test office with external blinds (Reinhart \& Walkenhorst, 2001). This study found that the DAYSIM daylight coefficients can predict Daylight Autonomy values to within a few percentage points. The errors result from building features like shading and glare protection devices, however these design features will not be in this research. 


\section{Appendix 2 - Individual Variable Analysis}

This appendix provides the detailed analysis of the 11 variables independently. This outlines the steps taken for each variable to reach a relationship type and equation. This was the first type of analysis used in this research and was used to aid the development of the prediction equations. 


\section{A2.1 Apartment Type}

16 variations of apartment type were simulated. The analysis of this variable is more complex than that of the other 10 variables because it cannot be expressed in linear increments. A scatter-plot was produced for all simulations to determine the range of simulation results. If it was found that there was a large amount of variations, then equations would need to have been produced for each of the 16 different apartment types. However from the scatter-plot, Figure 1, it can be seen that most of the apartments are within a $10 \%$ range (30\%-40\%). The apartments within this range are also the apartments most likely to be located on the lower floors. The apartments that had DA values lower than this range $(13,14,15$ and 16$)$ were 3 bedroom apartments and would typically be located on the top floors.

Table 3 - Key for Figure 1

\begin{tabular}{|l|l|}
\hline 1 & Studio (Small Wide) \\
\hline 2 & Studio (Large Wide) \\
\hline 3 & Studio (Small Deep) \\
\hline 4 & Studio (Large Deep) \\
\hline 5 & 1 Bedroom (Small Wide) \\
\hline 6 & 1 Bedroom (Large Wide) \\
\hline 7 & 1 Bedroom (Small Deep) \\
\hline 8 & 1 Bedroom (Large Deep) \\
\hline 9 & 2 Bedroom (Small Wide) \\
\hline 10 & 2 Bedroom (Large Wide) \\
\hline 11 & 2 Bedroom (Small Deep) \\
\hline 12 & 2 Bedroom (Large Deep) \\
\hline 13 & 3 Bedroom (Small Wide) \\
\hline 14 & 3 Bedroom (Large Wide) \\
\hline 15 & 3 Bedroom (Small Deep) \\
\hline 16 & 3 Bedroom (Large Deep) \\
\hline
\end{tabular}




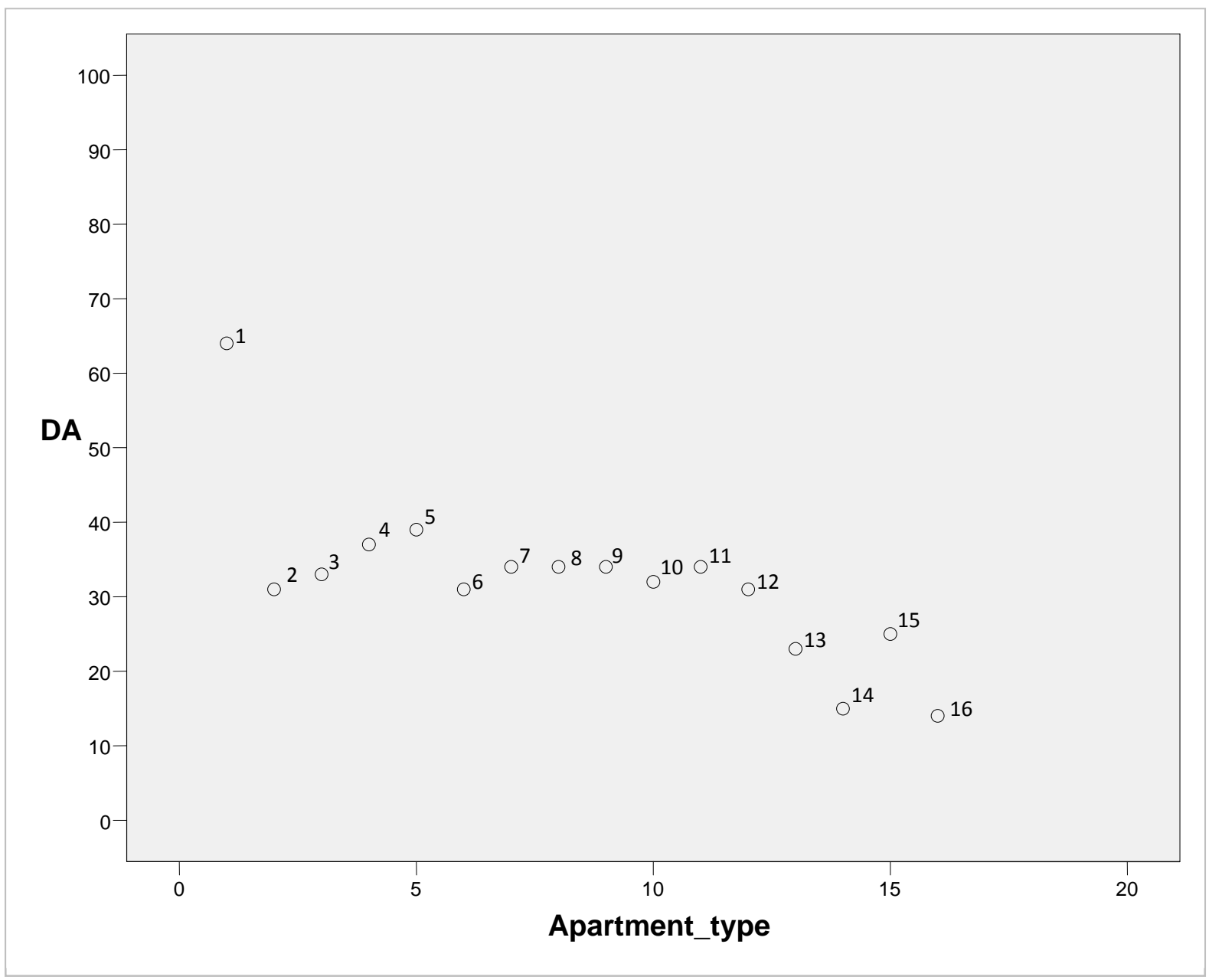

Figure 1 - Apartment Type: Scatter Diagram

Because most of the apartments were within a $10 \%$ range, it was decided to exclude this variable from further analysis. One equation for all apartment type was sufficient. This decision is not expected to have a major effect on the overall results and the time required to produce an equation for all apartments is not realistic given the small variation in results. 


\section{A2.2 Building Height}

Seven variations of building height were simulated and analysed using regression analysis. The following analysis investigates the relationship between building height and daylight autonomy. Figure 2 shows the best three models for the relationship between daylight autonomy (y-axis) and building height ( $x$-axis). From this figure, it can be sent that the exponential model provides the best description of this relationship.

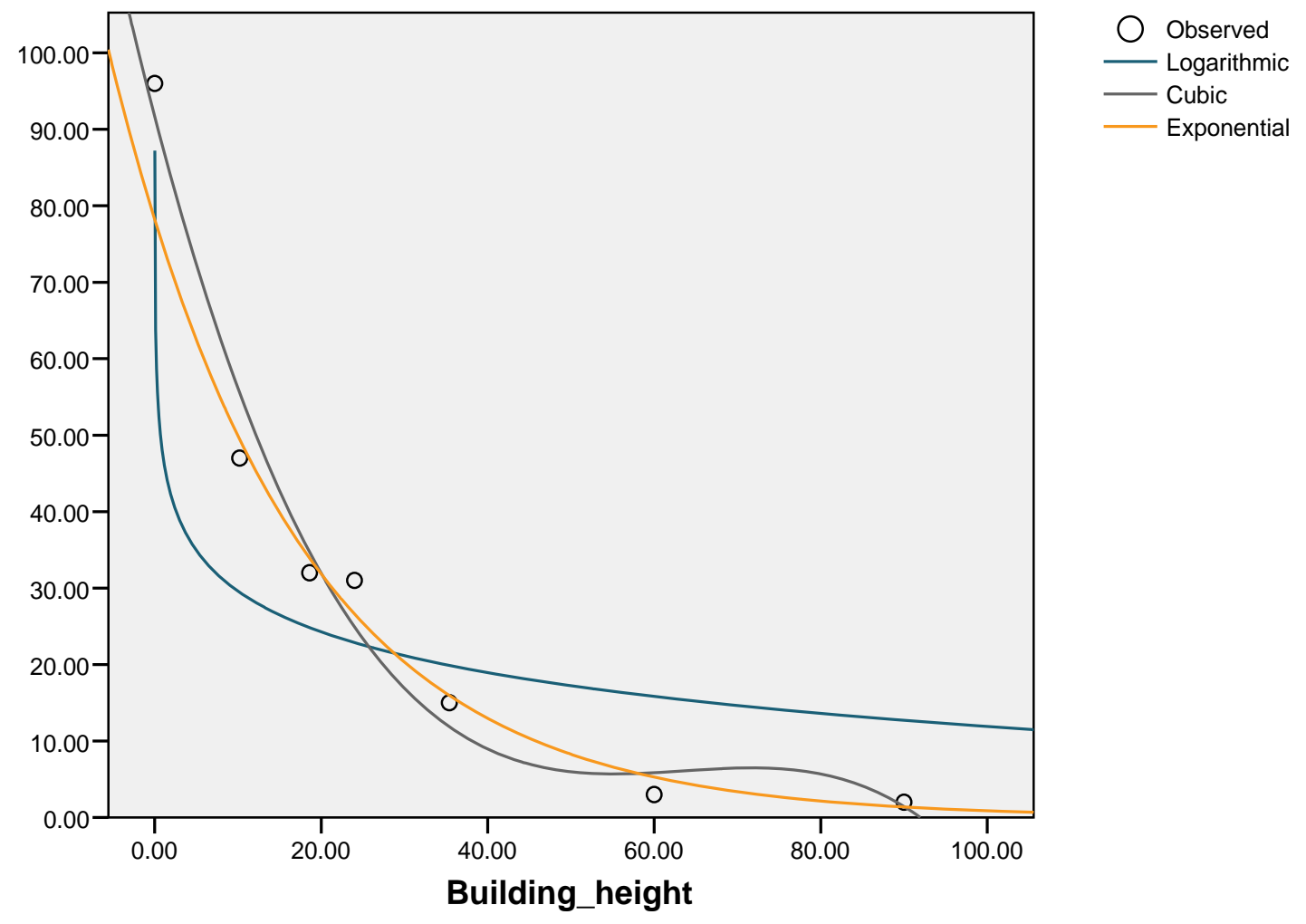

Figure 2 - Building Height: Regression Models

To check that the exponential model was the most reliable model for the relationship between daylight autonomy and building height, the statistics provided by SPSS on the strength of the model were consults. Table 4 shows this statistical analysis, where the key information is the R Square values and the Significance (Sig) values. 
Table 4 - Building Height: Model Summary

Dependent Variable: DA

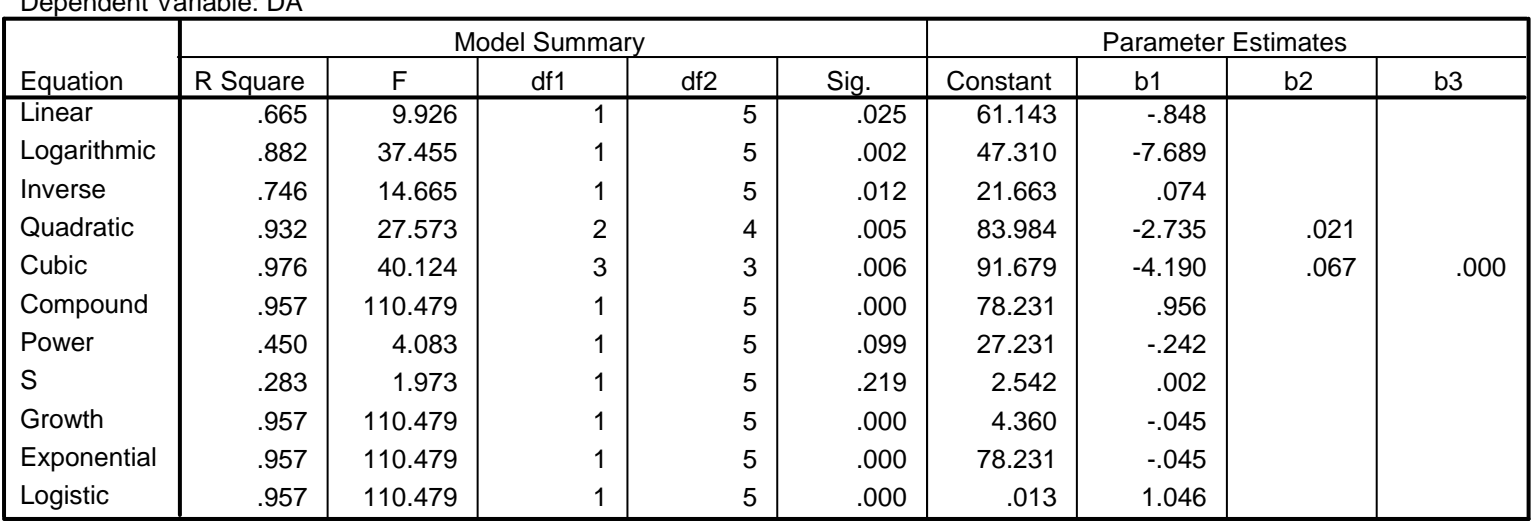

The independent variable is Building_height.

In Table 4, the model with the best $\mathrm{R}$ Square value was the Cubic model, closely followed by the Exponential model. The Exponential model had a Significance level of 0 which indicates that this model perfectly describes this relationship. Referring back to Figure 2, the exponential model also makes the most sense. In reality, the illuminance levels would be expected to decrease as the building height increases due to less visible sky and shading. However, the cubic model suggests that a slight increase in the daylight performance at the back of the room, in the range of $3 \% \mathrm{DA}$, would be expected for building heights around $70 \mathrm{~m}$, but this would not be likely in reality.

Using the parameter information provided in Table 4, an equation was developed for the relationship between daylight performance and opposite building height, this equation is shown below:

\section{$D A=78.231 \epsilon^{-0.04 E B H}$}

From the analysis of the relationship between opposite building height and daylight autonomy, the statistics indicated that there is a strong correlation between these two variables. The relationship is curvilinear and where the model that best describes the relationship is an exponential model. 


\section{A2.3 Ceiling Height}

Five variations of ceiling height were simulated and analysed using regression analysis. The following analysis investigates the relationship between ceiling height and daylight autonomy. Figure 3 shows the two models that predicted the relationship between daylight autonomy ( $y$-axis) and ceiling height (x-axis) best. From this figure, it can be seen that neither of these models represent this relationship particularly well, with just two of the 5 cases being described accurately with this model.

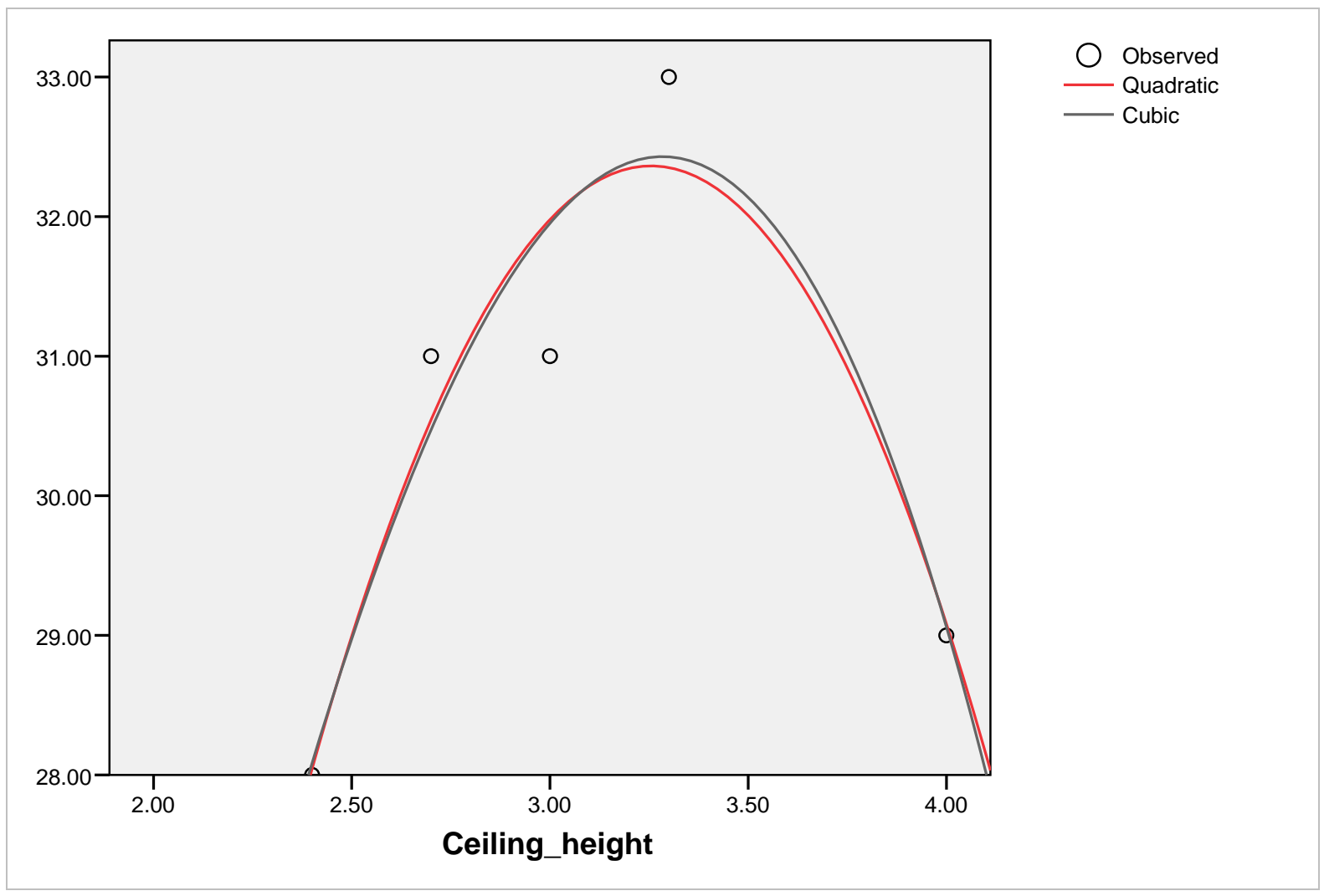

Figure 3 - Ceiling Height: Regression Models

The statistics provided by SPSS were check to determine if any of the models had acceptable $R$ Square values and Significance values to suggest a relationship between these two variables. From Table 5 it can be seen that while the Quadratic and Cubic models have acceptable $R$ Square values (greater than 0.7) the Significance levels are over the acceptable level of 0.05 . This indicates that the relationship between ceiling height and daylight performance cannot be reliably described by any of the 11 statistical models. 
Table 5 - Ceiling Height: Model Summary

Dependent Variable: DA

\begin{tabular}{|c|c|c|c|c|c|c|c|c|c|}
\hline \multirow[b]{2}{*}{ Equation } & \multicolumn{5}{|c|}{ Model Summary } & \multicolumn{4}{|c|}{ Parameter Estimates } \\
\hline & R Square & $\mathrm{F}$ & df1 & $\mathrm{df} 2$ & Sig. & Constant & b1 & $\mathrm{b} 2$ & b3 \\
\hline Linear & .018 & .055 & 1 & 3 & .830 & 29.093 & .424 & & \\
\hline Logarithmic & .044 & .137 & 1 & 3 & .736 & 28.086 & 2.086 & & \\
\hline Inverse & .080 & .260 & 1 & 3 & .646 & 33.296 & -8.652 & & \\
\hline Quadratic & .895 & 8.518 & 2 & 2 & .105 & -30.235 & 38.462 & -5.908 & \\
\hline Cubic & .900 & 8.967 & 2 & 2 & .100 & -11.517 & 20.076 & .000 & -.621 \\
\hline Compound & .019 & .057 & 1 & 3 & .826 & 29.040 & 1.014 & & \\
\hline Power & .045 & .142 & 1 & 3 & .731 & 28.084 & .070 & & \\
\hline $\mathrm{S}$ & .082 & .268 & 1 & 3 & .640 & 3.510 & -.289 & & \\
\hline Growth & .019 & .057 & 1 & 3 & .826 & 3.369 & .014 & & \\
\hline Exponential & .019 & .057 & 1 & 3 & .826 & 29.040 & .014 & & \\
\hline Logistic & .019 & .057 & 1 & 3 & .826 & .034 & .986 & & \\
\hline
\end{tabular}

The independent variable is Ceiling_height.

Because none of the 11 models can reliably describe the relationship between daylight performance and ceiling height, it was decided to exclude this variable from further analysis. Including this variable in the final model would compromise the reliability of results and because there is a variation of $5 \%$ DA between all 5 cases the exclusion of this variable will have a negligible effect on the overall results. 


\section{A2.4 Glazed Area}

Four variations of glazed area were simulated and analysed using regression analysis. The following analysis investigates the relationship between glazed area and daylight autonomy. Figure 4 investigates this relationship by comparing the found best models. Glazed area is shown of the xaxis as a percentage of the floor area and daylight autonomy is shown on the $y$-axis. From Figure 4 it can be seen that any of these models could be used to describe this relationship.

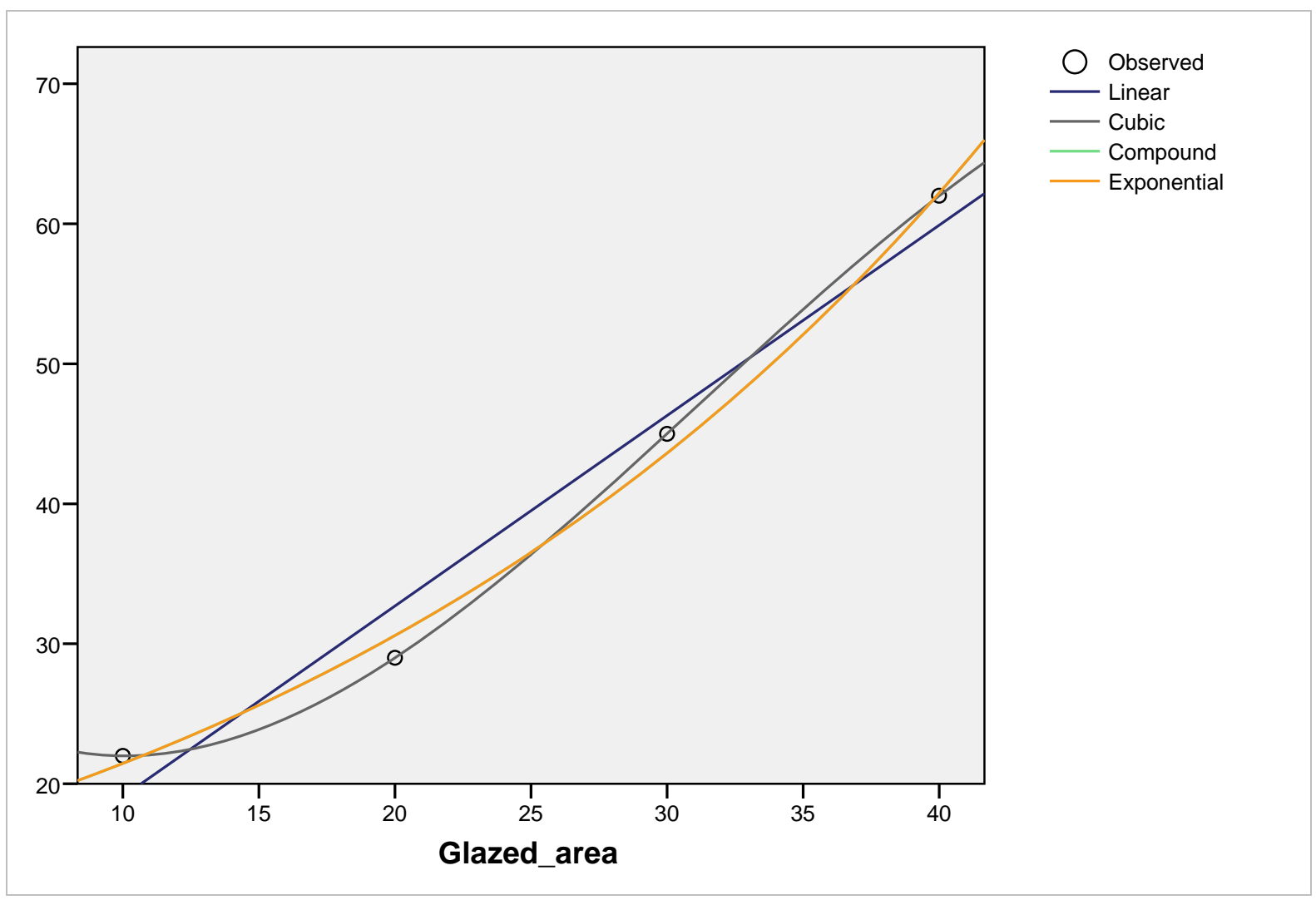

Figure 4-Glazed Area: Regression Models

To determine which of these models describes this relationship best, these statistics in Table 6 were consulted. From this table, the model that appears to provide the best results with an $\mathrm{R}$ Square value and Significance value that suggest a perfect relationship is the Cubic model. Although in reality this is not the best model. Daylight autonomy values would be expected to steadily increase as the glazed areas increased, more glazing equal more light, but the cubic model indicates that only minor increases would be expected for glazed areas greater than $40 \%$. The exponential model also had a high R Square value and low Significance level and more closely matches the expected relationship between these two variables. Statistically, the linear model also has an acceptable $R$ Square value and Significance value. 
Table 6 - Glazed Area: Model Summary

\begin{tabular}{|c|c|c|c|c|c|c|c|c|c|}
\hline \multirow[b]{2}{*}{ Equation } & \multicolumn{5}{|c|}{ Model Summary } & \multicolumn{4}{|c|}{ Parameter Estimates } \\
\hline & R Square & $\mathrm{F}$ & df1 & $\mathrm{df} 2$ & Sig. & Constant & b1 & b2 & b3 \\
\hline Linear & .970 & 65.589 & 1 & 2 & .015 & 5.500 & 1.360 & & \\
\hline Logarithmic & .868 & 13.207 & 1 & 2 & .068 & -46.071 & 27.629 & & \\
\hline Inverse & .725 & 5.267 & 1 & 2 & .149 & 63.026 & -451.692 & & \\
\hline Quadratic & .997 & 148.406 & 2 & 1 & .058 & 18.000 & .110 & .025 & \\
\hline Cubic & 1.000 & & 3 & 0 & . & 32.000 & -2.117 & .125 & -.001 \\
\hline Compound & .993 & 281.861 & 1 & 2 & .004 & 15.046 & 1.036 & & \\
\hline Power & .938 & 30.181 & 1 & 2 & .032 & 3.687 & .740 & & \\
\hline S & .824 & 9.383 & 1 & 2 & .092 & 4.245 & -12.422 & & \\
\hline Growth & .993 & 281.861 & 1 & 2 & .004 & 2.711 & .035 & & \\
\hline Exponential & .993 & 281.861 & 1 & 2 & .004 & 15.046 & .035 & & \\
\hline Logistic & .993 & 281.861 & 1 & 2 & .004 & .066 & .965 & & \\
\hline
\end{tabular}

The independent variable is Glazed area.

It was decided that the model the represented the relationship between daylight autonomy and glazing area was exponential, the equation for this is shown below and was created using the parameter values given in Table 6.

$$
D A=15.046 a^{2.0356 A}
$$

From the analysis of the relationship between glazed area and daylight autonomy, it can be concluded that there is a very strong correlation between these two variables. The relationship is curvilinear, where the model that best describes the relationship is an exponential model. However, it would be acceptable to simplify this relationship through the use of the linear model. 


\section{A2.5 Glazing Transmittance}

Five variations of glazing transmittance were simulated and analysed using regression analysis. The following analysis investigates the relationship between glazing transmittance and daylight autonomy. Figure 5 shows the four models that describe this relationship best, where daylight autonomy is shown on the $y$-axis and glazing transmittance (in percent) is shown on the $x$-axis. From Figure 5, it appears that three of the four models have an almost identical form for glazed area between $40 \%$ and $90 \%$. Of these three models, the linear model is the simplest equation.

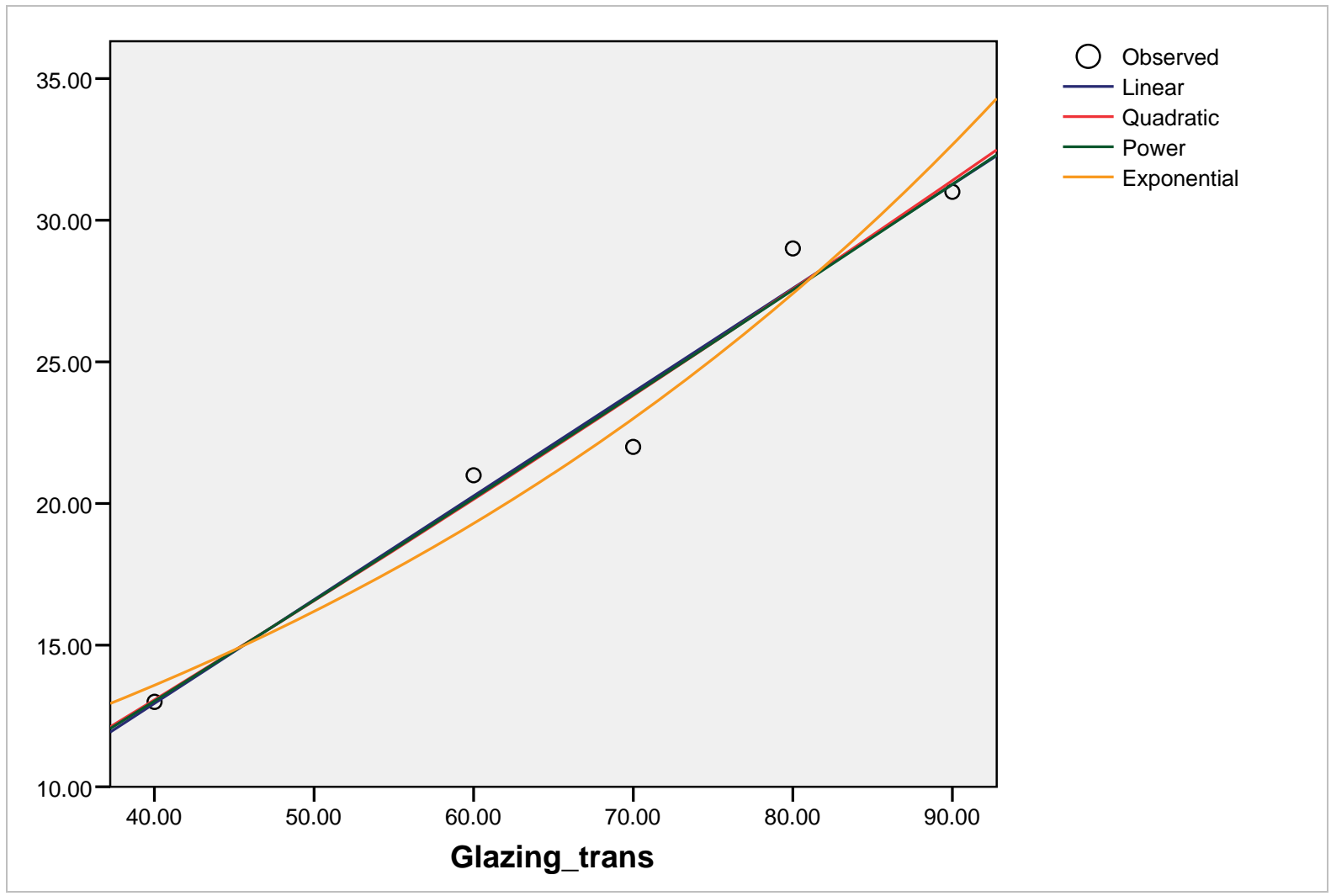

Figure 5 - Glazing Transmittance: Regression Models

The statistics provided by SPSS were consulted to check the strength of these models and the relationship between daylight autonomy and glazing transmittance. Table 7 shows these statistics, where the key values are the R Square and Significance values. The R Square values for all 11 models were very strong with values over 0.9 , indicating that the models statistically fit the data very well and that this is a near perfect relationship. The significance values are also under the maximum acceptable value of 0.05 . From Table 7 , the model with the highest $R$ Square value and lowest Significance level was the power model. However, the linear model also has a very high $\mathrm{R}$ Square and low Significance level but is a much simpler model than the power. 
Table 7 - Glazing Transmittance: Model Summary

Dependent Variable: DA

\begin{tabular}{|c|c|c|c|c|c|c|c|c|c|}
\hline \multirow[b]{2}{*}{ Equation } & \multicolumn{5}{|c|}{ Model Summary } & \multicolumn{4}{|c|}{ Parameter Estimates } \\
\hline & R Square & $\mathrm{F}$ & df1 & df2 & Sig. & Constant & b1 & b2 & b3 \\
\hline Linear & .969 & 94.357 & 1 & 3 & .002 & -1.703 & .366 & & \\
\hline Logarithmic & .950 & 57.321 & 1 & 3 & .005 & -69.492 & 22.161 & & \\
\hline Inverse & .907 & 29.404 & 1 & 3 & .012 & 42.962 & -1241.887 & & \\
\hline Quadratic & .970 & 31.812 & 2 & 2 & .030 & -.024 & .310 & .000 & \\
\hline Cubic & .970 & 31.812 & 2 & 2 & .030 & -.024 & .310 & .000 & .000 \\
\hline Compound & .964 & 80.431 & 1 & 3 & .003 & 6.736 & 1.018 & & \\
\hline Power & .977 & 128.486 & 1 & 3 & .001 & .243 & 1.079 & & \\
\hline S & .963 & 78.795 & 1 & 3 & .003 & 4.078 & -61.457 & & \\
\hline Growth & .964 & 80.431 & 1 & 3 & .003 & 1.907 & .018 & & \\
\hline Exponential & .964 & 80.431 & 1 & 3 & .003 & 6.736 & .018 & & \\
\hline Logistic & .964 & 80.431 & 1 & 3 & .003 & .148 & .983 & & \\
\hline
\end{tabular}

The independent variable is Glazing_trans.

Although the Power model provided the best fit for this relationship, the difference between this model and the linear model is minimal and the linear model was a less complex. It was decided that the best model to use in the equation for the tool was the linear model because of its simplicity. The following equation shows the equation for this relationship using the parameters drawn from Table 7.

\section{$D A=-1.703+0.366 G T$}

From the analysis of the relationship between glazing transmission and daylight autonomy, it can be concluded that there is a very strong correlation between these two variables. The relationship can be linear or curvilinear depending on the desired complexity of the model, where the model to describe this relationship in the regression models is a simple linear model. 


\section{A2.6 Geographical Location}

11 variations of geographical location were simulated and analysed using regression analysis. The following analysis investigates the relationship between geographical location and daylight autonomy. Figure 6 is a scatter plot which shows the results from the simulations where the daylight autonomy values are shown of the $y$-axis and the location values are shown on the $x$-axis. The location values were ranked based on their latitudes, where the location that was expected to have the highest DA values was located closest to the equator and the location with the lowest DA values was located at the largest distance from the equator. The key for this graph is shown below in Table 8.

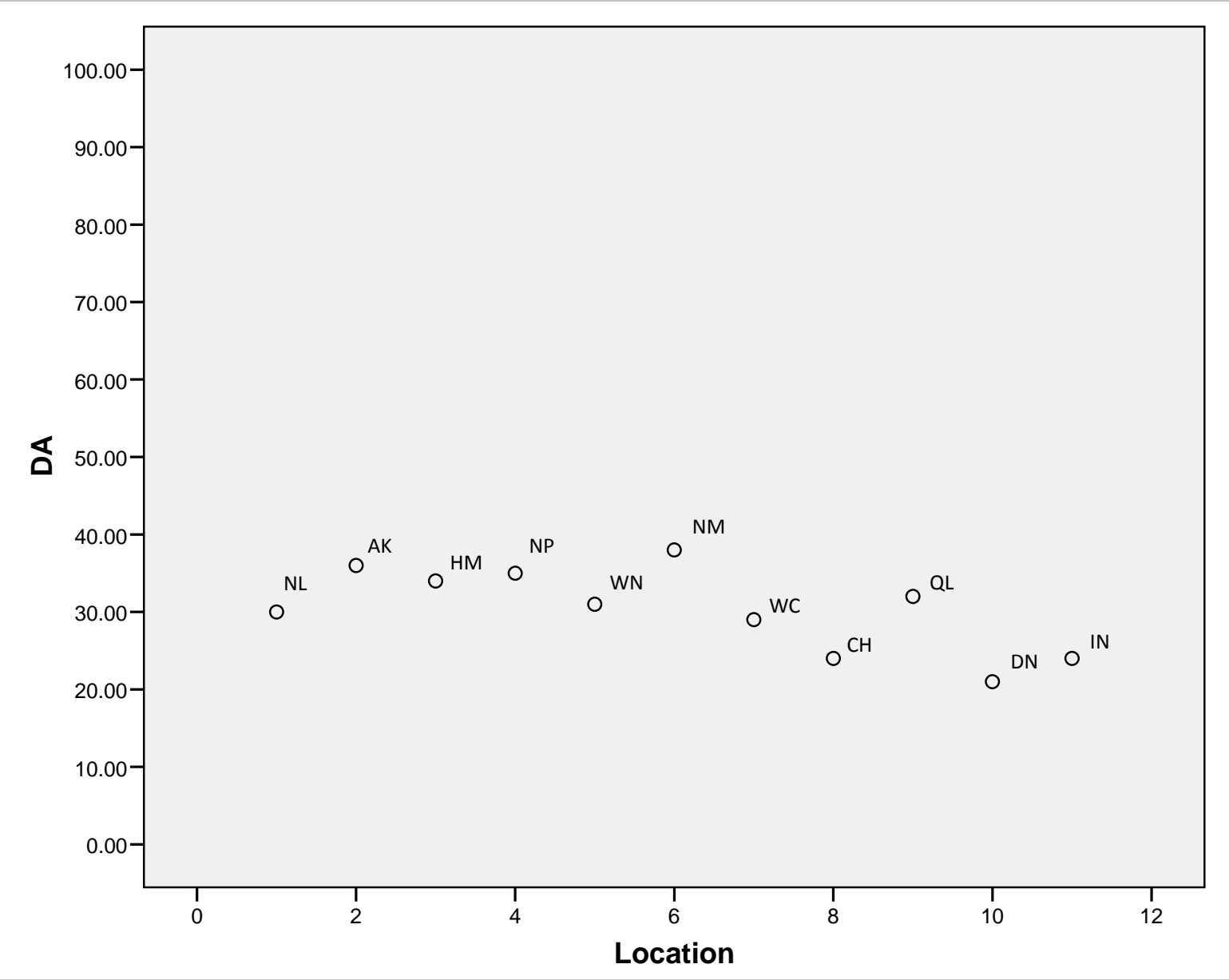

Figure 6 - Geographical Location: Scatter Diagram 
Table 8 - Key for Figure 6

\begin{tabular}{|l|l|}
\hline NL & Northland \\
\hline AK & Auckland \\
\hline HM & Hamilton \\
\hline NP & New Plymouth \\
\hline WN & Wellington \\
\hline NM & Nelson/Marlborough \\
\hline WC & West Coast \\
\hline CH & Christchurch \\
\hline QL & Queenstown Lakes \\
\hline DN & Dunedin \\
\hline IN & Invercargill \\
\hline
\end{tabular}

Table 9 shows the SPSS statistics for the relationship between daylight autonomy and geographical location, using the aforementioned ranking. From this table, it can be seen that none of the models have acceptable R Square values. This meant that using this ranking system, there was no linear relationship between daylight autonomy and geographical location.

Table 9 - Geographical Location: Model Summary

\begin{tabular}{|c|c|c|c|c|c|c|c|c|c|}
\hline \multirow[b]{2}{*}{ Equation } & \multicolumn{5}{|c|}{ Model Summary } & \multicolumn{4}{|c|}{ Parameter Estimates } \\
\hline & R Square & $\mathrm{F}$ & df1 & $\mathrm{df} 2$ & Sig. & Constant & b1 & b2 & b3 \\
\hline Linear & .438 & 7.028 & 1 & 9 & .026 & 36.909 & -1.091 & & \\
\hline Logarithmic & .243 & 2.891 & 1 & 9 & .123 & 36.117 & -3.616 & & \\
\hline Inverse & .070 & .677 & 1 & 9 & .432 & 28.894 & 5.353 & & \\
\hline Quadratic & .576 & 5.444 & 2 & 8 & .032 & 31.212 & 1.538 & -.219 & \\
\hline Cubic & .620 & 3.800 & 3 & 7 & .066 & 26.227 & 5.656 & -1.041 & .046 \\
\hline Compound & .459 & 7.650 & 1 & 9 & .022 & 37.735 & .962 & & \\
\hline Power & .262 & 3.187 & 1 & 9 & .108 & 36.785 & -.131 & & \\
\hline S & .082 & .807 & 1 & 9 & .392 & 3.342 & .202 & & \\
\hline Growth & .459 & 7.650 & 1 & 9 & .022 & 3.631 & -.039 & & \\
\hline Exponential & .459 & 7.650 & 1 & 9 & .022 & 37.735 & -.039 & & \\
\hline Logistic & .459 & 7.650 & 1 & 9 & .022 & .027 & 1.040 & & \\
\hline
\end{tabular}

The independent variable is Location.

Because geographical location considers two different factors, latitudes and solar radiation, there were other types of ranking that could have been used. Table 10 shows these different ranking systems, where the order of results for each systems is shown. The latitude method used early is shown in the first column. A ranking based on the simulation result is shown in column two, where the location with the highest DA values is shown first, through to the last being the lowest DA values, this was used to compare to the other ranking systems, the best system was the ranking that was closes to the DA ranking. The other three ranking systems tested used the weather data for each location. The Total Annual radiation was combined in column 3, the diffuse radiation component was shown in column 4 and the direct radiation component was shown in column 5 . The location with the highest radiation values are shown first, through to the locations with the lowest radiation values. 
Table 10 - Geographical Location Ranking Methods

\begin{tabular}{|c|c|c|c|c|}
\hline Latitudes & $\begin{array}{c}\text { Simulations } \\
\text { (DA) }\end{array}$ & $\begin{array}{c}\text { Total Annual } \\
\text { Radiation }\end{array}$ & Diffuse Radiation & Direct Radiation \\
\hline NL & NM & NM & NM & NL \\
\hline AK & AK & QL & QL & HM \\
\hline HM & NP & NP & NP & AK \\
\hline NP & HM & AK & AK & NP \\
\hline WN & QL & HM & WC & CH \\
\hline NM & WN & WC & HM & IN \\
\hline WC & NL & CH & CH & NM \\
\hline CH & WC & NL & WN & DN \\
\hline QL & CH & WN & NL & WN \\
\hline DN & IN & IN & DN & WC \\
\hline IN & DN & DN & IN & QL \\
\hline
\end{tabular}

From Table 10 it can be seen that none of the ranking methods have found an appropriate way to assess the relationship between location and daylight autonomy. Originally it was expected that the rankings for location (latitudes) would have given the best results. The next ranking that was expected to display some similarity to the simulation results was the combined total annual radiation, the data used in simulation to provide the daylight availability. However as can be seen in 
Table 10, this ranking method does not fit the results appropriately either. From Table 10 it was concluded that the relationship between the method for ranking locations is complex and would involved consideration of both latitude and daylight availability. It was decided that the best method to rank the locations would be based on the simulations results, so the location with the highest result was ranked first and the location with the lowest result would be ranked last. Table 11 shows the rankings used for the remainder of this research.

Table 11 - Geographical Location Selected Ranking Method

\begin{tabular}{|c|l|}
\hline 1 & NM (Nelson/Marlborough) \\
\hline 2 & AK (Auckland) \\
\hline 3 & NP (New Plymouth) \\
\hline 4 & HM (Hamilton) \\
\hline 5 & QL (Queenstown Lakes) \\
\hline 6 & WN (Wellington) \\
\hline 7 & NL (Northland) \\
\hline 8 & WC (West Coast) \\
\hline 9 & $\mathrm{CH}$ (Christchurch) \\
\hline 10 & $\mathrm{IN} \mathrm{(Invercargill)}$ \\
\hline 11 & $\mathrm{DN}$ (Dunedin) \\
\hline
\end{tabular}

Figure 7 is a scatter plot showing the relationship between geographical location and daylight autonomy, using the new rankings to order the results. 


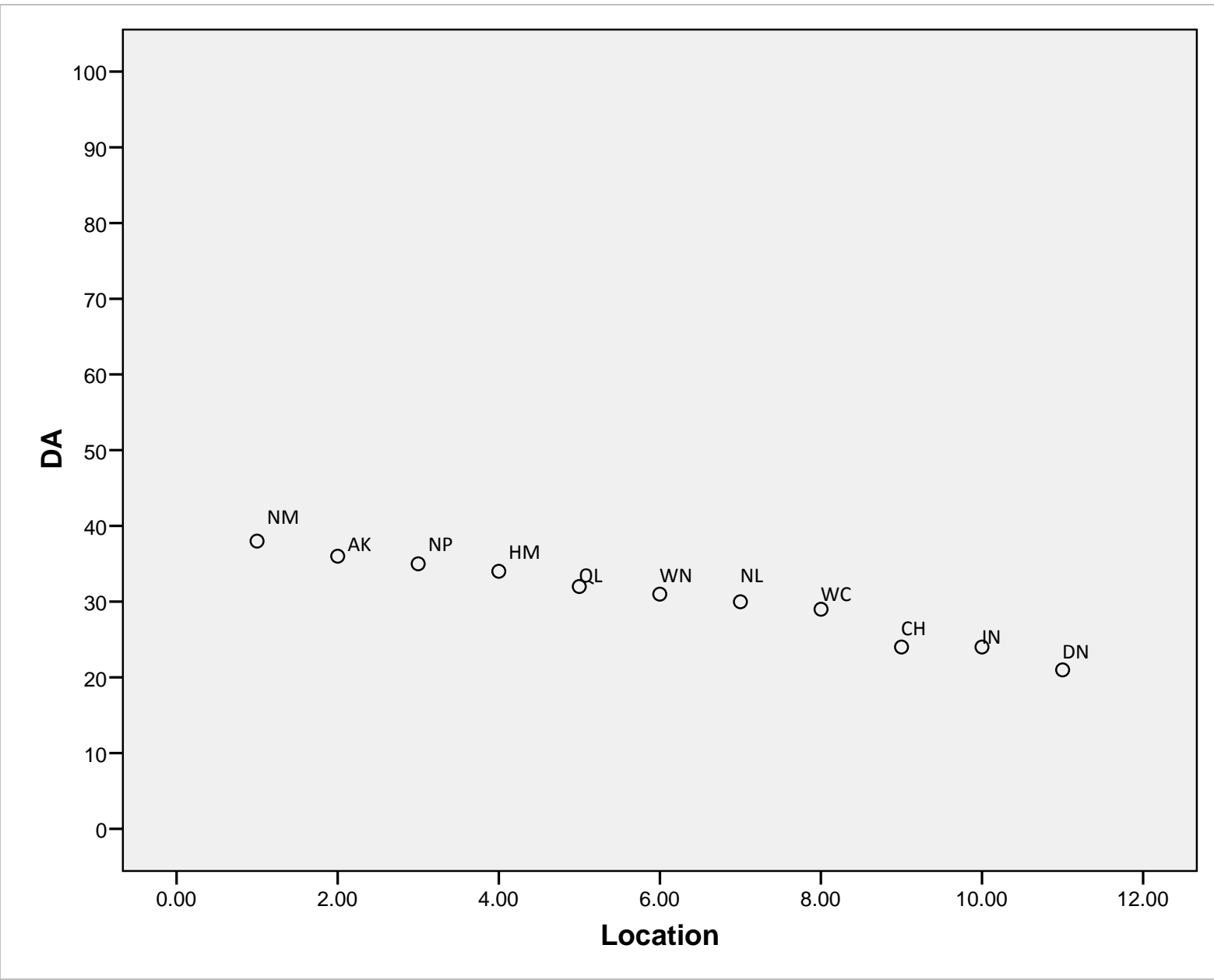

Figure 7 - Geographical Location: Scatter Diagram (alternative ranking method)

Using this ranking method, the relationship between daylight autonomy and the geographical relationships was explored. Figure 8 shows the four best models to describe this relationship, where daylight autonomy values are shown on the $y$-axis and geographical locations are shown to the $x$ axis. From Figure 8 , the models with the best predictions were the quadratic and cubic models but all models fit the results reasonably well. 


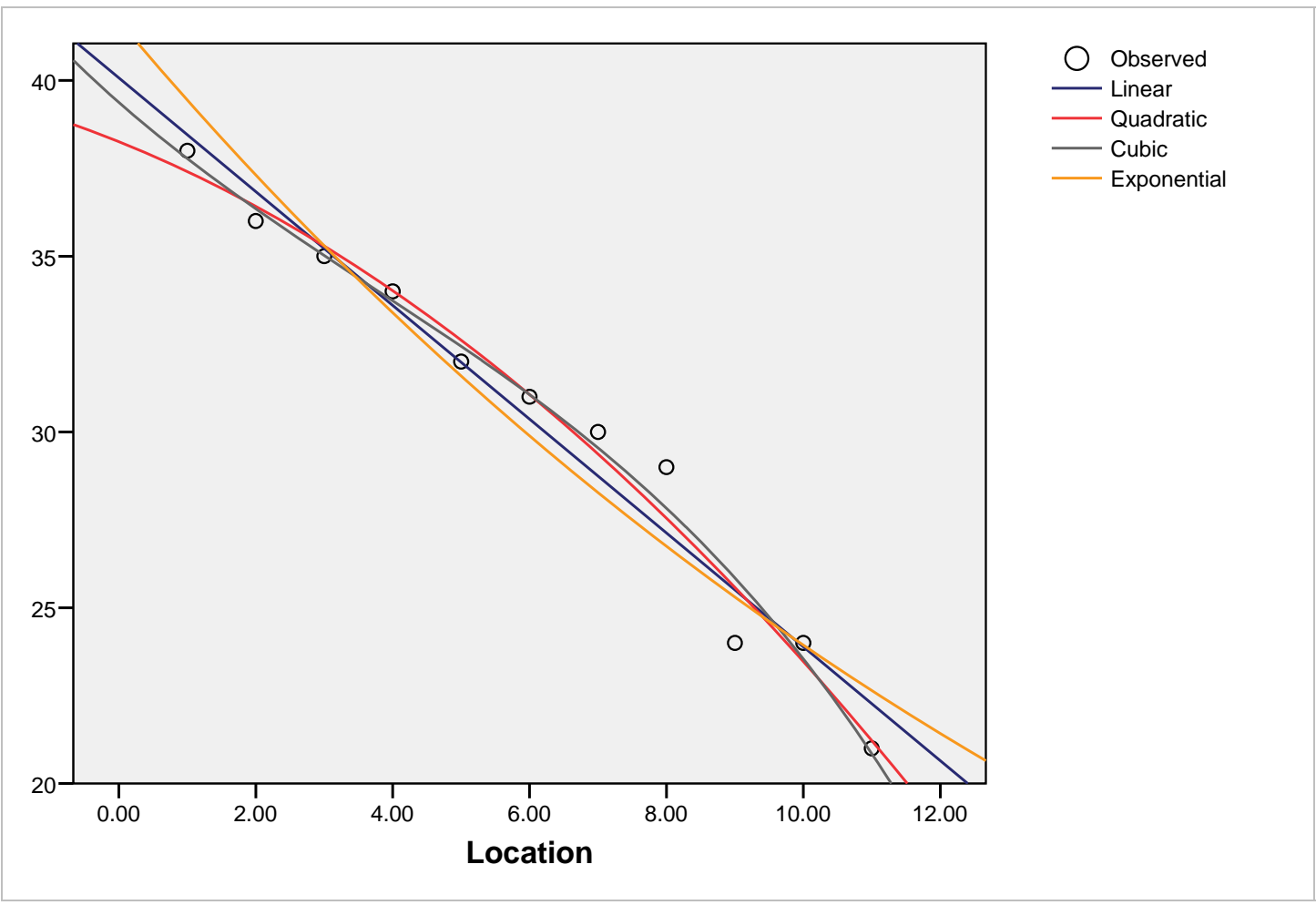

Figure 8 - Geographical Location: Regression Models

The SPSS statistical output was consulted to determine with model had the best fit. From Table 12 it can be seen that there is a considerably stronger correlation for this ranking method. The $R$ Square values are almost all over the minimum value of 0.7 , with many having high $R$ Square value, greater than 0.9. Significance values were also very strong, with all values less than the maximum Significance level of 0.05 . The statistics confirm that the cubic and quadratic models do provide the best fit for this relationship. However, the linear model has a very good $R$ Square value and Significance level which indicates that this model could also be used.

Table 12 - Geographical Location: Model Summary (alternative ranking method)

\begin{tabular}{|c|c|c|c|c|c|c|c|c|c|}
\hline \multirow[b]{2}{*}{ Equation } & \multicolumn{5}{|c|}{ Model Summary } & \multicolumn{4}{|c|}{ Parameter Estimates } \\
\hline & R Square & $\mathrm{F}$ & df1 & $\mathrm{df} 2$ & Sig. & Constant & b1 & b2 & b3 \\
\hline Linear & .965 & 246.675 & 1 & 9 & .000 & 40.073 & -1.618 & & \\
\hline Logarithmic & .818 & 40.384 & 1 & 9 & .000 & 40.915 & -6.631 & & \\
\hline Inverse & .548 & 10.932 & 1 & 9 & .009 & 26.249 & 14.987 & & \\
\hline Quadratic & .979 & 185.154 & 2 & 8 & .000 & 38.255 & -.779 & -.070 & \\
\hline Cubic & .981 & 120.774 & 3 & 7 & .000 & 39.379 & -1.708 & .115 & -.010 \\
\hline Compound & .936 & 131.752 & 1 & 9 & .000 & 41.689 & .946 & & \\
\hline Power & .756 & 27.890 & 1 & 9 & .001 & 42.542 & -.222 & & \\
\hline S & .481 & 8.355 & 1 & 9 & .018 & 3.263 & .489 & & \\
\hline Growth & .936 & 131.752 & 1 & 9 & .000 & 3.730 & -.055 & & \\
\hline Exponential & .936 & 131.752 & 1 & 9 & .000 & 41.689 & -.055 & & \\
\hline Logistic & .936 & 131.752 & 1 & 9 & .000 & .024 & 1.057 & & \\
\hline
\end{tabular}

The independent variable is Location.

The linear model was selected for this relationship because it has very good $\mathrm{R}$ Square and Significance values and is a less complex model than the cubic and quadratic models. Using the 
Parameter values given in Table 12, the following equation was created for this relationship to be used in the second stage of analysis:

$$
D A=40.073-1.618 L
$$

From the analysis of the relationship between geographical location and daylight autonomy, it can be concluded that there is a reasonably strong correlation between these two variables, however there are some complexities in the ranking system for the various locations. It was decided that the model to describe this relationship is linear, however a cubic model was also capable of accurately predicting the relationship. 


\section{A2.7 Orientation}

Seven variations of orientation were simulated and analysed using regression analysis. The following analysis investigates the relationship between orientation and daylight autonomy.

All 7 orientations were analysed using SPSS, Table 13 shows the statistical strength of this relationship. It can be seen that none of the R Square values or Significance levels are acceptable. This indicates the there in no relationship can accurately predict the relationship between daylight autonomy and orientation.

Table 13 - Orientation: Model Summary

\begin{tabular}{|c|c|c|c|c|c|c|c|c|c|}
\hline \multirow[b]{2}{*}{ Equation } & \multicolumn{5}{|c|}{ Model Summary } & \multicolumn{4}{|c|}{ Parameter Estimates } \\
\hline & R Square & $\mathrm{F}$ & df1 & $\mathrm{df} 2$ & Sig. & Constant & b1 & b2 & b3 \\
\hline Linear & .155 & .919 & 1 & 5 & .382 & 32.211 & .013 & & \\
\hline Logarithmic & .280 & 1.946 & 1 & 5 & .222 & 32.601 & .242 & & \\
\hline Inverse & .260 & 1.758 & 1 & 5 & .242 & 33.667 & -.003 & & \\
\hline Quadratic & .372 & 1.184 & 2 & 4 & .394 & 30.857 & .061 & .000 & \\
\hline Cubic & .399 & .663 & 3 & 3 & 628 & 30.545 & .095 & -.001 & 2.00E-006 \\
\hline Compound & 163 & .973 & 1 & 5 & .369 & 32.152 & 1.000 & & \\
\hline Power & .287 & 2.016 & 1 & 5 & .215 & 32.549 & .007 & & \\
\hline S & .266 & 1.816 & 1 & 5 & .236 & 3.515 & -8.1E-005 & & \\
\hline Growth & . 163 & .973 & 1 & 5 & 369 & 3.470 & .000 & & \\
\hline Exponential & 163 & .973 & 1 & 5 & .369 & 32.152 & .000 & & \\
\hline Logistic & .163 & .973 & 1 & 5 & .369 & .031 & 1.000 & & \\
\hline
\end{tabular}

The independent variable is Orientation.

Because orientation was identified as a major factor that affects daylight performance, it was decided to assess this relationship with just the four cardinal orientations: North, East, South and West. Figure 9 shows the four best models for the relationship between daylight autonomy (y-axis) and orientation (x-axis), given in angles off North. From Figure 9 the best to models are clearly the cubic and quadratic models, however the fit was made difficult because of the $1 \%$ DA difference between the East and West case, both 90 degrees off the azimuth. 


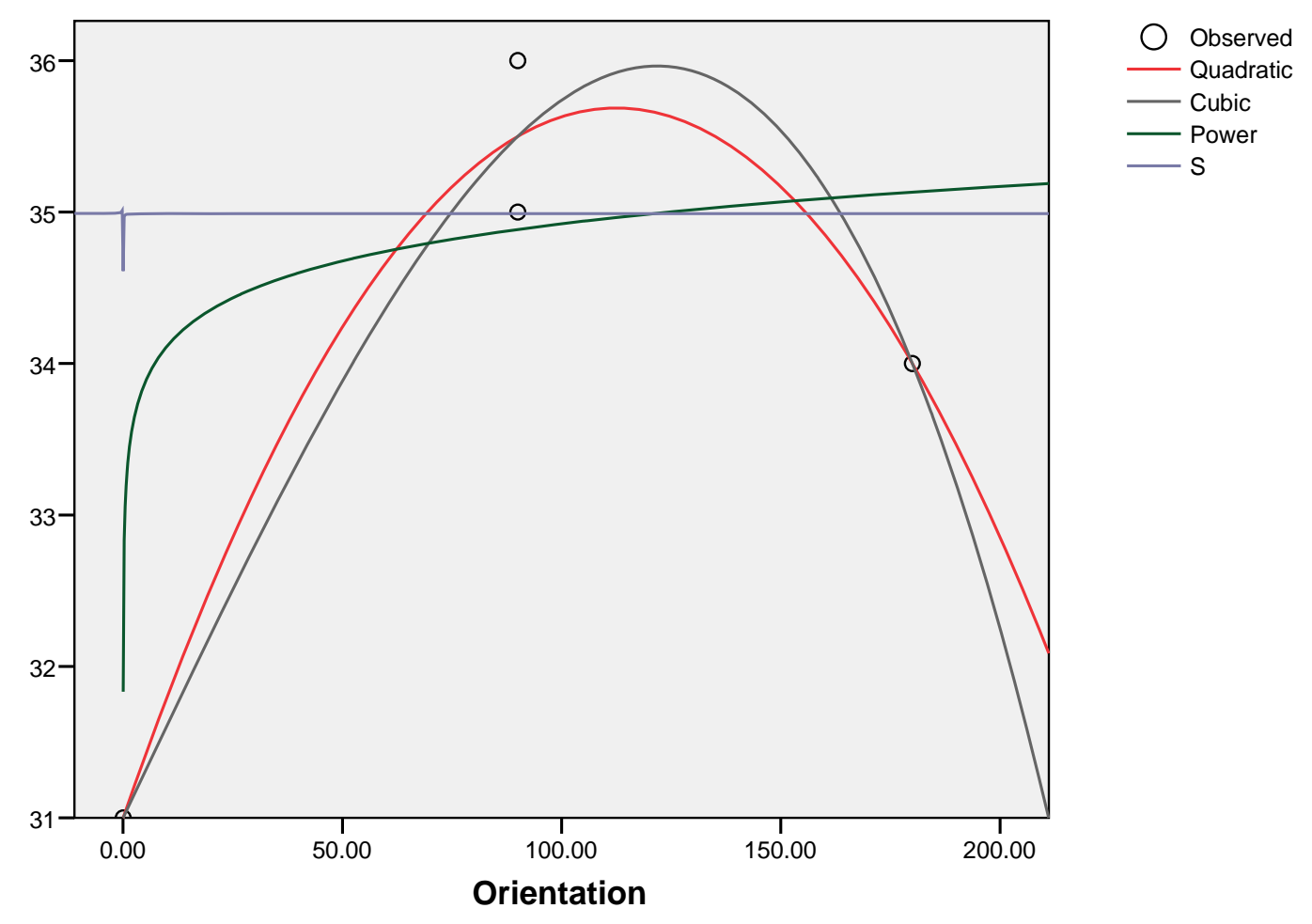

Figure 9 - Orientation: Regression Models

In Table 14 the statistics for these models were consults. It can be seen that while the cubic and quadratic model had very high $\mathrm{R}$ Square values, the significance levels were above the recommended 0.05 .

Table 14 - Orientation: Model Summary (cardinal orientations only)

\begin{tabular}{|c|c|c|c|c|c|c|c|c|c|}
\hline \multirow[b]{2}{*}{ Equation } & \multicolumn{5}{|c|}{ Model Summary } & \multicolumn{4}{|c|}{ Parameter Estimates } \\
\hline & R Square & $\mathrm{F}$ & df1 & $\mathrm{df} 2$ & Sig. & Constant & b1 & b2 & b3 \\
\hline Linear & .321 & .947 & 1 & 2 & .433 & 32.500 & .017 & & \\
\hline Logarithmic & .821 & 9.164 & 1 & 2 & .094 & 33.388 & .336 & & \\
\hline Inverse & .857 & 12.000 & 1 & 2 & .074 & 35.000 & -.004 & & \\
\hline Quadratic & .964 & 13.500 & 2 & 1 & .189 & 31.000 & .083 & .000 & \\
\hline Cubic & .964 & 13.500 & 2 & 1 & .189 & 31.000 & .061 & .000 & $-1.4 \mathrm{E}-006$ \\
\hline Compound & .338 & 1.020 & 1 & 2 & 419 & 32.415 & 1.001 & & \\
\hline Power & .836 & 10.161 & 1 & 2 & .086 & 33.324 & .010 & & \\
\hline$S$ & .871 & 13.463 & 1 & 2 & .067 & 3.555 & .000 & & \\
\hline Growth & .338 & 1.020 & 1 & 2 & .419 & 3.479 & .001 & & \\
\hline Exponential & .338 & 1.020 & 1 & 2 & .419 & 32.415 & .001 & & \\
\hline Logistic & .338 & 1.020 & 1 & 2 & .419 & .031 & .999 & & \\
\hline
\end{tabular}

The independent variable is Orientation.

Because of the unacceptable Significance values there was not model that could reliably predict the relationship between orientation and daylight autonomy. Orientation is a factor that affects daylight performance in urban environments so it was decided that this factor should remain in the further analysis despite no model being found in this stage of the analysis. Due to the limited variation in results, a variation of just 5\% DA, it is not expected that this will have an impact on the accuracy of the final equation. 


\section{A2.8 Proposed Building Height}

Five variations of proposed building height were simulated and analysed using regression analysis. The following analysis investigates the relationship between proposed building height and daylight autonomy. Figure 10 shows the four models with the best fit for this relationship where daylight autonomy is shown on the $y$-axis and proposed building height is shown on the $x$-axis in metres. In this graph it is difficult to see which of these models is best, therefore the SPSS statistical analysis was needed to determine which model best described this relationship.

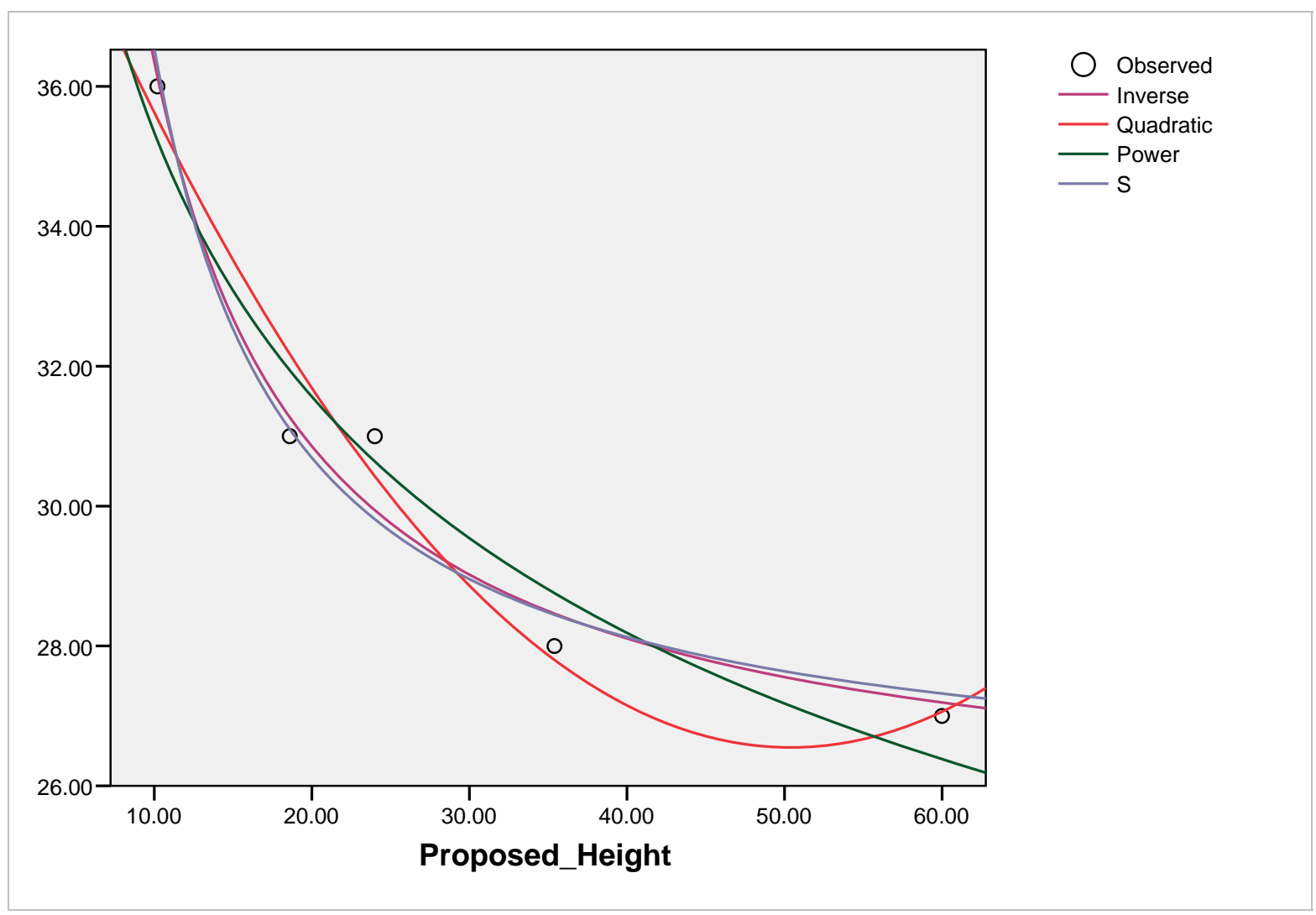

Figure 10 - Proposed Building Height: Regression Models

Table 15 shows the statistics on the fit of each of the models for the relationship between daylight autonomy and proposed building height. From this table it can be seen that all the R Square values were above the minimum acceptable level for statistical significance of 0.7 . The Significance level were not as good, with 2 being above the acceptable level of 0.05 and 5 being near this value. From this table the model with the best overall result was the inverse model. 
Table 15 - Proposed Building Height: Model Summary

Dependent Variable: DA

\begin{tabular}{|c|c|c|c|c|c|c|c|c|c|}
\hline \multirow[b]{2}{*}{ Equation } & \multicolumn{5}{|c|}{ Model Summary } & \multicolumn{4}{|c|}{ Parameter Estimates } \\
\hline & R Square & $\mathrm{F}$ & df1 & $\mathrm{df} 2$ & Sig. & Constant & b1 & b2 & b3 \\
\hline Linear & .761 & 9.553 & 1 & 3 & .054 & 35.304 & -.159 & & \\
\hline Logarithmic & .931 & 40.684 & 1 & 3 & .008 & 46.921 & -5.073 & & \\
\hline Inverse & .970 & 97.537 & 1 & 3 & .002 & 25.358 & 109.946 & & \\
\hline Quadratic & .960 & 24.041 & 2 & 2 & .040 & 40.676 & -.561 & .006 & \\
\hline Cubic & .969 & 10.561 & 3 & 1 & .222 & 43.477 & -.926 & .018 & .000 \\
\hline Compound & .792 & 11.451 & 1 & 3 & .043 & 35.488 & .995 & & \\
\hline Power & .945 & 51.925 & 1 & 3 & .006 & 51.470 & -.163 & & \\
\hline $\mathrm{s}$ & .960 & 72.451 & 1 & 3 & .003 & 3.249 & 3.493 & & \\
\hline Growth & .792 & 11.451 & 1 & 3 & .043 & 3.569 & -.005 & & \\
\hline Exponential & .792 & 11.451 & 1 & 3 & .043 & 35.488 & -.005 & & \\
\hline Logistic & .792 & 11.451 & 1 & 3 & .043 & .028 & 1.005 & & \\
\hline
\end{tabular}

The independent variable is Proposed_Height.

The inverse model was selected as the best model for this relationship, so the following equation was created using the parameter values from Table 15.

$$
D A=29.398+109.946+P B H
$$

From the analysis of the relationship between proposed building height and daylight autonomy, it can be concluded that there is a strong correlation between these two variables. The relationship is curvilinear and the model that best describes the relationship is an inverse model. 


\section{A2.9 Reflectance}

10 variations of reflectance were simulated and analysed using regression analysis. The following analysis investigates the relationship between reflectance and daylight autonomy. Figure 11 shows the four models that provide the best fit for this relationship, where the daylight autonomy values are given on the $y$-axis and the reflectance values are given on the $x$-axis in percentages. This figure shows that the curvilinear models fit the data better, but the linear model also has a reasonable fit.

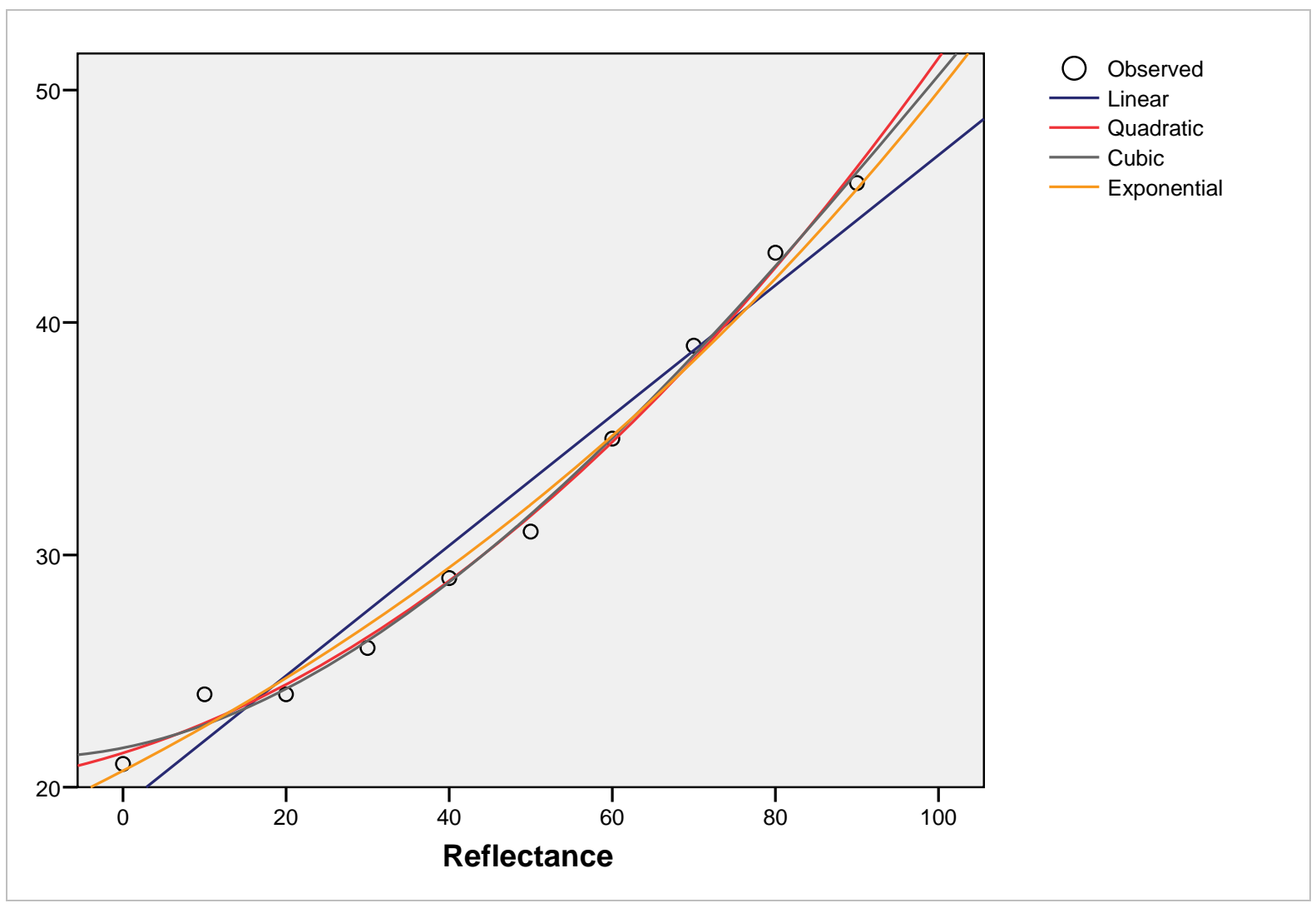

Figure 11 - Reflectance: Regression Models

The SPSS analysis of fit is shown in Table 16, where R Square value and significance levels were used to select the best model. From this table it can be seen that the quadratic and cubic model have a near perfect fit. The fit of the linear model is not as strong as the $\mathrm{R}$ Square value is slightly lower but the statistics indicate that this model would still provide a strong prediction of results. 
Table 16 - Reflectance: Model Summary

Dependent Variable: DA

\begin{tabular}{|c|c|c|c|c|c|c|c|c|c|}
\hline \multirow[b]{2}{*}{ Equation } & \multicolumn{5}{|c|}{ Model Summary } & \multicolumn{4}{|c|}{ Parameter Estimates } \\
\hline & R Square & $\mathrm{F}$ & df1 & $\mathrm{df} 2$ & Sig. & Constant & b1 & b2 & b3 \\
\hline Linear & .966 & 226.958 & 1 & 8 & .000 & 19.200 & .280 & & \\
\hline Logarithmic & .347 & 4.243 & 1 & 8 & .073 & 27.860 & 1.480 & & \\
\hline Inverse & .194 & 1.921 & 1 & 8 & .203 & 33.001 & -.012 & & \\
\hline Quadratic & .994 & 603.539 & 2 & 7 & .000 & 21.473 & .110 & .002 & \\
\hline Cubic & .995 & 366.412 & 3 & 6 & .000 & 21.688 & .070 & .003 & $-8.5 E-006$ \\
\hline Compound & .987 & 602.290 & 1 & 8 & .000 & 20.712 & 1.009 & & \\
\hline Power & .419 & 5.770 & 1 & 8 & .043 & 26.900 & .051 & & \\
\hline S & .251 & 2.678 & 1 & 8 & .140 & 3.469 & .000 & & \\
\hline Growth & .987 & 602.290 & 1 & 8 & .000 & 3.031 & .009 & & \\
\hline Exponential & .987 & 602.290 & 1 & 8 & .000 & 20.712 & .009 & & \\
\hline Logistic & .987 & 602.290 & 1 & 8 & .000 & .048 & .991 & & \\
\hline
\end{tabular}

The independent variable is Reflectance.

The model with the best fit for the relationship between reflectance of the opposite building and daylight autonomy was the cubic model but the quadratic model was selected because it was less complex and had a difference in R Square of only 0.001 (or $0.1 \%$ ). Using the parameter values in Table 16 the equation for this relationship was formed, as shown below:

$$
D A=21.473+0.110 R+0.002 R^{2}
$$

From the analysis of the relationship between reflectance of the opposite building and daylight autonomy, it can be concluded that there is a very strong correlation between these two variables. The relationship is curvilinear and the model that best describes the relationship is a quadratic model, however a linear model could be used for simplicity. 


\section{A2.10 Street Width}

Seven variations of street width were simulated and analysed using regression analysis. The following analysis investigates the relationship between street width and daylight autonomy. Figure 12 shows the four models that fit the simulation results best. The daylight autonomy values are given on the $y$-axis and the street widths are given on the $x$-axis in metres. From Figure 12 it was unclear which was the best model and whether this relationship was linear or curvilinear.

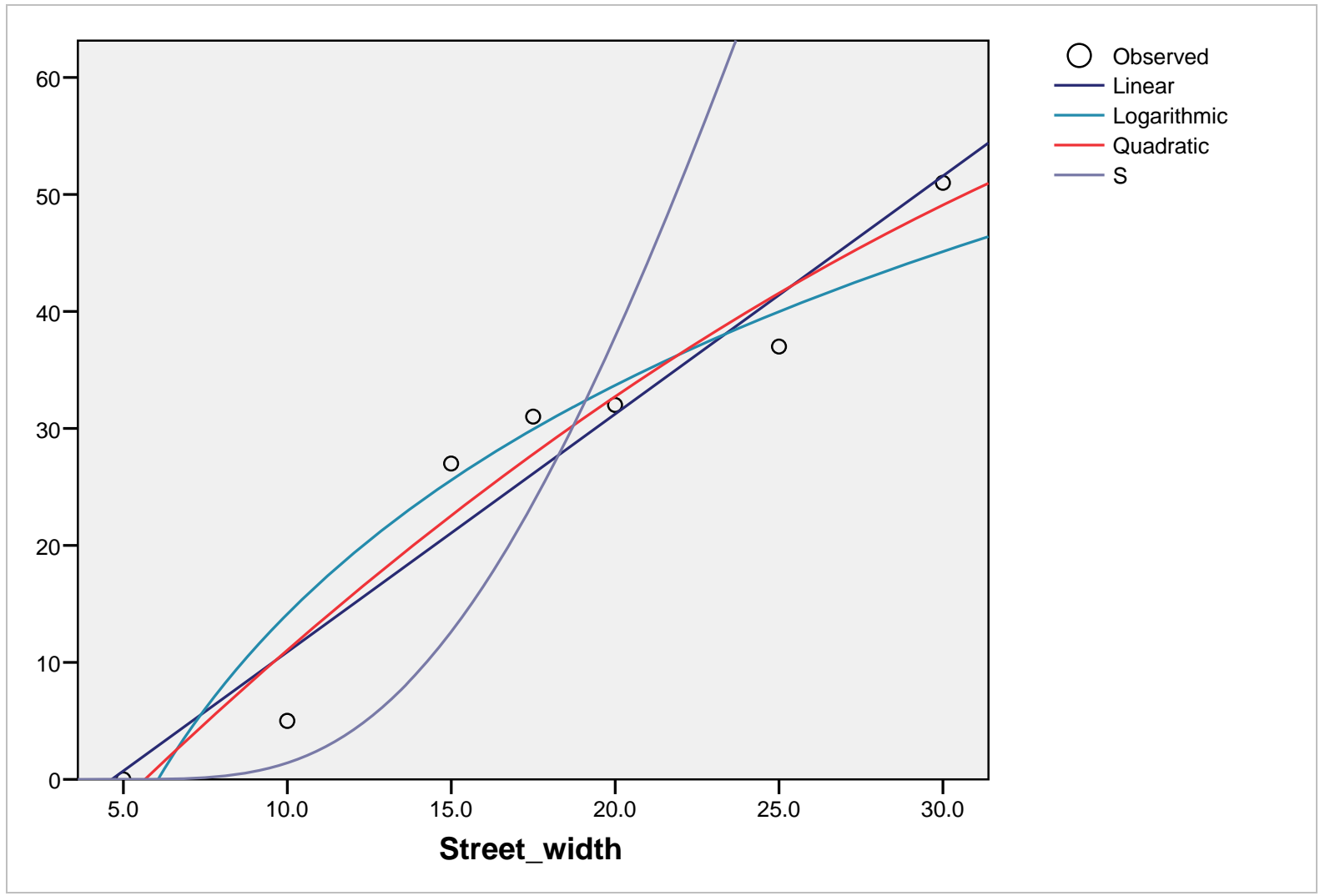

\section{Figure 12 - Street Width: Regression Models}

To determine which model provided the best fit for the relationship between daylight autonomy and street with, the statistical analysis provided by SPSS was consulted. Table 17 shows this analysis, where the key values were the $R$ Square and Significance values. The model with the best $R$ Square and Significance levels was the $S$ model, however by referring back to Figure 12 this model clear does not fit the data points well. The models with the next highest $R$ Square values were cubic and quadratic. Of these models the cubic model had a high Significance level meaning that this model was not as strong as the quadratic model which had a near perfect significance level of 0.002 . The linear model also had a high $\mathrm{R}$ Square and had a Significance level of 0 which indicates a perfect model. 
Table 17 - Street Width: Model Summary

Dependent Variable: DA

\begin{tabular}{|c|c|c|c|c|c|c|c|c|c|}
\hline \multirow[b]{2}{*}{ Equation } & \multicolumn{5}{|c|}{ Model Summary } & \multicolumn{4}{|c|}{ Parameter Estimates } \\
\hline & R Square & $\mathrm{F}$ & df1 & $\mathrm{df} 2$ & Sig. & Constant & b1 & b2 & b3 \\
\hline Linear & .941 & 79.169 & 1 & 5 & .000 & -9.456 & 2.034 & & \\
\hline Logarithmic & .916 & 54.310 & 1 & 5 & .001 & -50.794 & 28.200 & & \\
\hline Inverse & .766 & 16.396 & 1 & 5 & .010 & 47.288 & -270.528 & & \\
\hline Quadratic & .951 & 38.588 & 2 & 4 & .002 & -15.901 & 2.960 & -.026 & \\
\hline Cubic & .951 & 19.439 & 3 & 3 & .018 & -18.467 & 3.605 & -.069 & .001 \\
\hline Compound & .570 & 6.625 & 1 & 5 & .050 & .014 & 1.412 & & \\
\hline Power & .807 & 20.861 & 1 & 5 & .006 & 8.69E-007 & 5.766 & & \\
\hline $\mathrm{s}$ & .958 & 114.248 & 1 & 5 & .000 & 6.928 & -65.902 & & \\
\hline Growth & .570 & 6.625 & 1 & 5 & .050 & -4.260 & .345 & & \\
\hline Exponential & .570 & 6.625 & 1 & 5 & .050 & .014 & .345 & & \\
\hline Logistic & .570 & 6.625 & 1 & 5 & .050 & 70.816 & .708 & & \\
\hline
\end{tabular}

The independent variable is Street_width.

It was decided that the best model to represent the relationship between street width and daylight autonomy was the linear model because it had the best combination of $R$ Square and Significance level and is simpler than the quadratic model, the equation for this relationship is shown below:

\section{$D A=-9.456+2.034 S W$}

From the analysis of the relationship between street width and daylight autonomy, it can be concluded that there is a very strong correlation between these two variables. The model that best describes the relationship is a linear model. 


\section{A2.11 Vertical Location}

Five variations of vertical location were simulated and analysed using regression analysis. The following analysis investigates the relationship between vertical location and daylight autonomy. Figure 13 shows the four models that had the best fit with vertical location (x-axis) and daylight autonomy. The cubic model stands out as having the best fit for this relationship.

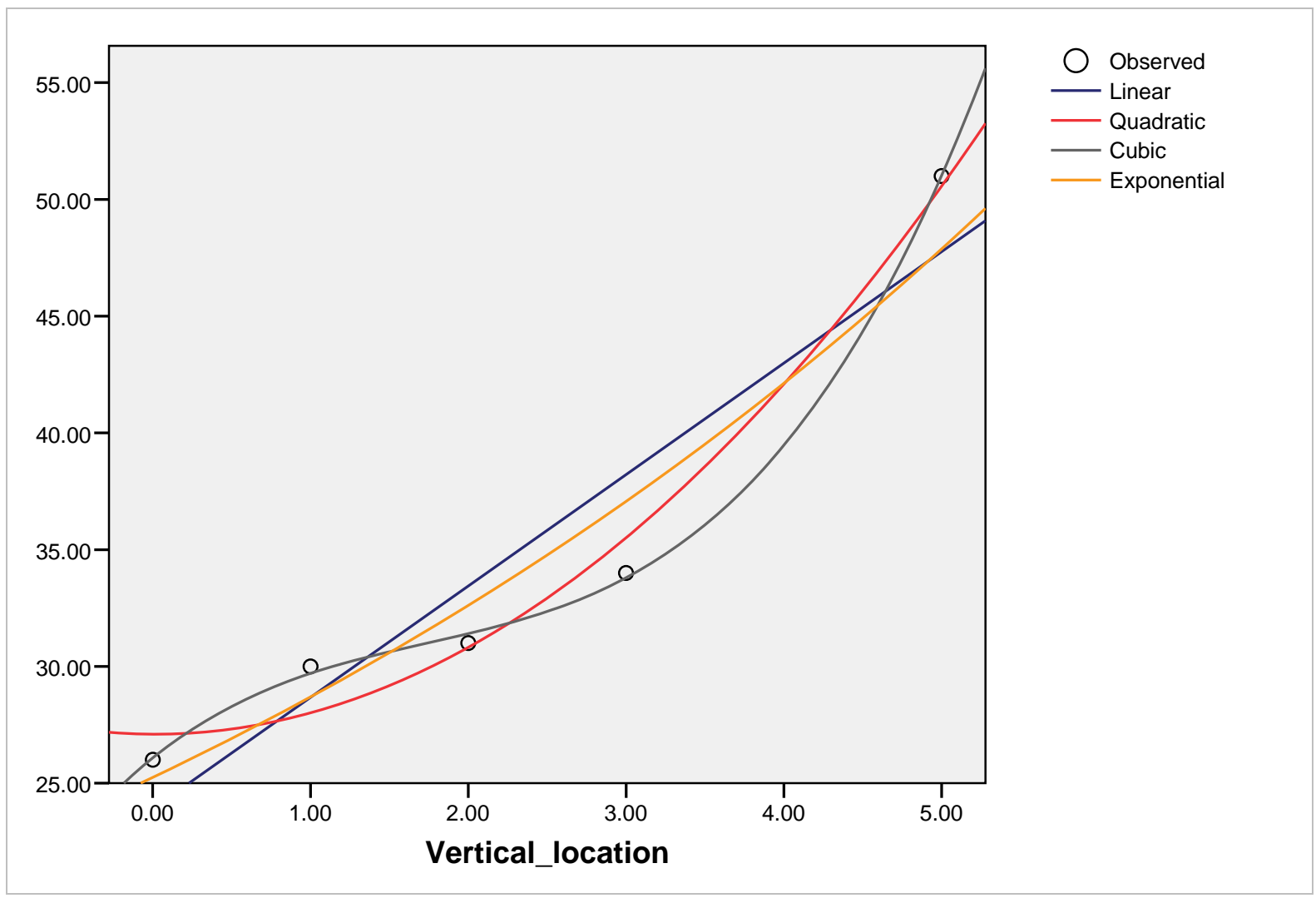

Figure 13 - Vertical Location: Regression Models

The statistics provided by SPSS were check to make sure that the cubic model was the best model for this relationship. Table 18 shows the statistics on the fit of these models. This table confirmed that the cubic model was the best model for this relationship as it had a near perfect $\mathrm{R}$ Square value of 0.999. The Significance value for the cubic model is not the best but is still within the acceptable range. The exponential model also fits the data reasonably well with a high $\mathrm{R}$ Square value and low Significance level. 
Table 18 - Vertical Location: Model Summary

Dependent Variable: DA

\begin{tabular}{|c|c|c|c|c|c|c|c|c|c|}
\hline \multirow[b]{2}{*}{ Equation } & \multicolumn{5}{|c|}{ Model Summary } & \multicolumn{4}{|c|}{ Parameter Estimates } \\
\hline & R Square & $\mathrm{F}$ & df1 & df2 & Sig. & Constant & b1 & b2 & b3 \\
\hline Linear & .893 & 25.011 & 1 & 3 & .015 & 23.903 & 4.771 & & \\
\hline Logarithmic & .359 & 1.683 & 1 & 3 & .285 & 35.560 & 1.654 & & \\
\hline Inverse & .234 & .918 & 1 & 3 & .409 & 36.507 & -.011 & & \\
\hline Quadratic & .980 & 48.254 & 2 & 2 & .020 & 27.098 & -.031 & .945 & \\
\hline Cubic & .999 & 413.107 & 3 & 1 & .036 & 26.076 & 5.442 & -2.254 & .433 \\
\hline Compound & .935 & 43.168 & 1 & 3 & .007 & 25.246 & 1.137 & & \\
\hline Power & .443 & 2.386 & 1 & 3 & .220 & 34.607 & .048 & & \\
\hline $\mathrm{s}$ & .307 & 1.330 & 1 & 3 & .332 & 3.574 & .000 & & \\
\hline Growth & .935 & 43.168 & 1 & 3 & .007 & 3.229 & .128 & & \\
\hline Exponential & .935 & 43.168 & 1 & 3 & .007 & 25.246 & .128 & & \\
\hline Logistic & .935 & 43.168 & 1 & 3 & .007 & .040 & .880 & & \\
\hline
\end{tabular}

The independent variable is Vertical_location.

Based on the results from Figure 13 and the statistics of these models it was decided that the best model for the relationship between daylight autonomy and vertical location was the cubic model. The cubic model visually had the best fit and this was confirmed with the $\mathrm{R}$ Square value. The parameter values from Table 18 were used to develop the following equation for this relationship:

$$
D A=26.076+5.442 V L-2.254 V L^{2}+0.438 V L^{3}
$$

From the analysis of the relationship between vertical location and daylight autonomy, it can be concluded that there is a reasonably strong correlation between these two variables. The relationship is curvilinear, where the model that best describes the relationship is a cubic model. 


\section{Appendix 3 - Analysis of Regression Models}

This appendix provides a detailed analysis of the 7 regression models. Within this appendix, each model is analysed using the SPSS statistics on the fit of the model to the simulation results. Each of these models was also compared against the simulation results to determine the range of errors and how well the model predicted each case. The process of selecting the best models is also summarised. 


\section{Linear Model 1A}

This stage involved the analysis of a simple linear regression prediction model for the relationship between the 9 independent variables and daylight autonomy (dependent variable). The variables were: opposite building height, street width, orientation, glazed area, geographical location, reflectance of the opposite building, vertical location, proposed building height and glazing transmittance.

Table 19 give a summary of how well the model represents the relationship between all variables. The key information drawn from this table is the $\mathrm{R}$ Square value and the Standard Error of the Estimate.

Table 19-1A: Model Summary

\begin{tabular}{|l|r|r|r|r|}
\hline Model & $\mathrm{R}$ & $\mathrm{R}$ Square & $\begin{array}{c}\text { Adjusted } \\
\text { R Square }\end{array}$ & $\begin{array}{r}\text { Std. Error of } \\
\text { the Estimate }\end{array}$ \\
\hline 1 & $.891^{\mathrm{a}}$ & .794 & .788 & 8.5304949 \\
\hline
\end{tabular}

From Table 19 it can be seen that the R Square value is greater than the minimum of 0.7. However, the value of 0.794 is at the lower end of acceptability, indicating that only $79 \%$ of the cases are explained by the model. The standard error for the model is 8.5 , as accuracy is a criteria for the development of this tool, it was hoped that the standard error values were considerably lower, around 2-3\% DA.

Table 20 assesses the acceptability of the model for representing the relationship between the independent variables and daylight autonomy. The key information drawn from this table is the Significance level.

Table 20-1A: Analysis of Variance (ANOVA)

\begin{tabular}{|ll|r|r|r|r|r|}
\hline \multicolumn{2}{|c|}{} & \multicolumn{1}{|c|}{ Sum of } & & & & \\
\hline 1 & Squares & df & Mean Square & \multicolumn{1}{c|}{$\mathrm{F}$} & Sig. \\
\hline & Regression & 89497.578 & 9 & 9944.175 & 136.653 & $.000^{\mathrm{a}}$ \\
& Residual & 23286.190 & 320 & 72.769 & & \\
& Total & 112783.8 & 329 & & & \\
\hline
\end{tabular}

From Table 20 it can be seen that the Significance level of this model is 0 , which is less than the maximum acceptance value of 0.05 . This indicates that the model describes the relationship very well. 
Table 21 provides the coefficient values for each variable. The key information drawn from this table is the coefficients, the Standard Errors and Significance level for each variable and the constant.

Table $21-1 \mathrm{~A}$ : Coefficients

\begin{tabular}{|c|c|c|c|c|c|c|}
\hline \multirow{2}{*}{\multicolumn{2}{|c|}{ Model }} & \multicolumn{2}{|c|}{$\begin{array}{c}\text { Unstandardized } \\
\text { Coefficients }\end{array}$} & \multirow{2}{*}{$\begin{array}{c}\begin{array}{c}\text { Standardized } \\
\text { Coefficients }\end{array} \\
\text { Beta }\end{array}$} & \multirow[b]{2}{*}{$\mathrm{t}$} & \multirow[b]{2}{*}{ Sig. } \\
\hline & & B & Std. Error & & & \\
\hline \multirow[t]{10}{*}{1} & (Constant) & -49.269 & 19.777 & & -2.491 & .013 \\
\hline & Building_height & -.477 & .025 & -.492 & -19.334 & .000 \\
\hline & Street_width & 1.873 & .088 & .540 & 21.250 & .000 \\
\hline & Orientation & .026 & .010 & .069 & 2.714 & .007 \\
\hline & Glazed_area & 1.290 & .082 & .402 & 15.761 & .000 \\
\hline & Location & -1.392 & .263 & -.134 & -5.290 & .000 \\
\hline & Reflectance & .251 & .025 & .261 & 10.255 & .000 \\
\hline & Vertical_location & 4.185 & 1.825 & .058 & 2.293 & .022 \\
\hline & Prop_BH & -.060 & .127 & -.016 & -.470 & .639 \\
\hline & Transmittance & .299 & .199 & .051 & 1.504 & .134 \\
\hline
\end{tabular}

On first glance at Table 21 it appear that the standard errors are generally quite low with the exception of the Constant and Vertical Location, when these errors ranges are explored in Table 22 it becomes apparent that a number of these standard errors are quite significant. The factors with high standard errors were: the Constant, Orientation, Location, Vertical Location, Proposed Building Height and Glazing Transmittance. The Significance levels found in Table 21 were generally quite good. Most Significance levels were below the maximum acceptable level of 0.05, with 5 of the 9 variables have significance levels of 0 . Two variables stood out as having unacceptably high significance levels, these were Proposed Building Height and Glazing Transmittance.

Table 22 -1A: Coefficient Errors

\begin{tabular}{|l|c|c|c|}
\hline \multicolumn{1}{|c|}{ Variables } & Coefficients & Upper Error & Lower Error \\
\hline Constant & -49.269 & -29.492 & -69.046 \\
\hline Building Height & -0.477 & -0.452 & -0.502 \\
\hline Street Width & 1.873 & 1.961 & 1.785 \\
\hline Orientation & 0.026 & 0.036 & 0.016 \\
\hline Glazed Area & 1.290 & 1.372 & 1.208 \\
\hline Location & -1.392 & -1.129 & -1.655 \\
\hline Reflectance & 0.251 & 0.276 & 0.226 \\
\hline Vertical Location & 4.185 & 6.010 & 2.360 \\
\hline Proposed Building Height & -0.060 & 0.067 & -0.187 \\
\hline Glazing Transmittance & 0.299 & 0.498 & 0.100 \\
\hline
\end{tabular}

Using the coefficients given in Table 21, the following equation was developed for this model.

$$
\begin{gathered}
D A=-49-0.48 B H+1.9 S W+0.0260 r+1.3 G A-1.4 L+0.25 E+4.2 V L \\
-0.06 P B H+0.30 T
\end{gathered}
$$

Figure 14 is a scatter diagram showing the range of errors present between the simulated result and the predicted result. These error values were found by subtracting the predicted value from the 
simulated value. In this graph, the values below 0 are over-predictions and the values above 0 are under-predictions.

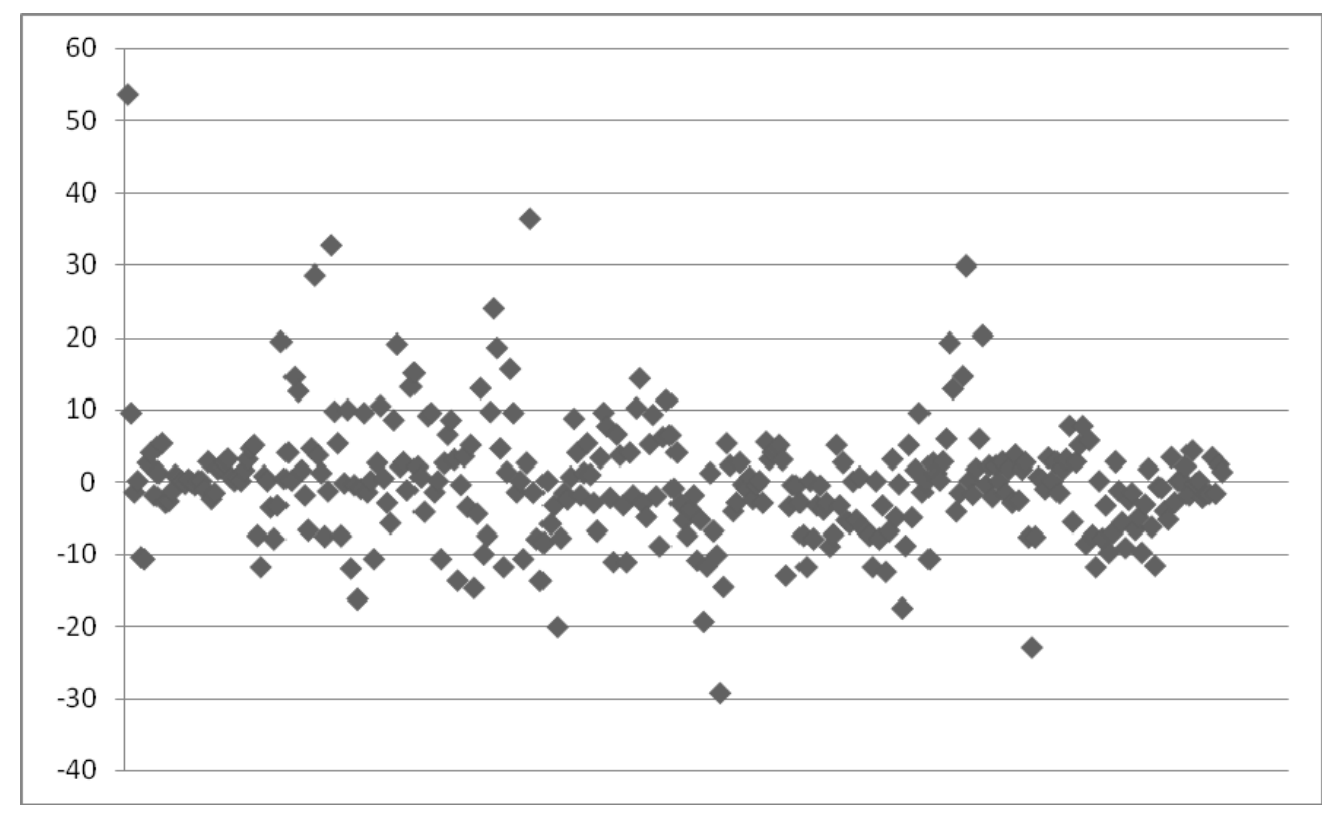

Figure $14-1 \mathrm{~A}$ : Errors in Predictions

From Figure 14 it can be seen that the errors range from -29 to 54\% DA, with most of the cases having errors less than 15\% DA. Approximately half the cases were under-predicted and half were over-predicted. Given the wide error range, it was felt that this model was not suitable for use in the compliance assessment tool. Another concern with this model was the 50/50 split, because this prediction model needs to clearly discriminate between passing and failing apartments, overpredictions could result in apartments being given a pass result when they would in reality not meet the minimum requirement. The ideal result from this graph would have been errors less than 5 , with the majority of cases above 0 (under-predictions).

Figure 15 is a scatter plot that shows the relationship between the simulated results ( $x$-axis) and predicted results (y-axis). The ideal trend line is shown in black, where the predicted results are directly proportional to the simulated results. 


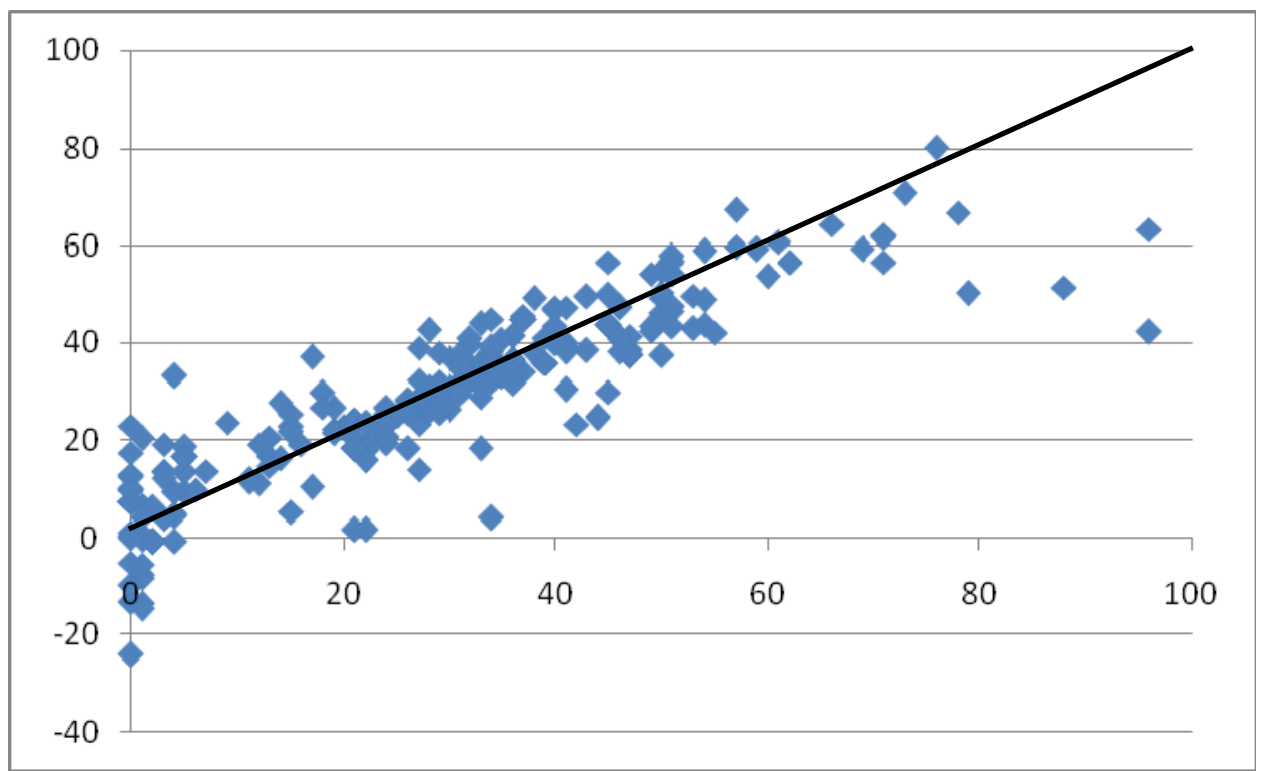

Figure 15-1A: Prediction Accuracy compared to Simulation Results

Figure 15 identifies that generally the prediction model is performing reasonably well. However, there are two sections in particular where the results do not follow the trend line, these being for the best and worst performing apartments. The worst performing apartments (less than $10 \%$ DA) have significant errors in the prediction, with errors up to $20 \%$ DA. However the errors in the lowest range are not of concern because they would still be well under the threshold value of $75 \%$ DA and would consequently still be give a fail result. The inaccurate predictions in the upper range are of great concern. One of the aims of the tool was to identify when apartment would not need simulations as they were not at risk of failing, but if the tool is under-predicting the result in the higher range then these good apartment would be required to conduct simulations when they were not necessary to prove compliance.

The results from the regression analysis indicated that generally this prediction model would perform reasonably well, with a Significance level of 0 and a number of the coefficients having significance levels of 0 and small standard errors. However the R Square value and Standard Error for the prediction model indicated that there may be some inaccuracies in the predictions. This was confirmed when the predicted results were compared to the simulated results for each case, where high error rates and inaccuracy in the apartments with the highest simulated results were observed. An aspect of the prediction model that stood out was the poor Significance level and extremely high Standard error for Proposed Building Height. Glazing transmittance also had a poor significance level and reasonably high standard error, but was not as bad as that of proposed building height. 


\section{Linear Model 1B}

This stage involved the analysis of a simple linear regression prediction model for the relationship between 8 independent variables and daylight autonomy (dependent variable), with the exclusion of proposed building height following the analysis from Model $1 \mathrm{~A}$.

Table 23 give a summary of how well the model represents the relationship between all variables. The key information drawn from this table is the $\mathrm{R}$ Square value and the Standard Error of the Estimate.

Table 23 -1B: Model Summary

\begin{tabular}{|l|r|r|r|r|}
\hline Model & R & R Square & $\begin{array}{c}\text { Adjusted } \\
\text { R Square }\end{array}$ & $\begin{array}{r}\text { Std. Error of } \\
\text { the Estimate }\end{array}$ \\
\hline 1 & $.891^{\mathrm{a}}$ & .793 & .788 & 8.5201311 \\
\hline
\end{tabular}

From Table 23 it can be seen that the R Square value is again greater than the minimum of 0.7 . However, the value of 0.793 is at the lower end of acceptability again and has a fractionally less successful prediction rate than the model from Stage 1A, with a difference of just 0.001. Again, the standard error is 8.5 which is well above the desired error of $2-3 \%$ DA.

Table 24 assesses the acceptability of the model for representing the relationship between the independent variables and daylight autonomy. The key information drawn from this table is the Significance level.

Table 24 -1B: Analysis of Variance (ANOVA)

\begin{tabular}{|ll|r|r|r|r|r|}
\hline & & Sum of & & & & \\
Model & & Squares & df & Mean Square & \multicolumn{1}{c|}{$\mathrm{F}$} & Sig. \\
\hline 1 & Regression & 89481.532 & 8 & 11185.191 & 154.082 & $.000^{\mathrm{a}}$ \\
& Residual & 23302.235 & 321 & 72.593 & & \\
& Total & 112783.8 & 329 & & & \\
\hline
\end{tabular}

From Table 24 it can be seen that the Significance level of this model is 0 again, which is less than the maximum acceptance value of 0.05 . This indicates that the model describes the relationship very well. 
Table 25 provides the coefficient values for each variable. The key information drawn from this table is the coefficients, the Standard Errors and Significance level for each variable and the constant.

Table 25 -1B: Coefficients

\begin{tabular}{|c|c|c|c|c|c|c|}
\hline \multirow{2}{*}{\multicolumn{2}{|c|}{ Model }} & \multicolumn{2}{|c|}{$\begin{array}{c}\text { Unstandardized } \\
\text { Coefficients }\end{array}$} & \multirow{2}{*}{$\begin{array}{c}\begin{array}{c}\text { Standardized } \\
\text { Coefficients }\end{array} \\
\text { Beta }\end{array}$} & \multirow[b]{2}{*}{$\mathrm{t}$} & \multirow[b]{2}{*}{ Sig. } \\
\hline & & B & Std. Error & & & \\
\hline \multirow[t]{9}{*}{1} & (Constant) & -56.089 & 13.408 & & -4.183 & .000 \\
\hline & Building_height & -.477 & .025 & -.492 & -19.352 & .000 \\
\hline & Street_width & 1.873 & .088 & .540 & 21.276 & .000 \\
\hline & Orientation & .026 & .010 & .070 & 2.740 & .006 \\
\hline & Glazed_area & 1.291 & .082 & .402 & 15.795 & .000 \\
\hline & Location & -1.391 & .263 & -.134 & -5.295 & .000 \\
\hline & Reflectance & .251 & .024 & .260 & 10.264 & .000 \\
\hline & Vertical_location & 4.193 & 1.823 & .058 & 2.300 & .022 \\
\hline & Transmittance & .360 & .150 & .061 & 2.407 & .017 \\
\hline
\end{tabular}

Again, it appears that the standard errors in Table 25 are generally quite low with the exception of the Constant and Vertical Location. Table 26 identified that the factors with high standard errors were almost unchanged from the Stage $1 \mathrm{~A}$ analysis, these factors were: the Constant, Orientation, Location, Vertical Location and Glazing Transmittance. The Significance levels found in Table 25 were generally quite good. All Significance levels were below the maximum acceptable level of 0.05 , with 5 of the 8 variables have significance levels of 0 . Considerable reductions were seen in significance levels for the constant and glazing transmittance.

Table 26 -1B: Coefficient Errors

\begin{tabular}{|l|c|c|c|}
\hline \multicolumn{1}{|c|}{ Variables } & Coefficients & Upper Error & Lower Error \\
\hline Constant & -56.089 & -42.681 & -69.497 \\
\hline Building Height & -0.477 & -0.452 & -0.502 \\
\hline Street Width & 1.873 & 1.961 & 1.785 \\
\hline Orientation & 0.026 & 0.036 & 0.016 \\
\hline Glazed Area & 1.291 & 1.373 & 1.209 \\
\hline Location & -1.391 & -1.128 & -1.654 \\
\hline Reflectance & 0.251 & 0.275 & 0.227 \\
\hline Vertical Location & 4.193 & 6.016 & 2.370 \\
\hline Glazing Transmittance & 0.360 & 0.510 & 0.210 \\
\hline
\end{tabular}

Using the coefficients given in Table 25, the following equation was developed for this model.

$$
\begin{gathered}
E A=-56-0.48 B E+1.9 S W+0.0260 x+1.3 G A-1.4 L+0.25 R+4.2 V E \\
+0.3 E T
\end{gathered}
$$

Figure 16 is a scatter diagram showing the range of errors present between the simulated result and the predicted result. This graph follows the format used in Figure 14, where the results below 0 are over-predictions and the results above 0 are under-predictions. 


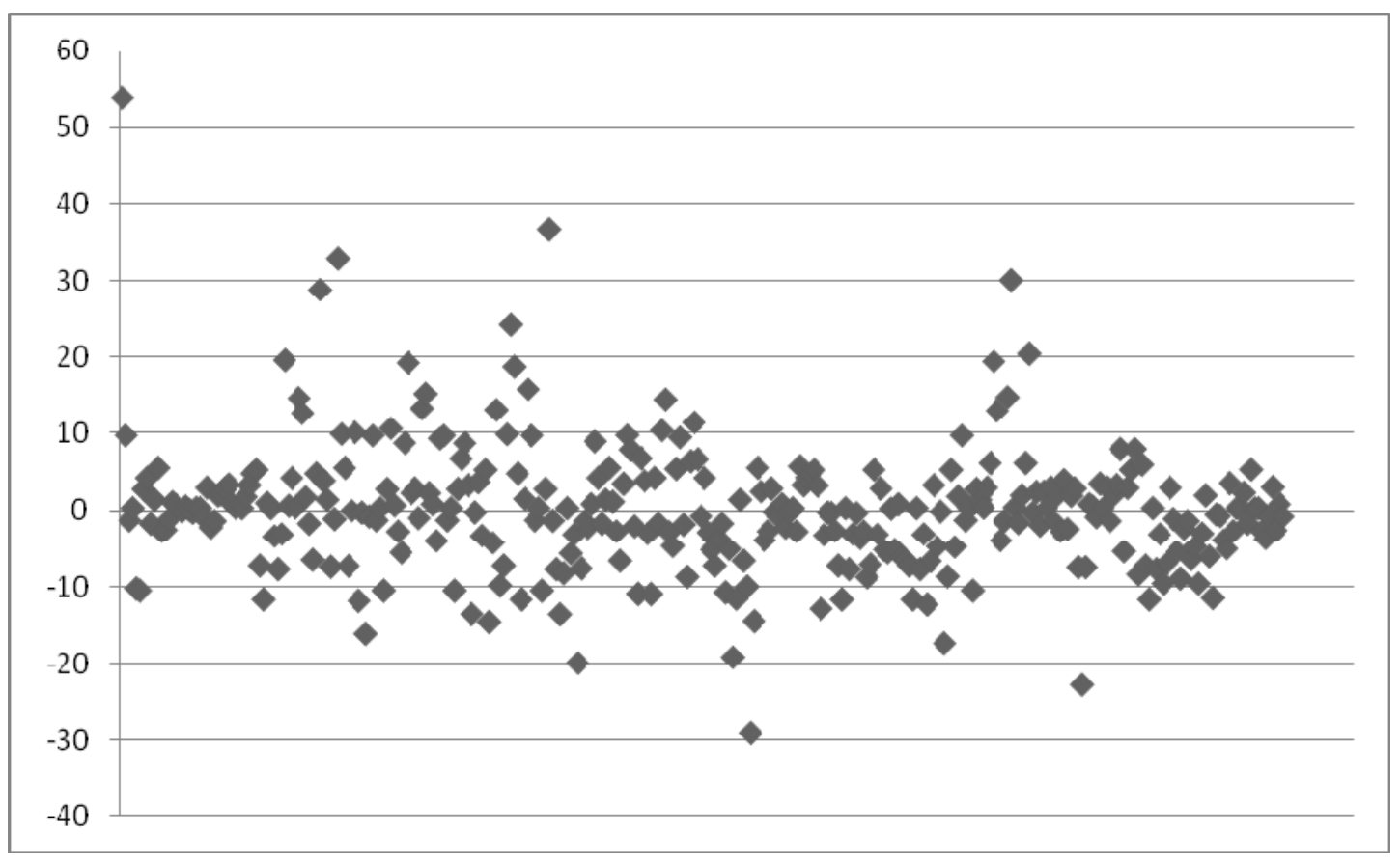

Figure $16-1 B$ : Errors in Predictions

Figure 16 shows no significant change from Figure 14 . The errors still range from -29 to $54 \%$ DA, with most cases having errors of $15 \%$ DA or less. Also, around half the cases are under-predicted and half over-predicted. This indicates that the changes made to the prediction model, removing proposed building height, has had little to no effect on the error in predictions.

Figure 17 is a scatter plot that shows the relationship between the simulated results ( $x$-axis) and predicted results (y-axis). The ideal trend line is shown in black, where the predicted results are directly proportional to the simulated results.

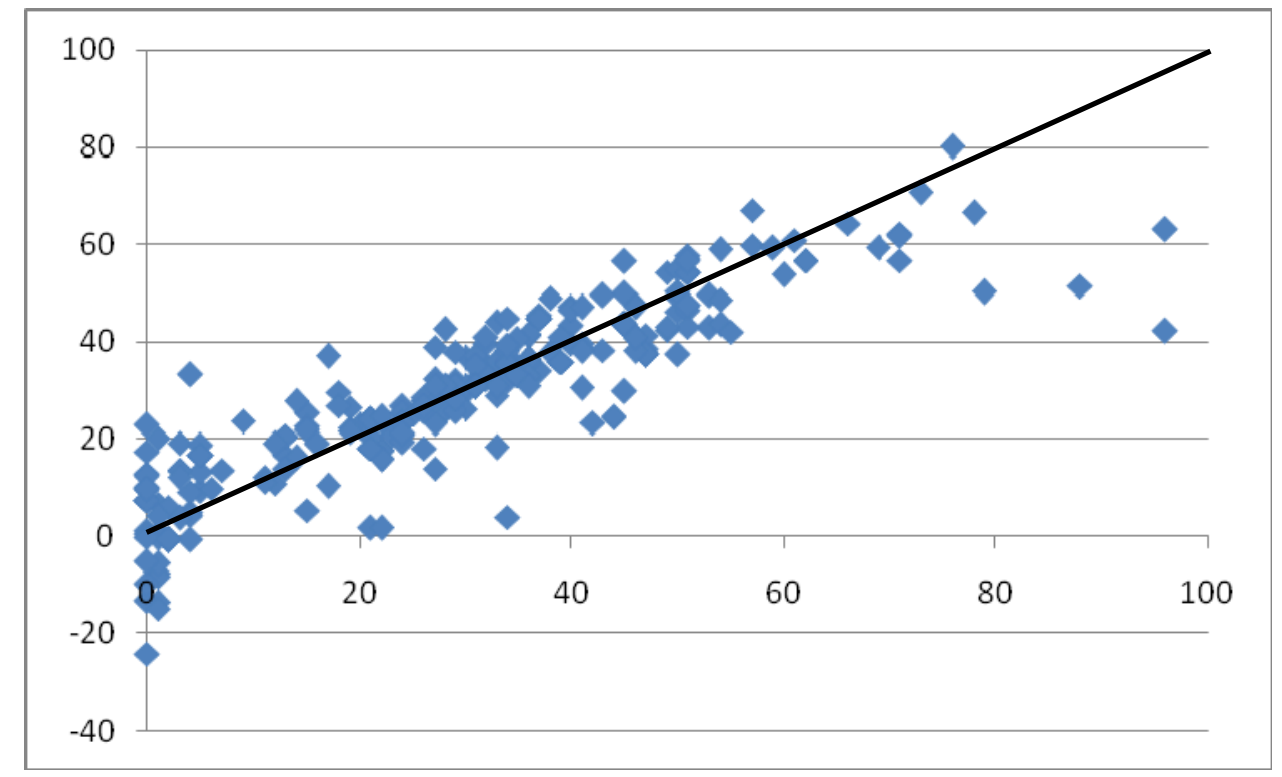

Figure 17-1B: Prediction Accuracy compared to Simulation Results 
Figure 17 identifies that there has been no significant improvement in the accuracy of the prediction model. The inaccuracies in the high range are still present which means this model is not suitable for use in the tool.

As with the model produced in $1 \mathrm{~A}$, it was expected the model would perform reasonably well because of the Significance level of 0 for the overall model and a number of the coefficients. A significant improvement was seen for the constant, with the Significance level decreasing to 0 and the Standard error also decreasing considerably. However, little change was observed in the comparisons between the simulated results and the predicted results, with high errors and inaccuracies in the high range still present. 


\section{Linear Model 1C}

This stage involved the analysis of a simple linear regression prediction model for the relationship between 7 independent variables and daylight autonomy (dependent variable), with the exclusion of proposed building height and glazing transmittance following the analysis from $1 \mathrm{~A}$.

Table 27 give a summary of how well the model represents the relationship between all variables. The key information drawn from this table is the $\mathrm{R}$ Square value and the Standard Error of the Estimate.

Table 27-1C: Model Summary

\begin{tabular}{|l|r|r|r|r|}
\hline Model & R & R Square & $\begin{array}{c}\text { Adjusted } \\
\text { R Square }\end{array}$ & $\begin{array}{r}\text { Std. Error of } \\
\text { the Estimate }\end{array}$ \\
\hline 1 & $.889^{\mathrm{a}}$ & .790 & .785 & 8.5833447 \\
\hline
\end{tabular}

From Table 27 it can be seen that the R Square value is again greater than the minimum of 0.7 . However, the value of 0.790 is at the lower end of acceptability, as with the two previous models, this value indicates that just over $20 \%$ of the cases are not explained by this equation. Again, the standard error is 8.5 which is well above the desired error of $2-3 \%$ DA.

Table 28 assesses the acceptability of the model for representing the relationship between the independent variables and daylight autonomy. The key information drawn from this table is the Significance level.

Table 28 -1C: Analysis of Variance (ANOVA)

\begin{tabular}{|ll|r|r|r|r|r|}
\hline & & Sum of & & & & \\
\hline 1 & & Squares & df & Mean Square & \multicolumn{1}{c|}{$\mathrm{F}$} & Sig. \\
\hline & Regression & 89060.802 & 7 & 12722.972 & 172.693 & $.000^{\mathrm{a}}$ \\
& Residual & 23722.966 & 322 & 73.674 & & \\
& Total & 112783.8 & 329 & & & \\
\hline
\end{tabular}

From Table 28 it can be seen that the Significance level of this model is also 0 , which is less than the maximum acceptance value of 0.05 . This indicates that the model describes the relationship very well.

provides the coefficient values for each variable. The key information drawn from this table is the coefficients, the Standard Errors and Significance level for each variable and the constant. 
Table 29 -1C: Coefficients

\begin{tabular}{|c|c|c|c|c|c|c|}
\hline \multirow{2}{*}{\multicolumn{2}{|c|}{ Model }} & \multicolumn{2}{|c|}{$\begin{array}{c}\text { Unstandardized } \\
\text { Coefficients }\end{array}$} & \multirow{2}{*}{$\begin{array}{c}\text { Standardized } \\
\text { Coefficients } \\
\text { Beta }\end{array}$} & \multirow[b]{2}{*}{$\mathrm{t}$} & \multirow[b]{2}{*}{ Sig. } \\
\hline & & $\mathrm{B}$ & Std. Error & & & \\
\hline \multirow[t]{8}{*}{1} & (Constant) & -25.077 & 3.746 & & -6.694 & .000 \\
\hline & Building_height & -.475 & .025 & -.490 & -19.150 & .000 \\
\hline & Street_width & 1.873 & .089 & .540 & 21.119 & .000 \\
\hline & Orientation & .027 & .010 & .073 & 2.825 & .005 \\
\hline & Glazed_area & 1.295 & .082 & .404 & 15.736 & .000 \\
\hline & Location & -1.389 & .265 & -.134 & -5.246 & .000 \\
\hline & Reflectance & .251 & .025 & .260 & 10.171 & .000 \\
\hline & Vertical_location & 4.231 & 1.836 & .059 & 2.304 & .022 \\
\hline
\end{tabular}

From Table 29 the Standard Errors appear to be quite low, when these errors are explored further in Table 30 it becomes apparent that a number of these standard errors are quite significant. The factors with high standard errors were: the Constant, Orientation, Location and Vertical Location. The Significance levels found in Table 29 were generally good. All Significance levels were below the maximum acceptable level of 0.05 , with a 5 of the 7 variables having significance levels of 0 . The only variable with a noticeably high Significance level was Vertical Location.

Table $30-1 \mathrm{C}$ : Coefficient Errors

\begin{tabular}{|l|c|c|c|}
\hline \multicolumn{1}{|c|}{ Variables } & Coefficients & Upper Error & Lower Error \\
\hline Constant & -25.077 & -21.331 & -28.823 \\
\hline Building Height & -0.475 & -0.450 & -0.500 \\
\hline Street Width & 1.873 & 1.962 & 1.784 \\
\hline Orientation & 0.027 & 0.037 & 0.017 \\
\hline Glazed Area & 1.295 & 1.377 & 1.213 \\
\hline Location & -1.389 & -1.124 & -1.654 \\
\hline Reflectance & 0.251 & 0.276 & 0.226 \\
\hline Vertical Location & 4.231 & 6.067 & 2.395 \\
\hline
\end{tabular}

Using the coefficients given in Table 29, the following equation was developed for this model.

$$
D A-25 \quad 0.400 L\left|1.2 S W^{*}\right| 0.027 Q_{N}|1.39 A \quad 1.4 L| 0.25 R \mid 4.2 V L
$$

Figure 18 is a scatter diagram showing the range of errors present between the simulated result and the predicted result. This graph follows the format used in Figure 14, where the results below 0 are over-predictions and the results above 0 are under-predictions. 


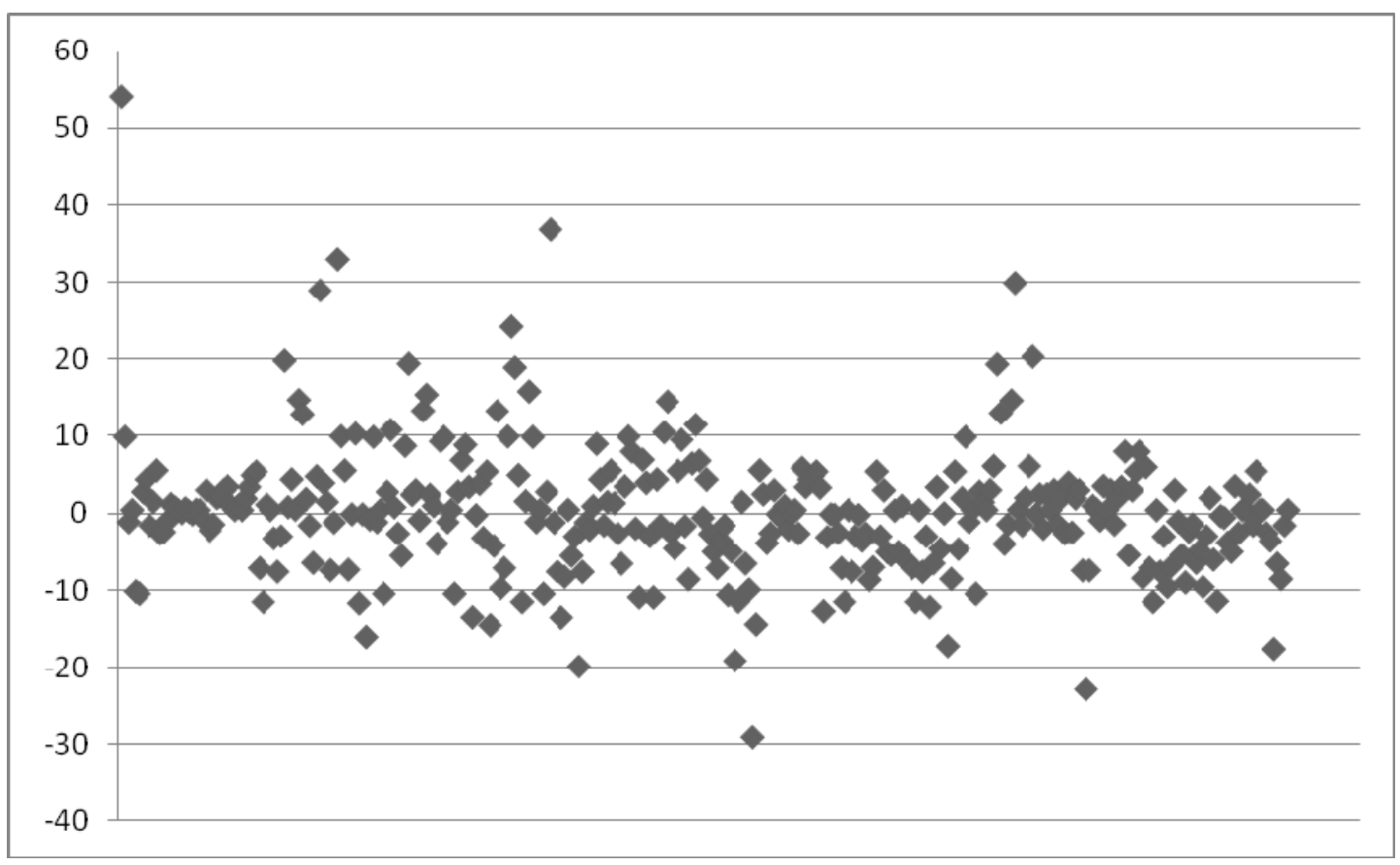

Figure 18-1C: Errors in Predictions

Figure 18 shows no significant change from Figure 14 and Figure 16 . The errors still range from -29 to $54 \%$ DA, with most cases having errors of 15\% DA or less. Again, around half the cases are underpredicted and half over-predicted. This indicates that the changes made to the prediction model, removing proposed building height and glazing transmittance, has had little to no effect on the error in predictions.

Figure 19 is a scatter plot that shows the relationship between the simulated results ( $x$-axis) and predicted results ( $y$-axis). The ideal trend line is shown in black, where the predicted results are directly proportional to the simulated results.

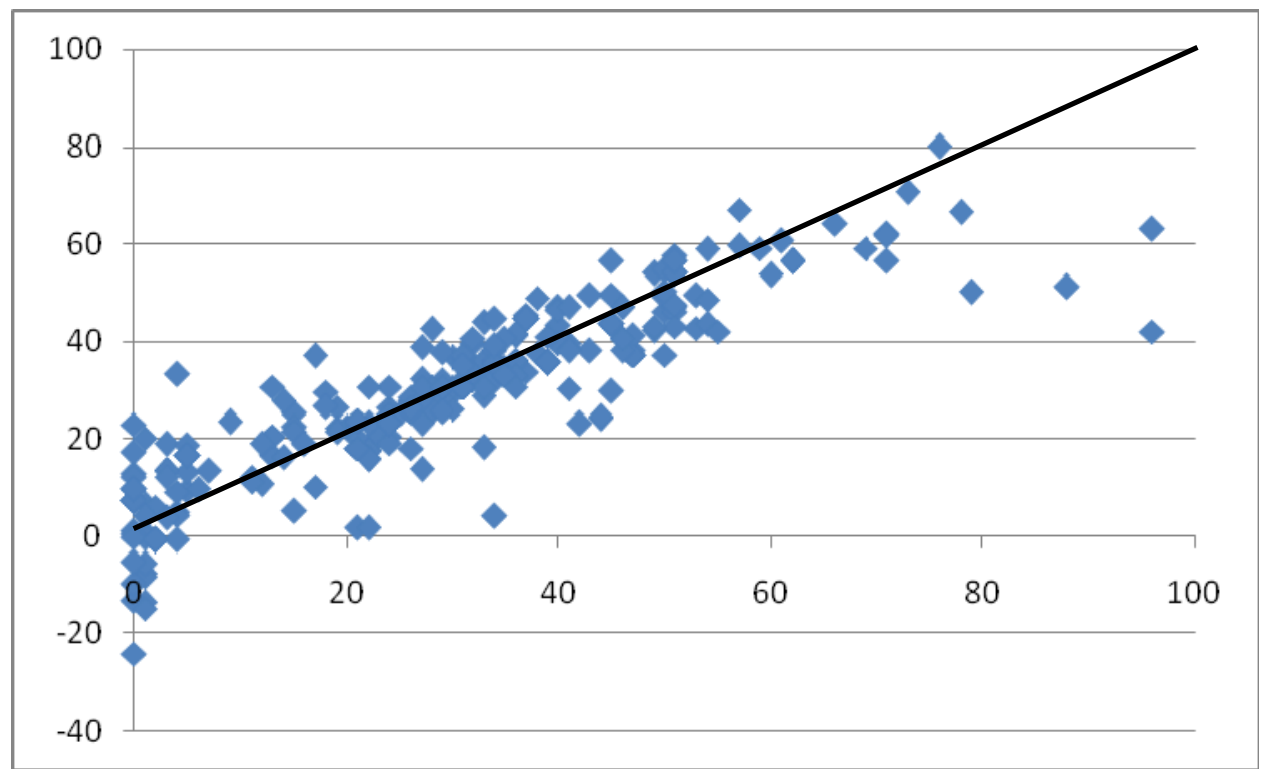

Figure 19-1C: Prediction Accuracy compared to Simulation Results 
Figure 19 found that again, there has been no significant improvement in the accuracy of the prediction model. The inaccuracies in the high range are still present which means this model is not suitable for use in the tool.

As with the model produced in stages $1 \mathrm{~A}$ and $1 \mathrm{~B}$, it was expected the model would perform reasonably well because of the Significance level of 0 for the overall model and a number of the coefficients. Further improvement was found in the standard error for the constant. But a decrease in the R Square value and an increase in the Standard Error meant that this was the weakest of the models so far and as such was not expected to yield any better result that the previous models. 


\section{Linear Model 1D}

This stage involved the analysis of a simple linear regression prediction model for the relationship between the 6 primary independent variables and daylight autonomy (dependent variable). The variables were: opposite building height, street width, orientation, glazed area, geographical location, reflectance of the opposite building.

Table 31 give a summary of how well the model represents the relationship between all variables. The key information drawn from this table is the $\mathrm{R}$ Square value and the Standard Error of the Estimate.

Table 31 -1D: Model Summary

\begin{tabular}{|l|r|r|r|r|}
\hline Model & \multicolumn{1}{|c|}{$\mathrm{R}$} & R Square & $\begin{array}{c}\text { Adjusted } \\
\text { R Square }\end{array}$ & $\begin{array}{r}\text { Std. Error of } \\
\text { the Estimate }\end{array}$ \\
\hline 1 & $.887^{\mathrm{a}}$ & .786 & .782 & 8.6404098 \\
\hline
\end{tabular}

From Table 31 it can be seen that the R Square value is greater than the minimum of 0.7. Again, this means over $20 \%$ of the cases are not explained by this model. This is the least successful of the four models tested in terms of $\mathrm{R}$ Square values, as there is a difference of 0.008 between the R Square for this model and that of the first model. The standard error is 8.6 which is slightly higher than the standard error of 8.5 for the other models and is well above the desired error of $2-3 \%$ DA.

Table 32 assesses the acceptability of the model for representing the relationship between the independent variables and daylight autonomy. The key information drawn from this table is the Significance level.

Table 32 -1D: Analysis of Variance (ANOVA)

\begin{tabular}{|ll|r|r|r|r|r|}
\hline \multicolumn{1}{|c|}{} & \multicolumn{1}{c|}{$\begin{array}{c}\text { Sum of } \\
\text { Sodel }\end{array}$} & \multicolumn{1}{c|}{ Squares } & df & Mean Square & \multicolumn{1}{c|}{$\mathrm{F}$} & Sig. \\
\hline 1 & Regression & 88668.886 & 6 & 14778.148 & 197.948 & $.000^{\mathrm{a}}$ \\
& Residual & 24114.108 & 323 & 74.657 & & \\
& Total & 112783.0 & 329 & & & \\
\hline
\end{tabular}

From Table 32 it can be seen that the Significance level of this model is also 0 , which is less than the maximum acceptance value of 0.05 , indicating that the model describes the relationship very well.

Table 33 provides the coefficient values for each variable. The key information drawn from this table is the coefficients, the Standard Errors and Significance level for each variable and the constant. 
Table 33 -1D: Coefficients

\begin{tabular}{|c|c|c|c|c|c|c|}
\hline \multirow{2}{*}{\multicolumn{2}{|c|}{ Model }} & \multicolumn{2}{|c|}{$\begin{array}{c}\text { Unstandardized } \\
\text { Coefficients }\end{array}$} & \multirow{2}{*}{$\begin{array}{c}\text { Standardized } \\
\text { Coefficients }\end{array}$} & \multirow[b]{2}{*}{$\mathrm{t}$} & \multirow[b]{2}{*}{ Sig. } \\
\hline & & $\mathrm{B}$ & Std. Error & & & \\
\hline \multirow[t]{7}{*}{1} & (Constant) & -20.652 & 3.238 & & -6.378 & .000 \\
\hline & Building_height & -.476 & .025 & -.491 & -19.077 & .000 \\
\hline & Street_width & 1.873 & .089 & .540 & 20.979 & .000 \\
\hline & Orientation & .027 & .010 & .071 & 2.732 & .007 \\
\hline & Glazed_area & 1.292 & .083 & .403 & 15.595 & .000 \\
\hline & Location & -1.391 & .266 & -.134 & -5.219 & .000 \\
\hline & Reflectance & .251 & .025 & .260 & 10.117 & .000 \\
\hline
\end{tabular}

From Table 33 it appears that the standard errors are generally quite low with the exception of the Constant, when these errors ranges are explored in Table 34 it becomes apparent that some of these standard errors are quite significant. The factors with high standard errors were: the Constant, Orientation and Location. All Significance levels found in Table 33 were found to be well under the maximum acceptable level of 0.05 , with all factors except Orientation having significance levels of 0 .

Table 34 -1D: Coefficient Errors

\begin{tabular}{|l|c|c|c|}
\hline \multicolumn{1}{|c|}{ Variables } & Coefficients & Upper Error & Lower Error \\
\hline Constant & -20.652 & -17.414 & -23.890 \\
\hline Building Height & -0.476 & -0.451 & -0.501 \\
\hline Street Width & 1.873 & 1.962 & 1.784 \\
\hline Orientation & 0.027 & 0.037 & 0.017 \\
\hline Glazed Area & 1.292 & 1.375 & 1.209 \\
\hline Location & -1.391 & -1.125 & -1.657 \\
\hline Reflectance & 0.251 & 0.276 & 0.226 \\
\hline
\end{tabular}

Using the coefficients given in Table 33, the following equation was developed for this model.

$$
D A=-20-0.48 E H+1.9 S W+0.0270 r+1.3 G A-1.4 E+0.25 R
$$

Figure 20 is a scatter diagram showing the range of errors present between the simulated result and the predicted result. This graph follows the format used in Figure 14, where the results below 0 are over-predictions and the results above 0 are under-predictions. 


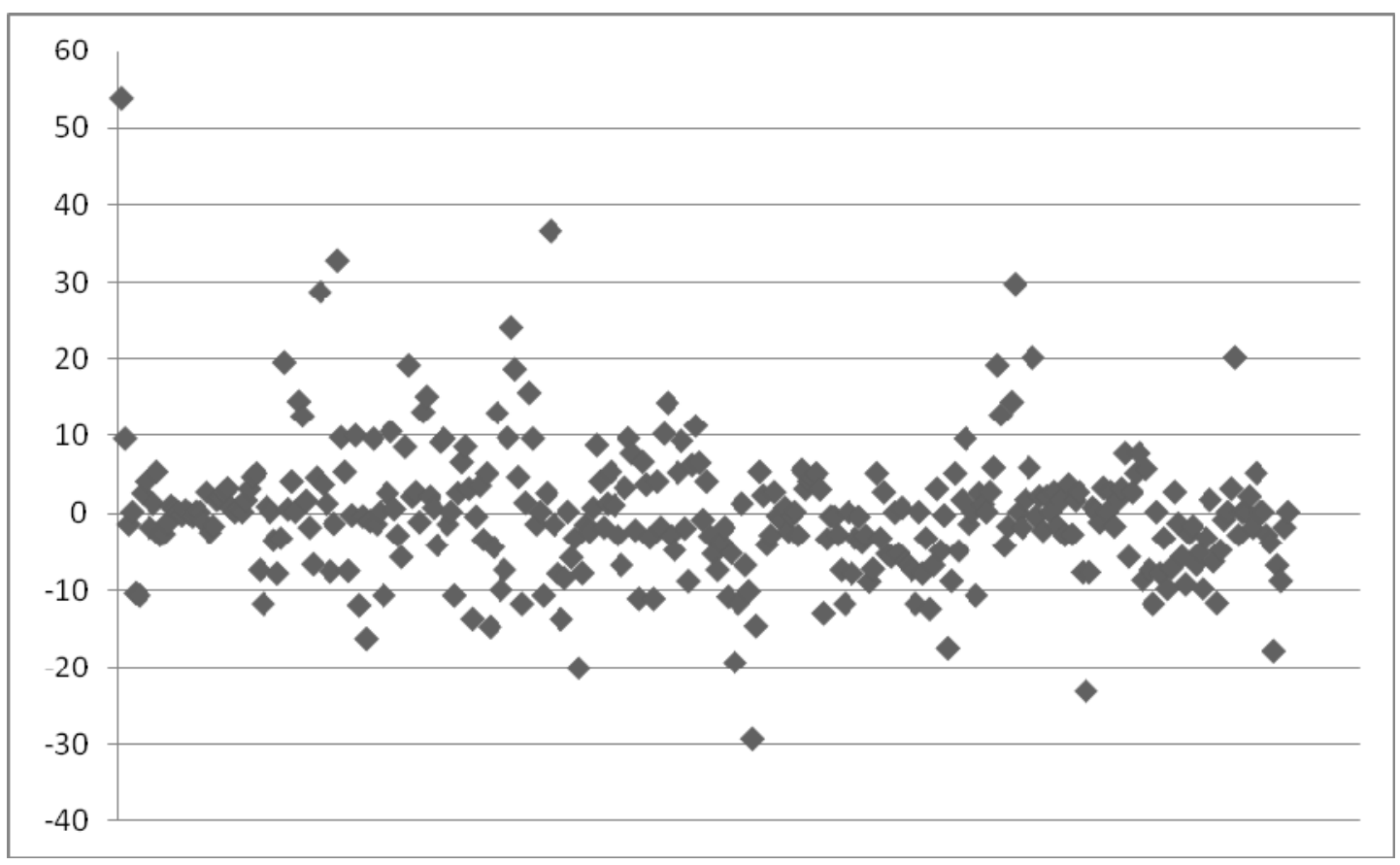

Figure 20 -1D: Errors in Predictions

Figure 20 shows no significant change from Figure 14, Figure 16 and Figure 18. The errors still range from -29 to $54 \%$ DA, with most cases having errors of $15 \%$ DA or less. And, around half the cases are under-predicted and half over-predicted. This indicates that the changes made to the prediction model, removing the secondary variables, has had little to no effect on the error in predictions.

Figure 21 is a scatter plot that shows the relationship between the simulated results ( $x$-axis) and predicted results (y-axis). The ideal trend line is shown in black, where the predicted results are directly proportional to the simulated results.

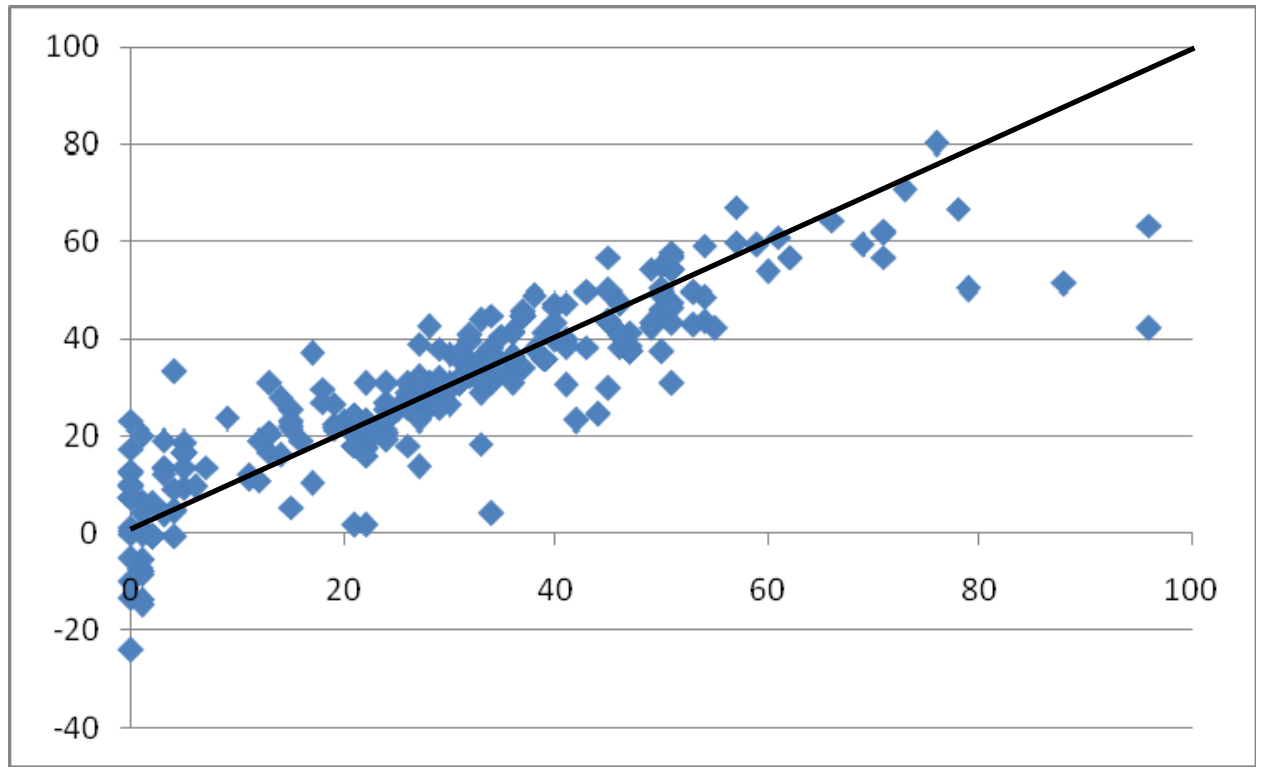

Figure 21 -1D: Prediction Accuracy compared to Simulation Results 
Figure 21 identifies that there has been no significant improvement in the accuracy of the prediction model. The inaccuracies in the high range are still present which means this model is not suitable for use in the tool.

Stage 1D had similar results to the previous models. It was expected that by removing the factors with higher significance levels and standard errors it would significantly improve the performance of the model, but the findings do not support this initial thought. Further decreases in the R Square and increases in the Standard Error were observed when compared to stage $1 \mathrm{C}$, therefore statistically this was the weakest of the models. The analysis found no noticeable change in the results compared to previous model. 


\section{Summary of Linear Models}

Table 35 provides a comparison of the various measures used to assess the strength of the models. From the analysis none of the models stood out as being better than the others, this information was used to select the best linear model to represent the relations between the various factors and daylight autonomy.

Table 35 - Comparison of Linear Models

\begin{tabular}{|c|c|c|c|c|}
\hline \multirow{2}{*}{ R Square } & $1 \mathrm{~A}$ & 1B & $1 \mathrm{C}$ & 1D \\
\hline & 0.794 & 0.793 & 0.790 & 0.786 \\
\hline Standard Error of Estimate & 8.530 & 8.520 & 8.580 & 8.640 \\
\hline Significance Level & 0.00 & 0.00 & 0.00 & 0.00 \\
\hline \multicolumn{5}{|l|}{ Coefficients } \\
\hline \multicolumn{5}{|l|}{ Constant } \\
\hline Standard Error & $40 \%$ & $24 \%$ & $15 \%$ & $16 \%$ \\
\hline Significance Level & 0.013 & 0.000 & 0.000 & 0.000 \\
\hline \multicolumn{5}{|l|}{ Building Height } \\
\hline Standard Error & $5 \%$ & $5 \%$ & $5 \%$ & $5 \%$ \\
\hline Significance Level & 0.000 & 0.000 & 0.000 & 0.000 \\
\hline \multicolumn{5}{|l|}{ Street Width } \\
\hline Standard Error & $4.7 \%$ & $4.7 \%$ & $4.7 \%$ & $4.7 \%$ \\
\hline Significance Level & 0.000 & 0.000 & 0.000 & 0.000 \\
\hline \multicolumn{5}{|l|}{ Orientation } \\
\hline Standard Error & $38 \%$ & $38 \%$ & $37 \%$ & $37 \%$ \\
\hline Significance Level & 0.007 & 0.006 & 0.005 & 0.007 \\
\hline \multicolumn{5}{|l|}{ Glazed Area } \\
\hline Standard Error & $6.3 \%$ & $6.3 \%$ & $6.3 \%$ & $6.4 \%$ \\
\hline Significance Level & 0.000 & 0.000 & 0.000 & 0.000 \\
\hline \multicolumn{5}{|l|}{ Location } \\
\hline Standard Error & $19 \%$ & $19 \%$ & $19 \%$ & $19 \%$ \\
\hline Significance Level & 0.000 & 0.000 & 0.000 & 0.000 \\
\hline \multicolumn{5}{|l|}{ Reflectance } \\
\hline Standard Error & $9.9 \%$ & $9.5 \%$ & $10 \%$ & $10 \%$ \\
\hline Significance Level & 0.000 & 0.000 & 0.000 & 0.000 \\
\hline \multicolumn{5}{|l|}{ Vertical Location } \\
\hline Standard Error & $44 \%$ & $43 \%$ & $43 \%$ & - \\
\hline Significance Level & 0.022 & 0.022 & 0.022 & - \\
\hline \multicolumn{5}{|l|}{ Proposed Building Height } \\
\hline Standard Error & $212 \%$ & - & - & - \\
\hline Significance Level & 0.639 & - & - & - \\
\hline \multicolumn{5}{|l|}{ Glazing Transmittance } \\
\hline Standard Error & $66 \%$ & $42 \%$ & - & - \\
\hline Significance Level & 0.134 & 0.017 & - & - \\
\hline
\end{tabular}

From Table 35 the strongest overall model was between $1 \mathrm{~A}$ and $1 \mathrm{~B}$. Model $1 \mathrm{~A}$ had the highest $\mathrm{R}$ Square value, but model $1 B$ had the Standard Error. The coefficients were needed to determine which of these two models was strongest. Model $1 \mathrm{~A}$, with all variables, had some high Standard errors, especially for the constant, proposed building height and glazing transmittance. The 
Significance levels for model $1 \mathrm{~A}$ were generally acceptable, however 2 of the coefficients had unacceptably high levels, in particular proposed building height. Model 1B had lower Standard errors, particularly for the constant and glazing transmittance. Noticeable improvements were seen for the Significance levels, the constant went from 0.013 to 0 and glazing transmittance went from an unacceptable 0.134 to 0.017 which is well within the acceptable range. From this analysis it was concluded that the strongest model was $1 \mathrm{~B}$. 


\section{Nonlinear Model 2A}

This stage involved the analysis of a more complex nonlinear regression prediction model for the relationship between 8 independent variables and daylight autonomy (dependent variable). Proposed building height was not included in this analysis as it was found in Stage 1 that it does not correlate well with the other variables, which would compromise the strength of the models if it was included. The variables analysed were: opposite building height, street width, orientation, glazed area, geographical location, reflectance of the opposite building, vertical location, and glazing transmittance.

Based on the models established for each variable separately, the following equation was used developed to describe the relationship.

$$
\begin{gathered}
D A=\beta_{0}+\beta_{1} \oplus \exp \left(\beta_{E H} B H\right)+\beta_{S W} S W+\beta_{O H} O V+\beta_{2} \Theta x p\left(\beta_{C L} G A\right)+\beta_{L} L+\beta_{E 1} R \\
+\beta_{E 2} R^{2}+\beta_{V L 1} V L-\beta_{V L 2} V L^{2}+\beta_{V L 2} V L^{3}+\beta_{T} T
\end{gathered}
$$

Table 36 gives the estimated coefficient values for the equation above. The key information drawn from this table was: the estimates; the standard errors and confidence intervals. The standard errors and confidence intervals were used to assess the reliability of the estimates.

Table 36 -2A: Parameter Estimates

\begin{tabular}{|l|r|r|r|r|}
\hline & & & \multicolumn{2}{|c|}{$95 \%$ Confidence Interval } \\
\cline { 4 - 5 } Parameter & Estimate & Std. Error & Lower Bound & Upper Bound \\
\hline alpha1 & -90.251 & 19.691 & -128.993 & -51.509 \\
bBH & -.050 & .003 & -.056 & -.044 \\
bSW & 1.873 & .069 & 1.737 & 2.010 \\
bOr & .036 & .008 & .021 & .051 \\
bGA & .032 & .011 & .010 & .053 \\
bloc & -1.368 & .206 & -1.774 & -.962 \\
bRef1 & .177 & .051 & .076 & .278 \\
bVL1 & 2.160 & 10.678 & -18.850 & 23.170 \\
btrans & .276 & .118 & .044 & .508 \\
bRef2 & .001 & .001 & .000 & .002 \\
bVL2 & .311 & 5.243 & -10.004 & 10.626 \\
bVL3 & .177 & .717 & -1.234 & 1.587 \\
alpha2 & 77.821 & 3.471 & 70.991 & 84.650 \\
alpha3 & 17.611 & 11.705 & -5.419 & 40.640 \\
\hline
\end{tabular}

From Table 36 it can be seen there is quite a lot of variation in errors, with some reasonably small errors and some very large errors. The constant (alpha1), Vertical location1 (bVL1), Glazing Transmittance (btrans), Vertical location2 (bVL2), Vertical location3 (bVL3) and Glazed area constant (alpha3) were all factors that had high Standard Errors. This indicates that some aspects of this model may not be reliable, vertical location in particular. 
Table 37 gives the analysis of how well the variation in the dependent variable is accounted for by the model. The key information drawn from this table was the R Square value.

Table $37-2 A$ : Analysis of Variance (ANOVA)

\begin{tabular}{|l|r|r|r|}
\hline Source & \multicolumn{1}{|c|}{$\begin{array}{c}\text { Sum of } \\
\text { Squares }\end{array}$} & df & \multicolumn{1}{c|}{$\begin{array}{c}\text { Mean } \\
\text { Squares }\end{array}$} \\
\hline Regression & 390666.2 & 14 & 27904.729 \\
Residual & 14158.794 & 316 & 44.806 \\
Uncorrected Total & 404825.0 & 330 & \\
Corrected Total & 112783.0 & 329 & \\
\hline
\end{tabular}

Dependent variable: DA

a. R squared = 1 - (Residual Sum of Squares) / (Corrected Sum of Squares) $=.874$.

From Table 37 it can be seen that the $R$ Square value of 0.874 is well above the minimum acceptable $R$ Square value of 0.7 . This indicates that this model represents the relationship between the independent variables and daylight autonomy quite well.

Using the parameters given in Table 37, the following equation was developed for this model.

$$
\begin{gathered}
D A=-90+78 \exp (-0.05 B H)+1.9 S W+0.0360 \mathrm{~V}+17 \exp (0.032 G A)-1.4 L \\
+0.18 R+0.001 R^{2}+2.2 V L-0.31 V L^{2}+0.18 V L^{2}+0.27 T
\end{gathered}
$$

Figure 22 is a scatter diagram showing the range of errors present between the simulated result and the predicted result. This graph follows the format used in Figure 14, where the results above 0 are under-predictions and the results below 0 are over-predictions.

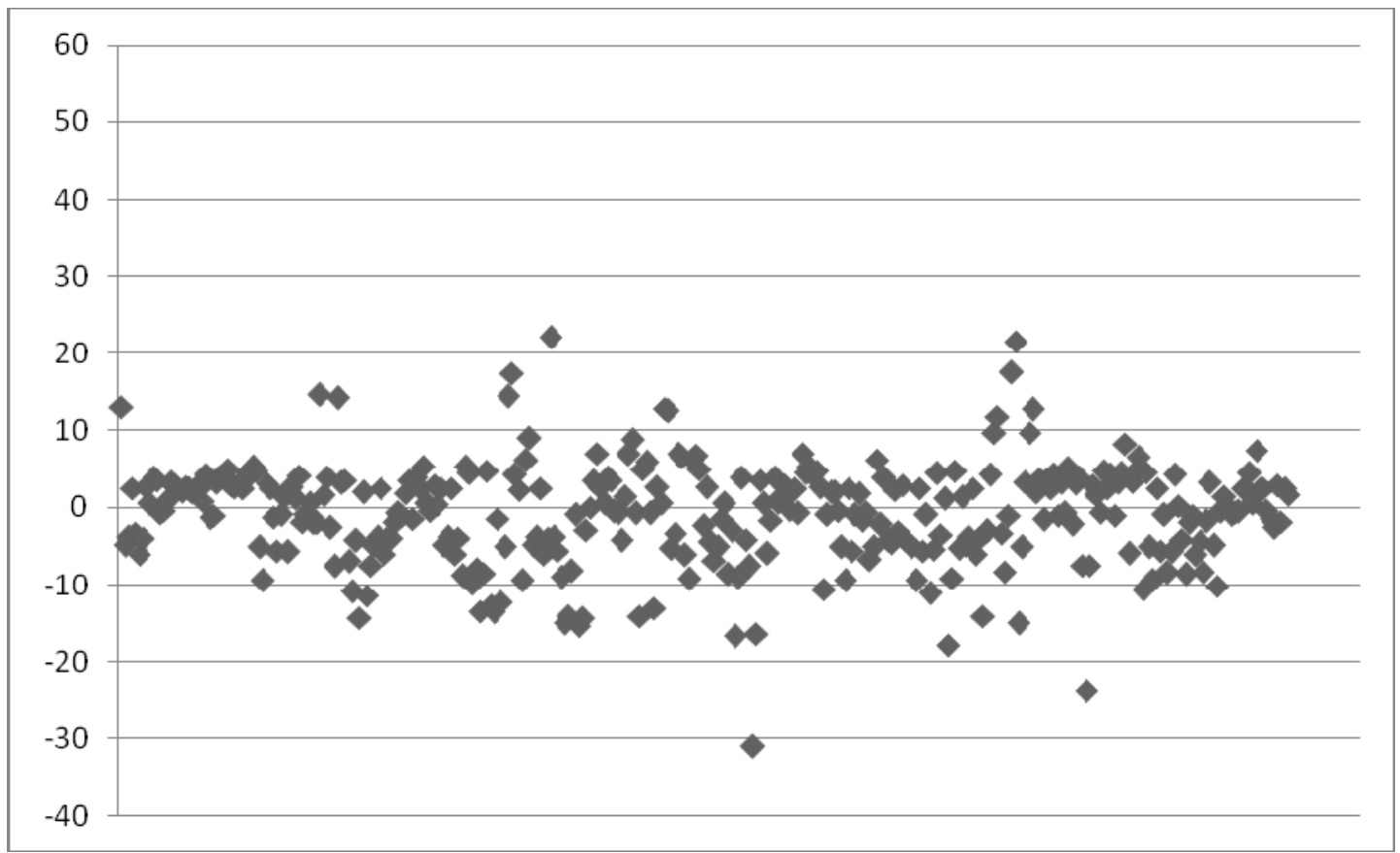

Figure 22 -2A: Errors in Predictions 
From Figure 22 it can be seen that the errors range from -31 to $22 \%$ DA, where most of the cases had errors less than $10 \%$ DA. Approximately half the cases were under-predicted and half were overpredicted. The concerns relating to the wide error range and 50/50 split of results discussed in Stage 1 are still present in this nonlinear model, however, the reduced error range is a definite improvement.

Figure 23 is a scatter plot that shows the relationship between the simulated results ( $x$-axis) and predicted results ( $y$-axis). The ideal trend line is shown in black, where the predicted results are directly proportional to the simulated results.

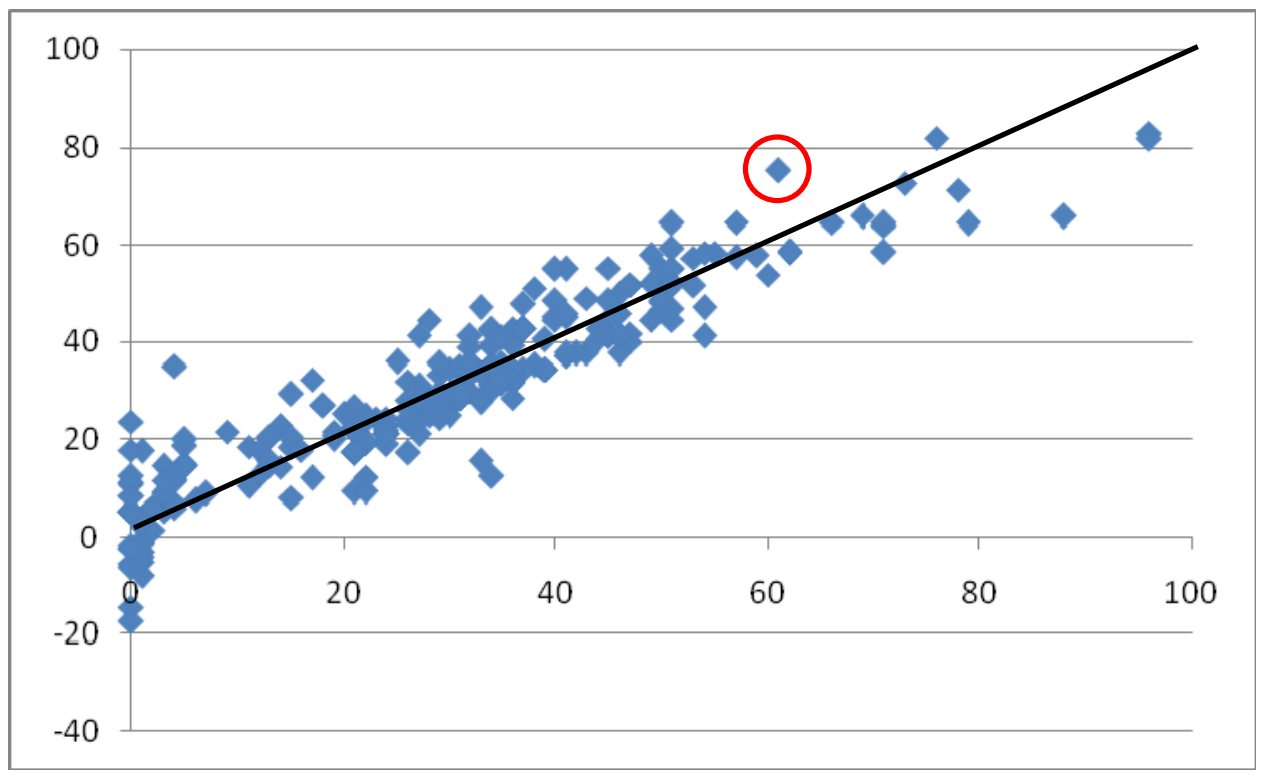

Figure 23-2A: Prediction Accuracy compared to Simulation Results

Figure 23 identifies that generally the prediction model is performing well. However, as with the linear models, there are two sections where the results do not follow the trend line, these being for the best and worst performing apartments. The worst performing apartments (less than $10 \% \mathrm{DA}$ ) have significant errors in the prediction, with errors up to $20 \%$ DA. However the errors in the lowest range are not of concern because they would still be well under the threshold value of $75 \%$ DA and would consequently still be give a fail result. The inaccurate predictions in the upper range are of great concern. As was identified in the analysis of Stage 1, it is essential that accurate results are provided in the upper range of simulated results. The errors in this top range of situations are considerably smaller than that of the linear regression models and in some of these situations would still provide a pass result. Another point of concern with this model is the point circled in red, this case had a simulated result of $60 \%$ DA, but the prediction model provided a result of $75 \%$ DA. Although this is just one out of 330 cases, this is one situation that would receive a pass result but in fact needed simulation.

The results from the regression analysis indicated that overall this prediction model would perform well, but some predictions errors may occur due to large standard errors for some parameters. The comparison of the predicted results and simulated results found that the model typically yielded error rates less than $10 \%$ DA which is not ideal but is an improvement of on the $15 \%$ DA of the linear 
regression models. The best apartments, as identified through simulations, are still a concern, but are noticeably better than the linear regression models. 


\section{Nonlinear Model 2B}

The second nonlinear model tested in stage 2 was a simplified version of the model from stage $2 \mathrm{~A}$. In the development of the model for this stage, variable were separated into two relationship types, linear and nonlinear. The linear variables had the standard linear equation and all nonlinear models were assumed to be exponential equations as the exponential model featured in the best four models for 6 of the 8 variables. The equation used for this stage is shown below:

$$
\begin{gathered}
D A=\beta_{0} \times E X P\left(\beta_{D H} B H\right) \times E X P\left(\beta_{G A} G A\right) \times E X P\left(\beta_{E} R\right) \times E X P\left(\beta_{V L} V L\right)+\beta_{S W} S W \\
+\beta_{L} L+\beta_{O r} Q_{r}+\beta_{T} T
\end{gathered}
$$

Table 38 gives the estimated coefficient values for the equation above. The key information drawn from this table was: the estimates; the standard errors and confidence intervals. The standard errors and confidence intervals were used to assess the reliability of the estimates.

Table 38 -2B: Parameter Estimates

\begin{tabular}{|l|r|r|r|r|}
\hline & & & \multicolumn{2}{|c|}{$95 \%$ Confidence Interval } \\
\cline { 4 - 5 } Parameter & Estimate & Std. Error & Lower Bound & Upper Bound \\
\hline alpha & 16.159 & 1.730 & 12.756 & 19.563 \\
betaBH & -.047 & .003 & -.053 & -.041 \\
betaSW & 1.839 & .070 & 1.701 & 1.977 \\
betaGA & .040 & .002 & .036 & .045 \\
betaOr & .036 & .008 & .021 & .051 \\
betaLoc & -1.472 & .210 & -1.885 & -1.060 \\
betaRef & .010 & .001 & .008 & .012 \\
betatrans & -.212 & .025 & -.261 & -.163 \\
betaVL & .167 & .037 & .094 & .239 \\
\hline
\end{tabular}

From Table 38 it can be seen the variation in errors is relatively small compared to the estimates, this result is an improvement on the errors observed for model 2A. The constant (alpha), Geographical location (betaLoc), Glazing Transmittance (betatrans) and Vertical location (betaVL) still had higher Standard Errors and wider confidence intervals. This indicates that there are still some aspects of this model that may not be reliable.

Table 39 gives the analysis of how well the variation in the dependent variable is accounted for by the model. The key information drawn from this table was the R Square value.

Table 39-2B: Analysis of Variance (ANOVA)

\begin{tabular}{|l|r|r|r|}
\hline Source & \multicolumn{1}{|c|}{$\begin{array}{c}\text { Sum of } \\
\text { Squares }\end{array}$} & \multicolumn{1}{c|}{ df } & \multicolumn{1}{c|}{$\begin{array}{c}\text { Mean } \\
\text { Squares }\end{array}$} \\
\hline Regression & 389775.6 & 9 & 43308.404 \\
Residual & 15049.366 & 321 & 46.883 \\
Uncorrected Total & 404825.0 & 330 & \\
Corrected Total & 112783.8 & 329 & \\
\hline
\end{tabular}

Dependent variable: DA

a. $\mathrm{R}$ squared $=1$ - (Residual Sum of Squares) /

(Corrected Sum of Squares) $=.867$. 
From Table 39 it can be seen that the $R$ Square value of 0.867 is well above the minimum acceptable $R$ Square value of 0.7 , but is slightly less than the $R$ Square value for model $2 A(0.874)$. This indicates that this model represents the relationship between the independent variables and daylight autonomy reasonably well, however model $2 \mathrm{~A}$ provides slightly better results.

Using the coefficients given in Table 39, the following equation was developed for this model.

$$
\begin{gathered}
D A=16 \times E X P(-0.047 B H) \times E X P(0.04 G A) \times E X P(0.01 R) \times E X P(0.176 V L)+1.85 W-1.5 L \\
+0.0360 r-0.21 T
\end{gathered}
$$

Figure 24 is a scatter diagram showing the range of errors present between the simulated result and the predicted result. This graph follows the format used in Figure 14, where the results below 0 are over-predictions and the results above 0 are under-predictions.

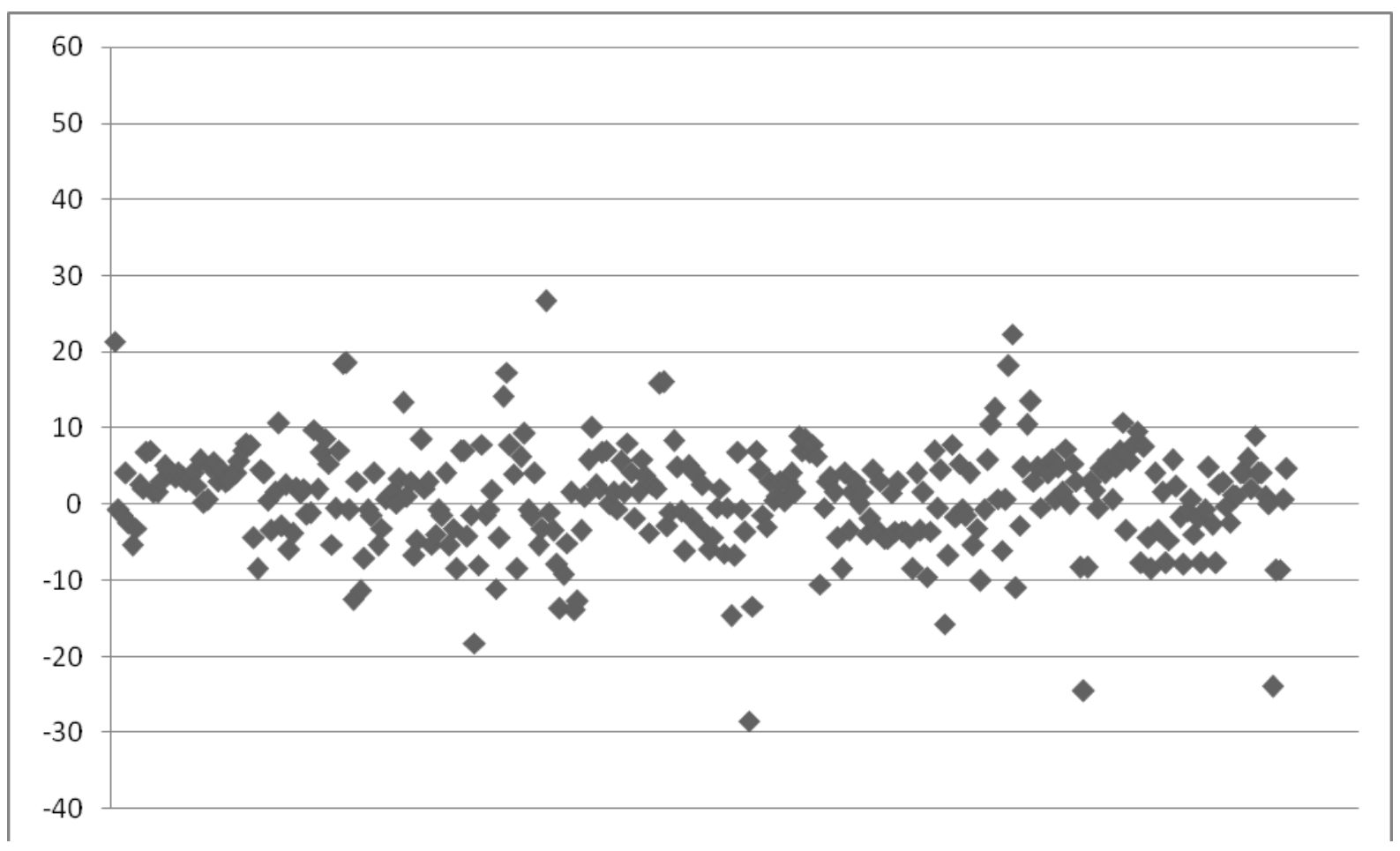

Figure $24-2 B$ : Errors in Predictions

From Figure 24 it can be seen that the errors range from -28 to $26 \%$ DA, with most of the cases having errors less than $10 \%$ DA. Only 134 of the 330 cases were over-predictions, this is an good result, as it was better to have under-predictions than over-predictions, meaning that it was less likely that cases could receive false pass results. However, it is important to identify the accuracy of the predictions around the threshold value of $75 \%$, which can be seen in Figure 25 .

Figure 25 is a scatter plot that shows the relationship between the simulated results ( $x$-axis) and predicted results (y-axis). The ideal trend line is shown in black, where the predicted results are directly proportional to the simulated results. 


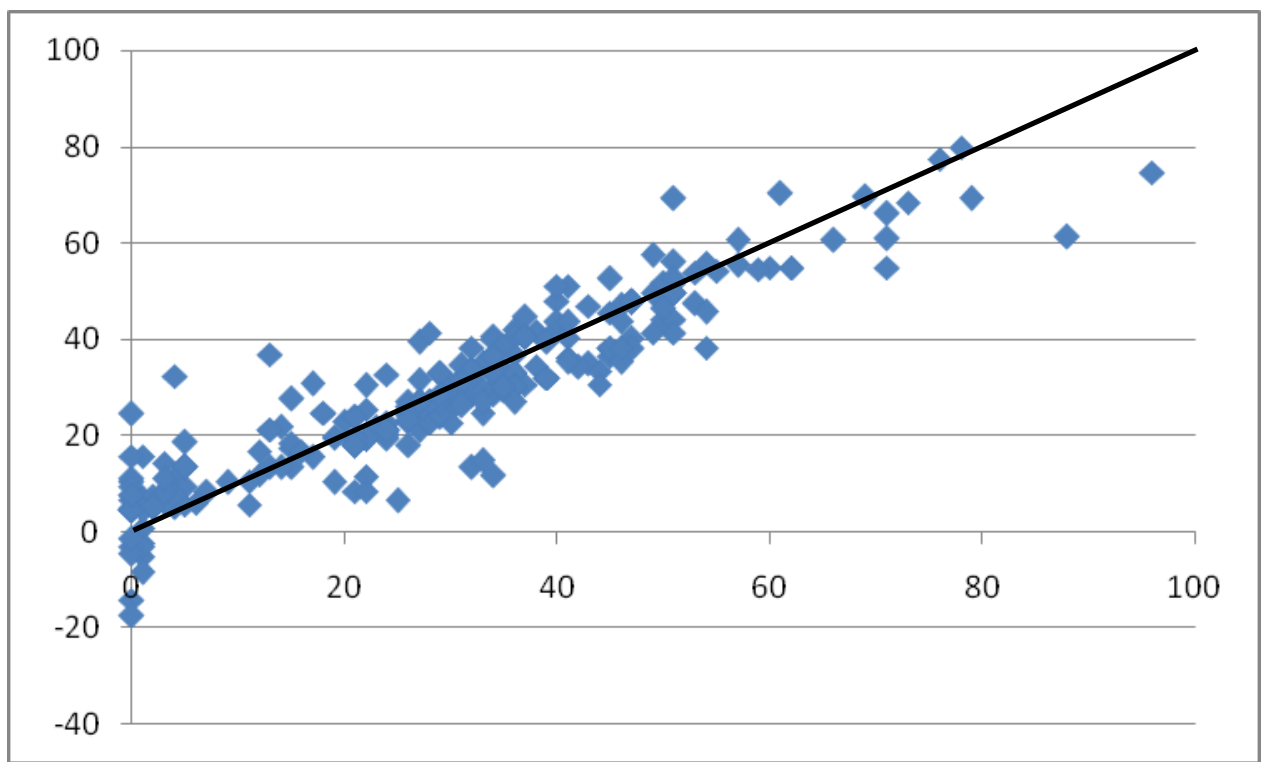

Figure 25 -2B: Prediction Accuracy compared to Simulation Results

Figure 25 identifies that generally the prediction model is performing reasonably well, and confirms the findings from Figure 24 , where $60 \%$ of the results were under-predictions. However, from Figure 25 it can be seen that the predictions around the threshold value of $75 \%$ of significantly underpredicted. This would result in a number apartment buildings requiring simulations that were not necessary to prove compliance. It should also be noted that unlike Figure 23 , there were no cases that received false pass results.

The results from the regression analysis indicated that overall this prediction model would perform reasonably well. The advantage of this model to represent nonlinear variables was the smaller errors found in Table 38. The comparison of the predicted results and simulated results found that the model typically yielded error rates less than $-10 \%$ DA and $20 \%$ DA which is similar to the $15 \%$ DA of the linear regression models. The major concern with this model is the under-predictions for cases above the threshold value. 


\section{Nonlinear Model 2C}

The third nonlinear model tested in stage 2 was similar to model $2 \mathrm{~A}$ as the analysis from Appendix 2 was used to develop the equation. However, to make this model less complex, it was decided to use the least complex functions for each variable (except orientation). Aspects like building height and glazed area, that were exponential function, became quadratic or cubic functions. The orientation function, which had been in the simplest form was given a more complex function to check this wasn't effecting the strength of the model. The equation used for this stage is shown below:

$$
\begin{gathered}
D A=\beta_{Q}+\beta_{D H Q} B H+\beta_{D H L} B H^{2}+\beta_{D H Q} B H^{8}+\beta_{S W} S W+\beta_{Q r}{ }^{O r}+\beta_{G A} G A+\beta_{L} L \\
+\beta_{E Q} R+\beta_{E L} R^{2}+\beta_{V L Q} V L-\beta_{V L E} V L^{2}+\beta_{V L Q} V L^{8}+\beta_{T} T
\end{gathered}
$$

Table 40 gives the estimated coefficient values for the equation above. The key information drawn from this table was: the estimates; the standard errors and confidence intervals. The standard errors and confidence intervals were used to assess the reliability of the estimates.

Table 40 -2C: Parameter Estimates

\begin{tabular}{|l|r|r|r|r|}
\hline & & & \multicolumn{2}{|c|}{$95 \%$ Confidence Interval } \\
\cline { 4 - 5 } Parameter & Estimate & Std. Error & Lower Bound & Upper Bound \\
\hline alpha & -15.407 & .000 & -15.407 & -15.407 \\
bBHa & -2.816 & .213 & -3.235 & -2.396 \\
bSW & 1.873 & 11.016 & -19.801 & 23.548 \\
bOr & 1.010 & .001 & 1.007 & 1.013 \\
bGAa & 1.307 & .071 & 1.166 & 1.447 \\
bloc & 1.374 & .739 & -.081 & 2.829 \\
bRefa & .193 & 5.407 & -10.447 & 10.832 \\
bVLa & 3.680 & .121 & 3.441 & 3.919 \\
btrans & .300 & .006 & .289 & .312 \\
bRefb & .001 & .053 & -.104 & .105 \\
bVLb & 1.212 & .001 & 1.210 & 1.213 \\
bVLc & .295 & .246 & -.189 & .779 \\
bBHb & .039 & .066 & -.091 & .169 \\
bGAb & .035 & .000 & .035 & .035 \\
bBHc & .000 & 13.394 & -26.354 & 26.354 \\
\hline
\end{tabular}

From Table 40 it can be seen there is quite a lot of variation in errors, with some reasonably small errors and some very large errors. In contrast to the two previous models, the estimate for the constant was perfect, with a standard error of 0.000. Unfortunately, this was not the case for all factors, with street width (bSW), geographical location (bloc), Reflectance (bRefa and bRefb) and building height (bBHb and $\mathrm{bBHc}$ ) all having unacceptably high standard errors. As these factors are the most important factors in the prediction of daylight performance in urban environments, these errors will likely cause a large number of incorrect prediction.

Table 41 gives the analysis of how well the variation in the dependent variable is accounted for by the model. The key information drawn from this table was the $\mathrm{R}$ Square value. 
Table 41 - Stage 2C: Analysis of Variance (ANOVA)

\begin{tabular}{|l|r|r|r|}
\hline Source & \multicolumn{1}{|c|}{$\begin{array}{c}\text { Sum of } \\
\text { Squares }\end{array}$} & df & \multicolumn{1}{c|}{$\begin{array}{c}\text { Mean } \\
\text { Squares }\end{array}$} \\
\hline Regression & 389791.2 & 15 & 25986.081 \\
Residual & 15033.783 & 315 & 47.726 \\
Uncorrected Total & 404825.0 & 330 & \\
Corrected Total & 112783.8 & 329 & \\
\hline
\end{tabular}

Dependent variable: DA

a. R squared = 1 - (Residual Sum of Squares) /

(Corrected Sum of Squares) $=.867$.

From Table 41 it can be seen that the $R$ Square value of 0.867 is well above the minimum acceptable $R$ Square value of 0.7 , and is exactly the same as the $R$ Square value of model $2 B$. This indicates that this model represents the relationship between the independent variables and daylight autonomy reasonably well, however model $2 \mathrm{~A}$ provides slightly better results.

Using the coefficients given in Table 41, the following equation was developed for this model.

$$
\begin{gathered}
D A=-15-2.8 B H+0.039 B H^{2}+0 B H^{2}+1.9 S W+1.0^{0 r}+1.3 G A-1.4 L \\
+0.19 R+0.001 R^{2}+3.7 V L-1.2 V L^{2}+0.29 V L^{2}+0.3 T
\end{gathered}
$$

Figure 26 is a scatter diagram showing the range of errors present between the simulated result and the predicted result. This graph follows the format used in Figure 14, where the results below 0 are over-predictions and the results above 0 are under-predictions.

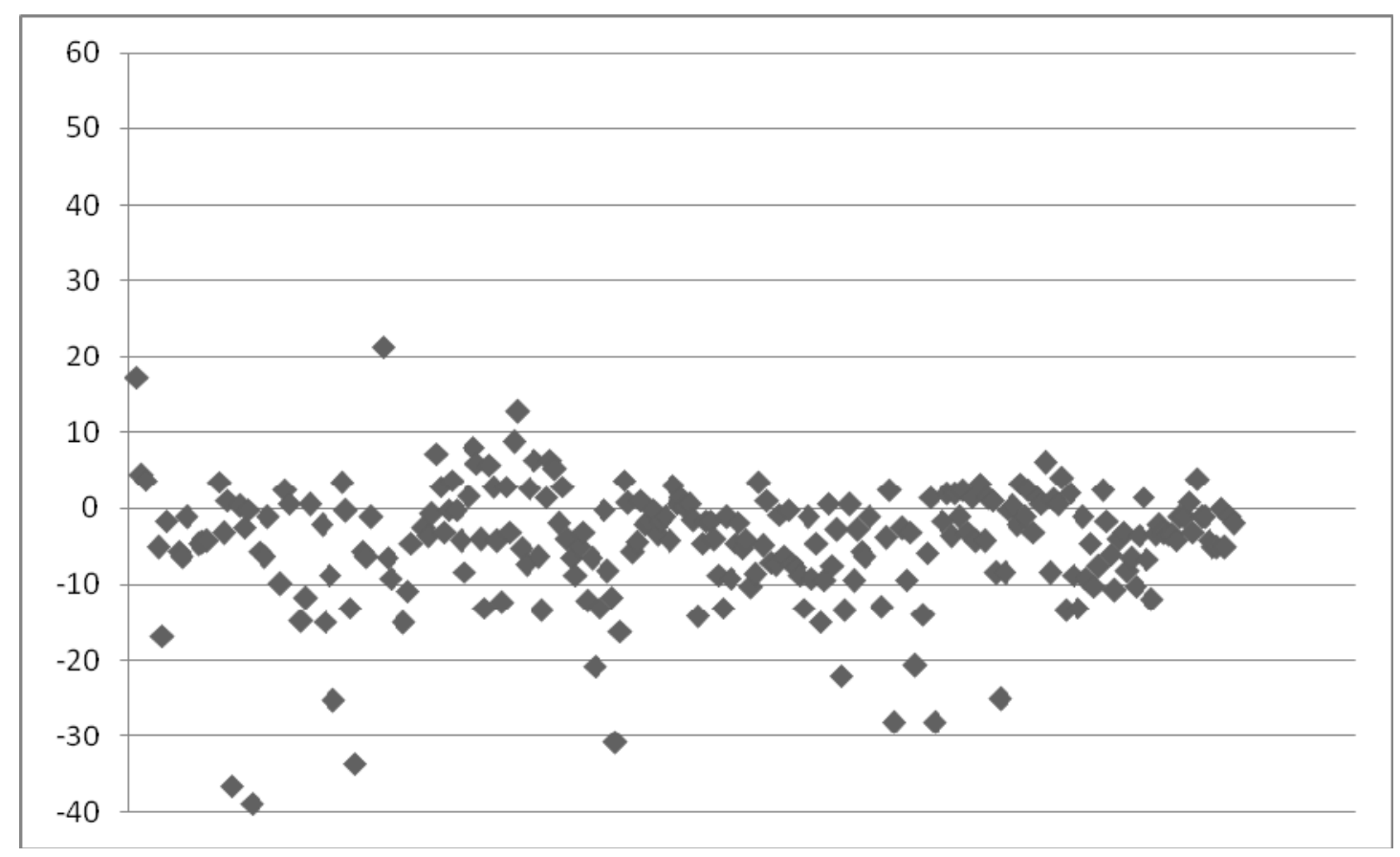

Figure 26-2C: Errors in Predictions

From Figure 26 it can be seen that the errors range from -148 to 21\% DA, where most of the cases had errors less than $15 \%$ DA. 253 of the 330 cases were over-predicted which is too high to be considered acceptable for discriminating between compliant and non-compliant apartments. 
Figure 27 is a scatter plot that shows the relationship between the simulated results ( $x$-axis) and predicted results ( $y$-axis). The ideal trend line is shown in black, where the predicted results are directly proportional to the simulated results.

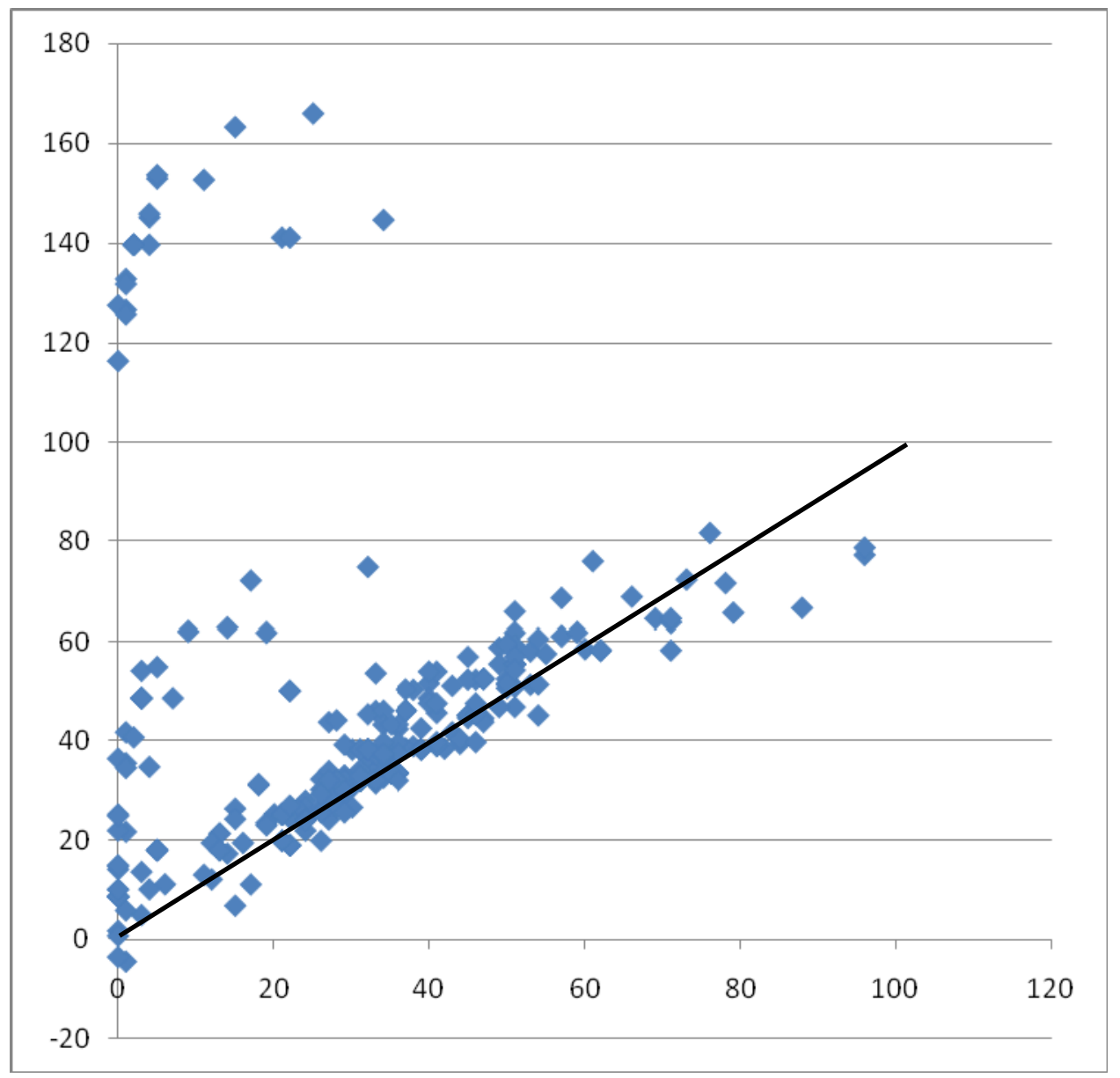

Figure 27-2C: Prediction Accuracy compared to Simulation Results

Figure 27 identifies that the prediction model is performing poorly. While there are still a number of cases close to the trend line, most of the cases with simulated values less than 30\% DA were predicted to have significantly higher results, with around 16 of the cases having prediction over $100 \%$ DA. This would result in some of the worst performing apartments being given a pass result which is not acceptable for this situation.

The results from the regression analysis indicated that generally this prediction model would not perform particularly well, with large standard errors for the variable that have the largest effect on daylight performance in urban environments. However, a relatively high $\mathrm{R}$ Square value indicated that model represented the relationships between the variable quite well. The comparison of the predicted results and simulated confirmed the finding from the standard errors. Over-predictions of up to $148 \%$ DA were observed and three quarters of the cases were over-predicted which is unacceptably high. Incorrect predictions for the lower apartments (from simulation) meant that these apartments would not have required simulations but in reality would have been well below the minimum requirement. 


\section{Summary of Nonlinear Models}

Table 42 provides a comparison of the various measures used to assess the strength of the models. From the analysis it was difficult to determine whether model $2 \mathrm{~A}$ or $2 \mathrm{~B}$ should be used to represent the relationship between the various factors and daylight autonomy. From the following table, the nonlinear model to best represent this relationship was found.

Table 42 - Comparison of Nonlinear Models

\begin{tabular}{|c|c|c|c|}
\hline & Stage 2A & Stage 2B & Stage 2C \\
\hline R Square & 0.874 & 0.867 & 0.867 \\
\hline \multicolumn{4}{|c|}{ Parameter Standard Errors } \\
\hline \multirow[t]{3}{*}{ Constant } & $22 \%$ & $10 \%$ & $0 \%$ \\
\hline & 4.5\% $(\alpha \mathrm{BH})$ & & \\
\hline & $66 \%(\alpha G A)$ & & \\
\hline \multirow[t]{3}{*}{ Building Height } & $6 \%(\beta \mathrm{BH})$ & $6 \%$ & 7.5\% (ßBHa) \\
\hline & & & $169 \%(\beta \mathrm{BHb})$ \\
\hline & & & $1339 \%(\beta B H c)$ \\
\hline Street Width & $3.7 \%$ & $3.8 \%$ & $588 \%$ \\
\hline Orientation & $22 \%$ & $22 \%$ & $0.09 \%$ \\
\hline \multirow[t]{2}{*}{ Glazed Area } & $34 \%$ & $5 \%$ & $5.4 \%(\beta G A a)$ \\
\hline & & & $0 \%(\beta G A b)$ \\
\hline Location & $15 \%$ & $14 \%$ & $54 \%$ \\
\hline \multirow[t]{2}{*}{ Reflectance } & $28 \%$ ( $\beta$ Refa) & $10 \%$ & 2802\% ( $\beta$ Refa) \\
\hline & $100 \%$ ( $\beta$ Refb) & & $5300 \%$ ( $\beta$ Refb) \\
\hline \multirow[t]{3}{*}{ Vertical Location } & $494 \%$ ( $\beta$ VLa) & $22 \%$ & 3.3\% ( $\beta$ VLa) \\
\hline & $1686 \%(\beta \vee L b)$ & & $0.08 \%(\beta \vee L b)$ \\
\hline & $405 \%(\beta V L c)$ & & $83 \%(\beta \bigvee L C)$ \\
\hline Glazing Transmittance & $42 \%$ & $12 \%$ & $2 \%$ \\
\hline
\end{tabular}

From Table 42 the strongest overall model was between 2A and 2B. Model $2 \mathrm{~A}$ had the highest $\mathrm{R}$ Square value, but had some extremely high errors. Model $2 B$ had considerably lower errors but also had a lower $R$ Square value. It was decided that model $2 B$ would most likely yield the best results, however due to the difficulty in deciding between models $2 \mathrm{~A}$ and $2 \mathrm{~B}$, both models were continued for further testing in the calibration stage, section 6.2. 


\section{Appendix 4 - Full Calibration Analysis}

This appendix shows the calibration results for each of the 97 apartments measured in this research. For each apartment, a graph is provided showing the illuminance exceeded for $75 \%$ of the standard year at the measurement points, used to determine if the apartment fails or complies with the minimum building code requirement of 30 lux for $75 \%$ of the standard year. A copy of the tool is shown with the inputs for each apartment and a YES or NO result is given where all three of the equations for the tool agree. Finally a table is provided to compare the measurement results (Pass or Fail) and the prediction results from each of the equations. 


\section{Apartment 1}

Measurement Results:

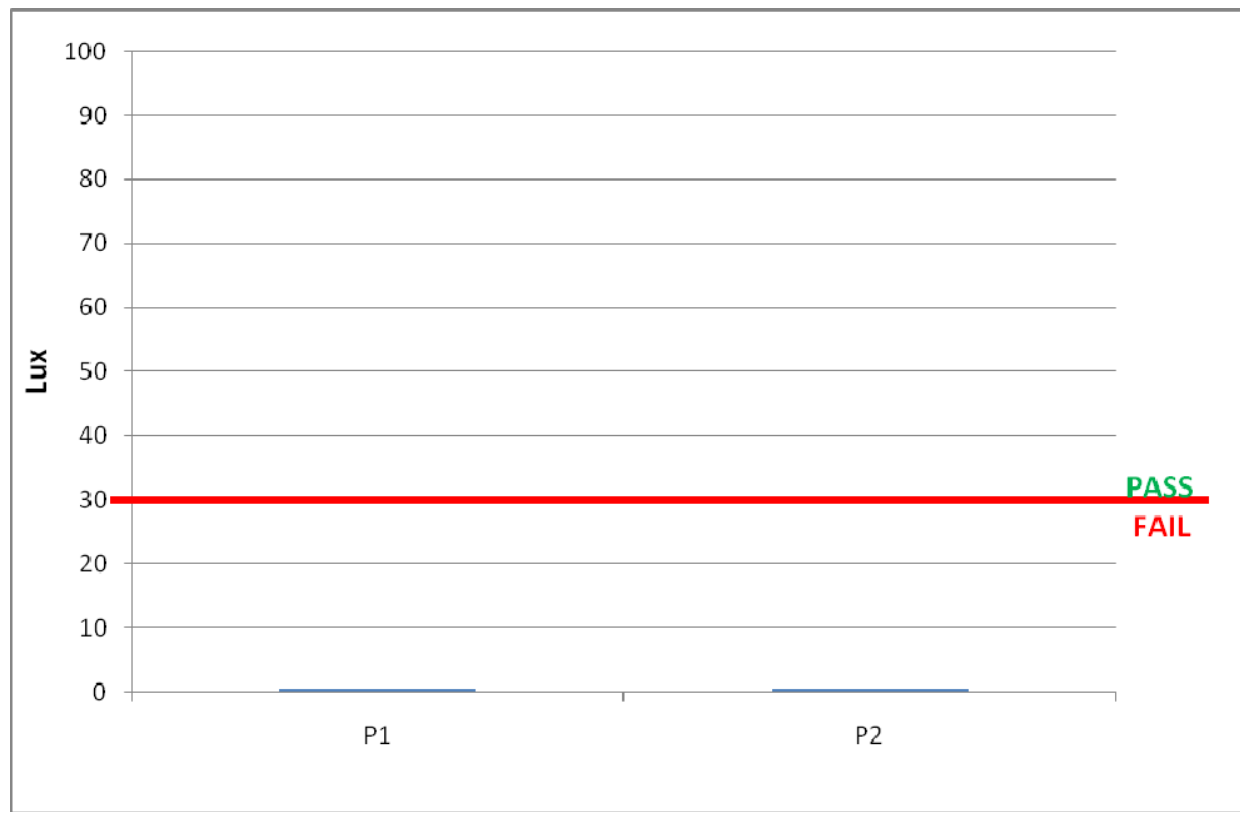

Application of the Tool:

\begin{tabular}{|l|c|}
\hline \multicolumn{2}{|c|}{ NZBC G7 Compliance Assessment Tool } \\
\hline \multicolumn{2}{|l|}{} \\
\hline Variable: & 35 \\
\hline Opposite Building Height & 3 \\
\hline Street Width & 32 \\
\hline Glazed Area & East \\
\hline Orientation & Wellington \\
\hline Location & 40 \\
\hline Reflectance of Opposite Building & 87 \\
\hline Glazing Transmittance & 0 \\
\hline Vertical Location of lowest apartment \\
\hline \multicolumn{2}{|}{} \\
\hline Are simulations required to prove compliance: \\
\hline
\end{tabular}

Comparison of Results:

\begin{tabular}{|c|c|c|c|}
\hline Measurements & Linear Model & Model 2A & Model 2B \\
\hline Fail & 44 & 5 & -1.8 \\
\hline
\end{tabular}




\section{Apartment 2}

Measurement Results:

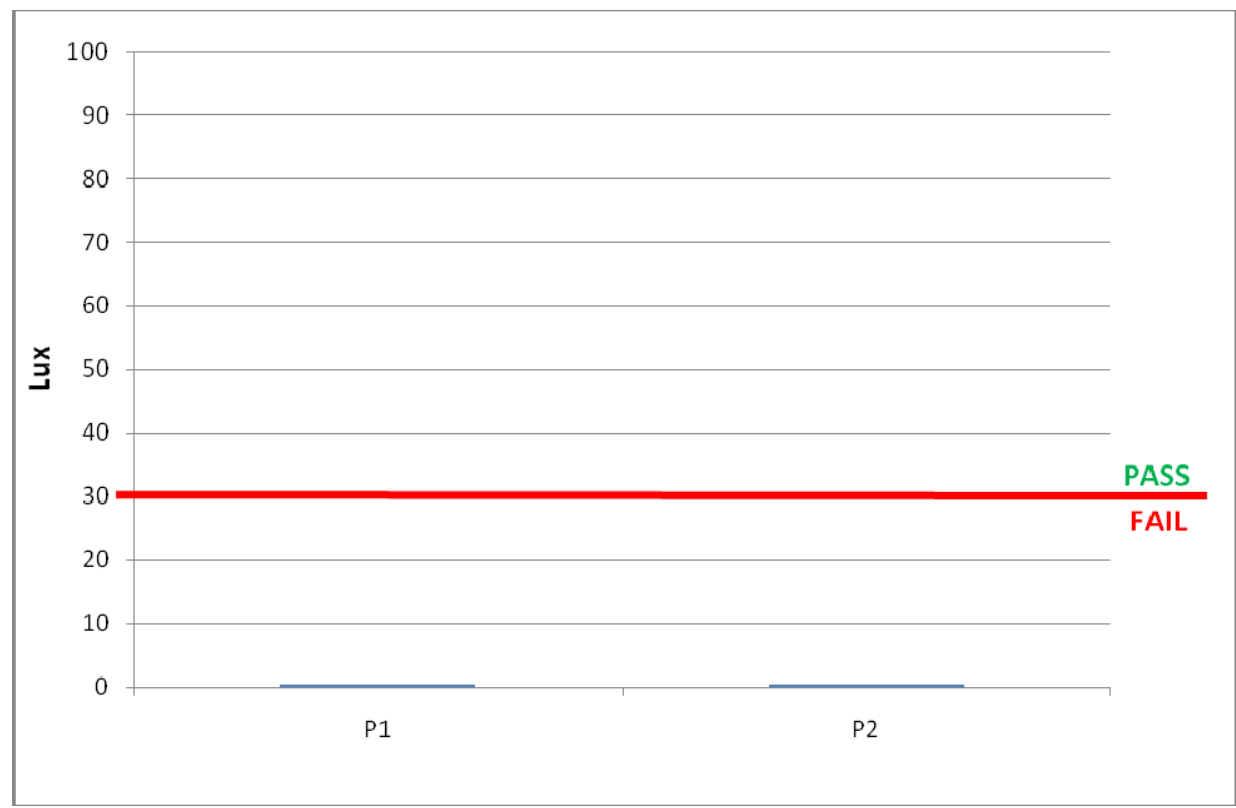

Application of the Tool:

\begin{tabular}{|l|c|}
\hline \multicolumn{2}{|c|}{ NZBC G7 Compliance Assessment Tool } \\
\hline \multicolumn{2}{|l|}{} \\
\hline Variable: & 35 \\
\hline Opposite Building Height & 3 \\
\hline Street Width & 16 \\
\hline Glazed Area & East \\
\hline Orientation & Wellington \\
\hline Location & 40 \\
\hline Reflectance of Opposite Building & 87 \\
\hline Glazing Transmittance & 5 \\
\hline Vertical Location of lowest apartment \\
\hline \multicolumn{2}{|l}{} \\
\hline Are simulations required to prove compliance: \\
\hline
\end{tabular}

Comparison of Results:

\begin{tabular}{|c|c|c|c|}
\hline Measurements & Linear Model & Model 2A & Model 2B \\
\hline Fail & 44 & 11 & 2.7 \\
\hline
\end{tabular}




\section{Apartment 3}

Measurement Results:

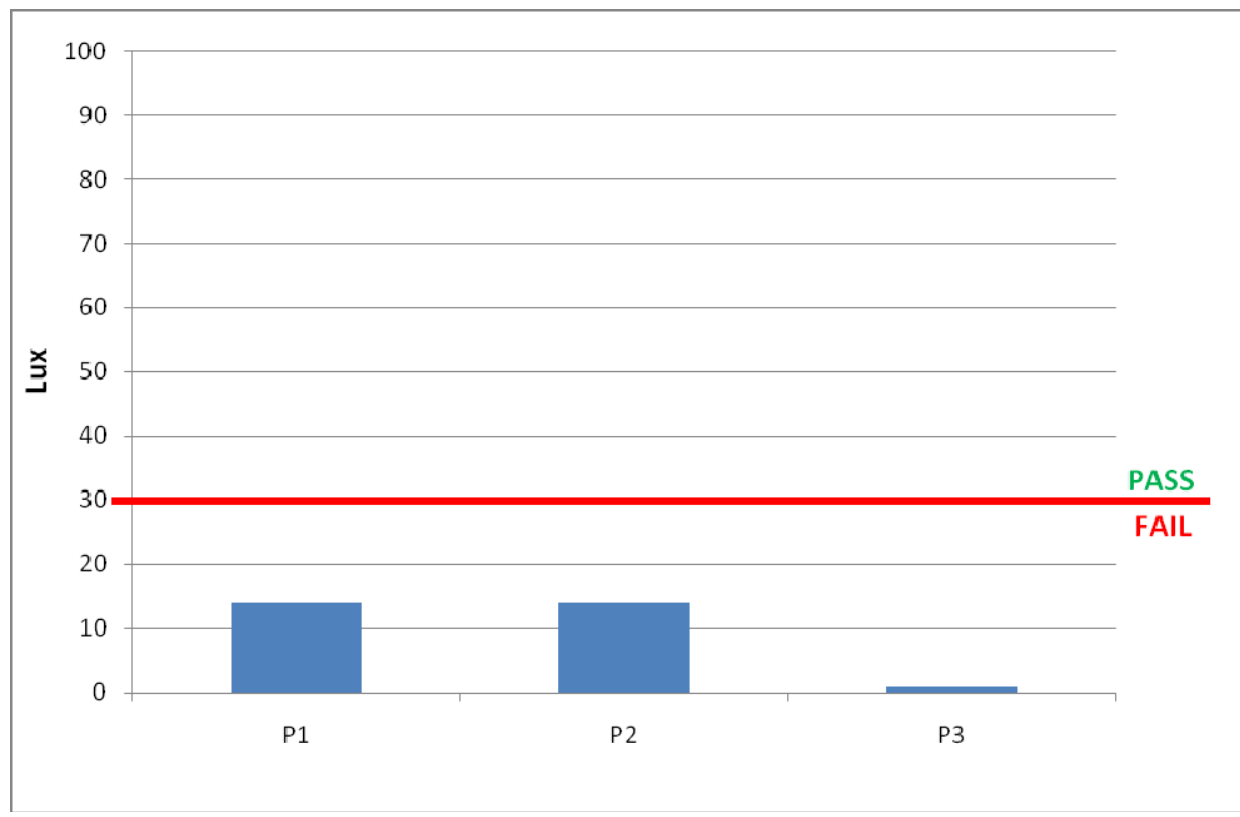

Application of the Tool:

\begin{tabular}{|l|c|}
\hline \multicolumn{2}{|c|}{ NZBC G7 Compliance Assessment Tool } \\
\hline \multicolumn{2}{|l|}{} \\
\hline Variable: & 5 \\
\hline Opposite Building Height & 11 \\
\hline Street Width & 15 \\
\hline Glazed Area & West \\
\hline Orientation & Wellington \\
\hline Location & 25 \\
\hline Reflectance of Opposite Building & 87 \\
\hline Glazing Transmittance & 2 \\
\hline Vertical Location of lowest apartment \\
\hline \multicolumn{2}{|l}{} \\
\hline Are simulations required to prove compliance: \\
\hline
\end{tabular}

Comparison of Results:

\begin{tabular}{|c|c|c|c|}
\hline Measurements & Linear Model & Model 2A & Model 2B \\
\hline Fail & 56 & 48 & 38 \\
\hline
\end{tabular}




\section{Apartment 4}

Measurement Results:

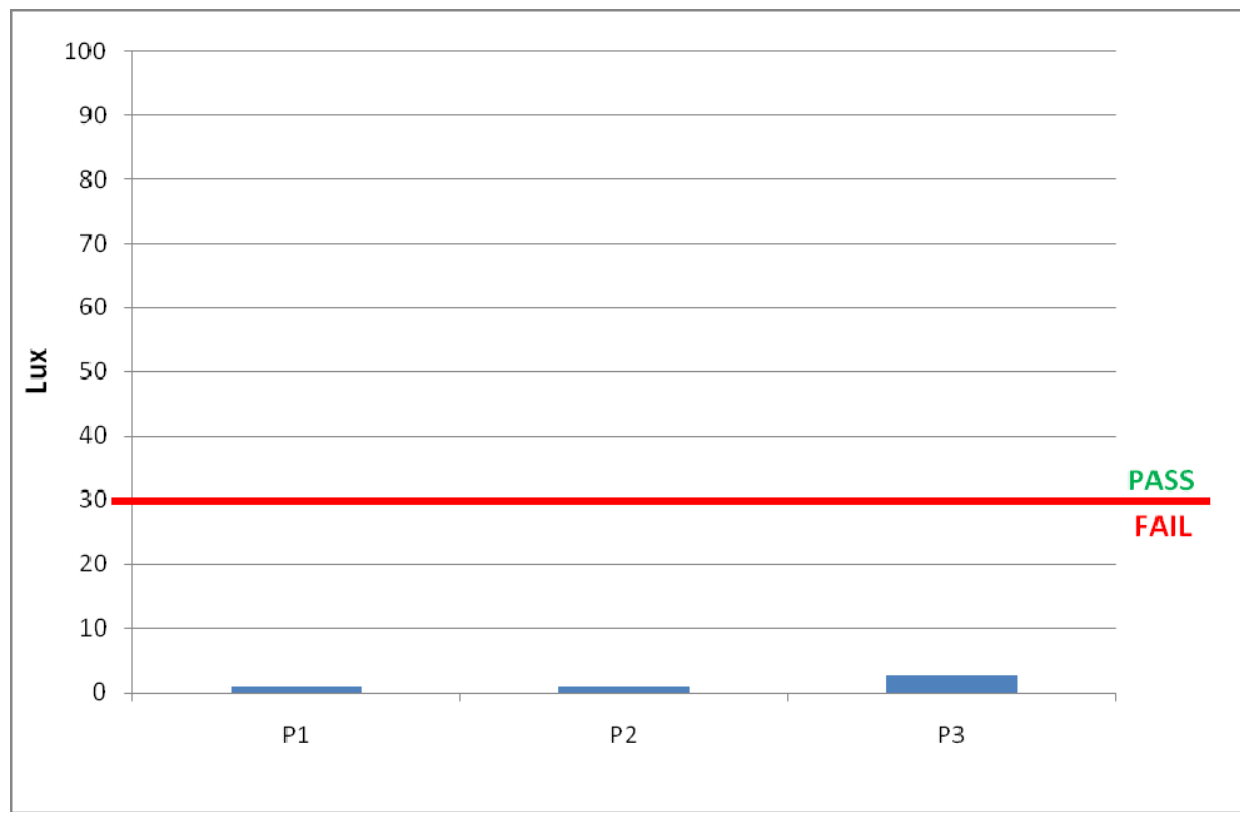

Application of the Tool:

\begin{tabular}{|l|c|}
\hline \multicolumn{2}{|c|}{ NZBC G7 Compliance Assessment Tool } \\
\hline \multicolumn{2}{|l|}{} \\
\hline Variable: & 39.5 \\
\hline Opposite Building Height & 5 \\
\hline Street Width & 50 \\
\hline Glazed Area & South \\
\hline Orientation & Wellington \\
\hline Location & 13.5 \\
\hline Reflectance of Opposite Building & 87 \\
\hline Glazing Transmittance & 1 \\
\hline Vertical Location of lowest apartment \\
\hline \multicolumn{2}{|l}{} \\
\hline Are simulations required to prove compliance: \\
\hline
\end{tabular}

Comparison of Results:

\begin{tabular}{|c|c|c|c|}
\hline Measurements & Linear Model & Model 2A & Model 2B \\
\hline Fail & 69 & 44 & 13 \\
\hline
\end{tabular}




\section{Apartment 5}

Measurement Results:

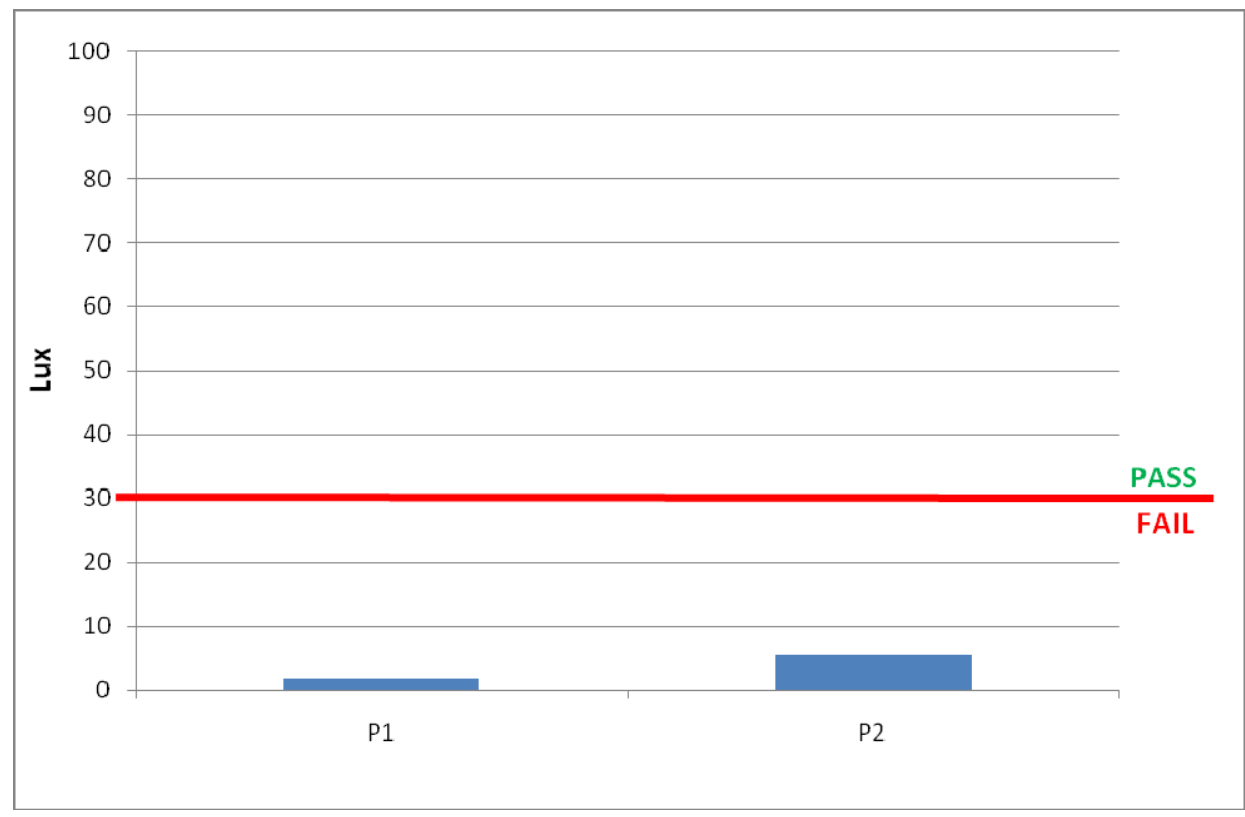

Application of the Tool:

\begin{tabular}{|l|c|}
\hline \multicolumn{2}{|c|}{ NZBC G7 Compliance Assessment Tool } \\
\hline \multicolumn{2}{|l|}{} \\
\hline Variable: & 35 \\
\hline Opposite Building Height & 3 \\
\hline Street Width & 10 \\
\hline Glazed Area & West \\
\hline Orientation & Wellington \\
\hline Location & 25 \\
\hline Reflectance of Opposite Building & 87 \\
\hline Glazing Transmittance & 3 \\
\hline Vertical Location of lowest apartment \\
\hline \multicolumn{2}{|l}{} \\
\hline Are simulations required to prove compliance: \\
\hline
\end{tabular}

Comparison of Results:

\begin{tabular}{|c|c|c|c|}
\hline Measurements & Linear Model & Model 2A & Model 2B \\
\hline Fail & 24 & -14 & -8 \\
\hline
\end{tabular}




\section{Apartment 6}

Measurement Results:

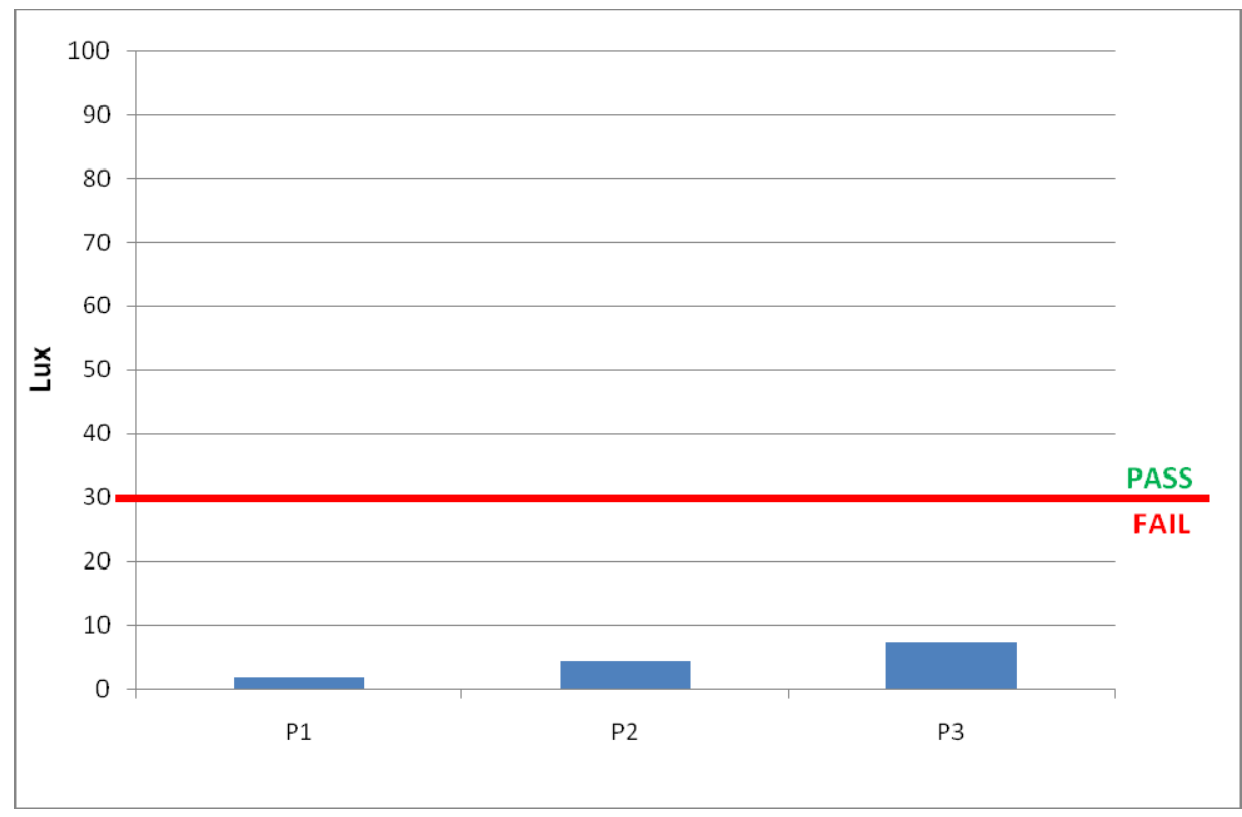

Application of the Tool:

\begin{tabular}{|l|c|}
\hline \multicolumn{2}{|c|}{ NZBC G7 Compliance Assessment Tool } \\
\hline \multicolumn{2}{|l|}{} \\
\hline Variable: & 75 \\
\hline Opposite Building Height & 9.5 \\
\hline Street Width & 12 \\
\hline Glazed Area & North \\
\hline Orientation & Wellington \\
\hline Location & 12 \\
\hline Reflectance of Opposite Building & 87 \\
\hline Glazing Transmittance & 1 \\
\hline Vertical Location of lowest apartment \\
\hline \multicolumn{2}{|c|}{} \\
\hline Are simulations required to prove compliance: \\
\hline
\end{tabular}

Comparison of Results:

\begin{tabular}{|c|c|c|c|}
\hline Measurements & Linear Model & Model 2A & Model 2B \\
\hline Fail & 6 & -24 & -8 \\
\hline
\end{tabular}




\section{Apartment 7}

Measurement Results:

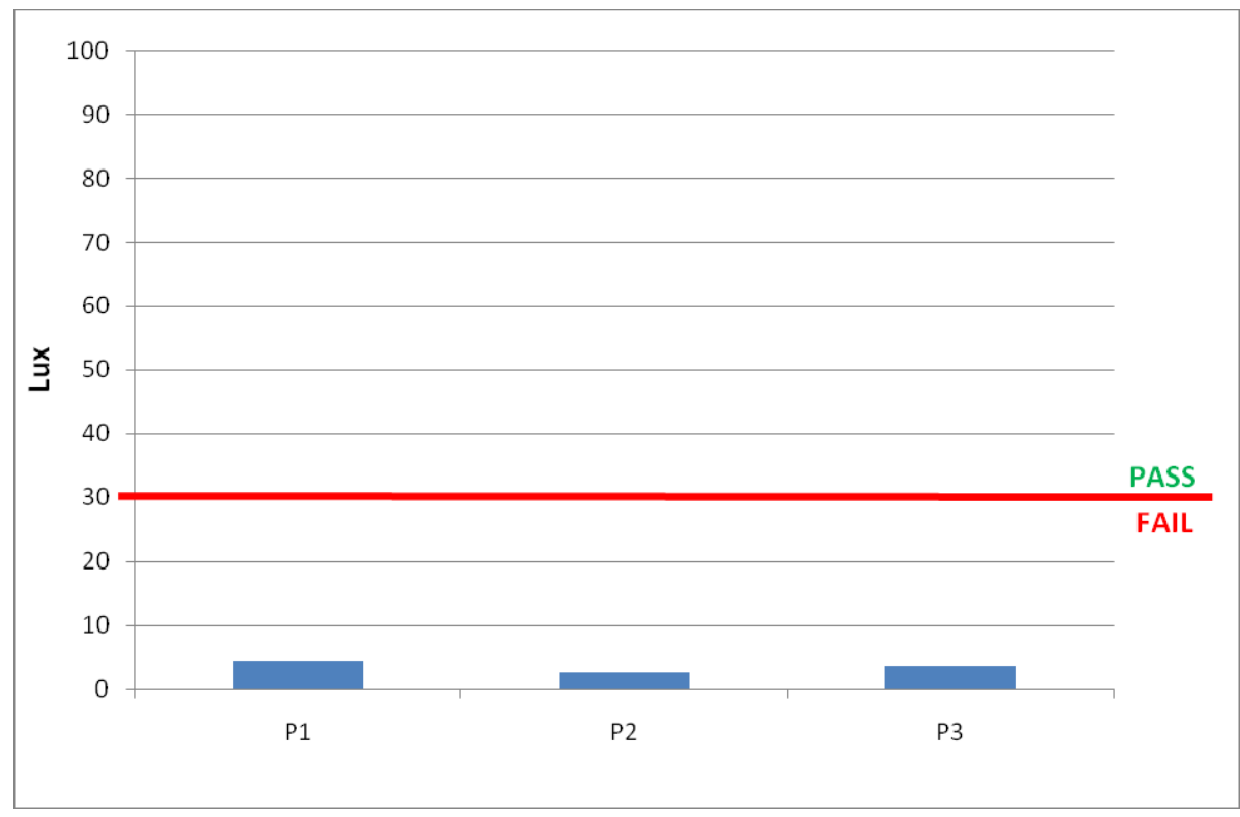

Application of the Tool:

\begin{tabular}{|l|c|}
\hline \multicolumn{2}{|c|}{ NZBC G7 Compliance Assessment Tool } \\
\hline \multicolumn{2}{|l|}{ Variable: } \\
\hline Opposite Building Height & 18 \\
\hline Street Width & 16 \\
\hline Glazed Area & 16.6 \\
\hline Orientation & North \\
\hline Location & Wellington \\
\hline Reflectance of Opposite Building & 10 \\
\hline Glazing Transmittance & 87 \\
\hline Vertical Location of lowest apartment & 1 \\
\hline \multicolumn{2}{|c|}{} \\
\hline Are simulations required to prove compliance: \\
\hline
\end{tabular}

Comparison of Results:

\begin{tabular}{|c|c|c|c|}
\hline Measurements & Linear Model & Model 2A & Model 2B \\
\hline Fail & 51 & 20 & 20 \\
\hline
\end{tabular}




\section{Apartment 8}

Measurement Results:

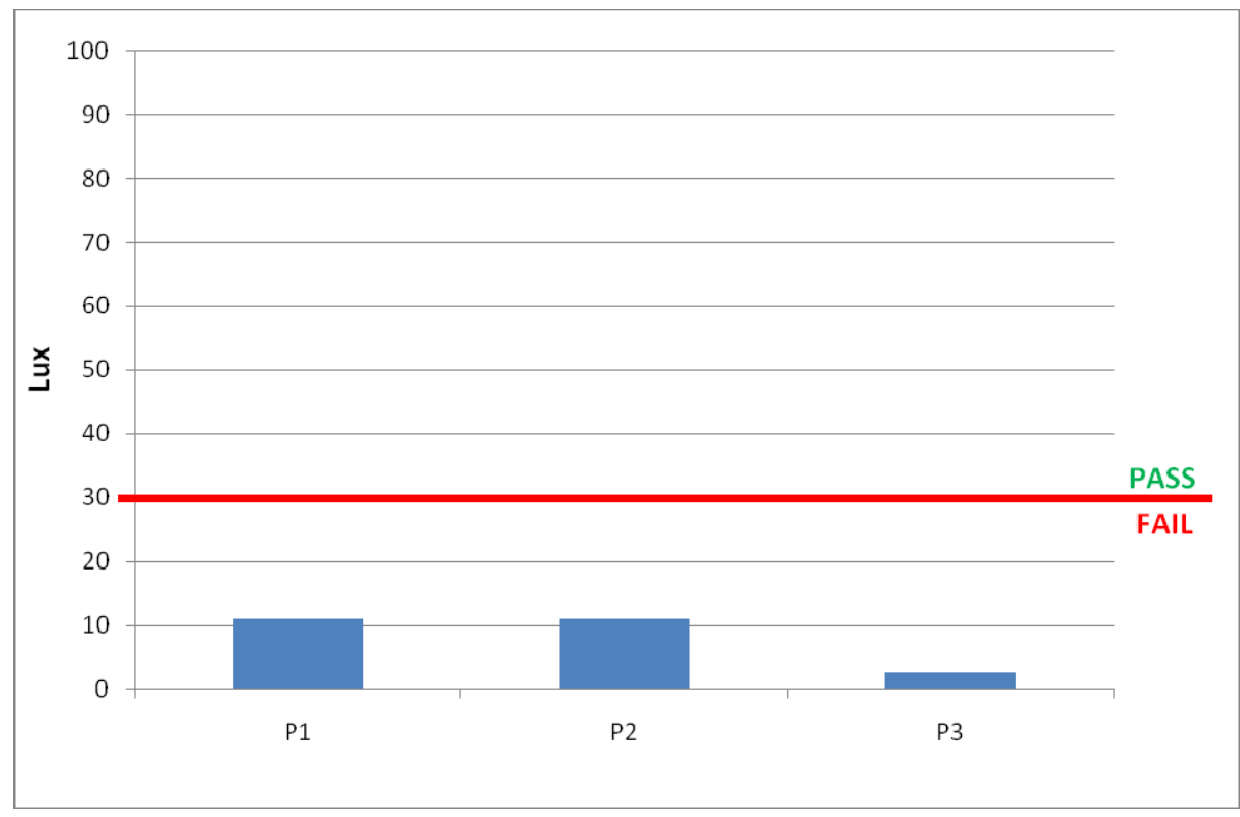

Application of the Tool:

\begin{tabular}{|l|c|}
\hline \multicolumn{2}{|c|}{ NZBC G7 Compliance Assessment Tool } \\
\hline \multicolumn{2}{|l|}{} \\
\hline Variable: & 39.5 \\
\hline Opposite Building Height & 5 \\
\hline Street Width & 50 \\
\hline Glazed Area & South \\
\hline Orientation & Wellington \\
\hline Location & 13.5 \\
\hline Reflectance of Opposite Building & 87 \\
\hline Glazing Transmittance & 1 \\
\hline Vertical Location of lowest apartment \\
\hline \multicolumn{2}{|c|}{} \\
\hline Are simulations required to prove compliance: \\
\hline
\end{tabular}

Comparison of Results:

\begin{tabular}{|c|c|c|c|}
\hline Measurements & Linear Model & Model 2A & Model 2B \\
\hline Fail & 69 & 44 & 13 \\
\hline
\end{tabular}




\section{Apartment 9}

Measurement Results:

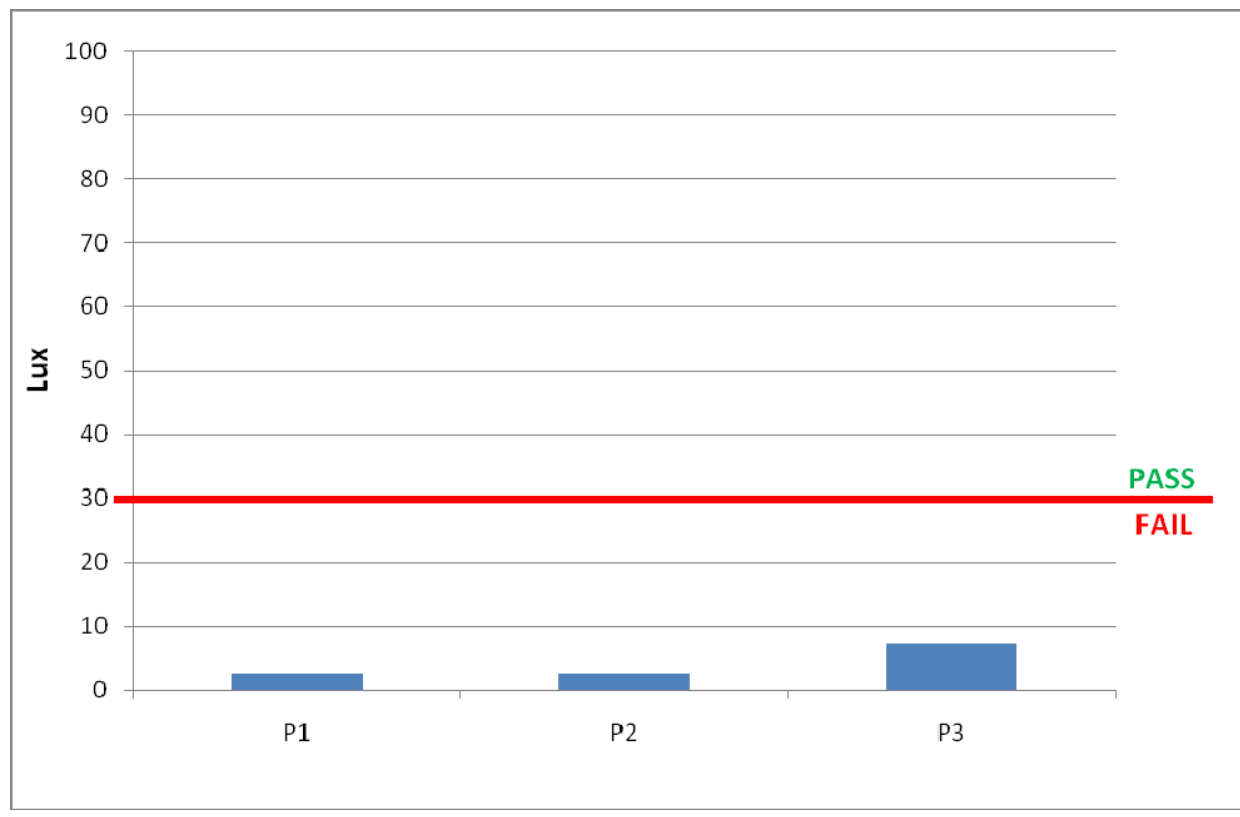

Application of the Tool:

\begin{tabular}{|l|c|}
\hline \multicolumn{2}{|c|}{ NZBC G7 Compliance Assessment Tool } \\
\hline \multicolumn{2}{|l|}{} \\
\hline Variable: & 35 \\
\hline Opposite Building Height & 3 \\
\hline Street Width & 32 \\
\hline Glazed Area & East \\
\hline Orientation & Wellington \\
\hline Location & 40 \\
\hline Reflectance of Opposite Building & 87 \\
\hline Glazing Transmittance & 1 \\
\hline Vertical Location of lowest apartment \\
\hline \multicolumn{2}{|c|}{} \\
\hline Are simulations required to prove compliance: \\
\hline
\end{tabular}

Comparison of Results:

\begin{tabular}{|c|c|c|c|}
\hline Measurements & Linear Model & Model 2A & Model 2B \\
\hline Fail & 74 & 51 & 1.4 \\
\hline
\end{tabular}




\section{Apartment 10}

Measurement Results:

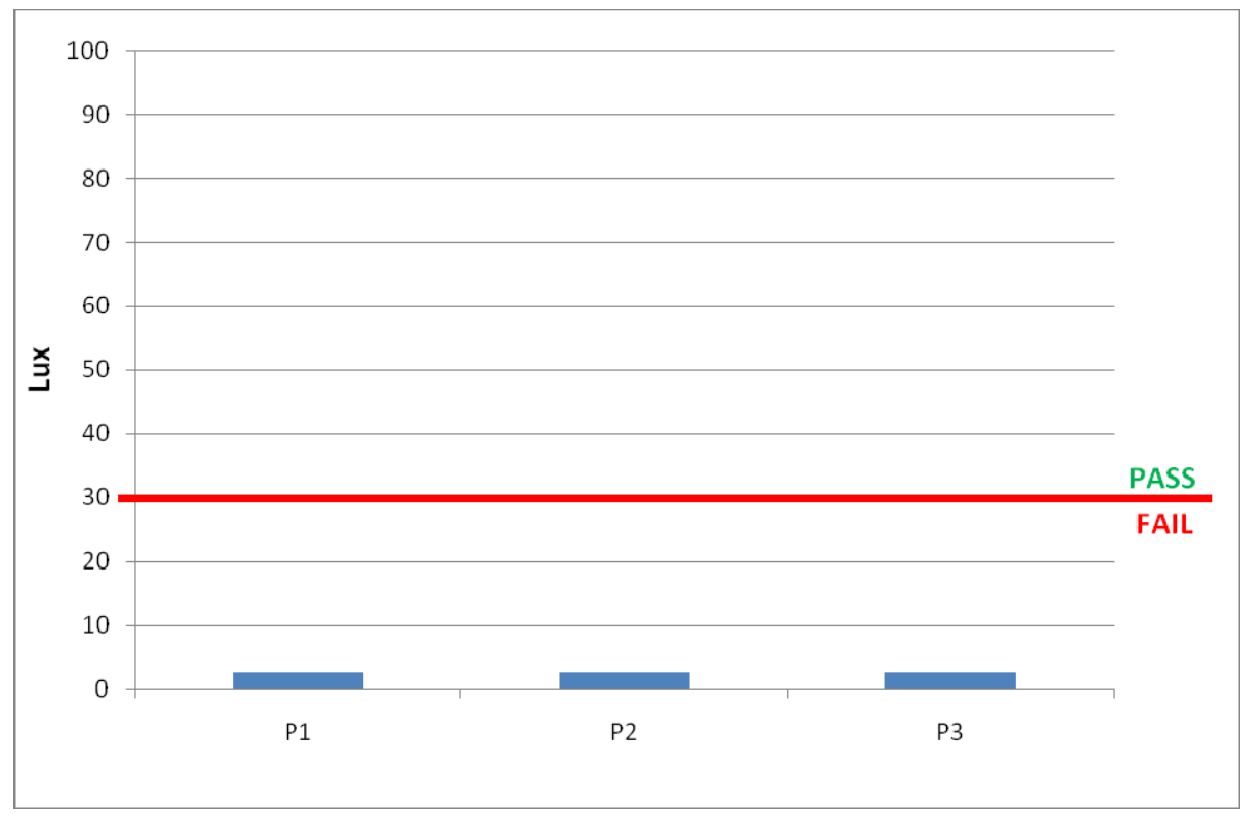

Application of the Tool:

\begin{tabular}{|l|c|}
\hline \multicolumn{2}{|c|}{ NZBC G7 Compliance Assessment Tool } \\
\hline \multicolumn{2}{|l|}{} \\
\hline Variable: & 20 \\
\hline Opposite Building Height & 5 \\
\hline Street Width & 21 \\
\hline Glazed Area & North \\
\hline Orientation & Wellington \\
\hline Location & 33.3 \\
\hline Reflectance of Opposite Building & 87 \\
\hline Glazing Transmittance & 3 \\
\hline Vertical Location of lowest apartment \\
\hline \multicolumn{2}{|c|}{} \\
\hline Are simulations required to prove compliance: \\
\hline
\end{tabular}

Comparison of Results:

\begin{tabular}{|c|c|c|c|}
\hline Measurements & Linear Model & Model 2A & Model 2B \\
\hline Fail & 49 & 13 & 16 \\
\hline
\end{tabular}




\section{Apartment 11}

Measurement Results:

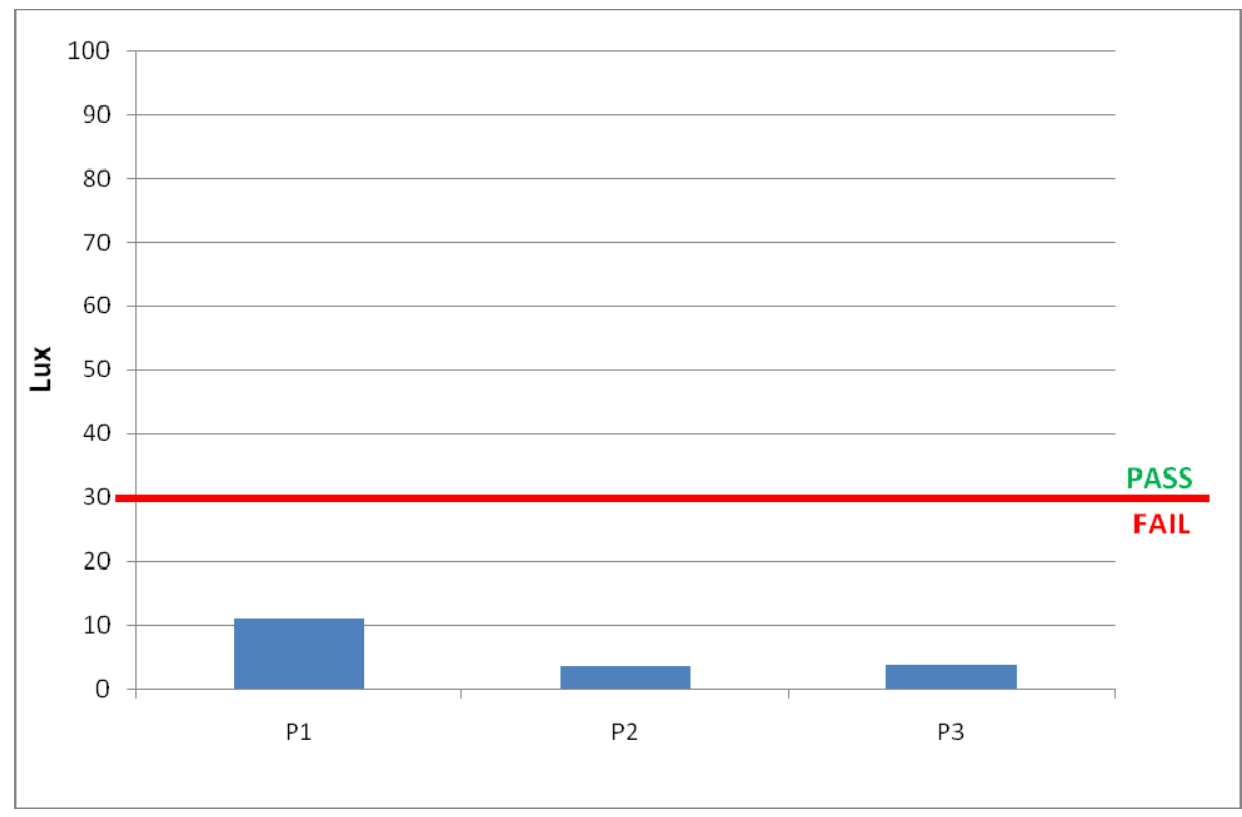

Application of the Tool:

\begin{tabular}{|l|c|}
\hline \multicolumn{2}{|c|}{ NZBC G7 Compliance Assessment Tool } \\
\hline \multicolumn{2}{|l|}{} \\
\hline Variable: & 75 \\
\hline Opposite Building Height & 9.5 \\
\hline Street Width & 14 \\
\hline Glazed Area & West \\
\hline Orientation & Wellington \\
\hline Location & 12 \\
\hline Reflectance of Opposite Building & 87 \\
\hline Glazing Transmittance & 1 \\
\hline Vertical Location of lowest apartment \\
\hline \multicolumn{2}{|c|}{} \\
\hline Are simulations required to prove compliance: \\
\hline
\end{tabular}

Comparison of Results:

\begin{tabular}{|c|c|c|c|}
\hline Measurements & Linear Model & Model 2A & Model 2B \\
\hline Fail & 11 & -19 & -5 \\
\hline
\end{tabular}




\section{Apartment 12}

Measurement Results:

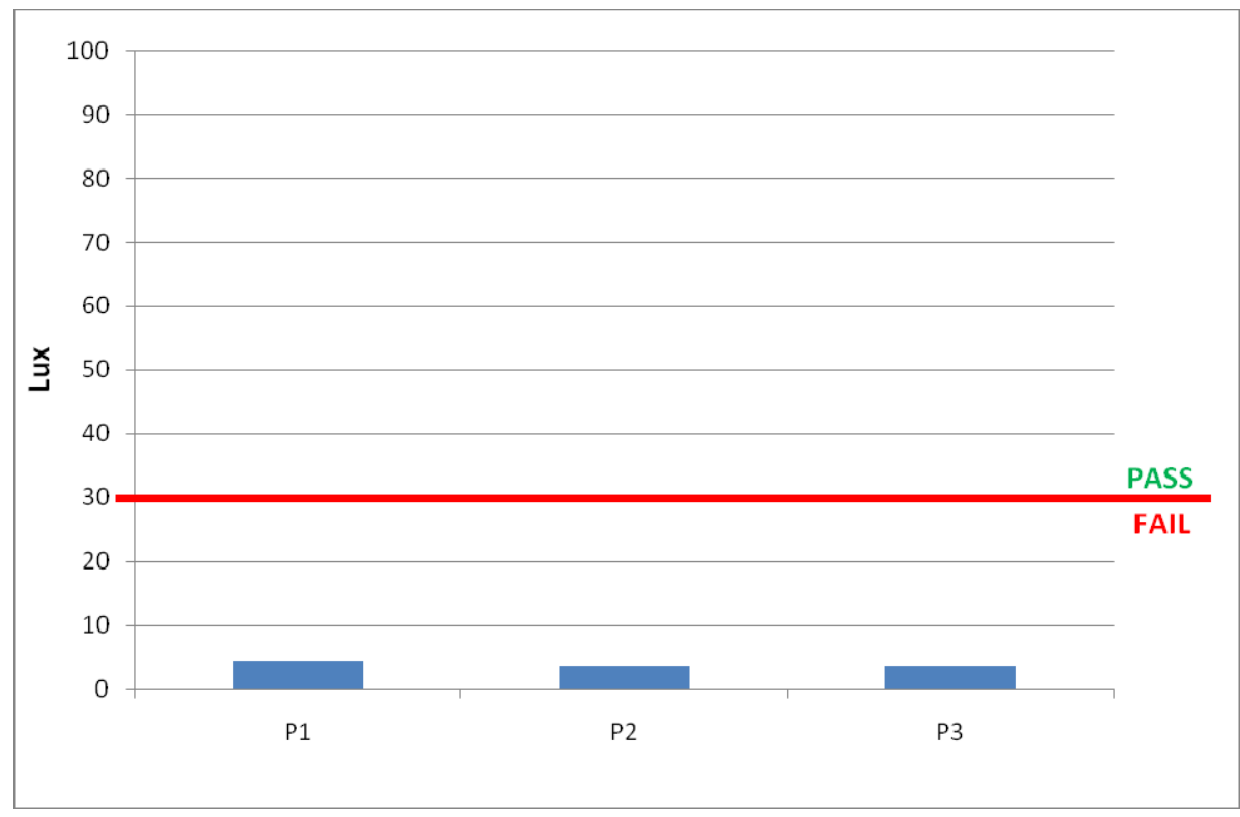

Application of the Tool:

\begin{tabular}{|l|c|}
\hline \multicolumn{2}{|c|}{ NZBC G7 Compliance Assessment Tool } \\
\hline \multicolumn{2}{|l|}{} \\
\hline Variable: & 35 \\
\hline Opposite Building Height & 3 \\
\hline Street Width & 32 \\
\hline Glazed Area & East \\
\hline Orientation & Wellington \\
\hline Location & 40 \\
\hline Reflectance of Opposite Building & 87 \\
\hline Glazing Transmittance & 0 \\
\hline Vertical Location of lowest apartment \\
\hline \multicolumn{2}{|c|}{} \\
\hline Are simulations required to prove compliance: \\
\hline
\end{tabular}

Comparison of Results:

\begin{tabular}{|c|c|c|c|}
\hline Measurements & Linear Model & Model 2A & Model 2B \\
\hline Fail & 44 & 5 & -1.8 \\
\hline
\end{tabular}




\section{Apartment 13}

Measurement Results:

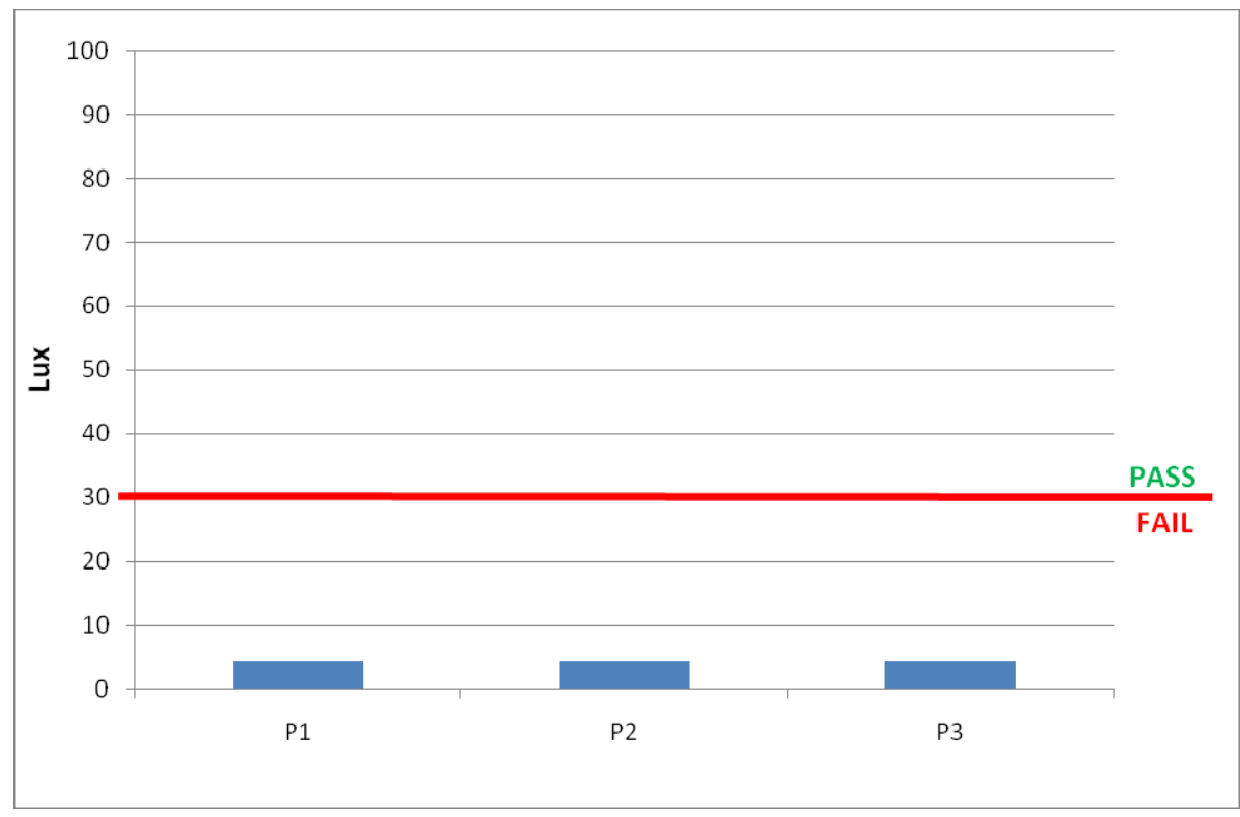

Application of the Tool:

\begin{tabular}{|l|c|}
\hline \multicolumn{2}{|c|}{ NZBC G7 Compliance Assessment Tool } \\
\hline \multicolumn{2}{|l|}{ Variable: } \\
\hline Opposite Building Height & 28 \\
\hline Street Width & 3 \\
\hline Glazed Area & 34 \\
\hline Orientation & North \\
\hline Location & Wellington \\
\hline Reflectance of Opposite Building & 24 \\
\hline Glazing Transmittance & 87 \\
\hline Vertical Location of lowest apartment & 6 \\
\hline \multicolumn{2}{|c|}{} \\
\hline Are simulations required to prove compliance: \\
\hline
\end{tabular}

Comparison of Results:

\begin{tabular}{|c|c|c|c|}
\hline Measurements & Linear Model & Model 2A & Model 2B \\
\hline Fail & 69 & 47 & 39 \\
\hline
\end{tabular}




\section{Apartment 14}

Measurement Results:

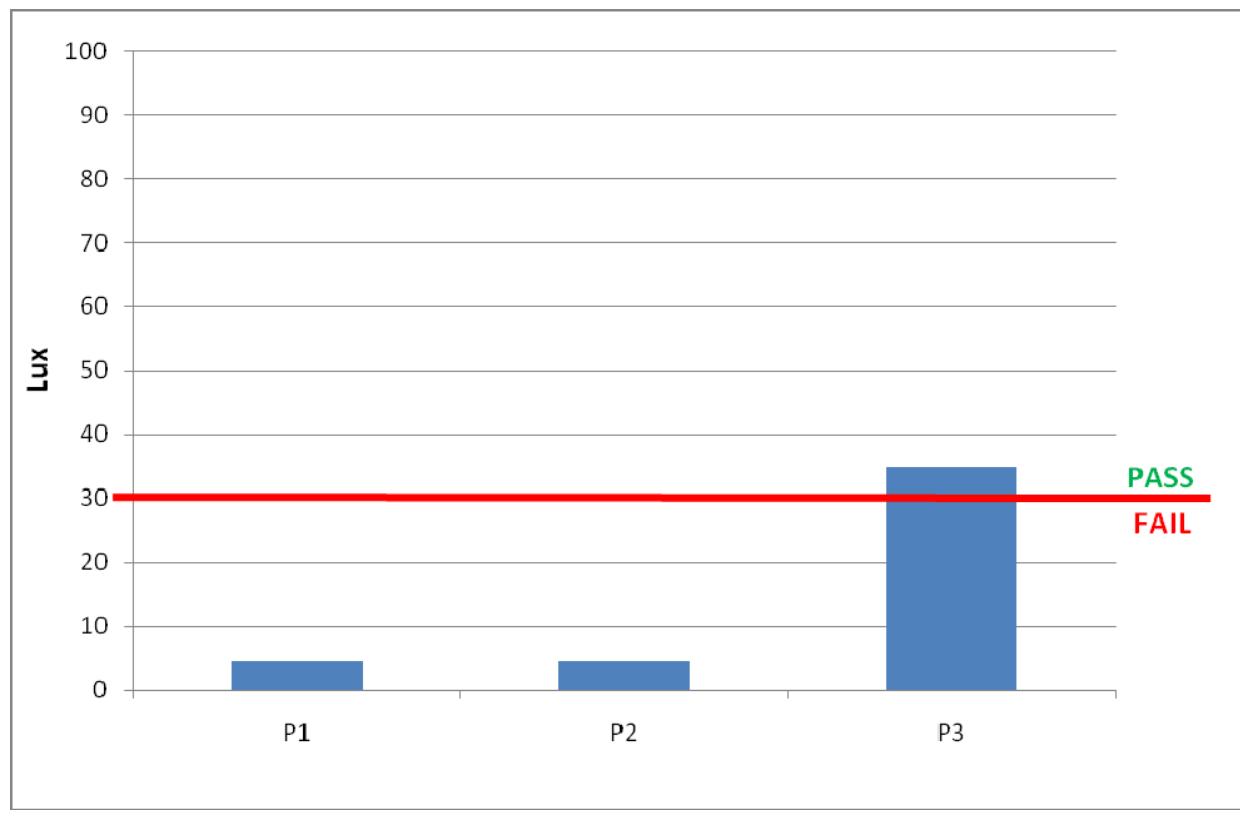

Application of the Tool:

\begin{tabular}{|l|c|}
\hline \multicolumn{2}{|c|}{ NZBC G7 Compliance Assessment Tool } \\
\hline \multicolumn{2}{|l|}{ Variable: } \\
\hline Opposite Building Height & 14 \\
\hline Street Width & 44 \\
\hline Glazed Area & 21 \\
\hline Orientation & South \\
\hline Location & Wellington \\
\hline Reflectance of Opposite Building & 21 \\
\hline Glazing Transmittance & 40 \\
\hline Vertical Location of lowest apartment & 7 \\
\hline \multicolumn{2}{|c|}{} \\
\hline Are simulations required to prove compliance: \\
\hline
\end{tabular}

Comparison of Results:

\begin{tabular}{|c|c|c|c|}
\hline Measurements & Linear Model & Model 2A & Model 2B \\
\hline Fail & 126 & 139 & 152 \\
\hline
\end{tabular}




\section{Apartment 15}

Measurement Results:

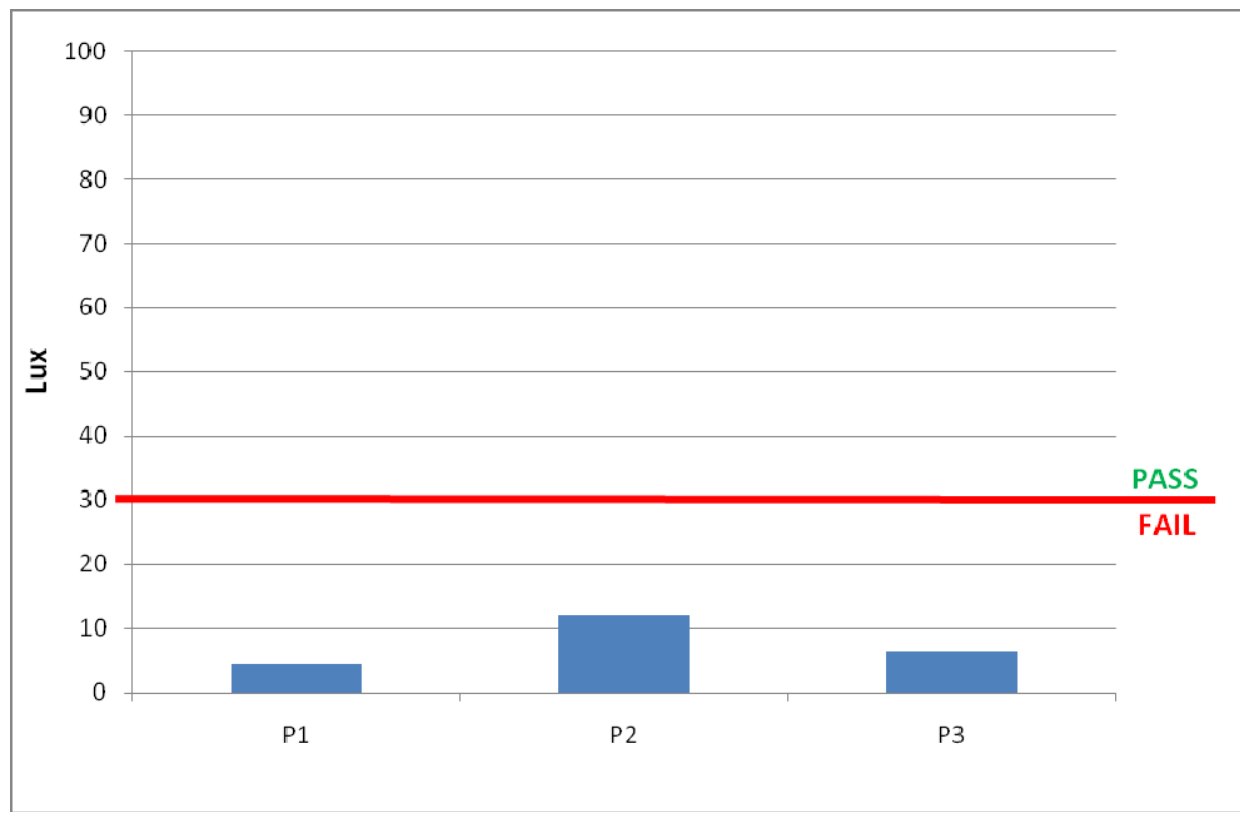

Application of the Tool:

\begin{tabular}{|l|c|}
\hline \multicolumn{2}{|c|}{ NZBC G7 Compliance Assessment Tool } \\
\hline \multicolumn{2}{|l|}{} \\
\hline Variable: & 31 \\
\hline Opposite Building Height & 16 \\
\hline Street Width & 24 \\
\hline Glazed Area & South \\
\hline Orientation & Wellington \\
\hline Location & 10.8 \\
\hline Reflectance of Opposite Building & 87 \\
\hline Glazing Transmittance & 1 \\
\hline Vertical Location of lowest apartment \\
\hline \multicolumn{2}{|}{} \\
\hline Are simulations required to prove compliance: \\
\hline
\end{tabular}

Comparison of Results:

\begin{tabular}{|c|c|c|c|}
\hline Measurements & Linear Model & Model 2A & Model 2B \\
\hline Fail & 59 & 20 & 21 \\
\hline
\end{tabular}




\section{Apartment 16}

Measurement Results:

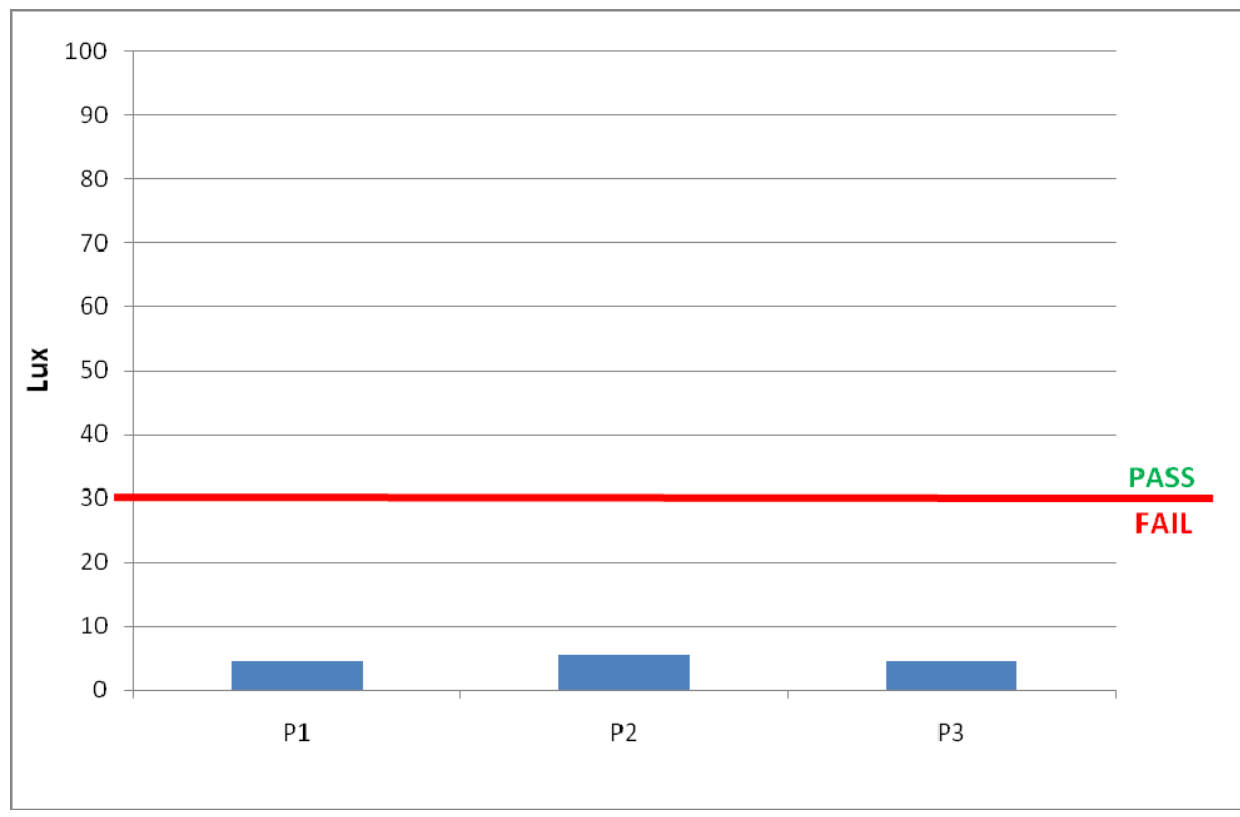

Application of the Tool:

\begin{tabular}{|l|c|}
\hline \multicolumn{2}{|c|}{ NZBC G7 Compliance Assessment Tool } \\
\hline \multicolumn{2}{|l|}{} \\
\hline Variable: & 20 \\
\hline Opposite Building Height & 5 \\
\hline Street Width & 23 \\
\hline Glazed Area & North \\
\hline Orientation & Wellington \\
\hline Location & 33.3 \\
\hline Reflectance of Opposite Building & 87 \\
\hline Glazing Transmittance & 3 \\
\hline Vertical Location of lowest apartment \\
\hline \multicolumn{2}{|}{} \\
\hline Are simulations required to prove compliance: \\
\hline
\end{tabular}

Comparison of Results:

\begin{tabular}{|c|c|c|c|}
\hline Measurements & Linear Model & Model 2A & Model 2B \\
\hline Fail & 52 & 15 & 19 \\
\hline
\end{tabular}




\section{Apartment 17}

Measurement Results:

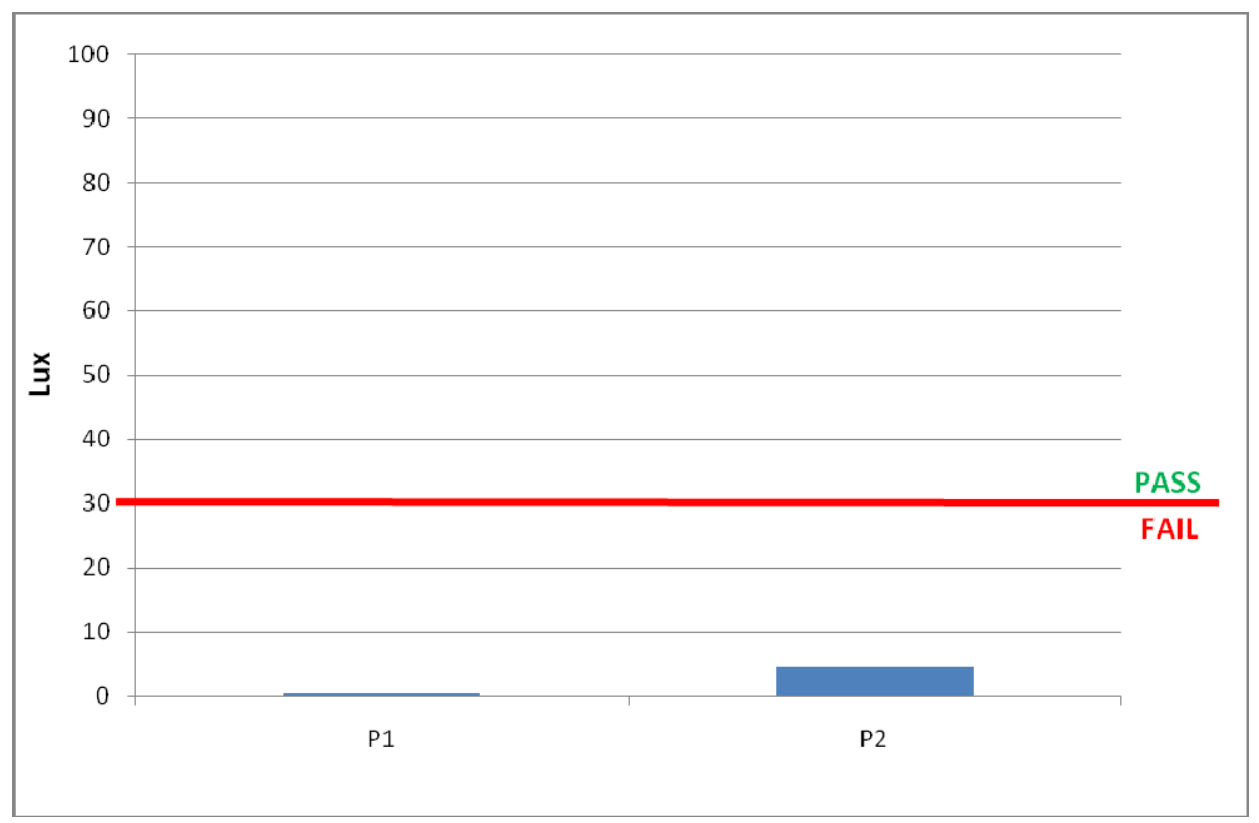

Application of the Tool:

\begin{tabular}{|l|c|}
\hline \multicolumn{2}{|c|}{ NZBC G7 Compliance Assessment Tool } \\
\hline \multicolumn{2}{|l|}{ Variable: } \\
\hline Opposite Building Height & 69 \\
\hline Street Width & 15 \\
\hline Glazed Area & 10 \\
\hline Orientation & South \\
\hline Location & Wellington \\
\hline Reflectance of Opposite Building & 20.8 \\
\hline Glazing Transmittance & 87 \\
\hline Vertical Location of lowest apartment & 4 \\
\hline \multicolumn{2}{|l}{} \\
\hline Are simulations required to prove compliance: \\
\hline
\end{tabular}

Comparison of Results:

\begin{tabular}{|c|c|c|c|}
\hline Measurements & Linear Model & Model 2A & Model 2B \\
\hline Fail & 36 & 5 & 9 \\
\hline
\end{tabular}




\section{Apartment 18}

Measurement Results:

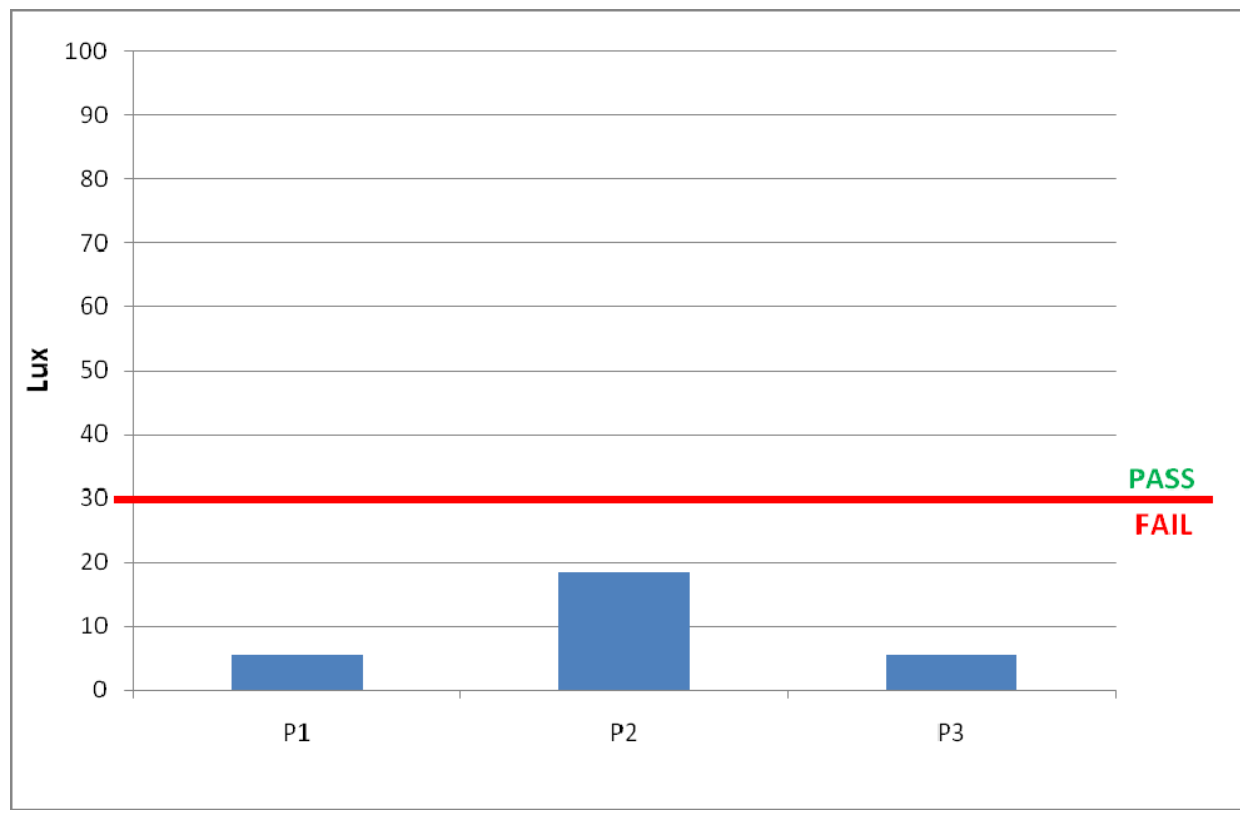

Application of the Tool:

\begin{tabular}{|l|c|}
\hline \multicolumn{2}{|c|}{ NZBC G7 Compliance Assessment Tool } \\
\hline \multicolumn{2}{|l|}{} \\
\hline Variable: & 3 \\
\hline Opposite Building Height & 1.8 \\
\hline Street Width & 30 \\
\hline Glazed Area & South \\
\hline Orientation & Wellington \\
\hline Location & 38.5 \\
\hline Reflectance of Opposite Building & 87 \\
\hline Glazing Transmittance & 0 \\
\hline Vertical Location of lowest apartment \\
\hline \multicolumn{2}{|}{} \\
\hline Are simulations required to prove compliance: \\
\hline
\end{tabular}

Comparison of Results:

\begin{tabular}{|c|c|c|c|}
\hline Measurements & Linear Model & Model 2A & Model 2B \\
\hline Fail & 56 & 56 & 51 \\
\hline
\end{tabular}




\section{Apartment 19}

Measurement Results:

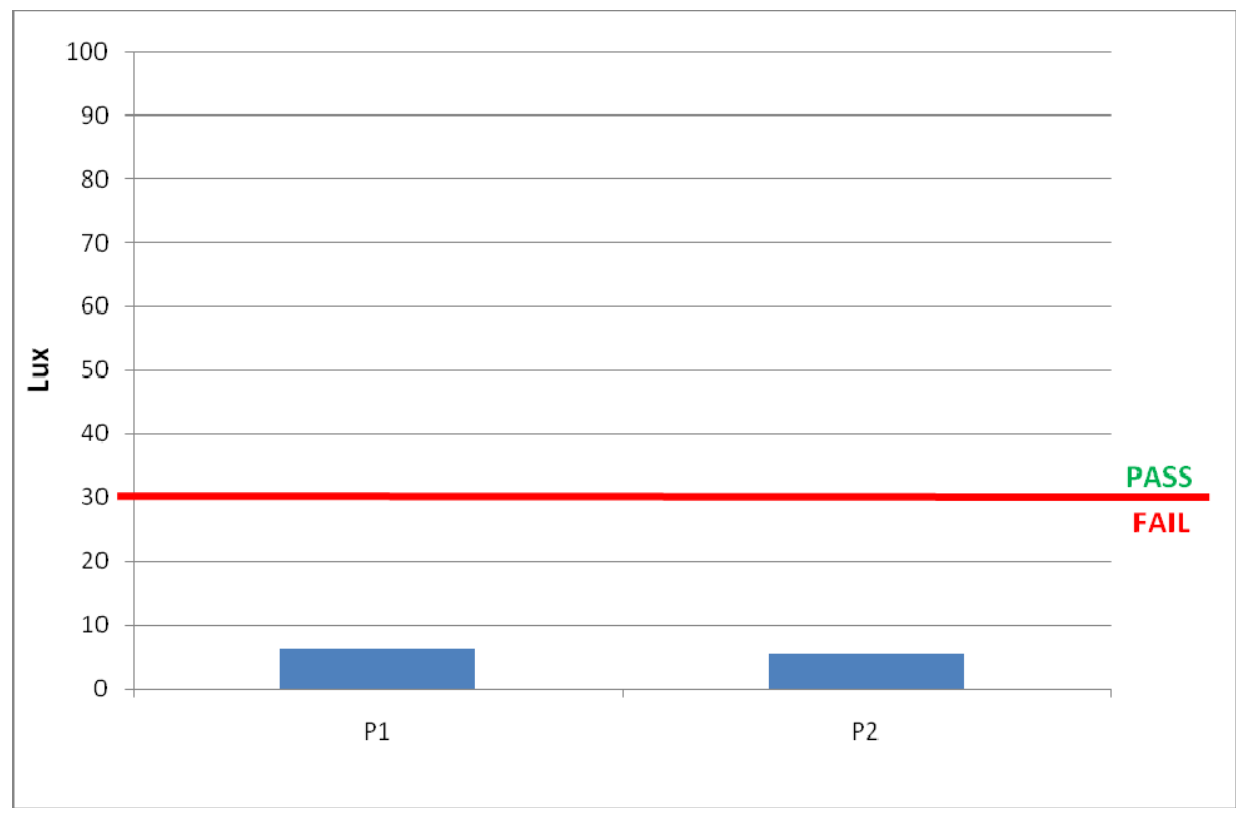

Application of the Tool:

\begin{tabular}{|l|c|}
\hline \multicolumn{2}{|c|}{ NZBC G7 Compliance Assessment Tool } \\
\hline \multicolumn{2}{|l|}{} \\
\hline Variable: & 3 \\
\hline Opposite Building Height & 1.8 \\
\hline Street Width & 32 \\
\hline Glazed Area & South \\
\hline Orientation & Wellington \\
\hline Location & 38.5 \\
\hline Reflectance of Opposite Building & 87 \\
\hline Glazing Transmittance & 0 \\
\hline Vertical Location of lowest apartment \\
\hline \multicolumn{2}{|l}{} \\
\hline Are simulations required to prove compliance: \\
\hline
\end{tabular}

Comparison of Results:

\begin{tabular}{|c|c|c|c|}
\hline Measurements & Linear Model & Model 2A & Model 2B \\
\hline Fail & 59 & 59 & 56 \\
\hline
\end{tabular}




\section{Apartment 20}

Measurement Results:

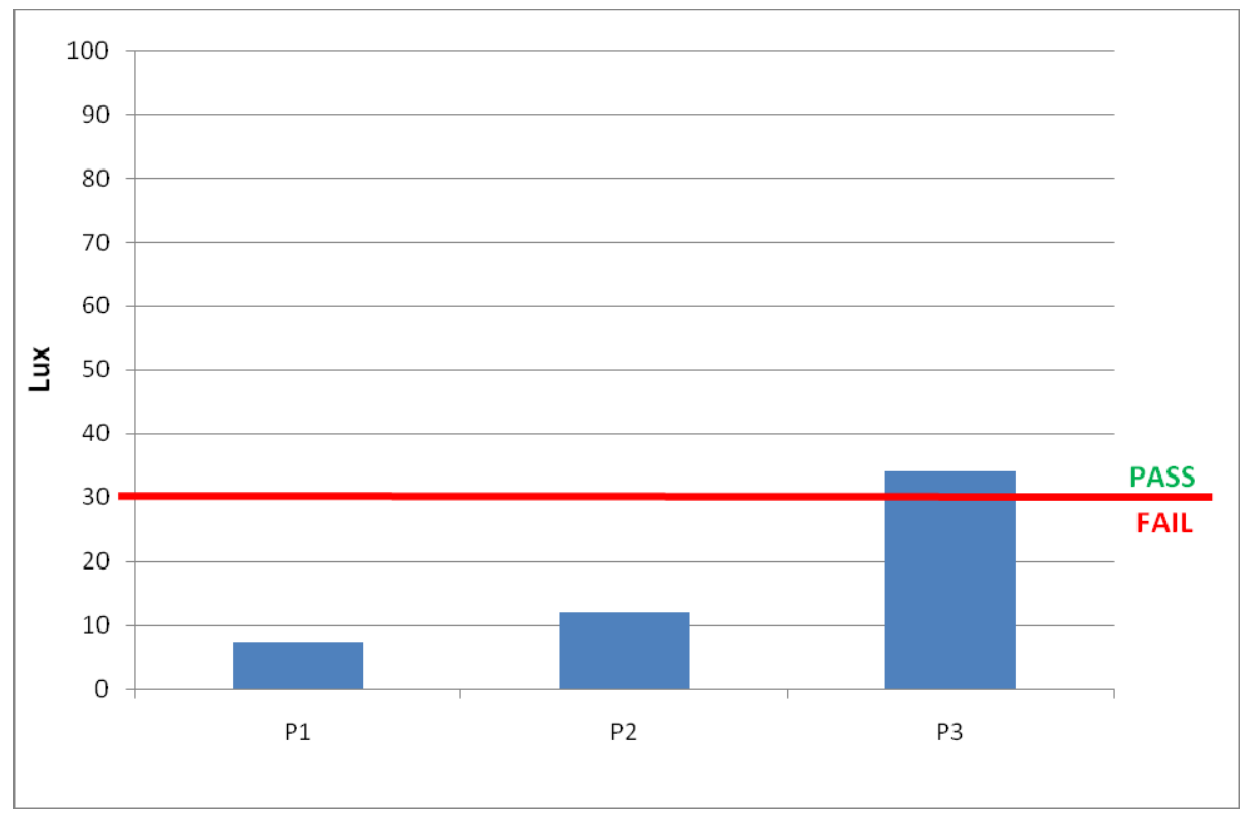

Application of the Tool:

\begin{tabular}{|l|c|}
\hline \multicolumn{2}{|c|}{ NZBC G7 Compliance Assessment Tool } \\
\hline \multicolumn{2}{|l|}{ Variable: } \\
\hline Opposite Building Height & 60 \\
\hline Street Width & 30 \\
\hline Glazed Area & 36 \\
\hline Orientation & East \\
\hline Location & Wellington \\
\hline Reflectance of Opposite Building & 30 \\
\hline Glazing Transmittance & 87 \\
\hline Vertical Location of lowest apartment & 4 \\
\hline \multicolumn{2}{|c|}{} \\
\hline Are simulations required to prove compliance: \\
\hline
\end{tabular}

Comparison of Results:

\begin{tabular}{|c|c|c|c|}
\hline Measurements & Linear Model & Model 2A & Model 2B \\
\hline Fail & 102 & 65 & 42 \\
\hline
\end{tabular}




\section{Apartment 21}

Measurement Results:

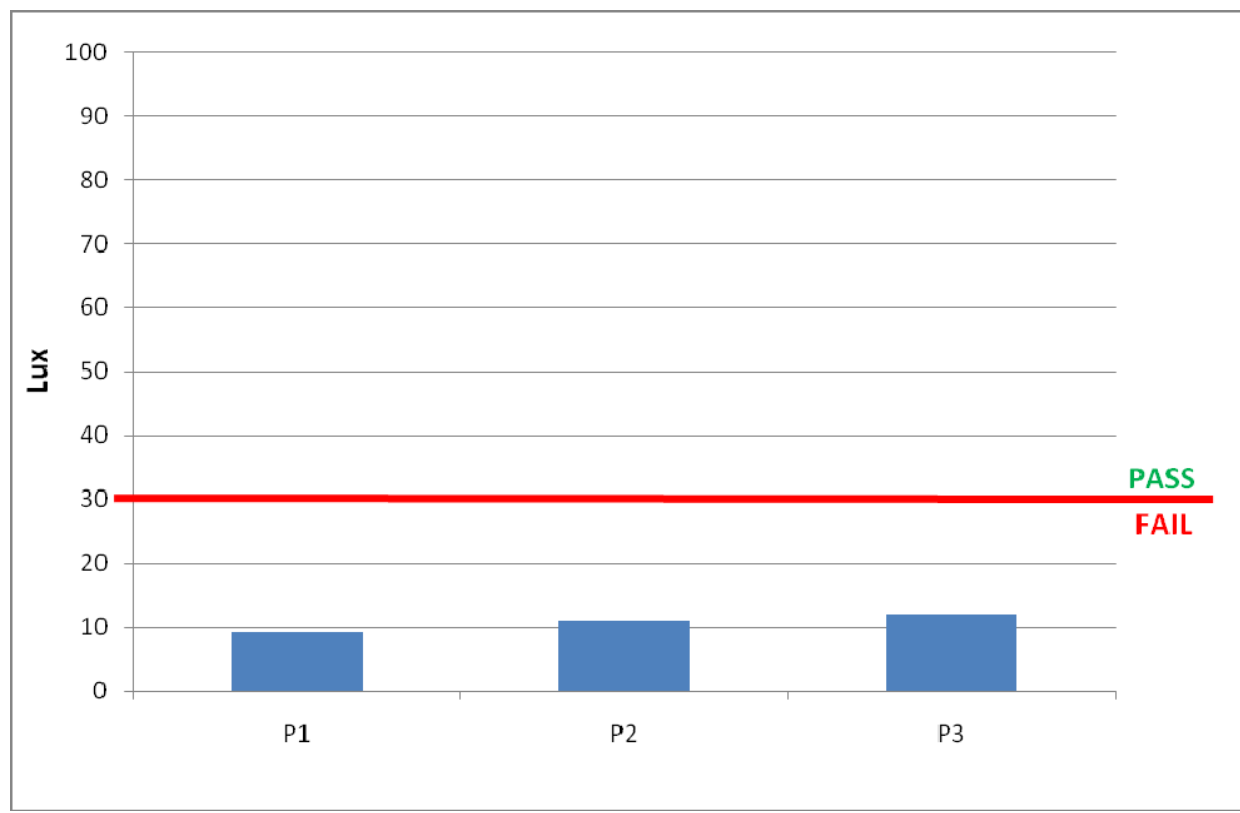

Application of the Tool:

\begin{tabular}{|l|c|}
\hline \multicolumn{2}{|c|}{ NZBC G7 Compliance Assessment Tool } \\
\hline \multicolumn{2}{|l|}{} \\
\hline Variable: & 75 \\
\hline Opposite Building Height & 9.5 \\
\hline Street Width & 11.8 \\
\hline Glazed Area & East \\
\hline Orientation & Wellington \\
\hline Location & 12 \\
\hline Reflectance of Opposite Building & 87 \\
\hline Glazing Transmittance & 3 \\
\hline Vertical Location of lowest apartment \\
\hline \multicolumn{2}{|}{} \\
\hline Are simulations required to prove compliance: \\
\hline
\end{tabular}

Comparison of Results:

\begin{tabular}{|c|c|c|c|}
\hline Measurements & Linear Model & Model 2A & Model 2B \\
\hline Fail & 17 & -15 & -5 \\
\hline
\end{tabular}




\section{Apartment 22}

Measurement Results:

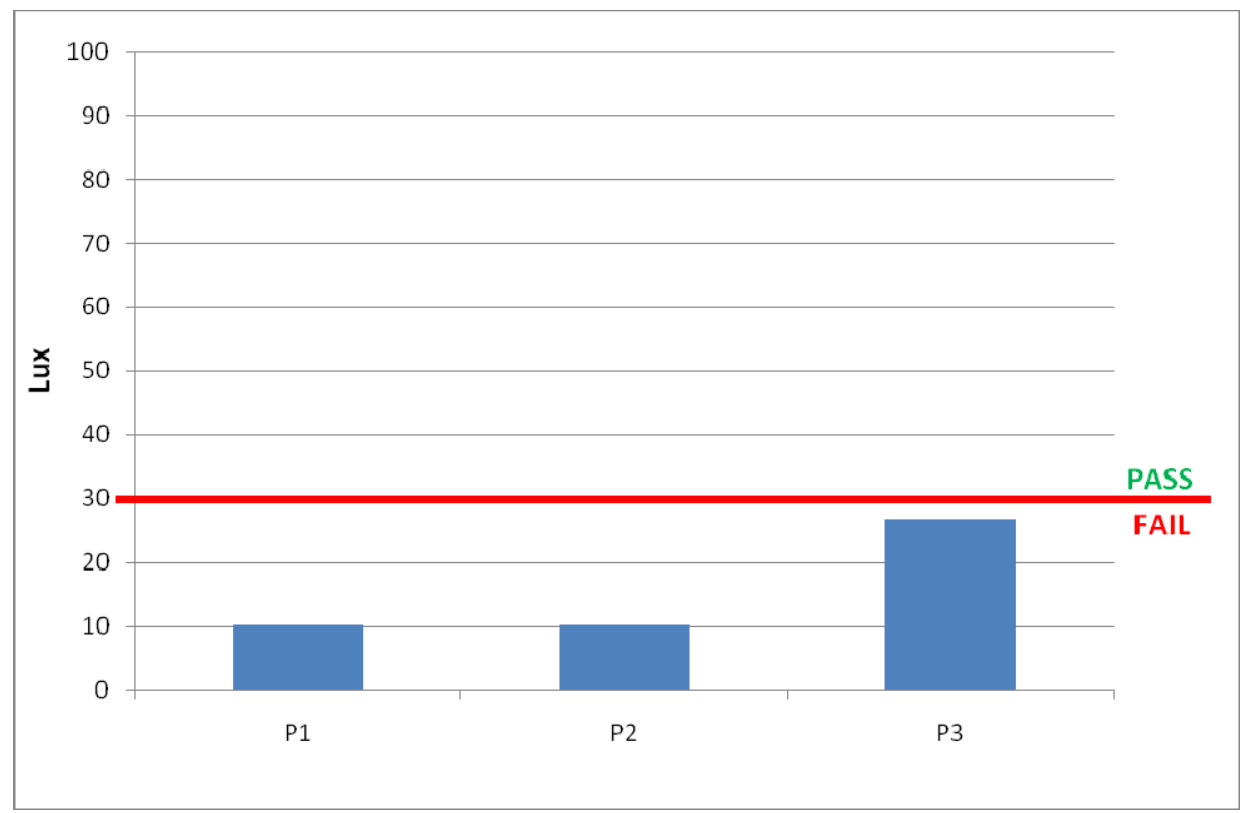

Application of the Tool:

\begin{tabular}{|l|c|}
\hline \multicolumn{2}{|c|}{ NZBC G7 Compliance Assessment Tool } \\
\hline \multicolumn{2}{|l|}{ Variable: } \\
\hline Opposite Building Height & 12 \\
\hline Street Width & 3 \\
\hline Glazed Area & 54 \\
\hline Orientation & North \\
\hline Location & Wellington \\
\hline Reflectance of Opposite Building & 21 \\
\hline Glazing Transmittance & 87 \\
\hline Vertical Location of lowest apartment & 7 \\
\hline \multicolumn{2}{|c|}{} \\
\hline Are simulations required to prove compliance: \\
\hline
\end{tabular}

Comparison of Results:

\begin{tabular}{|c|c|c|c|}
\hline Measurements & Linear Model & Model 2A & Model 2B \\
\hline Fail & 105 & 137 & 315 \\
\hline
\end{tabular}




\section{Apartment 23}

Measurement Results:

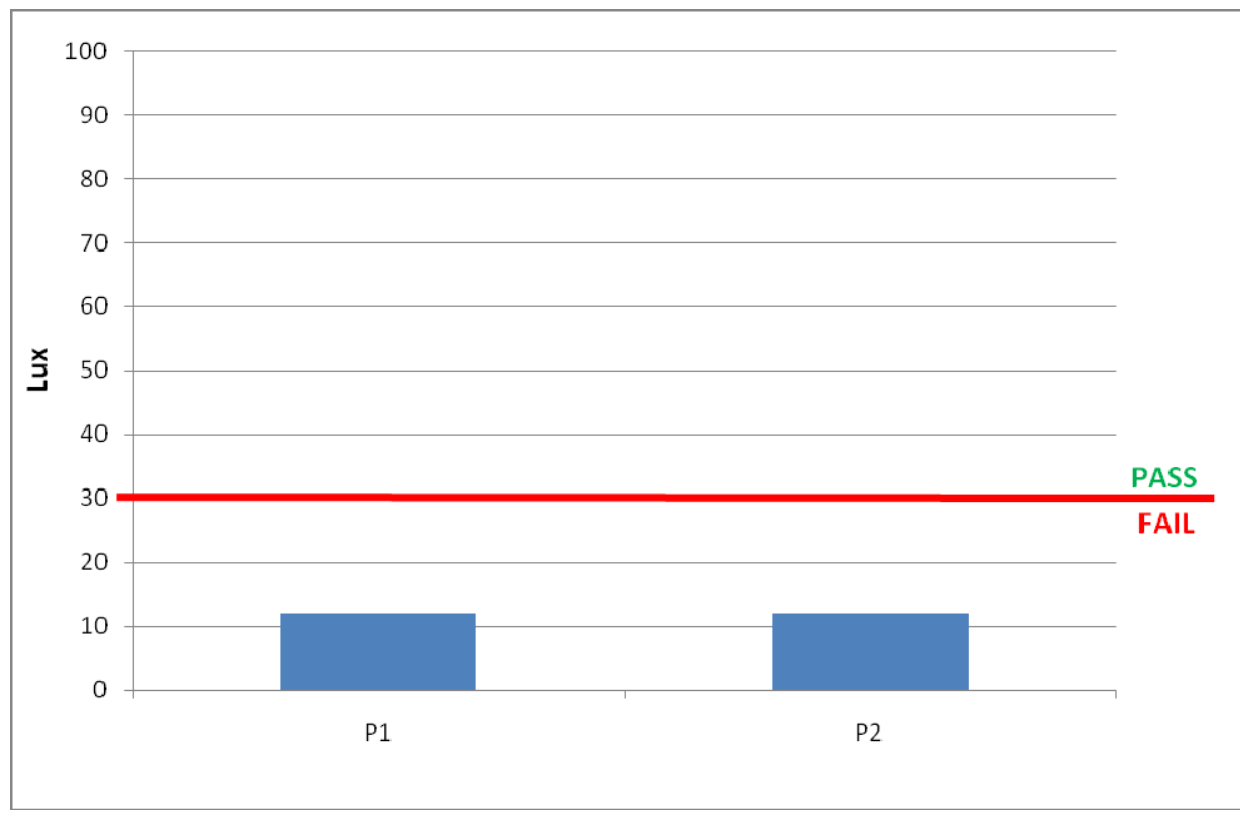

Application of the Tool:

\begin{tabular}{|l|c|}
\hline \multicolumn{2}{|c|}{ NZBC G7 Compliance Assessment Tool } \\
\hline \multicolumn{2}{|l|}{} \\
\hline Variable: & 20 \\
\hline Opposite Building Height & 5 \\
\hline Street Width & 26 \\
\hline Glazed Area & North \\
\hline Orientation & Wellington \\
\hline Location & 33.3 \\
\hline Reflectance of Opposite Building & 87 \\
\hline Glazing Transmittance & 3 \\
\hline Vertical Location of lowest apartment \\
\hline \multicolumn{2}{|}{} \\
\hline Are simulations required to prove compliance: \\
\hline
\end{tabular}

Comparison of Results:

\begin{tabular}{|c|c|c|c|}
\hline Measurements & Linear Model & Model 2A & Model 2B \\
\hline Fail & 56 & 19 & 24 \\
\hline
\end{tabular}




\section{Apartment 24}

Measurement Results:

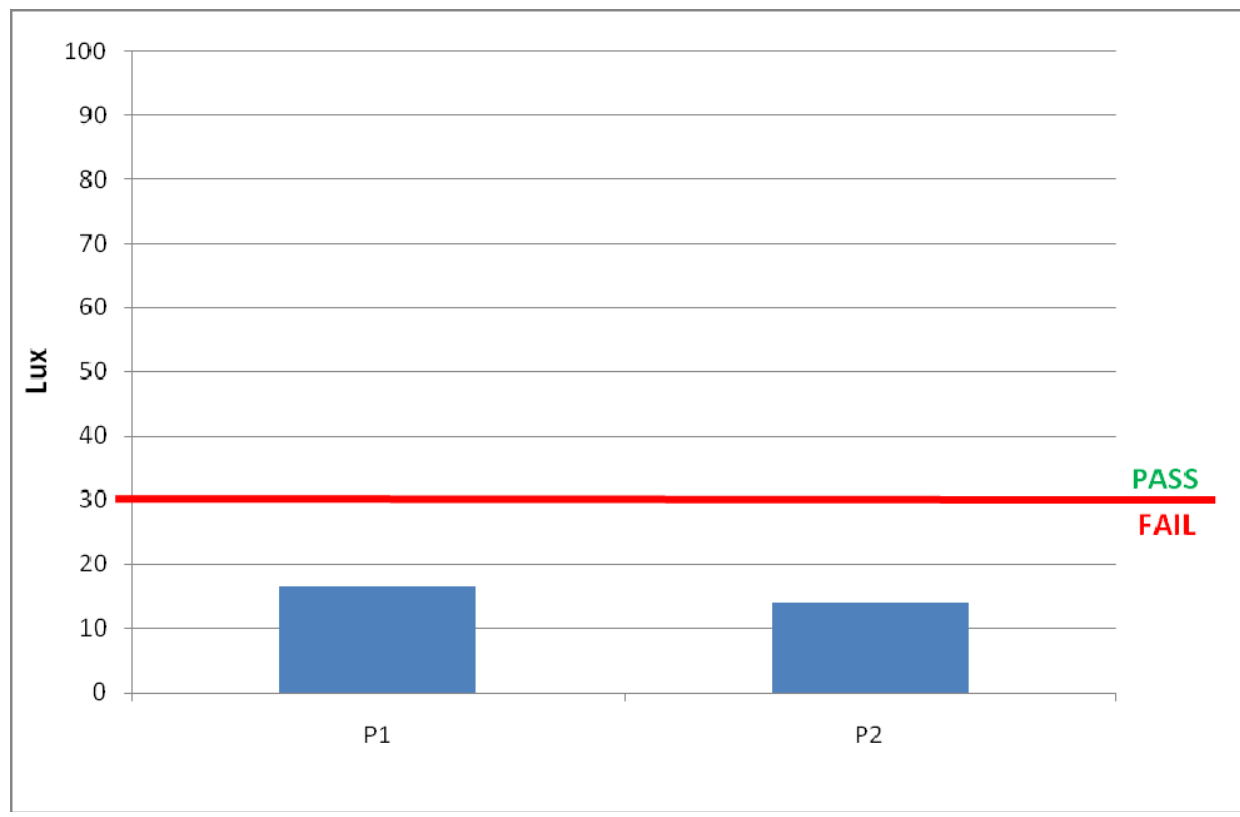

Application of the Tool:

\begin{tabular}{|l|c|}
\hline \multicolumn{2}{|c|}{ NZBC G7 Compliance Assessment Tool } \\
\hline \multicolumn{2}{|l|}{} \\
\hline Variable: & 0 \\
\hline Opposite Building Height & 0 \\
\hline Street Width & 11 \\
\hline Glazed Area & North \\
\hline Orientation & Wellington \\
\hline Location & 0 \\
\hline Reflectance of Opposite Building & 87 \\
\hline Glazing Transmittance & 1 \\
\hline Vertical Location of lowest apartment \\
\hline \multicolumn{2}{|}{} \\
\hline Are simulations required to prove compliance: \\
\hline
\end{tabular}

Comparison of Results:

\begin{tabular}{|c|c|c|c|}
\hline Measurements & Linear Model & Model 2A & Model 2B \\
\hline Fail & 20 & 30 & 2 \\
\hline
\end{tabular}




\section{Apartment 25}

Measurement Results:

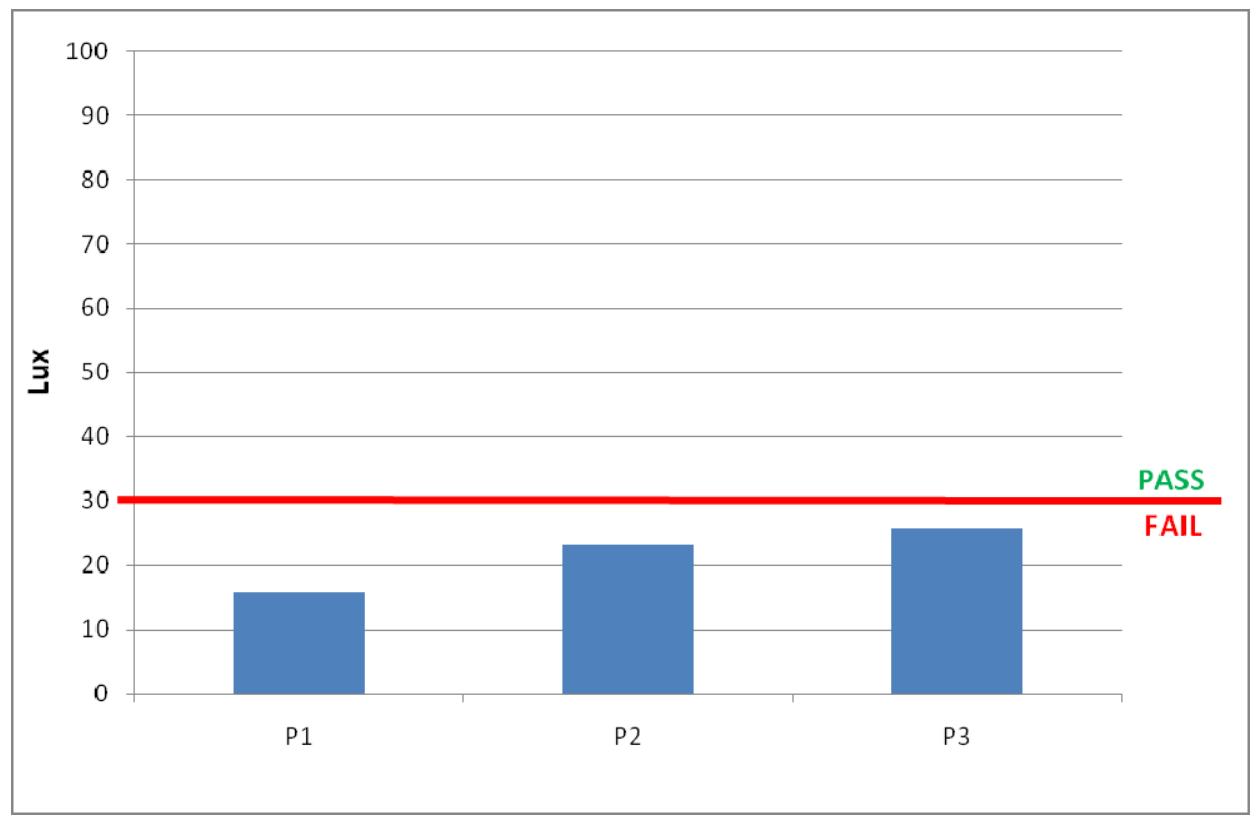

Application of the Tool:

\begin{tabular}{|l|c|}
\hline \multicolumn{2}{|c|}{ NZBC G7 Compliance Assessment Tool } \\
\hline \multicolumn{2}{|l|}{} \\
\hline Variable: & 20 \\
\hline Opposite Building Height & 14.5 \\
\hline Street Width & 17 \\
\hline Glazed Area & South \\
\hline Orientation & Wellington \\
\hline Location & 13.5 \\
\hline Reflectance of Opposite Building & 87 \\
\hline Glazing Transmittance & 1 \\
\hline Vertical Location of lowest apartment \\
\hline \multicolumn{2}{|c|}{} \\
\hline Are simulations required to prove compliance: & YES \\
\hline
\end{tabular}

Comparison of Results:

\begin{tabular}{|c|c|c|c|}
\hline Measurements & Linear Model & Model 2A & Model 2B \\
\hline Fail & 53 & 22 & 22 \\
\hline
\end{tabular}




\section{Apartment 26}

Measurement Results:

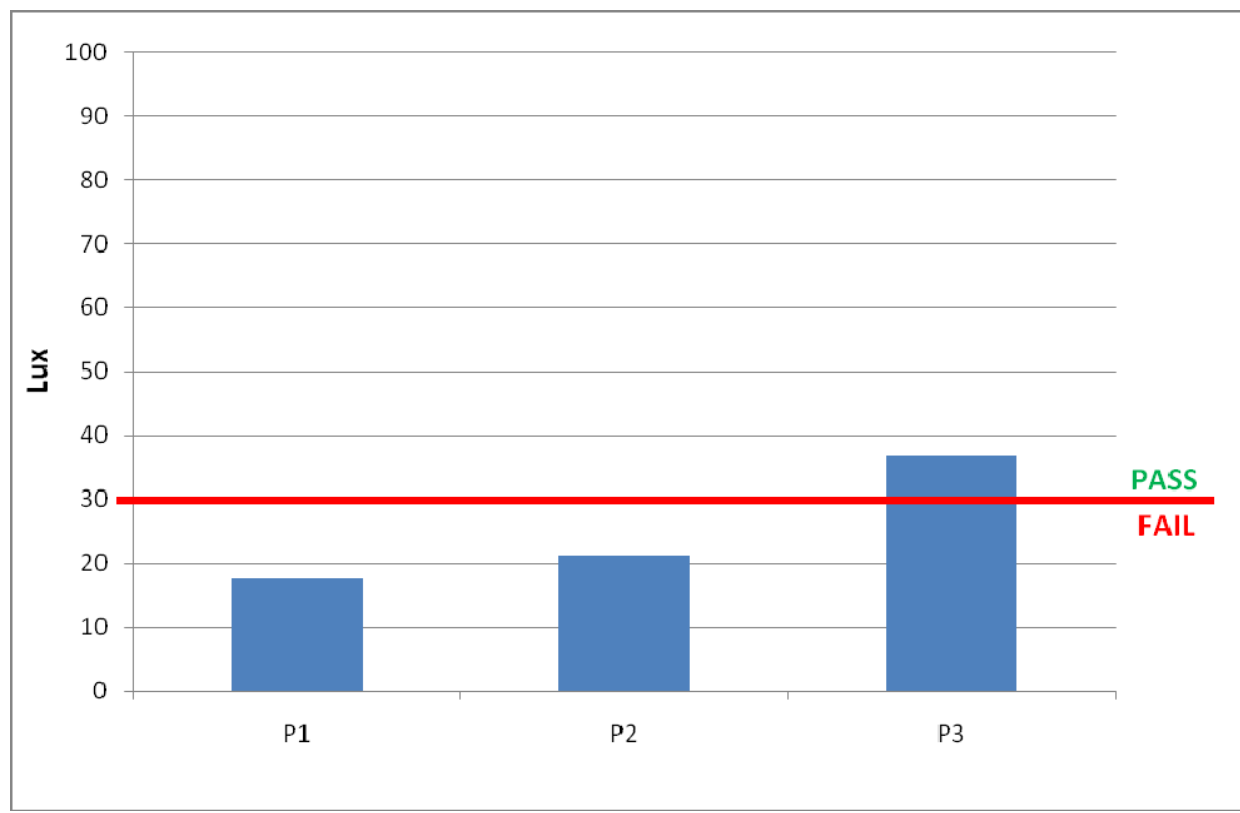

Application of the Tool:

\begin{tabular}{|l|c|}
\hline \multicolumn{2}{|c|}{ NZBC G7 Compliance Assessment Tool } \\
\hline \multicolumn{2}{|l|}{ Variable: } \\
\hline Opposite Building Height & 16 \\
\hline Street Width & 9 \\
\hline Glazed Area & 22 \\
\hline Orientation & East \\
\hline Location & Wellington \\
\hline Reflectance of Opposite Building & 14.8 \\
\hline Glazing Transmittance & 87 \\
\hline Vertical Location of lowest apartment & 1 \\
\hline \multicolumn{2}{|c|}{} \\
\hline Are simulations required to prove compliance: \\
\hline
\end{tabular}

Comparison of Results:

\begin{tabular}{|c|c|c|c|}
\hline Measurements & Linear Model & Model 2A & Model 2B \\
\hline Fail & 49 & 21 & 17 \\
\hline
\end{tabular}




\section{Apartment 27}

Measurement Results:

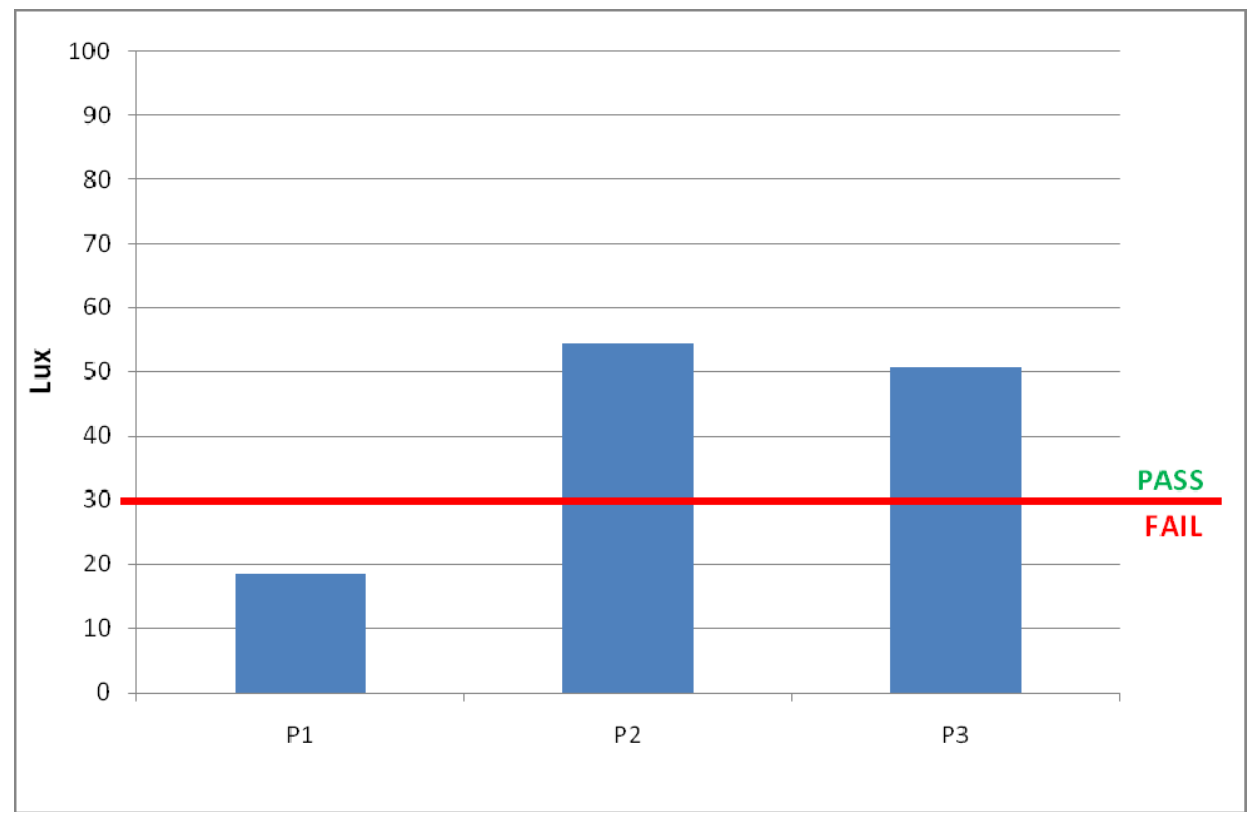

Application of the Tool:

\begin{tabular}{|l|c|}
\hline \multicolumn{2}{|c|}{ NZBC G7 Compliance Assessment Tool } \\
\hline \multicolumn{2}{|l|}{} \\
\hline Variable: & 0 \\
\hline Opposite Building Height & 0 \\
\hline Street Width & 20 \\
\hline Glazed Area & North \\
\hline Orientation & Wellington \\
\hline Location & 0 \\
\hline Reflectance of Opposite Building & 87 \\
\hline Glazing Transmittance & 1 \\
\hline Vertical Location of lowest apartment & \\
\hline \multicolumn{2}{|c|}{} \\
\hline Are simulations required to prove compliance: & YES \\
\hline
\end{tabular}

Comparison of Results:

\begin{tabular}{|c|c|c|c|}
\hline Measurements & Linear Model & Model 2A & Model 2B \\
\hline Fail & 31 & 38 & 15 \\
\hline
\end{tabular}




\section{Apartment 28}

Measurement Results:

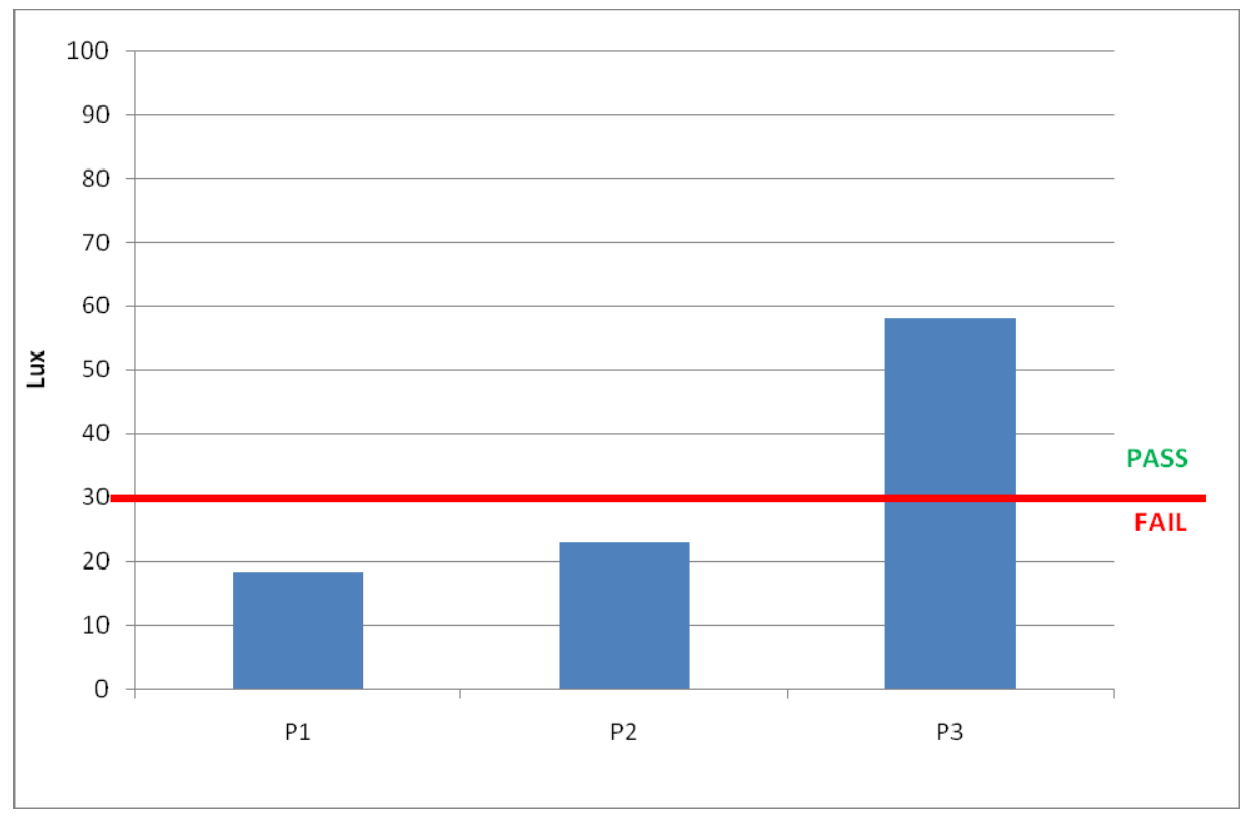

Application of the Tool:

\begin{tabular}{|l|c|}
\hline \multicolumn{2}{|c|}{ NZBC G7 Compliance Assessment Tool } \\
\hline \multicolumn{2}{|c|}{} \\
\hline Variable: & 26 \\
\hline Opposite Building Height & 24 \\
\hline Street Width & 26 \\
\hline Glazed Area & West \\
\hline Orientation & Christchurch \\
\hline Location & 20.8 \\
\hline Reflectance of Opposite Building & 87 \\
\hline Glazing Transmittance & 8 \\
\hline Vertical Location of lowest apartment \\
\hline \multicolumn{2}{|}{} \\
\hline Are simulations required to prove compliance: \\
\hline
\end{tabular}

Comparison of Results:

\begin{tabular}{|c|c|c|c|}
\hline Measurements & Linear Model & Model 2A & Model 2B \\
\hline Fail & 104 & 123 & 83 \\
\hline
\end{tabular}




\section{Apartment 29}

Measurement Results:

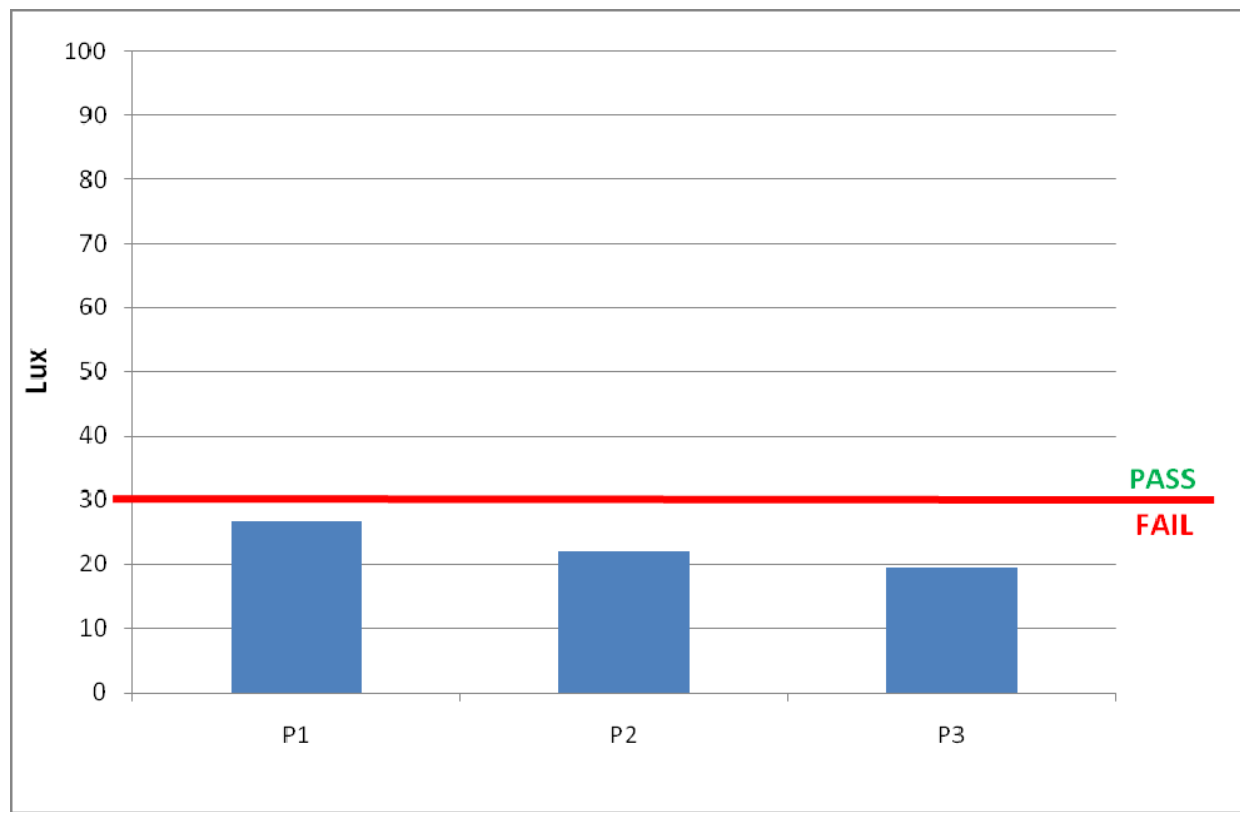

Application of the Tool:

\begin{tabular}{|l|c|}
\hline \multicolumn{2}{|c|}{ NZBC G7 Compliance Assessment Tool } \\
\hline \multicolumn{2}{|l|}{ Variable: } \\
\hline Opposite Building Height & 75 \\
\hline Street Width & 9.5 \\
\hline Glazed Area & 11.8 \\
\hline Orientation & East \\
\hline Location & Wellington \\
\hline Reflectance of Opposite Building & 12 \\
\hline Glazing Transmittance & 87 \\
\hline Vertical Location of lowest apartment & 3 \\
\hline \multicolumn{2}{|}{} \\
\hline Are simulations required to prove compliance: \\
\hline
\end{tabular}

Comparison of Results:

\begin{tabular}{|c|c|c|c|}
\hline Measurements & Linear Model & Model 2A & Model 2B \\
\hline Fail & 16 & -15 & -5 \\
\hline
\end{tabular}




\section{Apartment 30}

Measurement Results:

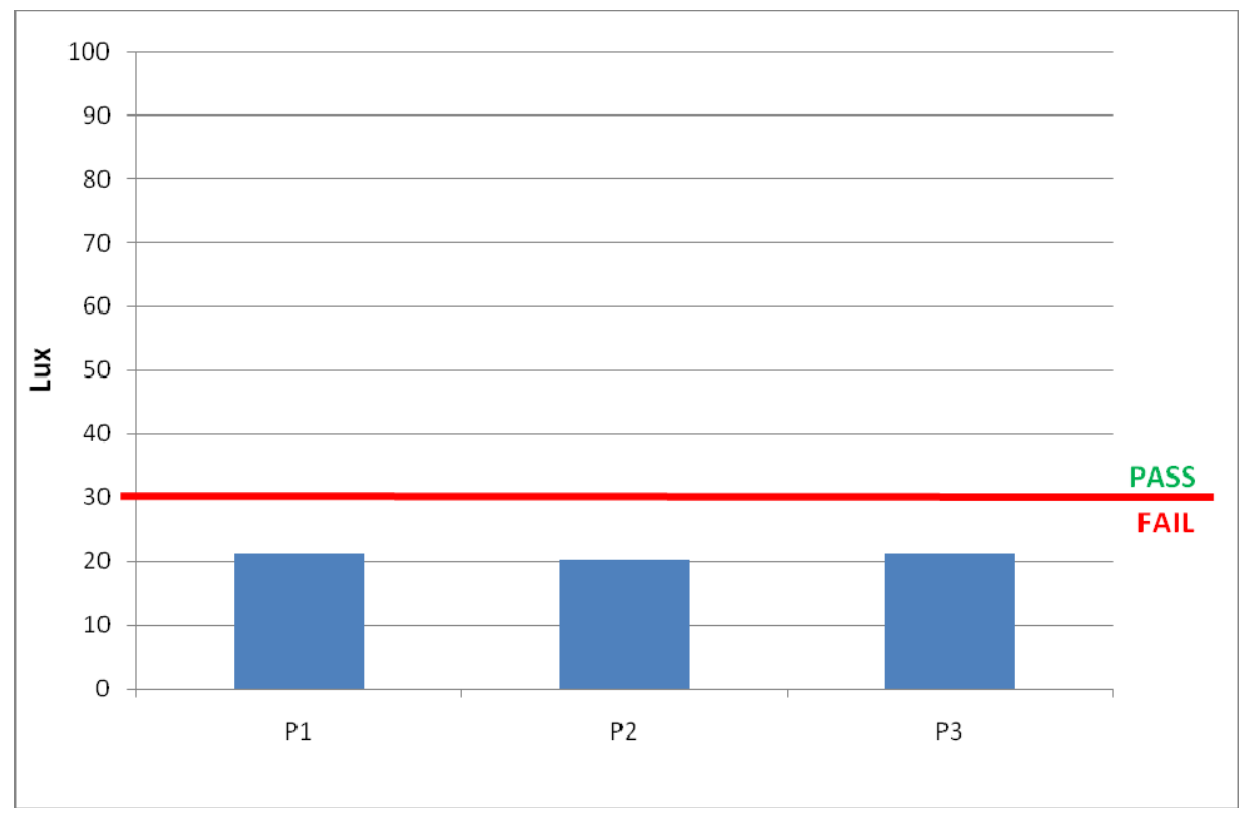

Application of the Tool:

\begin{tabular}{|l|c|}
\hline \multicolumn{2}{|c|}{ NZBC G7 Compliance Assessment Tool } \\
\hline \multicolumn{2}{|l|}{} \\
\hline Variable: & 60 \\
\hline Opposite Building Height & 30 \\
\hline Street Width & 13 \\
\hline Glazed Area & East \\
\hline Orientation & Wellington \\
\hline Location & 30 \\
\hline Reflectance of Opposite Building & 87 \\
\hline Glazing Transmittance & 4 \\
\hline Vertical Location of lowest apartment \\
\hline \multicolumn{2}{|l}{} \\
\hline Are simulations required to prove compliance: \\
\hline
\end{tabular}

Comparison of Results:

\begin{tabular}{|c|c|c|c|}
\hline Measurements & Linear Model & Model 2A & Model 2B \\
\hline Fail & 72 & 36 & 35 \\
\hline
\end{tabular}




\section{Apartment 31}

Measurement Results:

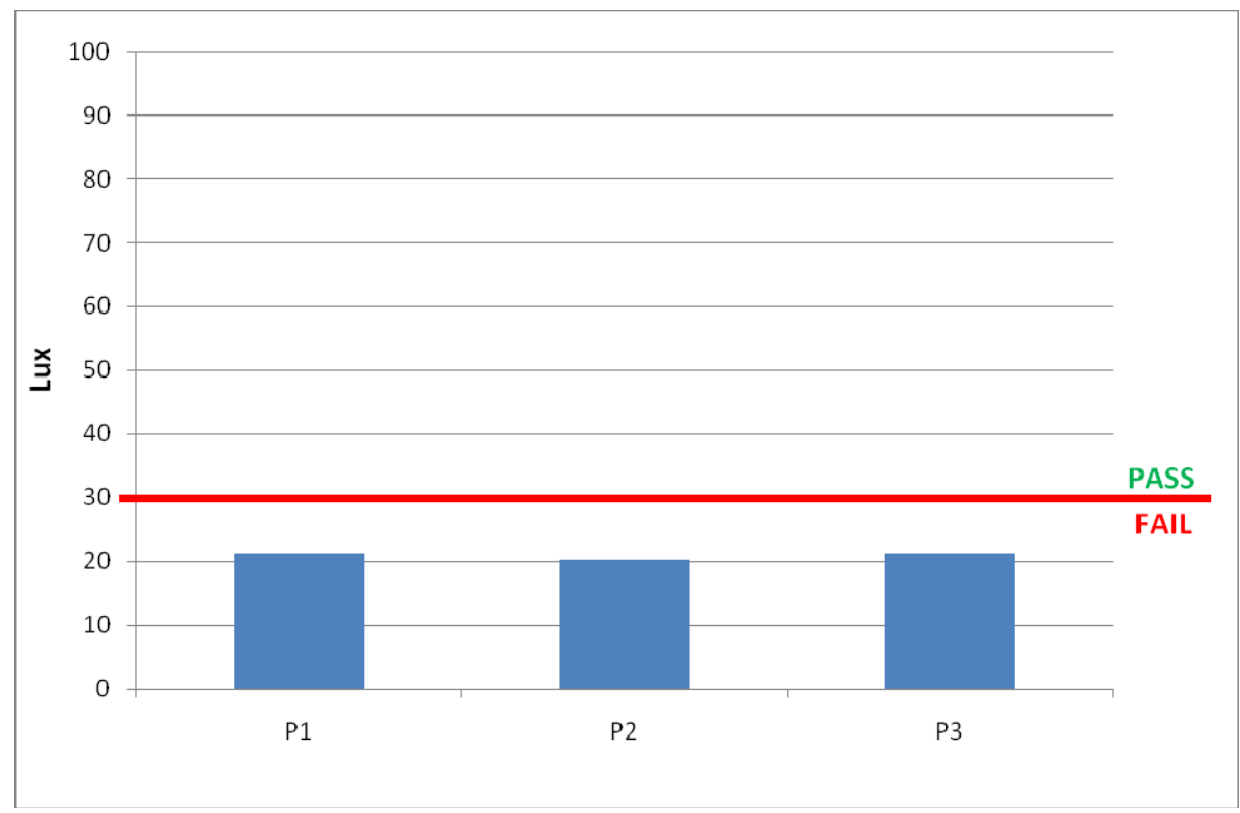

Application of the Tool:

\begin{tabular}{|l|c|}
\hline \multicolumn{2}{|c|}{ NZBC G7 Compliance Assessment Tool } \\
\hline \multicolumn{2}{|l|}{} \\
\hline Variable: & 0 \\
\hline Opposite Building Height & 9 \\
\hline Street Width & 31 \\
\hline Glazed Area & South \\
\hline Orientation & Auckland \\
\hline Location & 5 \\
\hline Reflectance of Opposite Building & 87 \\
\hline Glazing Transmittance & 11 \\
\hline Vertical Location of lowest apartment \\
\hline \multicolumn{2}{|c|}{} \\
\hline Are simulations required to prove compliance: \\
\hline
\end{tabular}

Comparison of Results:

\begin{tabular}{|c|c|c|c|}
\hline Measurements & Linear Model & Model 2A & Model 2B \\
\hline Fail & 116 & 302 & 408 \\
\hline
\end{tabular}




\section{Apartment 32}

Measurement Results:

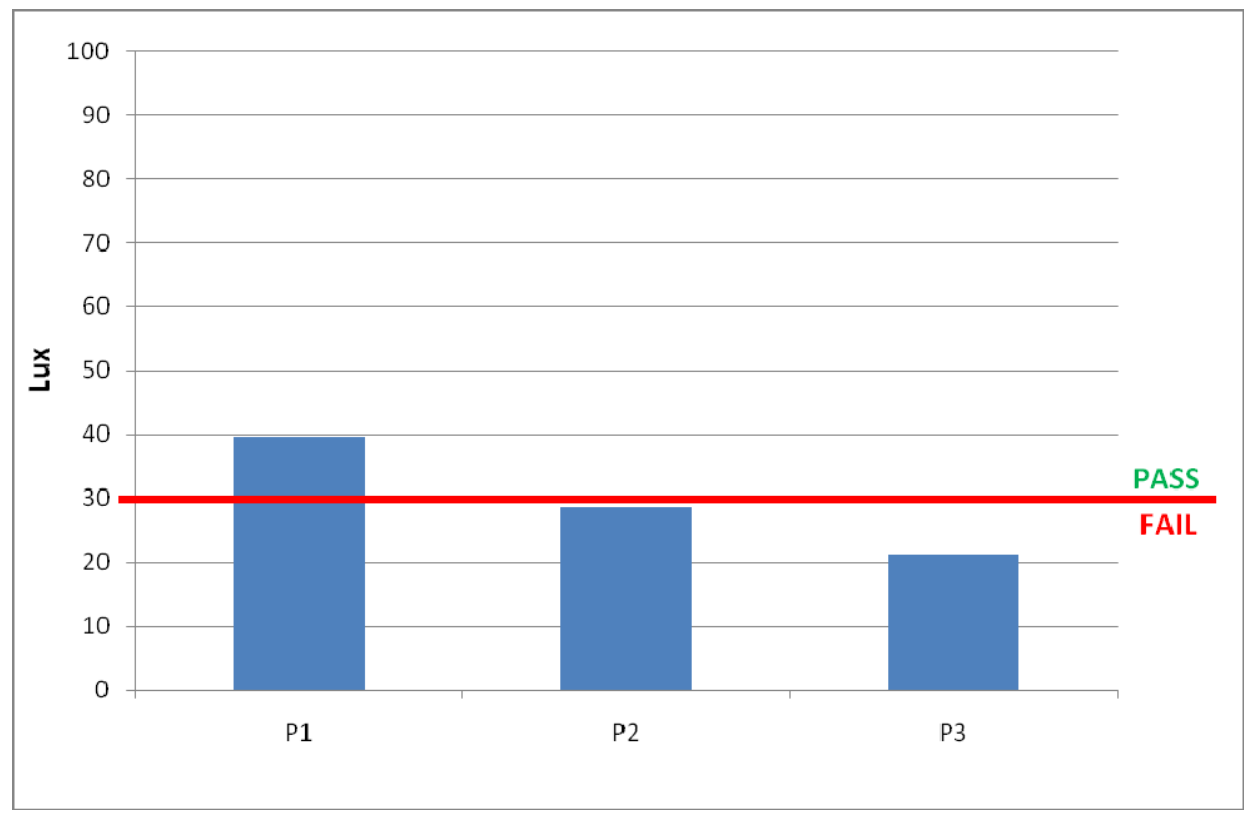

Application of the Tool:

\begin{tabular}{|l|c|}
\hline \multicolumn{2}{|c|}{ NZBC G7 Compliance Assessment Tool } \\
\hline \multicolumn{2}{|l|}{ Variable: } \\
\hline Opposite Building Height & 35 \\
\hline Street Width & 2.6 \\
\hline Glazed Area & 25 \\
\hline Orientation & South \\
\hline Location & Wellington \\
\hline Reflectance of Opposite Building & 25 \\
\hline Glazing Transmittance & 87 \\
\hline Vertical Location of lowest apartment & 4 \\
\hline \multicolumn{2}{|c|}{} \\
\hline Are simulations required to prove compliance: \\
\hline
\end{tabular}

Comparison of Results:

\begin{tabular}{|c|c|c|c|}
\hline Measurements & Linear Model & Model 2A & Model 2B \\
\hline Fail & 50 & 9 & 5 \\
\hline
\end{tabular}




\section{Apartment 33}

Measurement Results:

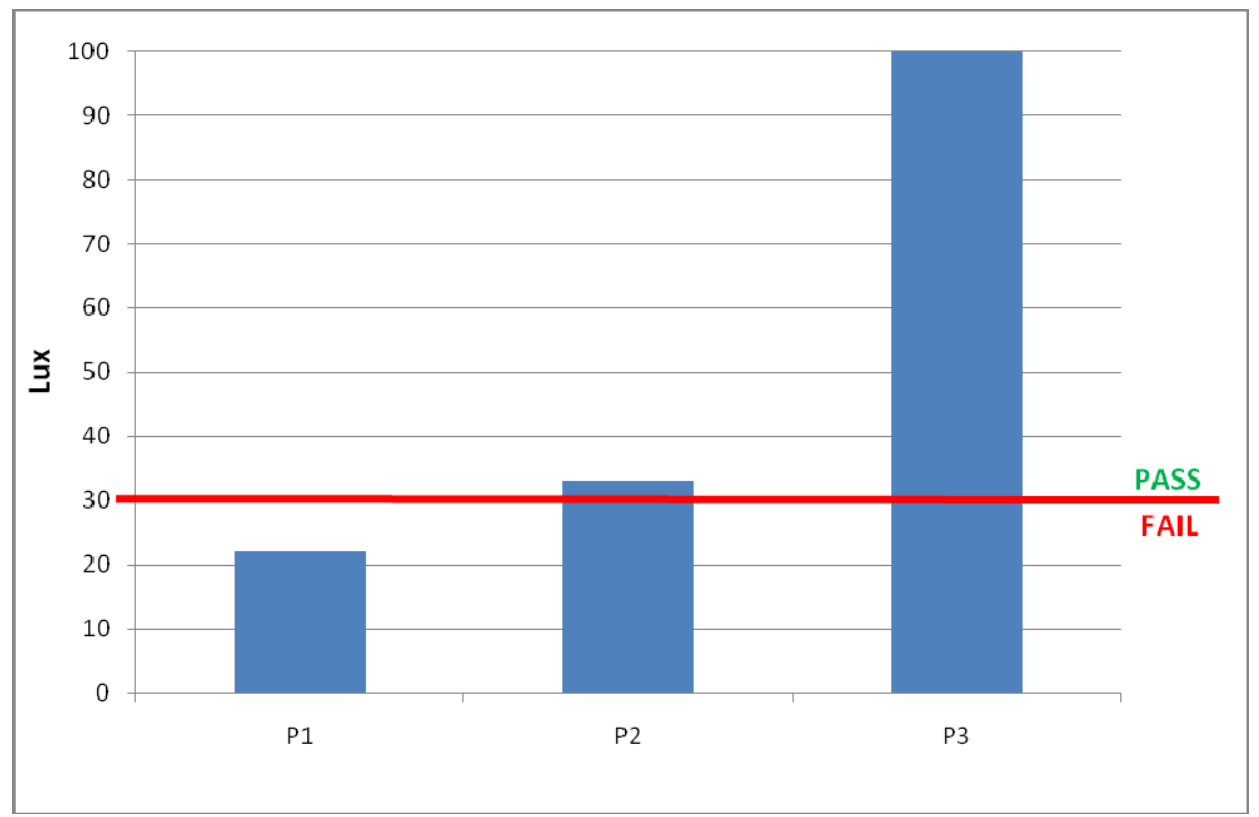

Application of the Tool:

\begin{tabular}{|l|c|}
\hline \multicolumn{2}{|c|}{ NZBC G7 Compliance Assessment Tool } \\
\hline \multicolumn{2}{|l|}{ Variable: } \\
\hline Opposite Building Height & 75 \\
\hline Street Width & 9.5 \\
\hline Glazed Area & 11.8 \\
\hline Orientation & East \\
\hline Location & Wellington \\
\hline Reflectance of Opposite Building & 12 \\
\hline Glazing Transmittance & 87 \\
\hline Vertical Location of lowest apartment & 11 \\
\hline \multicolumn{2}{|}{} \\
\hline Are simulations required to prove compliance: \\
\hline
\end{tabular}

Comparison of Results:

\begin{tabular}{|c|c|c|c|}
\hline Measurements & Linear Model & Model 2A & Model 2B \\
\hline Fail & 50 & 198 & -0.6 \\
\hline
\end{tabular}




\section{Apartment 34}

Measurement Results:

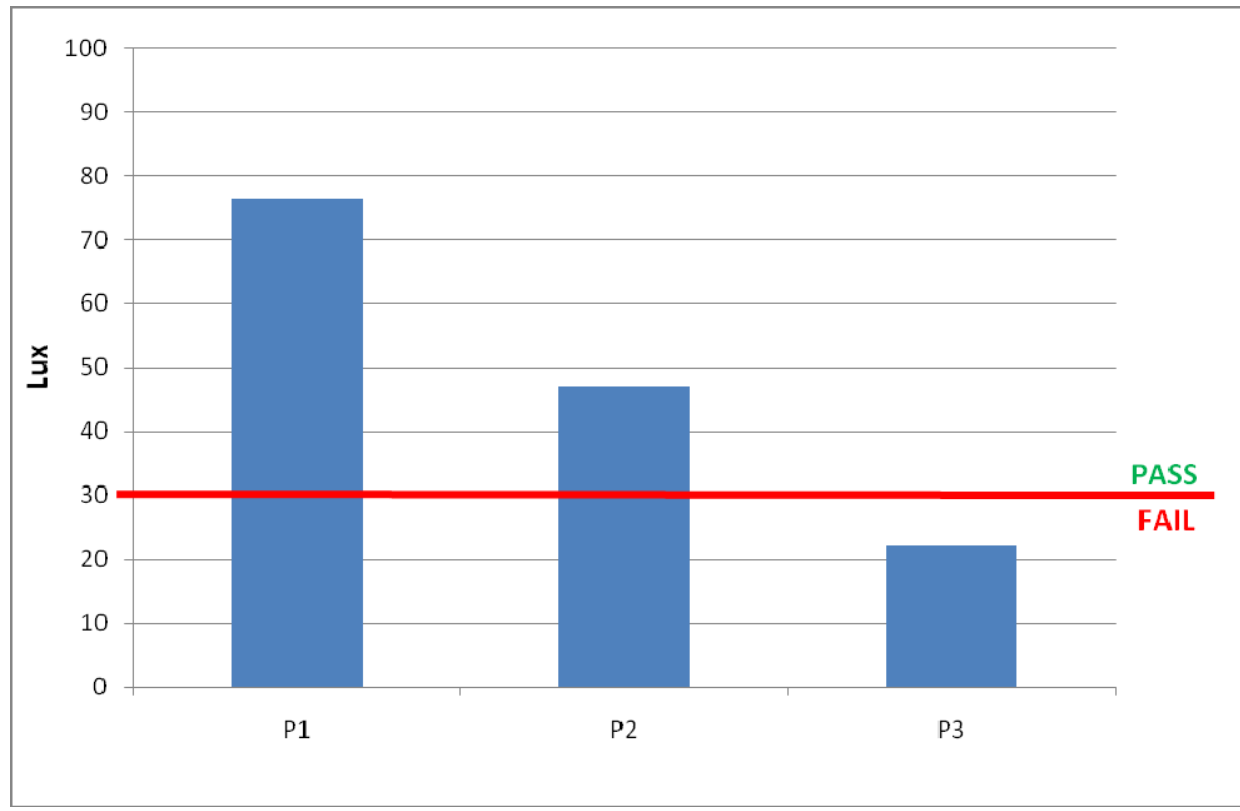

Application of the Tool:

\begin{tabular}{|l|c|}
\hline \multicolumn{2}{|c|}{ NZBC G7 Compliance Assessment Tool } \\
\hline \multicolumn{2}{|l|}{ Variable: } \\
\hline Opposite Building Height & 32 \\
\hline Street Width & 50 \\
\hline Glazed Area & 32 \\
\hline Orientation & West \\
\hline Location & Wellington \\
\hline Reflectance of Opposite Building & 33.3 \\
\hline Glazing Transmittance & 87 \\
\hline Vertical Location of lowest apartment & 0 \\
\hline \multicolumn{2}{|c|}{} \\
\hline Are simulations required to prove compliance: \\
\hline
\end{tabular}

Comparison of Results:

\begin{tabular}{|c|c|c|c|}
\hline Measurements & Linear Model & Model 2A & Model 2B \\
\hline Fail & 132 & 94 & 86 \\
\hline
\end{tabular}


Apartment 34 - Courtyard

Application of the Tool:

\begin{tabular}{|l|c|}
\hline \multicolumn{2}{|c|}{ NZBC G7 Compliance Assessment Tool } \\
\hline Variable: & 2 \\
\hline Opposite Building Height & 6 \\
\hline Street Width & 32 \\
\hline Glazed Area & West \\
\hline Orientation & Wellington \\
\hline Location & 38.5 \\
\hline Reflectance of Opposite Building & 87 \\
\hline Glazing Transmittance & 0 \\
\hline Vertical Location of lowest apartment & \\
\hline \multicolumn{2}{|l|}{} \\
\hline Are simulations required to prove compliance: & YES \\
\hline
\end{tabular}

Comparison of Results:

\begin{tabular}{|c|c|c|c|}
\hline Measurements & Linear Model & Model 2A & Model 2B \\
\hline Fail & 65 & 67 & 64 \\
\hline
\end{tabular}




\section{Apartment 35}

Measurement Results:

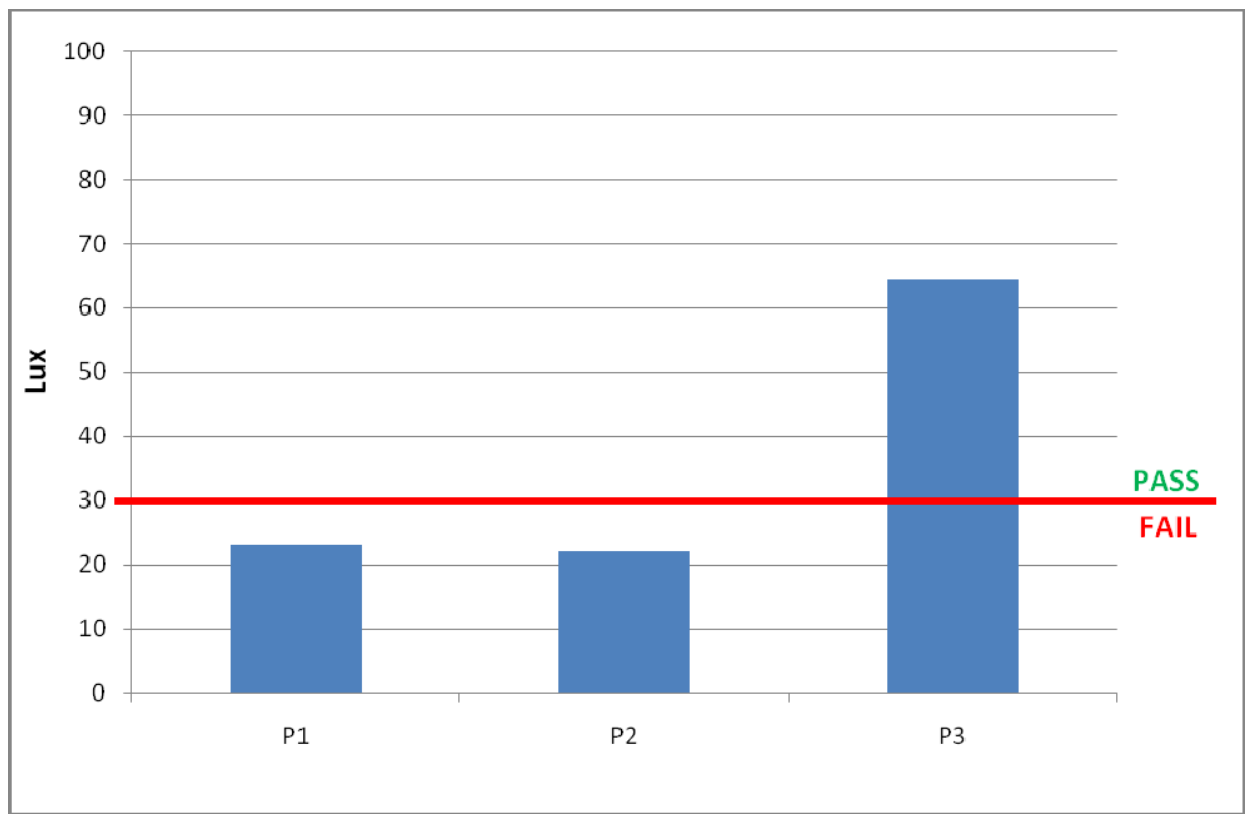

Application of the Tool:

\begin{tabular}{|l|c|}
\hline \multicolumn{2}{|c|}{ NZBC G7 Compliance Assessment Tool } \\
\hline \multicolumn{2}{|l|}{} \\
\hline Variable: & 0 \\
\hline Opposite Building Height & 0 \\
\hline Street Width & 26 \\
\hline Glazed Area & South \\
\hline Orientation & Christchurch \\
\hline Location & 0 \\
\hline Reflectance of Opposite Building & 87 \\
\hline Glazing Transmittance & 11 \\
\hline Vertical Location of lowest apartment \\
\hline \multicolumn{2}{|c|}{} \\
\hline Are simulations required to prove compliance: \\
\hline
\end{tabular}

Comparison of Results:

\begin{tabular}{|c|c|c|c|}
\hline Measurements & Linear Model & Model 2A & Model 2B \\
\hline Fail & 81 & 267 & 291 \\
\hline
\end{tabular}




\section{Apartment 36}

Measurement Results:

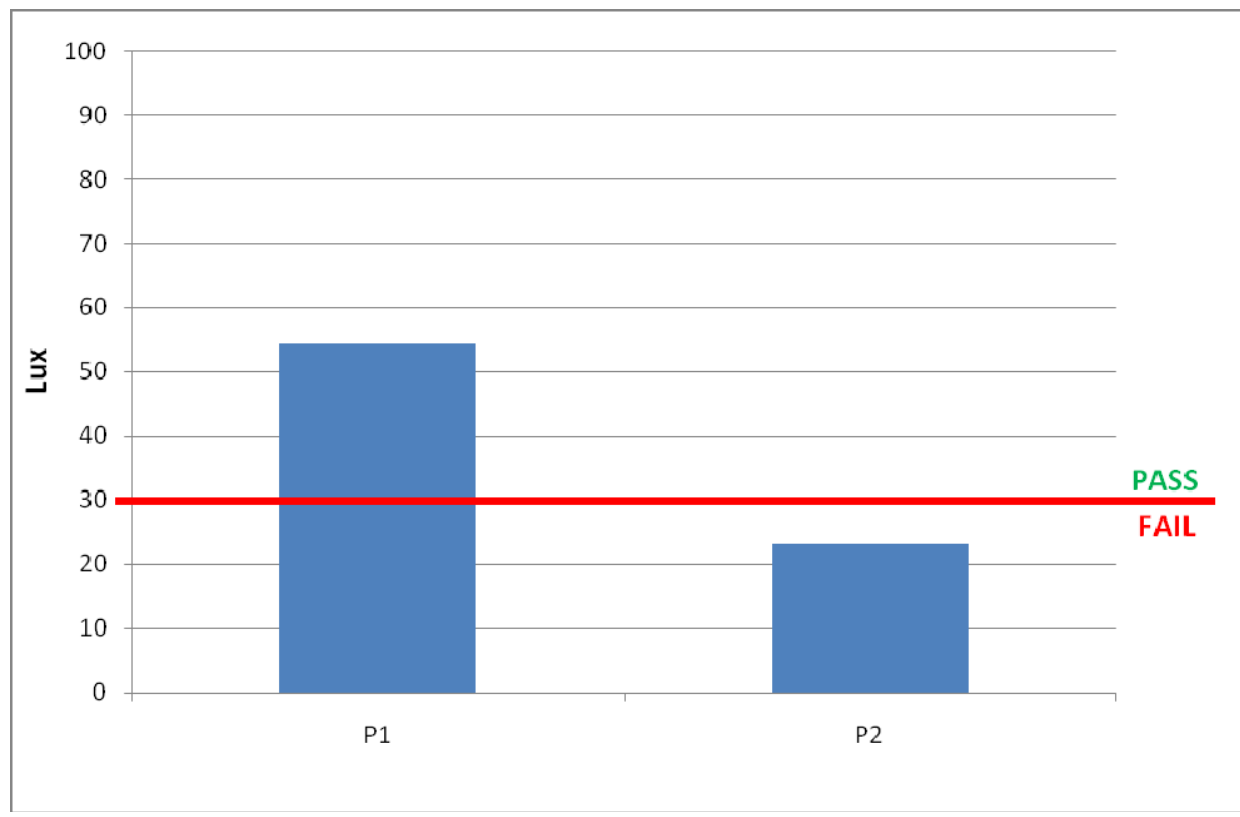

Application of the Tool:

\begin{tabular}{|l|c|}
\hline \multicolumn{2}{|c|}{ NZBC G7 Compliance Assessment Tool } \\
\hline \multicolumn{2}{|l|}{ Variable: } \\
\hline Opposite Building Height & 13.3 \\
\hline Street Width & 14 \\
\hline Glazed Area & 15 \\
\hline Orientation & West \\
\hline Location & Wellington \\
\hline Reflectance of Opposite Building & 12 \\
\hline Glazing Transmittance & 87 \\
\hline Vertical Location of lowest apartment & 2 \\
\hline \multicolumn{2}{|}{} \\
\hline Are simulations required to prove compliance: \\
\hline
\end{tabular}

Comparison of Results:

\begin{tabular}{|c|c|c|c|}
\hline Measurements & Linear Model & Model 2A & Model 2B \\
\hline Fail & 54 & 30 & 27 \\
\hline
\end{tabular}




\section{Apartment 37}

Measurement Results:

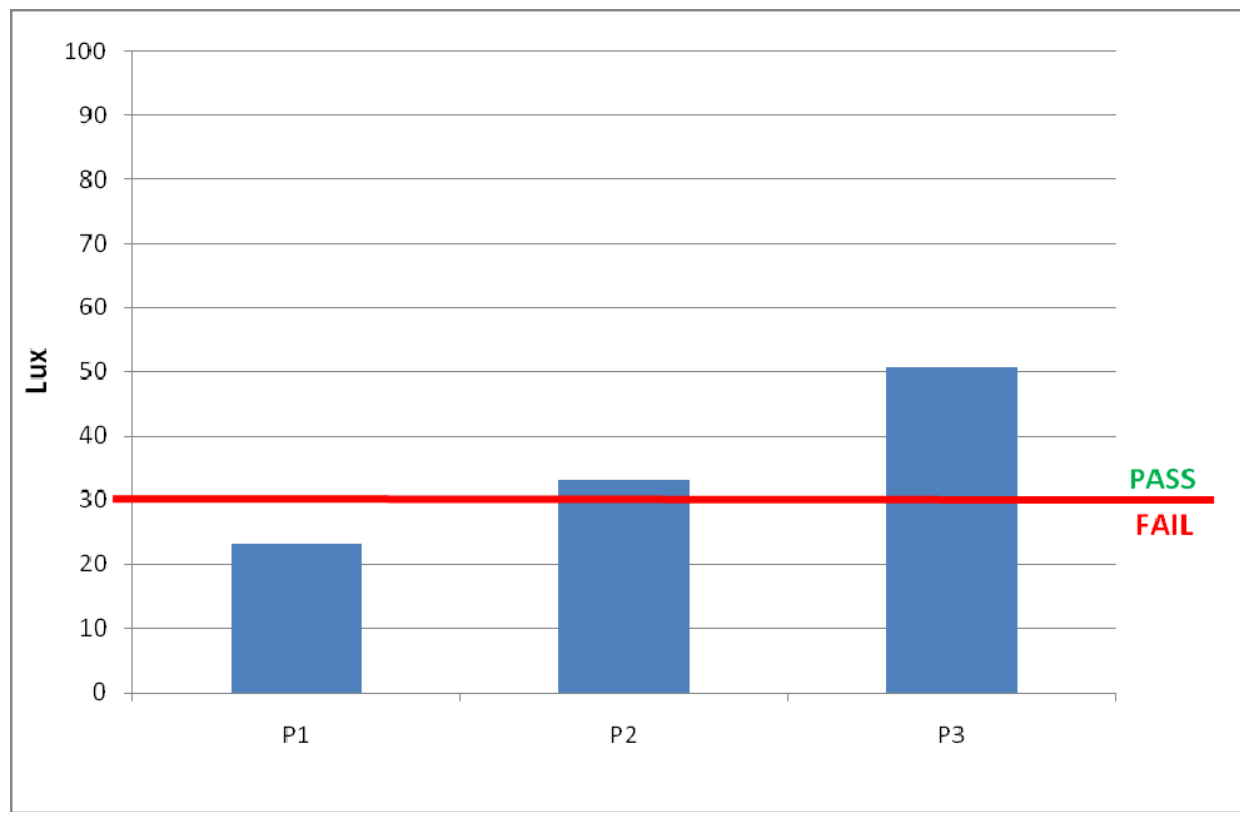

Application of the Tool:

\begin{tabular}{|l|c|}
\hline \multicolumn{2}{|c|}{ NZBC G7 Compliance Assessment Tool } \\
\hline \multicolumn{2}{|l|}{} \\
\hline Variable: & 11.5 \\
\hline Opposite Building Height & 52 \\
\hline Street Width & 32 \\
\hline Glazed Area & North \\
\hline Orientation & Wellington \\
\hline Location & 38.5 \\
\hline Reflectance of Opposite Building & 87 \\
\hline Glazing Transmittance & 0 \\
\hline Vertical Location of lowest apartment \\
\hline \multicolumn{2}{|l}{} \\
\hline Are simulations required to prove compliance: \\
\hline
\end{tabular}

Comparison of Results:

\begin{tabular}{|c|c|c|c|}
\hline Measurements & Linear Model & Model 2A & Model 2B \\
\hline Fail & 144 & 124 & 118 \\
\hline
\end{tabular}


Apartment 37 - Courtyard

Application of the Tool:

\begin{tabular}{|l|c|}
\hline \multicolumn{2}{|c|}{ NZBC G7 Compliance Assessment Tool } \\
\hline \multicolumn{2}{|c|}{} \\
\hline Variable: & 2 \\
\hline Opposite Building Height & 6 \\
\hline Street Width & 32 \\
\hline Glazed Area & North \\
\hline Orientation & Wellington \\
\hline Location & 38.5 \\
\hline Reflectance of Opposite Building & 87 \\
\hline Glazing Transmittance & 0 \\
\hline Vertical Location of lowest apartment & \\
\hline & YES \\
\hline
\end{tabular}

Comparison of Results:

\begin{tabular}{|c|c|c|c|}
\hline Measurements & Linear Model & Model 2A & Model 2B \\
\hline Fail & 63 & 64 & 61 \\
\hline
\end{tabular}




\section{Apartment 38}

Measurement Results:

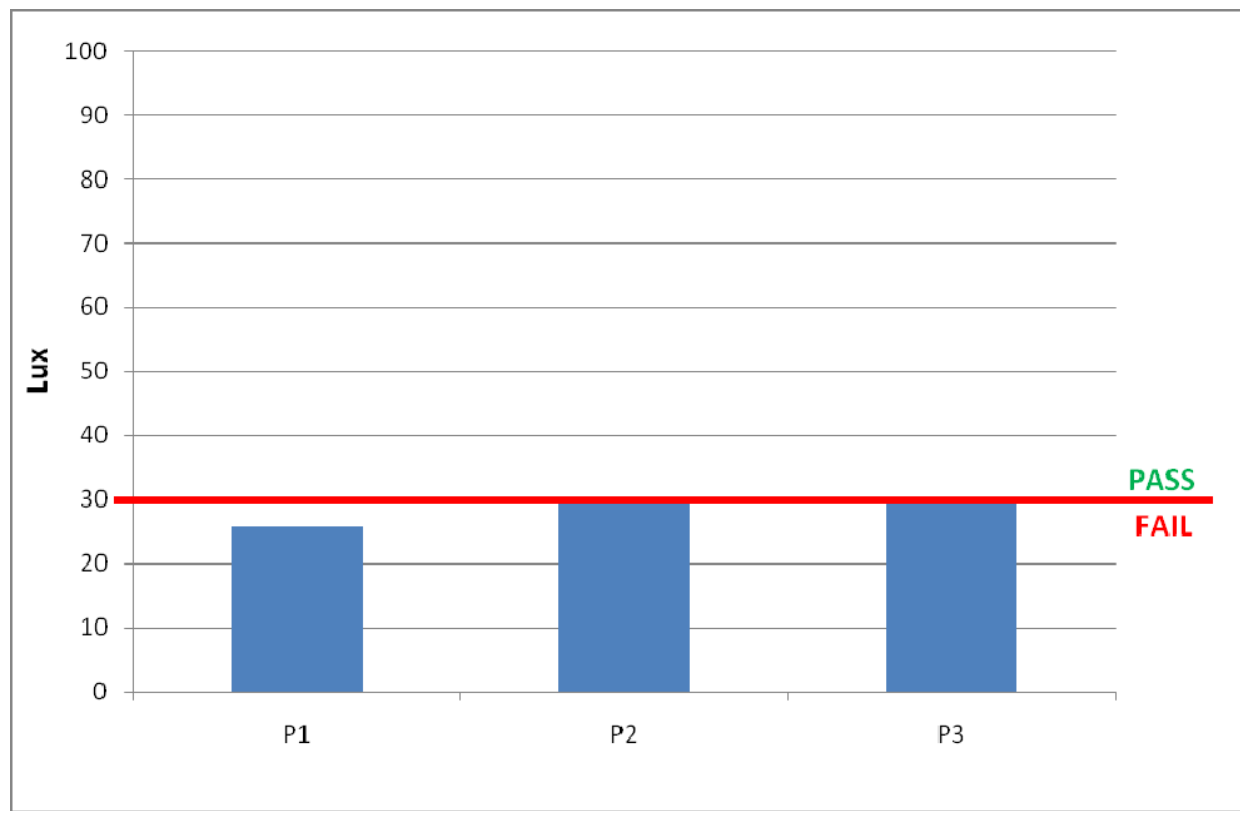

Application of the Tool:

\begin{tabular}{|l|c|}
\hline \multicolumn{2}{|c|}{ NZBC G7 Compliance Assessment Tool } \\
\hline \multicolumn{2}{|l|}{ Variable: } \\
\hline Opposite Building Height & 62 \\
\hline Street Width & 9.5 \\
\hline Glazed Area & 48 \\
\hline Orientation & West \\
\hline Location & Auckland \\
\hline Reflectance of Opposite Building & 5 \\
\hline Glazing Transmittance & 87 \\
\hline Vertical Location of lowest apartment & 3 \\
\hline \multicolumn{2}{|l}{} \\
\hline Are simulations required to prove compliance: & YES \\
\hline
\end{tabular}

Comparison of Results:

\begin{tabular}{|c|c|c|c|}
\hline Measurements & Linear Model & Model 2A & Model 2B \\
\hline Borderline Fail & 73 & 46 & 10 \\
\hline
\end{tabular}




\section{Apartment 39}

Measurement Results:

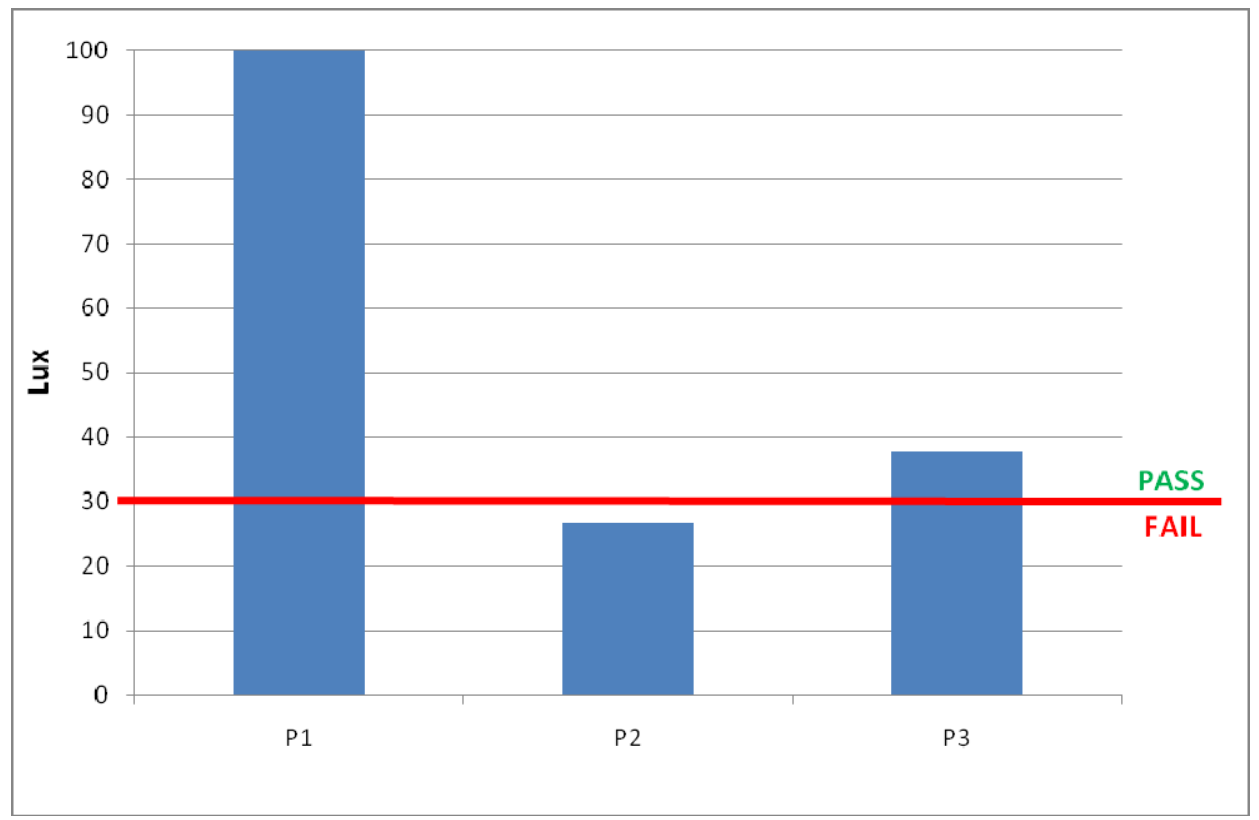

Application of the Tool:

\begin{tabular}{|l|c|}
\hline \multicolumn{2}{|c|}{ NZBC G7 Compliance Assessment Tool } \\
\hline \multicolumn{2}{|l|}{} \\
\hline Variable: & 32 \\
\hline Opposite Building Height & 50 \\
\hline Street Width & 48 \\
\hline Glazed Area & West \\
\hline Orientation & Wellington \\
\hline Location & 33.3 \\
\hline Reflectance of Opposite Building & 87 \\
\hline Glazing Transmittance & 2 \\
\hline Vertical Location of lowest apartment \\
\hline \multicolumn{2}{|c|}{} \\
\hline Are simulations required to prove compliance: \\
\hline
\end{tabular}

Comparison of Results:

\begin{tabular}{|c|c|c|c|}
\hline Measurements & Linear Model & Model 2A & Model 2B \\
\hline Borderline Fail & 161 & 131 & 116 \\
\hline
\end{tabular}




\section{Apartment 40}

Measurement Results:

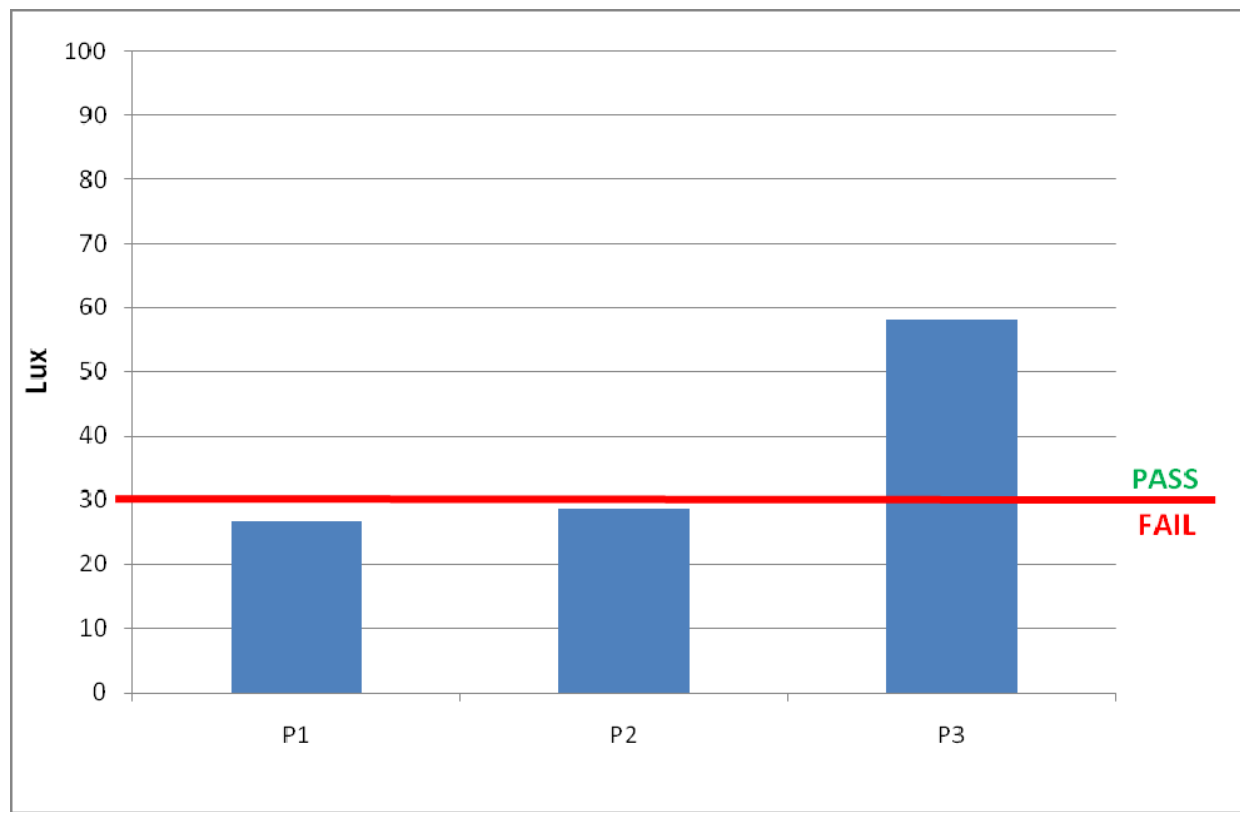

Application of the Tool:

\begin{tabular}{|l|c|}
\hline \multicolumn{2}{|c|}{ NZBC G7 Compliance Assessment Tool } \\
\hline \multicolumn{2}{|c|}{} \\
\hline Variable: & 13 \\
\hline Opposite Building Height & 28 \\
\hline Street Width & 26 \\
\hline Glazed Area & North \\
\hline Orientation & Christchurch \\
\hline Location & 25 \\
\hline Reflectance of Opposite Building & 87 \\
\hline Glazing Transmittance & 9 \\
\hline Vertical Location of lowest apartment \\
\hline \multicolumn{2}{|c|}{} \\
\hline Are simulations required to prove compliance: \\
\hline
\end{tabular}

Comparison of Results:

\begin{tabular}{|c|c|c|c|}
\hline Measurements & Linear Model & Model 2A & Model 2B \\
\hline Borderline Fail & 121 & 183 & 175 \\
\hline
\end{tabular}




\section{Apartment 41}

Measurement Results:

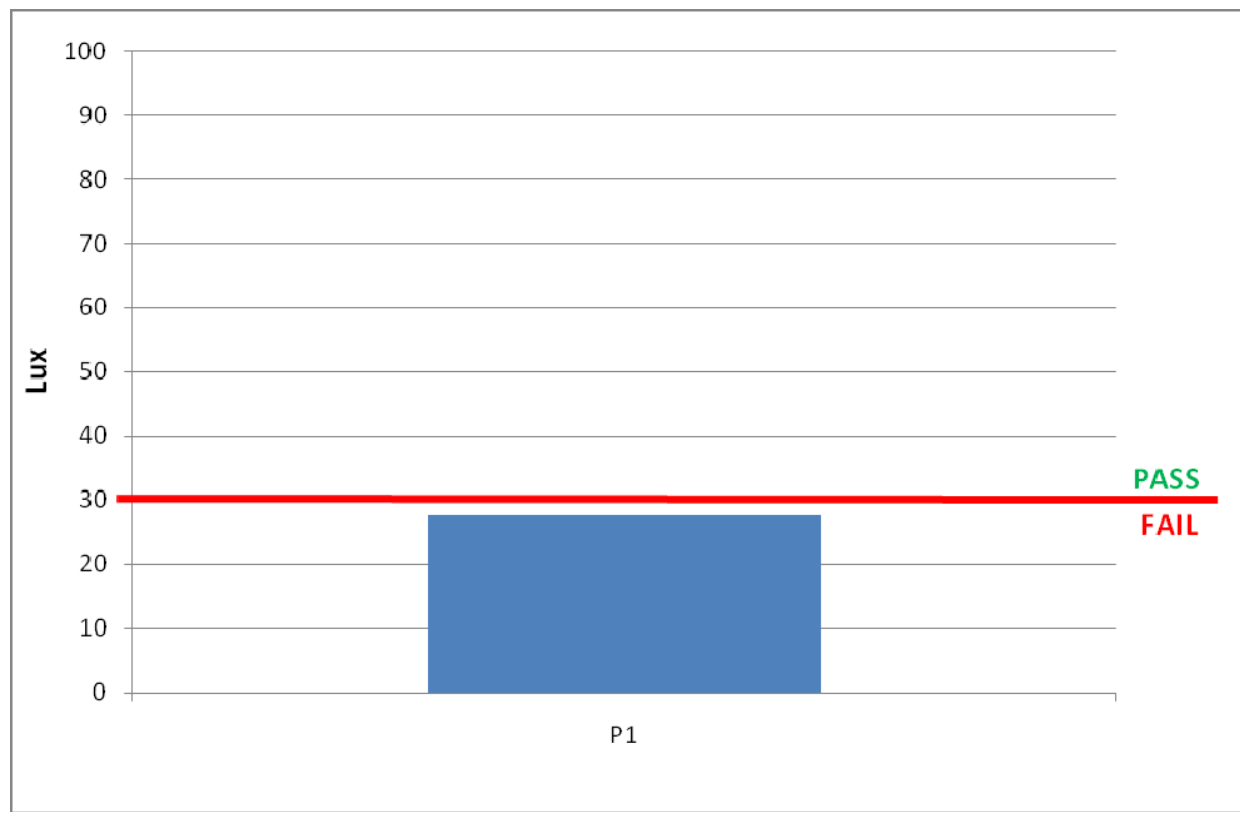

Application of the Tool:

\begin{tabular}{|l|c|}
\hline \multicolumn{2}{|c|}{ NZBC G7 Compliance Assessment Tool } \\
\hline \multicolumn{2}{|l|}{} \\
\hline Variable: & 30 \\
\hline Opposite Building Height & 27 \\
\hline Street Width & 10 \\
\hline Glazed Area & East \\
\hline Orientation & Wellington \\
\hline Location & 38 \\
\hline Reflectance of Opposite Building & 87 \\
\hline Glazing Transmittance & 7 \\
\hline Vertical Location of lowest apartment \\
\hline \multicolumn{2}{|l}{} \\
\hline Are simulations required to prove compliance: \\
\hline
\end{tabular}

Comparison of Results:

\begin{tabular}{|c|c|c|c|}
\hline Measurements & Linear Model & Model 2A & Model 2B \\
\hline Borderline Fail & 92 & 89 & 55 \\
\hline
\end{tabular}




\section{Apartment 42}

Measurement Results:

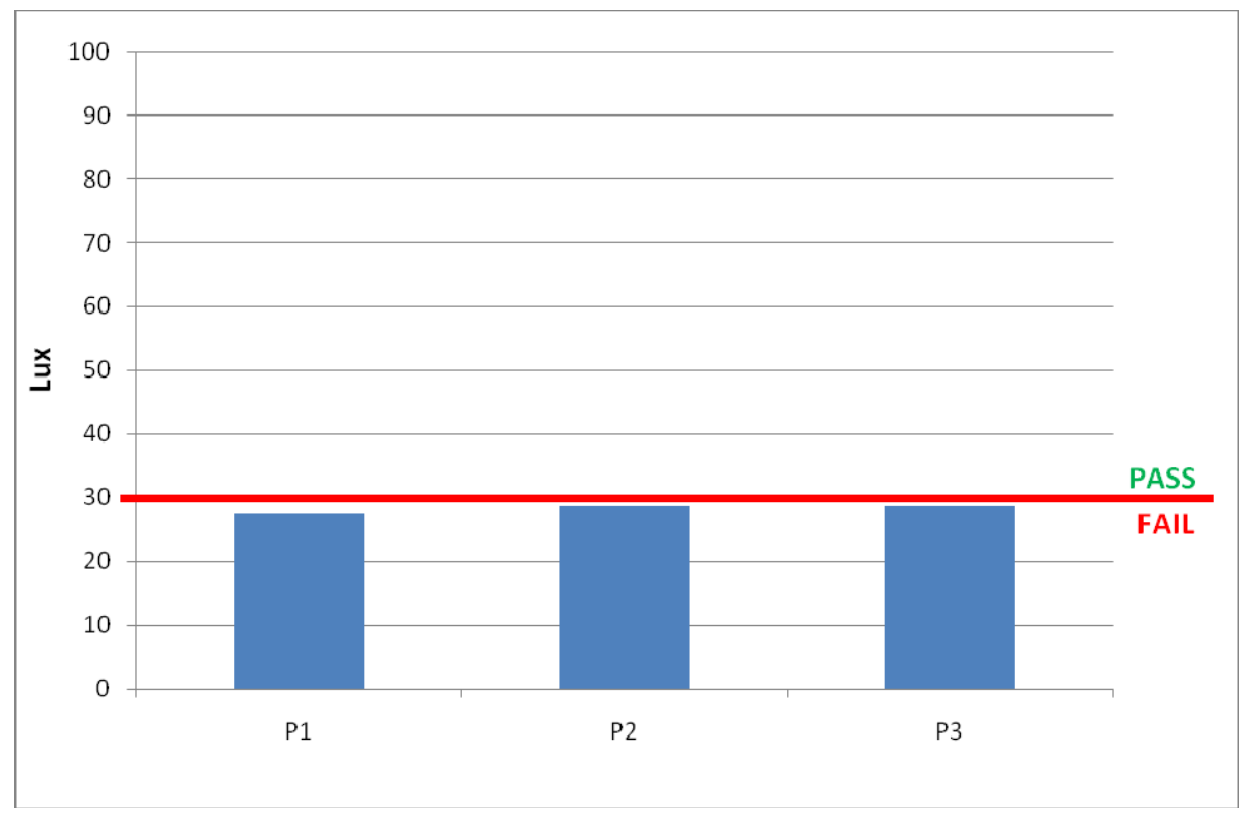

Application of the Tool:

\begin{tabular}{|l|c|}
\hline \multicolumn{2}{|c|}{ NZBC G7 Compliance Assessment Tool } \\
\hline \multicolumn{2}{|l|}{} \\
\hline Variable: & 28 \\
\hline Opposite Building Height & 3 \\
\hline Street Width & 34 \\
\hline Glazed Area & North \\
\hline Orientation & Wellington \\
\hline Location & 24 \\
\hline Reflectance of Opposite Building & 87 \\
\hline Glazing Transmittance & 8 \\
\hline Vertical Location of lowest apartment \\
\hline \multicolumn{2}{|c|}{} \\
\hline Are simulations required to prove compliance: \\
\hline
\end{tabular}

Comparison of Results:

\begin{tabular}{|c|c|c|c|}
\hline Measurements & Linear Model & Model 2A & Model 2B \\
\hline Borderline Fail & 77 & 95 & 65 \\
\hline
\end{tabular}




\section{Apartment 43}

Measurement Results:

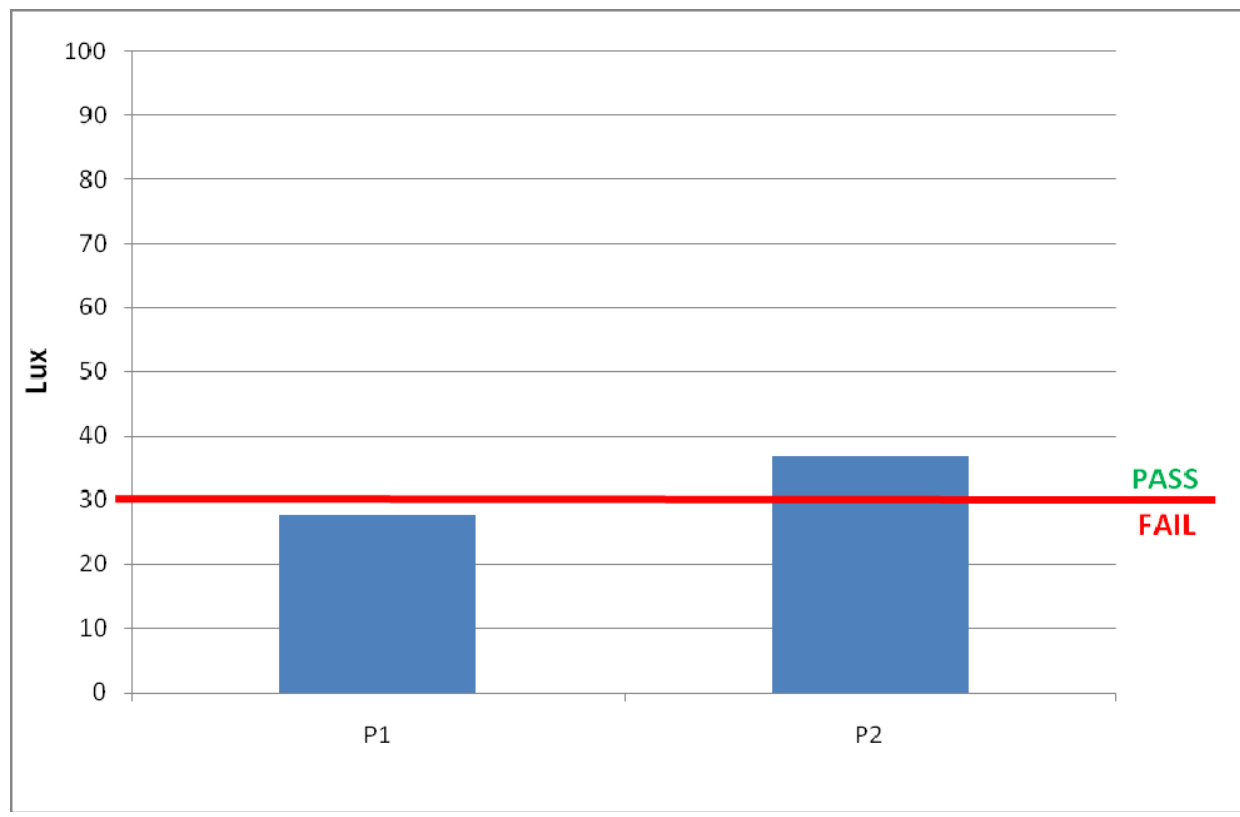

Application of the Tool:

\begin{tabular}{|l|c|}
\hline \multicolumn{2}{|c|}{ NZBC G7 Compliance Assessment Tool } \\
\hline \multicolumn{2}{|l|}{} \\
\hline Variable: & 18 \\
\hline Opposite Building Height & 16 \\
\hline Street Width & 17.5 \\
\hline Glazed Area & North \\
\hline Orientation & Wellington \\
\hline Location & 10 \\
\hline Reflectance of Opposite Building & 87 \\
\hline Glazing Transmittance & 6 \\
\hline Vertical Location of lowest apartment \\
\hline \multicolumn{2}{|l}{} \\
\hline Are simulations required to prove compliance: \\
\hline
\end{tabular}

Comparison of Results:

\begin{tabular}{|c|c|c|c|}
\hline Measurements & Linear Model & Model 2A & Model 2B \\
\hline Borderline Fail & 73 & 59 & 46 \\
\hline
\end{tabular}




\section{Apartment 44}

Measurement Results:

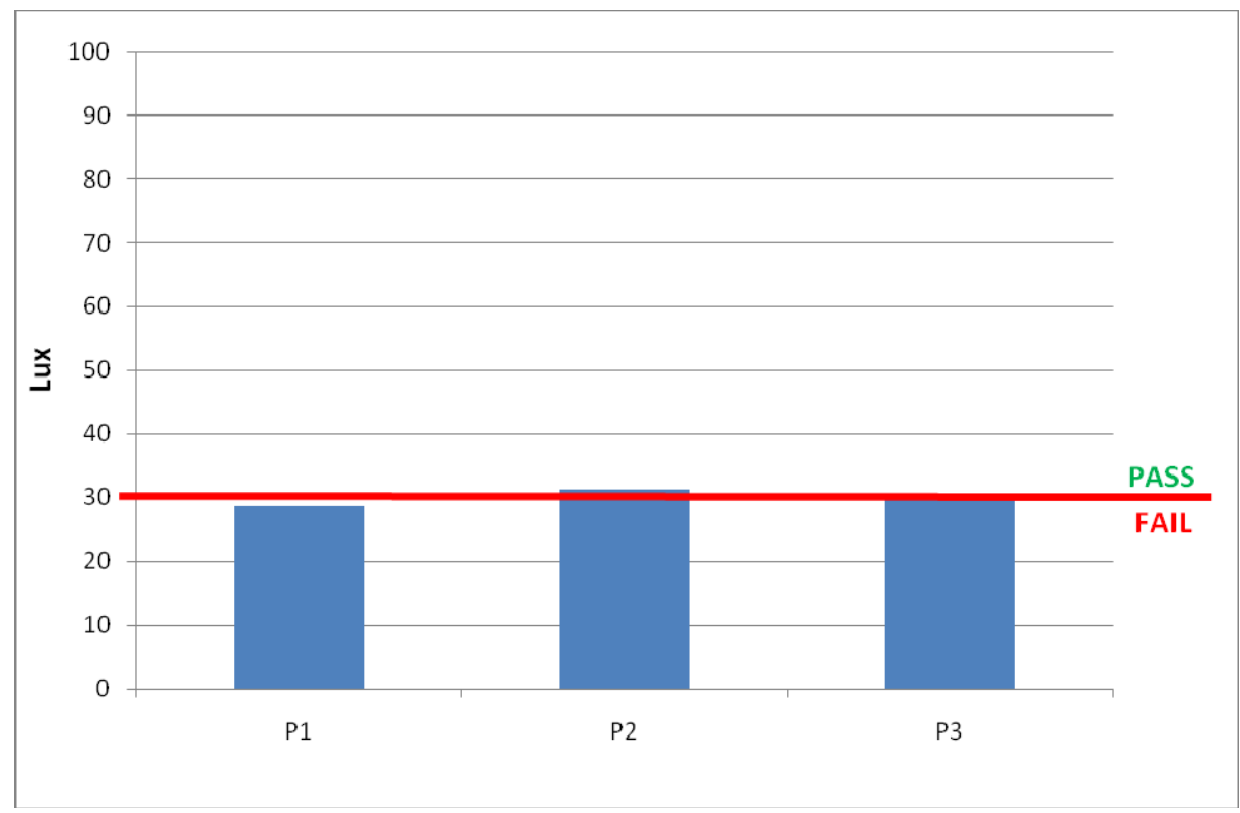

Application of the Tool:

\begin{tabular}{|l|c|}
\hline \multicolumn{2}{|c|}{ NZBC G7 Compliance Assessment Tool } \\
\hline \multicolumn{2}{|l|}{ Variable: } \\
\hline Opposite Building Height & 12 \\
\hline Street Width & 3 \\
\hline Glazed Area & 35 \\
\hline Orientation & South \\
\hline Location & Wellington \\
\hline Reflectance of Opposite Building & 21 \\
\hline Glazing Transmittance & 87 \\
\hline Vertical Location of lowest apartment & 10 \\
\hline \multicolumn{2}{|c|}{} \\
\hline Are simulations required to prove compliance: \\
\hline
\end{tabular}

Comparison of Results:

\begin{tabular}{|c|c|c|c|}
\hline Measurements & Linear Model & Model 2A & Model 2B \\
\hline Borderline Fail & 98 & 205 & 252 \\
\hline
\end{tabular}




\section{Apartment 45}

Measurement Results:

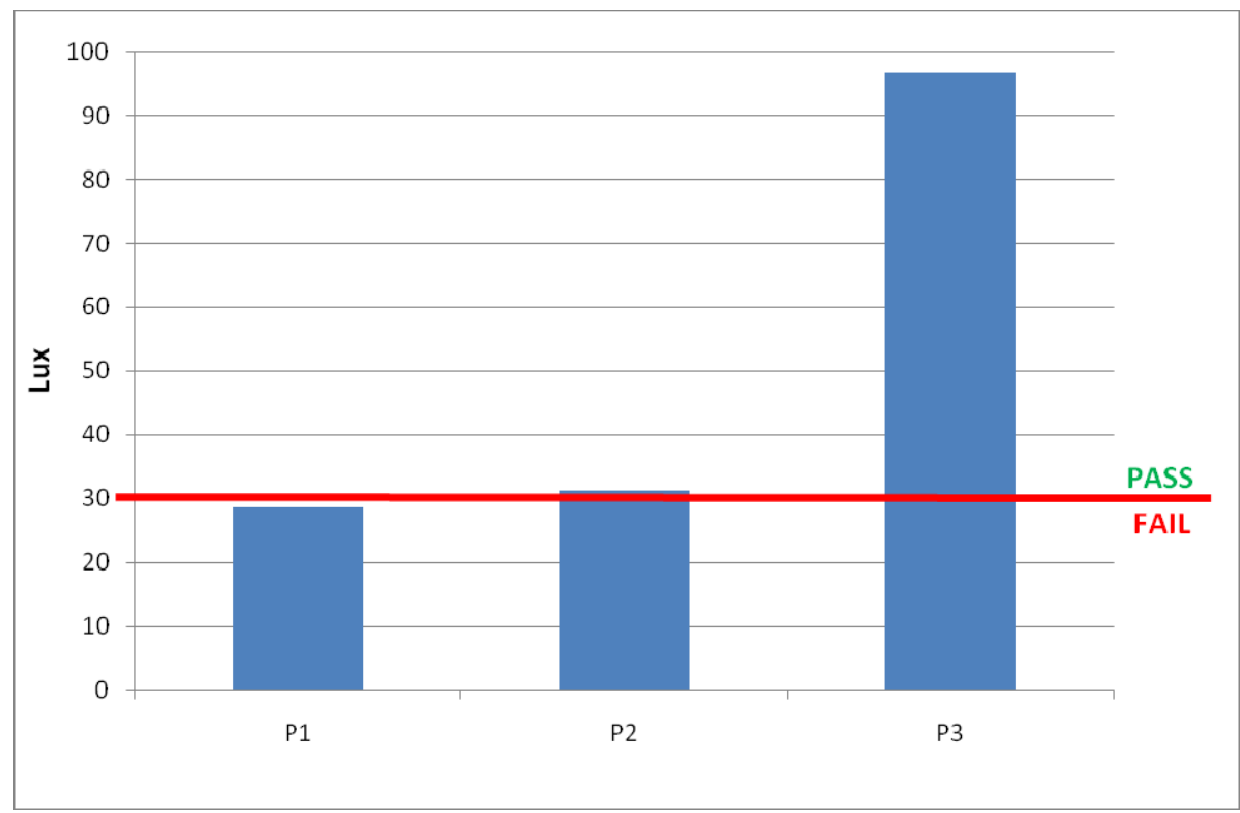

Application of the Tool:

\begin{tabular}{|l|c|}
\hline \multicolumn{2}{|c|}{ NZBC G7 Compliance Assessment Tool } \\
\hline \multicolumn{2}{|l|}{ Variable: } \\
\hline Opposite Building Height & 37 \\
\hline Street Width & 25 \\
\hline Glazed Area & 45 \\
\hline Orientation & West \\
\hline Location & Wellington \\
\hline Reflectance of Opposite Building & 24 \\
\hline Glazing Transmittance & 87 \\
\hline Vertical Location of lowest apartment & 5 \\
\hline \multicolumn{2}{|c|}{} \\
\hline Are simulations required to prove compliance: \\
\hline
\end{tabular}

Comparison of Results:

\begin{tabular}{|c|c|c|c|}
\hline Measurements & Linear Model & Model 2A & Model 2B \\
\hline Borderline Fail & 118 & 92 & 74 \\
\hline
\end{tabular}




\section{Apartment 46}

Measurement Results:

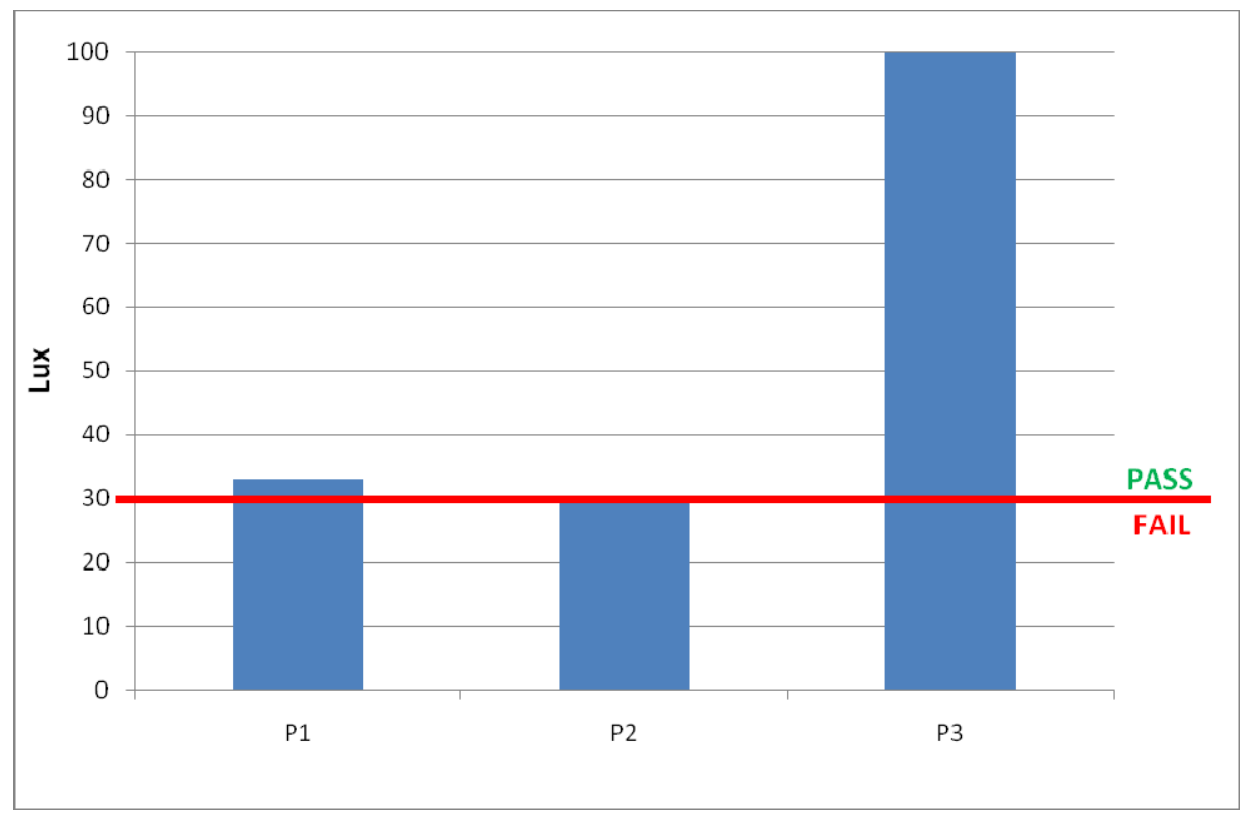

Application of the Tool:

\begin{tabular}{|l|c|}
\hline \multicolumn{2}{|c|}{ NZBC G7 Compliance Assessment Tool } \\
\hline \multicolumn{2}{|l|}{ Variable: } \\
\hline Opposite Building Height & 19 \\
\hline Street Width & 25 \\
\hline Glazed Area & 18 \\
\hline Orientation & South \\
\hline Location & Wellington \\
\hline Reflectance of Opposite Building & 20 \\
\hline Glazing Transmittance & 87 \\
\hline Vertical Location of lowest apartment & 1 \\
\hline \multicolumn{2}{|c|}{} \\
\hline Are simulations required to prove compliance: \\
\hline
\end{tabular}

Comparison of Results:

\begin{tabular}{|c|c|c|c|}
\hline Measurements & Linear Model & Model 2A & Model 2B \\
\hline Borderline Fail & 76 & 46 & 44 \\
\hline
\end{tabular}




\section{Apartment 47}

Measurement Results:

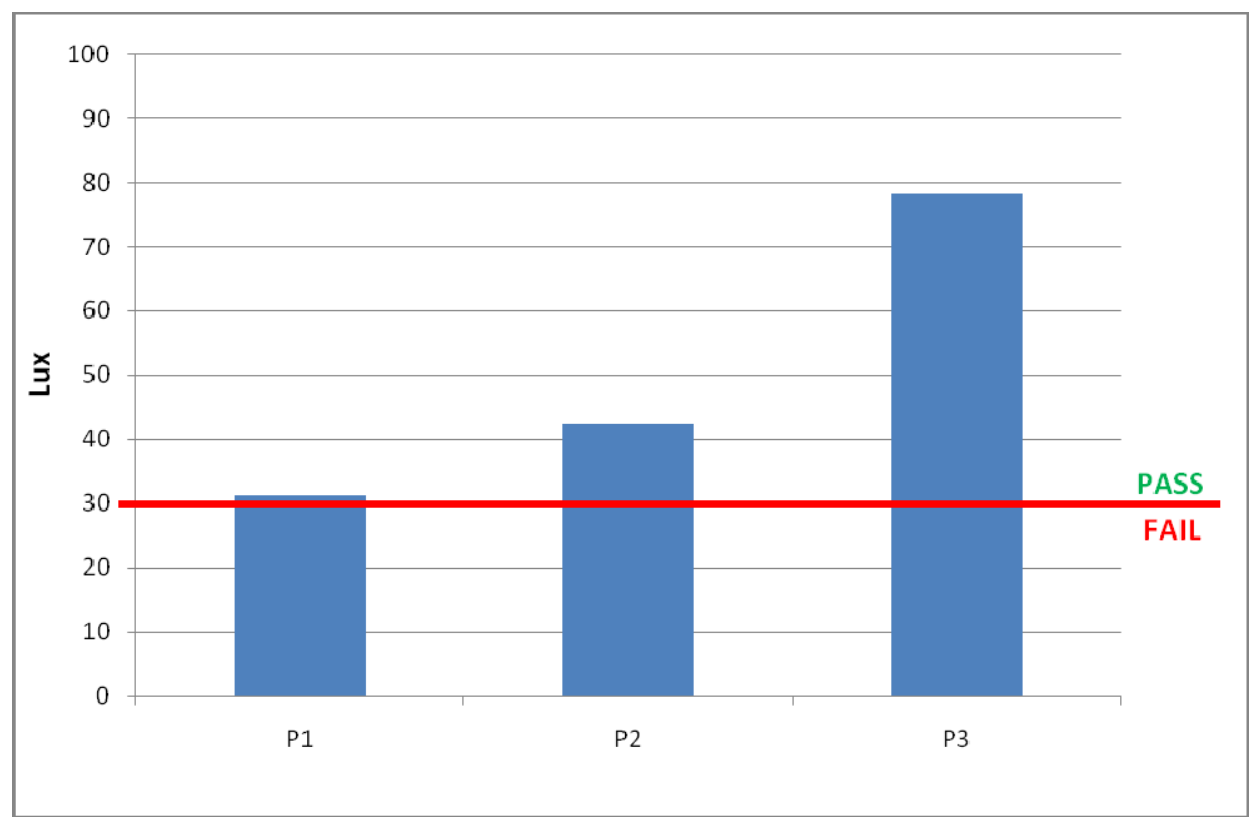

Application of the Tool:

\begin{tabular}{|l|c|}
\hline \multicolumn{2}{|c|}{ NZBC G7 Compliance Assessment Tool } \\
\hline \multicolumn{2}{|l|}{} \\
\hline Variable: & 0 \\
\hline Opposite Building Height & 9 \\
\hline Street Width & 32 \\
\hline Glazed Area & South \\
\hline Orientation & Auckland \\
\hline Location & 5 \\
\hline Reflectance of Opposite Building & 87 \\
\hline Glazing Transmittance & 1 \\
\hline Vertical Location of lowest apartment \\
\hline \multicolumn{2}{|l}{} \\
\hline Are simulations required to prove compliance: & NO \\
\hline
\end{tabular}

Comparison of Results:

\begin{tabular}{|c|c|c|c|}
\hline Measurements & Linear Model & Model 2A & Model 2B \\
\hline Borderline Pass & 75 & 84 & 74 \\
\hline
\end{tabular}




\section{Apartment 48}

Measurement Results:

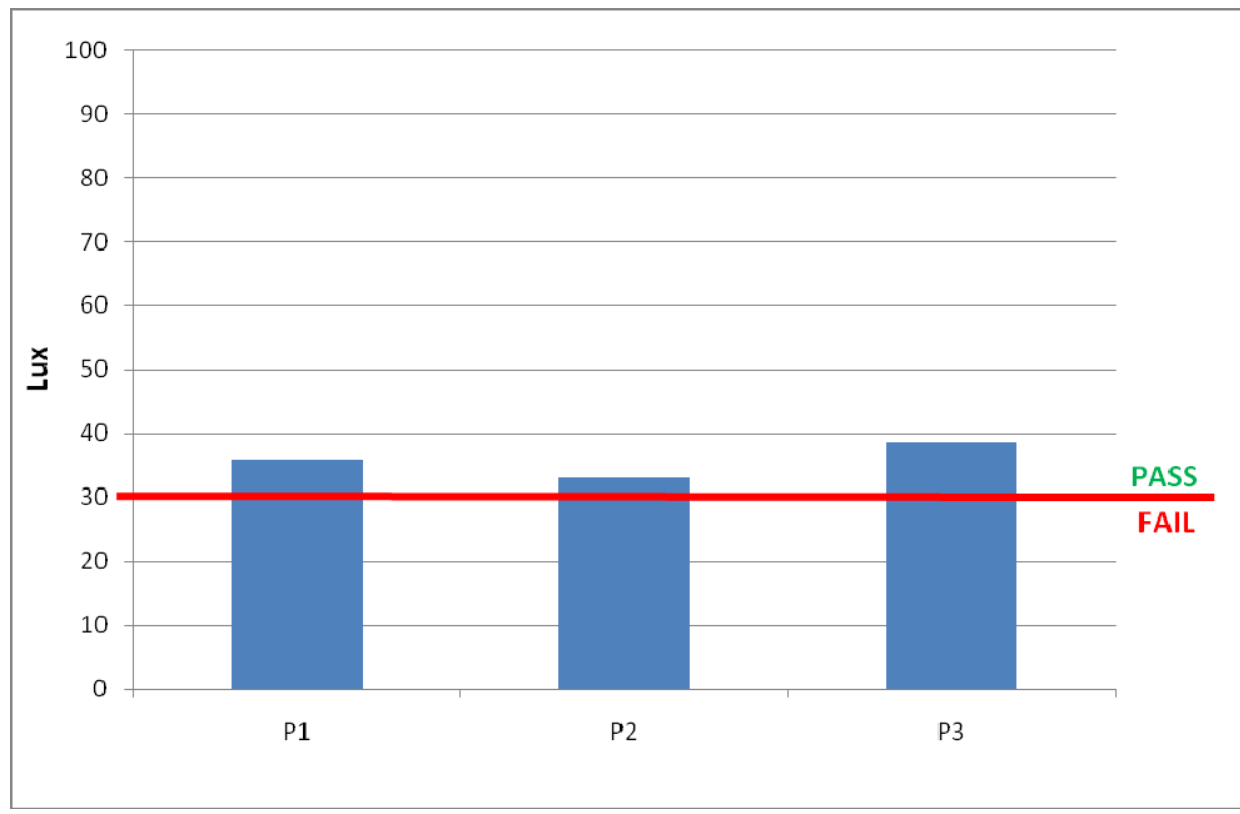

Application of the Tool:

\begin{tabular}{|l|c|}
\hline \multicolumn{2}{|c|}{ NZBC G7 Compliance Assessment Tool } \\
\hline \multicolumn{2}{|l|}{ Variable: } \\
\hline Opposite Building Height & 23.8 \\
\hline Street Width & 40 \\
\hline Glazed Area & 24 \\
\hline Orientation & West \\
\hline Location & Wellington \\
\hline Reflectance of Opposite Building & 13.5 \\
\hline Glazing Transmittance & 87 \\
\hline Vertical Location of lowest apartment & 2 \\
\hline \multicolumn{2}{|c|}{} \\
\hline Are simulations required to prove compliance: \\
\hline
\end{tabular}

Comparison of Results:

\begin{tabular}{|c|c|c|c|}
\hline Measurements & Linear Model & Model 2A & Model 2B \\
\hline Borderline Pass & 110 & 72 & 71 \\
\hline
\end{tabular}




\section{Apartment 49}

Measurement Results:

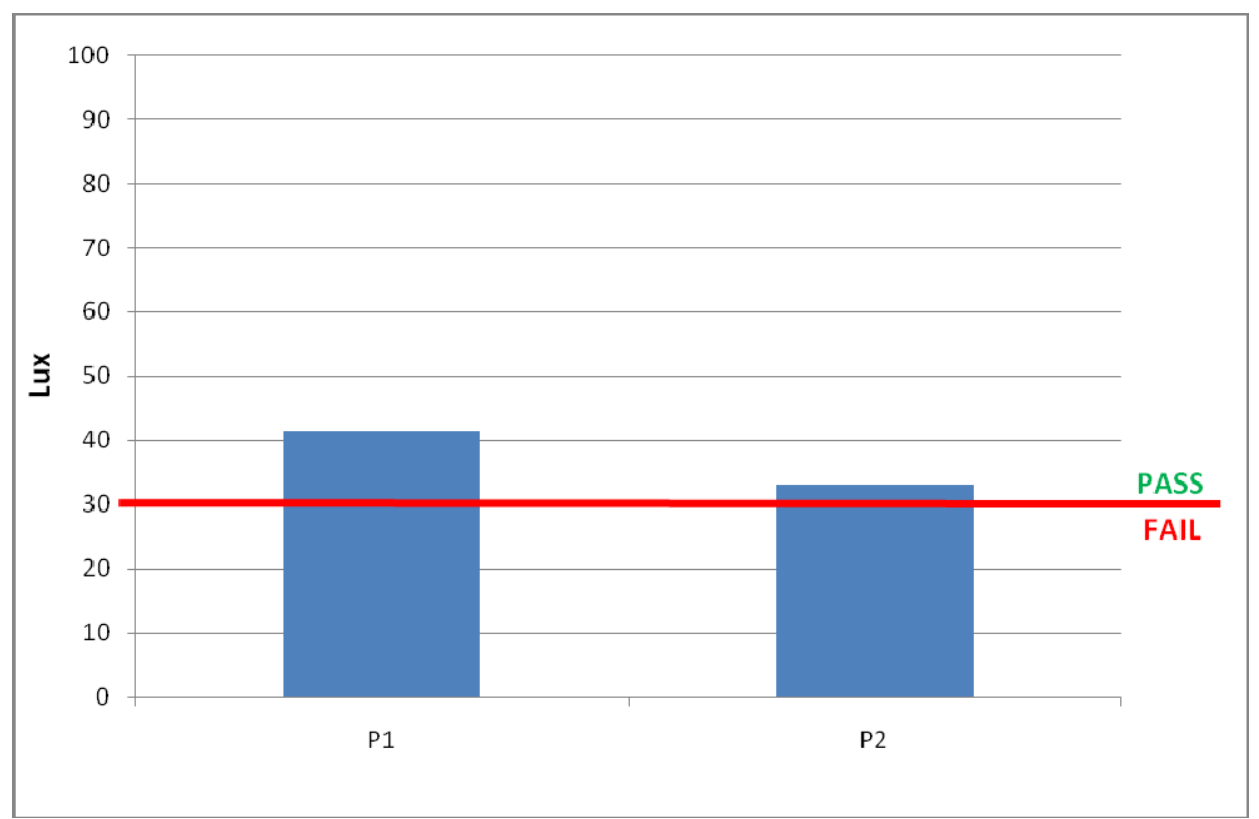

Application of the Tool:

\begin{tabular}{|l|c|}
\hline \multicolumn{2}{|c|}{ NZBC G7 Compliance Assessment Tool } \\
\hline \multicolumn{2}{|l|}{ Variable: } \\
\hline Opposite Building Height & 18 \\
\hline Street Width & 15.9 \\
\hline Glazed Area & 30 \\
\hline Orientation & North \\
\hline Location & Wellington \\
\hline Reflectance of Opposite Building & 10 \\
\hline Glazing Transmittance & 87 \\
\hline Vertical Location of lowest apartment & 7 \\
\hline \multicolumn{2}{|c}{} \\
\hline Are simulations required to prove compliance: \\
\hline
\end{tabular}

Comparison of Results:

\begin{tabular}{|c|c|c|c|}
\hline Measurements & Linear Model & Model 2A & Model 2B \\
\hline Borderline Pass & 93 & 95 & 89 \\
\hline
\end{tabular}




\section{Apartment 50}

Measurement Results:

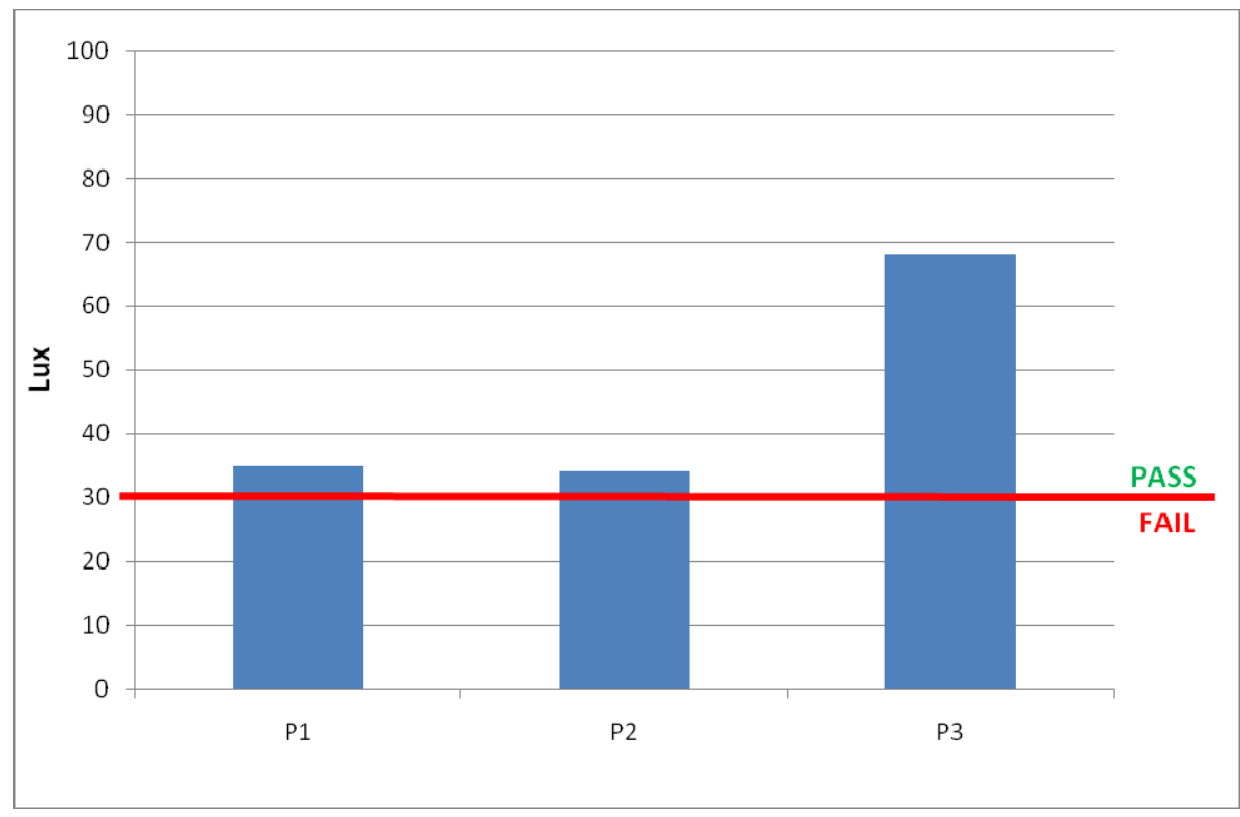

Application of the Tool:

\begin{tabular}{|l|c|}
\hline \multicolumn{2}{|c|}{ NZBC G7 Compliance Assessment Tool } \\
\hline \multicolumn{2}{|l|}{} \\
\hline Variable: & 0 \\
\hline Opposite Building Height & 0 \\
\hline Street Width & 26 \\
\hline Glazed Area & East \\
\hline Orientation & Christchurch \\
\hline Location & 0 \\
\hline Reflectance of Opposite Building & 87 \\
\hline Glazing Transmittance & 10 \\
\hline Vertical Location of lowest apartment \\
\hline \multicolumn{2}{|c|}{} \\
\hline Are simulations required to prove compliance: \\
\hline
\end{tabular}

Comparison of Results:

\begin{tabular}{|c|c|c|c|}
\hline Measurements & Linear Model & Model 2A & Model 2B \\
\hline Borderline Pass & 75 & 210 & 237 \\
\hline
\end{tabular}




\section{Apartment 5}

Measurement Results:

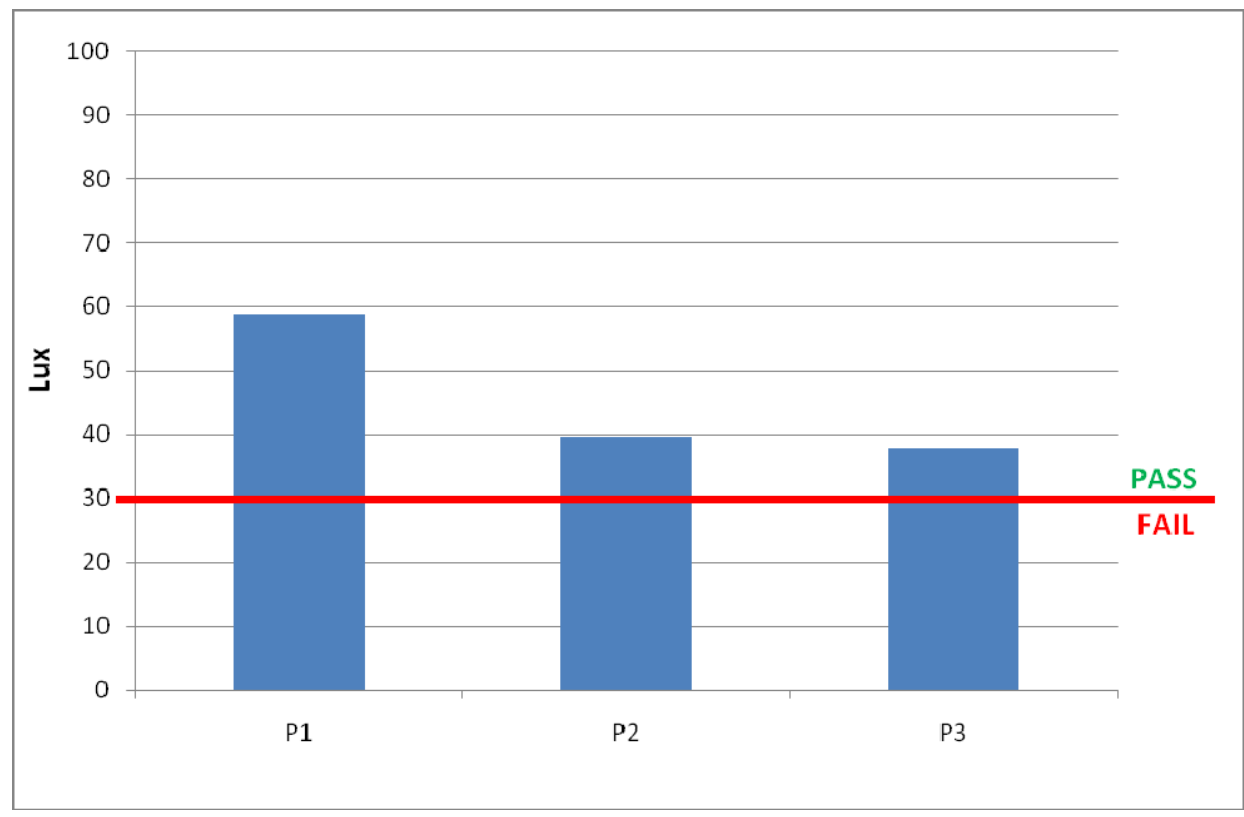

Application of the Tool:

\begin{tabular}{|l|c|}
\hline \multicolumn{2}{|c|}{ NZBC G7 Compliance Assessment Tool } \\
\hline \multicolumn{2}{|l|}{} \\
\hline Variable: & 20 \\
\hline Opposite Building Height & 5 \\
\hline Street Width & 23 \\
\hline Glazed Area & North \\
\hline Orientation & Wellington \\
\hline Location & 33.3 \\
\hline Reflectance of Opposite Building & 87 \\
\hline Glazing Transmittance & 3 \\
\hline Vertical Location of lowest apartment \\
\hline \multicolumn{2}{|}{} \\
\hline Are simulations required to prove compliance: \\
\hline
\end{tabular}

Comparison of Results:

\begin{tabular}{|c|c|c|c|}
\hline Measurements & Linear Model & Model 2A & Model 2B \\
\hline Pass & 52 & 15 & 19 \\
\hline
\end{tabular}




\section{Apartment 52}

Measurement Results:

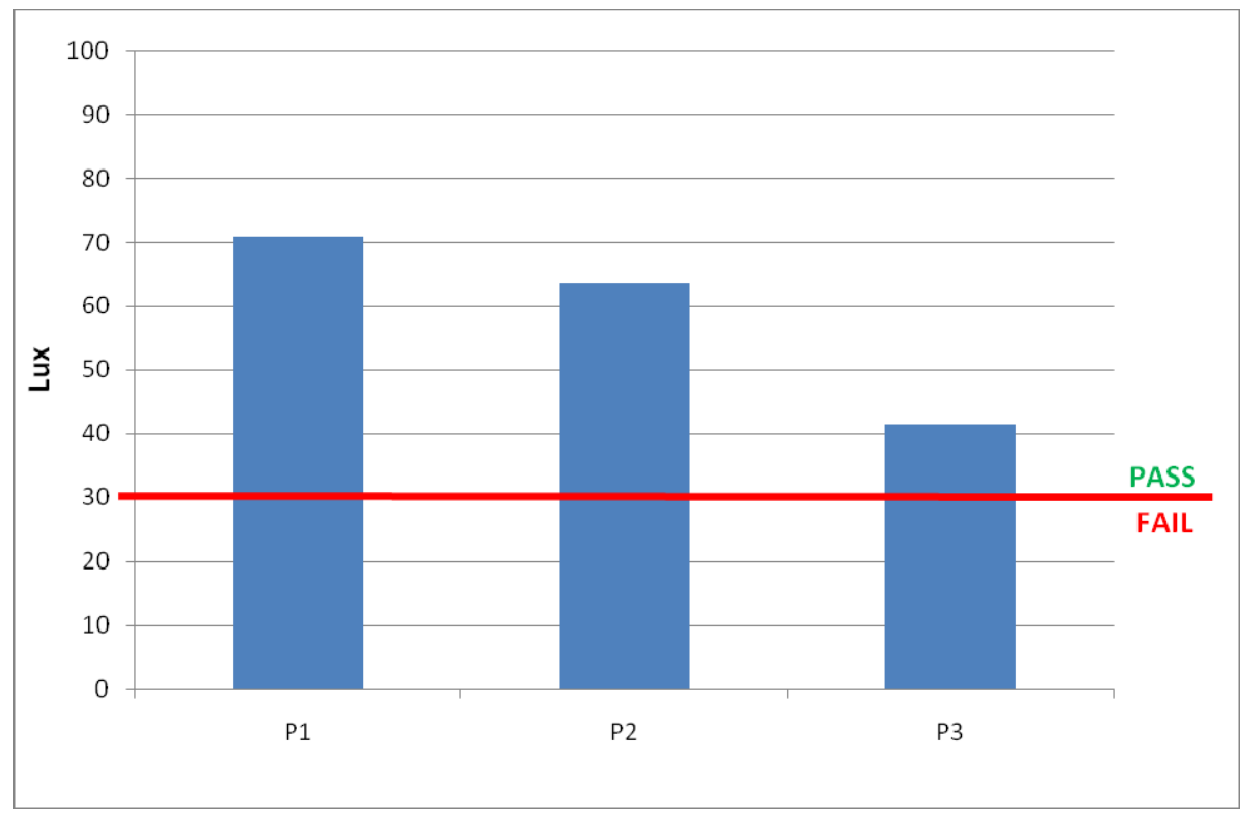

Application of the Tool:

\begin{tabular}{|l|c|}
\hline \multicolumn{2}{|c|}{ NZBC G7 Compliance Assessment Tool } \\
\hline \multicolumn{2}{|l|}{} \\
\hline Variable: & 20 \\
\hline Opposite Building Height & 5 \\
\hline Street Width & 34 \\
\hline Glazed Area & North \\
\hline Orientation & Wellington \\
\hline Location & 12 \\
\hline Reflectance of Opposite Building & 87 \\
\hline Glazing Transmittance & 11 \\
\hline Vertical Location of lowest apartment \\
\hline \multicolumn{2}{|c|}{} \\
\hline Are simulations required to prove compliance: \\
\hline
\end{tabular}

Comparison of Results:

\begin{tabular}{|c|c|c|c|}
\hline Measurements & Linear Model & Model 2A & Model 2B \\
\hline Pass & 94 & 239 & 174 \\
\hline
\end{tabular}




\section{Apartment 53}

Measurement Results:

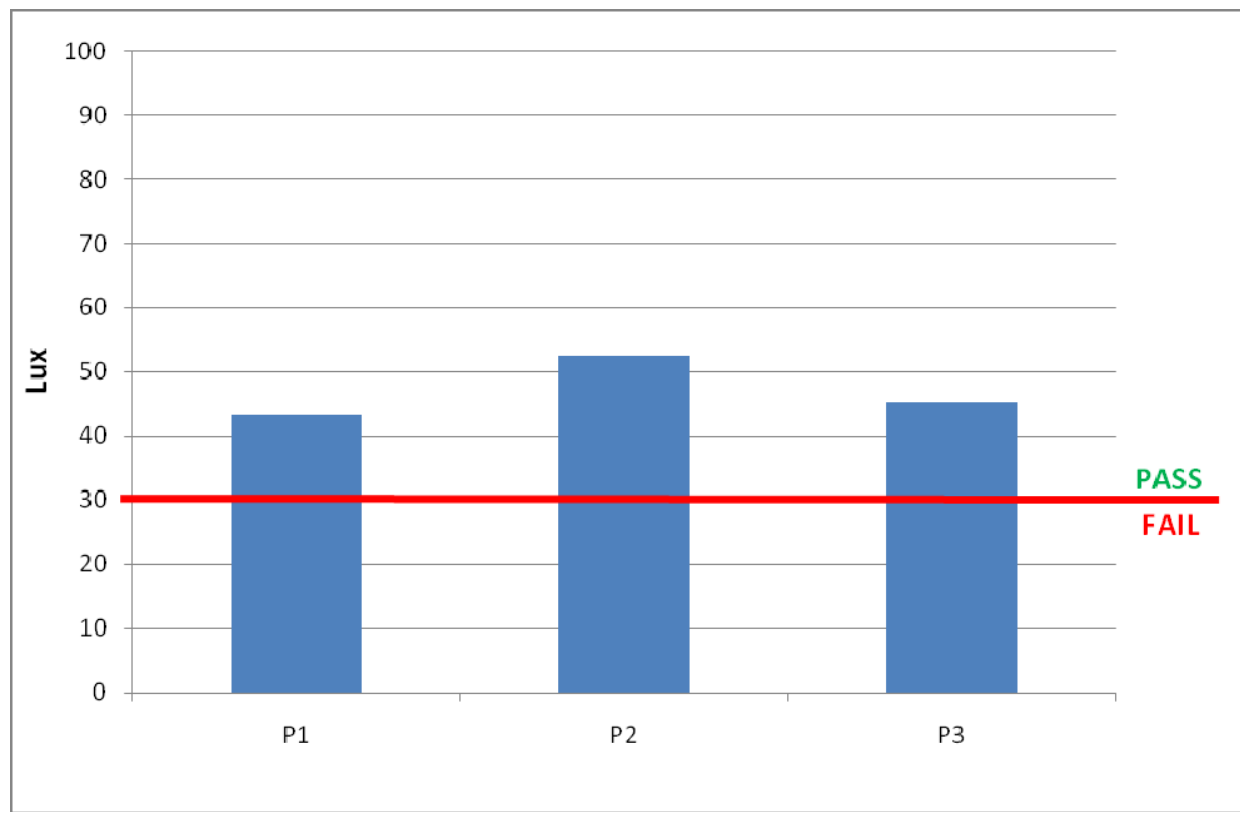

Application of the Tool:

\begin{tabular}{|l|c|}
\hline \multicolumn{2}{|c|}{ NZBC G7 Compliance Assessment Tool } \\
\hline \multicolumn{2}{|l|}{} \\
\hline Variable: & 11.5 \\
\hline Opposite Building Height & 15.5 \\
\hline Street Width & 24 \\
\hline Glazed Area & North \\
\hline Orientation & Wellington \\
\hline Location & 13.5 \\
\hline Reflectance of Opposite Building & 87 \\
\hline Glazing Transmittance & 4 \\
\hline Vertical Location of lowest apartment \\
\hline \multicolumn{2}{|c|}{} \\
\hline Are simulations required to prove compliance: \\
\hline
\end{tabular}

Comparison of Results:

\begin{tabular}{|c|c|c|c|}
\hline Measurements & Linear Model & Model 2A & Model 2B \\
\hline Pass & 76 & 53 & 58 \\
\hline
\end{tabular}




\section{Apartment 54}

Measurement Results:

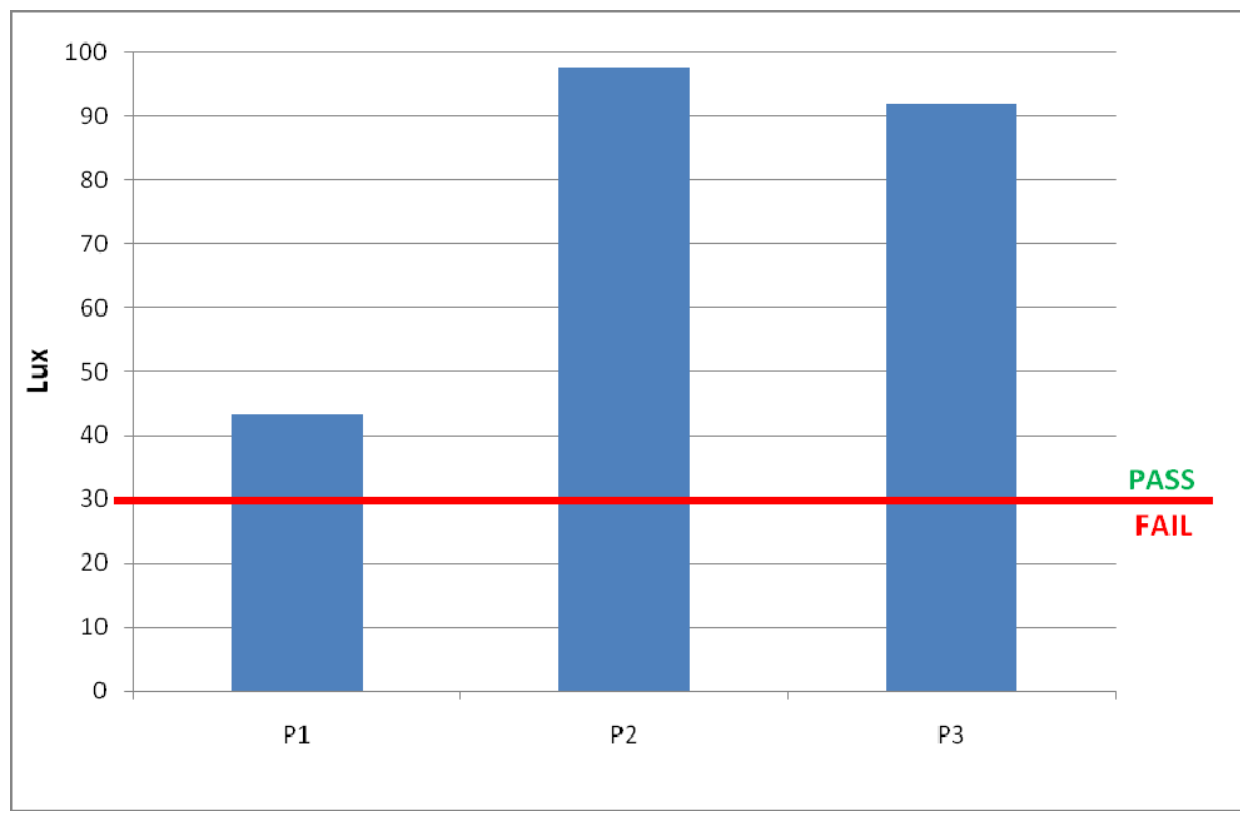

Application of the Tool:

\begin{tabular}{|l|c|}
\hline \multicolumn{2}{|c|}{ NZBC G7 Compliance Assessment Tool } \\
\hline \multicolumn{2}{|l|}{ Variable: } \\
\hline Opposite Building Height & 32 \\
\hline Street Width & 50 \\
\hline Glazed Area & 43 \\
\hline Orientation & West \\
\hline Location & Wellington \\
\hline Reflectance of Opposite Building & 33.3 \\
\hline Glazing Transmittance & 87 \\
\hline Vertical Location of lowest apartment & 6 \\
\hline \multicolumn{2}{|c|}{} \\
\hline Are simulations required to prove compliance: \\
\hline
\end{tabular}

Comparison of Results:

\begin{tabular}{|c|c|c|c|}
\hline Measurements & Linear Model & Model 2A & Model 2B \\
\hline Pass & 171 & 154 & 148 \\
\hline
\end{tabular}




\section{Apartment 55}

Measurement Results:

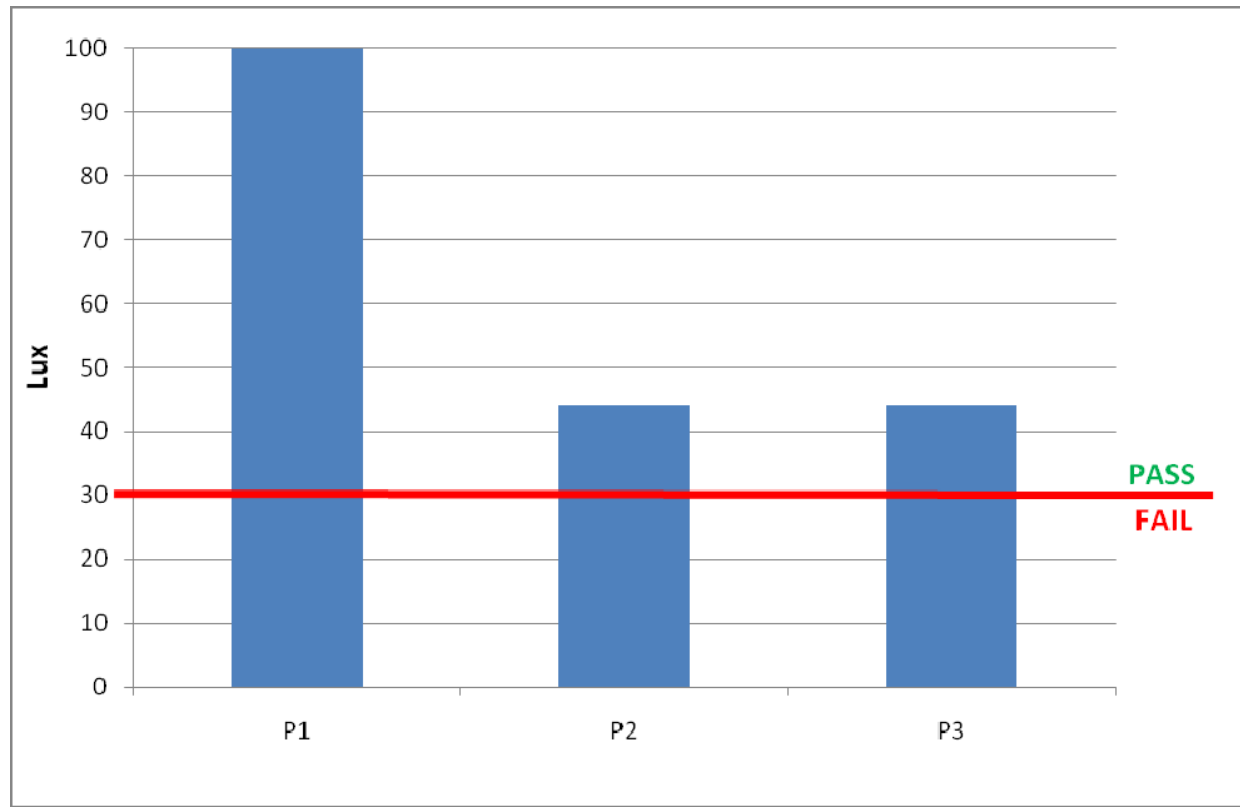

Application of the Tool:

\begin{tabular}{|l|c|}
\hline \multicolumn{2}{|c|}{ NZBC G7 Compliance Assessment Tool } \\
\hline \multicolumn{2}{|l|}{ Variable: } \\
\hline Opposite Building Height & 17.5 \\
\hline Street Width & 10 \\
\hline Glazed Area & 48 \\
\hline Orientation & West \\
\hline Location & Wellington \\
\hline Reflectance of Opposite Building & 38 \\
\hline Glazing Transmittance & 87 \\
\hline Vertical Location of lowest apartment & 4 \\
\hline \multicolumn{2}{|c|}{} \\
\hline Are simulations required to prove compliance: \\
\hline
\end{tabular}

Comparison of Results:

\begin{tabular}{|c|c|c|c|}
\hline Measurements & Linear Model & Model 2A & Model 2B \\
\hline Pass & 102 & 84 & 137 \\
\hline
\end{tabular}




\section{Apartment 56}

Measurement Results:

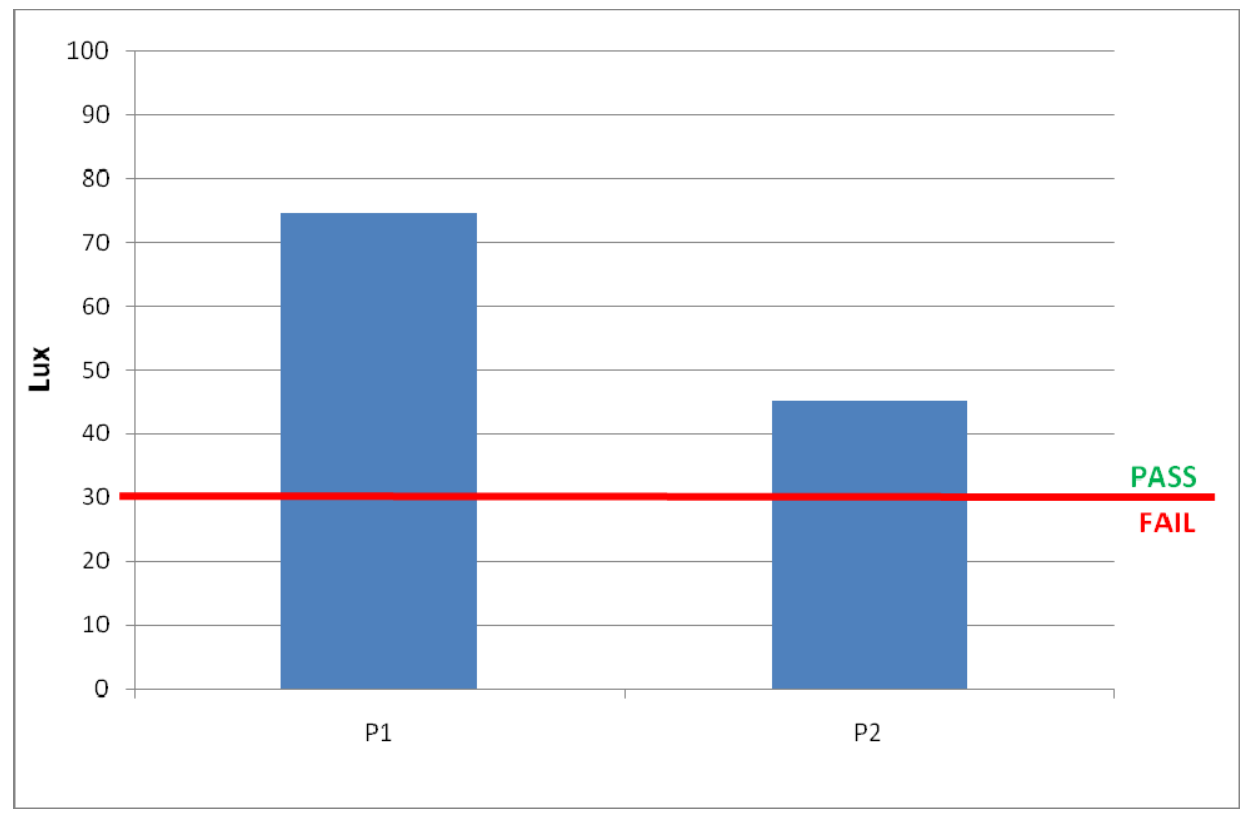

Application of the Tool:

\begin{tabular}{|l|c|}
\hline \multicolumn{2}{|c|}{ NZBC G7 Compliance Assessment Tool } \\
\hline \multicolumn{2}{|l|}{ Variable: } \\
\hline Opposite Building Height & 20 \\
\hline Street Width & 5 \\
\hline Glazed Area & 21 \\
\hline Orientation & North \\
\hline Location & Wellington \\
\hline Reflectance of Opposite Building & 12 \\
\hline Glazing Transmittance & 87 \\
\hline Vertical Location of lowest apartment & 6 \\
\hline \multicolumn{2}{|c|}{} \\
\hline Are simulations required to prove compliance: \\
\hline
\end{tabular}

Comparison of Results:

\begin{tabular}{|c|c|c|c|}
\hline Measurements & Linear Model & Model 2A & Model 2B \\
\hline Pass & 56 & 40 & 29 \\
\hline
\end{tabular}




\section{Apartment 57}

Measurement Results:

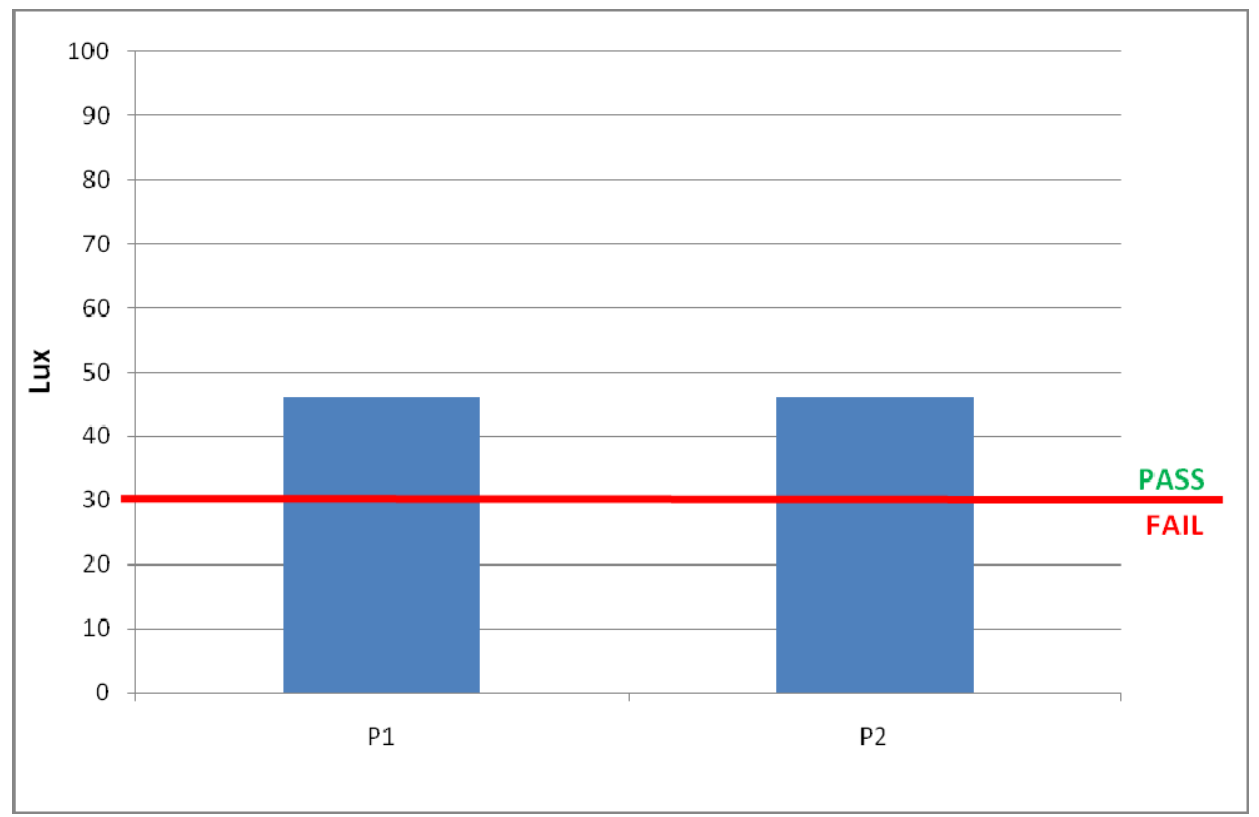

Application of the Tool:

\begin{tabular}{|l|c|}
\hline \multicolumn{2}{|c|}{ NZBC G7 Compliance Assessment Tool } \\
\hline \multicolumn{2}{|l|}{ Variable: } \\
\hline Opposite Building Height & 23.8 \\
\hline Street Width & 40 \\
\hline Glazed Area & 24 \\
\hline Orientation & West \\
\hline Location & Wellington \\
\hline Reflectance of Opposite Building & 13.5 \\
\hline Glazing Transmittance & 87 \\
\hline Vertical Location of lowest apartment & 7 \\
\hline \multicolumn{2}{|c|}{} \\
\hline Are simulations required to prove compliance: \\
\hline
\end{tabular}

Comparison of Results:

\begin{tabular}{|c|c|c|c|}
\hline Measurements & Linear Model & Model 2A & Model 2B \\
\hline Pass & 131 & 128 & 103 \\
\hline
\end{tabular}




\section{Apartment 58}

Measurement Results:

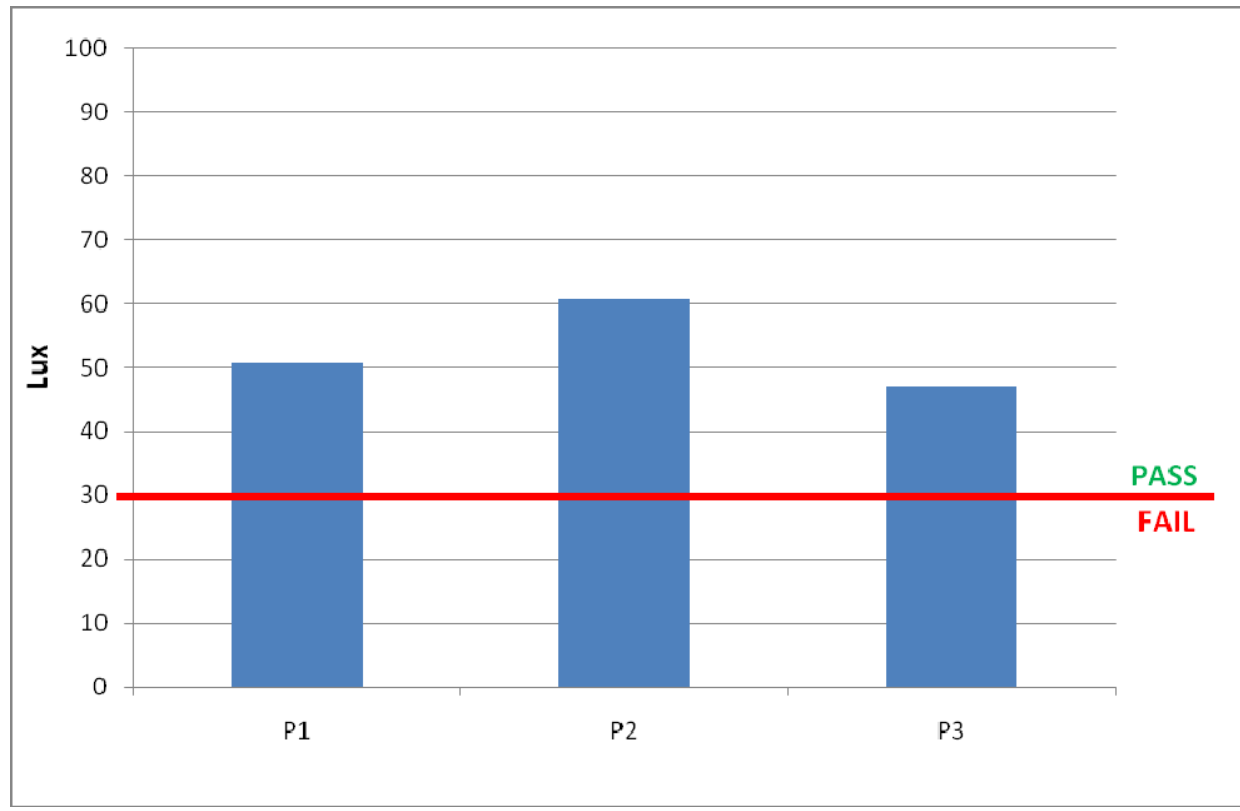

Application of the Tool:

\begin{tabular}{|l|c|}
\hline \multicolumn{2}{|c|}{ NZBC G7 Compliance Assessment Tool } \\
\hline \multicolumn{2}{|l|}{ Variable: } \\
\hline Opposite Building Height & 11.6 \\
\hline Street Width & 9 \\
\hline Glazed Area & 53 \\
\hline Orientation & North \\
\hline Location & Wellington \\
\hline Reflectance of Opposite Building & 30 \\
\hline Glazing Transmittance & 87 \\
\hline Vertical Location of lowest apartment & 6 \\
\hline \multicolumn{2}{|c|}{} \\
\hline Are simulations required to prove compliance: \\
\hline
\end{tabular}

Comparison of Results:

\begin{tabular}{|c|c|c|c|}
\hline Measurements & Linear Model & Model 2A & Model 2B \\
\hline Pass & 114 & 128 & 292 \\
\hline
\end{tabular}




\section{Apartment 59}

Measurement Results:

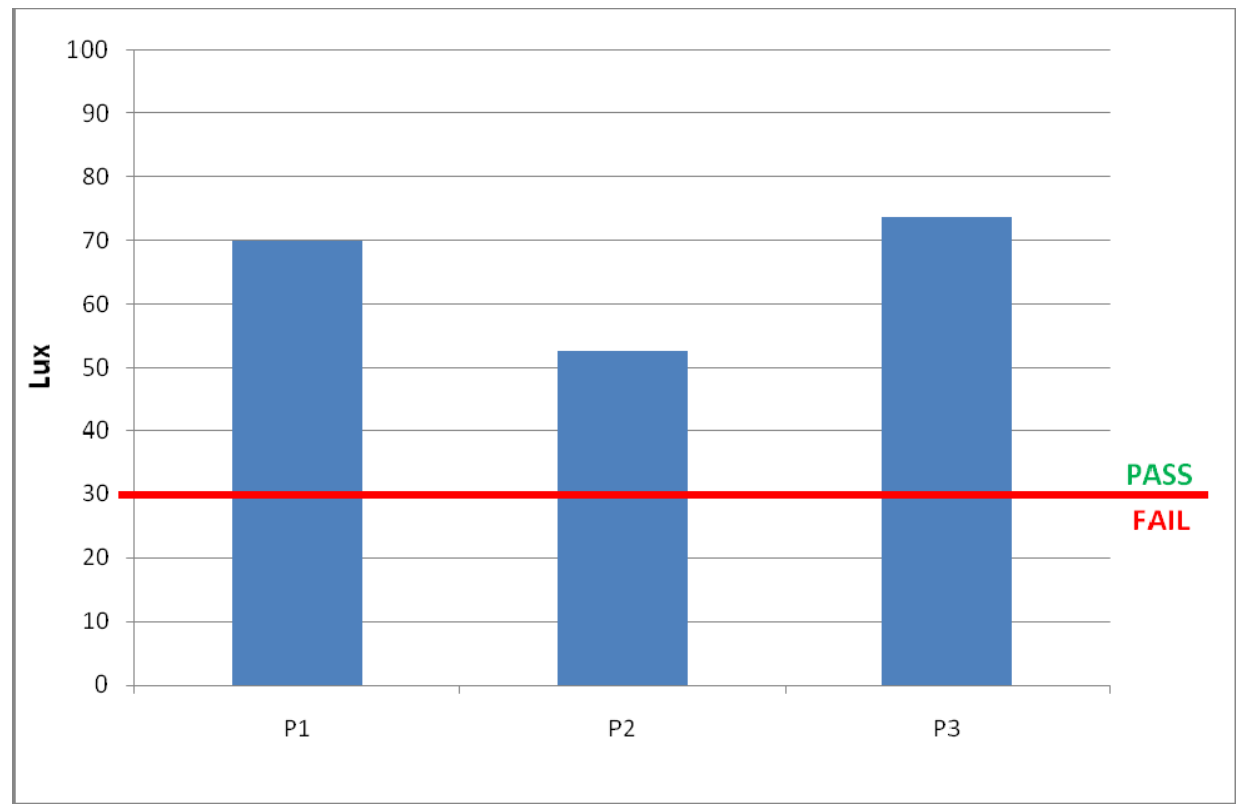

Application of the Tool:

\begin{tabular}{|l|c|}
\hline \multicolumn{2}{|c|}{ NZBC G7 Compliance Assessment Tool } \\
\hline \multicolumn{2}{|l|}{} \\
\hline Variable: & 20 \\
\hline Opposite Building Height & 37 \\
\hline Street Width & 24 \\
\hline Glazed Area & East \\
\hline Orientation & Wellington \\
\hline Location & 38 \\
\hline Reflectance of Opposite Building & 87 \\
\hline Glazing Transmittance & 7 \\
\hline Vertical Location of lowest apartment \\
\hline \multicolumn{2}{|l}{} \\
\hline Are simulations required to prove compliance: \\
\hline
\end{tabular}

Comparison of Results:

\begin{tabular}{|c|c|c|c|}
\hline Measurements & Linear Model & Model 2A & Model 2B \\
\hline Pass & 133 & 133 & 126 \\
\hline
\end{tabular}




\section{Apartment 60}

Measurement Results:

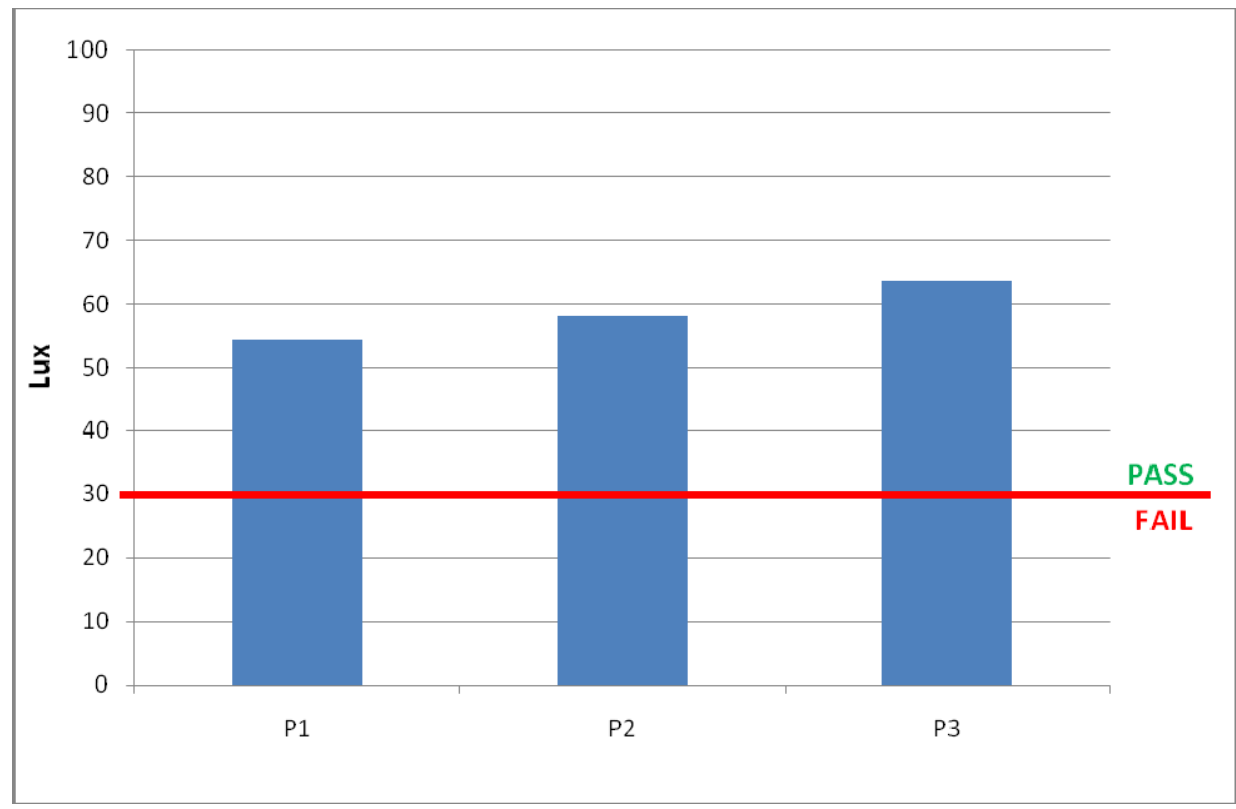

Application of the Tool:

\begin{tabular}{|l|c|}
\hline \multicolumn{2}{|c|}{ NZBC G7 Compliance Assessment Tool } \\
\hline \multicolumn{2}{|l|}{} \\
\hline Variable: & 42 \\
\hline Opposite Building Height & 66 \\
\hline Street Width & 10 \\
\hline Glazed Area & South \\
\hline Orientation & Wellington \\
\hline Location & 14.8 \\
\hline Reflectance of Opposite Building & 87 \\
\hline Glazing Transmittance & 9 \\
\hline Vertical Location of lowest apartment \\
\hline \multicolumn{2}{|c|}{} \\
\hline Are simulations required to prove compliance: \\
\hline
\end{tabular}

Comparison of Results:

\begin{tabular}{|c|c|c|c|}
\hline Measurements & Linear Model & Model 2A & Model 2B \\
\hline Pass & 164 & 215 & 119 \\
\hline
\end{tabular}




\section{Apartment 61}

Measurement Results:

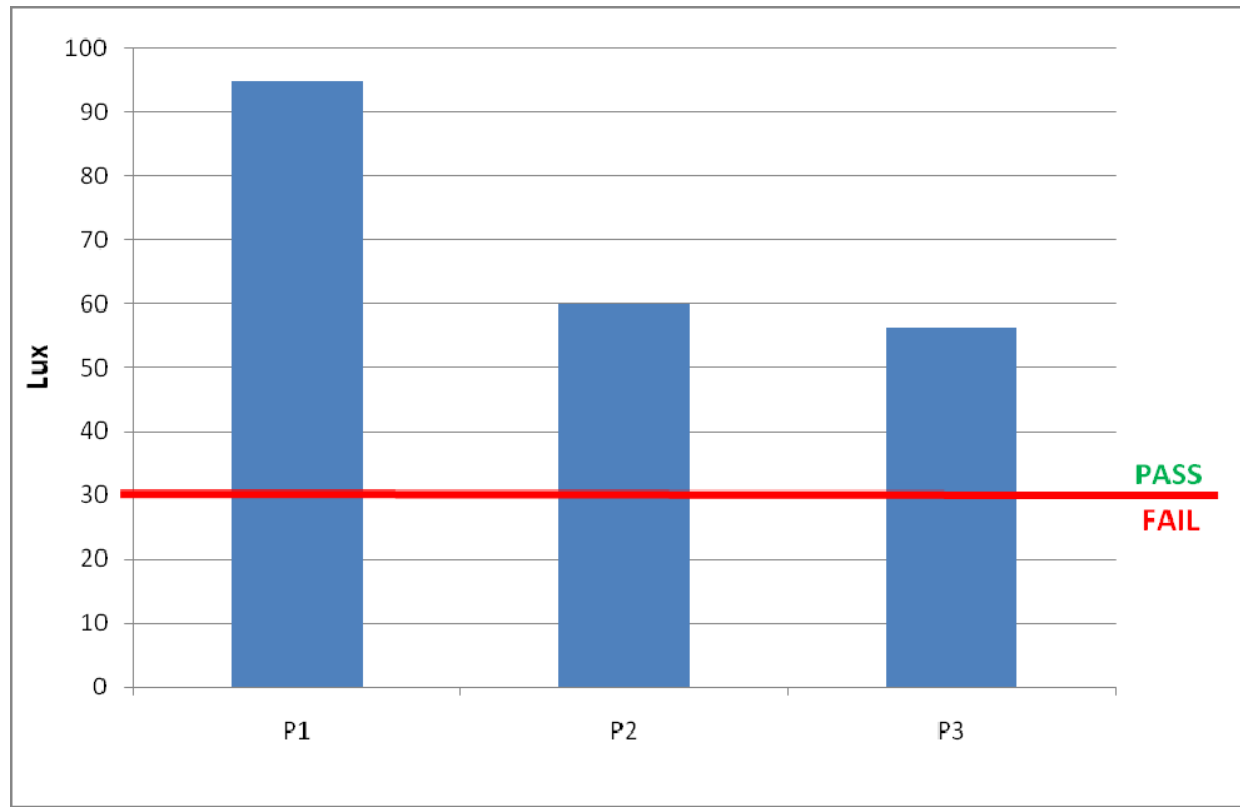

Application of the Tool:

\begin{tabular}{|l|c|}
\hline \multicolumn{2}{|c|}{ NZBC G7 Compliance Assessment Tool } \\
\hline \multicolumn{2}{|l|}{ Variable: } \\
\hline Opposite Building Height & 14 \\
\hline Street Width & 44 \\
\hline Glazed Area & 34 \\
\hline Orientation & South \\
\hline Location & Wellington \\
\hline Reflectance of Opposite Building & 21 \\
\hline Glazing Transmittance & 87 \\
\hline Vertical Location of lowest apartment & 7 \\
\hline \multicolumn{2}{|c|}{} \\
\hline Are simulations required to prove compliance: \\
\hline
\end{tabular}

Comparison of Results:

\begin{tabular}{|c|c|c|c|}
\hline Measurements & Linear Model & Model 2A & Model 2B \\
\hline Pass & 160 & 170 & 198 \\
\hline
\end{tabular}




\section{Apartment 62}

Measurement Results:

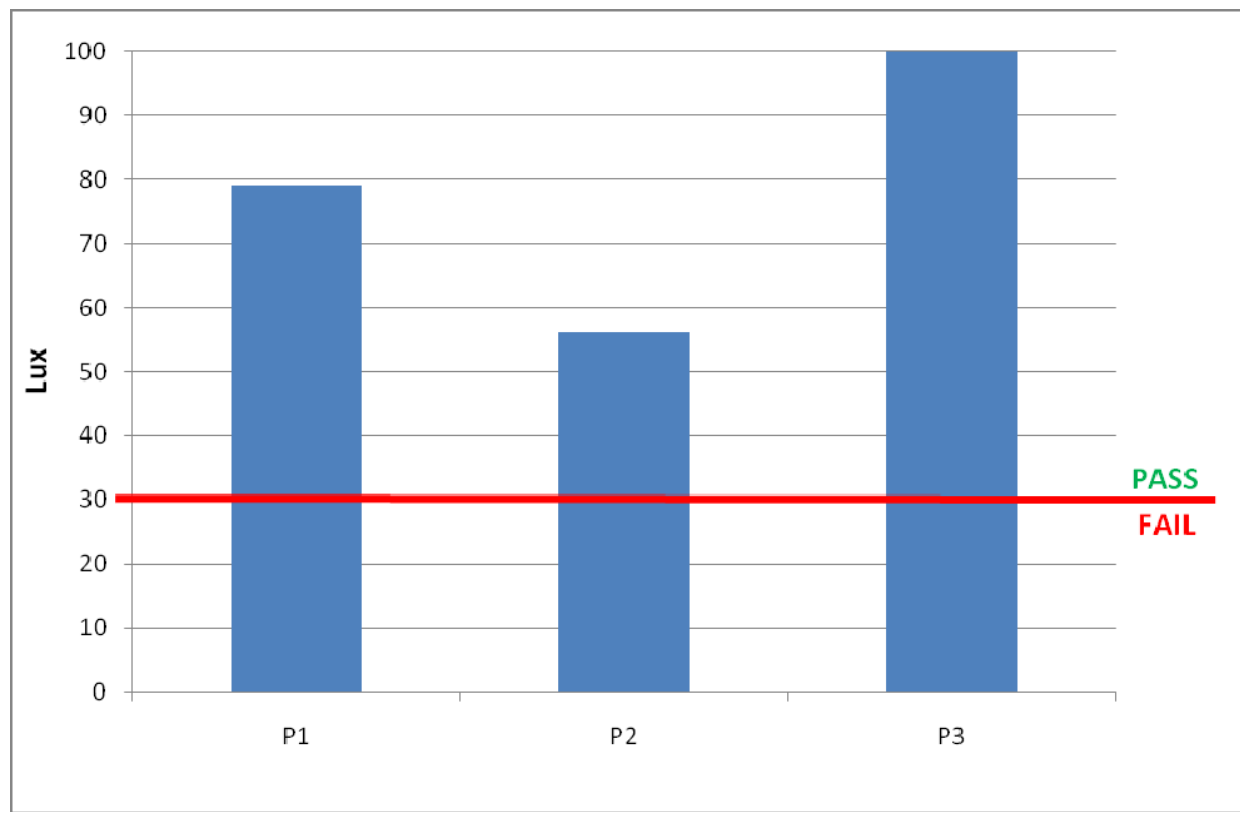

Application of the Tool:

\begin{tabular}{|l|c|}
\hline \multicolumn{2}{|c|}{ NZBC G7 Compliance Assessment Tool } \\
\hline \multicolumn{2}{|l|}{ Variable: } \\
\hline Opposite Building Height & 8 \\
\hline Street Width & 44 \\
\hline Glazed Area & 35 \\
\hline Orientation & North \\
\hline Location & Wellington \\
\hline Reflectance of Opposite Building & 20 \\
\hline Glazing Transmittance & 87 \\
\hline Vertical Location of lowest apartment & 1 \\
\hline \multicolumn{2}{|c}{} \\
\hline Are simulations required to prove compliance: & NO \\
\hline
\end{tabular}

Comparison of Results:

\begin{tabular}{|c|c|c|c|}
\hline Measurements & Linear Model & Model 2A & Model 2B \\
\hline Pass & 134 & 120 & 119 \\
\hline
\end{tabular}




\section{Apartment 63}

Measurement Results:

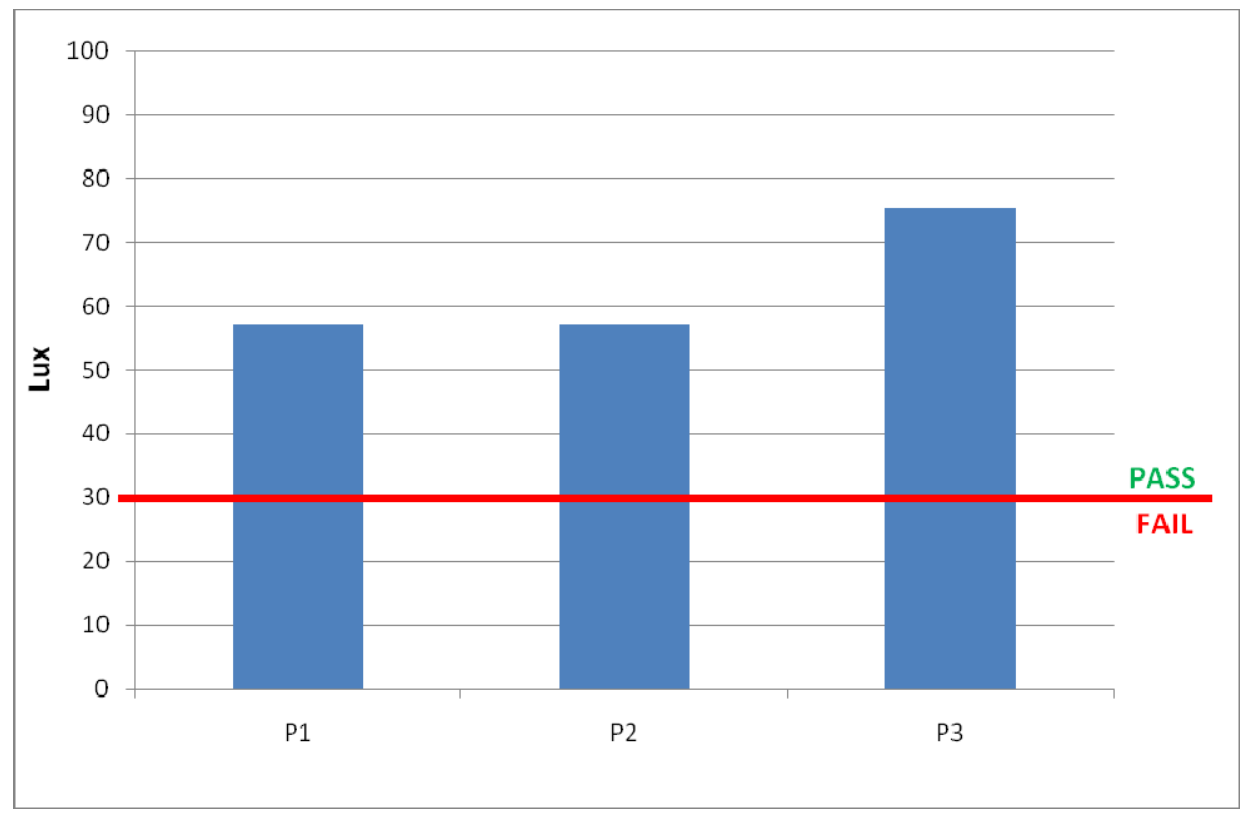

Application of the Tool:

\begin{tabular}{|l|c|}
\hline \multicolumn{2}{|c|}{ NZBC G7 Compliance Assessment Tool } \\
\hline \multicolumn{2}{|l|}{ Variable: } \\
\hline Opposite Building Height & 0 \\
\hline Street Width & 0 \\
\hline Glazed Area & 23 \\
\hline Orientation & North \\
\hline Location & 0 \\
\hline Reflectance of Opposite Building & 87 \\
\hline Glazing Transmittance & 16 \\
\hline Vertical Location of lowest apartment \\
\hline \multicolumn{2}{|c|}{} \\
\hline Are simulations required to prove compliance: \\
\hline
\end{tabular}

Comparison of Results:

\begin{tabular}{|c|c|c|c|}
\hline Measurements & Linear Model & Model 2A & Model 2B \\
\hline Pass & 98 & 720 & 650 \\
\hline
\end{tabular}




\section{Apartment 64}

Measurement Results:

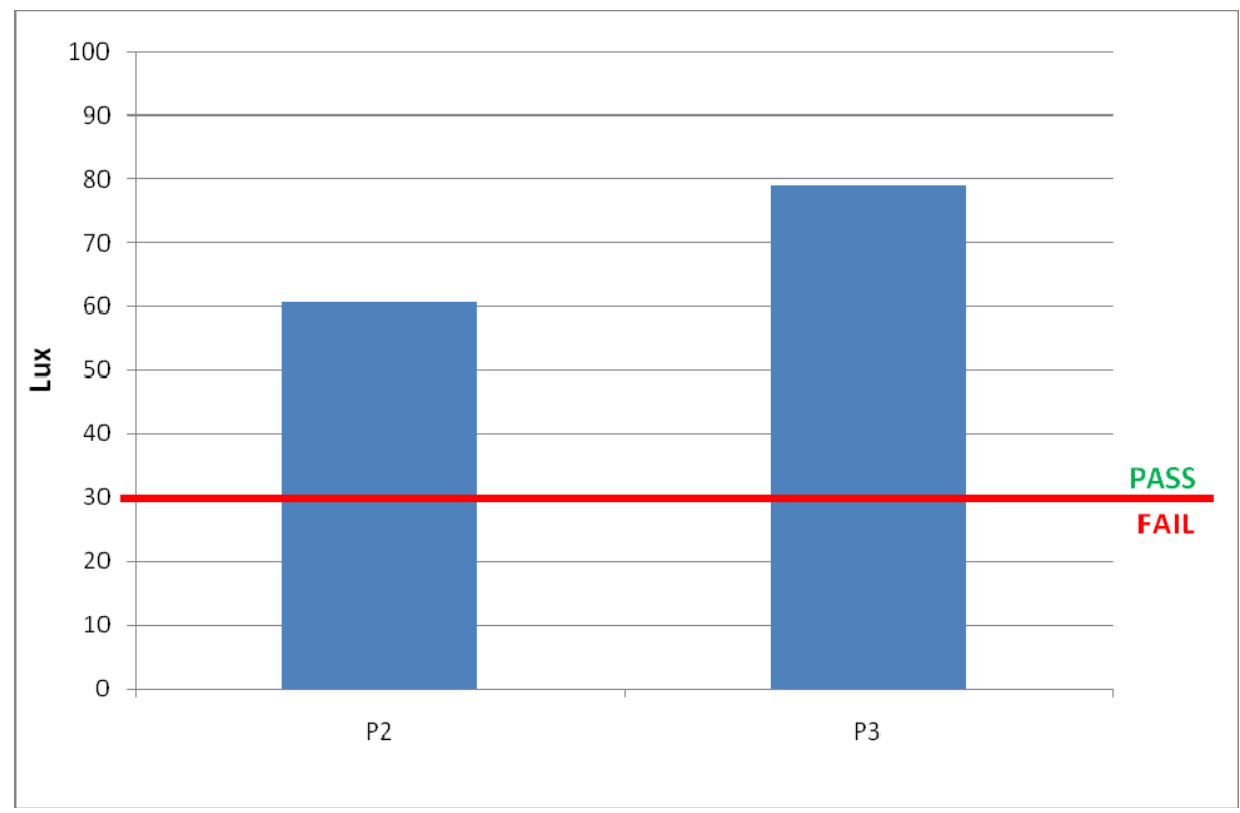

Application of the Tool:

\begin{tabular}{|l|c|}
\hline \multicolumn{2}{|c|}{ NZBC G7 Compliance Assessment Tool } \\
\hline \multicolumn{2}{|l|}{} \\
\hline Variable: & 28 \\
\hline Opposite Building Height & 24 \\
\hline Street Width & 10 \\
\hline Glazed Area & South \\
\hline Orientation & Wellington \\
\hline Location & 10 \\
\hline Reflectance of Opposite Building & 87 \\
\hline Glazing Transmittance & 16 \\
\hline Vertical Location of lowest apartment \\
\hline \multicolumn{2}{|l}{} \\
\hline Are simulations required to prove compliance: \\
\hline
\end{tabular}

Comparison of Results:

\begin{tabular}{|c|c|c|c|}
\hline Measurements & Linear Model & Model 2A & Model 2B \\
\hline Pass & 120 & 702 & 142 \\
\hline
\end{tabular}




\section{Apartment 65}

Measurement Results:

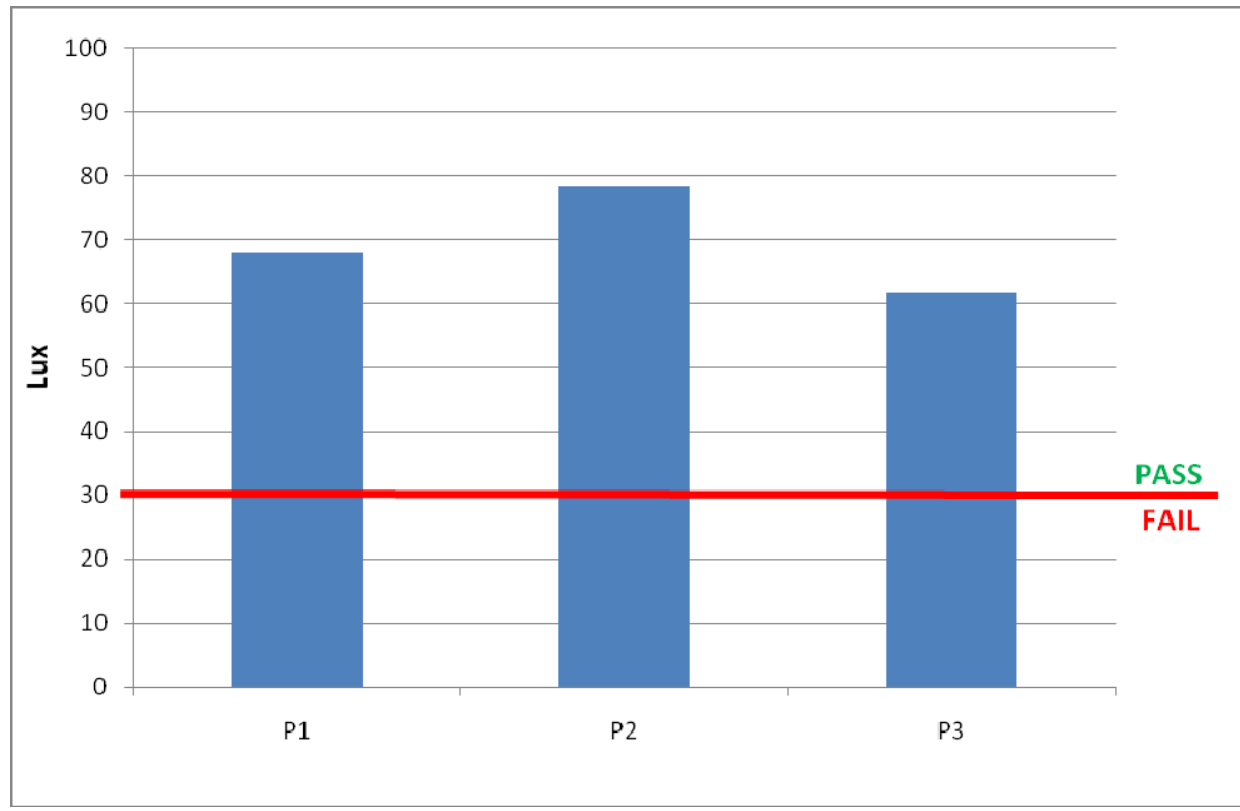

Application of the Tool:

\begin{tabular}{|l|c|}
\hline \multicolumn{2}{|c|}{ NZBC G7 Compliance Assessment Tool } \\
\hline \multicolumn{2}{|l|}{} \\
\hline Variable: & 20 \\
\hline Opposite Building Height & 37 \\
\hline Street Width & 24 \\
\hline Glazed Area & East \\
\hline Orientation & Wellington \\
\hline Location & 38 \\
\hline Reflectance of Opposite Building & 87 \\
\hline Glazing Transmittance & 1 \\
\hline Vertical Location of lowest apartment \\
\hline \multicolumn{2}{|c|}{} \\
\hline Are simulations required to prove compliance: \\
\hline
\end{tabular}

Comparison of Results:

\begin{tabular}{|c|c|c|c|}
\hline Measurements & Linear Model & Model 2A & Model 2B \\
\hline Pass & 108 & 74 & 72 \\
\hline
\end{tabular}




\section{Apartment 66}

Measurement Results:

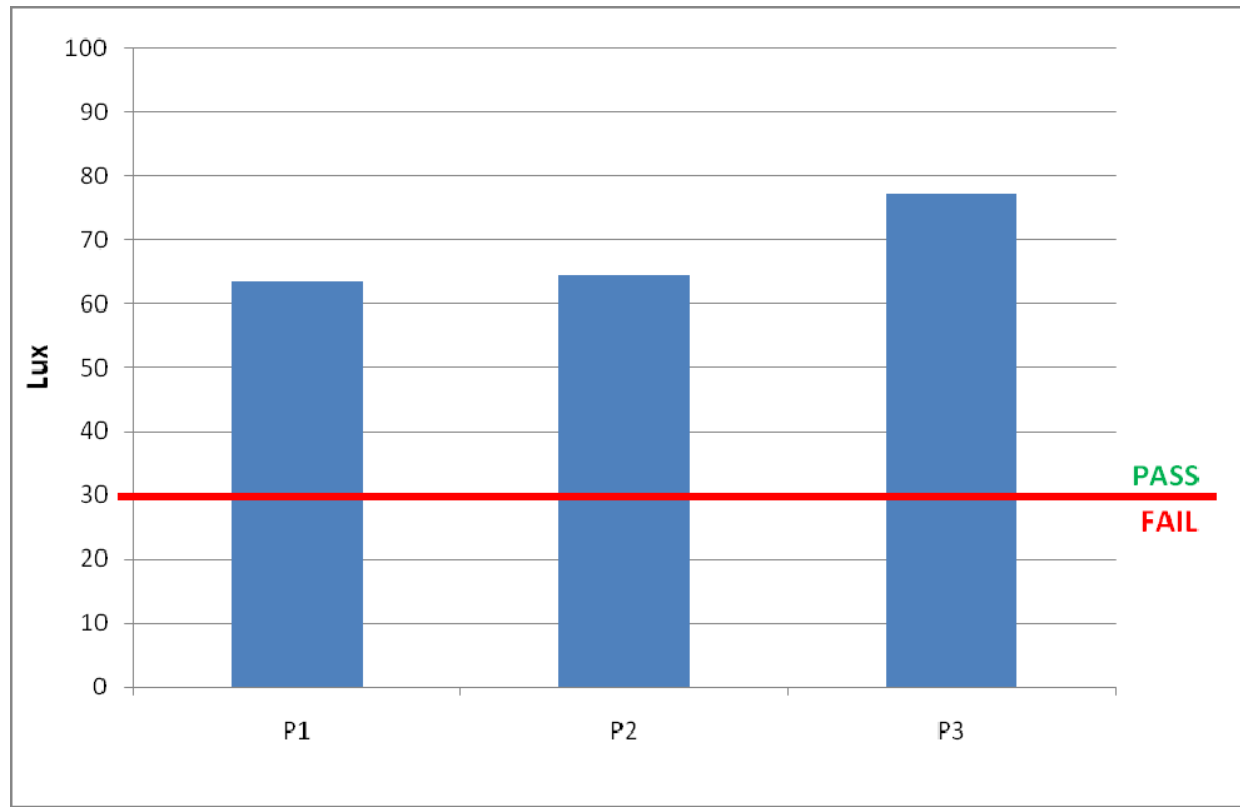

Application of the Tool:

\begin{tabular}{|l|c|}
\hline \multicolumn{2}{|c|}{ NZBC G7 Compliance Assessment Tool } \\
\hline \multicolumn{2}{|l|}{ Variable: } \\
\hline Opposite Building Height & 19 \\
\hline Street Width & 25 \\
\hline Glazed Area & 12 \\
\hline Orientation & South \\
\hline Location & Wellington \\
\hline Reflectance of Opposite Building & 20 \\
\hline Glazing Transmittance & 87 \\
\hline Vertical Location of lowest apartment & 1 \\
\hline \multicolumn{2}{|l}{} \\
\hline Are simulations required to prove compliance: \\
\hline
\end{tabular}

Comparison of Results:

\begin{tabular}{|c|c|c|c|}
\hline Measurements & Linear Model & Model 2A & Model 2B \\
\hline Pass & 68 & 40 & 40 \\
\hline
\end{tabular}




\section{Apartment 67}

Measurement Results:

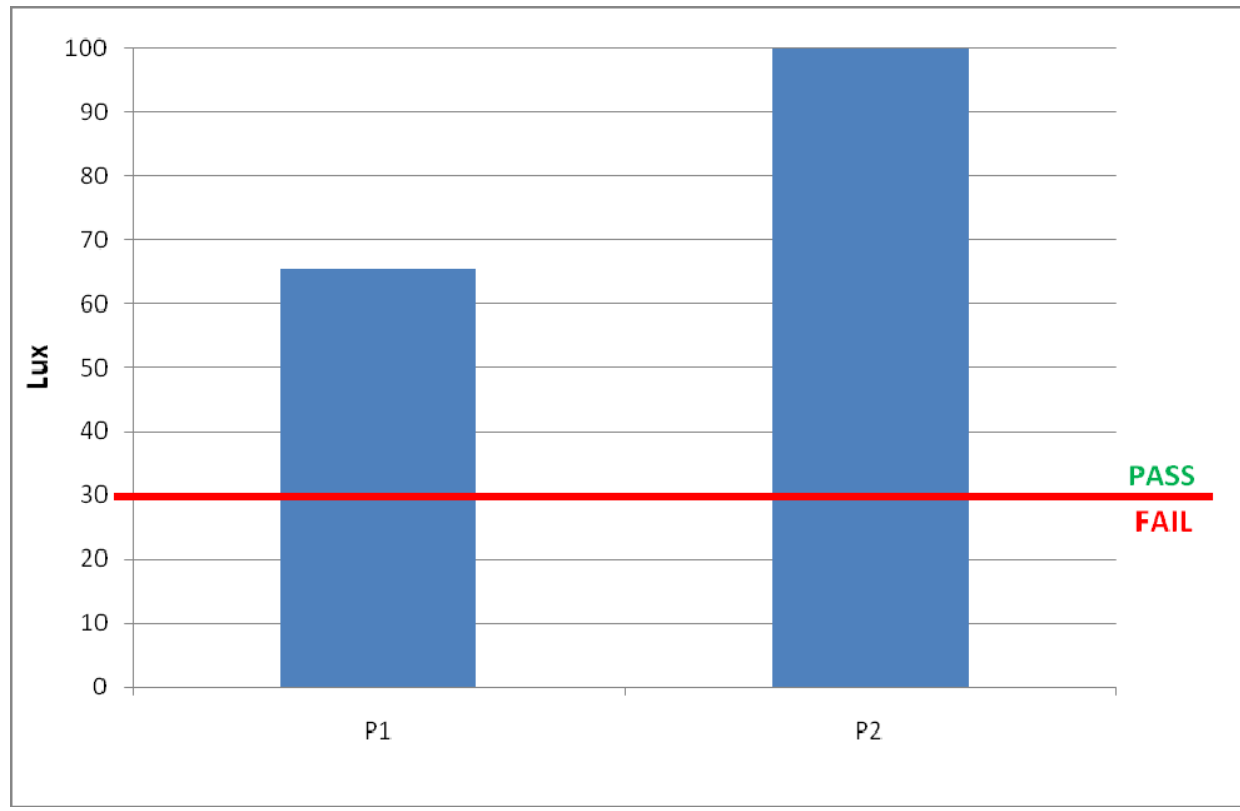

Application of the Tool:

\begin{tabular}{|l|c|}
\hline \multicolumn{2}{|c|}{ NZBC G7 Compliance Assessment Tool } \\
\hline \multicolumn{2}{|l|}{ Variable: } \\
\hline Opposite Building Height & 67 \\
\hline Street Width & 15 \\
\hline Glazed Area & 61 \\
\hline Orientation & West \\
\hline Location & Wellington \\
\hline Reflectance of Opposite Building & 20.8 \\
\hline Glazing Transmittance & 87 \\
\hline Vertical Location of lowest apartment & 4 \\
\hline \multicolumn{2}{|c|}{} \\
\hline Are simulations required to prove compliance: \\
\hline
\end{tabular}

Comparison of Results:

\begin{tabular}{|c|c|c|c|}
\hline Measurements & Linear Model & Model 2A & Model 2B \\
\hline Pass & 100 & 102 & 23 \\
\hline
\end{tabular}




\section{Apartment 68}

Measurement Results:

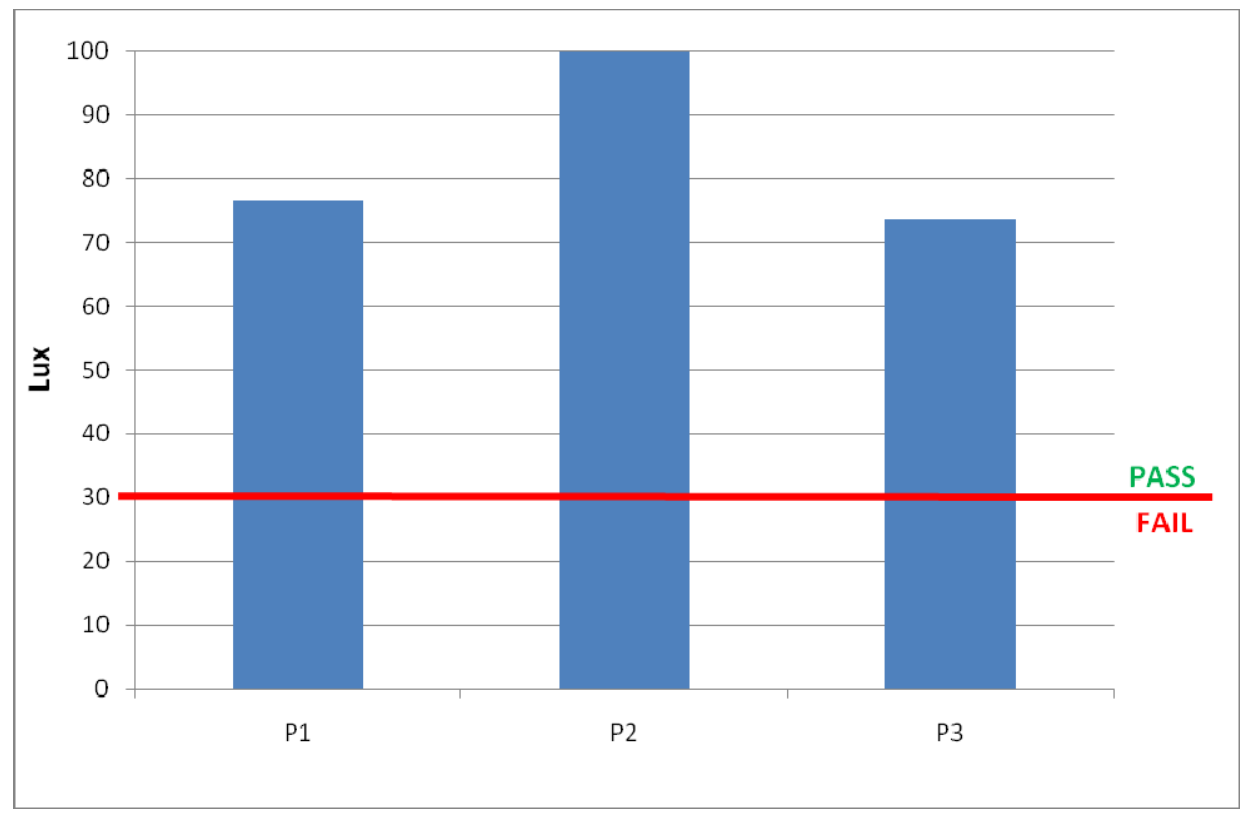

Application of the Tool:

\begin{tabular}{|l|c|}
\hline \multicolumn{2}{|c|}{ NZBC G7 Compliance Assessment Tool } \\
\hline \multicolumn{2}{|l|}{ Variable: } \\
\hline Opposite Building Height & 35 \\
\hline Street Width & 3 \\
\hline Glazed Area & 10 \\
\hline Orientation & East \\
\hline Location & Wellington \\
\hline Reflectance of Opposite Building & 25 \\
\hline Glazing Transmittance & 87 \\
\hline Vertical Location of lowest apartment & 8 \\
\hline \multicolumn{2}{|c|}{} \\
\hline Are simulations required to prove compliance: \\
\hline
\end{tabular}

Comparison of Results:

\begin{tabular}{|c|c|c|c|}
\hline Measurements & Linear Model & Model 2A & Model 2B \\
\hline Pass & 45 & 65 & 5 \\
\hline
\end{tabular}




\section{Apartment 69}

Measurement Results:

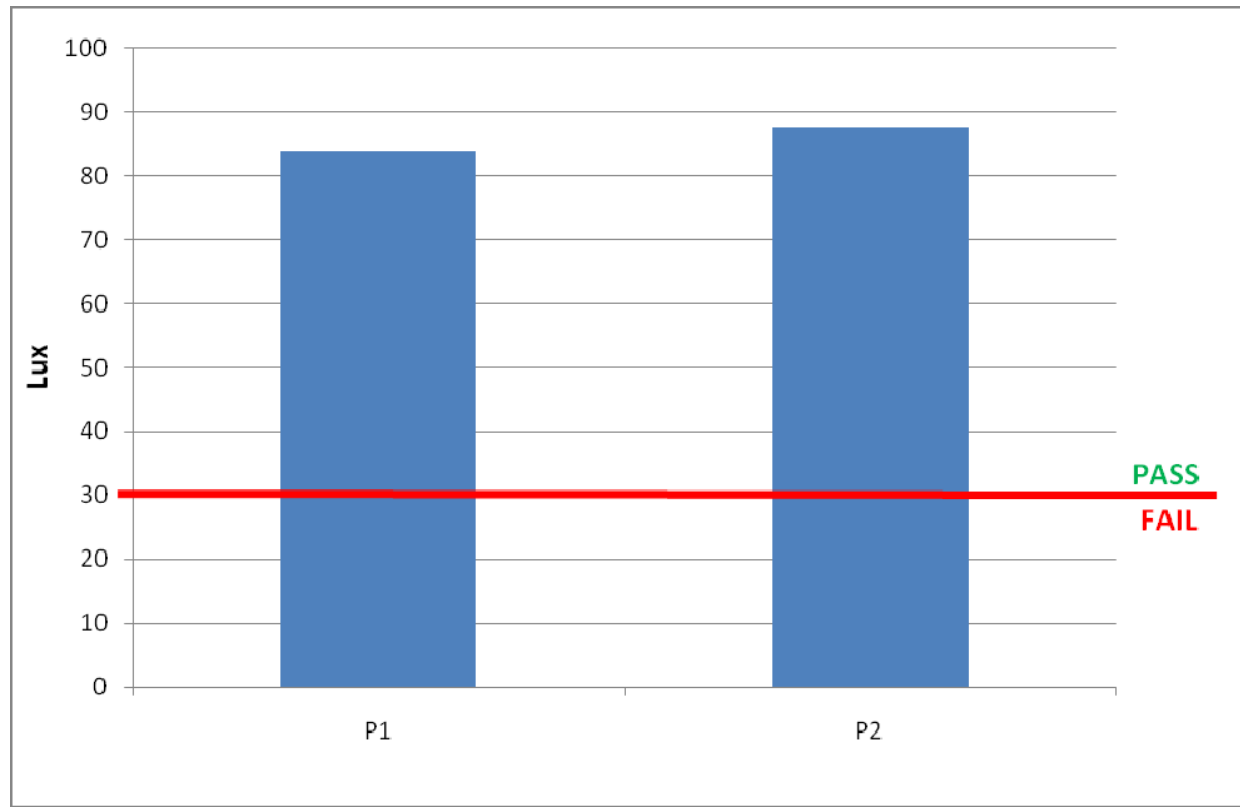

Application of the Tool:

\begin{tabular}{|l|c|}
\hline \multicolumn{2}{|c|}{ NZBC G7 Compliance Assessment Tool } \\
\hline \multicolumn{2}{|l|}{} \\
\hline Variable: & 4 \\
\hline Opposite Building Height & 20 \\
\hline Street Width & 32 \\
\hline Glazed Area & North \\
\hline Orientation & Wellington \\
\hline Location & 40 \\
\hline Reflectance of Opposite Building & 87 \\
\hline Glazing Transmittance & 3 \\
\hline Vertical Location of lowest apartment \\
\hline \multicolumn{2}{|c|}{} \\
\hline Are simulations required to prove compliance: \\
\hline
\end{tabular}

Comparison of Results:

\begin{tabular}{|c|c|c|c|}
\hline Measurements & Linear Model & Model 2A & Model 2B \\
\hline Pass & 101 & 92 & 131 \\
\hline
\end{tabular}




\section{Apartment 70}

Measurement Results:

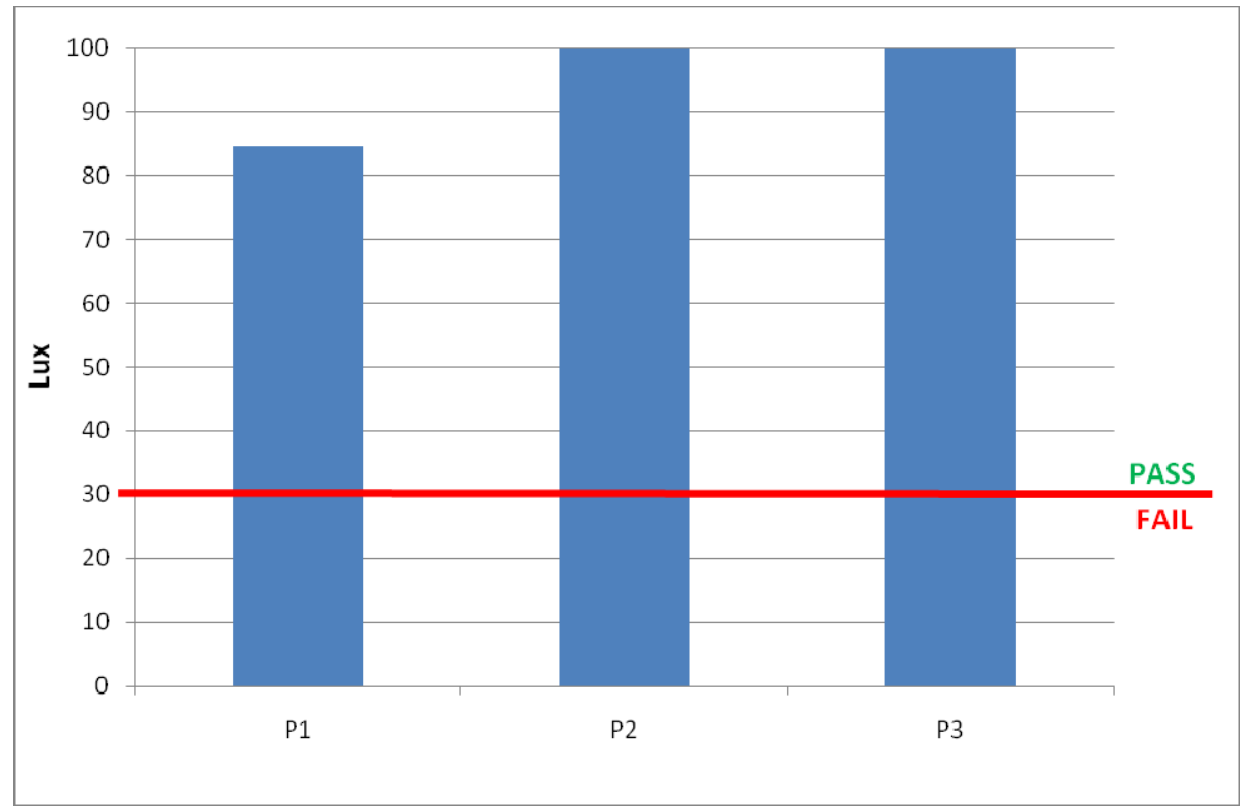

Application of the Tool:

\begin{tabular}{|l|c|}
\hline \multicolumn{2}{|c|}{ NZBC G7 Compliance Assessment Tool } \\
\hline \multicolumn{2}{|l|}{ Variable: } \\
\hline Opposite Building Height & 42 \\
\hline Street Width & 66 \\
\hline Glazed Area & 11.7 \\
\hline Orientation & South \\
\hline Location & Wellington \\
\hline Reflectance of Opposite Building & 14.8 \\
\hline Glazing Transmittance & 87 \\
\hline Vertical Location of lowest apartment & 9 \\
\hline \multicolumn{2}{|c|}{} \\
\hline Are simulations required to prove compliance: \\
\hline
\end{tabular}

Comparison of Results:

\begin{tabular}{|c|c|c|c|}
\hline Measurements & Linear Model & Model 2A & Model 2B \\
\hline Pass & 166 & 216 & 120 \\
\hline
\end{tabular}




\section{Apartment 71}

Measurement Results:

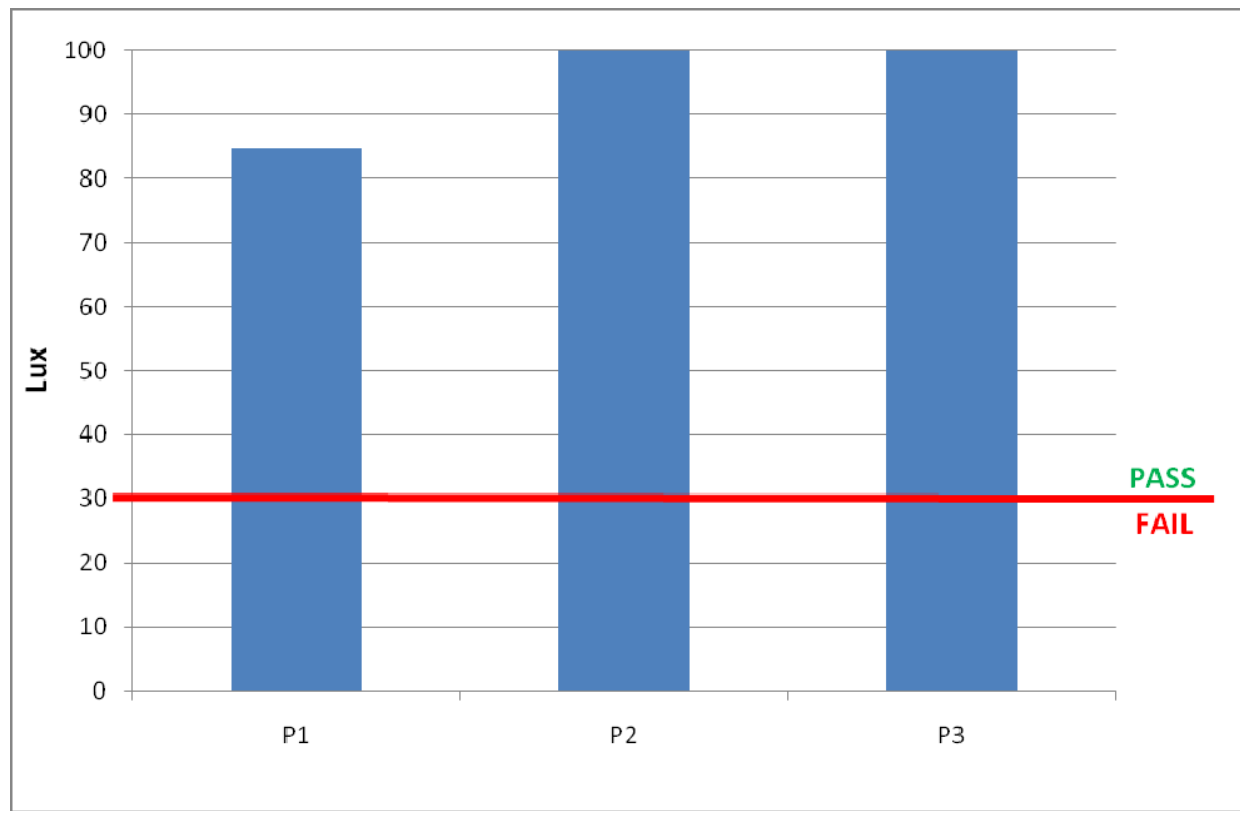

Application of the Tool:

\begin{tabular}{|l|c|}
\hline \multicolumn{2}{|c|}{ NZBC G7 Compliance Assessment Tool } \\
\hline \multicolumn{2}{|l|}{} \\
\hline Variable: & 11.6 \\
\hline Opposite Building Height & 9 \\
\hline Street Width & 41 \\
\hline Glazed Area & North \\
\hline Orientation & Wellington \\
\hline Location & 30 \\
\hline Reflectance of Opposite Building & 87 \\
\hline Glazing Transmittance & 6 \\
\hline Vertical Location of lowest apartment \\
\hline \multicolumn{2}{|l}{} \\
\hline Are simulations required to prove compliance: \\
\hline
\end{tabular}

Comparison of Results:

\begin{tabular}{|c|c|c|c|}
\hline Measurements & Linear Model & Model 2A & Model 2B \\
\hline Pass & 98 & 97 & 176 \\
\hline
\end{tabular}




\section{Apartment 72}

Measurement Results:

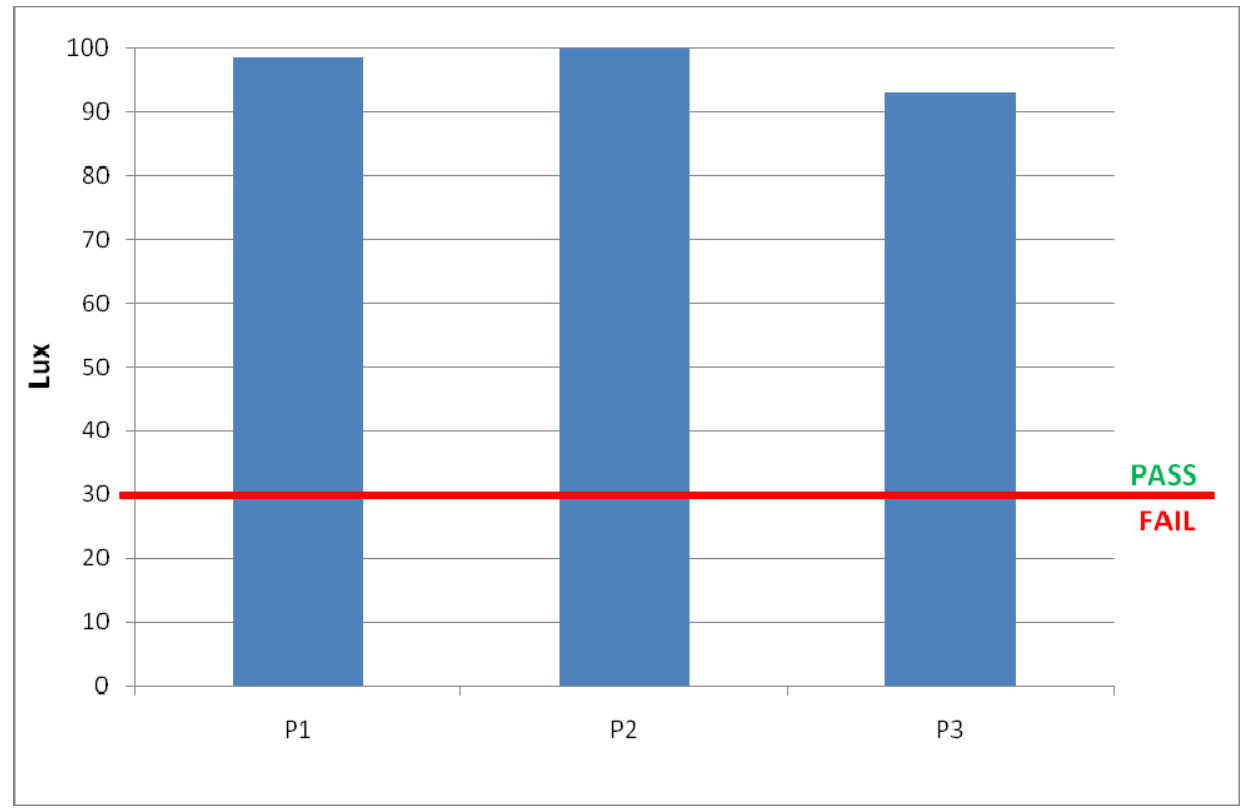

Application of the Tool:

\begin{tabular}{|l|c|}
\hline \multicolumn{2}{|c|}{ NZBC G7 Compliance Assessment Tool } \\
\hline \multicolumn{2}{|l|}{ Variable: } \\
\hline Opposite Building Height & 15 \\
\hline Street Width & 45 \\
\hline Glazed Area & 48 \\
\hline Orientation & East \\
\hline Location & Auckland \\
\hline Reflectance of Opposite Building & 12 \\
\hline Glazing Transmittance & 87 \\
\hline Vertical Location of lowest apartment & 0 \\
\hline \multicolumn{2}{|c|}{} \\
\hline Are simulations required to prove compliance: & NO \\
\hline
\end{tabular}

Comparison of Results:

\begin{tabular}{|c|c|c|c|}
\hline Measurements & Linear Model & Model 2A & Model 2B \\
\hline Pass & 151 & 139 & 126 \\
\hline
\end{tabular}




\section{Apartment 73}

Measurement Results:

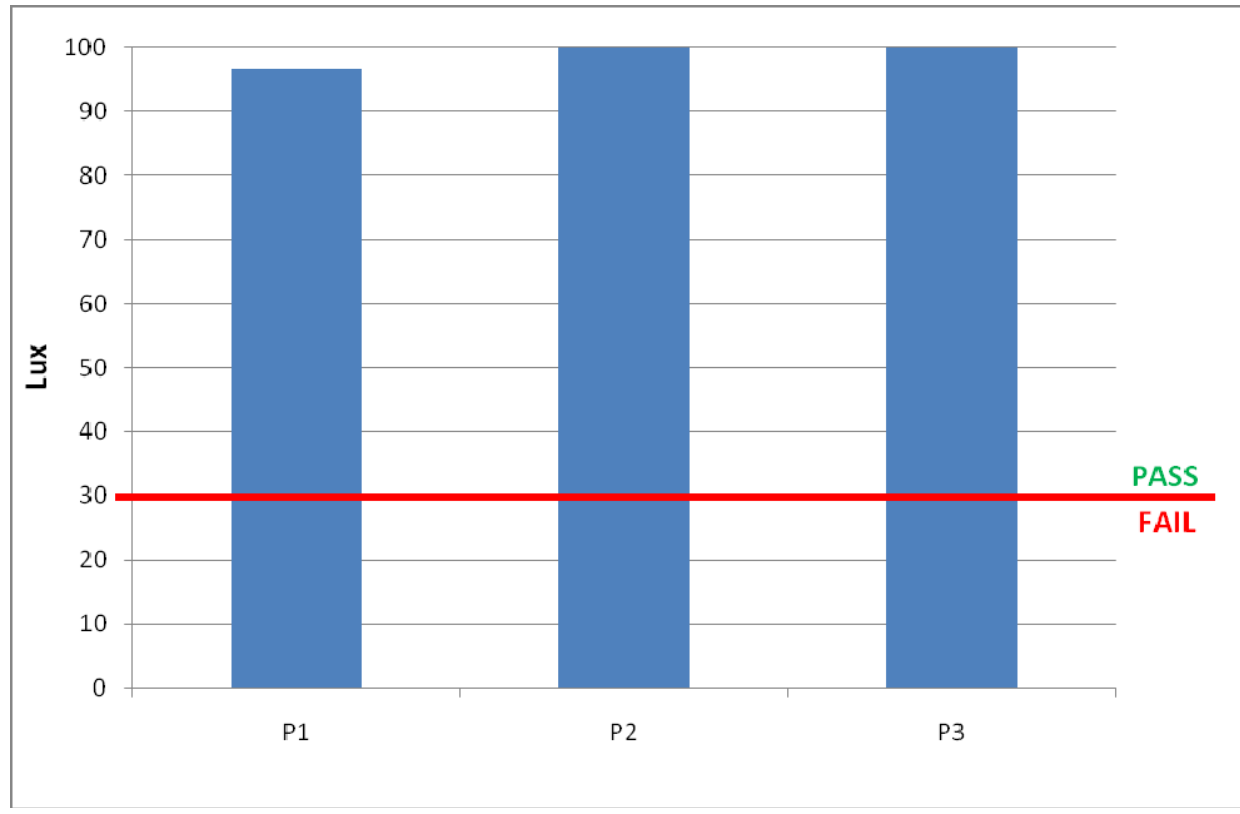

Application of the Tool:

\begin{tabular}{|l|c|}
\hline \multicolumn{2}{|c|}{ NZBC G7 Compliance Assessment Tool } \\
\hline \multicolumn{2}{|l|}{ Variable: } \\
\hline Opposite Building Height & 70 \\
\hline Street Width & 31 \\
\hline Glazed Area & 43 \\
\hline Orientation & East \\
\hline Location & Wellington \\
\hline Reflectance of Opposite Building & 12 \\
\hline Glazing Transmittance & 87 \\
\hline Vertical Location of lowest apartment & 1 \\
\hline \multicolumn{2}{|c|}{} \\
\hline Are simulations required to prove compliance: \\
\hline
\end{tabular}

Comparison of Results:

\begin{tabular}{|c|c|c|c|}
\hline Measurements & Linear Model & Model 2A & Model 2B \\
\hline Pass & 91 & 63 & 37 \\
\hline
\end{tabular}




\section{Apartment 74}

Measurement Results:

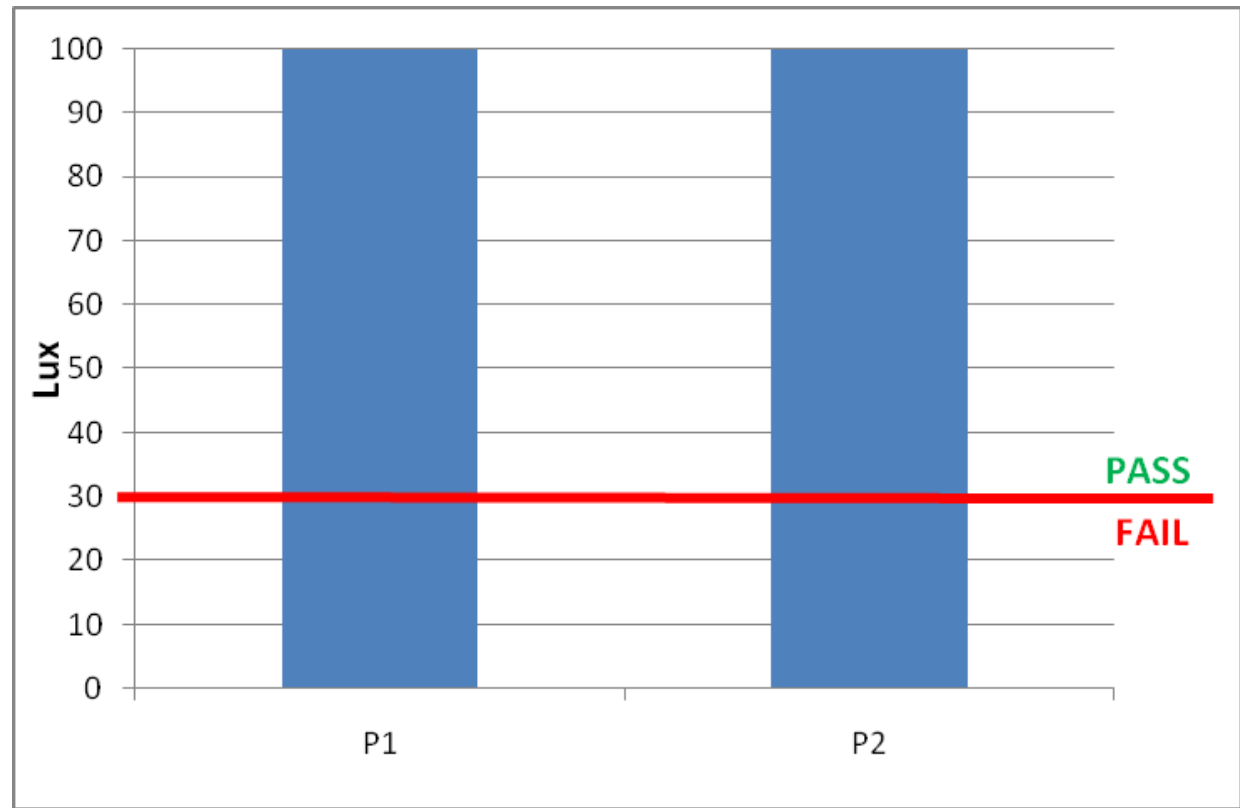

Application of the Tool:

\begin{tabular}{|l|c|}
\hline \multicolumn{2}{|c|}{ NZBC G7 Compliance Assessment Tool } \\
\hline \multicolumn{2}{|l|}{ Variable: } \\
\hline Opposite Building Height & 12 \\
\hline Street Width & 14.5 \\
\hline Glazed Area & 28.6 \\
\hline Orientation & West \\
\hline Location & Wellington \\
\hline Reflectance of Opposite Building & 10.8 \\
\hline Glazing Transmittance & 87 \\
\hline Vertical Location of lowest apartment & 4 \\
\hline \multicolumn{2}{|c|}{} \\
\hline Are simulations required to prove compliance: \\
\hline
\end{tabular}

Comparison of Results:

\begin{tabular}{|c|c|c|c|}
\hline Measurements & Linear Model & Model 2A & Model 2B \\
\hline Pass & 81 & 59 & 67 \\
\hline
\end{tabular}




\section{Apartment 75}

Measurement Results:

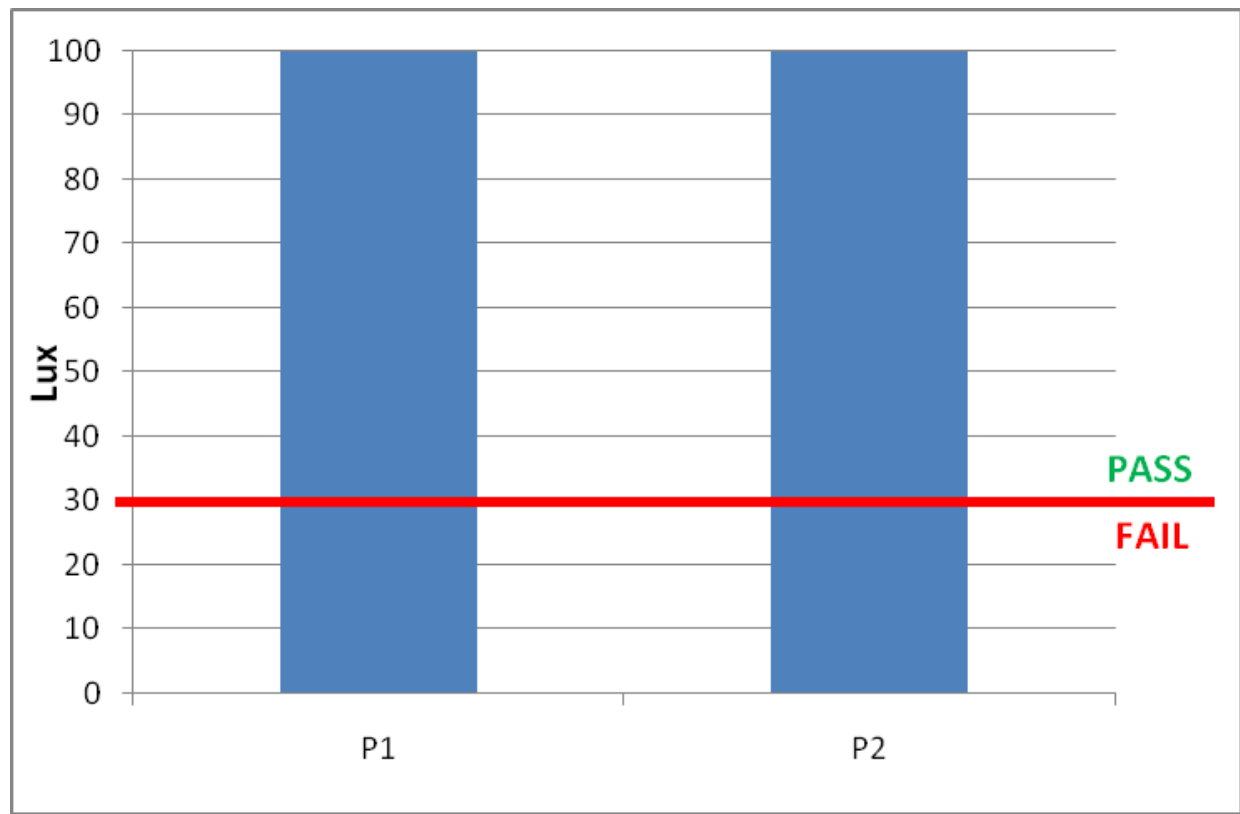

Application of the Tool:

\begin{tabular}{|l|c|}
\hline \multicolumn{2}{|c|}{ NZBC G7 Compliance Assessment Tool } \\
\hline \multicolumn{2}{|l|}{ Variable: } \\
\hline Opposite Building Height & 16 \\
\hline Street Width & 9 \\
\hline Glazed Area & 22 \\
\hline Orientation & East \\
\hline Location & Wellington \\
\hline Reflectance of Opposite Building & 14.8 \\
\hline Glazing Transmittance & 87 \\
\hline Vertical Location of lowest apartment & 1 \\
\hline \multicolumn{2}{|}{} \\
\hline Are simulations required to prove compliance: \\
\hline
\end{tabular}

Comparison of Results:

\begin{tabular}{|c|c|c|c|}
\hline Measurements & Linear Model & Model 2A & Model 2B \\
\hline Pass & 49 & 21 & 17 \\
\hline
\end{tabular}




\section{Apartment 76}

Measurement Results:

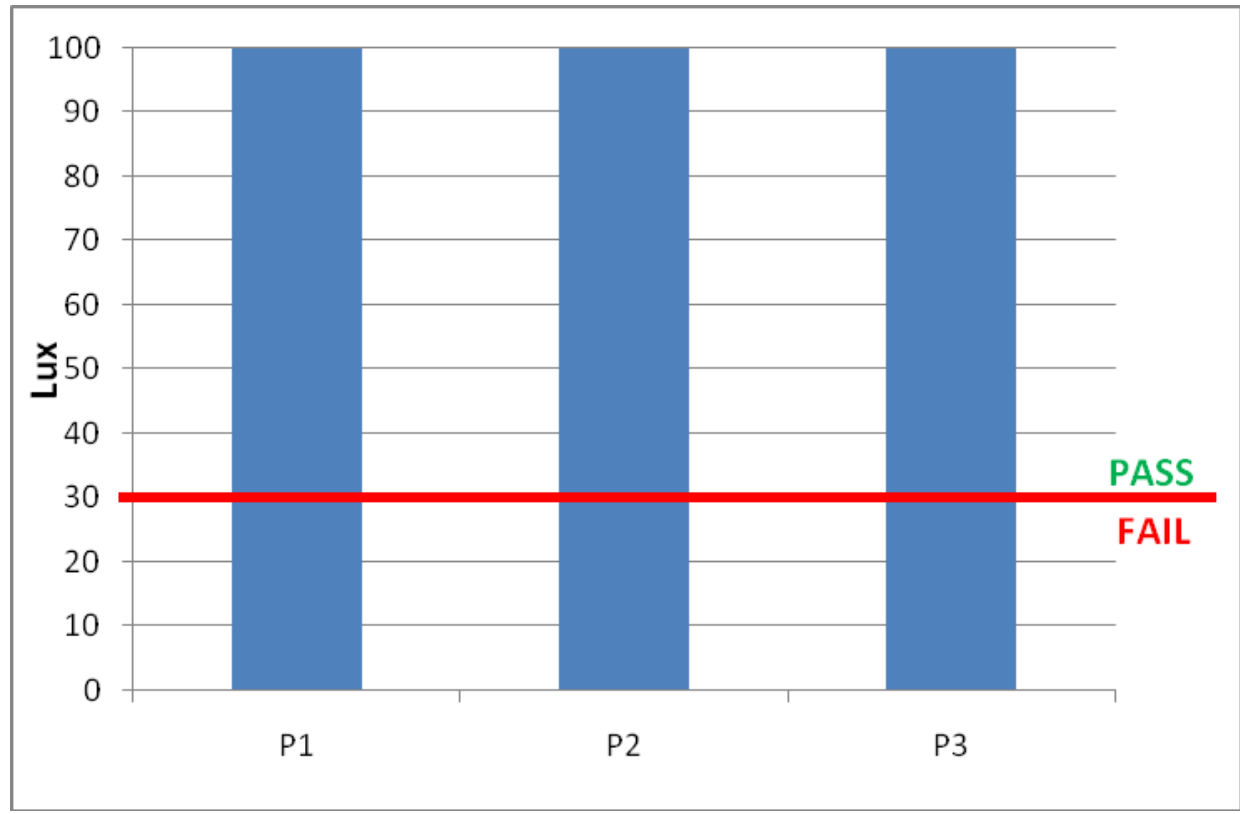

Application of the Tool:

\begin{tabular}{|l|c|}
\hline \multicolumn{2}{|c|}{ NZBC G7 Compliance Assessment Tool } \\
\hline \multicolumn{2}{|l|}{ Variable: } \\
\hline Opposite Building Height & 4 \\
\hline Street Width & 15 \\
\hline Glazed Area & 36 \\
\hline Orientation & North \\
\hline Location & Wellington \\
\hline Reflectance of Opposite Building & 40 \\
\hline Glazing Transmittance & 87 \\
\hline Vertical Location of lowest apartment & 4 \\
\hline \multicolumn{2}{|c|}{} \\
\hline Are simulations required to prove compliance: \\
\hline
\end{tabular}

Comparison of Results:

\begin{tabular}{|c|c|c|c|}
\hline Measurements & Linear Model & Model 2A & Model 2B \\
\hline Pass & 101 & 96 & 170 \\
\hline
\end{tabular}




\section{Apartment 77}

Measurement Results:

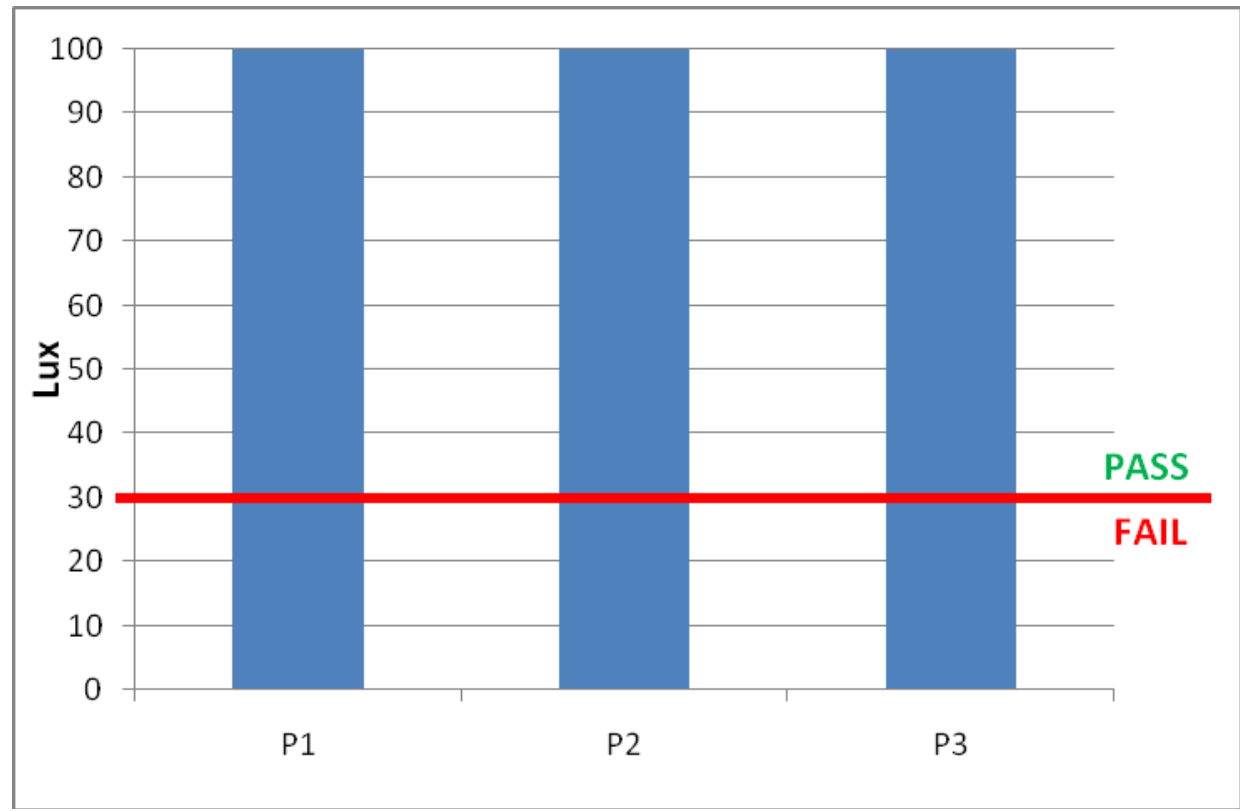

Application of the Tool:

\begin{tabular}{|l|c|}
\hline \multicolumn{2}{|c|}{ NZBC G7 Compliance Assessment Tool } \\
\hline \multicolumn{2}{|l|}{ Variable: } \\
\hline Opposite Building Height & 32 \\
\hline Street Width & 68 \\
\hline Glazed Area & 50 \\
\hline Orientation & North \\
\hline Location & Wellington \\
\hline Reflectance of Opposite Building & 33.3 \\
\hline Glazing Transmittance & 87 \\
\hline Vertical Location of lowest apartment & 5 \\
\hline \multicolumn{2}{|c|}{} \\
\hline Are simulations required to prove compliance: \\
\hline
\end{tabular}

Comparison of Results:

\begin{tabular}{|c|c|c|c|}
\hline Measurements & Linear Model & Model 2A & Model 2B \\
\hline Pass & 207 & 188 & 187 \\
\hline
\end{tabular}




\section{Apartment 78}

Measurement Results:

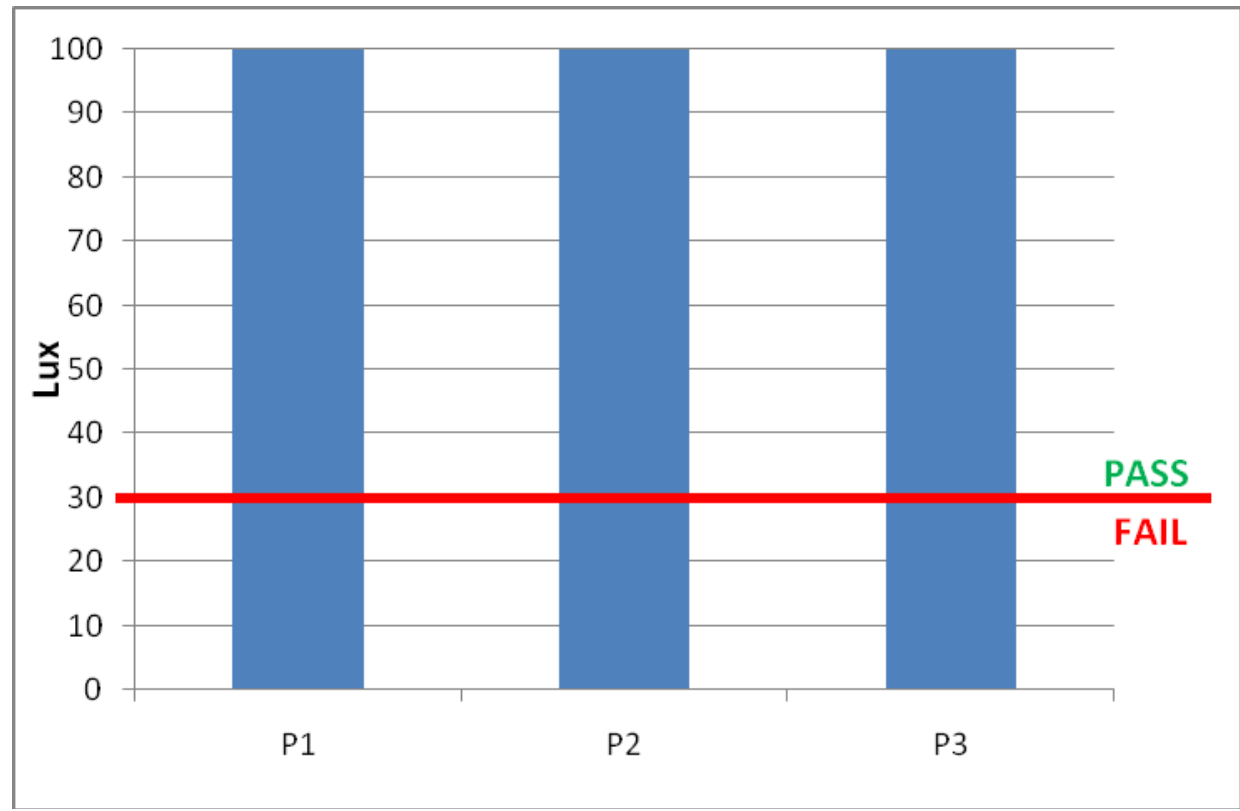

Application of the Tool:

\begin{tabular}{|l|c|}
\hline \multicolumn{2}{|c|}{ NZBC G7 Compliance Assessment Tool } \\
\hline \multicolumn{2}{|l|}{ Variable: } \\
\hline Opposite Building Height & 15 \\
\hline Street Width & 33 \\
\hline Glazed Area & 26 \\
\hline Orientation & South \\
\hline Location & Wellington \\
\hline Reflectance of Opposite Building & 13.5 \\
\hline Glazing Transmittance & 87 \\
\hline Vertical Location of lowest apartment & 7 \\
\hline \multicolumn{2}{|c|}{} \\
\hline Are simulations required to prove compliance: \\
\hline
\end{tabular}

Comparison of Results:

\begin{tabular}{|c|c|c|c|}
\hline Measurements & Linear Model & Model 2A & Model 2B \\
\hline Pass & 127 & 134 & 128 \\
\hline
\end{tabular}




\section{Apartment 79}

Measurement Results:

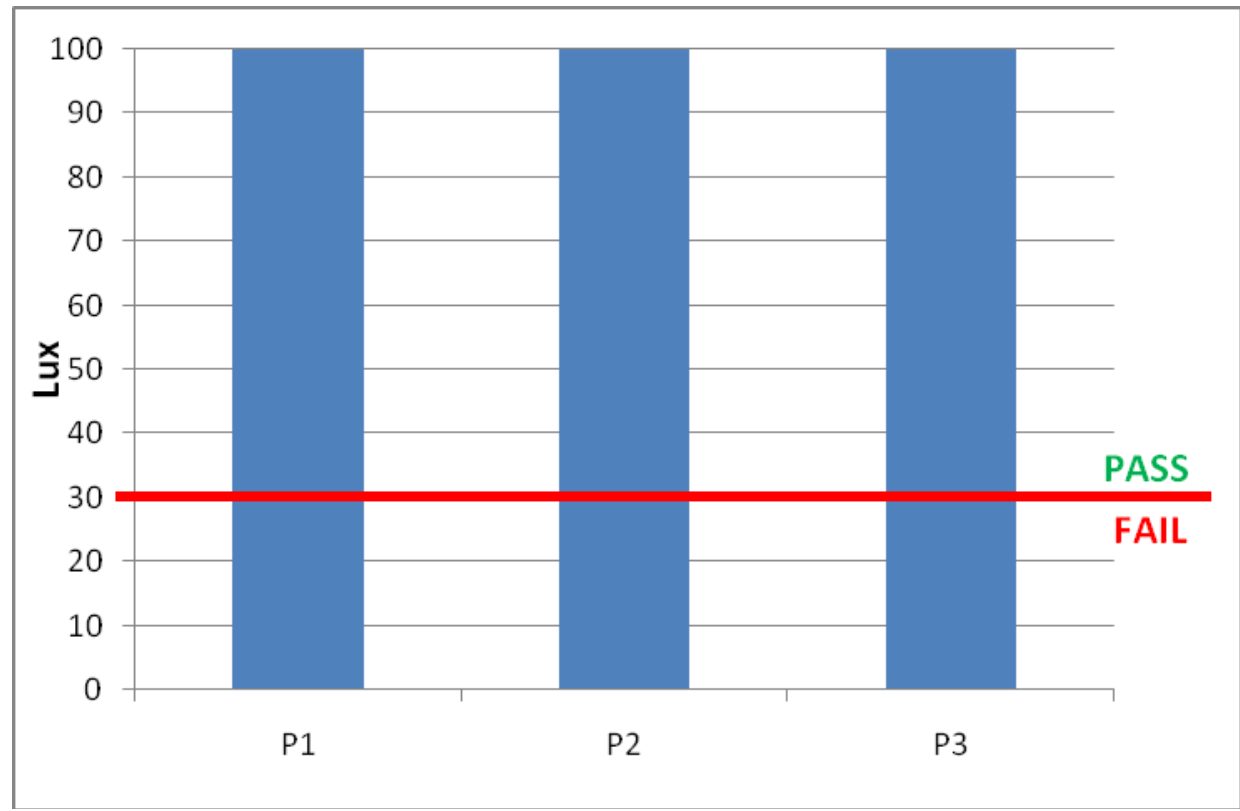

Application of the Tool:

\begin{tabular}{|l|c|}
\hline \multicolumn{2}{|c|}{ NZBC G7 Compliance Assessment Tool } \\
\hline \multicolumn{2}{|l|}{ Variable: } \\
\hline Opposite Building Height & 28 \\
\hline Street Width & 24 \\
\hline Glazed Area & 45 \\
\hline Orientation & South \\
\hline Location & Wellington \\
\hline Reflectance of Opposite Building & 10 \\
\hline Glazing Transmittance & 87 \\
\hline Vertical Location of lowest apartment & 16 \\
\hline \multicolumn{2}{|c|}{} \\
\hline Are simulations required to prove compliance: \\
\hline
\end{tabular}

Comparison of Results:

\begin{tabular}{|c|c|c|c|}
\hline Measurements & Linear Model & Model 2A & Model 2B \\
\hline Pass & 165 & 752 & 507 \\
\hline
\end{tabular}




\section{Apartment 80}

Measurement Results:

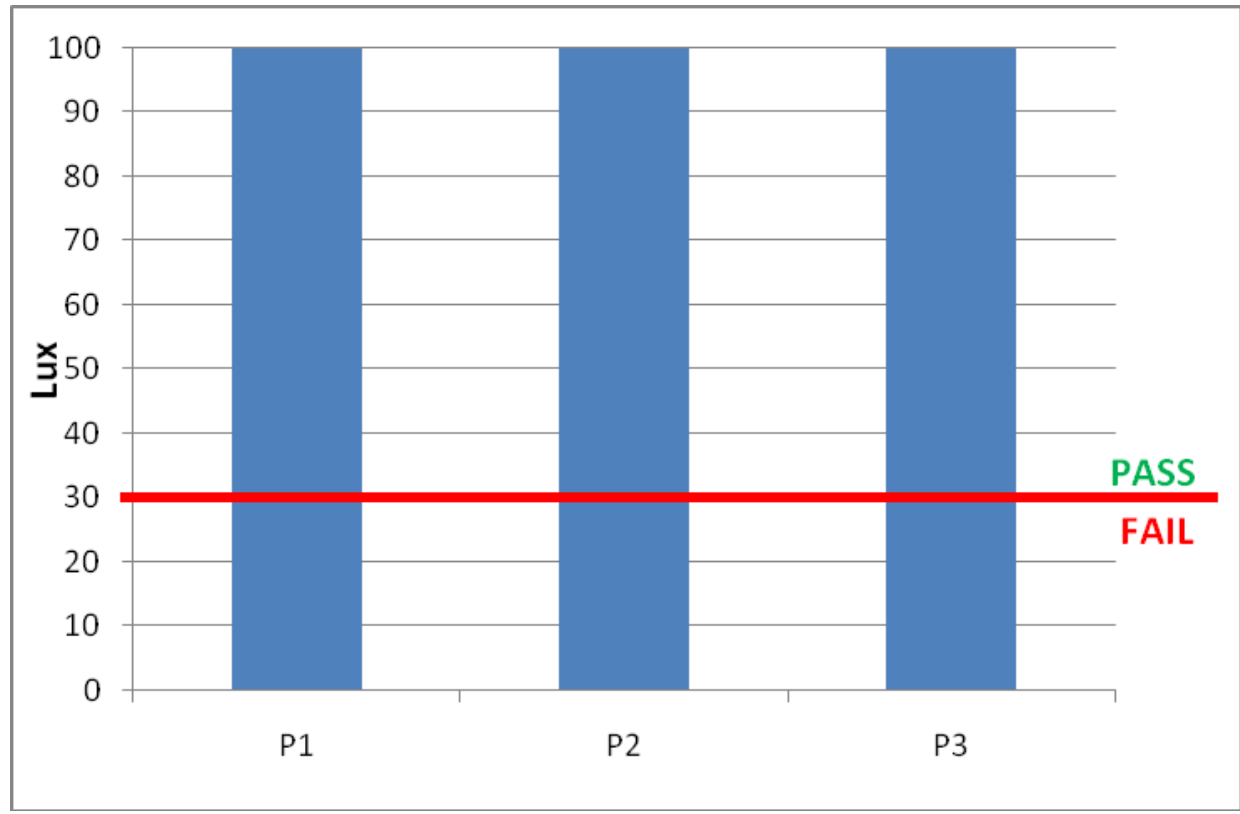

Application of the Tool:

\begin{tabular}{|l|c|}
\hline \multicolumn{2}{|c|}{ NZBC G7 Compliance Assessment Tool } \\
\hline \multicolumn{2}{|l|}{ Variable: } \\
\hline Opposite Building Height & 70 \\
\hline Street Width & 31 \\
\hline Glazed Area & 63 \\
\hline Orientation & East \\
\hline Location & Wellington \\
\hline Reflectance of Opposite Building & 12 \\
\hline Glazing Transmittance & 87 \\
\hline Vertical Location of lowest apartment & 1 \\
\hline \multicolumn{2}{|l}{} \\
\hline Are simulations required to prove compliance: \\
\hline
\end{tabular}

Comparison of Results:

\begin{tabular}{|c|c|c|c|}
\hline Measurements & Linear Model & Model 2A & Model 2B \\
\hline Pass & 117 & 125 & 43 \\
\hline
\end{tabular}




\section{Apartment 81}

Measurement Results:

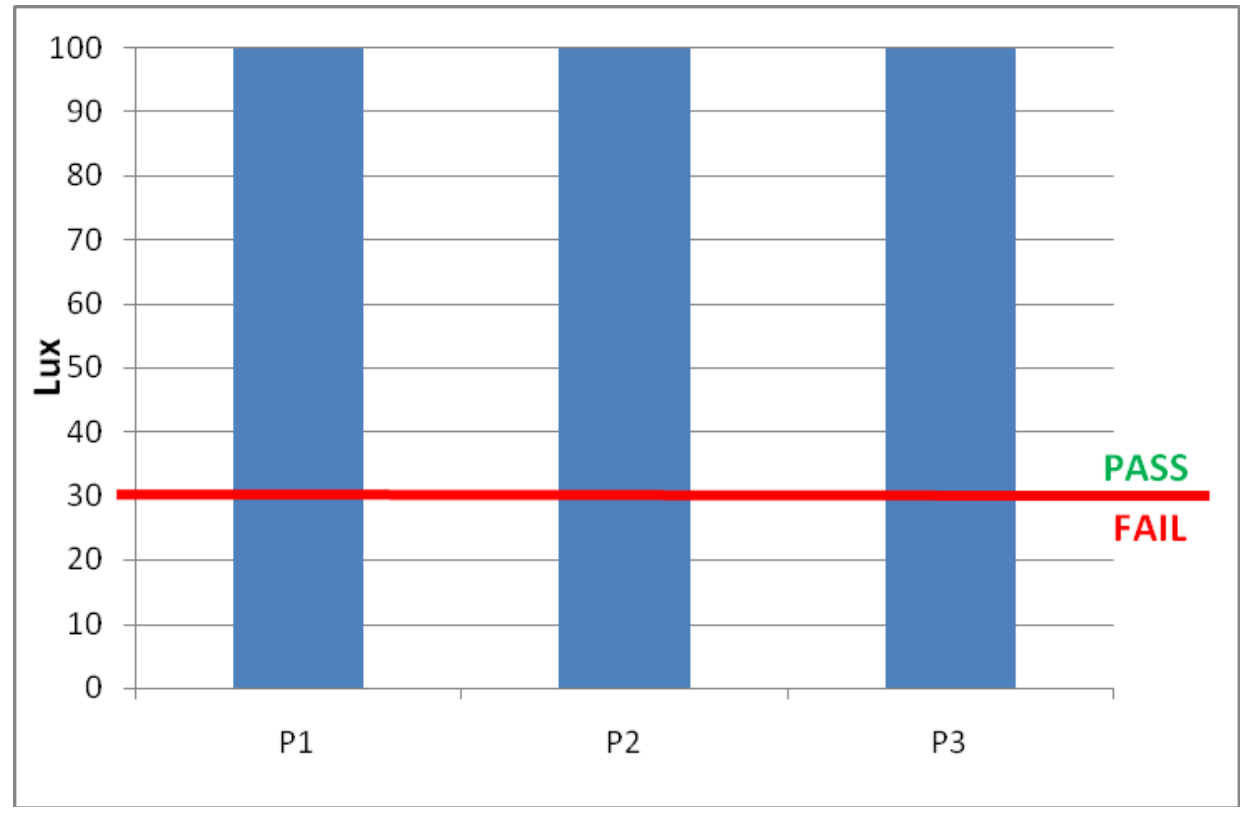

Application of the Tool:

\begin{tabular}{|l|c|}
\hline \multicolumn{2}{|c|}{ NZBC G7 Compliance Assessment Tool } \\
\hline \multicolumn{2}{|l|}{ Variable: } \\
\hline Opposite Building Height & 0 \\
\hline Street Width & 0 \\
\hline Glazed Area & 66 \\
\hline Orientation & North \\
\hline Location & Wellington \\
\hline Reflectance of Opposite Building & 0 \\
\hline Glazing Transmittance & 87 \\
\hline Vertical Location of lowest apartment & 16 \\
\hline \multicolumn{2}{|c|}{} \\
\hline Are simulations required to prove compliance: \\
\hline
\end{tabular}

Comparison of Results:

\begin{tabular}{|c|c|c|c|}
\hline Measurements & Linear Model & Model 2A & Model 2B \\
\hline Pass & 153 & 288 & 3756 \\
\hline
\end{tabular}




\section{Apartment 82}

Measurement Results:

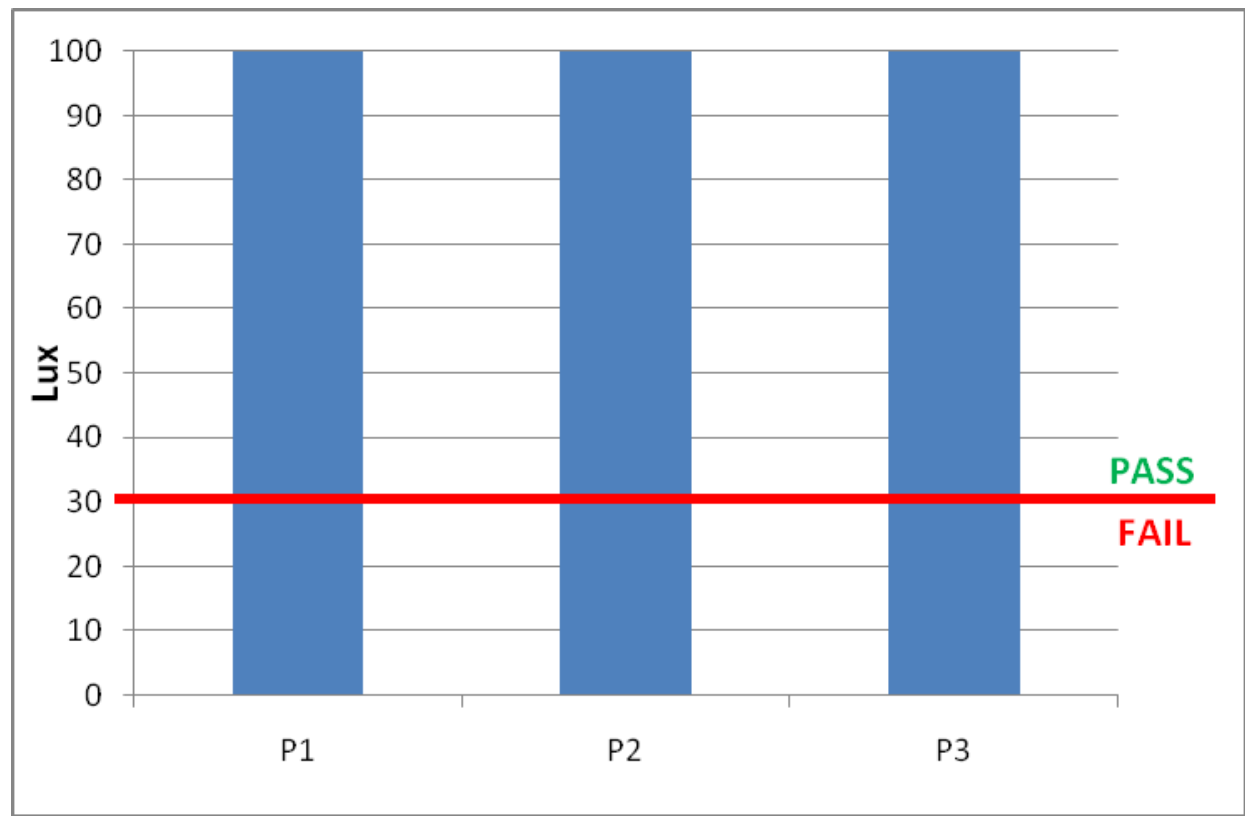

Application of the Tool:

\begin{tabular}{|l|c|}
\hline \multicolumn{2}{|c|}{ NZBC G7 Compliance Assessment Tool } \\
\hline \multicolumn{2}{|l|}{ Variable: } \\
\hline Opposite Building Height & 20 \\
\hline Street Width & 5 \\
\hline Glazed Area & 50 \\
\hline Orientation & North \\
\hline Location & Wellington \\
\hline Reflectance of Opposite Building & 12 \\
\hline Glazing Transmittance & 87 \\
\hline Vertical Location of lowest apartment & 11 \\
\hline \multicolumn{2}{|c}{} \\
\hline Are simulations required to prove compliance: \\
\hline
\end{tabular}

Comparison of Results:

\begin{tabular}{|c|c|c|c|}
\hline Measurements & Linear Model & Model 2A & Model 2B \\
\hline Pass & 115 & 274 & 346 \\
\hline
\end{tabular}




\section{Apartment 83}

Measurement Results:

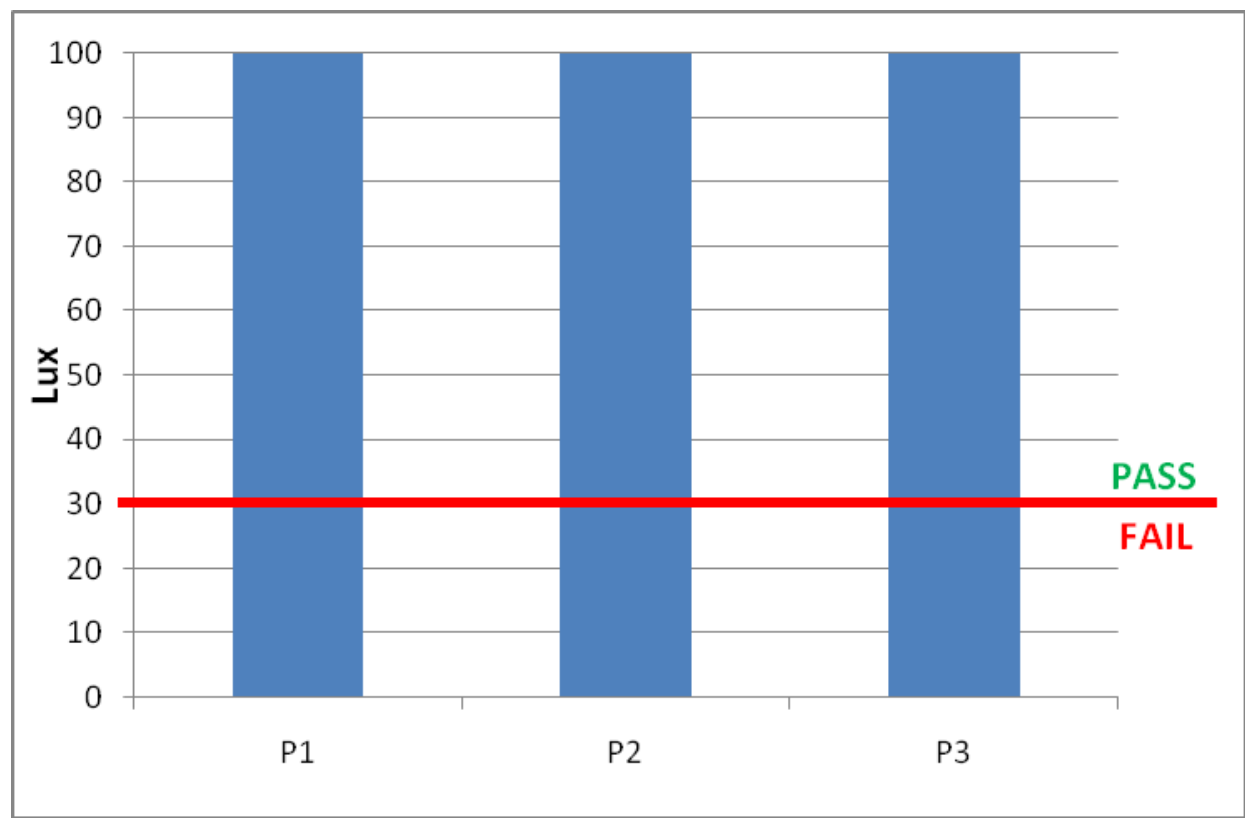

Application of the Tool:

\begin{tabular}{|l|c|}
\hline \multicolumn{2}{|c|}{ NZBC G7 Compliance Assessment Tool } \\
\hline \multicolumn{2}{|l|}{ Variable: } \\
\hline Opposite Building Height & 29 \\
\hline Street Width & 30 \\
\hline Glazed Area & 54 \\
\hline Orientation & South \\
\hline Location & Wellington \\
\hline Reflectance of Opposite Building & 20 \\
\hline Glazing Transmittance & 87 \\
\hline Vertical Location of lowest apartment & 6 \\
\hline \multicolumn{2}{|c}{} \\
\hline Are simulations required to prove compliance: \\
\hline
\end{tabular}

Comparison of Results:

\begin{tabular}{|c|c|c|c|}
\hline Measurements & Linear Model & Model 2A & Model 2B \\
\hline Pass & 148 & 149 & 160 \\
\hline
\end{tabular}




\section{Apartment 84}

Measurement Results:

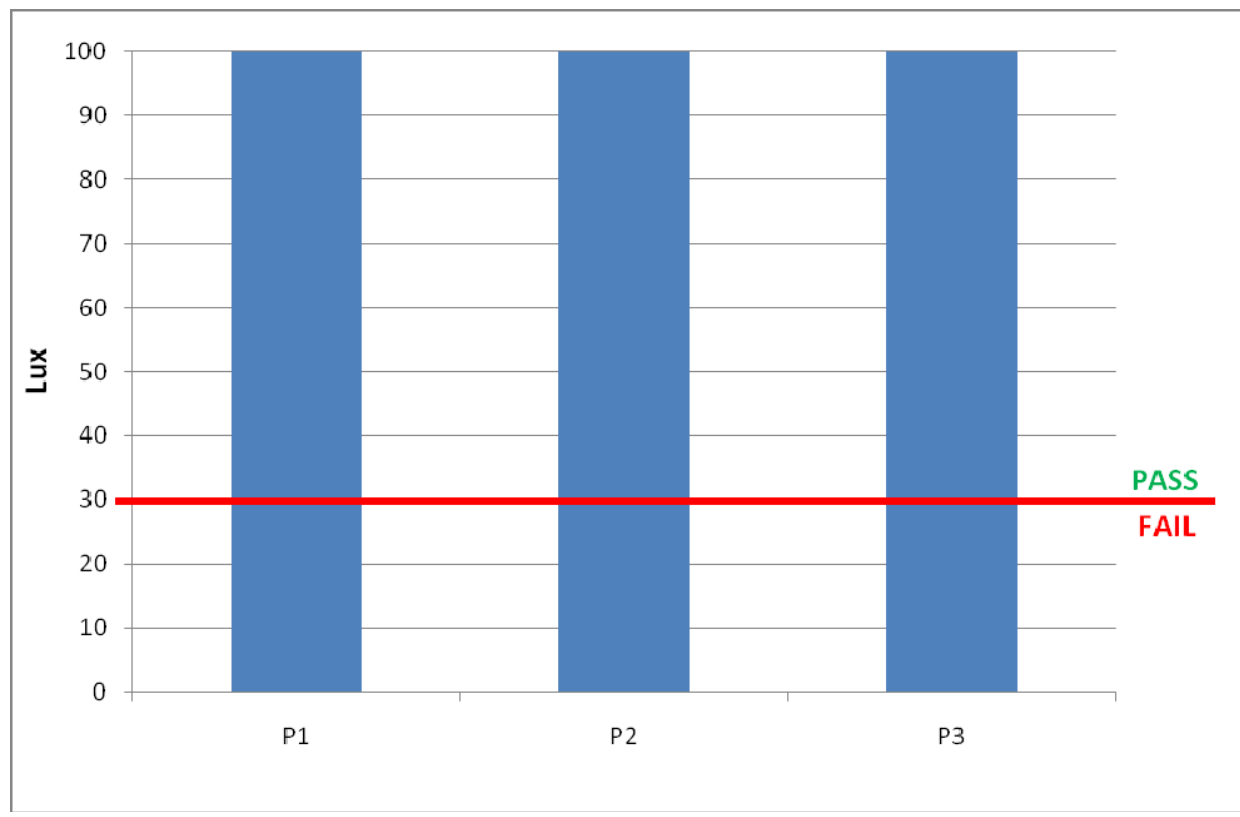

Application of the Tool:

\begin{tabular}{|l|c|}
\hline \multicolumn{2}{|c|}{ NZBC G7 Compliance Assessment Tool } \\
\hline \multicolumn{2}{|l|}{} \\
\hline Variable: & 11.6 \\
\hline Opposite Building Height & 9 \\
\hline Street Width & 45 \\
\hline Glazed Area & North \\
\hline Orientation & Wellington \\
\hline Location & 30 \\
\hline Reflectance of Opposite Building & 87 \\
\hline Glazing Transmittance & 8 \\
\hline Vertical Location of lowest apartment \\
\hline \multicolumn{2}{|c|}{} \\
\hline Are simulations required to prove compliance: \\
\hline
\end{tabular}

Comparison of Results:

\begin{tabular}{|c|c|c|c|}
\hline Measurements & Linear Model & Model 2A & Model 2B \\
\hline Pass & 112 & 154 & 301 \\
\hline
\end{tabular}




\section{Apartment 85}

Measurement Results:

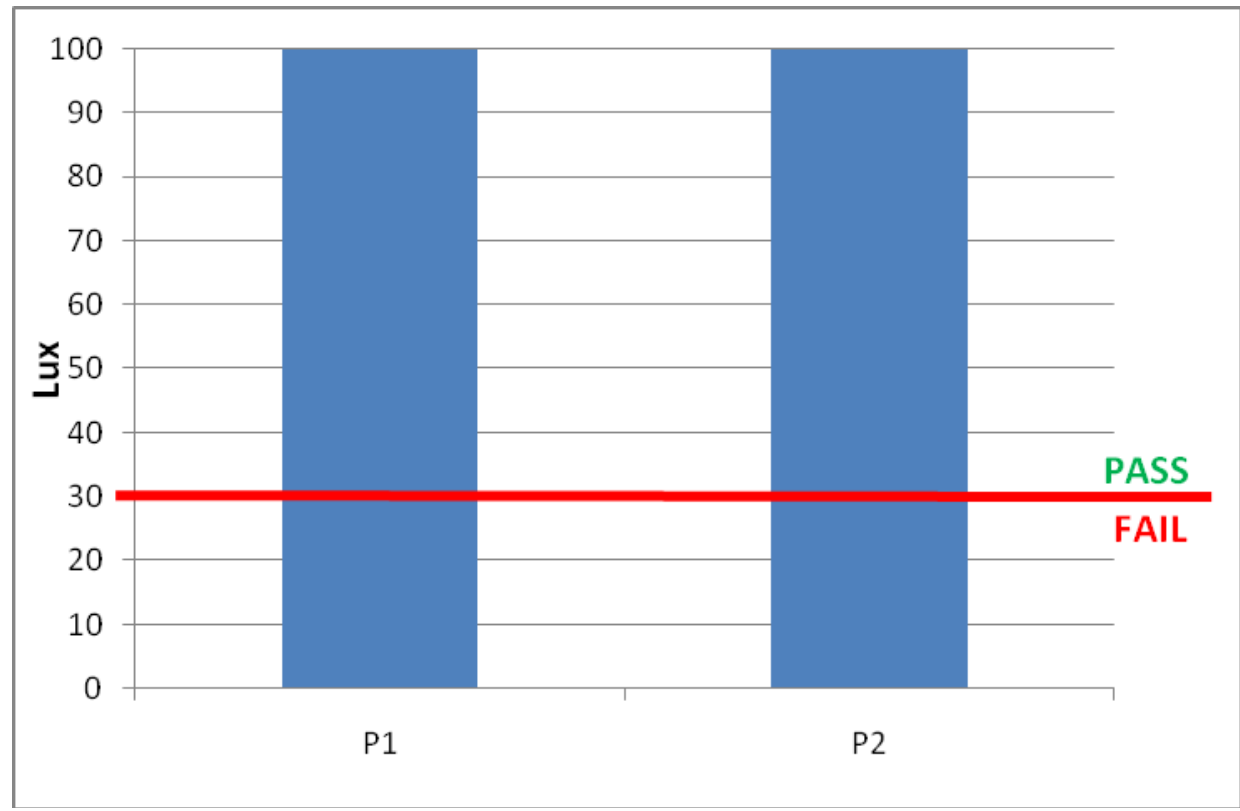

Application of the Tool:

\begin{tabular}{|l|c|}
\hline \multicolumn{2}{|c|}{ NZBC G7 Compliance Assessment Tool } \\
\hline \multicolumn{2}{|l|}{ Variable: } \\
\hline Opposite Building Height & 43 \\
\hline Street Width & 94.5 \\
\hline Glazed Area & 14 \\
\hline Orientation & North \\
\hline Location & Wellington \\
\hline Reflectance of Opposite Building & 12 \\
\hline Glazing Transmittance & 87 \\
\hline Vertical Location of lowest apartment & 8 \\
\hline \multicolumn{2}{|c|}{} \\
\hline Are simulations required to prove compliance: \\
\hline
\end{tabular}

Comparison of Results:

\begin{tabular}{|c|c|c|c|}
\hline Measurements & Linear Model & Model 2A & Model 2B \\
\hline Pass & 212 & 229 & 163 \\
\hline
\end{tabular}




\section{Apartment 86}

Measurement Results:

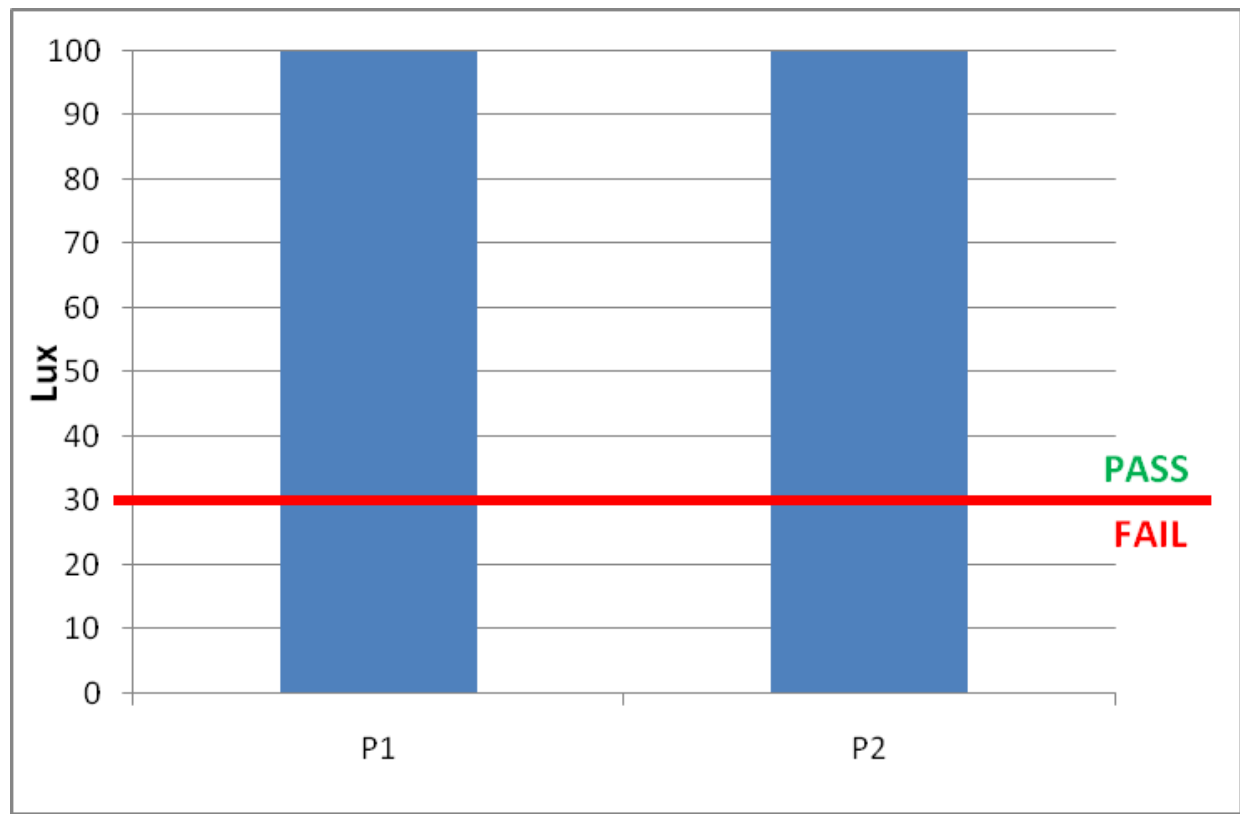

Application of the Tool:

\begin{tabular}{|l|c|}
\hline \multicolumn{2}{|c|}{ NZBC G7 Compliance Assessment Tool } \\
\hline \multicolumn{2}{|l|}{ Variable: } \\
\hline Opposite Building Height & 4 \\
\hline Street Width & 15 \\
\hline Glazed Area & 44 \\
\hline Orientation & North \\
\hline Location & Wellington \\
\hline Reflectance of Opposite Building & 40 \\
\hline Glazing Transmittance & 87 \\
\hline Vertical Location of lowest apartment & 3 \\
\hline \multicolumn{2}{|c|}{} \\
\hline Are simulations required to prove compliance: \\
\hline
\end{tabular}

Comparison of Results:

\begin{tabular}{|c|c|c|c|}
\hline Measurements & Linear Model & Model 2A & Model 2B \\
\hline Pass & 107 & 106 & 197 \\
\hline
\end{tabular}




\section{Apartment 87}

Measurement Results:

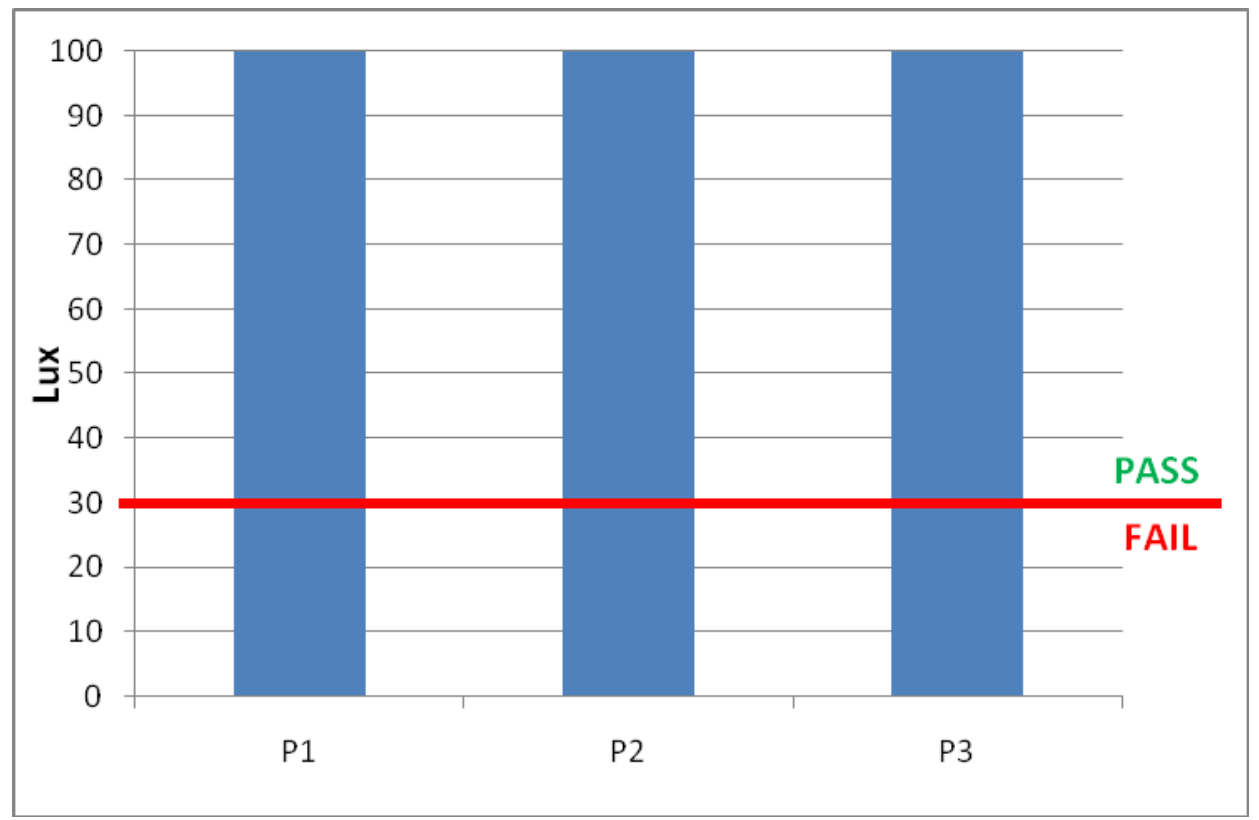

Application of the Tool:

\begin{tabular}{|l|c|}
\hline \multicolumn{2}{|c|}{ NZBC G7 Compliance Assessment Tool } \\
\hline \multicolumn{2}{|l|}{ Variable: } \\
\hline Opposite Building Height & 29 \\
\hline Street Width & 30 \\
\hline Glazed Area & 36 \\
\hline Orientation & South \\
\hline Location & Wellington \\
\hline Reflectance of Opposite Building & 20 \\
\hline Glazing Transmittance & 87 \\
\hline Vertical Location of lowest apartment & 6 \\
\hline \multicolumn{2}{|c|}{} \\
\hline Are simulations required to prove compliance: \\
\hline
\end{tabular}

Comparison of Results:

\begin{tabular}{|c|c|c|c|}
\hline Measurements & Linear Model & Model 2A & Model 2B \\
\hline Pass & 125 & 106 & 95 \\
\hline
\end{tabular}




\section{Apartment 88}

Measurement Results:

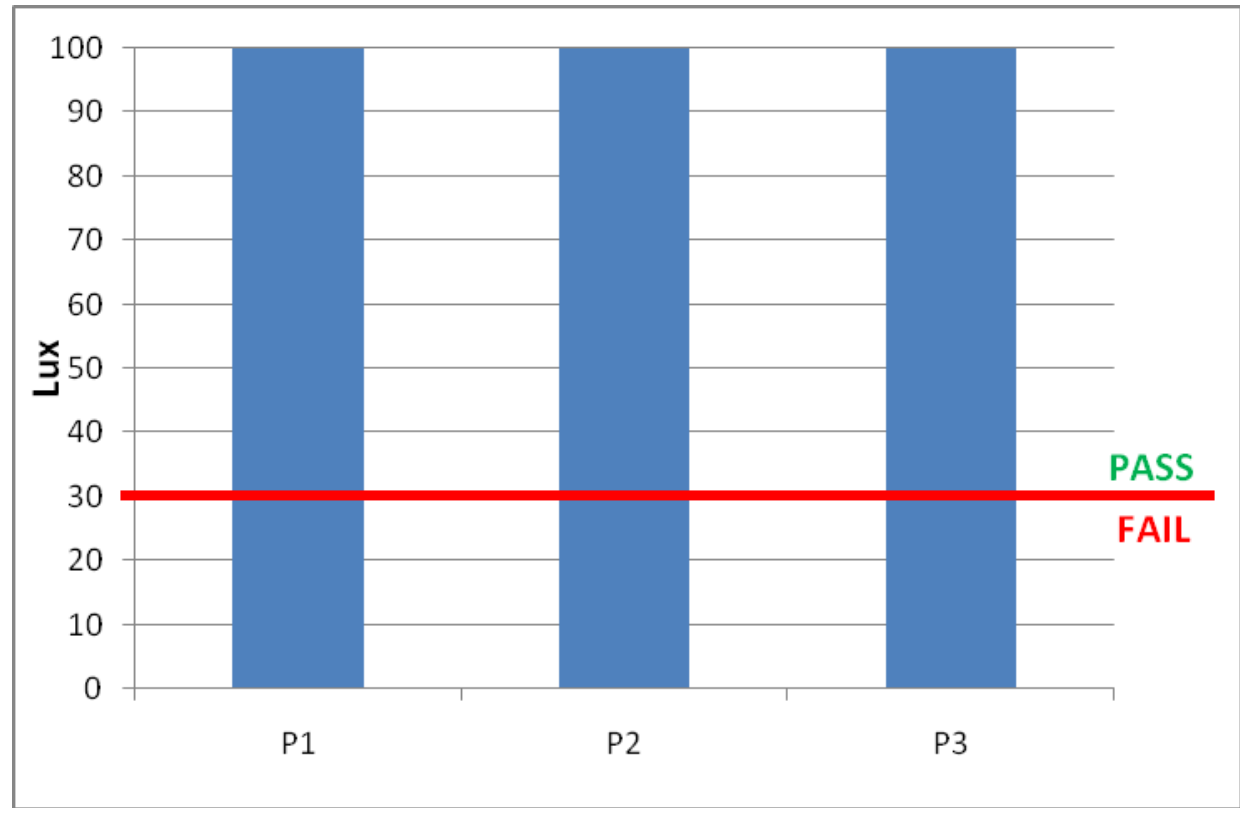

Application of the Tool:

\begin{tabular}{|l|c|}
\hline \multicolumn{2}{|c|}{ NZBC G7 Compliance Assessment Tool } \\
\hline \multicolumn{2}{|l|}{} \\
\hline Variable: & 30 \\
\hline Opposite Building Height & 29 \\
\hline Street Width & 32 \\
\hline Glazed Area & North \\
\hline Orientation & Auckland \\
\hline Location & 21 \\
\hline Reflectance of Opposite Building & 87 \\
\hline Glazing Transmittance & 16 \\
\hline Vertical Location of lowest apartment \\
\hline \multicolumn{2}{|c|}{} \\
\hline Are simulations required to prove compliance: \\
\hline
\end{tabular}

Comparison of Results:

\begin{tabular}{|c|c|c|c|}
\hline Measurements & Linear Model & Model 2A & Model 2B \\
\hline Pass & 160 & 735 & 324 \\
\hline
\end{tabular}




\section{Apartment 89}

Measurement Results:

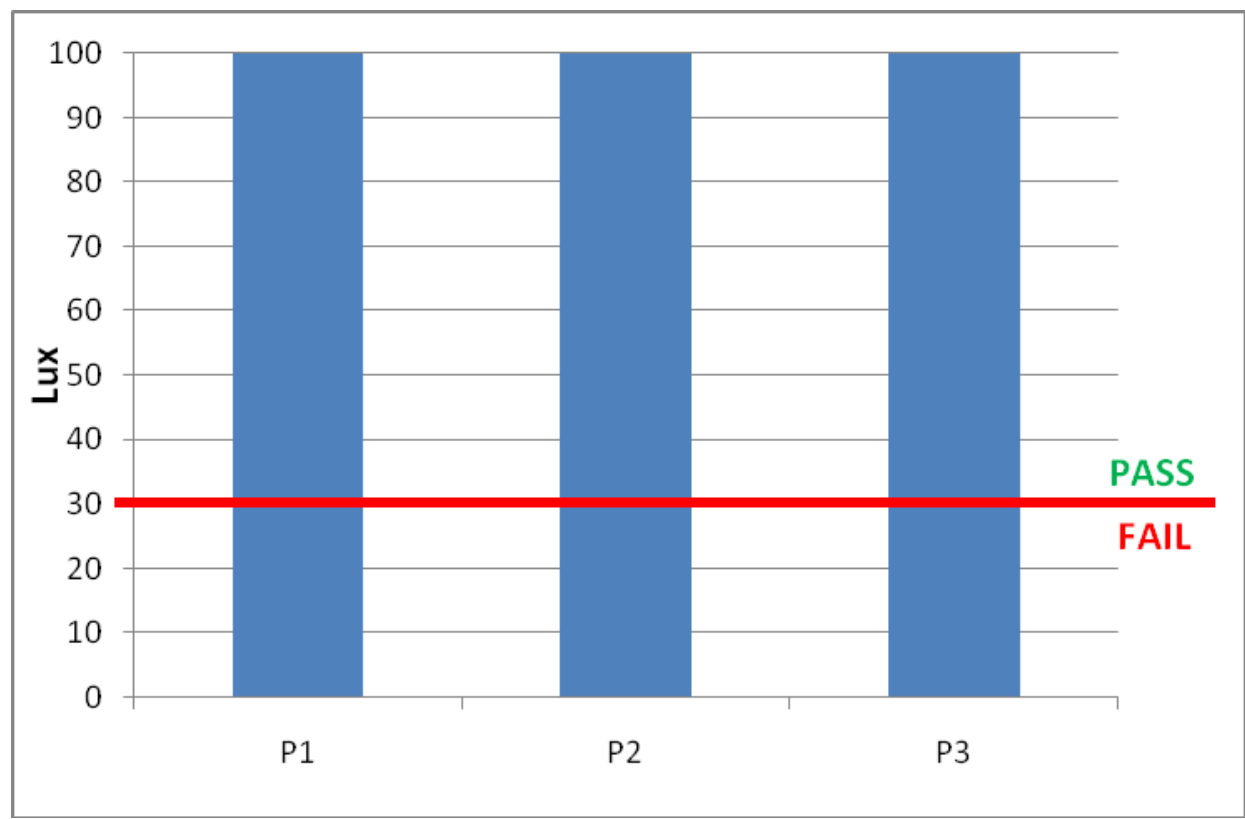

Application of the Tool:

\begin{tabular}{|l|c|}
\hline \multicolumn{2}{|c|}{ NZBC G7 Compliance Assessment Tool } \\
\hline \multicolumn{2}{|l|}{ Variable: } \\
\hline Opposite Building Height & 13.3 \\
\hline Street Width & 94.5 \\
\hline Glazed Area & 31 \\
\hline Orientation & North \\
\hline Location & Wellington \\
\hline Reflectance of Opposite Building & 12 \\
\hline Glazing Transmittance & 87 \\
\hline Vertical Location of lowest apartment & 9 \\
\hline \multicolumn{2}{|c|}{} \\
\hline Are simulations required to prove compliance: \\
\hline
\end{tabular}

Comparison of Results:

\begin{tabular}{|c|c|c|c|}
\hline Measurements & Linear Model & Model 2A & Model 2B \\
\hline Pass & 253 & 315 & 310 \\
\hline
\end{tabular}




\section{Apartment 90}

Measurement Results:

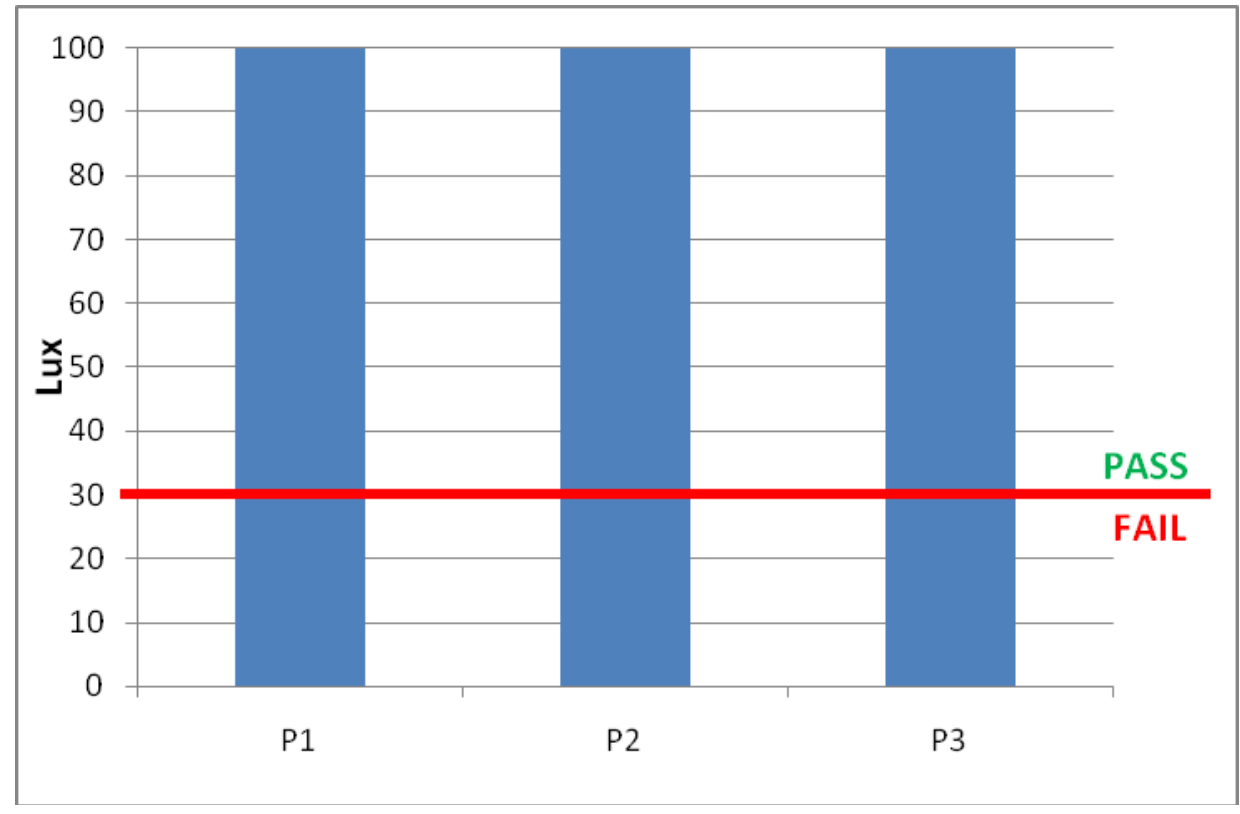

Application of the Tool:

\begin{tabular}{|l|c|}
\hline \multicolumn{2}{|c|}{ NZBC G7 Compliance Assessment Tool } \\
\hline \multicolumn{2}{|l|}{ Variable: } \\
\hline Opposite Building Height & 15 \\
\hline Street Width & 60 \\
\hline Glazed Area & 59 \\
\hline Orientation & West \\
\hline Location & Wellington \\
\hline Reflectance of Opposite Building & 30 \\
\hline Glazing Transmittance & 87 \\
\hline Vertical Location of lowest apartment & 3 \\
\hline \multicolumn{2}{|c|}{} \\
\hline Are simulations required to prove compliance: \\
\hline
\end{tabular}

Comparison of Results:

\begin{tabular}{|c|c|c|c|}
\hline Measurements & Linear Model & Model 2A & Model 2B \\
\hline Pass & 205 & 208 & 279 \\
\hline
\end{tabular}




\section{Apartment 91}

Measurement Results:

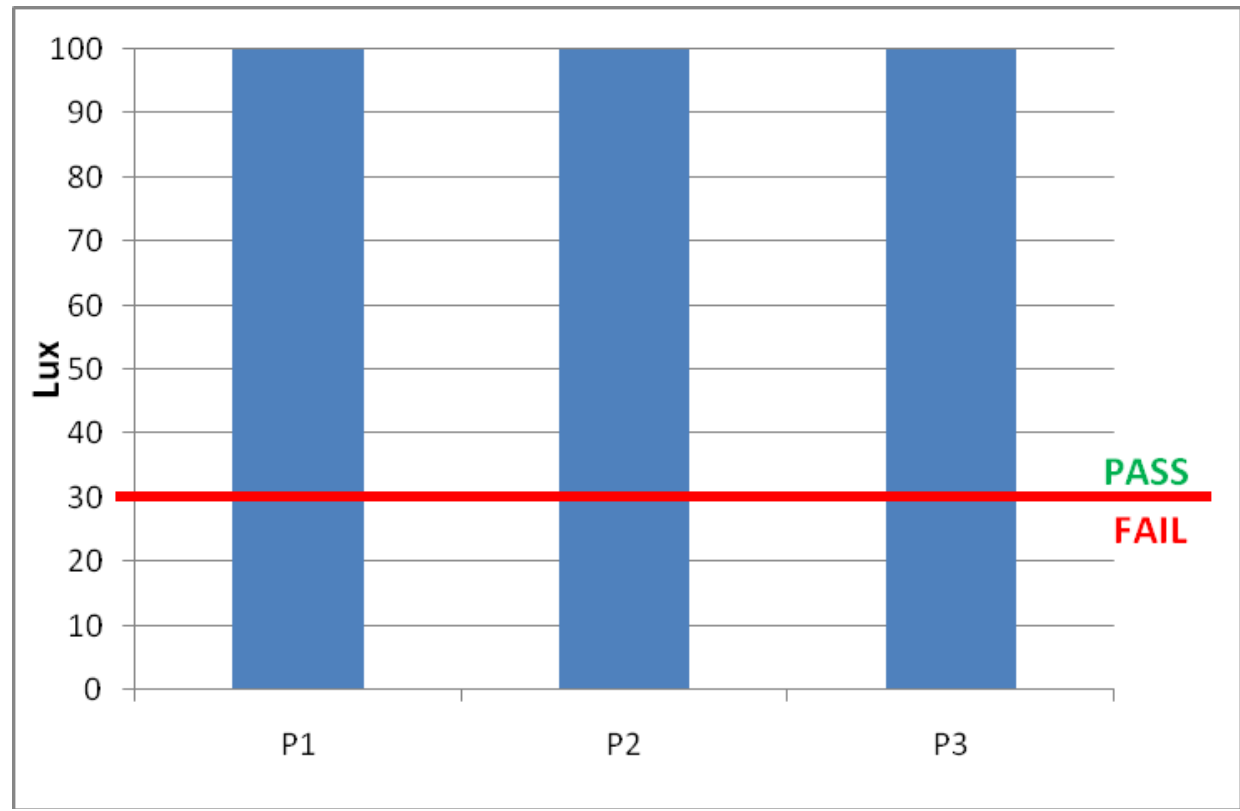

Application of the Tool:

\begin{tabular}{|l|c|}
\hline \multicolumn{2}{|c|}{ NZBC G7 Compliance Assessment Tool } \\
\hline \multicolumn{2}{|l|}{ Variable: } \\
\hline Opposite Building Height & 27 \\
\hline Street Width & 12 \\
\hline Glazed Area & 45 \\
\hline Orientation & East \\
\hline Location & Wellington \\
\hline Reflectance of Opposite Building & 24 \\
\hline Glazing Transmittance & 87 \\
\hline Vertical Location of lowest apartment & 12 \\
\hline \multicolumn{2}{|c|}{} \\
\hline Are simulations required to prove compliance: \\
\hline
\end{tabular}

Comparison of Results:

\begin{tabular}{|c|c|c|c|}
\hline Measurements & Linear Model & Model 2A & Model 2B \\
\hline Pass & 128 & 337 & 286 \\
\hline
\end{tabular}




\section{Apartment 92}

Measurement Results:

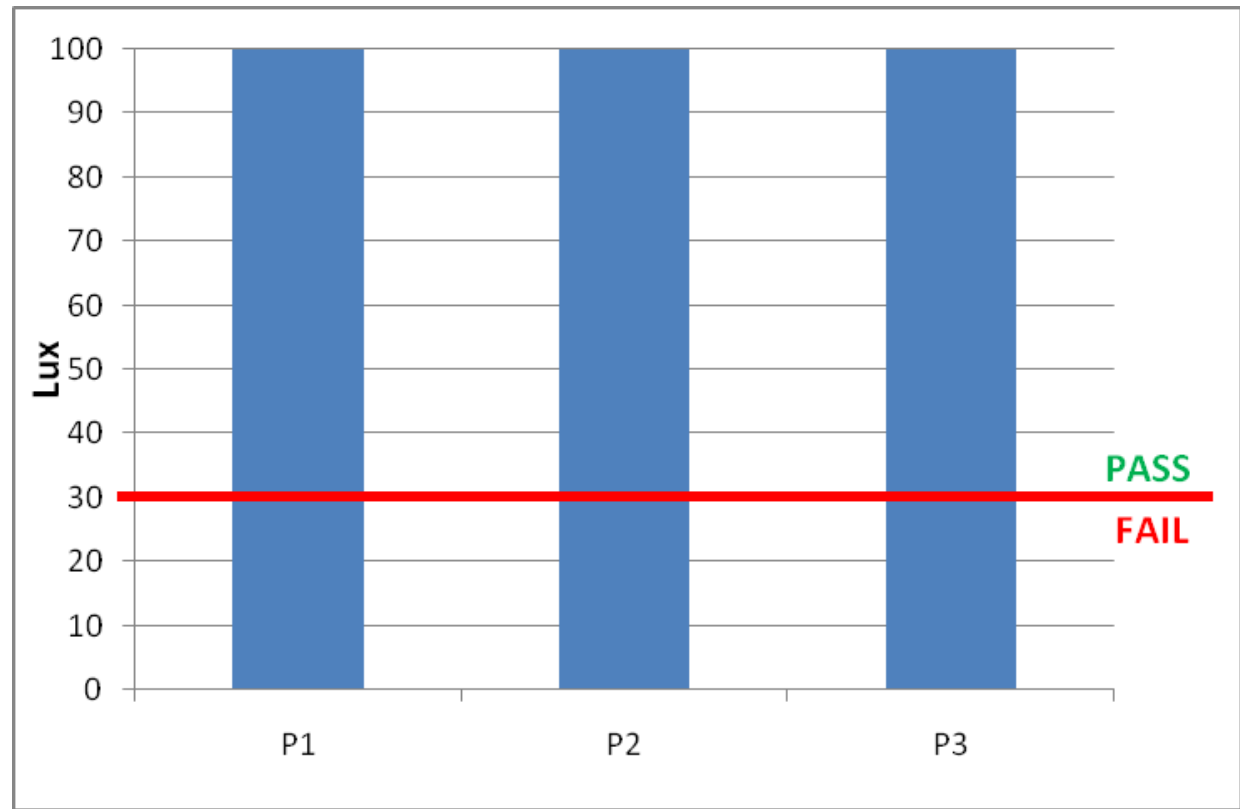

Application of the Tool:

\begin{tabular}{|l|c|}
\hline \multicolumn{2}{|c|}{ NZBC G7 Compliance Assessment Tool } \\
\hline \multicolumn{2}{|l|}{ Variable: } \\
\hline Opposite Building Height & 24.6 \\
\hline Street Width & 15 \\
\hline Glazed Area & 35 \\
\hline Orientation & South \\
\hline Location & Wellington \\
\hline Reflectance of Opposite Building & 33 \\
\hline Glazing Transmittance & 87 \\
\hline Vertical Location of lowest apartment & 8 \\
\hline \multicolumn{2}{|c|}{} \\
\hline Are simulations required to prove compliance: \\
\hline
\end{tabular}

Comparison of Results:

\begin{tabular}{|c|c|c|c|}
\hline Measurements & Linear Model & Model 2A & Model 2B \\
\hline Pass & 109 & 131 & 124 \\
\hline
\end{tabular}




\section{Apartment 93}

Measurement Results:

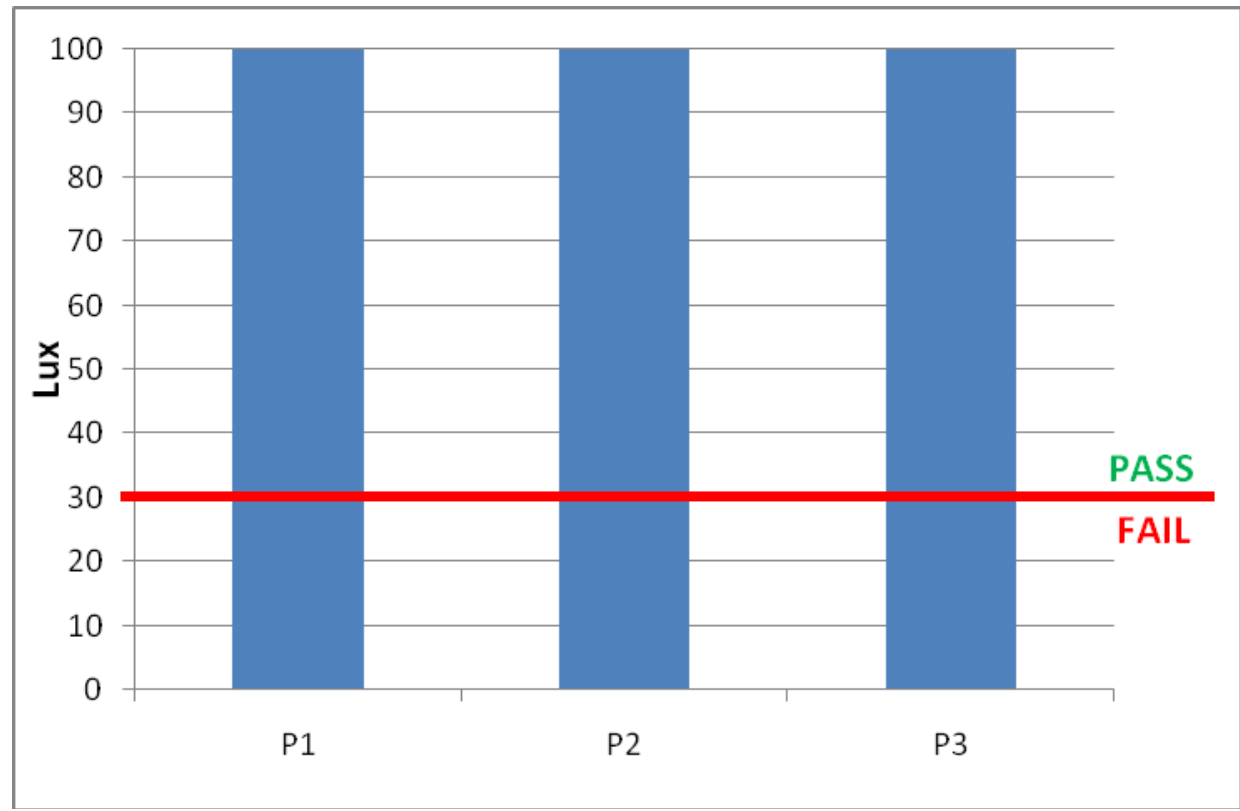

Application of the Tool:

\begin{tabular}{|l|c|}
\hline \multicolumn{2}{|c|}{ NZBC G7 Compliance Assessment Tool } \\
\hline \multicolumn{2}{|l|}{ Variable: } \\
\hline Opposite Building Height & 32 \\
\hline Street Width & 68 \\
\hline Glazed Area & 63 \\
\hline Orientation & North \\
\hline Location & Wellington \\
\hline Reflectance of Opposite Building & 33.3 \\
\hline Glazing Transmittance & 87 \\
\hline Vertical Location of lowest apartment & 5 \\
\hline \multicolumn{2}{|c|}{} \\
\hline Are simulations required to prove compliance: \\
\hline
\end{tabular}

Comparison of Results:

\begin{tabular}{|c|c|c|c|}
\hline Measurements & Linear Model & Model 2A & Model 2B \\
\hline Pass & 224 & 232 & 247 \\
\hline
\end{tabular}




\section{Apartment 94}

Measurement Results:

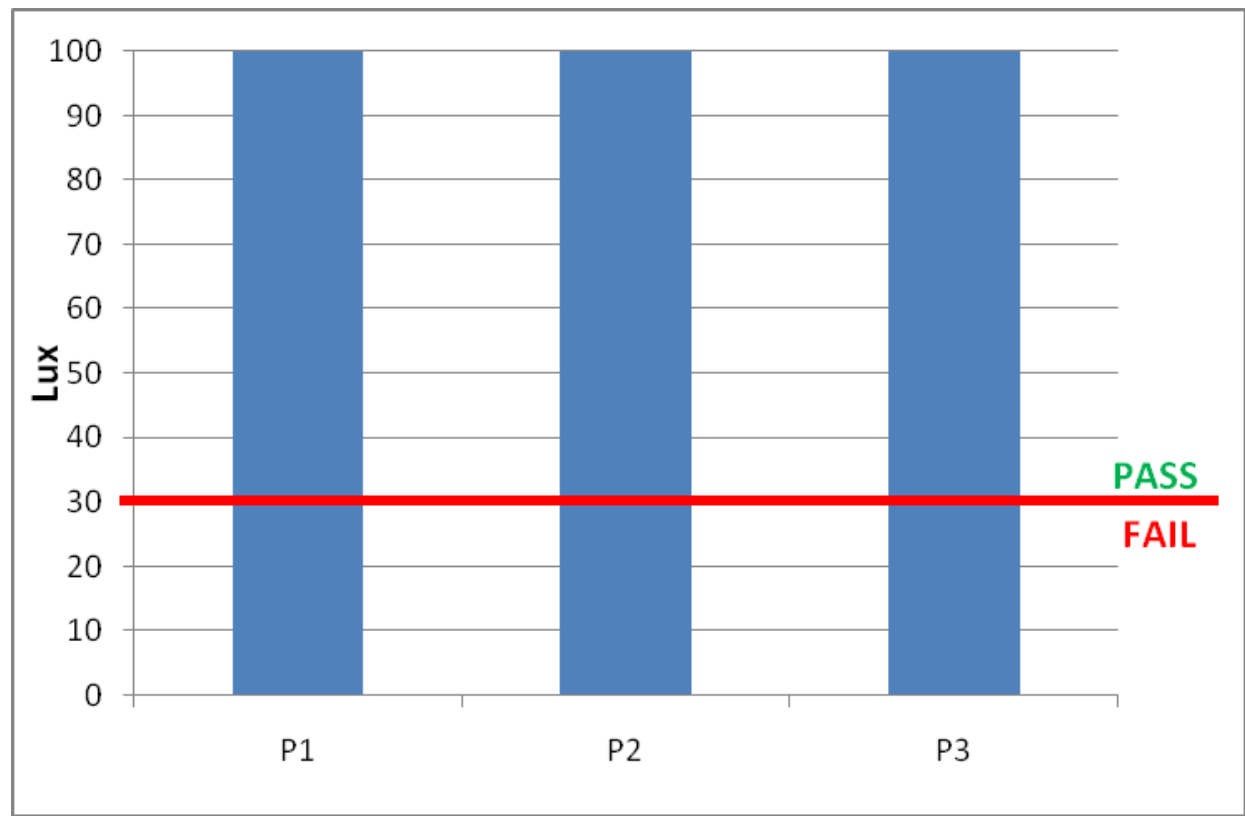

Application of the Tool:

\begin{tabular}{|l|c|}
\hline \multicolumn{2}{|c|}{ NZBC G7 Compliance Assessment Tool } \\
\hline \multicolumn{2}{|l|}{ Variable: } \\
\hline Opposite Building Height & 15 \\
\hline Street Width & 60 \\
\hline Glazed Area & 100 \\
\hline Orientation & West \\
\hline Location & Wellington \\
\hline Reflectance of Opposite Building & 30 \\
\hline Glazing Transmittance & 87 \\
\hline Vertical Location of lowest apartment & 10 \\
\hline \multicolumn{2}{|c}{} \\
\hline Are simulations required to prove compliance: \\
\hline
\end{tabular}

Comparison of Results:

\begin{tabular}{|c|c|c|c|}
\hline Measurements & Linear Model & Model 2A & Model 2B \\
\hline Pass & 287 & 683 & 3506 \\
\hline
\end{tabular}




\section{Apartment 95}

Measurement Results:

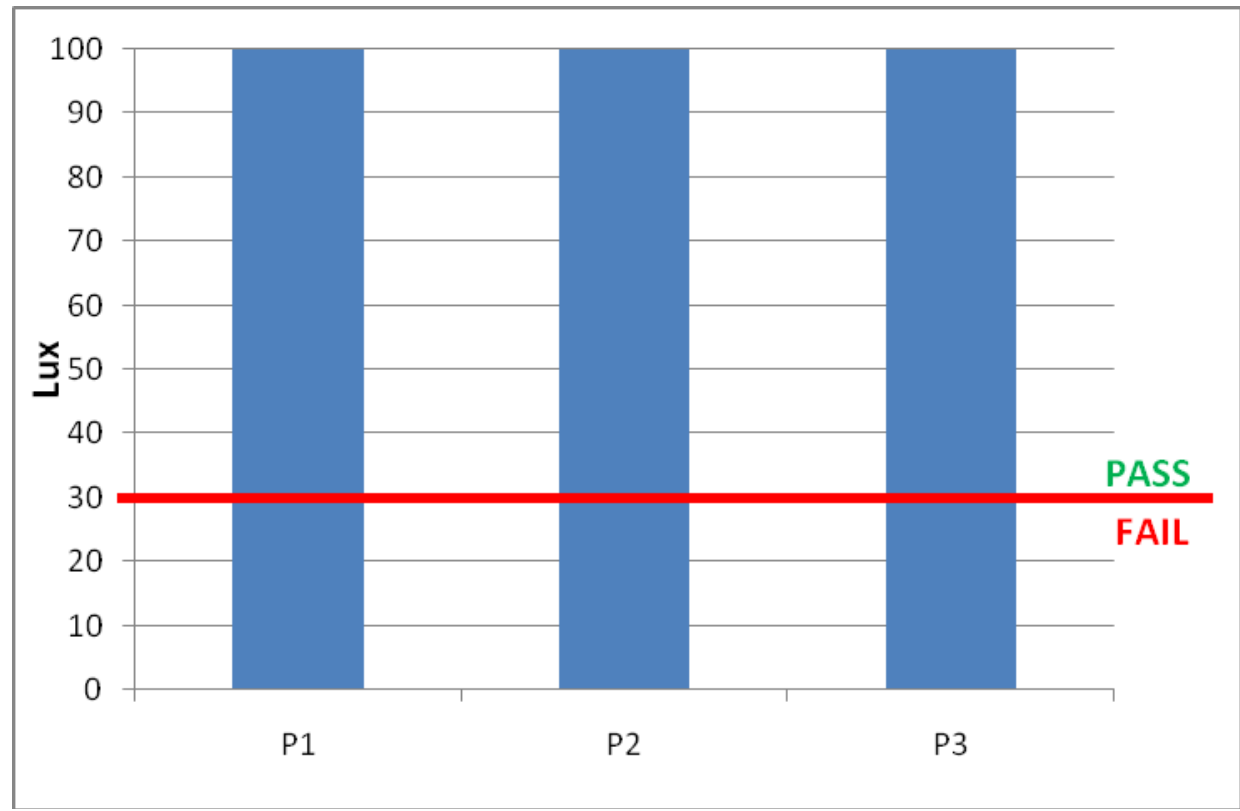

Application of the Tool:

\begin{tabular}{|l|c|}
\hline \multicolumn{2}{|c|}{ NZBC G7 Compliance Assessment Tool } \\
\hline \multicolumn{2}{|l|}{ Variable: } \\
\hline Opposite Building Height & 0 \\
\hline Street Width & 0 \\
\hline Glazed Area & 58 \\
\hline Orientation & West \\
\hline Location & Wellington \\
\hline Reflectance of Opposite Building & 0 \\
\hline Glazing Transmittance & 87 \\
\hline Vertical Location of lowest apartment & 9 \\
\hline \multicolumn{2}{|l}{} \\
\hline Are simulations required to prove compliance: \\
\hline
\end{tabular}

Comparison of Results:

\begin{tabular}{|c|c|c|c|}
\hline Measurements & Linear Model & Model 2A & Model 2B \\
\hline Pass & 116 & 242 & 777 \\
\hline
\end{tabular}




\section{Apartment 96}

Measurement Results:

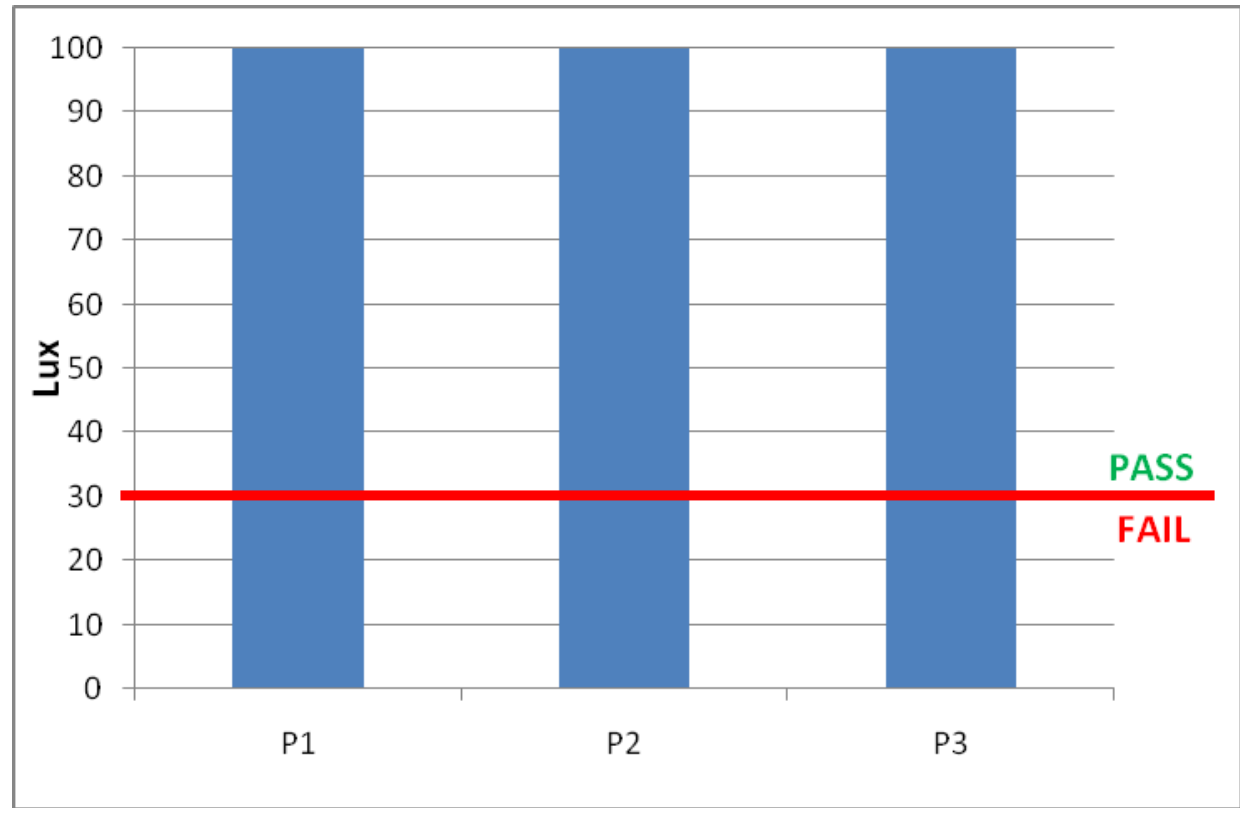

Application of the Tool:

\begin{tabular}{|l|c|}
\hline \multicolumn{2}{|c|}{ NZBC G7 Compliance Assessment Tool } \\
\hline \multicolumn{2}{|l|}{ Variable: } \\
\hline Opposite Building Height & 25 \\
\hline Street Width & 60 \\
\hline Glazed Area & 17.4 \\
\hline Orientation & West \\
\hline Location & Wellington \\
\hline Reflectance of Opposite Building & 13.5 \\
\hline Glazing Transmittance & 87 \\
\hline Vertical Location of lowest apartment & 7 \\
\hline \multicolumn{2}{|c|}{} \\
\hline Are simulations required to prove compliance: \\
\hline
\end{tabular}

Comparison of Results:

\begin{tabular}{|c|c|c|c|}
\hline Measurements & Linear Model & Model 2A & Model 2B \\
\hline Pass & 159 & 157 & 125 \\
\hline
\end{tabular}




\section{Apartment 97}

Measurement Results:

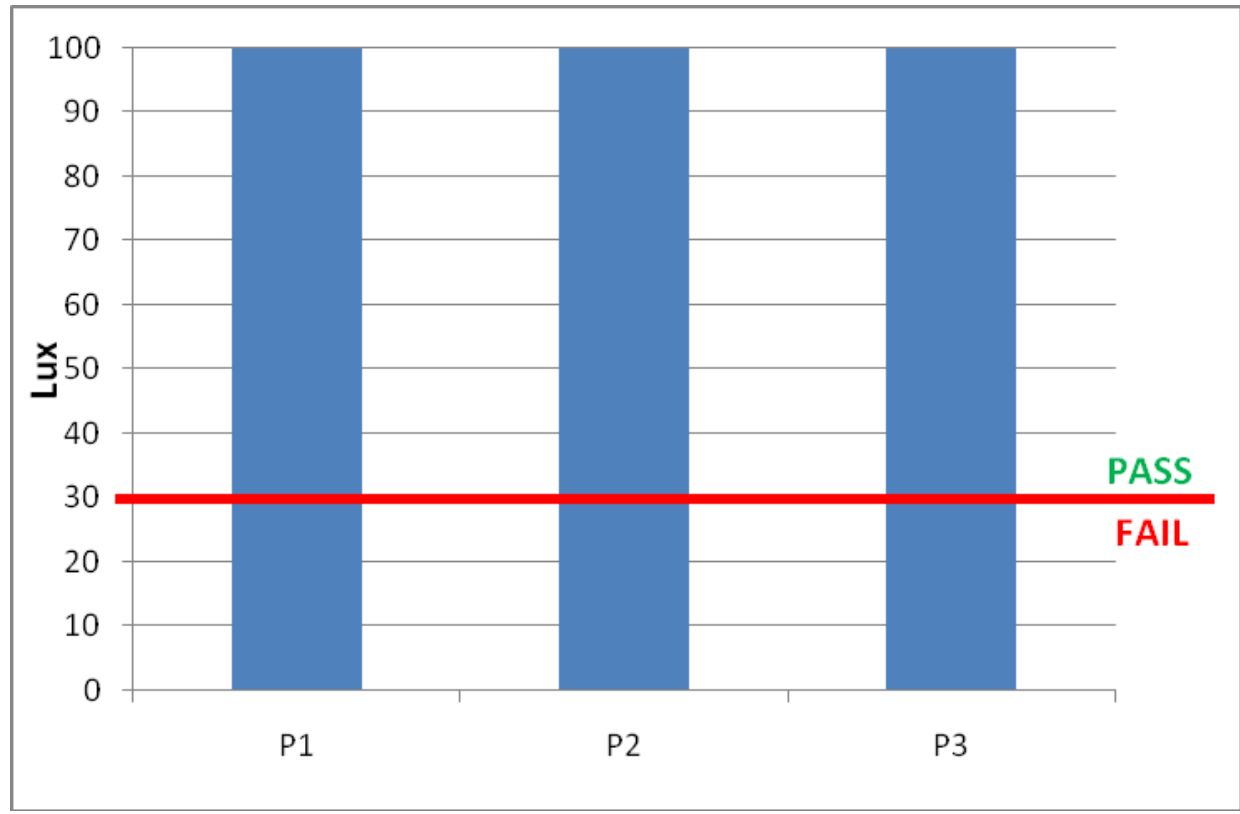

Application of the Tool:

\begin{tabular}{|l|c|}
\hline \multicolumn{2}{|c|}{ NZBC G7 Compliance Assessment Tool } \\
\hline \multicolumn{2}{|l|}{ Variable: } \\
\hline Opposite Building Height & 14 \\
\hline Street Width & 44 \\
\hline Glazed Area & 68 \\
\hline Orientation & South \\
\hline Location & Wellington \\
\hline Reflectance of Opposite Building & 21 \\
\hline Glazing Transmittance & 87 \\
\hline Vertical Location of lowest apartment & 10 \\
\hline \multicolumn{2}{|c|}{} \\
\hline Are simulations required to prove compliance: \\
\hline
\end{tabular}

Comparison of Results:

\begin{tabular}{|c|c|c|c|}
\hline Measurements & Linear Model & Model 2A & Model 2B \\
\hline Pass & 217 & 319 & 971 \\
\hline
\end{tabular}




\section{Appendix 5 - Analysis of Borderline Calibration Apartments}

The appendix provides the detail analysis of the 13 borderline apartments. These are the apartments that were within 5 lux of the threshold illuminance from the apartment measurements, i.e. between 25 and 35 lux at the poorest performing measurement point. The purpose of this analysis was to ensure that apartments around the threshold were receiving the correct outcomes. These were the most important apartments to assess because errors of 5\% DA could be expected in the tool and could result in incorrect outcomes for apartments within this range. 


\section{APARTMENT 38}

Figure 28 shows the illuminance levels exceeded for $75 \%$ of the standard year based on the daylight factor measurements taken in Apartment 38.

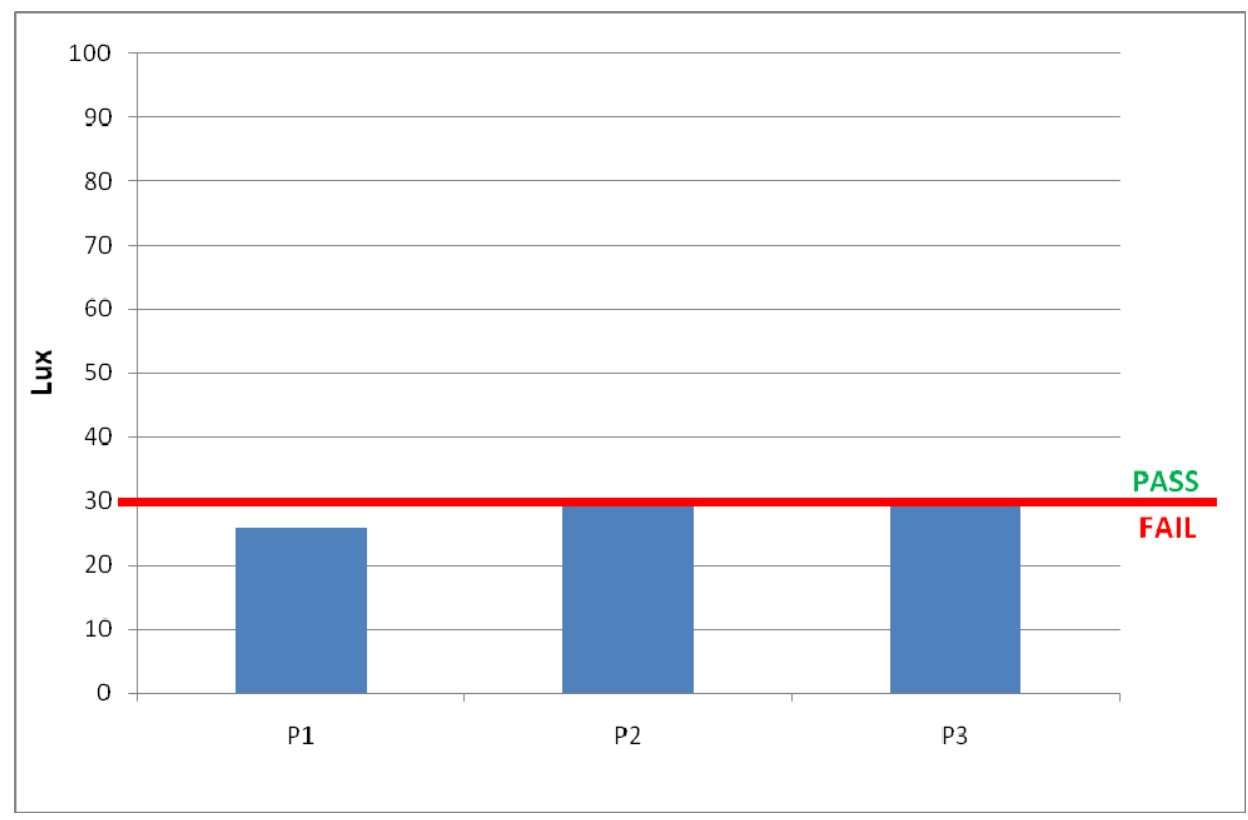

Figure 28 - Illuminance exceeded for $\mathbf{7 5 \%}$ of the Standard Year in Apartment 38

From Figure 28 it can be seen that this apartment fails to meet the minimum requirement. Two of the three measurement points were right on 30 lux, but the first point had an illuminance of 25 lux. Because all points are relatively similar and the maximum illuminance is right on 30 lux, it is likely furniture in this space had little effect on the overall daylight performance. 
Figure 29 shows the application of the compliance assessment tool for Apartment 38.

\begin{tabular}{|l|c|}
\hline \multicolumn{2}{|c|}{ NZBC G7 Compliance Assessment Tool } \\
\hline \multicolumn{2}{|l|}{} \\
\hline Variable: & 62 \\
\hline Opposite Building Height & 9.5 \\
\hline Street Width & 48 \\
\hline Glazed Area & West \\
\hline Orientation & Auckland \\
\hline Location & 5 \\
\hline Reflectance of Opposite Building & 87 \\
\hline Glazing Transmittance & 3 \\
\hline Vertical Location of lowest apartment \\
\hline \multicolumn{2}{|c}{} \\
\hline Are simulations required to prove compliance: \\
\hline
\end{tabular}

Figure 29 - Application of the tool for Apartment 38

The measured data in Figure 28 shows that apartment 38 fails to comply with NZBC Clause G7 and Figure 29 confirms that the tool agrees with this.

Table 43 provides a comparison of the results from each of the prediction models. All three of the models provided the correct outcome for this case, the linear model provided the best prediction for this case as it identified that the apartment is only just failing to meet the minimum requirement.

Table 43 - Prediction Model Results for Apartment 38

\begin{tabular}{|c|c|c|c|}
\hline Measurements & Linear Model & Model 2A & Model 2B \\
\hline Borderline Fail & 73 & 46 & 10 \\
\hline
\end{tabular}




\section{APARTMENT 39}

Figure 30 shows the illuminance levels exceeded for $75 \%$ of the standard year based on the daylight factor measurements taken in Apartment 39.

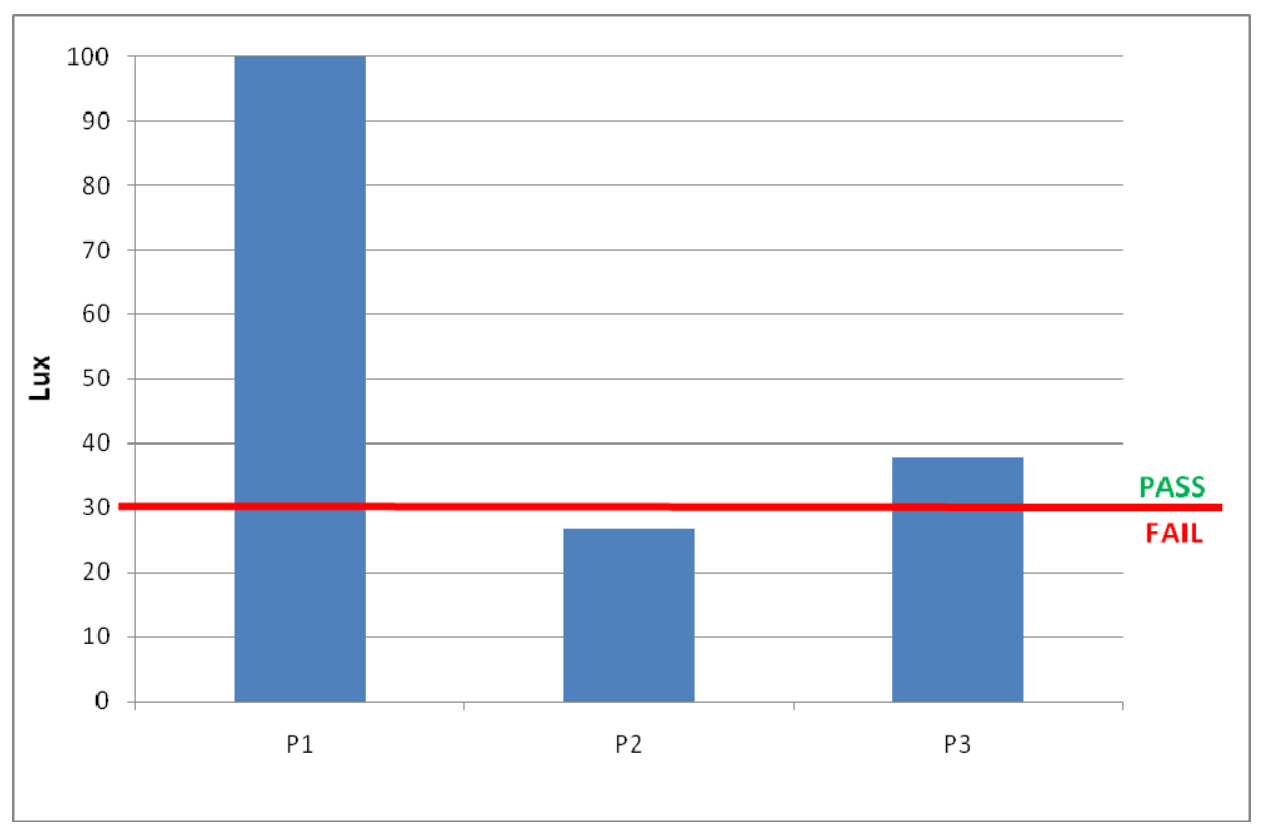

Figure 30 - Illuminance exceeded for $\mathbf{7 5 \%}$ of the Standard Year in Apartment 39

From Figure 30 it can be seen that measurement point 2 failed to meet the minimum requirement, meaning the apartment fails to meet the minimum requirement. Measurement point 1 has an illuminance of over 100 lux, this indicates that there is adequate natural light reaching some areas at the back of the apartment. While point 3 also exceeds the minimum requirement it was only by 7 lux. Due to the large variation in results, it is possible that furniture placement may have caused the fail result for point 2 and that as an empty space this apartment may exceed the minimum requirement. 
Figure 31 shows the application of the compliance assessment tool for Apartment 39.

\begin{tabular}{|l|c|}
\hline \multicolumn{2}{|c|}{ NZBC G7 Compliance Assessment Tool } \\
\hline \multicolumn{2}{|l|}{} \\
\hline Variable: \\
\hline Opposite Building Height & 32 \\
\hline Street Width & 50 \\
\hline Glazed Area & 48 \\
\hline Orientation & West \\
\hline Location & Wellington \\
\hline Reflectance of Opposite Building & 33.3 \\
\hline Glazing Transmittance & 87 \\
\hline Vertical Location of lowest apartment & 2 \\
\hline \multicolumn{2}{|c|}{} \\
\hline Are simulations required to prove compliance: \\
\hline
\end{tabular}

Figure 31 - Application of the tool for Apartment 39

The measured data in Figure 30 shows that apartment 39 fails to comply with NZBC Clause G7 and Figure 31 identified that the tool does not agree with this. However, as was mentioned earlier, it is likely that without furniture this apartment would exceed the minimum requirement.

Table 44 provides a comparison of the results from each of the prediction models. All three of the models provided the incorrect outcome for this case, Model 2B provided the best prediction for this case, but was still $41 \%$ DA over-predicted.

Table 44 - Prediction Model Results for Apartment 39

\begin{tabular}{|c|c|c|c|}
\hline Measurements & Linear Model & Model 2A & Model 2B \\
\hline Borderline Fail & 161 & 131 & 116 \\
\hline
\end{tabular}




\section{APARTMENT 40}

Figure 32 shows the illuminance levels exceeded for $75 \%$ of the standard year based on the daylight factor measurements taken in Apartment 40.

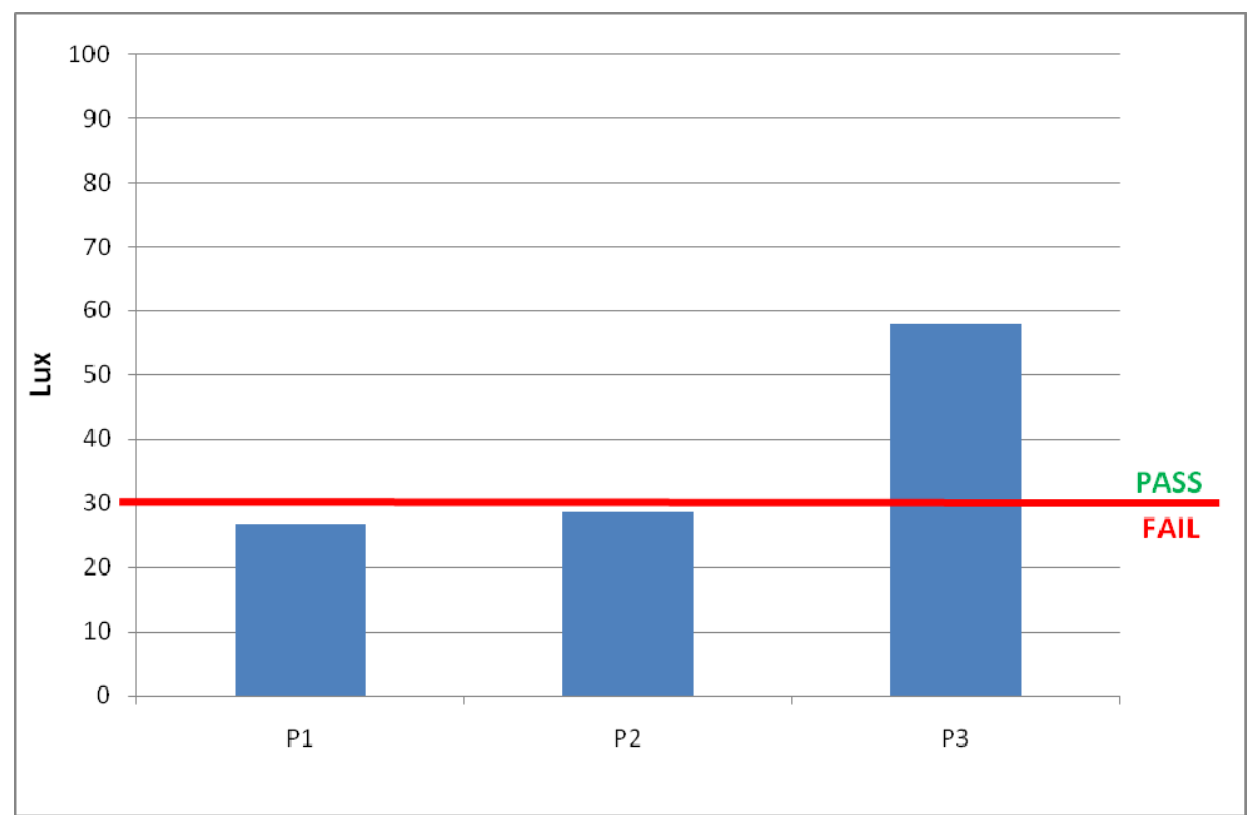

Figure 32 - Illuminance exceeded for $\mathbf{7 5 \%}$ of the Standard Year in Apartment 40

From Figure 32 it can be seen that this apartment fails to meet the minimum requirement as two of the three points fail to meet the minimum requirement. The third point is well above the minimum requirement of 30 lux. The first two measurement points were shaded by a bed which is most likely the reason for the lower illuminances. It is likely that if this apartment were unfurnished then it would exceed the minimum requirement. 
Figure 33 shows the application of the compliance assessment tool for Apartment 40.

\begin{tabular}{|l|c|}
\hline \multicolumn{2}{|c|}{ NZBC G7 Compliance Assessment Tool } \\
\hline \multicolumn{2}{|l|}{ Variable: } \\
\hline Opposite Building Height & 13 \\
\hline Street Width & 28 \\
\hline Glazed Area & 26 \\
\hline Orientation & North \\
\hline Location & Christchurch \\
\hline Reflectance of Opposite Building & 25 \\
\hline Glazing Transmittance & 87 \\
\hline Vertical Location of lowest apartment & 9 \\
\hline \multicolumn{2}{|}{} \\
\hline Are simulations required to prove compliance: \\
\hline
\end{tabular}

Figure 33 - Application of the tool for Apartment $\mathbf{4 0}$

The measured data in Figure 32 shows that apartment 40 fails to comply with NZBC Clause G7 and Figure 33 identifies that the tool does not agrees with this. Therefore, the incorrect result was given for this case, but it was identified that without furnishings this apartment would most likely meet the minimum requirement.

Table 45 provides a comparison of the results from each of the prediction models. All three of the models provided the incorrect outcome for this case, the linear model provided the prediction closest to the threshold, but was still $46 \%$ DA over-predicted.

Table 45 - Prediction Model Results for Apartment 40

\begin{tabular}{|c|c|c|c|}
\hline Measurements & Linear Model & Model 2A & Model 2B \\
\hline Borderline Fail & 121 & 183 & 175 \\
\hline
\end{tabular}




\section{APARTMENT 41}

Figure 34 shows the illuminance levels exceeded for $75 \%$ of the standard year based on the daylight factor measurements taken in Apartment 41.

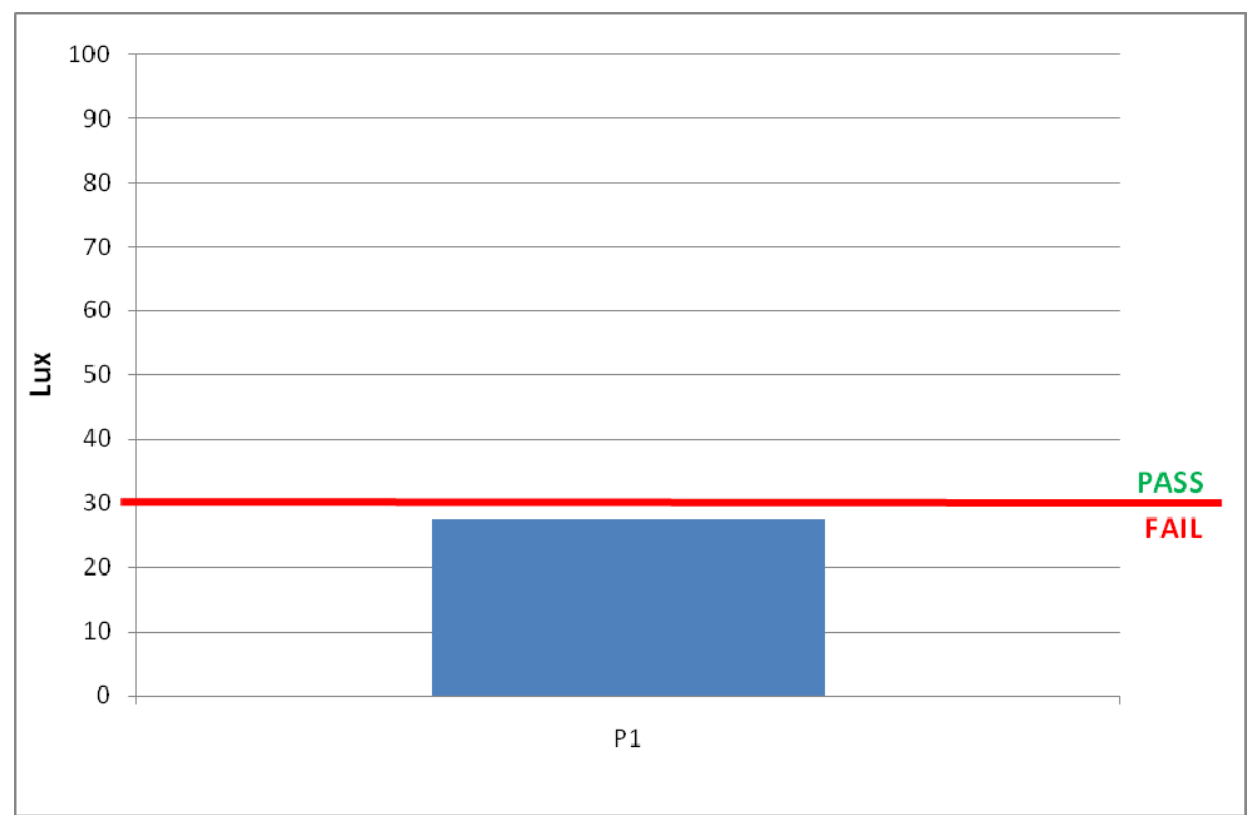

Figure 34 - Illuminance exceeded for $\mathbf{7 5 \%}$ of the Standard Year in Apartment 41

From Figure 34 it can be seen that this apartment fails to meet the minimum requirement. As only one measurement point was able to be measured in this apartment, it was not possible to consider the effect that furniture had on the results. 
Figure 35 shows the application of the compliance assessment tool for Apartment 41.

\begin{tabular}{|l|c|}
\hline \multicolumn{2}{|c|}{ NZBC G7 Compliance Assessment Tool } \\
\hline \multicolumn{2}{|l|}{} \\
\hline Variable: & 30 \\
\hline Opposite Building Height & 27 \\
\hline Street Width & 10 \\
\hline Glazed Area & East \\
\hline Orientation & Wellington \\
\hline Location & 38 \\
\hline Reflectance of Opposite Building & 87 \\
\hline Glazing Transmittance & 7 \\
\hline Vertical Location of lowest apartment \\
\hline \multicolumn{2}{|l}{} \\
\hline Are simulations required to prove compliance: \\
\hline
\end{tabular}

Figure 35 - Application of the tool for Apartment 41

The measured data in Figure 34 shows that apartment 41 fails to comply with NZBC Clause G7. No result was provided for Figure 35 as there was not a consensus between all prediction models. Table 46 identified that two of the three models provided incorrect predictions, while Model 2B was the only model to provide the correct result.

Table 46 - Prediction Model Results for Apartment 41

\begin{tabular}{|c|c|c|c|}
\hline Measurements & Linear Model & Model 2A & Model 2B \\
\hline Borderline Fail & 92 & 89 & 55 \\
\hline
\end{tabular}




\section{APARTMENT 42}

Figure 36 shows the illuminance levels exceeded for $75 \%$ of the standard year based on the daylight factor measurements taken in Apartment 42.

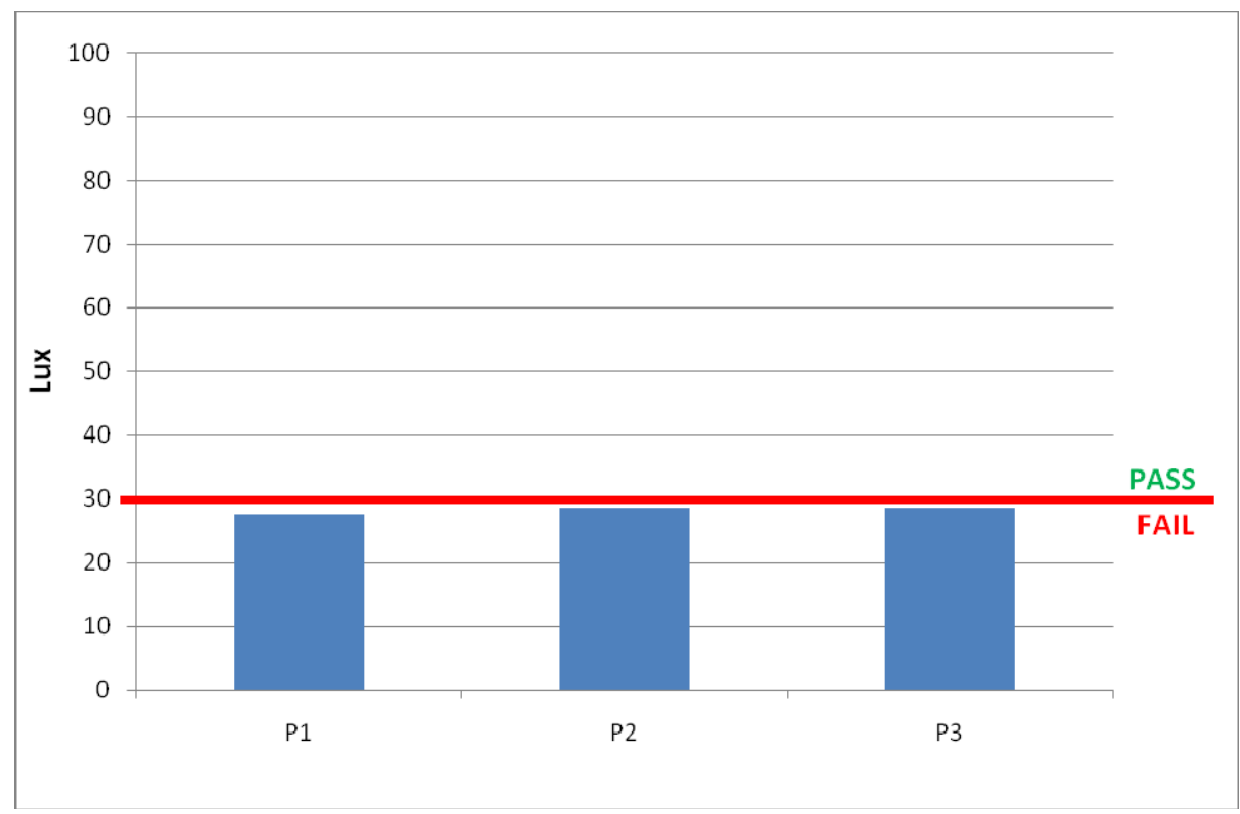

Figure 36 - Illuminance exceeded for 75\% of the Standard Year in Apartment 42

From Figure 36 it can be seen that all three points failed to meet the minimum requirement. It is possible that if this space were unfurnished, it may pass but as all three measurement points had a similar result, it is likely that this apartment would be a borderline pass at best. 
Figure 37 shows the application of the compliance assessment tool for Apartment 42.

\begin{tabular}{|l|c|}
\hline \multicolumn{2}{|c|}{ NZBC G7 Compliance Assessment Tool } \\
\hline \multicolumn{2}{|c|}{ Variable: } \\
\hline Opposite Building Height & 28 \\
\hline Street Width & 3 \\
\hline Glazed Area & 34 \\
\hline Orientation & North \\
\hline Location & Wellington \\
\hline Reflectance of Opposite Building & 24 \\
\hline Glazing Transmittance & 87 \\
\hline Vertical Location of lowest apartment & 8 \\
\hline \multicolumn{2}{|}{} \\
\hline Are simulations required to prove compliance: \\
\hline
\end{tabular}

Figure 37 - Application of the tool for Apartment 42

The measured data in Figure 36 shows that apartment 42 fails to comply with NZBC Clause G7. No result was provided for Figure 37 as all prediction models did not agree. Table 47 identified that two of the three models gave Apartment 42 a pass result which was the incorrect outcome for this case. Model 2B provided the correct result for this case.

Table 47 - Prediction Model Results for Apartment 42

\begin{tabular}{|c|c|c|c|}
\hline Measurements & Linear Model & Model 2A & Model 2B \\
\hline Borderline Fail & 77 & 95 & 65 \\
\hline
\end{tabular}




\section{APARTMENT 43}

Figure 38 shows the illuminance levels exceeded for $75 \%$ of the standard year based on the daylight factor measurements taken in Apartment 43.

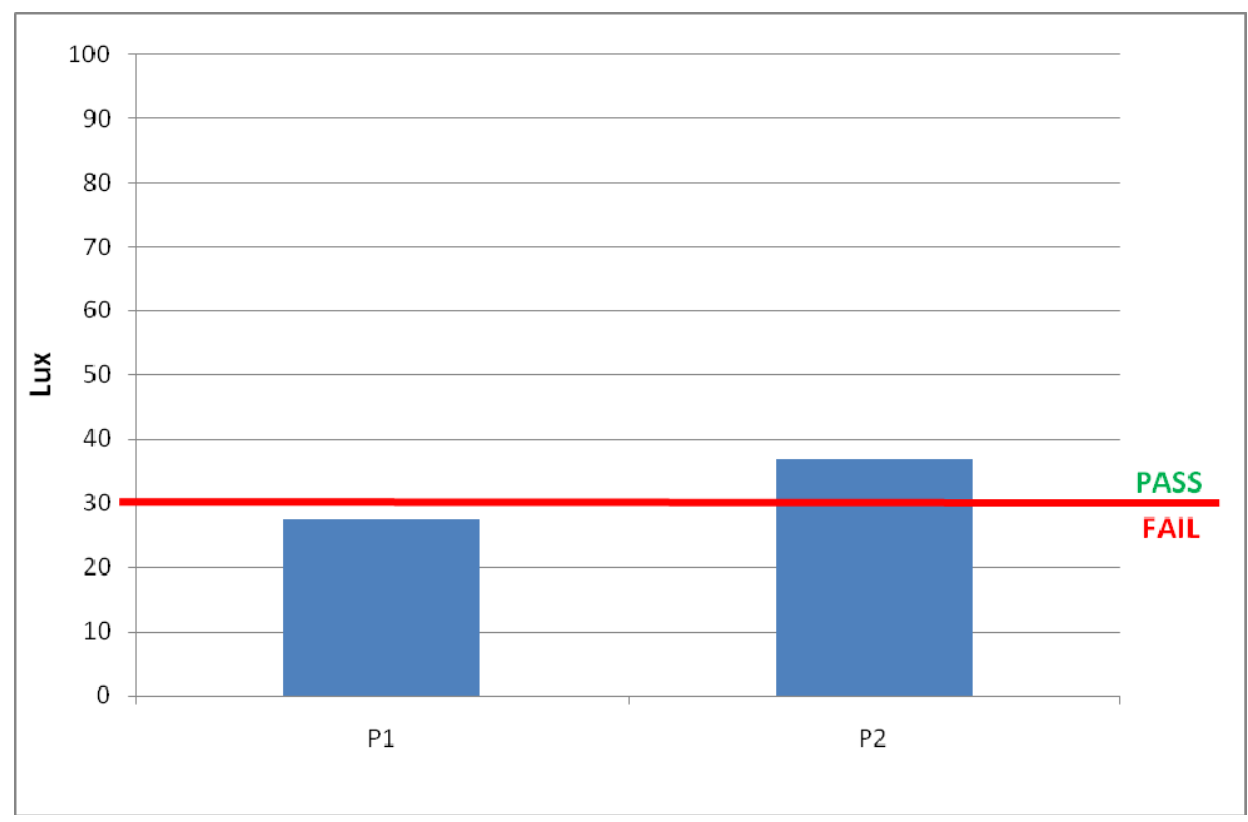

Figure 38 - Illuminance exceeded for $\mathbf{7 5 \%}$ of the Standard Year in Apartment 43

From Figure 38 it can be seen that point 1 fails to fails to meet the minimum requirement, meaning that this apartment receives a fail result overall. Because all points are relatively similar, it is likely furniture in this space had little effect on the overall daylight performance. 
Figure 39 shows the application of the compliance assessment tool for Apartment 43.

\begin{tabular}{|l|c|}
\hline \multicolumn{2}{|c|}{ NZBC G7 Compliance Assessment Tool } \\
\hline \multicolumn{2}{|l|}{} \\
\hline Variable: \\
\hline Opposite Building Height & 18 \\
\hline Street Width & 16 \\
\hline Glazed Area & 17.5 \\
\hline Orientation & North \\
\hline Location & Wellington \\
\hline Reflectance of Opposite Building & 10 \\
\hline Glazing Transmittance & 87 \\
\hline Vertical Location of lowest apartment & 6 \\
\hline \multicolumn{2}{|l}{} \\
\hline Are simulations required to prove compliance: \\
\hline
\end{tabular}

Figure 39 - Application of the tool for Apartment 43

The measured data in Figure 38 shows that apartment 43 fails to comply with NZBC Clause G7 and Figure 39 confirms that the tool agrees with this.

Table 48 provides a comparison of the results from each of the prediction models. All three of the models provided the correct outcome for Apartment 43, with the linear model providing the best prediction.

Table 48 - Prediction Model Results for Apartment 43

\begin{tabular}{|c|c|c|c|}
\hline Measurements & Linear Model & Model 2A & Model 2B \\
\hline Borderline Fail & 73 & 59 & 46 \\
\hline
\end{tabular}




\section{APARTMENT 44}

Figure 40 shows the illuminance levels exceeded for $75 \%$ of the standard year based on the daylight factor measurements taken in Apartment 44.

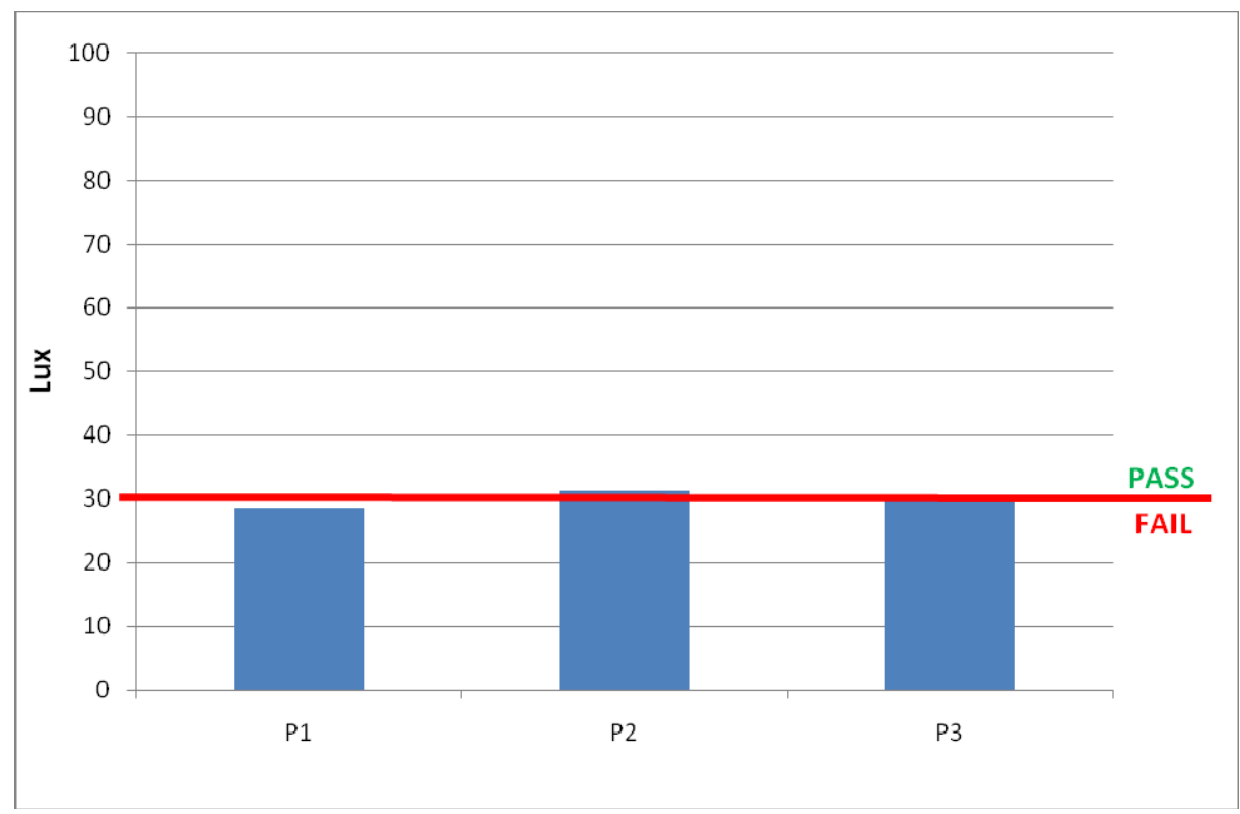

Figure 40 - Illuminance exceeded for $\mathbf{7 5 \%}$ of the Standard Year in Apartment 44

From Figure 40 it can be seen that point 1 is just under the minimum requirement and point 3 is right on the threshold of 30 lux. This results in a fail result for the overall apartment. Because all points are relatively similar, it is likely furniture in this space had little effect on the overall daylight performance. 
Figure 41 shows the application of the compliance assessment tool for Apartment 44.

\begin{tabular}{|l|c|}
\hline \multicolumn{2}{|c|}{ NZBC G7 Compliance Assessment Tool } \\
\hline \multicolumn{2}{|l|}{} \\
\hline Variable: & 12 \\
\hline Opposite Building Height & 3 \\
\hline Street Width & 35 \\
\hline Glazed Area & South \\
\hline Orientation & Wellington \\
\hline Location & 21 \\
\hline Reflectance of Opposite Building & 87 \\
\hline Glazing Transmittance & 10 \\
\hline Vertical Location of lowest apartment \\
\hline \multicolumn{2}{|c|}{} \\
\hline Are simulations required to prove compliance: \\
\hline
\end{tabular}

Figure 41 - Application of the tool for Apartment 44

The measured data in Figure 40 shows that apartment 44 fails to comply with NZBC Clause $\mathrm{G} 7$ and Figure 41 indicates that the tool does not agrees with this.

Table 49 provides a comparison of the results from each of the prediction models. All three of the models provided the incorrect outcome for this case, the linear model provided the closest prediction, but this was still $23 \%$ DA above the threshold.

Table 49 - Prediction Model Results for Apartment 44

\begin{tabular}{|c|c|c|c|}
\hline Measurements & Linear Model & Model 2A & Model 2B \\
\hline Borderline Fail & 98 & 205 & 252 \\
\hline
\end{tabular}




\section{APARTMENT 45}

Figure 42 shows the illuminance levels exceeded for $75 \%$ of the standard year based on the daylight factor measurements taken in Apartment 45.

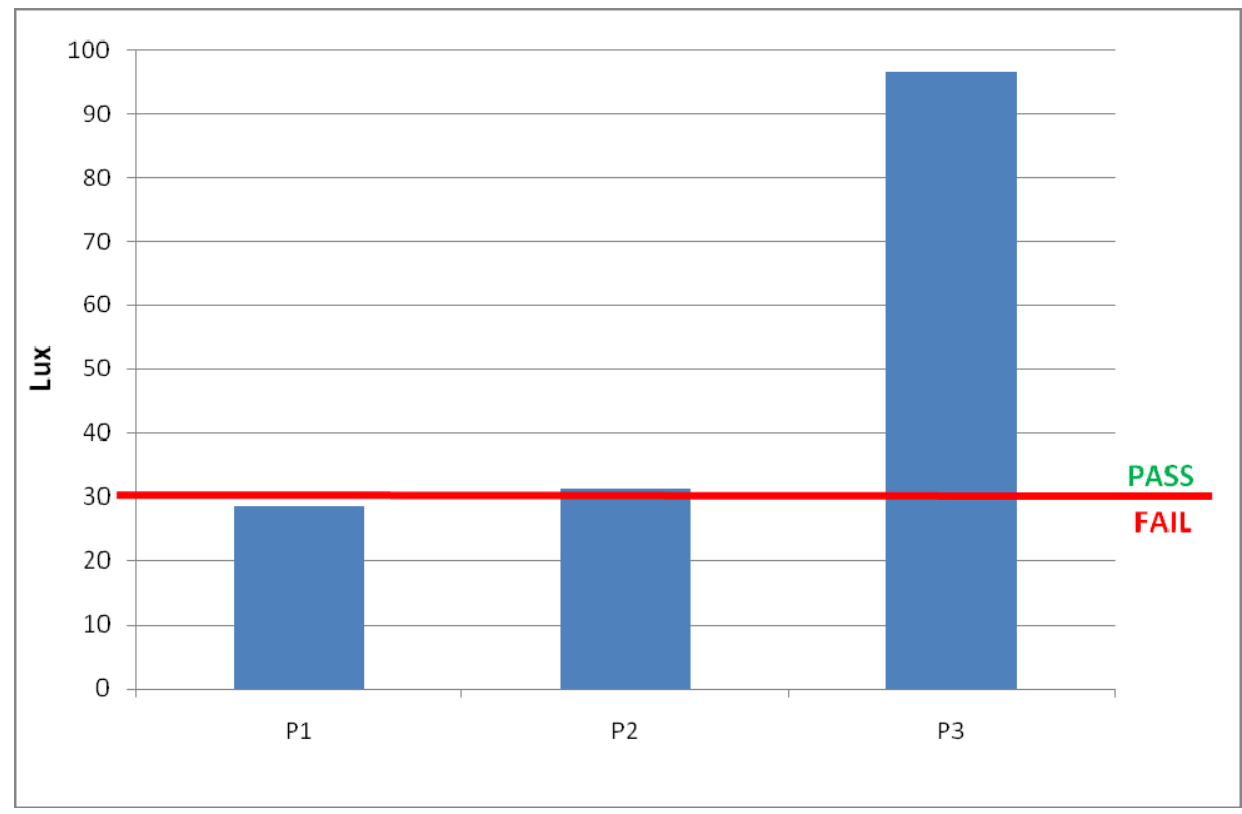

Figure 42 - Illuminance exceeded for $\mathbf{7 5 \%}$ of the Standard Year in Apartment 45

From Figure 42 it can be seen that this apartment fails to meet the minimum requirement. Two of the three measurement points were on the borderline, with point 1 being just below the threshold of 30 lux and point 2 being just above. Point 3 was well above the minimum requirement of 30 lux, with an illuminance of 96 lux for $75 \%$ of the standard year which suggests there is adequate natural light reaching some areas of apartment. Because of the high illuminance at point 3 , it is possible that if this apartment was unfurnished it would exceed the minimum requirement at all three points. 
Figure 43 shows the application of the compliance assessment tool for Apartment 45.

\begin{tabular}{|l|c|}
\hline \multicolumn{2}{|c|}{ NZBC G7 Compliance Assessment Tool } \\
\hline \multicolumn{2}{|c|}{ Variable: } \\
\hline Opposite Building Height & 37 \\
\hline Street Width & 25 \\
\hline Glazed Area & 45 \\
\hline Orientation & West \\
\hline Location & Wellington \\
\hline Reflectance of Opposite Building & 24 \\
\hline Glazing Transmittance & 87 \\
\hline Vertical Location of lowest apartment & 5 \\
\hline \multicolumn{2}{|}{} \\
\hline Are simulations required to prove compliance: \\
\hline
\end{tabular}

Figure 43 - Application of the tool for Apartment 45

The measured data in Figure 42 shows that apartment 45 fails to comply with NZBC Clause G7. No result was provided for Figure 43 as there was not a consensus between all prediction models. Table 50 identified that two of the three models provided the incorrect outcome, but Model 2B was the correct outcome for this case as is was a borderline fail.

Table 50 - Prediction Model Results for Apartment 45

\begin{tabular}{|c|c|c|c|}
\hline Measurements & Linear Model & Model 2A & Model 2B \\
\hline Borderline Fail & 118 & 92 & 74 \\
\hline
\end{tabular}




\section{APARTMENT 46}

Figure 44 shows the illuminance levels exceeded for $75 \%$ of the standard year based on the daylight factor measurements taken in Apartment 46.

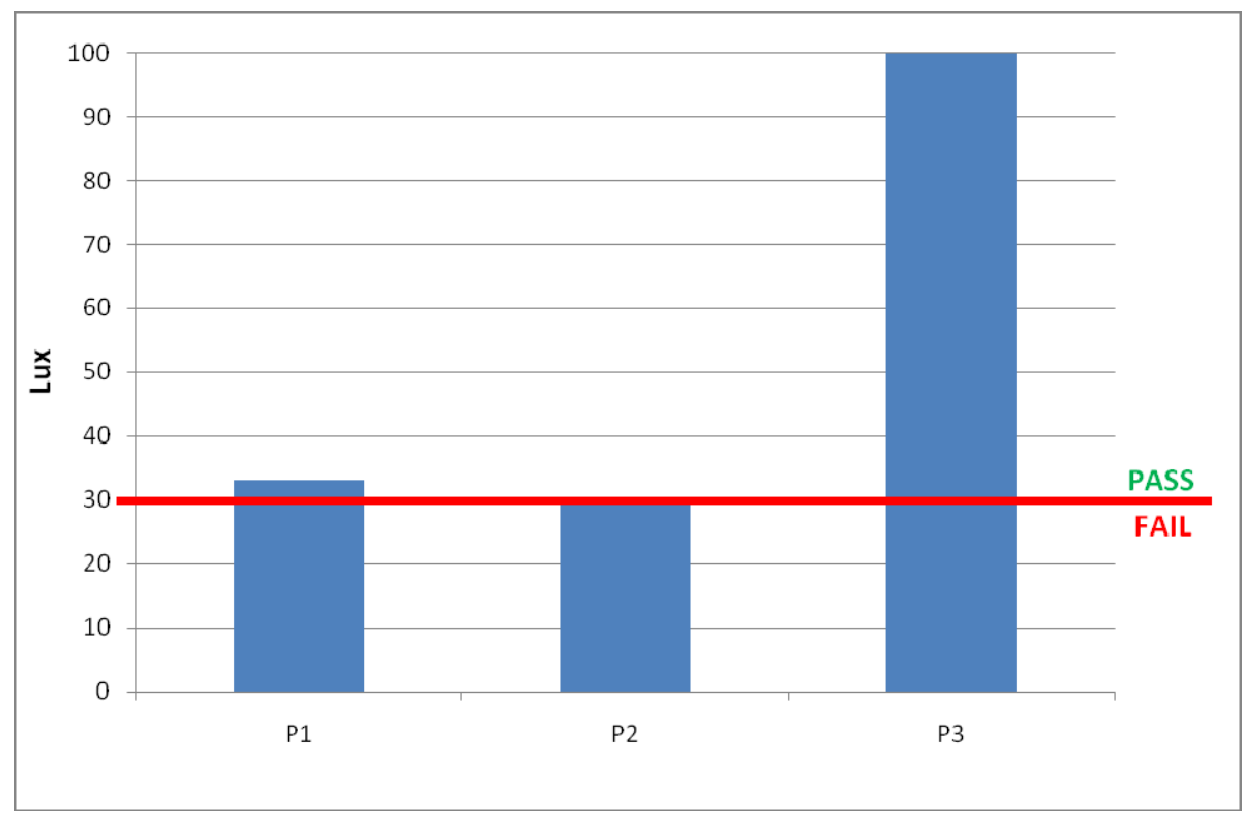

Figure 44 - Illuminance exceeded for $\mathbf{7 5 \%}$ of the Standard Year in Apartment 46

From Figure 44 it is difficult to determine whether this apartment fails or not. It is clear that measurement points 1 and 3 exceed the minimum requirement, but point 2 is right on the cusp with an illuminance of 29.4. Because point is just below the threshold this apartment does not meet the minimum requirement overall. The high illuminance at point 3 means that it is possible that if this apartment was unfurnished then it may exceed the minimum requirement at all three points. 
Figure 45 shows the application of the compliance assessment tool for Apartment 46.

\begin{tabular}{|l|c|}
\hline \multicolumn{2}{|c|}{ NZBC G7 Compliance Assessment Tool } \\
\hline \multicolumn{2}{|l|}{ Variable: } \\
\hline Opposite Building Height & 19 \\
\hline Street Width & 25 \\
\hline Glazed Area & 18 \\
\hline Orientation & South \\
\hline Location & Wellington \\
\hline Reflectance of Opposite Building & 20 \\
\hline Glazing Transmittance & 87 \\
\hline Vertical Location of lowest apartment & 1 \\
\hline \multicolumn{2}{|l}{} \\
\hline Are simulations required to prove compliance: \\
\hline
\end{tabular}

Figure 45 - Application of the tool for Apartment 46

The measured data in Figure 45 shows that apartment 46 fails to comply with NZBC Clause G7. No result was provided for Figure 45 as there was not a consensus between all prediction models. The comparison of the prediction model results in Table 51 indicated that the correct result is provided by the two nonlinear models ( $2 \mathrm{~A}$ and $2 \mathrm{~B}$ ), while the linear model slightly overestimated the result in this situation.

Table 51 - Prediction Model Results for Apartment 46

\begin{tabular}{|c|c|c|c|}
\hline Measurements & Linear Model & Model 2A & Model 2B \\
\hline Borderline Fail & 76 & 46 & 44 \\
\hline
\end{tabular}




\section{APARTMENT 47}

Figure 46 shows the illuminance levels exceeded for $75 \%$ of the standard year based on the daylight factor measurements taken in Apartment 47.

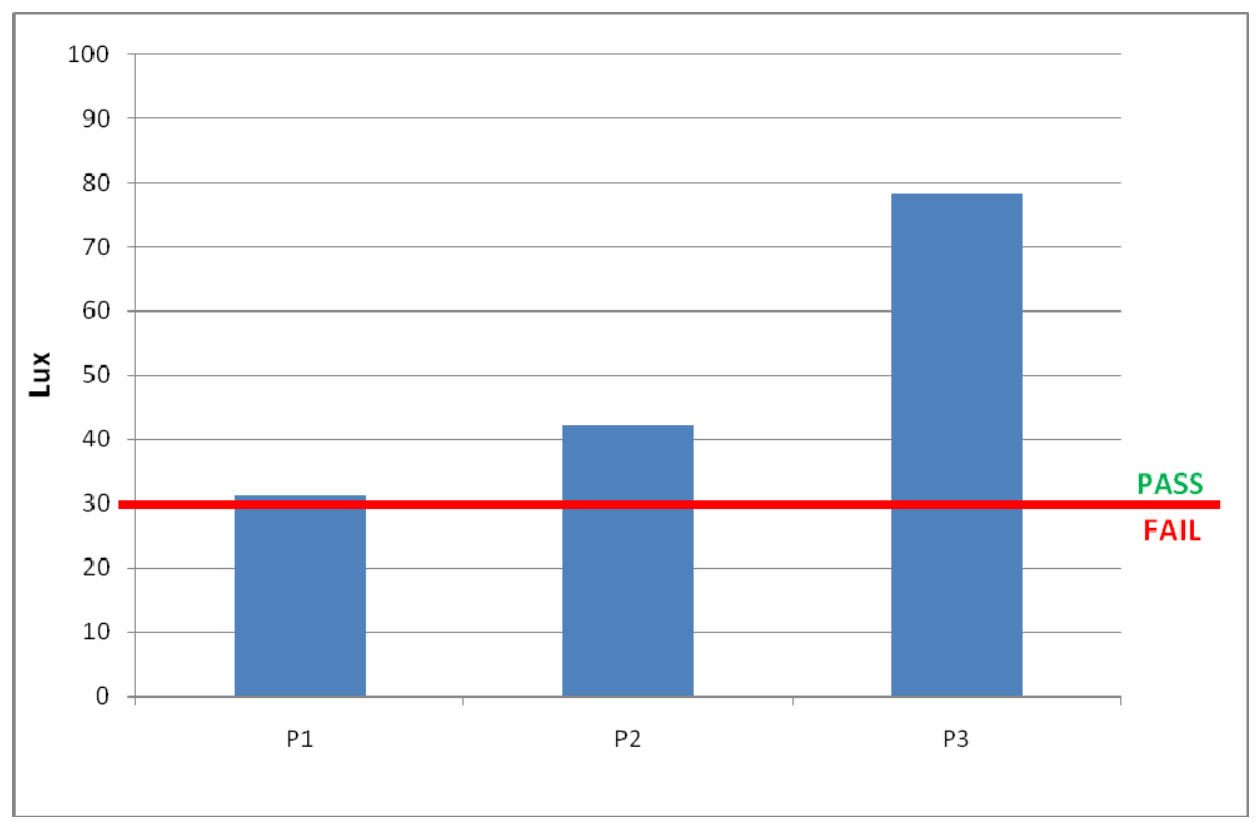

Figure 46 - Illuminance exceeded for $\mathbf{7 5 \%}$ of the Standard Year in Apartment 47

From Figure 46 it can be seen that this apartment exceeds the minimum requirement for all three points. Points 2 and 3 clearly exceed this requirement, but point 1 only just exceeds it with an illuminance of 31 lux, making this measurement point in particular susceptible to failing as a result of minor changes to the environment like changing the reflectance of the opposite building or dirt build up on the glazing reducing the transmittance. It should also be noted that with the significantly higher illuminance of point 3 it is possible that furniture has resulted in reduced performance at points 1 and 2 . 
Figure 47 shows the application of the compliance assessment tool for Apartment 47.

\begin{tabular}{|l|c|}
\hline \multicolumn{2}{|c|}{ NZBC G7 Compliance Assessment Tool } \\
\hline \multicolumn{2}{|l|}{} \\
\hline Variable: & 0 \\
\hline Opposite Building Height & 9 \\
\hline Street Width & 32 \\
\hline Glazed Area & South \\
\hline Orientation & Auckland \\
\hline Location & 5 \\
\hline Reflectance of Opposite Building & 87 \\
\hline Glazing Transmittance & 1 \\
\hline Vertical Location of lowest apartment & \\
\hline \multicolumn{2}{|l}{} \\
\hline Are simulations required to prove compliance: \\
\hline
\end{tabular}

Figure 47 - Application of the tool for Apartment 47

The measured data in Figure 46 shows that apartment 47 fails to comply with NZBC Clause G7. No result was provided for Figure 47 as the prediction models did not agree. The comparison of the prediction model results in Table 52 indicated that the linear model and model $2 \mathrm{~A}$ provided the correct results for this case, however model $2 \mathrm{~B}$ slightly underestimated the results for this case. The linear model provided the best result for this case as it gave a borderline pass result.

Table 52 - Prediction Model Results for Apartment 47

\begin{tabular}{|c|c|c|c|}
\hline Measurements & Linear Model & Model 2A & Model 2B \\
\hline Borderline Pass & 75 & 84 & 74 \\
\hline
\end{tabular}




\section{APARTMENT 48}

Figure 48 shows the illuminance levels exceeded for $75 \%$ of the standard year based on the daylight factor measurements taken in Apartment 48.

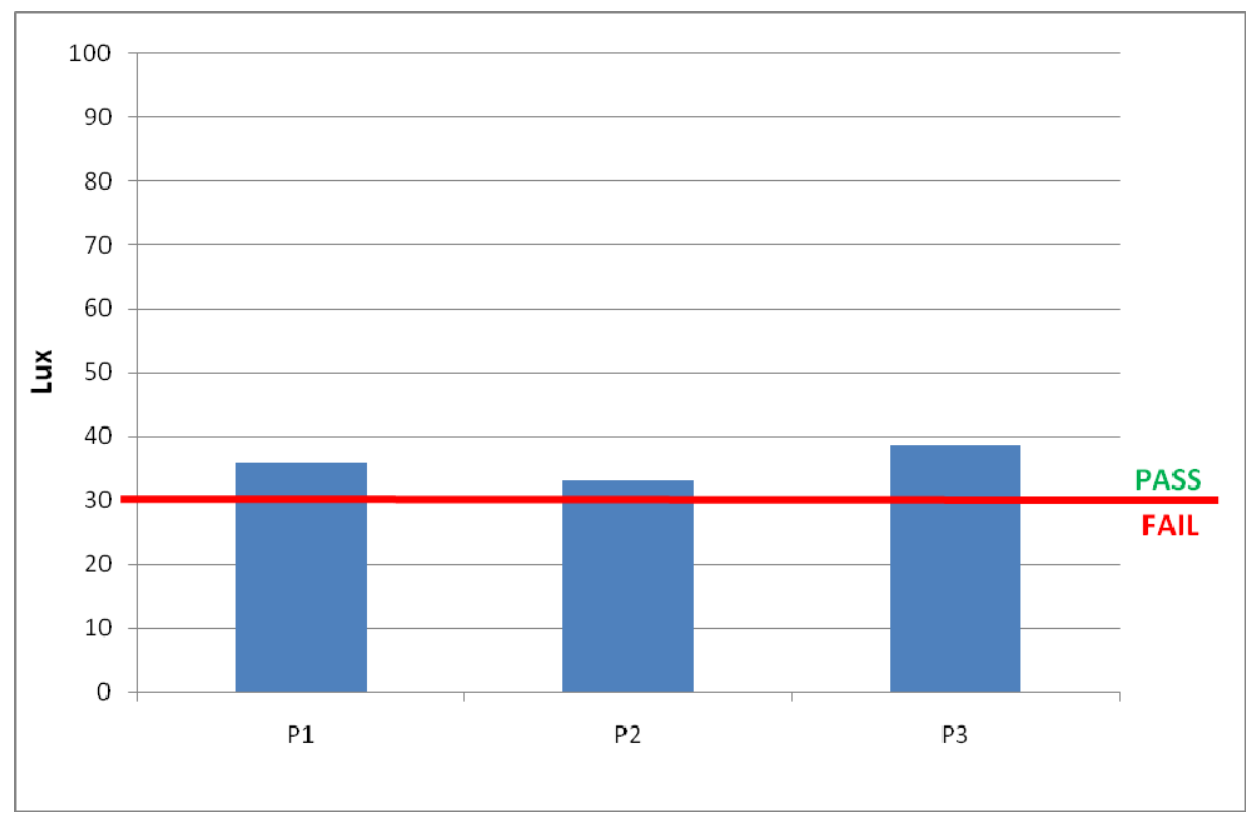

Figure 48 - Illuminance exceeded for $75 \%$ of the Standard Year in Apartment 48

From Figure 48 it can be seen that this apartment exceeds the minimum requirement. All three measurement points exceeded the minimum requirement of 30 lux but were all within 10 lux of the threshold level. Because all points are relatively similar, it is likely furniture in this space had little effect on the overall daylight performance. 
Figure 49 shows the application of the compliance assessment tool for Apartment 48.

\begin{tabular}{|l|c|}
\hline \multicolumn{2}{|c|}{ NZBC G7 Compliance Assessment Tool } \\
\hline \multicolumn{2}{|l|}{} \\
\hline Variable: & 23.8 \\
\hline Opposite Building Height & 40 \\
\hline Street Width & 24 \\
\hline Glazed Area & West \\
\hline Orientation & Wellington \\
\hline Location & 13.5 \\
\hline Reflectance of Opposite Building & 87 \\
\hline Glazing Transmittance & 2 \\
\hline Vertical Location of lowest apartment \\
\hline \multicolumn{2}{|}{} \\
\hline Are simulations required to prove compliance: \\
\hline
\end{tabular}

Figure 49 - Application of the tool for Apartment 48

The measured data in Figure 48 shows that apartment 48 fails to comply with NZBC Clause G7. No result was provided for Figure 49 as the prediction models did not agree. The comparison of the prediction model results in Table 53 identified that the linear model provided the correct result for this case, while models $2 \mathrm{~A}$ and $2 \mathrm{~B}$ slightly underestimated the results for this case.

Table 53 - Prediction Model Results for Apartment 48

\begin{tabular}{|c|c|c|c|}
\hline Measurements & Linear Model & Model 2A & Model 2B \\
\hline Borderline Pass & 110 & 72 & 71 \\
\hline
\end{tabular}




\section{APARTMENT 49}

Figure 50 shows the illuminance levels exceeded for $75 \%$ of the standard year based on the daylight factor measurements taken in Apartment 49.

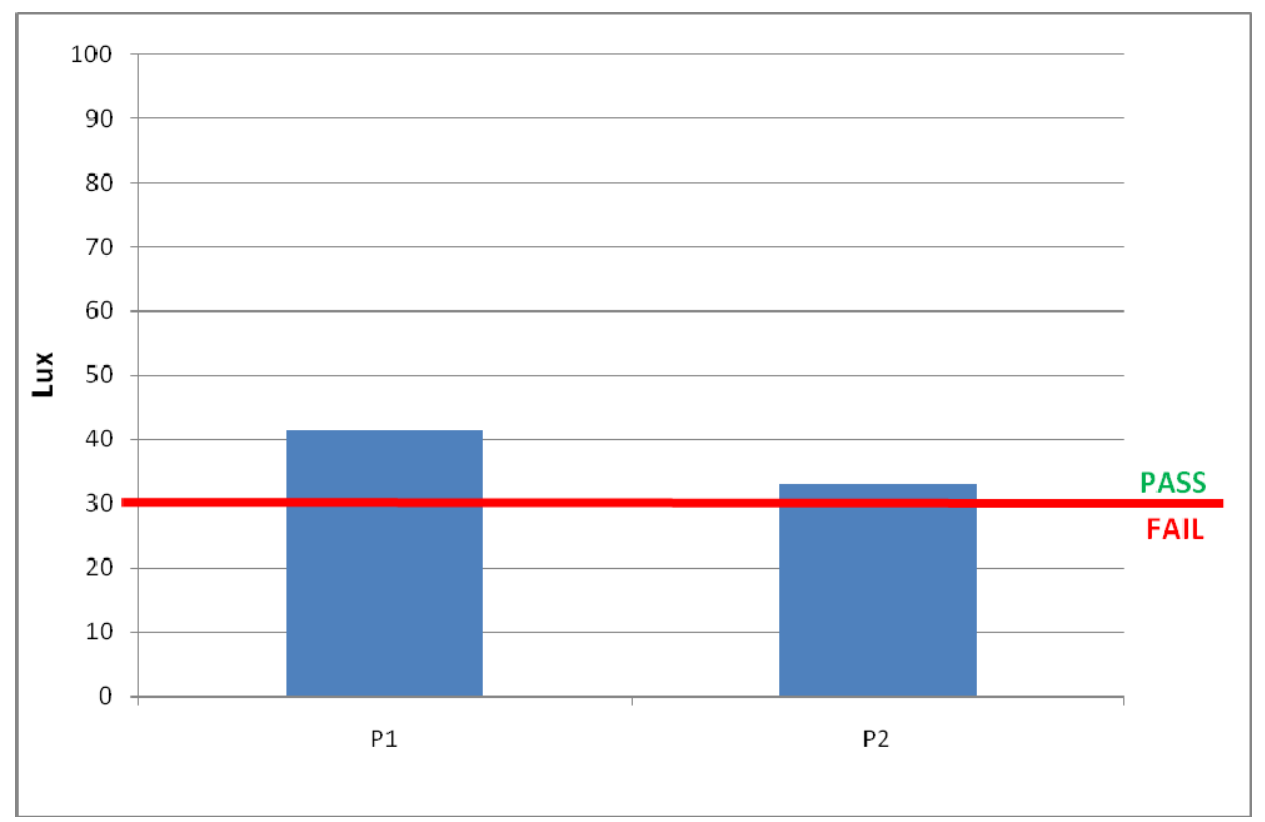

Figure 50 - Illuminance exceeded for $\mathbf{7 5 \%}$ of the Standard Year in Apartment 49

From Figure 50 it can be seen that this apartment exceeds the minimum requirement. Both measurement points were above 30 lux, but neither point greatly exceeded this value. Because both points were relatively similar, it is likely furniture in this space had little effect on the overall daylight performance. 
Figure 51 shows the application of the compliance assessment tool for Apartment 49.

\begin{tabular}{|l|c|}
\hline \multicolumn{2}{|c|}{ NZBC G7 Compliance Assessment Tool } \\
\hline \multicolumn{2}{|c|}{ Variable: } \\
\hline Opposite Building Height & 18 \\
\hline Street Width & 15.9 \\
\hline Glazed Area & 30 \\
\hline Orientation & North \\
\hline Location & Wellington \\
\hline Reflectance of Opposite Building & 10 \\
\hline Glazing Transmittance & 87 \\
\hline Vertical Location of lowest apartment & 7 \\
\hline \multicolumn{2}{|c|}{} \\
\hline Are simulations required to prove compliance: \\
\hline
\end{tabular}

Figure 51 - Application of the tool for Apartment 49

The measured data in Figure 50 shows that apartment 49 complies with NZBC Clause G7 and Figure 51 confirms that the tool agrees with this.

Table 54 provides a comparison of the results from each of the prediction models. All three of the models provided the correct outcome for this case.

Table 54 - Prediction Model Results for Apartment 49

\begin{tabular}{|l|c|c|c|}
\hline Measurements & Linear Model & Model 2A & Model 2B \\
\hline Borderline Pass & 93 & 95 & 89 \\
\hline
\end{tabular}




\section{APARTMENT 50}

Figure 52 shows the illuminance levels exceeded for $75 \%$ of the standard year based on the daylight factor measurements taken in Apartment 50.

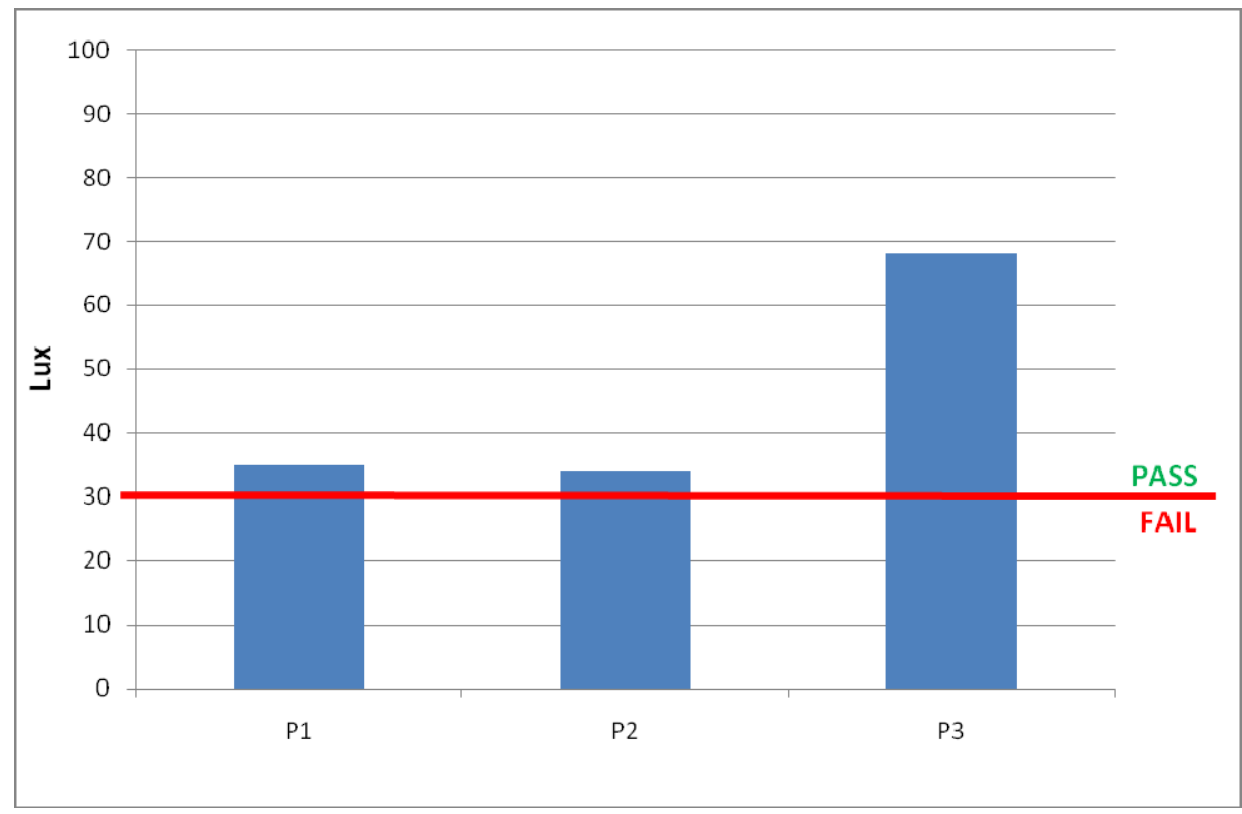

Figure 52 - Illuminance exceeded for $\mathbf{7 5 \%}$ of the Standard Year in Apartment 50

From Figure 52 it can be seen that this apartment met the minimum requirement. Two of the three measurement points exceeded the requirement by $4-5 \%$ DA, while measurement point 3 was just over twice the minimum requirement. As with a number of other apartments, it is possible that furniture reduced the performance slight at point 1 and 2, but this was expected to have a minimal effect on the overall performance of the apartment. 
Figure 53 shows the application of the compliance assessment tool for Apartment 50.

\begin{tabular}{|l|c|}
\hline \multicolumn{2}{|c|}{ NZBC G7 Compliance Assessment Tool } \\
\hline \multicolumn{2}{|l|}{} \\
\hline Variable: & 0 \\
\hline Opposite Building Height & 0 \\
\hline Street Width & 26 \\
\hline Glazed Area & East \\
\hline Orientation & Christchurch \\
\hline Location & 0 \\
\hline Reflectance of Opposite Building & 87 \\
\hline Glazing Transmittance & 10 \\
\hline Vertical Location of lowest apartment \\
\hline \multicolumn{2}{|c}{} \\
\hline Are simulations required to prove compliance: \\
\hline
\end{tabular}

Figure 53 - Application of the tool for Apartment 50

The measured data in Figure 52 shows that apartment 53 complies with NZBC Clause G7 and Figure 53 confirms that the tool agrees with this.

Table 55 provides a comparison of the results from each of the prediction models. All three of the models provided the correct outcome for this case, however the linear model provided the best prediction as it was a borderline pass result.

Table 55 - Prediction Model Results for Apartment 50

\begin{tabular}{|c|c|c|c|}
\hline Measurements & Linear Model & Model 2A & Model 2B \\
\hline Borderline Pass & 75 & 210 & 237 \\
\hline
\end{tabular}




\section{Appendix 6 - Analysis of False Pass Calibration Apartments}

Appendix 6 provides the analysis of the apartments that had false pass results given by at least one of the prediction equations. A false pass apartment is where the prediction equation said that simulation was not needed but the measurements identified that the apartment did NOT meet the minimum requirement. 


\section{APARTMENT 14}

Figure 54 shows the illuminance levels exceeded for $75 \%$ of the standard year based on the daylight factor measurements taken in Apartment 14.

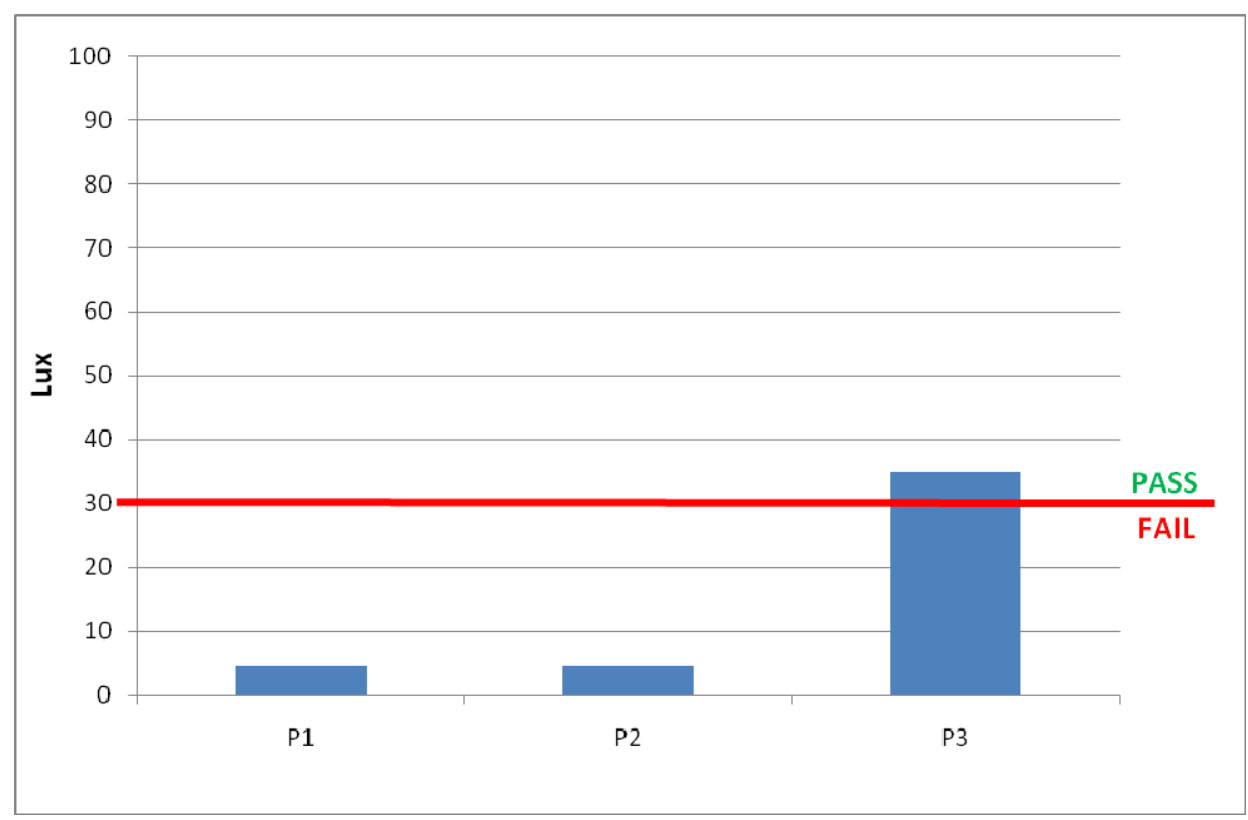

Figure 54 - Illuminance exceeded for $75 \%$ of the Standard Year in Apartment 14

From Figure 54 it can be seen that this apartment is well below the minimum requirement at two of the measurement points, while point 3 does exceed this minimum requirement it is only by 5\% DA. Because point 1 and 2 failed to meet the requirement, this apartment fails to meet the minimum requirement overall. 
Figure 55 shows the application of the compliance assessment tool for Apartment 14.

\begin{tabular}{|l|c|}
\hline \multicolumn{2}{|c|}{ NZBC G7 Compliance Assessment Tool } \\
\hline \multicolumn{2}{|l|}{ Variable: } \\
\hline Opposite Building Height & 14 \\
\hline Street Width & 44 \\
\hline Glazed Area & 21 \\
\hline Orientation & South \\
\hline Location & Wellington \\
\hline Reflectance of Opposite Building & 21 \\
\hline Glazing Transmittance & 40 \\
\hline Vertical Location of lowest apartment & 7 \\
\hline \multicolumn{2}{|c}{} \\
\hline Are simulations required to prove compliance: \\
\hline
\end{tabular}

Figure 55 - Application of the tool for Apartment 14

The measured data in Figure 54 shows that apartment 14 fails to comply with NZBC Clause G7 and Figure 55 indicates that the tool does not agree with this.

Table 56 provides a comparison of the results from each of the prediction models. All three of the models provided the incorrect outcome for this case, the model with the closest prediction was the linear model but this was more than 50\% DA over-predicted.

Table 56 - Prediction Model Results for Apartment 14

\begin{tabular}{|c|c|c|c|}
\hline Measurements & Linear Model & Model 2A & Model 2B \\
\hline Fail & 126 & 139 & 152 \\
\hline
\end{tabular}




\section{APARTMENT 20}

Figure 56 shows the illuminance levels exceeded for $75 \%$ of the standard year based on the daylight factor measurements taken in Apartment 20.

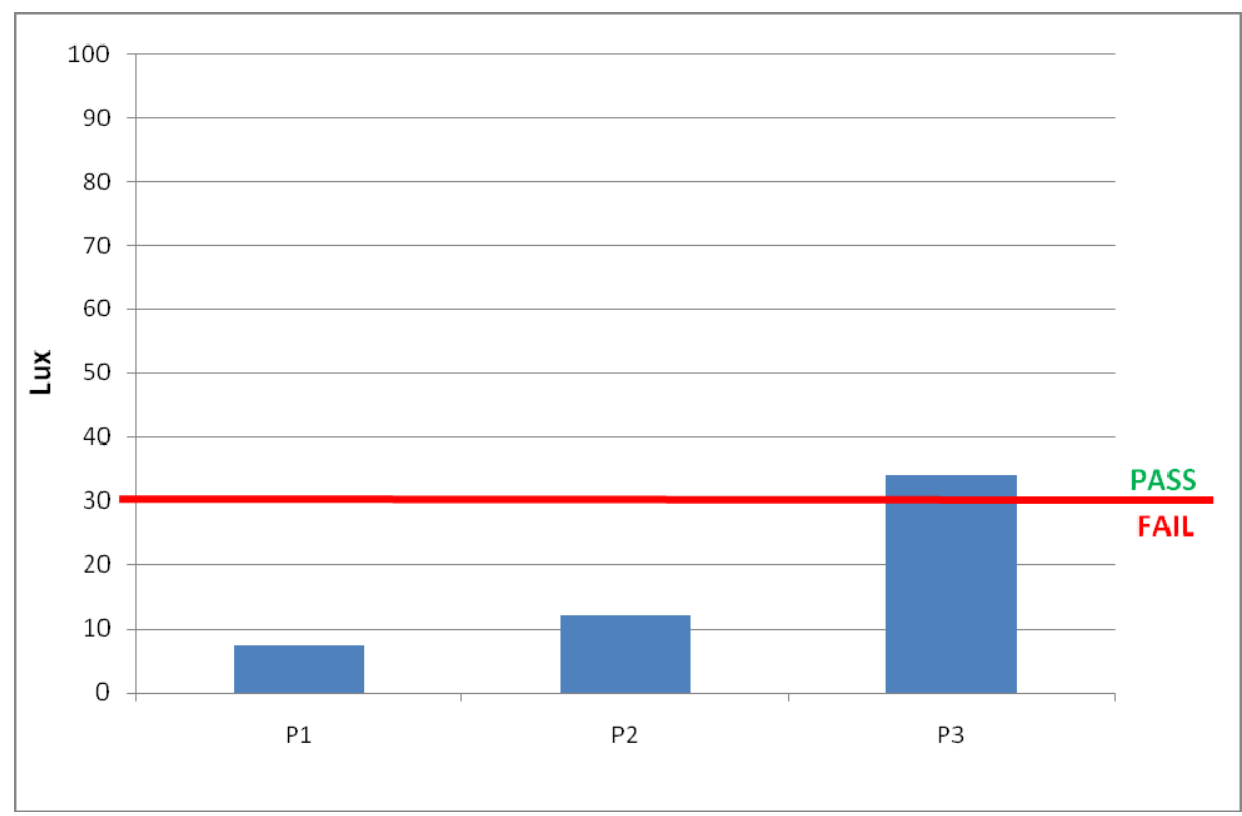

Figure 56 - Illuminance exceeded for $\mathbf{7 5 \%}$ of the Standard Year in Apartment 20

From Figure 56 it is clear that the apartment fails to meet the minimum requirement at points 1 and 2 , therefore the apartment as a whole fails. 
Figure 57 shows the application of the compliance assessment tool for Apartment 20.

\begin{tabular}{|l|c|}
\hline \multicolumn{2}{|c|}{ NZBC G7 Compliance Assessment Tool } \\
\hline \multicolumn{2}{|l|}{} \\
\hline Variable: & 60 \\
\hline Opposite Building Height & 30 \\
\hline Street Width & 36 \\
\hline Glazed Area & East \\
\hline Orientation & Wellington \\
\hline Location & 30 \\
\hline Reflectance of Opposite Building & 87 \\
\hline Glazing Transmittance & 4 \\
\hline Vertical Location of lowest apartment \\
\hline \multicolumn{2}{|l}{} \\
\hline Are simulations required to prove compliance: \\
\hline
\end{tabular}

Figure 57 - Application of the tool for Apartment 20

The measured data in Figure 56 shows that apartment 20 fails to comply with NZBC Clause G7. No result was provided for Figure 57 as the prediction models did not agree. The comparison of the prediction model results in Table 57 identified that models $2 \mathrm{~A}$ and $2 \mathrm{~B}$ provided the correct results for this case, however the linear model over-estimated the results for this case by more than $25 \%$.

Table 57 - Prediction Model Results for Apartment 20

\begin{tabular}{|c|c|c|c|}
\hline Measurements & Linear Model & Model 2A & Model 2B \\
\hline Fail & 102 & 65 & 42 \\
\hline
\end{tabular}




\section{APARTMENT 22}

Figure 58 shows the illuminance levels exceeded for $75 \%$ of the standard year based on the daylight factor measurements taken in Apartment 22.

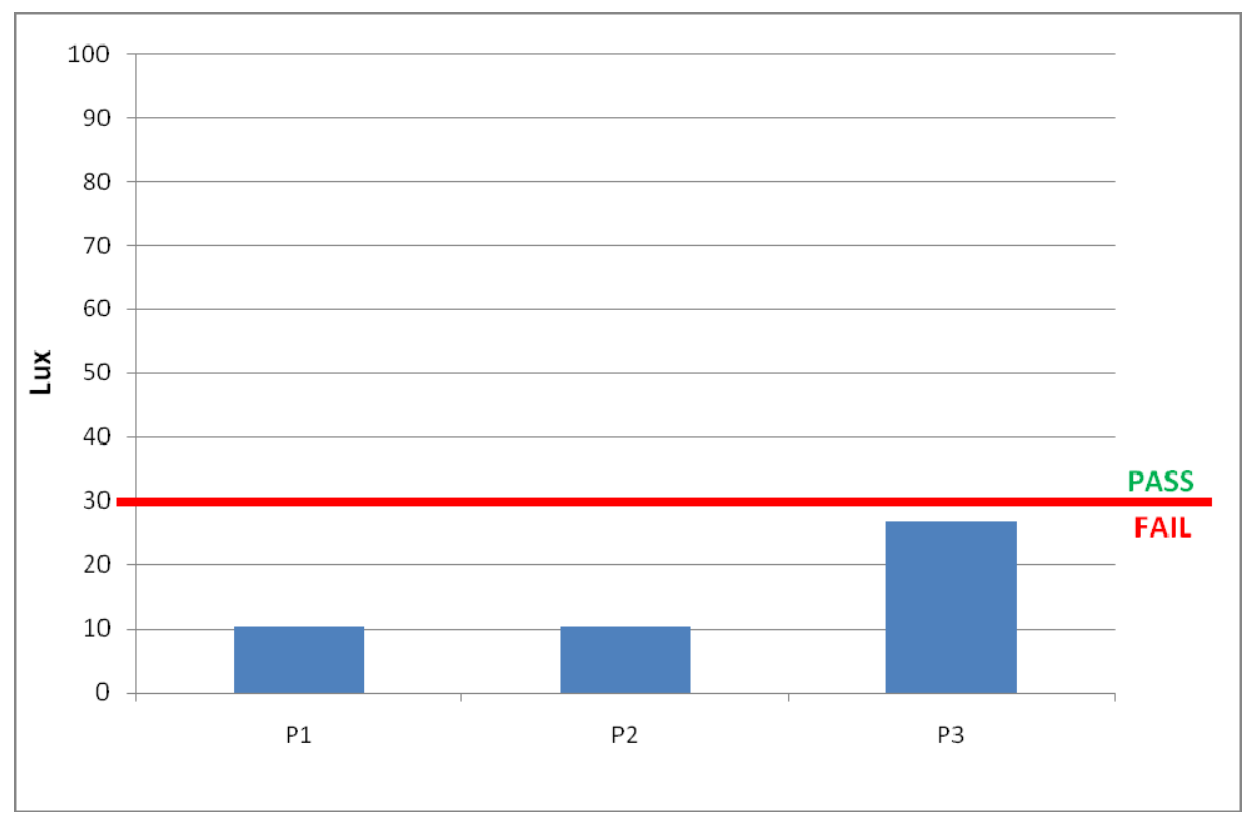

Figure 58 - Illuminance exceeded for $\mathbf{7 5 \%}$ of the Standard Year in Apartment 22

From Figure 58 it can be seen that this apartment fails to meet the minimum requirement, as all measurement points failed to meet this requirement. 
Figure 59 shows the application of the compliance assessment tool for Apartment 22.

\begin{tabular}{|l|c|}
\hline \multicolumn{2}{|c|}{ NZBC G7 Compliance Assessment Tool } \\
\hline \multicolumn{2}{|c|}{ Variable: } \\
\hline Opposite Building Height & 12 \\
\hline Street Width & 3 \\
\hline Glazed Area & 54 \\
\hline Orientation & North \\
\hline Location & Wellington \\
\hline Reflectance of Opposite Building & 21 \\
\hline Glazing Transmittance & 87 \\
\hline Vertical Location of lowest apartment & 7 \\
\hline \multicolumn{2}{|c|}{} \\
\hline Are simulations required to prove compliance: \\
\hline
\end{tabular}

Figure 59 - Application of the tool for Apartment 22

The measured data in Figure 58 shows that apartment 22 fails to comply with NZBC Clause G7 and Figure 59 identifies that the tool does not agree with this.

Table 58 provides a comparison of the results from each of the prediction models. All three of the models provided the incorrect outcome for this case. The linear model provided the best prediction but was still $30 \%$ DA over the threshold.

Table 58 - Prediction Model Results for Apartment 22

\begin{tabular}{|c|c|c|c|}
\hline Measurements & Linear Model & Model 2A & Model 2B \\
\hline Fail & 105 & 137 & 315 \\
\hline
\end{tabular}




\section{APARTMENT 28}

Figure 60 shows the illuminance levels exceeded for $75 \%$ of the standard year based on the daylight factor measurements taken in Apartment 28.

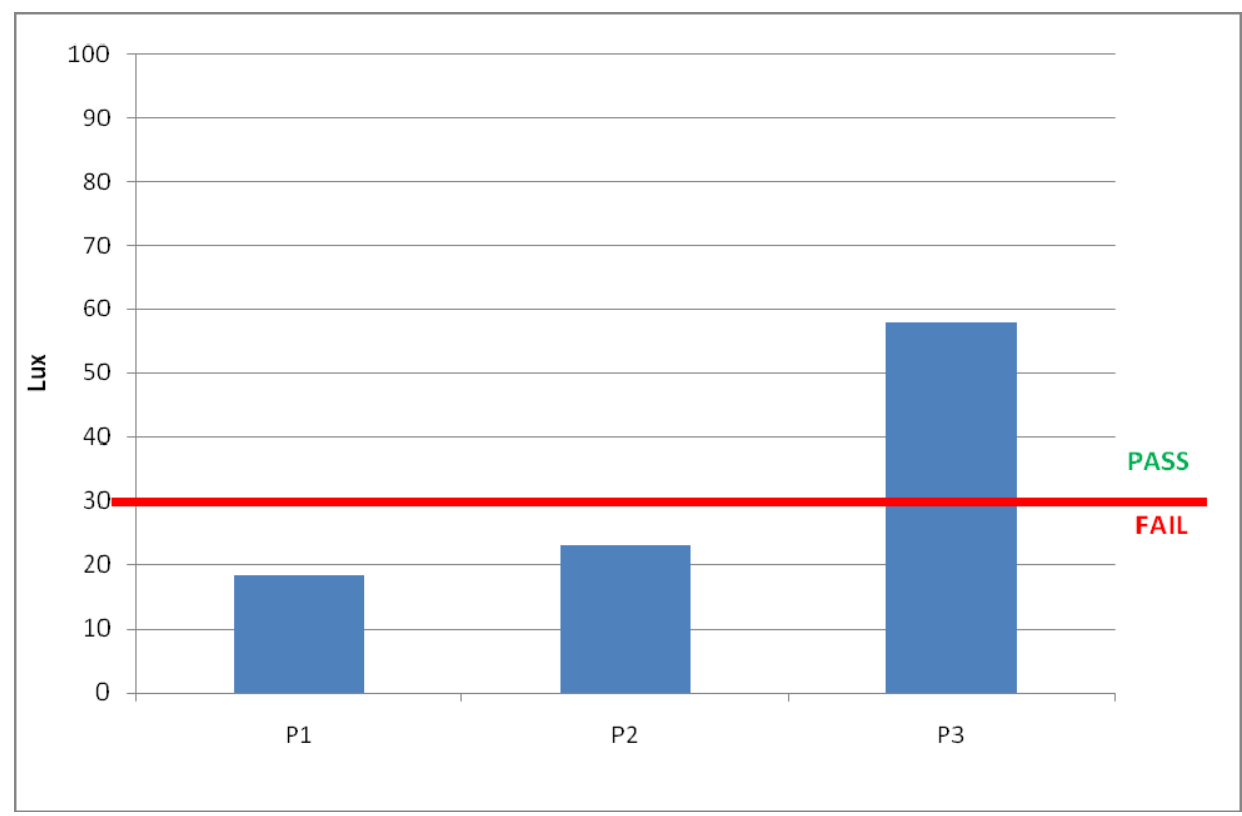

Figure 60 - Illuminance exceeded for $\mathbf{7 5 \%}$ of the Standard Year in Apartment 28

From Figure 60 it can be seen that measurement points 1 and 2 failed to meet the minimum requirement, meaning the apartment fails to meet the minimum requirement overall. Measurement point 3 has an illuminance of 57 lux, this indicates that there is adequate natural light reaching some areas at the back of the apartment. Because there is a noticeable difference between the illuminance at points 1 and 2 and point 3 it is possible the lower illuminances are due to shading from furniture. 
Figure 61 shows the application of the compliance assessment tool for Apartment 28.

\begin{tabular}{|l|c|}
\hline \multicolumn{2}{|c|}{ NZBC G7 Compliance Assessment Tool } \\
\hline \multicolumn{2}{|c|}{ Variable: } \\
\hline Opposite Building Height & 26 \\
\hline Street Width & 24 \\
\hline Glazed Area & 26 \\
\hline Orientation & West \\
\hline Location & Christchurch \\
\hline Reflectance of Opposite Building & 20.8 \\
\hline Glazing Transmittance & 87 \\
\hline Vertical Location of lowest apartment & 8 \\
\hline \multicolumn{2}{|c|}{} \\
\hline Are simulations required to prove compliance: \\
\hline
\end{tabular}

Figure 61 - Application of the tool for Apartment 28

The measured data in Figure 60 shows that apartment 28 fails to comply with NZBC Clause G7 and Figure 61 identifies that the tool does not agree with this.

Table 59 provides a comparison of the results from each of the prediction models. All three of the models provided the incorrect outcome for this case. Model 2B provided the best prediction for this case as it was within $10 \%$ DA of the threshold.

Table 59 - Prediction Model Results for Apartment 28

\begin{tabular}{|c|c|c|c|}
\hline Measurements & Linear Model & Model 2A & Model 2B \\
\hline Fail & 104 & 123 & 83 \\
\hline
\end{tabular}




\section{APARTMENT 31}

Figure 62 shows the illuminance levels exceeded for $75 \%$ of the standard year based on the daylight factor measurements taken in Apartment 31.

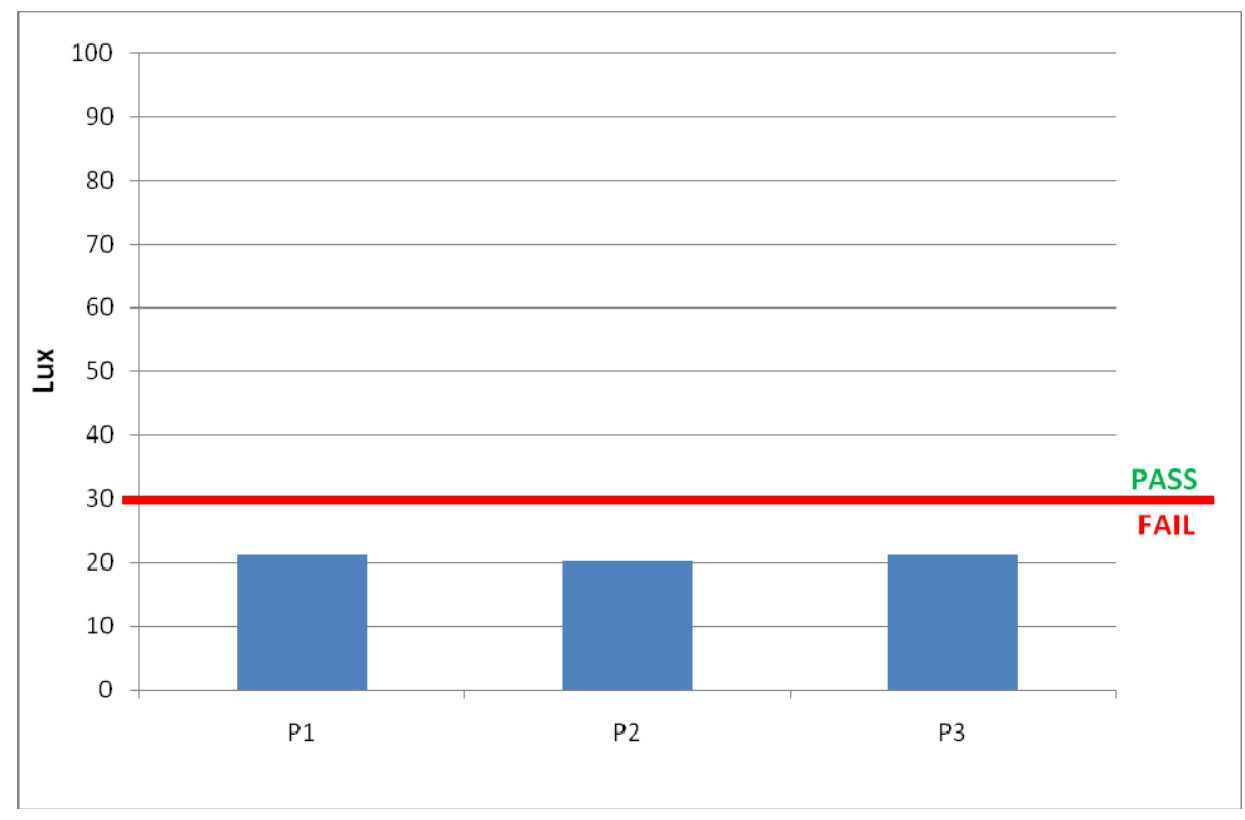

Figure 62 - Illuminance exceeded for $75 \%$ of the Standard Year in Apartment 31

From Figure 62 it can be seen that this apartment fails to meet the minimum requirement at all three measurement points. 
Figure 63 shows the application of the compliance assessment tool for Apartment 31.

\begin{tabular}{|l|c|}
\hline \multicolumn{2}{|c|}{ NZBC G7 Compliance Assessment Tool } \\
\hline \multicolumn{2}{|l|}{} \\
\hline Variable: & 0 \\
\hline Opposite Building Height & 9 \\
\hline Street Width & 31 \\
\hline Glazed Area & South \\
\hline Orientation & Auckland \\
\hline Location & 5 \\
\hline Reflectance of Opposite Building & 87 \\
\hline Glazing Transmittance & 11 \\
\hline Vertical Location of lowest apartment \\
\hline \multicolumn{2}{|c|}{} \\
\hline Are simulations required to prove compliance: \\
\hline
\end{tabular}

Figure 63 - Application of the tool for Apartment 31

The measured data in Figure 62 shows that apartment 31 fails to comply with NZBC Clause G7 and Figure 63 identifies that the tool does not agree with this.

Table 60 provides a comparison of the results from each of the prediction models. All three of the models provided the incorrect outcome for this case. The linear model provided the best prediction for this case, as it was 41\% DA out while the other two cases were $227 \%$ DA and $333 \%$ DA out respectively.

Table 60 - Prediction Model Results for Apartment 31

\begin{tabular}{|c|c|c|c|}
\hline Measurements & Linear Model & Model 2A & Model 2B \\
\hline Fail & 116 & 302 & 408 \\
\hline
\end{tabular}




\section{APARTMENT 33}

Figure 64 shows the illuminance levels exceeded for $75 \%$ of the standard year based on the daylight factor measurements taken in Apartment 33.

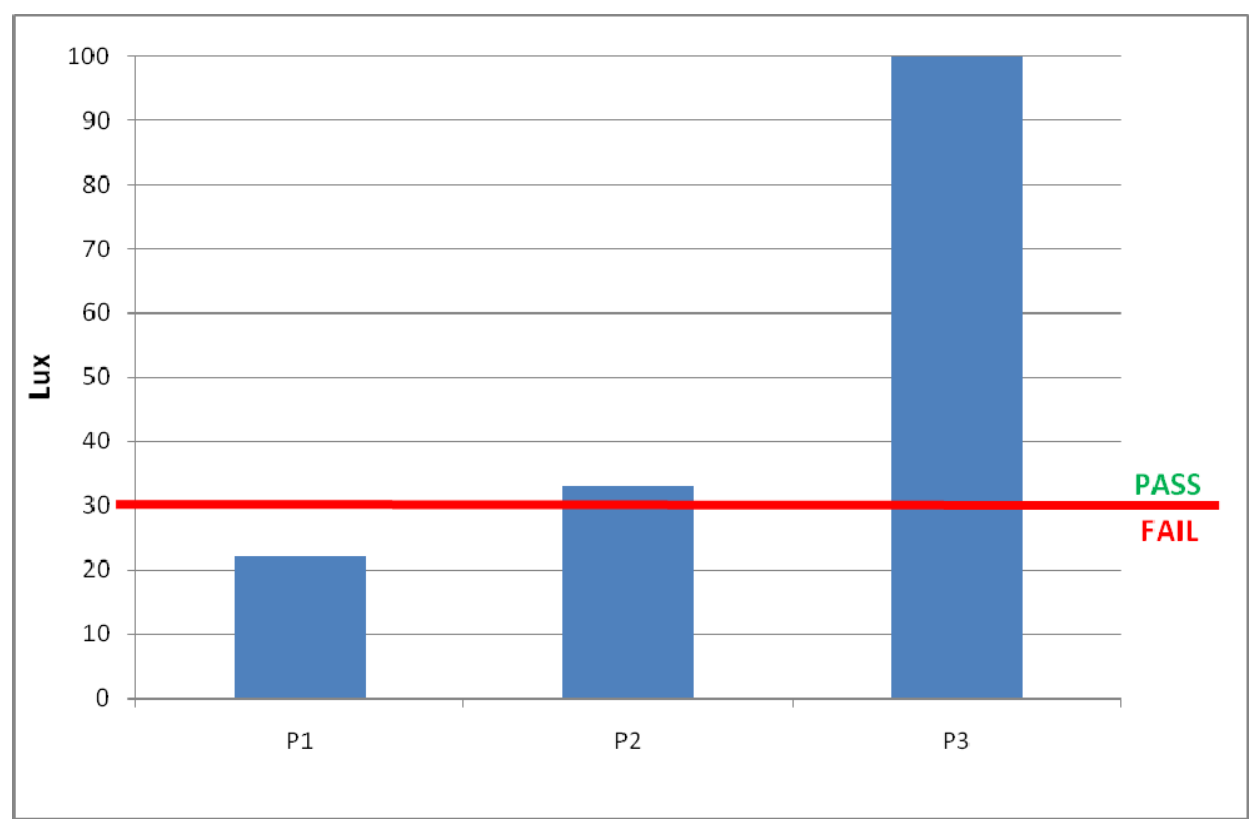

Figure 64 - Illuminance exceeded for $75 \%$ of the Standard Year in Apartment 33

From Figure 64 it can be seen that measurement point 1 failed to meet the minimum requirement, meaning the apartment fails to meet the minimum requirement. Measurement point 3 has an illuminance of over 100 lux, this indicates that there is adequate natural light reaching some areas at the back of the apartment. Due to the large variation in results, it is possible that furniture configuration may have caused the fail result for point 1 and that as an empty space this apartment may exceed the minimum requirement. 
Figure 65 shows the application of the compliance assessment tool for Apartment 33.

\begin{tabular}{|l|c|}
\hline \multicolumn{2}{|c|}{ NZBC G7 Compliance Assessment Tool } \\
\hline \multicolumn{2}{|l|}{ Variable: } \\
\hline Opposite Building Height & 75 \\
\hline Street Width & 9.5 \\
\hline Glazed Area & 11.8 \\
\hline Orientation & East \\
\hline Location & Wellington \\
\hline Reflectance of Opposite Building & 12 \\
\hline Glazing Transmittance & 87 \\
\hline Vertical Location of lowest apartment & 11 \\
\hline \multicolumn{2}{|c}{} \\
\hline Are simulations required to prove compliance: \\
\hline
\end{tabular}

Figure 65 - Application of the tool for Apartment 33

The measured data in Figure 64 shows that apartment 33 fails to comply with NZBC Clause G7. No result was provided for Figure 65 as the prediction models did not agree. The comparison of the prediction model results in Table 61 identified that the linear models and model 2B provided the correct results for this case, however model $2 \mathrm{~A}$ over-estimated the results for this case by more than $120 \%$ DA.

Table 61 - Prediction Model Results for Apartment 33

\begin{tabular}{|c|c|c|c|}
\hline Measurements & Linear Model & Model 2A & Model 2B \\
\hline Fail & 50 & 198 & -0.6 \\
\hline
\end{tabular}




\section{APARTMENT 34}

Figure 66 shows the illuminance levels exceeded for $75 \%$ of the standard year based on the daylight factor measurements taken in Apartment 34.

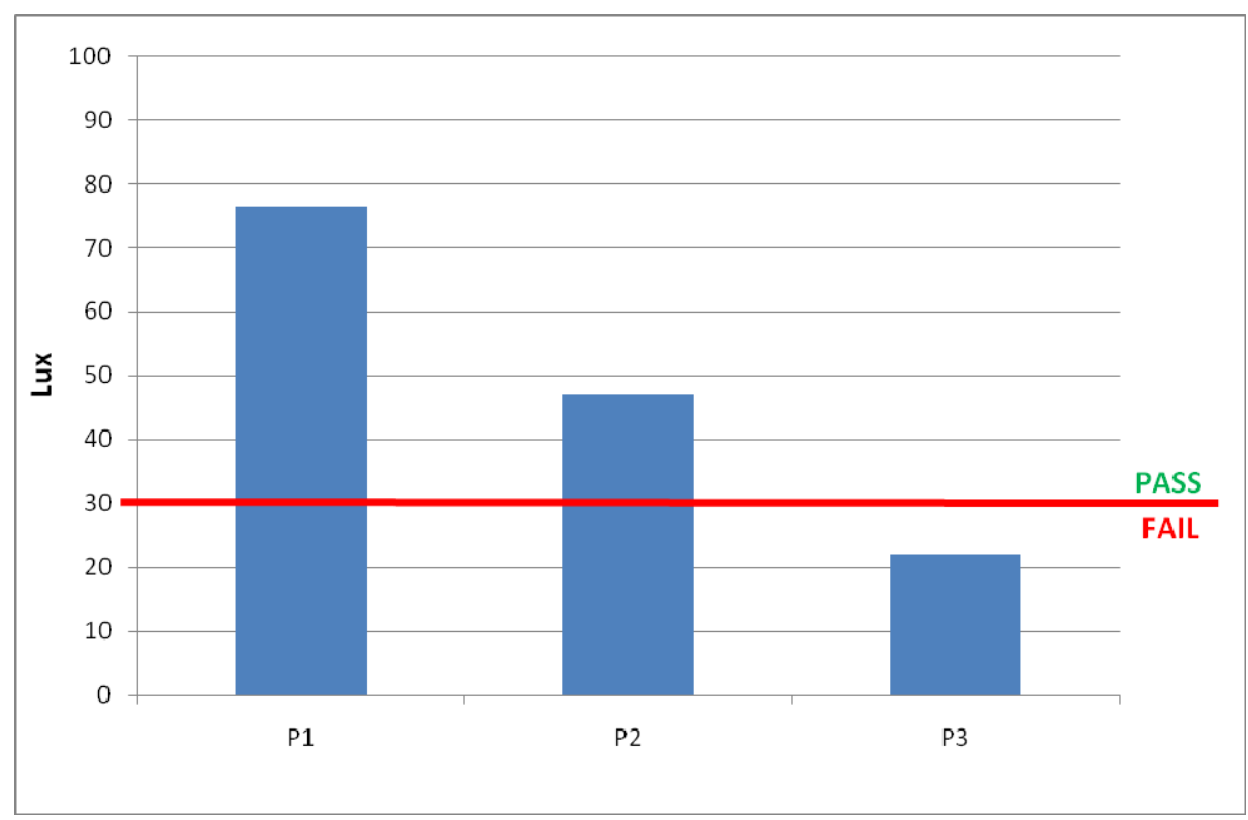

Figure 66 - Illuminance exceeded for $75 \%$ of the Standard Year in Apartment 34

From Figure 66 it can be seen that measurement point 3 failed to meet the minimum requirement, meaning the apartment fails to meet the minimum requirement overall. Measurement points 1 and 2 both exceed the minimum requirement indicating that there is adequate natural light reaching some areas at the back of the apartment. Due to the variation in results, it is possible that furniture configuration may have caused the fail result for point 3 and that as an unfurnished space this apartment may exceed the minimum requirement. 
Figure 67 shows the application of the compliance assessment tool for Apartment 34.

\begin{tabular}{|l|c|}
\hline \multicolumn{2}{|c|}{ NZBC G7 Compliance Assessment Tool } \\
\hline \multicolumn{2}{|l|}{ Variable: } \\
\hline Opposite Building Height & 32 \\
\hline Street Width & 50 \\
\hline Glazed Area & 32 \\
\hline Orientation & West \\
\hline Location & Wellington \\
\hline Reflectance of Opposite Building & 33.3 \\
\hline Glazing Transmittance & 87 \\
\hline Vertical Location of lowest apartment & 0 \\
\hline \multicolumn{2}{|c|}{} \\
\hline Are simulations required to prove compliance: \\
\hline
\end{tabular}

Figure 67 - Application of the tool for Apartment 34

The measured data in Figure 66 shows that apartment 34 fails to comply with NZBC Clause G7 and Figure 67 confirms that the tool does not agree with this.

Table 62 provides a comparison of the results from each of the prediction models. All three of the models provided the incorrect outcome for this case.

Table 62 - Prediction Model Results for Apartment 34

\begin{tabular}{|c|c|c|c|}
\hline Measurements & Linear Model & Model 2A & Model 2B \\
\hline Fail & 132 & 94 & 86 \\
\hline
\end{tabular}

Apartment 34 was situated at ground level and had an enclosed courtyard in front of the living area as shown in Figure 68.

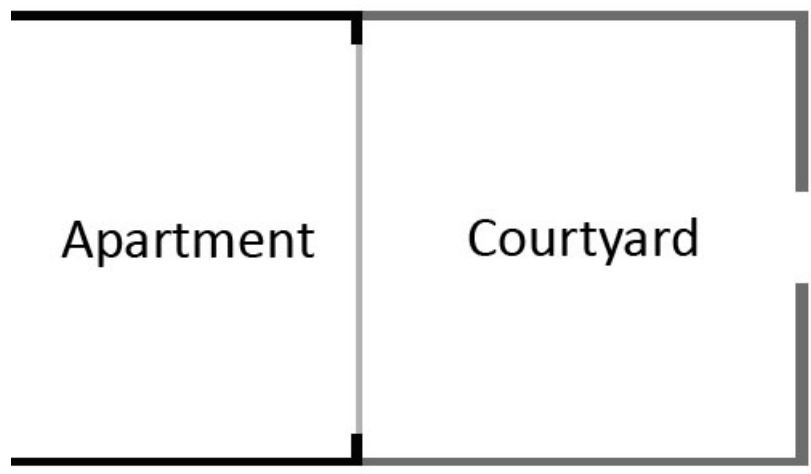

Figure 68- Location of Courtyard in reference to Apartment 34

The application of the tool in Figure 68 assumed that the courtyard was not there and the main obstruction for this apartment was adjacent buildings. Figure 69 shows the result given by the tool if the courtyard boundary walls were consider the main obstructions. 


\begin{tabular}{|l|c|}
\hline \multicolumn{2}{|c|}{ NZBC G7 Compliance Assessment Tool } \\
\hline \multicolumn{2}{|l|}{} \\
\hline Variable: & 2 \\
\hline Opposite Building Height & 6 \\
\hline Street Width & 32 \\
\hline Glazed Area & West \\
\hline Orientation & Wellington \\
\hline Location & 38.5 \\
\hline Reflectance of Opposite Building & 87 \\
\hline Glazing Transmittance & 0 \\
\hline Vertical Location of lowest apartment \\
\hline \multicolumn{2}{|c|}{} \\
\hline Are simulations required to prove compliance: \\
\hline
\end{tabular}

Figure 69 - Application of the tool for Apartment 34 with Courtyard

The measured data in Figure 66 shows that apartment 34 fails to comply with NZBC Clause G7 and Figure 69 confirms that when the courtyard is the main obstruction the tool agrees with this.

Table 63 provides a comparison of the results from each of the prediction models. All three of the models provided the correct outcome for this case.

Table 63 - Prediction Model Results for Apartment 34 with Courtyard

\begin{tabular}{|c|c|c|c|}
\hline Measurements & Linear Model & Model 2A & Model 2B \\
\hline Fail & 65 & 67 & 64 \\
\hline
\end{tabular}

The analysis of apartment 34 highlighted the importance of applying the tool correctly to receive the best results. It is crucial to document aspects like this in the instruction on how to use the tool. 


\section{APARTMENT 35}

Figure 70 shows the illuminance levels exceeded for $75 \%$ of the standard year based on the daylight factor measurements taken in Apartment 35.

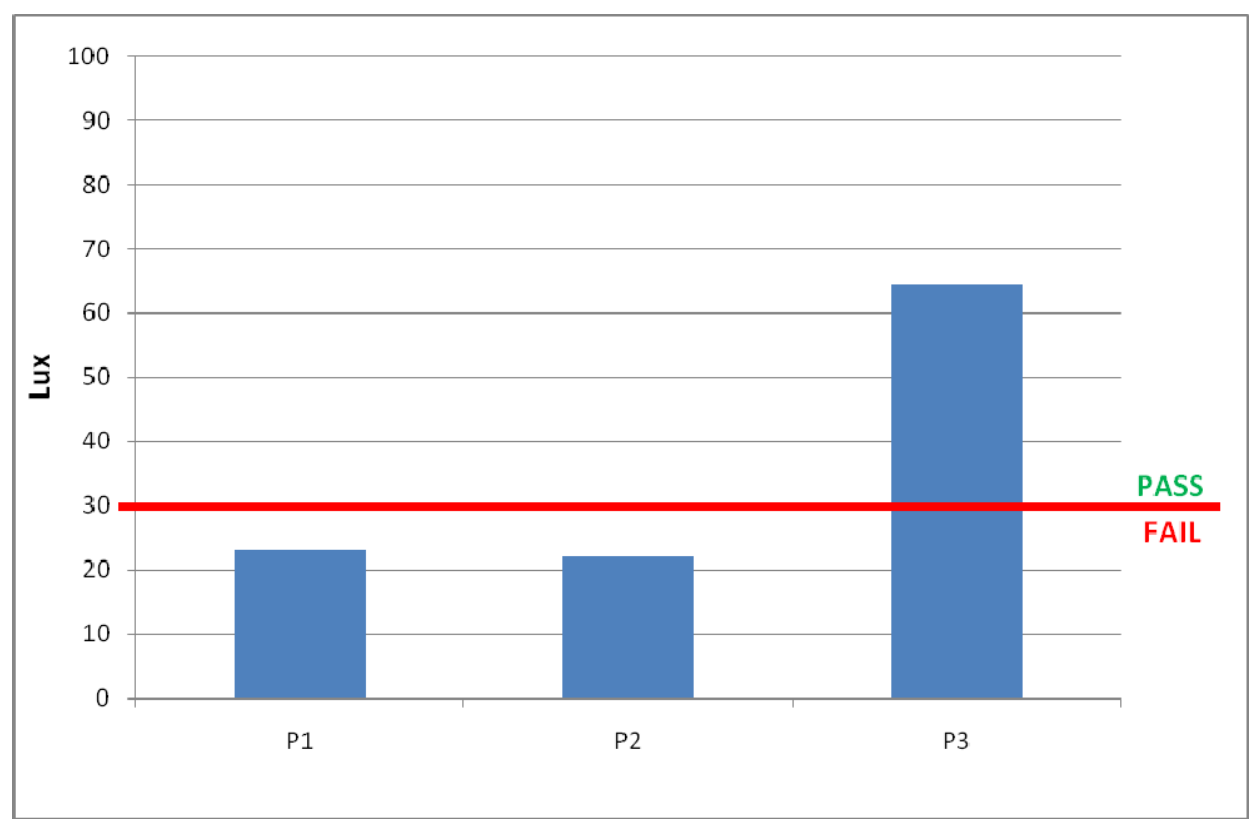

Figure 70 - Illuminance exceeded for $\mathbf{7 5 \%}$ of the Standard Year in Apartment 35

From Figure 70 it can be seen that measurement points 1 and 2 failed to meet the minimum requirement, meaning the apartment fails to meet the minimum requirement overall. Measurement point 3 has an illuminance just over twice the minimum requirement, indicating that there is adequate natural light reaching some areas at the back of the apartment. The variation in results is possibly due to furniture configuration and may have been responsible for the fail results for points 1 and 2 , as an unfurnished space this apartment may exceed the minimum requirement. 
Figure 71 shows the application of the compliance assessment tool for Apartment 35.

\begin{tabular}{|l|c|}
\hline \multicolumn{2}{|c|}{ NZBC G7 Compliance Assessment Tool } \\
\hline \multicolumn{2}{|l|}{} \\
\hline Variable: \\
\hline Opposite Building Height & 0 \\
\hline Street Width & 0 \\
\hline Glazed Area & 26 \\
\hline Orientation & South \\
\hline Location & Wellington \\
\hline Reflectance of Opposite Building & 0 \\
\hline Glazing Transmittance & 87 \\
\hline Vertical Location of lowest apartment & 11 \\
\hline \multicolumn{2}{|c|}{} \\
\hline Are simulations required to prove compliance: \\
\hline
\end{tabular}

Figure 71 - Application of the tool for Apartment 35

The measured data in Figure 70 shows that apartment 35 fails to comply with NZBC Clause G7 and Figure 71 identifies that the tool does not agree with this.

Table 64 provides a comparison of the results from each of the prediction models. All three of the models provided the incorrect outcome for this case. The model with the closest prediction is the linear model, which is $11 \%$ DA over the threshold.

Table 64 - Prediction Model Results for Apartment 35

\begin{tabular}{|c|c|c|c|}
\hline Measurements & Linear Model & Model 2A & Model 2B \\
\hline Fail & 86 & 272 & 296 \\
\hline
\end{tabular}




\section{APARTMENT 37}

Figure 72 shows the illuminance levels exceeded for $75 \%$ of the standard year based on the daylight factor measurements taken in Apartment 37.

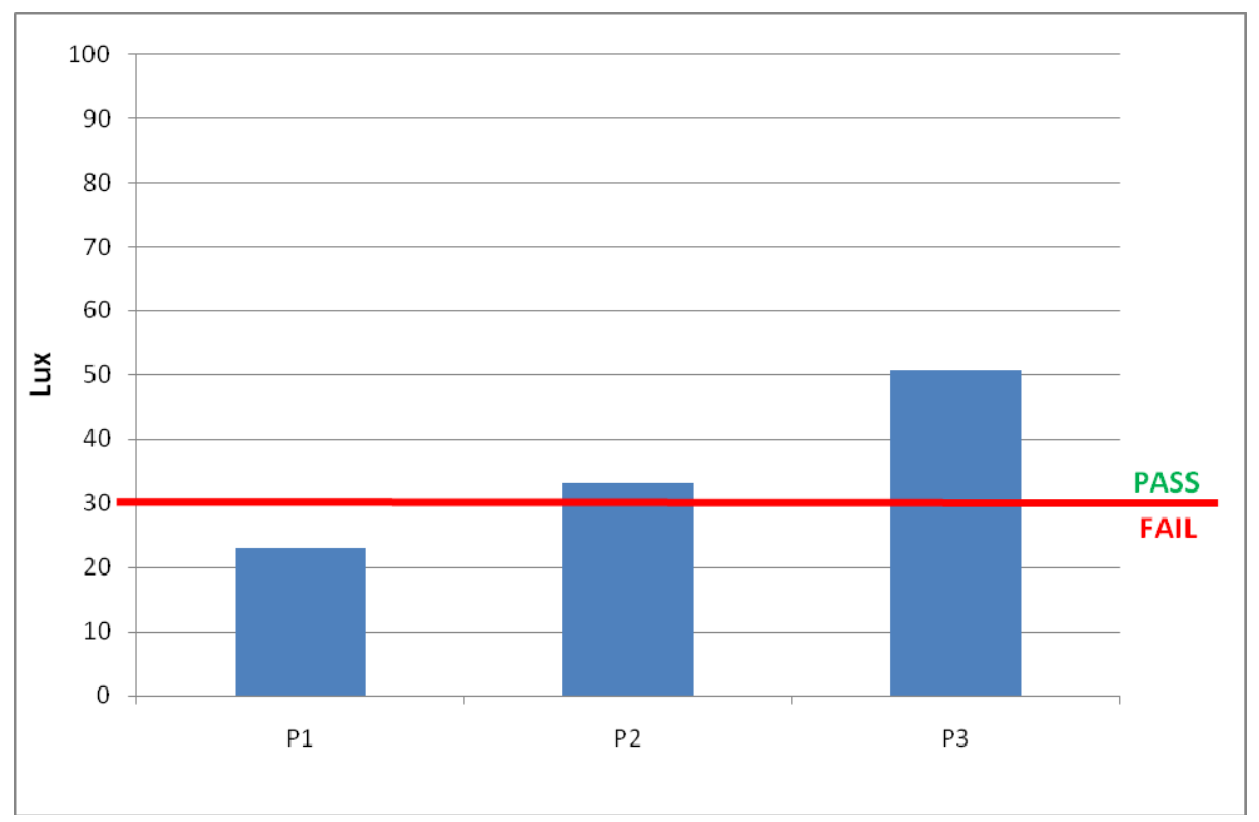

Figure 72 - Illuminance exceeded for $\mathbf{7 5 \%}$ of the Standard Year in Apartment 37

From Figure 72 it can be seen that measurement point 1 failed to meet the minimum requirement, meaning the apartment fails to meet the minimum requirement overall. The variation in results is possibly due to furniture configuration and may have been responsible for the fail results for points 1 , but as the highest illuminance exceeded for $75 \%$ of the standard in the apartment was 50 lux it is unlikely that the furniture had a major affect on the results. 
Figure 73 shows the application of the compliance assessment tool for Apartment 37.

\begin{tabular}{|l|c|}
\hline \multicolumn{2}{|c|}{ NZBC G7 Compliance Assessment Tool } \\
\hline \multicolumn{2}{|l|}{} \\
\hline Variable: \\
\hline Opposite Building Height & 11.5 \\
\hline Street Width & 52 \\
\hline Glazed Area & 32 \\
\hline Orientation & North \\
\hline Location & Wellington \\
\hline Reflectance of Opposite Building & 38.5 \\
\hline Glazing Transmittance & 87 \\
\hline Vertical Location of lowest apartment & 0 \\
\hline \multicolumn{2}{|c|}{} \\
\hline Are simulations required to prove compliance: \\
\hline
\end{tabular}

Figure 73 - Application of the tool for Apartment 37

The measured data in Figure 72 shows that apartment 37 fails to comply with NZBC Clause G7 and Figure 73 identifies that the tool does not agree with this.

Table 65 provides a comparison of the results from each of the prediction models. All three of the models provided the incorrect outcome for this case.

Table 65 - Prediction Model Results for Apartment 37

\begin{tabular}{|c|c|c|c|}
\hline Measurements & Linear Model & Model 2A & Model 2B \\
\hline Fail & 144 & 124 & 118 \\
\hline
\end{tabular}


Apartment 37 was situated at ground level and had an enclosed courtyard in front of the living area similar to Apartment 34. The application of the tool in Figure 74 assumed that the courtyard was not there and the main obstruction for this apartment was adjacent buildings. Figure 74 shows the result given by the tool if the courtyard boundary walls were consider the main obstructions.

\begin{tabular}{|l|c|}
\hline \multicolumn{2}{|c|}{ NZBC G7 Compliance Assessment Tool } \\
\hline \multicolumn{2}{|l|}{} \\
\hline Variable: & 2 \\
\hline Opposite Building Height & 6 \\
\hline Street Width & 32 \\
\hline Glazed Area & North \\
\hline Orientation & Wellington \\
\hline Location & 38.5 \\
\hline Reflectance of Opposite Building & 87 \\
\hline Glazing Transmittance & 0 \\
\hline Vertical Location of lowest apartment \\
\hline \multicolumn{2}{|}{} \\
\hline Are simulations required to prove compliance: \\
\hline
\end{tabular}

Figure 74 - Application of the tool for Apartment 37 with Courtyard

The measured data in Figure 72 shows that apartment 37 fails to comply with NZBC Clause G7 and Figure 74 confirms that when the courtyard is used as the main obstruction the tool agrees with this.

Table 66 provides a comparison of the results from each of the prediction models. All three of the models provided the correct outcome for this case.

Table 66 - Prediction Model Results for Apartment 37 with Courtyard

\begin{tabular}{|c|c|c|c|}
\hline Measurements & Linear Model & Model 2A & Model 2B \\
\hline Fail & 63 & 64 & 61 \\
\hline
\end{tabular}




\section{Appendix 7 - Analysis of False Fail Calibration Apartments}

Appendix 7 provides the analysis of the apartments that had false fail results given by at least one of the prediction equations. A false fail apartment is where the prediction equation said that simulation was needed but the measurements identified that the apartment exceeded the minimum requirement. 


\section{APARTMENT 51}

Figure 75 shows the illuminance levels exceeded for $75 \%$ of the standard year based on the daylight factor measurements taken in Apartment 51.

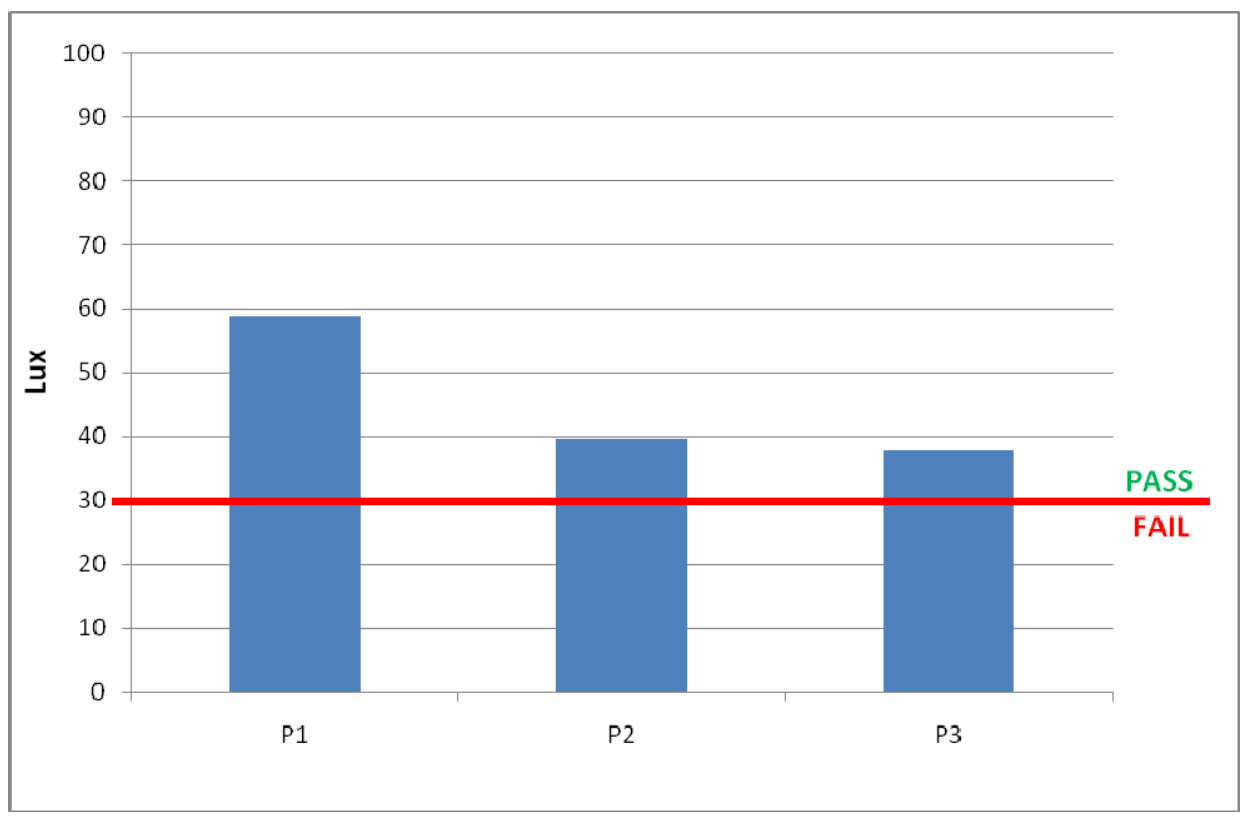

Figure 75 - Illuminance exceeded for $\mathbf{7 5 \%}$ of the Standard Year in Apartment 51

From Figure 75 it can be seen that this apartment exceeds the minimum requirement at all three measurement points. Variations in the results may have been cause by furniture configuration, but as the lowest points measured still exceed the minimum requirement, the effect of furniture on the results is less important. 
Figure 76 shows the application of the compliance assessment tool for Apartment 51.

\begin{tabular}{|l|c|}
\hline \multicolumn{2}{|c|}{ NZBC G7 Compliance Assessment Tool } \\
\hline \multicolumn{2}{|l|}{} \\
\hline Variable: & 20 \\
\hline Opposite Building Height & 5 \\
\hline Street Width & 23 \\
\hline Glazed Area & North \\
\hline Orientation & Wellington \\
\hline Location & 33.3 \\
\hline Reflectance of Opposite Building & 87 \\
\hline Glazing Transmittance & 3 \\
\hline Vertical Location of lowest apartment \\
\hline \multicolumn{2}{|}{} \\
\hline Are simulations required to prove compliance: \\
\hline
\end{tabular}

Figure 76 - Application of the tool for Apartment 51

The measured data in Figure 75 shows that apartment 51 complies with NZBC Clause G7 and Figure 76 identifies that the tool does not agree with this.

Table 67 provides a comparison of the results from each of the prediction models. All three of the models provided the incorrect outcome for this case. The linear model provided the best result but was still $23 \%$ DA away from the threshold.

Table 67 - Prediction Model Results for Apartment 51

\begin{tabular}{|c|c|c|c|}
\hline Measurements & Linear Model & Model 2A & Model 2B \\
\hline Pass & 52 & 15 & 19 \\
\hline
\end{tabular}




\section{APARTMENT 53}

Figure 77 shows the illuminance levels exceeded for $75 \%$ of the standard year based on the daylight factor measurements taken in Apartment 53.

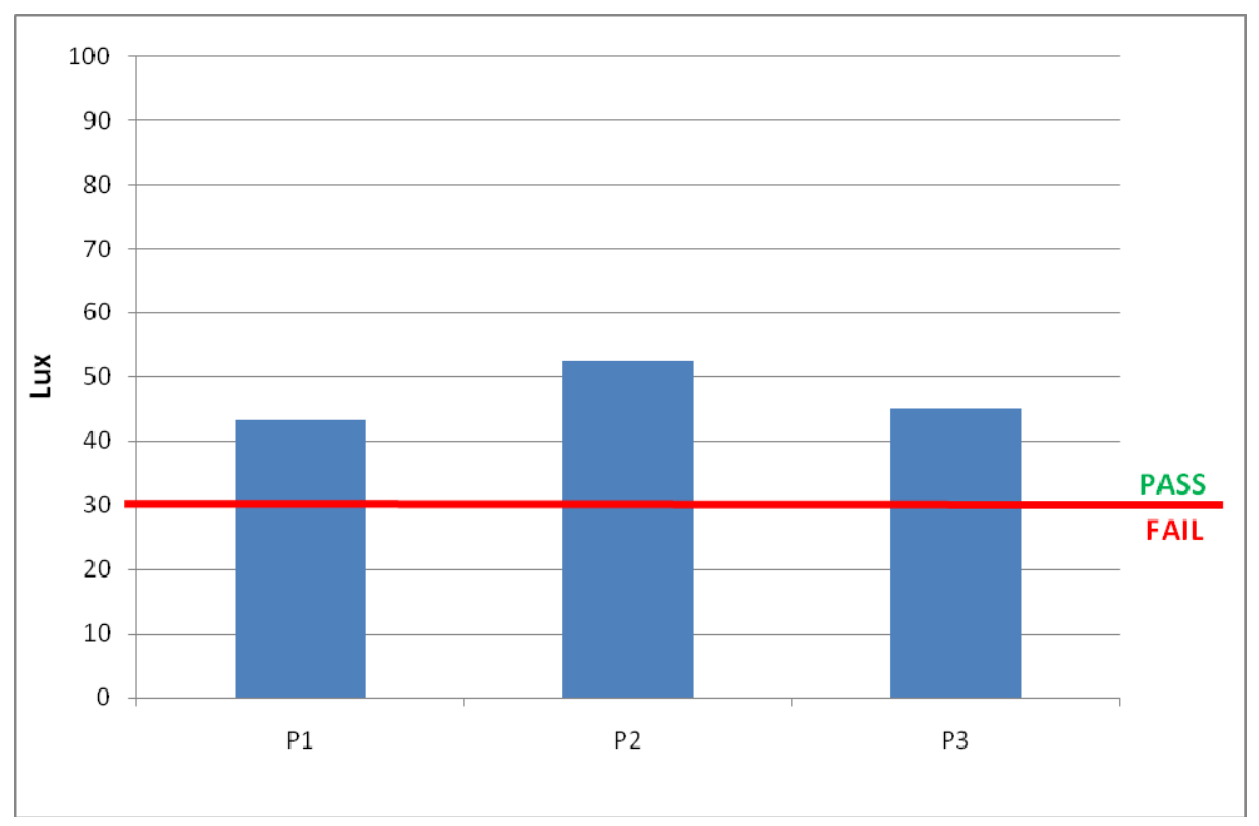

Figure 77 - Illuminance exceeded for $\mathbf{7 5 \%}$ of the Standard Year in Apartment 53

From Figure 77 it can be seen that this apartment exceeds the minimum requirement at all three measurement points 
Figure 78 shows the application of the compliance assessment tool for Apartment 53.

\begin{tabular}{|l|c|}
\hline \multicolumn{2}{|c|}{ NZBC G7 Compliance Assessment Tool } \\
\hline \multicolumn{2}{|l|}{} \\
\hline Variable: & 11.5 \\
\hline Opposite Building Height & 15.5 \\
\hline Street Width & 24 \\
\hline Glazed Area & North \\
\hline Orientation & Wellington \\
\hline Location & 13.5 \\
\hline Reflectance of Opposite Building & 87 \\
\hline Glazing Transmittance & 4 \\
\hline Vertical Location of lowest apartment & \\
\hline \multicolumn{2}{|c|}{} \\
\hline Are simulations required to prove compliance: \\
\hline
\end{tabular}

Figure 78 - Application of the tool for Apartment 53

The measured data in Figure 77 shows that apartment 53 complies with NZBC Clause G7. No result was provided for Figure 78 as the prediction models did not agree. The comparison of the prediction model results in Table 68 identified that the linear model provided the correct results for this case, however models $2 \mathrm{~A}$ and $2 \mathrm{~B}$ under-estimated the results for this case by more around $20 \% \mathrm{DA}$.

Table 68 - Prediction Model Results for Apartment 53

\begin{tabular}{|c|c|c|c|}
\hline Measurements & Linear Model & Model 2A & Model 2B \\
\hline Pass & 76 & 53 & 58 \\
\hline
\end{tabular}




\section{APARTMENT 56}

Figure 79 shows the illuminance levels exceeded for $75 \%$ of the standard year based on the daylight factor measurements taken in Apartment 56.

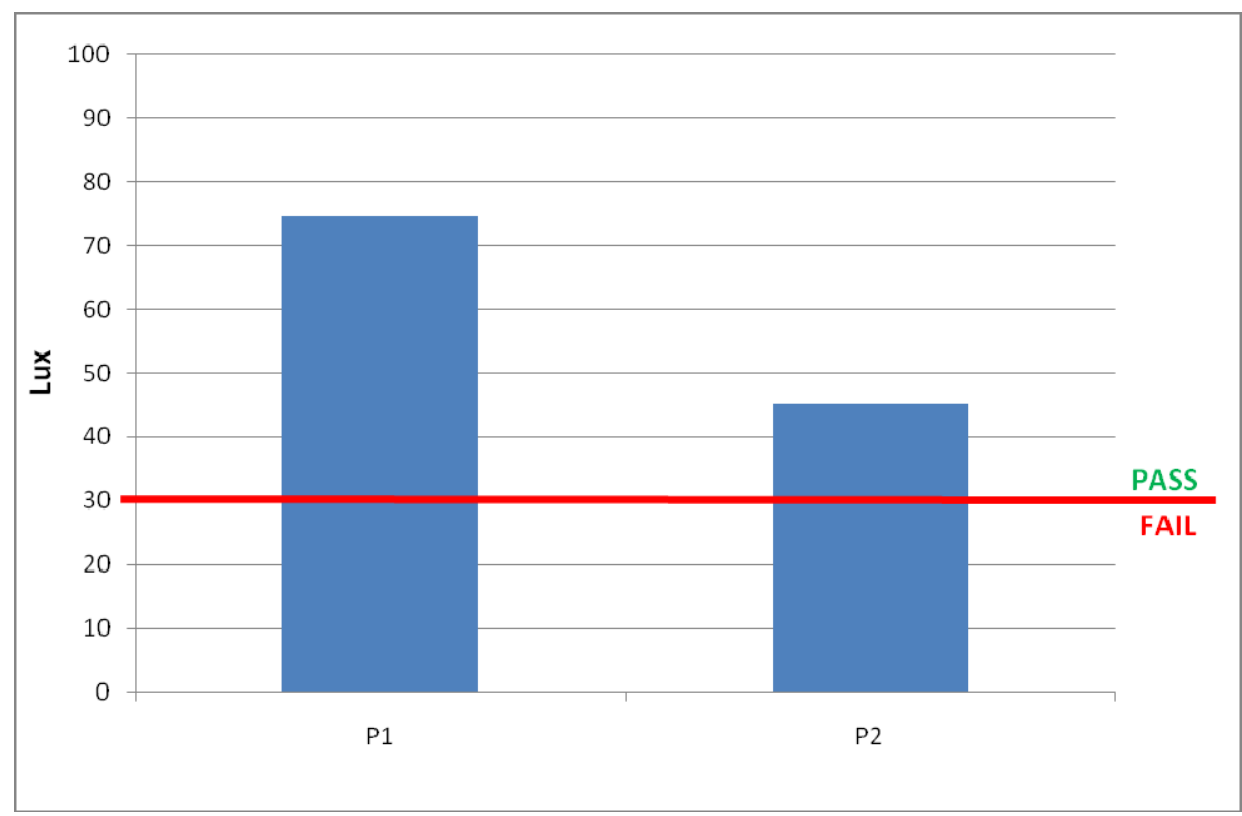

Figure 79 - Illuminance exceeded for $\mathbf{7 5 \%}$ of the Standard Year in Apartment 56

From Figure 79 it can be seen that this apartment exceeds the minimum requirement the minimum requirement at both measurement points. 
Figure 80 shows the application of the compliance assessment tool for Apartment 56.

\begin{tabular}{|l|c|}
\hline \multicolumn{2}{|c|}{ NZBC G7 Compliance Assessment Tool } \\
\hline \multicolumn{2}{|l|}{} \\
\hline Variable: & 20 \\
\hline Opposite Building Height & 5 \\
\hline Street Width & 21 \\
\hline Glazed Area & North \\
\hline Orientation & Wellington \\
\hline Location & 12 \\
\hline Reflectance of Opposite Building & 87 \\
\hline Glazing Transmittance & 6 \\
\hline Vertical Location of lowest apartment \\
\hline \multicolumn{2}{|}{} \\
\hline Are simulations required to prove compliance: \\
\hline
\end{tabular}

Figure 80 - Application of the tool for Apartment 56

The measured data in Figure 79 shows that apartment 56 complies with NZBC Clause G7 and Figure 80 identifies that the tool does not agree with this.

Table 69 provides a comparison of the results from each of the prediction models. All three of the models provided the incorrect outcome for this case. The linear model provided the best prediction for this case.

Table 69 - Prediction Model Results for Apartment 56

\begin{tabular}{|c|c|c|c|}
\hline Measurements & Linear Model & Model 2A & Model 2B \\
\hline Pass & 56 & 40 & 29 \\
\hline
\end{tabular}




\section{APARTMENT 65}

Figure 81 shows the illuminance levels exceeded for $75 \%$ of the standard year based on the daylight factor measurements taken in Apartment 65.

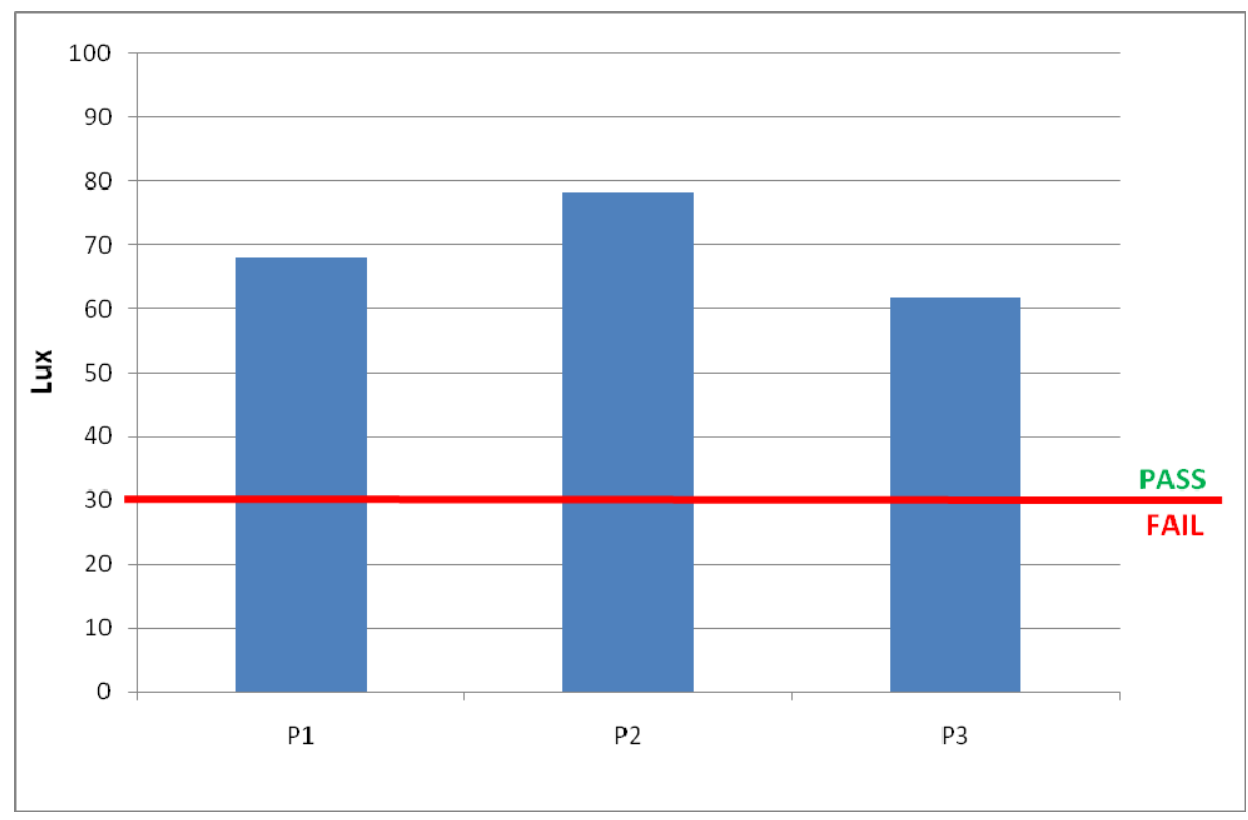

Figure 81 - Illuminance exceeded for $75 \%$ of the Standard Year in Apartment 65

From Figure 81 it can be seen that this apartment exceeds the minimum requirement with all measurement points having illuminance well over the minimum of 30 lux for $75 \%$ of the standard year. 
Figure 82 shows the application of the compliance assessment tool for Apartment 65.

\begin{tabular}{|l|c|}
\hline \multicolumn{2}{|c|}{ NZBC G7 Compliance Assessment Tool } \\
\hline \multicolumn{2}{|c|}{ Variable: } \\
\hline Opposite Building Height & 20 \\
\hline Street Width & 37 \\
\hline Glazed Area & 24 \\
\hline Orientation & East \\
\hline Location & Wellington \\
\hline Reflectance of Opposite Building & 38 \\
\hline Glazing Transmittance & 87 \\
\hline Vertical Location of lowest apartment & 1 \\
\hline \multicolumn{2}{|c|}{} \\
\hline Are simulations required to prove compliance: \\
\hline
\end{tabular}

Figure 82 - Application of the tool for Apartment 65

The measured data in Figure 81 shows that apartment 65 complies with NZBC Clause G7. No result was provided for Figure 82 as the prediction models did not agree. The comparison of the prediction model results in Table 70 identified that the linear model provided the correct results for this case, however models $2 \mathrm{~A}$ and $2 \mathrm{~B}$ under-estimated the results for this case by $1 \% \mathrm{DA}$ and $3 \% \mathrm{DA}$ respectively.

Table 70 - Prediction Model Results for Apartment 65

\begin{tabular}{|c|c|c|c|}
\hline Measurements & Linear Model & Model 2A & Model 2B \\
\hline Pass & 108 & 74 & 72 \\
\hline
\end{tabular}




\section{APARTMENT 66}

Figure 83 shows the illuminance levels exceeded for $75 \%$ of the standard year based on the daylight factor measurements taken in Apartment 66.

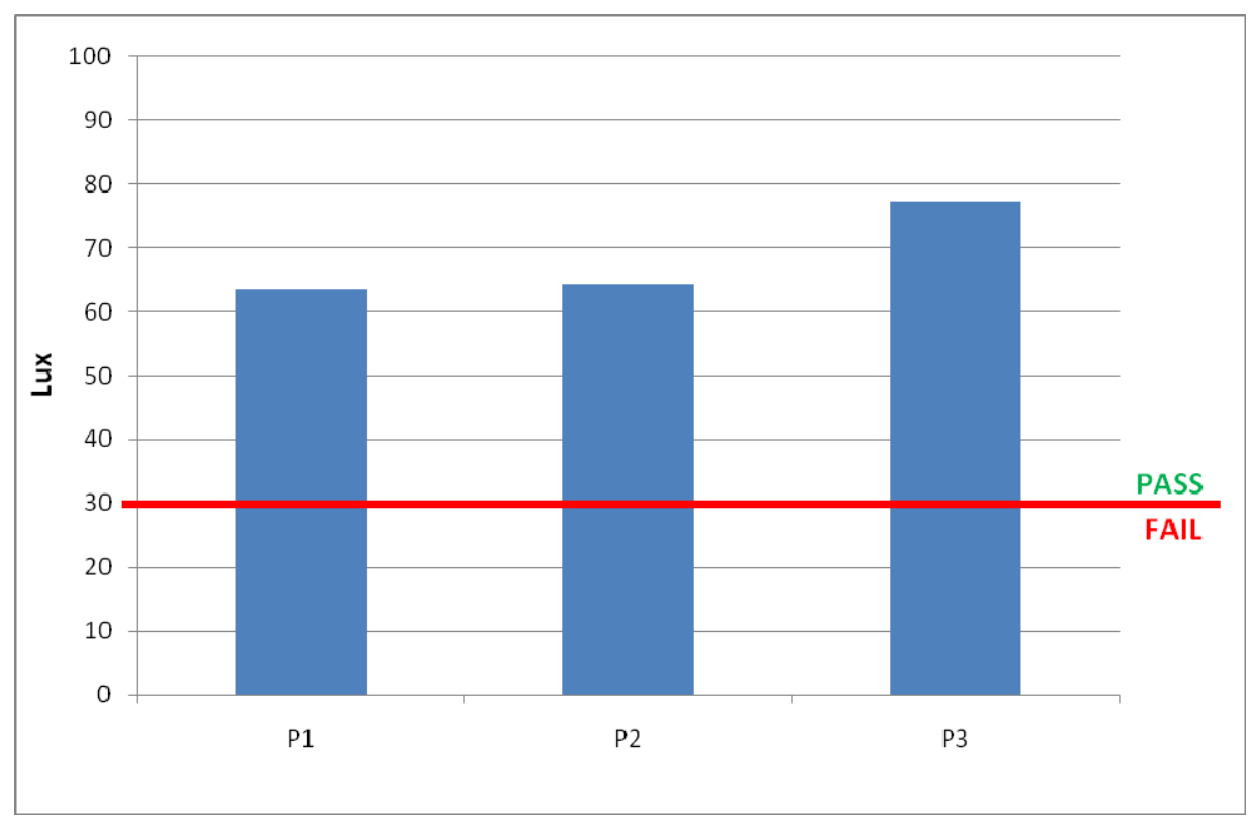

Figure 83 - Illuminance exceeded for $75 \%$ of the Standard Year in Apartment 66

From Figure 83 it can be seen that this apartment exceeds the minimum requirement, with all measurement points having adequate illuminance level for $75 \%$ of the standard year. 
Figure 84 shows the application of the compliance assessment tool for Apartment 66.

\begin{tabular}{|l|c|}
\hline \multicolumn{2}{|c|}{ NZBC G7 Compliance Assessment Tool } \\
\hline \multicolumn{2}{|l|}{ Variable: } \\
\hline Opposite Building Height & 19 \\
\hline Street Width & 25 \\
\hline Glazed Area & 12 \\
\hline Orientation & South \\
\hline Location & Wellington \\
\hline Reflectance of Opposite Building & 20 \\
\hline Glazing Transmittance & 87 \\
\hline Vertical Location of lowest apartment & 1 \\
\hline \multicolumn{2}{|c}{} \\
\hline Are simulations required to prove compliance: \\
\hline
\end{tabular}

Figure 84 - Application of the tool for Apartment 66

The measured data in Figure 83 shows that apartment 66 complies with NZBC Clause G7 and Figure 84 indicates that the tool does not agree with this.

Table 71 provides a comparison of the results from each of the prediction models. All three of the models provided the incorrect outcome for this case. The linear model provided the best prediction for this case but was at least 7\% DA under-predicted.

Table 71 - Prediction Model Results for Apartment 66

\begin{tabular}{|c|c|c|c|}
\hline Measurements & Linear Model & Model 2A & Model 2B \\
\hline Pass & 68 & 40 & 40 \\
\hline
\end{tabular}




\section{APARTMENT 67}

Figure 85 shows the illuminance levels exceeded for $75 \%$ of the standard year based on the daylight factor measurements taken in Apartment 67.

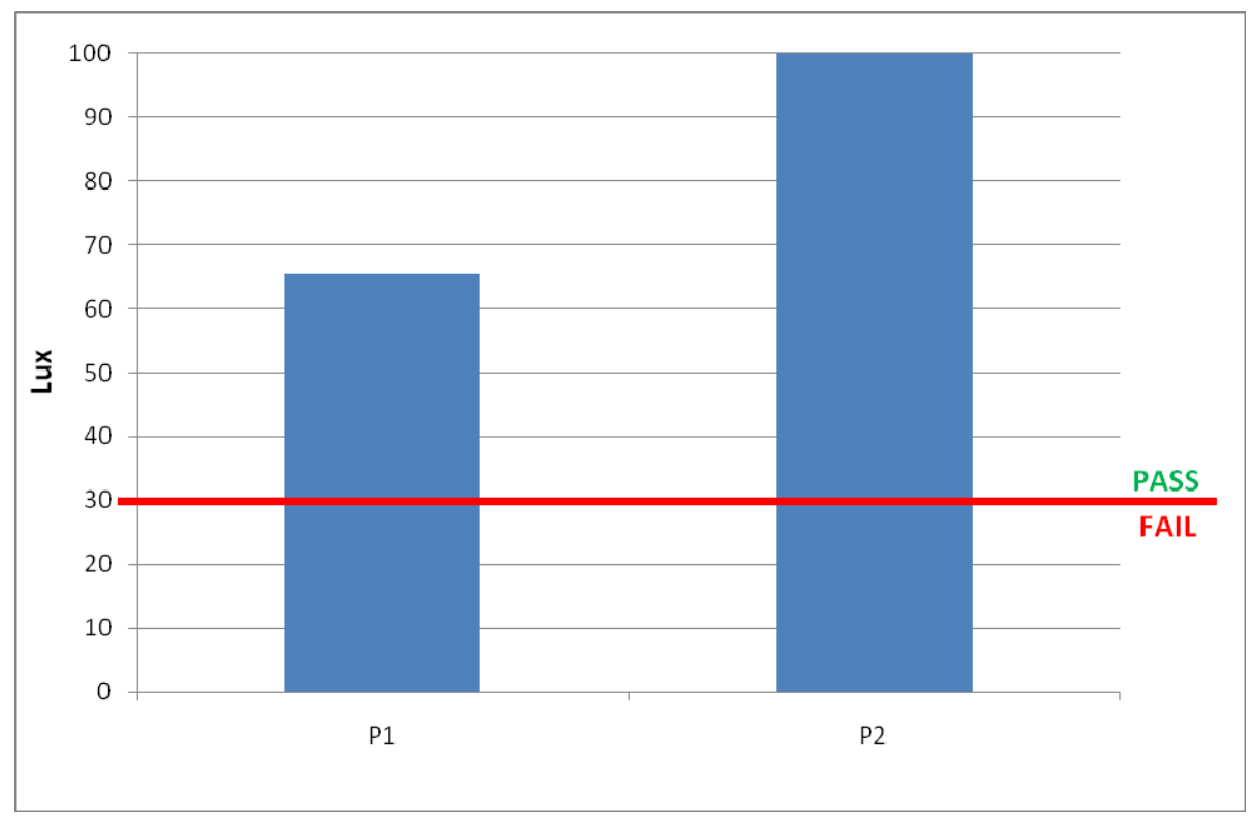

Figure 85 - Illuminance exceeded for $75 \%$ of the Standard Year in Apartment 67

From Figure 85 it can be seen that this apartment exceeds the minimum requirement, with both measurement points being well over the minimum of 30 lux for $75 \%$ of the standard year. 
Figure 86 shows the application of the compliance assessment tool for Apartment 67.

\begin{tabular}{|l|c|}
\hline \multicolumn{2}{|c|}{ NZBC G7 Compliance Assessment Tool } \\
\hline \multicolumn{2}{|l|}{ Variable: } \\
\hline Opposite Building Height & 67 \\
\hline Street Width & 15 \\
\hline Glazed Area & 61 \\
\hline Orientation & West \\
\hline Location & Wellington \\
\hline Reflectance of Opposite Building & 20.8 \\
\hline Glazing Transmittance & 87 \\
\hline Vertical Location of lowest apartment & 4 \\
\hline \multicolumn{2}{|c|}{} \\
\hline Are simulations required to prove compliance: \\
\hline
\end{tabular}

Figure 86 - Application of the tool for Apartment 67

The measured data in Figure 85 shows that apartment 67 fails to comply with NZBC Clause G7. No result was provided for Figure 86 as the prediction models did not agree. The comparison of the prediction model results in Table 72 identified that the linear model and model $2 \mathrm{~A}$ provided the correct results for this case, however model $2 \mathrm{~B}$ under-estimated the results for this case by $52 \% \mathrm{DA}$.

Table 72 - Prediction Model Results for Apartment 67

\begin{tabular}{|c|c|c|c|}
\hline Measurements & Linear Model & Model 2A & Model 2B \\
\hline Pass & 100 & 102 & 23 \\
\hline
\end{tabular}




\section{APARTMENT 68}

Figure 87 shows the illuminance levels exceeded for $75 \%$ of the standard year based on the daylight factor measurements taken in Apartment 68.

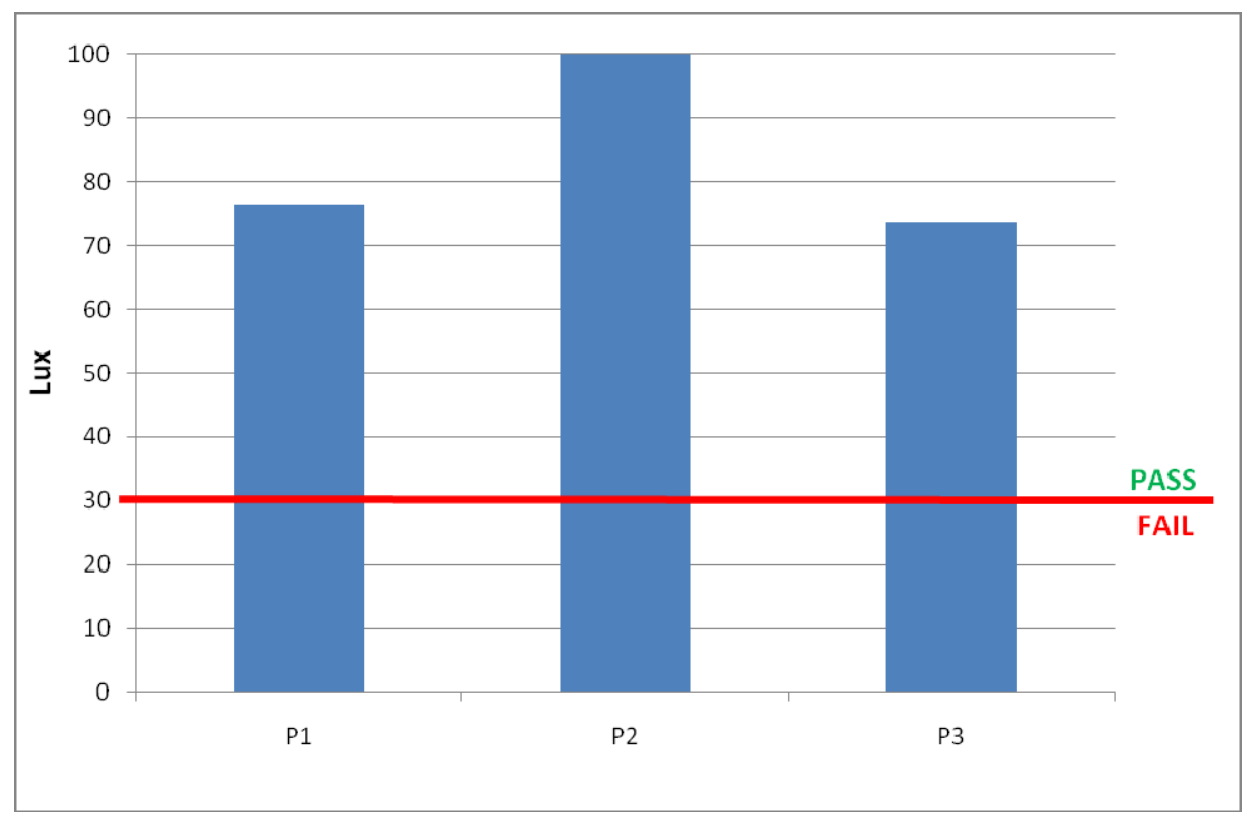

Figure 87 - Illuminance exceeded for $75 \%$ of the Standard Year in Apartment 68

From Figure 87 it can be seen that this apartment easily exceeds the minimum requirement, with illuminance levels greater than 70 lux for $75 \%$ of the standard year. 
Figure 88 shows the application of the compliance assessment tool for Apartment 68.

\begin{tabular}{|l|c|}
\hline \multicolumn{2}{|c|}{ NZBC G7 Compliance Assessment Tool } \\
\hline \multicolumn{2}{|l|}{ Variable: } \\
\hline Opposite Building Height & 35 \\
\hline Street Width & 3 \\
\hline Glazed Area & 10 \\
\hline Orientation & East \\
\hline Location & Wellington \\
\hline Reflectance of Opposite Building & 25 \\
\hline Glazing Transmittance & 87 \\
\hline Vertical Location of lowest apartment & 8 \\
\hline \multicolumn{2}{|}{} \\
\hline Are simulations required to prove compliance: \\
\hline
\end{tabular}

Figure 88 - Application of the tool for Apartment 68

The measured data in Figure 87 shows that apartment 68 complies with NZBC Clause G7 and Figure 88 identifies that the tool does not agree with this.

Table 73 provides a comparison of the results from each of the prediction models. All three of the models provided the incorrect outcome for this case. The best model was model $2 \mathrm{~A}$ as it was only 10\% DA under-predicted.

Table 73 - Prediction Model Results for Apartment 68

\begin{tabular}{|c|c|c|c|}
\hline Measurements & Linear Model & Model 2A & Model 2B \\
\hline Pass & 45 & 65 & 5 \\
\hline
\end{tabular}




\section{APARTMENT 73}

Figure 89 shows the illuminance levels exceeded for $75 \%$ of the standard year based on the daylight factor measurements taken in Apartment 73.

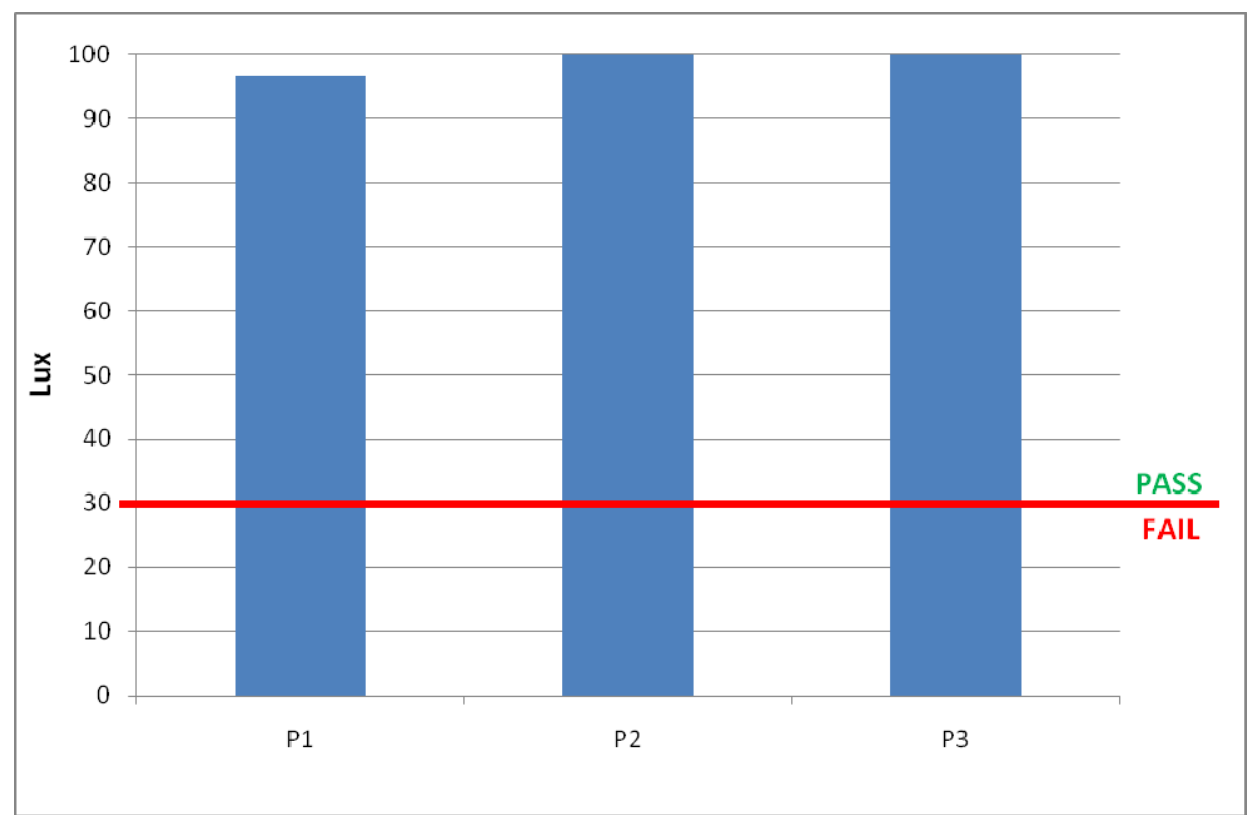

Figure 89 - Illuminance exceeded for $\mathbf{7 5 \%}$ of the Standard Year in Apartment $\mathbf{7 3}$

From Figure 89 it can be seen that this apartment easily exceeds the minimum requirement, with illuminance levels greater than 90 lux for $75 \%$ of the standard year at the back of the room. 
Figure 90 shows the application of the compliance assessment tool for Apartment 73.

\begin{tabular}{|l|c|}
\hline \multicolumn{2}{|c|}{ NZBC G7 Compliance Assessment Tool } \\
\hline \multicolumn{2}{|l|}{ Variable: } \\
\hline Opposite Building Height & 70 \\
\hline Street Width & 31 \\
\hline Glazed Area & 43 \\
\hline Orientation & East \\
\hline Location & Wellington \\
\hline Reflectance of Opposite Building & 12 \\
\hline Glazing Transmittance & 87 \\
\hline Vertical Location of lowest apartment & 1 \\
\hline \multicolumn{2}{|c|}{} \\
\hline Are simulations required to prove compliance: \\
\hline
\end{tabular}

Figure 90 - Application of the tool for Apartment 73

The measured data in Figure 89 shows that apartment 73 complies with NZBC Clause G7. No result was provided for Figure 90 as the prediction models did not agree. The comparison of the prediction model results in Table 74 identified that the linear model provided the correct results for this case, however models $2 \mathrm{~A}$ and $2 \mathrm{~B}$ under-estimated the results for this case by $12 \% \mathrm{DA}$ and $38 \% \mathrm{DA}$ respectively.

Table 74 - Prediction Model Results for Apartment 73

\begin{tabular}{|c|c|c|c|}
\hline Measurements & Linear Model & Model 2A & Model 2B \\
\hline Pass & 91 & 63 & 37 \\
\hline
\end{tabular}




\section{APARTMENT 74}

Figure 91 shows the illuminance levels exceeded for $75 \%$ of the standard year based on the daylight factor measurements taken in Apartment 74.

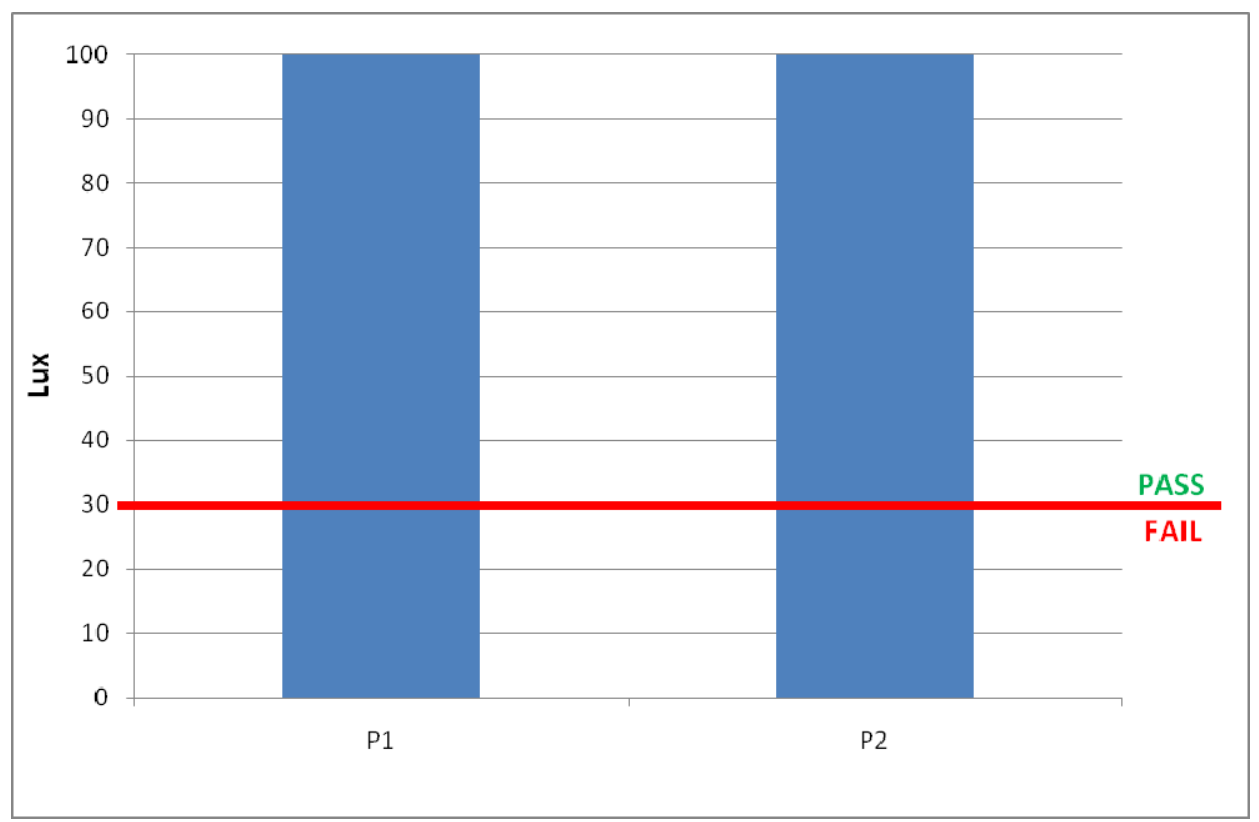

Figure 91 - Illuminance exceeded for $\mathbf{7 5 \%}$ of the Standard Year in Apartment 74

From Figure 91 it can be seen that this apartment easily exceeds the minimum requirement, with illuminance levels greater than 100 lux for $75 \%$ of the standard year at the back of the room. 
Figure 92 shows the application of the compliance assessment tool for Apartment 74.

\begin{tabular}{|l|c|}
\hline \multicolumn{2}{|c|}{ NZBC G7 Compliance Assessment Tool } \\
\hline \multicolumn{2}{|l|}{} \\
\hline Variable: & 12 \\
\hline Opposite Building Height & 14.5 \\
\hline Street Width & 28.6 \\
\hline Glazed Area & West \\
\hline Orientation & Wellington \\
\hline Location & 10.8 \\
\hline Reflectance of Opposite Building & 87 \\
\hline Glazing Transmittance & 4 \\
\hline Vertical Location of lowest apartment \\
\hline \multicolumn{2}{|}{} \\
\hline Are simulations required to prove compliance: \\
\hline
\end{tabular}

Figure 92 - Application of the tool for Apartment 74

The measured data in Figure 91 shows that apartment 74 complies with NZBC Clause G7. No result was provided for Figure 92 as the prediction models did not agree. The comparison of the prediction model results in Table 75 identified that the linear model provided the correct results for this case, however models $2 \mathrm{~A}$ and $2 \mathrm{~B}$ under-estimated the results for this case by $20 \% \mathrm{DA}$ and $8 \% \mathrm{DA}$ respectively.

Table 75 - Prediction Model Results for Apartment 74

\begin{tabular}{|c|c|c|c|}
\hline Measurements & Linear Model & Model 2A & Model 2B \\
\hline Pass & 81 & 59 & 67 \\
\hline
\end{tabular}




\section{APARTMENT 75}

Figure 93 shows the illuminance levels exceeded for $75 \%$ of the standard year based on the daylight factor measurements taken in Apartment 75.

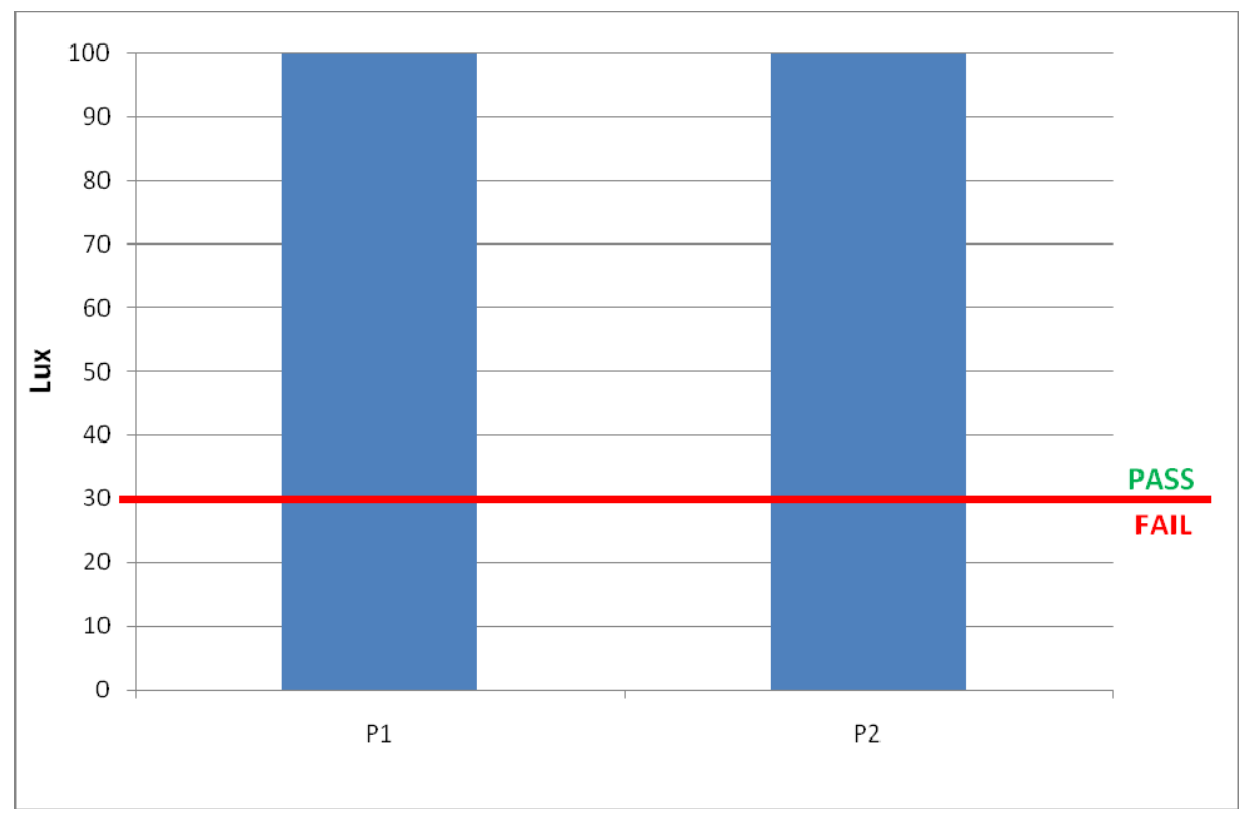

Figure 93 - Illuminance exceeded for $\mathbf{7 5 \%}$ of the Standard Year in Apartment 75

From Figure 93 it can be seen that this apartment easily exceeds the minimum requirement, with illuminance levels greater than 100 lux for $75 \%$ of the standard year. 
Figure 94 shows the application of the compliance assessment tool for Apartment 75.

\begin{tabular}{|l|c|}
\hline \multicolumn{2}{|c|}{ NZBC G7 Compliance Assessment Tool } \\
\hline \multicolumn{2}{|l|}{ Variable: } \\
\hline Opposite Building Height & 16 \\
\hline Street Width & 9 \\
\hline Glazed Area & 22 \\
\hline Orientation & East \\
\hline Location & Wellington \\
\hline Reflectance of Opposite Building & 14.8 \\
\hline Glazing Transmittance & 87 \\
\hline Vertical Location of lowest apartment & 1 \\
\hline \multicolumn{2}{|c|}{} \\
\hline Are simulations required to prove compliance: \\
\hline
\end{tabular}

Figure 94 - Application of the tool for Apartment 75

The measured data in Figure 93 shows that apartment 75 complies with NZBC Clause G7 and Figure 94 identifies that the tool does not agree with this.

Table 76 provides a comparison of the results from each of the prediction models. All three of the models provided the incorrect outcome for this case. The best model is the linear model as it is the closest to the threshold, but is still 26\% DA under-predicted.

Table 76 - Prediction Model Results for Apartment 75

\begin{tabular}{|c|c|c|c|}
\hline Measurements & Linear Model & Model 2A & Model 2B \\
\hline Pass & 49 & 21 & 17 \\
\hline
\end{tabular}




\section{APARTMENT 80}

Figure 95 shows the illuminance levels exceeded for $75 \%$ of the standard year based on the daylight factor measurements taken in Apartment 80.

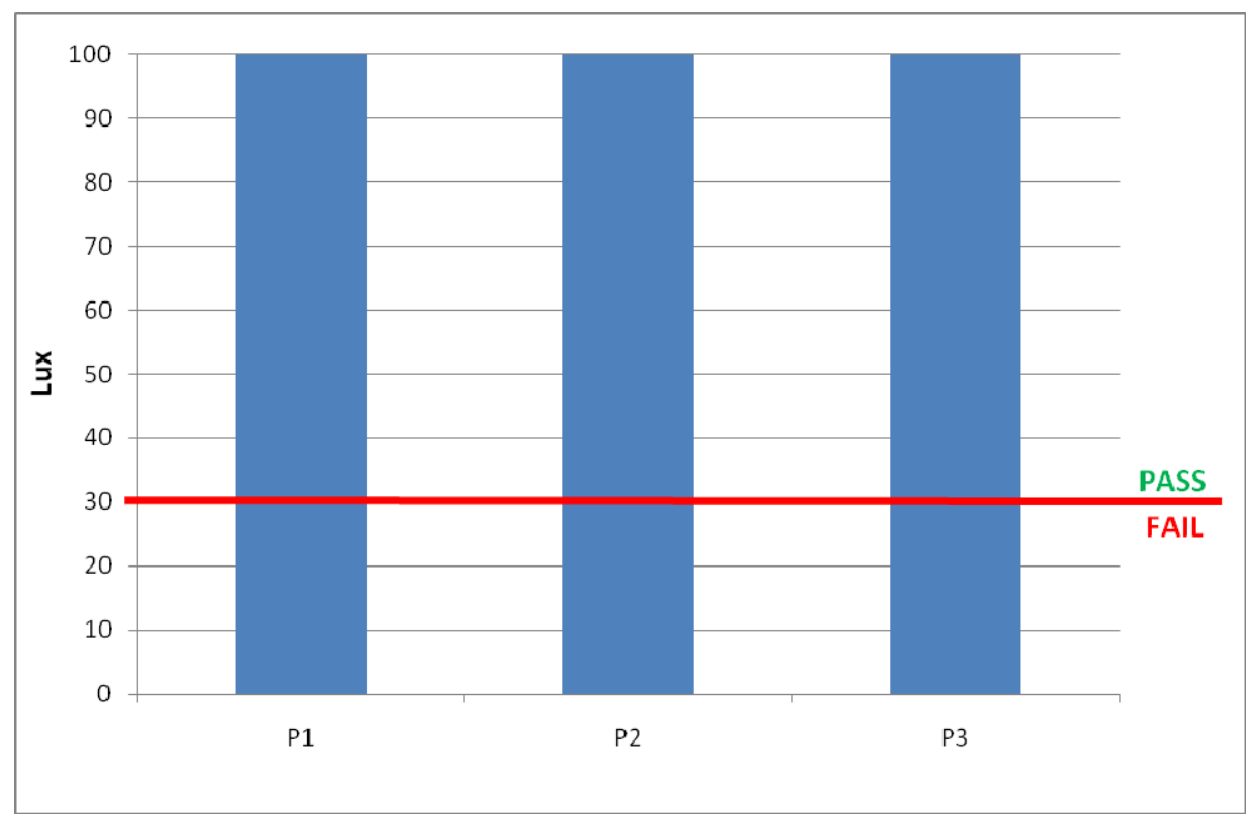

Figure 95 - Illuminance exceeded for $\mathbf{7 5 \%}$ of the Standard Year in Apartment 80

From Figure 95 it can be seen that this apartment easily exceeds the minimum requirement, with all measurement points having illuminance levels greater than 100 lux for $75 \%$ of the standard year. 
Figure 96 shows the application of the compliance assessment tool for Apartment 80.

\begin{tabular}{|l|c|}
\hline \multicolumn{2}{|c|}{ NZBC G7 Compliance Assessment Tool } \\
\hline \multicolumn{2}{|c|}{} \\
\hline Variable: & 70 \\
\hline Opposite Building Height & 31 \\
\hline Street Width & 63 \\
\hline Glazed Area & East \\
\hline Orientation & Wellington \\
\hline Location & 12 \\
\hline Reflectance of Opposite Building & 87 \\
\hline Glazing Transmittance & 1 \\
\hline Vertical Location of lowest apartment \\
\hline \multicolumn{2}{|}{} \\
\hline Are simulations required to prove compliance: \\
\hline
\end{tabular}

Figure 96 - Application of the tool for Apartment 80

The measured data in Figure 95 shows that apartment 80 complies with NZBC Clause G7. No result was provided for Figure 96 as the prediction models did not agree. The comparison of the prediction model results in Table 77 identified that the linear model and model $2 \mathrm{~A}$ provided the correct results for this case, however model $2 \mathrm{~B}$ under-estimated the result for this case by $32 \% \mathrm{DA}$.

Table 77 - Prediction Model Results for Apartment 80

\begin{tabular}{|c|c|c|c|}
\hline Measurements & Linear Model & Model 2A & Model 2B \\
\hline Pass & 117 & 125 & 43 \\
\hline
\end{tabular}




\section{Appendix 8 - Analysis of Overall Calibration Results for Models}

Appendix 8 contains information on the incorrect outcomes for the three equations: Linear, Model $2 \mathrm{~A}$ and Model 2B. This information includes a table with all the incorrect outcomes per equation that also identifies the variable inputs. Seven graphs were produced for each of the variables to determine if any of the variables were the cause of the incorrect outcomes. 


\section{A8.1 Linear Model}

\begin{tabular}{|c|c|c|c|c|c|c|c|c|c|c|c|c|c|c|c|}
\hline $\overrightarrow{\&}$ & 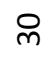 & $\grave{\sim}$ & 욱 & س & 3 & $\stackrel{\infty}{m}$ & $r$ & & & & & & & & \\
\hline 是 & $\stackrel{m}{\rightarrow}$ & $\stackrel{\infty}{\sim}$ & $\stackrel{\varphi}{N}$ & $z$ & $u$ & $\stackrel{\llcorner}{N}$ & $\sigma$ & & & & & & & & \\
\hline 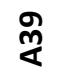 & $\tilde{m}$ & ㅇํ & $\stackrel{\infty}{+}$ & 3 & 3 & $\stackrel{\sim}{m}$ & $\sim$ & $\underset{⿱ ㇒}{\stackrel{n}{\alpha}}$ & $\underset{\sim}{\sigma}$ & $\sigma$ & $\approx$ & ш & 3 & $\stackrel{\llcorner}{\sim}$ & $r$ \\
\hline$\hat{\underline{\alpha}}$ & 기 & กี & $\tilde{m}$ & $z$ & 3 & $\stackrel{\infty}{m}$ & 0 & $\underset{\&}{\infty}$ & $\stackrel{n}{m}$ & $m$ & 어 & ш & 3 & $\stackrel{\llcorner}{\sim}$ & $\infty$ \\
\hline 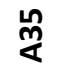 & 0 & 0 & $\stackrel{\varphi}{N}$ & $n$ & $u$ & 0 & $\exists$ & $\stackrel{\ell}{\&}$ & Э & $\stackrel{\llcorner}{N}$ & ษ & $n$ & 3 & ণ & $r$ \\
\hline 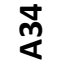 & $\tilde{m}$ & 오 & $\tilde{m}$ & 3 & 3 & $\stackrel{m}{m}$ & 0 & 占 & $\stackrel{\text { N }}{n^{\prime}}$ & in & $\vec{N}$ & $z$ & 3 & ㄱ & 0 \\
\hline$\stackrel{-\vec{m}}{\underline{\alpha}}$ & 0 & $\sigma$ & $\vec{m}$ & $\backsim$ & $\varangle$ & in & $\vec{\neg}$ & 苞 & $\stackrel{i}{N}$ & in & $\stackrel{\sim}{\sim}$ & z & 3 & $\stackrel{m}{m}$ & $m$ \\
\hline$\stackrel{\stackrel{0}{\Psi}}{\gtrless}$ & $\stackrel{\sim}{N}$ & $\stackrel{\sim}{\sim}$ & $\stackrel{\varphi}{N}$ & 3 & $u$ & ㄱ & $\infty$ & $\stackrel{0}{\&}$ & ને & $\stackrel{\llcorner}{\sim}$ & $\stackrel{\infty}{\sim}$ & $n$ & 3 & $\stackrel{\sim}{ }$ & $r$ \\
\hline 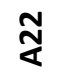 & ㄱ & $m$ & เே & $z$ & 3 & $\vec{N}$ & $r$ & $\frac{2}{8}$ & $\hat{m}$ & $\stackrel{\llcorner}{\sim}$ & $\stackrel{\text { ケ }}{\circ}$ & 3 & 3 & $\stackrel{d}{\sim}$ & in \\
\hline 芩 & 8 & ㅇ & $\stackrel{m}{m}$ & ш & 3 & 요 & $\nabla$ & $\underset{\&}{~}$ & テี & $m$ & $\stackrel{n}{m}$ & $\sim$ & 3 & $\vec{N}$ & 윽 \\
\hline \multirow[t]{2}{*}{$\underset{<}{\stackrel{J}{<}}$} & $\vec{r}$ & \& & $\vec{N}$ & $n$ & 3 & $\vec{N}$ & $r$ & ₹ & $\stackrel{\infty}{\sim}$ & $m$ & স্ & $z$ & 3 & $\stackrel{d}{\sim}$ & $\infty$ \\
\hline & $\frac{I}{\infty}$ & s & త & б̊ & త్ర & $\mathscr{\simeq}$ & $>$ & & $\frac{I}{\Phi}$ & 3 & త্ర & б̊ & 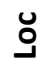 & $\underset{\mathscr{x}}{\mathscr{J}}$ & $\lessgtr$ \\
\hline
\end{tabular}




\section{Building Height}

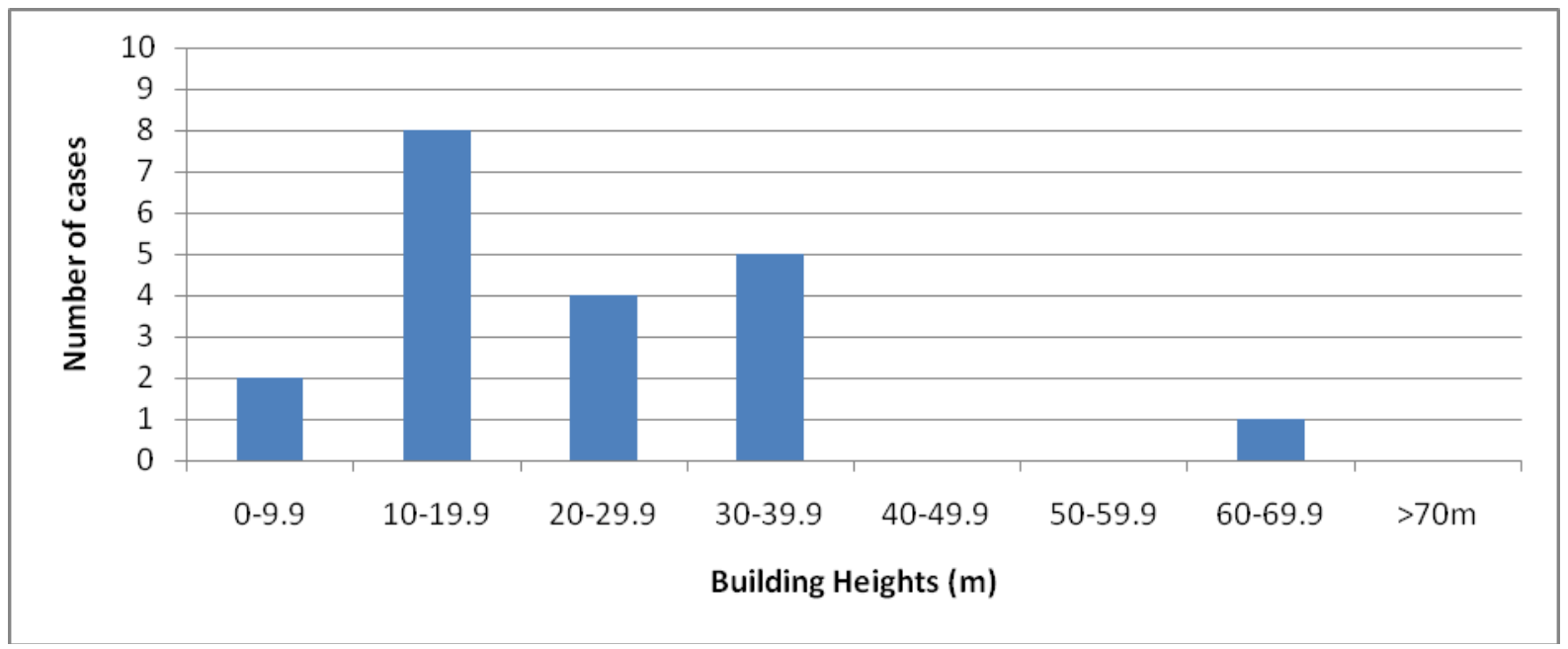

\section{Street Width}

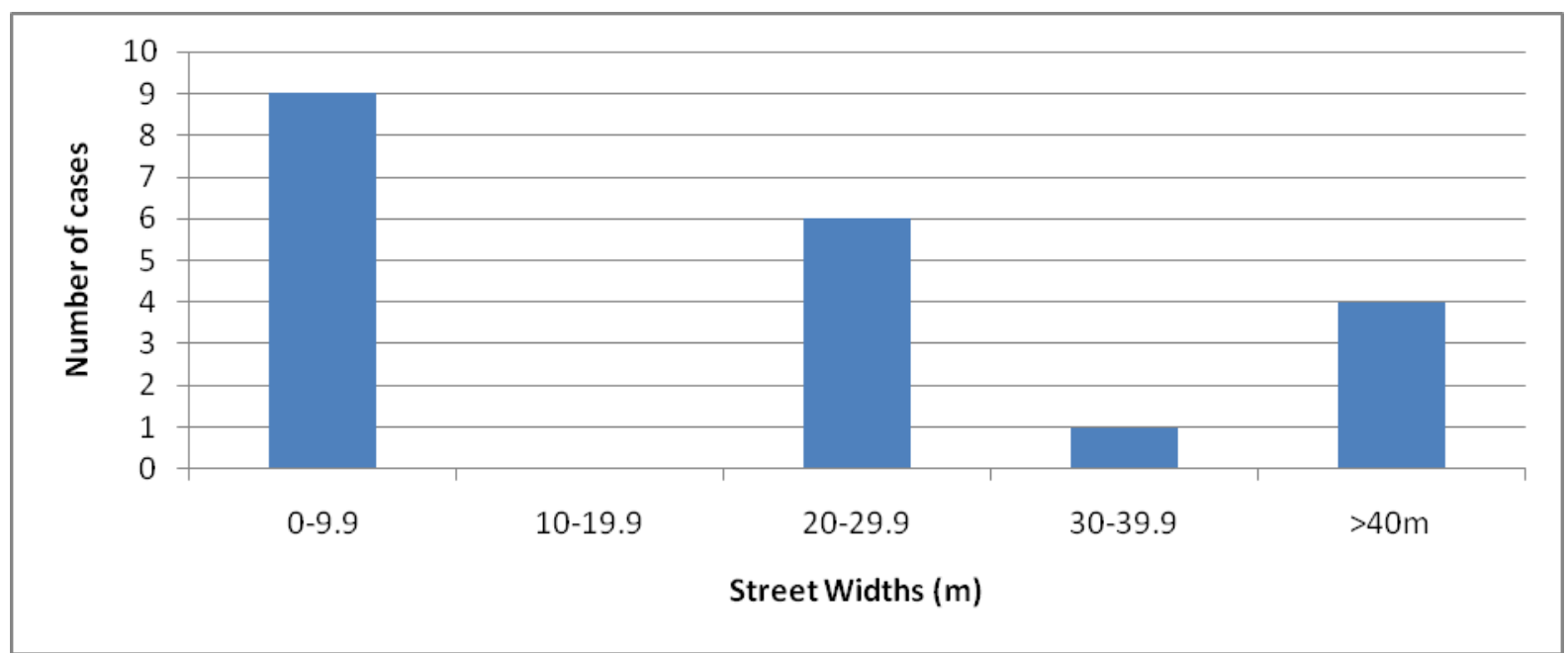

Glazed Area

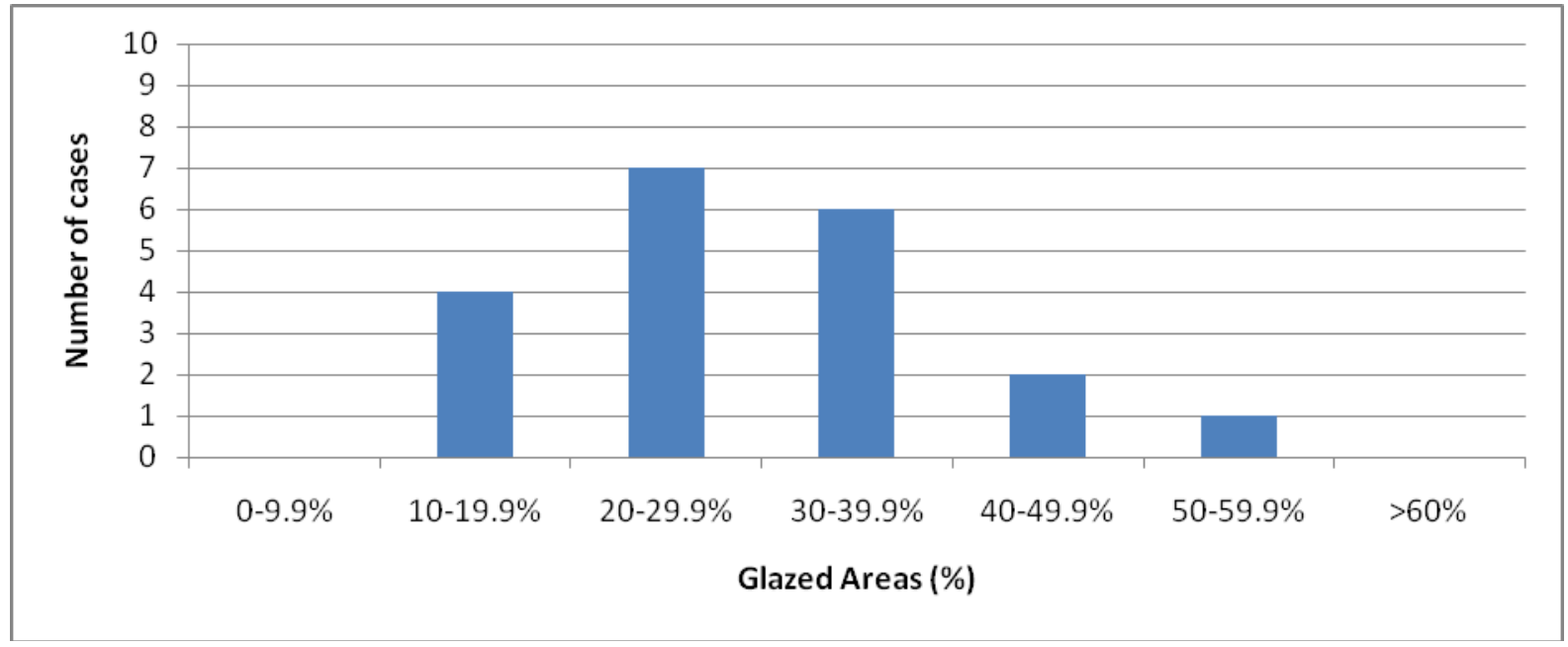




\section{Orientation}

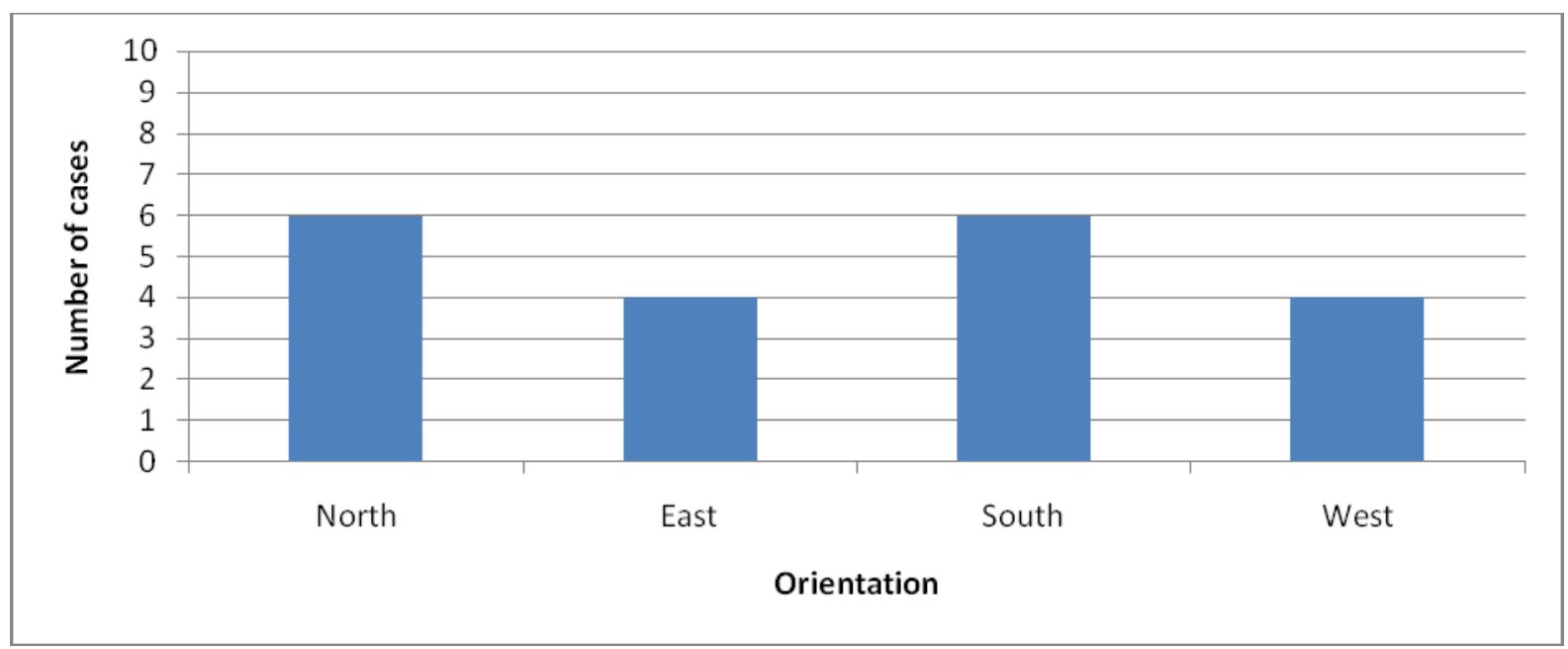

\section{Location}

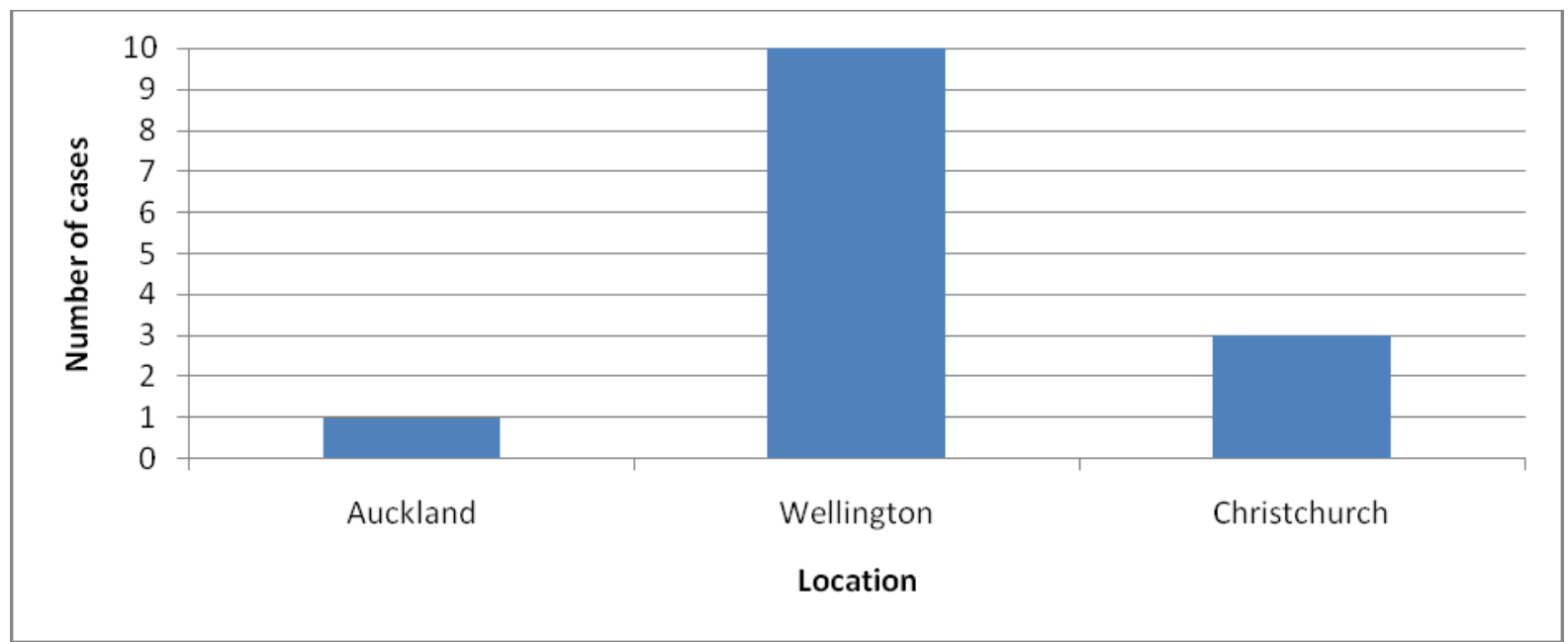

\section{Reflectance}

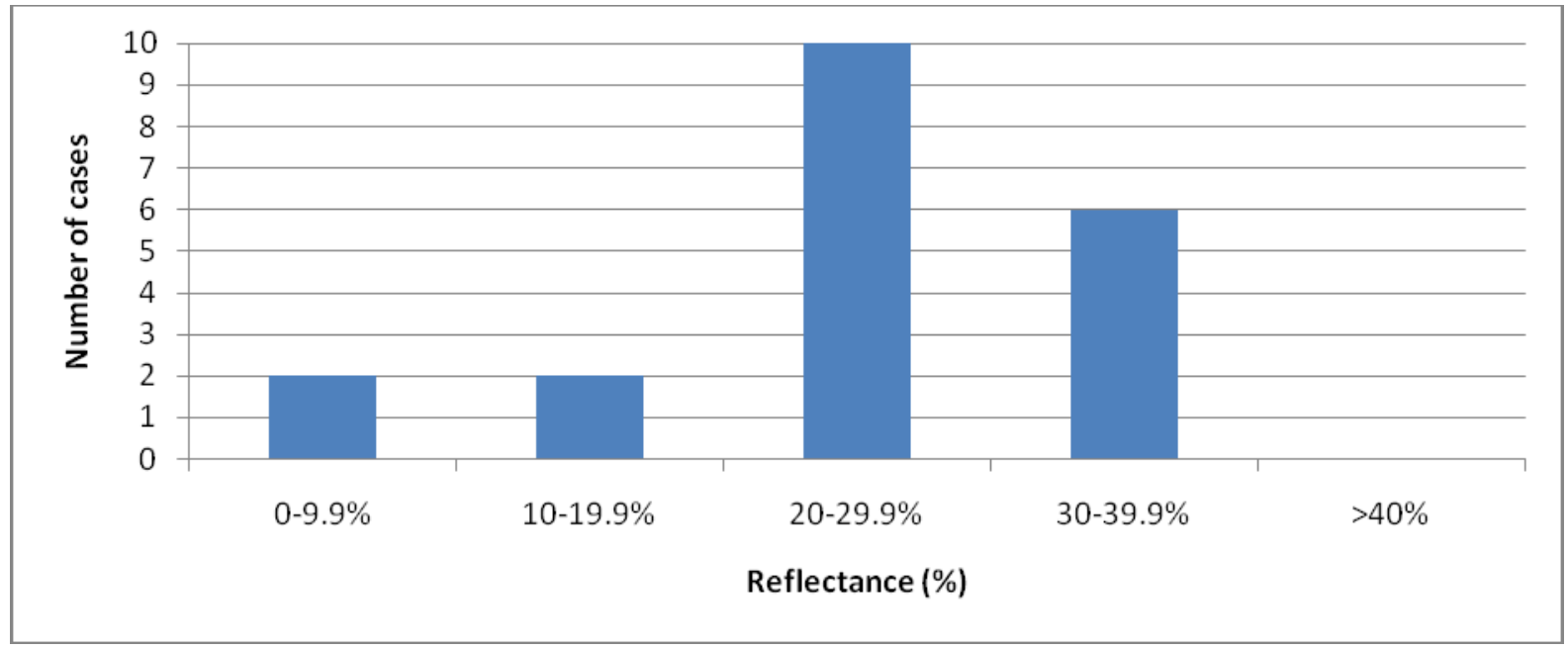


Vertical Location

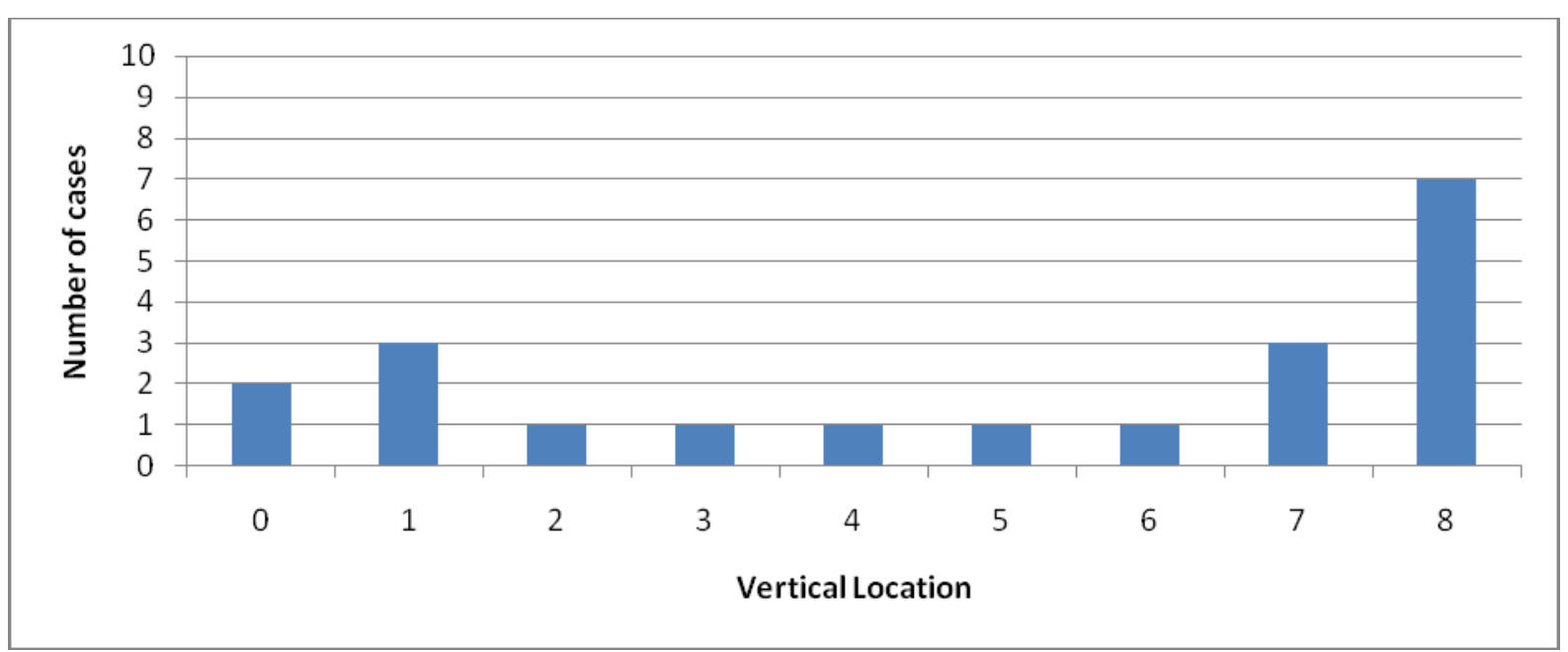




\section{A8.2 Model 2A}

\begin{tabular}{|c|c|c|c|c|c|c|c|c|c|c|c|c|c|c|c|}
\hline$\tilde{z}$ & $\stackrel{\infty}{\sim}$ & $m$ & m & $z$ & 3 & $\stackrel{\Xi}{\sim}$ & $\infty$ & & & & & & & & \\
\hline$\underset{\&}{+}$ & $\stackrel{\text { m }}{ }$ & $\widehat{へ}$ & 우 & ш & 3 & $\stackrel{\infty}{m}$ & $r$ & $\stackrel{n}{<}$ & $\underset{-1}{0}$ & $\sigma$ & N & ш & 3 & $\stackrel{\sim}{\sim}$ & - \\
\hline 是 & $\stackrel{m}{\rightarrow}$ & $\stackrel{\infty}{N}$ & $\stackrel{\stackrel{N}{N}}{ }$ & $z$ & $u$ & $\stackrel{\mathscr{N}}{N}$ & $a$ & $\stackrel{n}{\frac{m}{\alpha}}$ & ㅇ & $\vec{m}$ & $\stackrel{m}{\forall}$ & ш & 3 & ㄱ & -1 \\
\hline 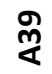 & $\tilde{m}$ & 으 & $\stackrel{\infty}{+}$ & 3 & 3 & $\stackrel{\sim}{m}$ & $\sim$ & $\stackrel{0}{\stackrel{0}{\varangle}}$ & $\stackrel{n}{m}$ & $m$ & 우 & ש & 3 & $\stackrel{\llcorner}{\sim}$ & $\infty$ \\
\hline$\stackrel{\hat{m}}{\underline{m}}$ & $\vec{r}$ & กี & $\tilde{m}$ & $z$ & 3 & $\stackrel{\infty}{m}$ & 0 & ஜ & 구 & $\stackrel{\sim}{\sim}$ & ㄱ & $\backsim$ & 3 & 尺 & -1 \\
\hline$\stackrel{\stackrel{n}{m}}{\underset{\alpha}{\alpha}}$ & 0 & 0 & $\stackrel{\sim}{N}$ & $n$ & $u$ & 0 & $\vec{\neg}$ & 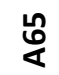 & ㅇ & $\hat{m}$ & $\stackrel{\searrow}{\sim}$ & ש & 3 & $\stackrel{\infty}{m}$ & -1 \\
\hline$\stackrel{\text { \} }{\alpha}} &{\tilde{m}} &{\text { 유 }} &{\tilde{m}} &{3} &{3} &{\stackrel{m}{m}} &{0} &{\text { 華 }} &{\stackrel{i}{ }} &{\text { Ln }} &{\vec{\sim}} &{z} &{3} &{\underset{\sim}{\sim}} &{6} \\
{\hline \stackrel{m}{\stackrel{m}{\alpha}}} &{\stackrel{\text { N }}{\wedge}} &{\tilde{n}} &{\text { 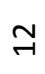 }} &{\text { ш }} &{3} &{\underset{\neg}{ }} &{\vec{\neg}} &{\text { 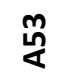 }} &{\exists} &{\stackrel{\llcorner}{\sim}} &{\stackrel{\searrow}{\sim}} &{z} &{3} &{\stackrel{m}{\rightarrow}} &{\nabla} \\
{\hline \overrightarrow{\underline{m}}} &{0} &{\sigma} &{\vec{m}} &{\sim} &{\ll} &{\text { in }} &{\vec{\neg}} &{\text { 르 }} &{\stackrel{i}{N}} &{\text { in }} &{\stackrel{\sim}{\sim}} &{z} &{3} &{\stackrel{m}{m}} &{m} \\
{\hline \text { 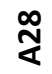 }} &{\ddot{N}} &{\stackrel{\sim}{\sim}} &{\stackrel{\bullet}{N}} &{3} &{u} &{\stackrel{i}{ }} &{\infty} &{\stackrel{\infty}{<}} &{\stackrel{m}{\sim}} &{\text { ㅇ }} &{\text { 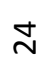 }} &{3} &{3} &{\underset{\sim}{+}} &{\sim} \\
{\hline \underset{\mathbb{N}}{\mathbb{N}}} &{\text { ๆี }} &{m} &{\text { 官 }} &{z} &{3} &{\vec{\sim}} &{r} &{\frac{\text { 䎹 }}{4}} &{\hat{m}} &{\stackrel{\llcorner}{\sim}} &{\stackrel{\stackrel{\sim}{\sigma}}{\circ}} &{3} &{3} &{\stackrel{\searrow}{\sim}} &{\text { in }} \\
{\hline \underset{\mathbb{\alpha}}{\stackrel{4}{\alpha}}} &{\stackrel{ナ}{\triangleleft}} &{\underset{\forall}{ষ}} &{\vec{\sim}} &{n} &{3} &{\vec{\sim}} &{r} &{\underset{8}{8}} &{\text { ㄱ }} &{m} &{\stackrel{\varphi}{m}} &{\backsim} &{3} &{\vec{\sim}} &{\text { 욱 }} \\
{\hline} &{\frac{I}{\infty}} &{\sum_{n}} &{\text { త্ড }} &{\text { 茴 }} &{\text { 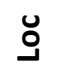 }} &{\underset{\propto}{\mathbb{\Phi}}} &{\lessgtr} &{ } &{\frac{I}{\infty}} &{\text { जे }} &{\mathbb{\circlearrowleft}} &{\text { ชั }} &{\text { 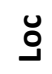 }} &{\text { 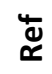 }} &{\overrightarrow{>}} \\
$\hline
\end{tabular}




\section{Building Height}

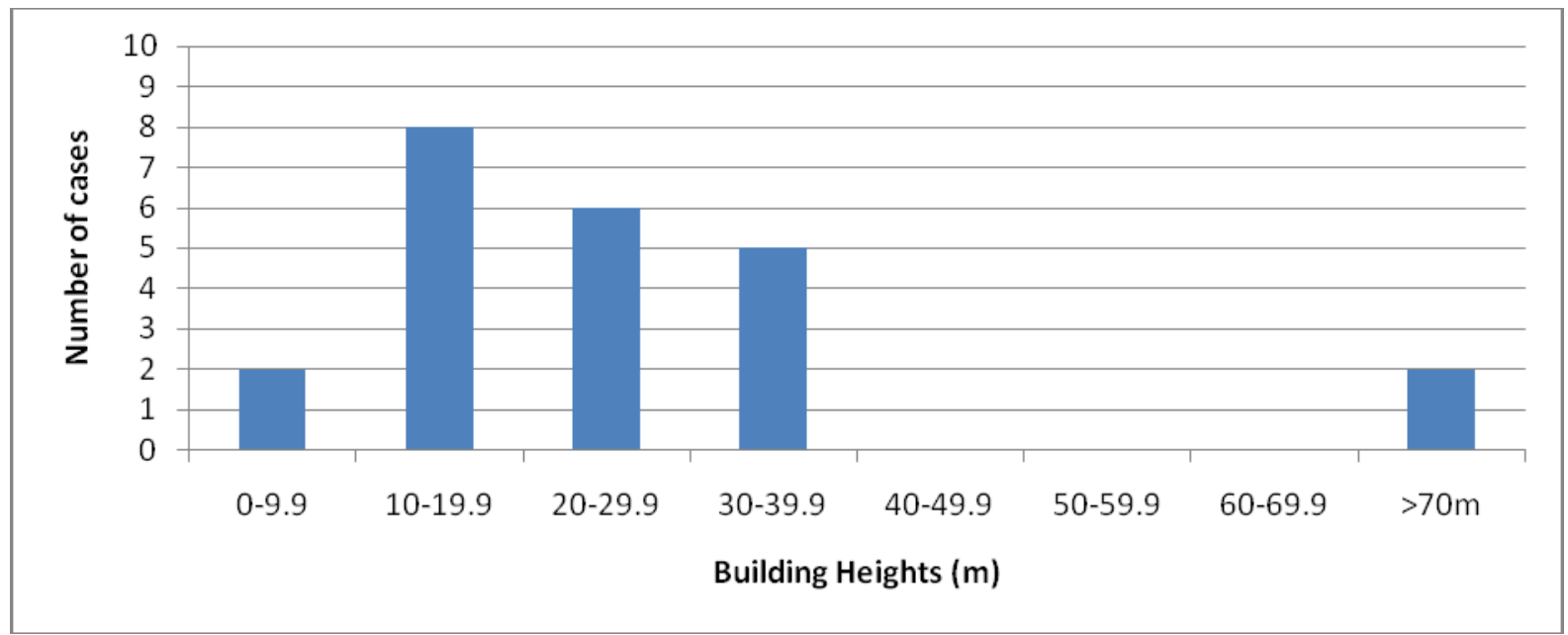

Street Width

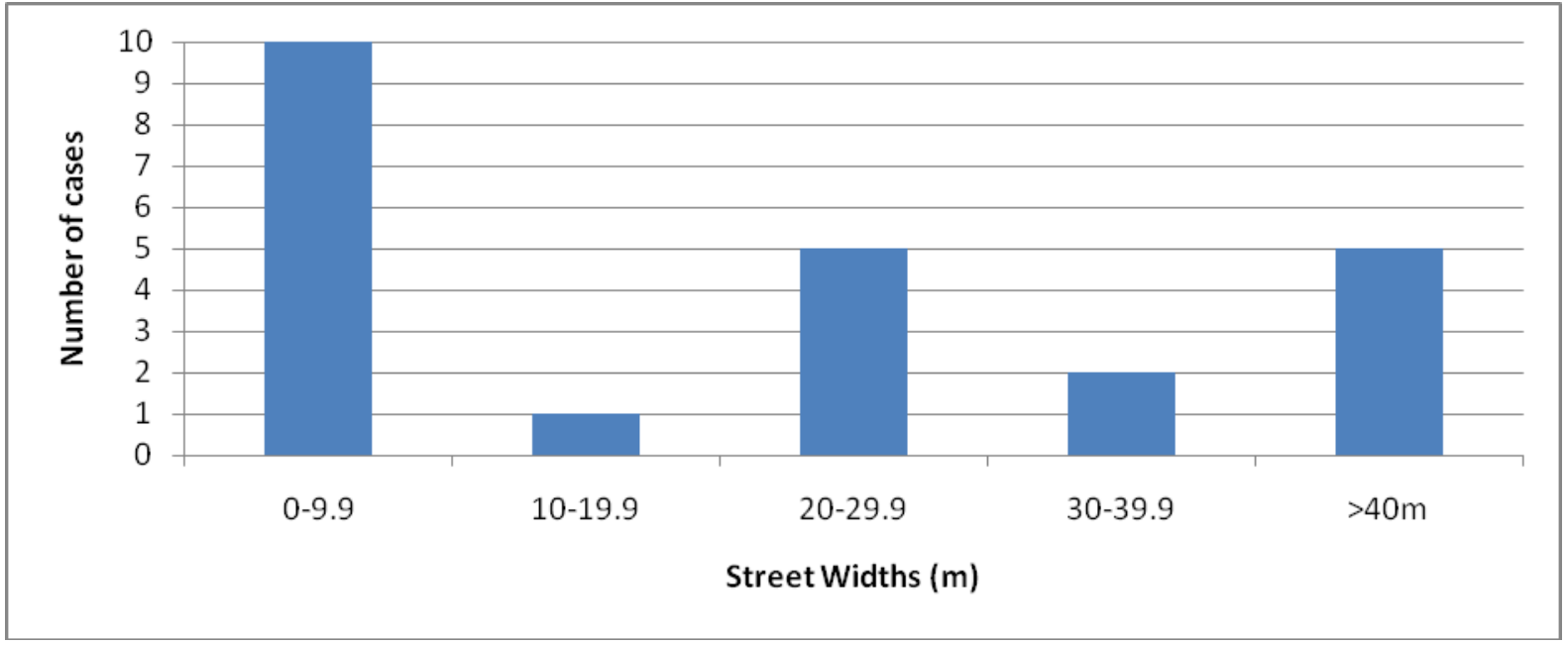

\section{Glazed Area}

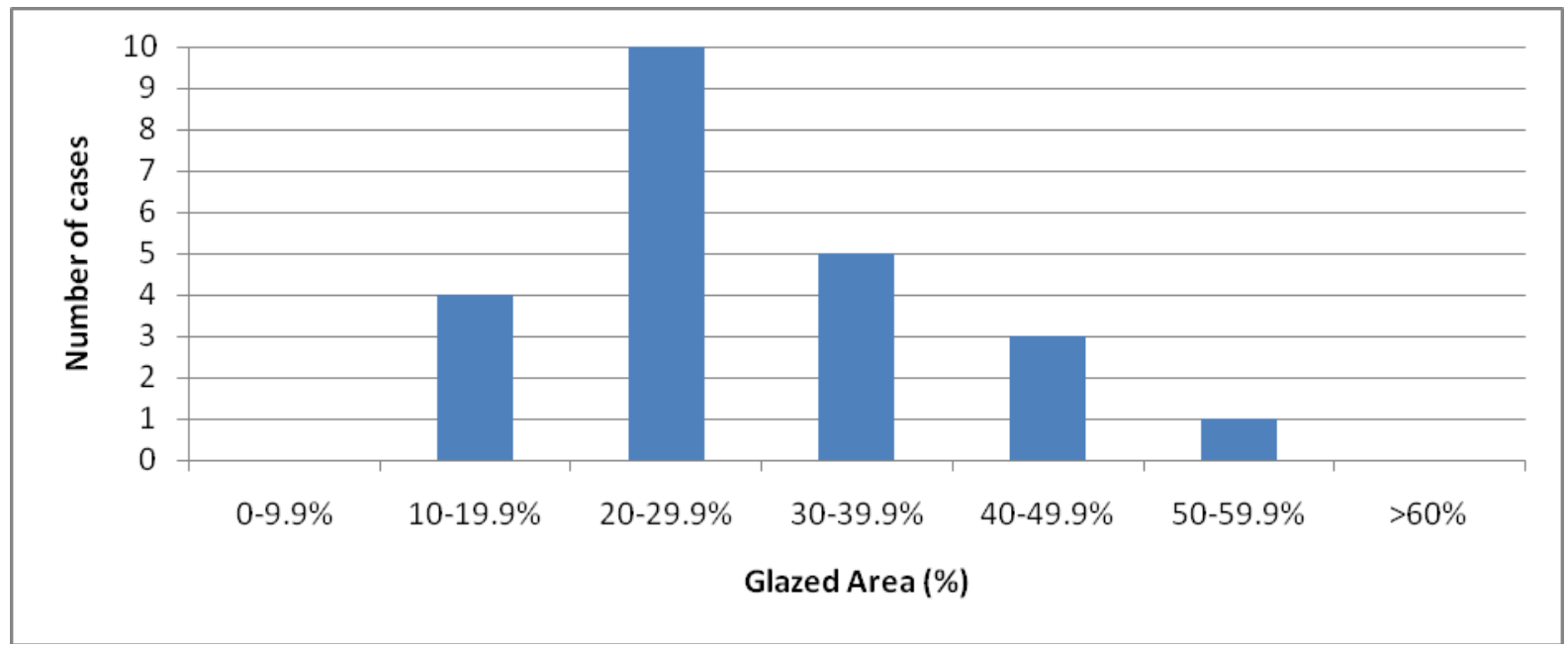




\section{Orientation}

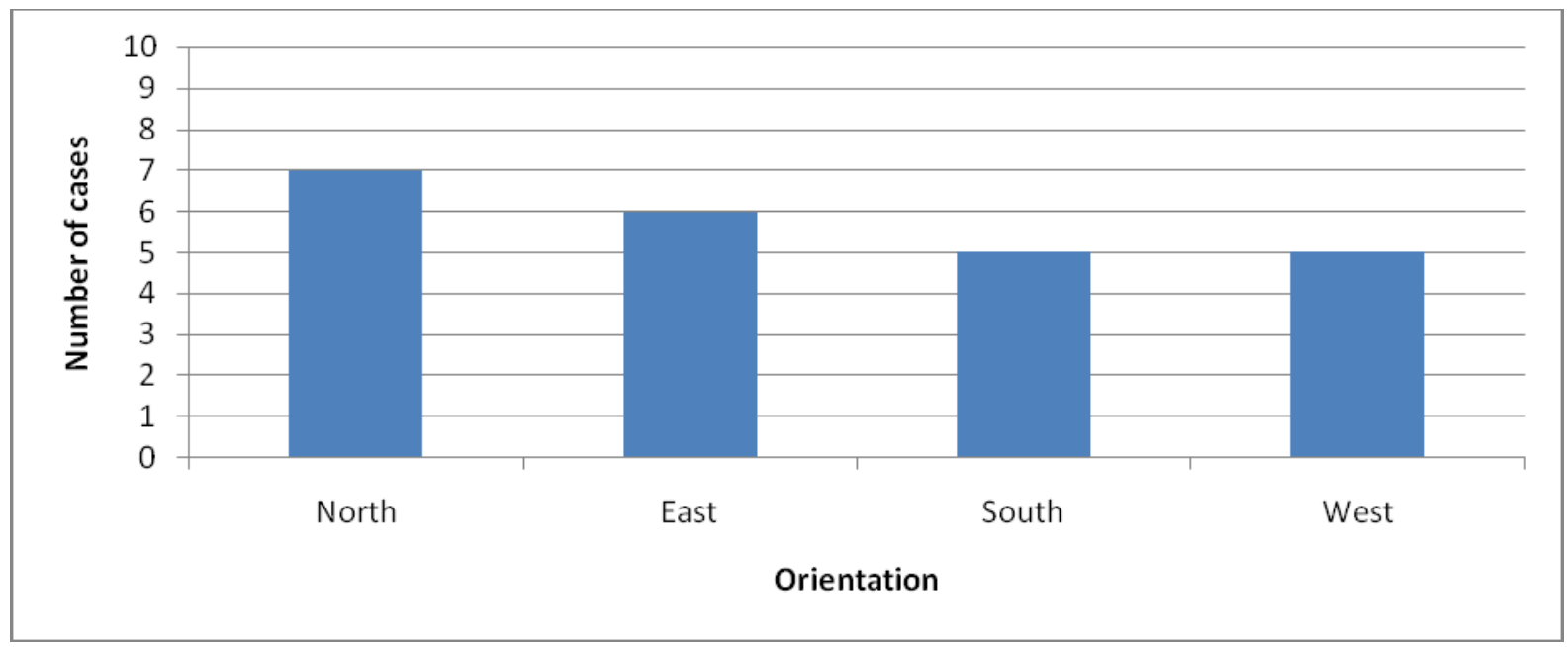

\section{Location}

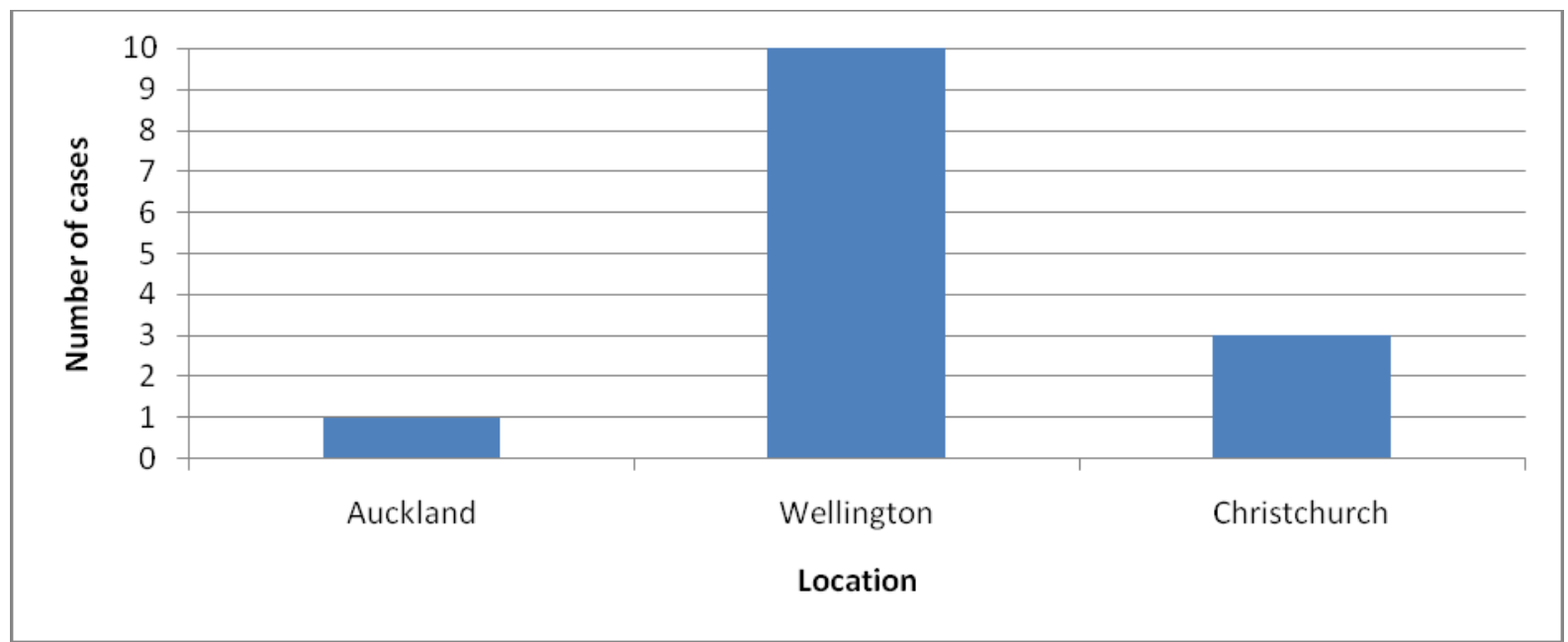

\section{Reflectance}

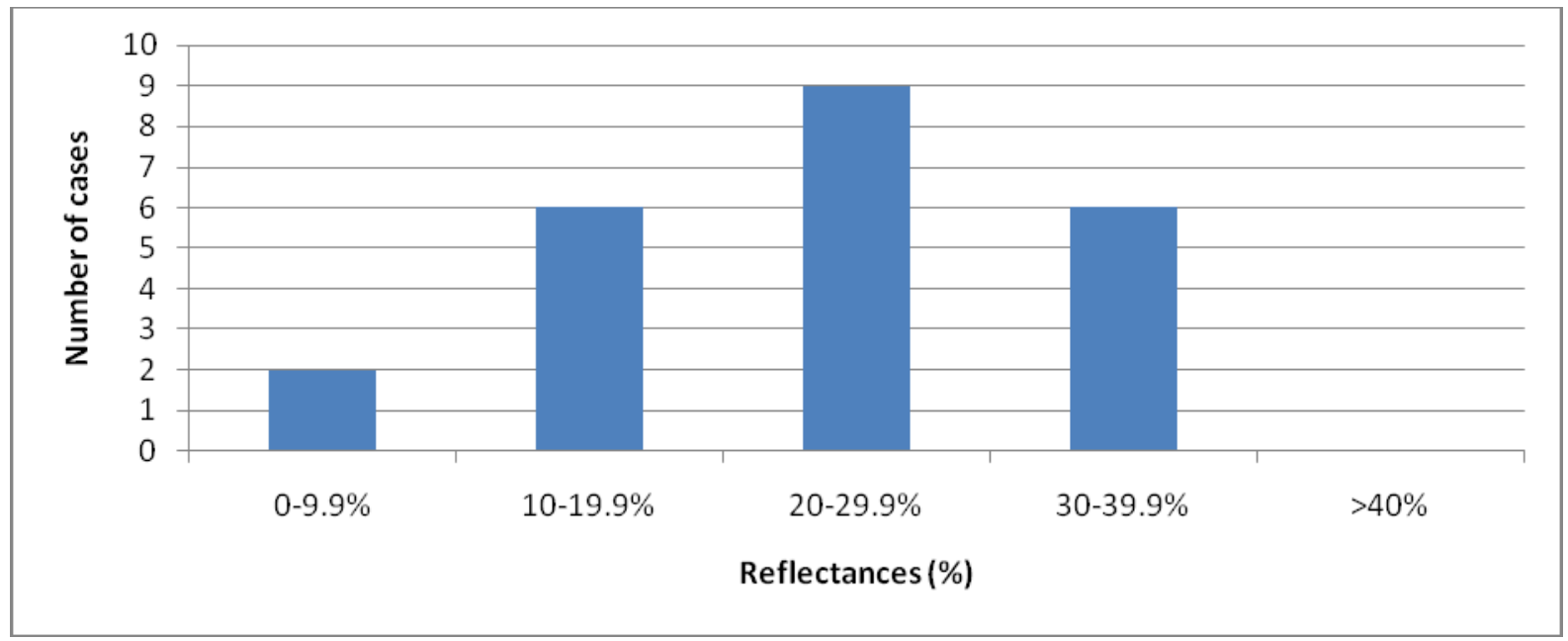


Vertical Location

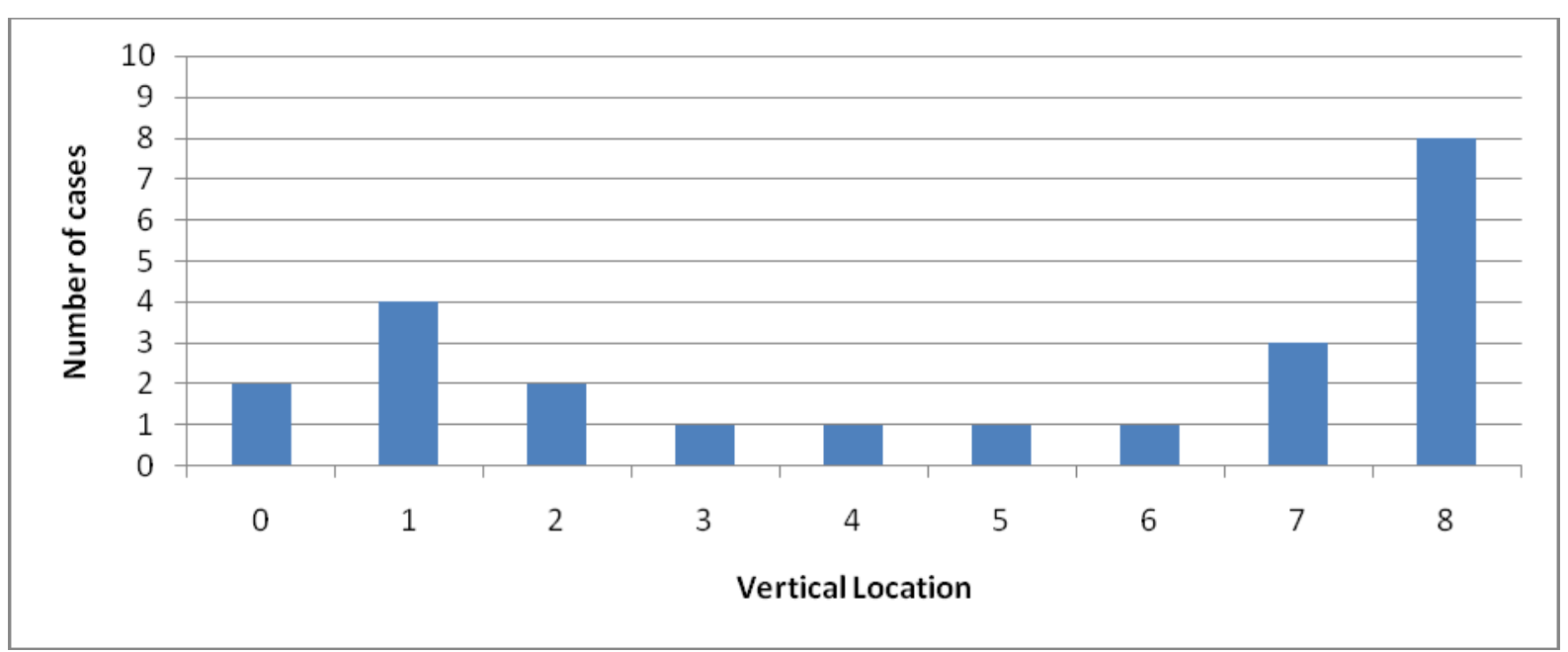




\section{A8.3 Model 2B}

\begin{tabular}{|c|c|c|c|c|c|c|c|c|c|c|c|c|c|c|c|}
\hline 主 & 0 & $\sigma$ & $\tilde{m}$ & $n$ & $\varangle$ & in & $r$ & $\stackrel{\infty}{\infty}$ & $R$ & $\vec{m}$ & $\tilde{\sigma}$ & ш & 3 & $\underset{7}{ }$ & $r$ \\
\hline 导 & $\underset{\neg}{ }$ & $m$ & $\stackrel{\llcorner}{m}$ & $n$ & 3 & $\vec{\sim}$ & 위 & $\stackrel{n}{\alpha}$ & $\stackrel{\bullet}{\sim}$ & $\sigma$ & $\approx$ & ш & 3 & $\stackrel{\llcorner}{\sim}$ & $r$ \\
\hline 导 & $\stackrel{m}{\rightarrow}$ & $\stackrel{\infty}{\sim}$ & $\stackrel{\sim}{N}$ & $z$ & $u$ & $\stackrel{\mathscr{N}}{\sim}$ & $\sigma$ & $\frac{m}{<}$ & ㅇ & $\vec{m}$ & $\stackrel{m}{q}$ & س & 3 & $\approx$ & $r$ \\
\hline$\underset{\dot{m}}{\stackrel{\alpha}{\alpha}}$ & $\tilde{m}$ & 유 & $\stackrel{\infty}{\rightarrow}$ & 3 & 3 & $\stackrel{\llcorner}{m}$ & $\sim$ & $\begin{array}{l}\infty \\
\stackrel{\leftrightarrow}{<}\end{array}$ & $\stackrel{\llcorner}{m}$ & $m$ & 위 & ш & 3 & $\stackrel{\llcorner}{\sim}$ & $\infty$ \\
\hline$\hat{\underline{q}}$ & $\exists$ & กี & $\tilde{m}$ & $z$ & 3 & $\stackrel{\infty}{m}$ & 0 & : & $\hat{\sigma}$ & $\stackrel{\Perp}{\sim}$ & $\overrightarrow{6}$ & 3 & 3 & શ & ナ \\
\hline$\stackrel{\mathscr{m}^{n}}{\alpha}$ & 0 & 0 & $\stackrel{\sim}{N}$ & $n$ & $u$ & 0 & $\vec{\gamma}$ & $\stackrel{8}{\&}$ & 구 & $\stackrel{\Perp}{\sim}$ & 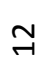 & $n$ & 3 & ㅇ & $r$ \\
\hline$\underset{m}{\stackrel{M}{\alpha}}$ & $\tilde{m}$ & 유 & $\tilde{m}$ & 3 & 3 & $\stackrel{m}{m}$ & 0 & 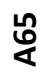 & $\stackrel{i}{ }$ & $\hat{m}$ & $\stackrel{\searrow}{\sim}$ & ш & 3 & $\stackrel{\infty}{m}$ & $r$ \\
\hline$\vec{m}$ & 0 & $\sigma$ & $\vec{m}$ & $n$ & $\ll$ & in & $\exists$ & 菖 & $\stackrel{\sim}{ }$ & in & $\vec{N}$ & $z$ & 3 & ㄱ & 0 \\
\hline$\underset{\sim}{\stackrel{\leftarrow}{\leftarrow}}$ & $\stackrel{\mathscr{N}}{\sim}$ & $\stackrel{d}{\sim}$ & $\stackrel{\sim}{N}$ & 3 & $u$ & ㅇ & $\infty$ & ஜn & $\vec{\exists}$ & $\stackrel{\sim}{\sim}$ & $\stackrel{\Delta}{N}$ & $z$ & 3 & $\stackrel{m}{\rightarrow}$ & 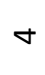 \\
\hline$\underset{\mathbb{Z}}{\mathbb{N}}$ & $\approx$ & $m$ & เ & $z$ & 3 & $\vec{\sim}$ & $r$ & 茎 & $\stackrel{N}{ }$ & เn & $\stackrel{\tilde{N}}{ }$ & z & 3 & $\stackrel{m}{m}$ & $m$ \\
\hline$\underset{⿱ 亠 乂}{\stackrel{J}{\alpha}}$ & $\underset{ন}{\stackrel{J}{*}}$ & 寸 & $\vec{N}$ & $n$ & 3 & $\vec{\sim}$ & $r$ & $\stackrel{\infty}{\&}$ & $\stackrel{m}{\sim}$ & q & $\stackrel{d}{N}$ & 3 & 3 & $\underset{\sim}{\star}$ & $\sim$ \\
\hline & $\frac{I}{D}$ & s & త్ర & ơ & $\stackrel{0}{0}$ & 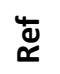 & 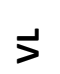 & & I & s & త్ & రั & ๑ & $\underset{\varpi}{\Phi}$ & 5 \\
\hline
\end{tabular}




\section{Building Height}

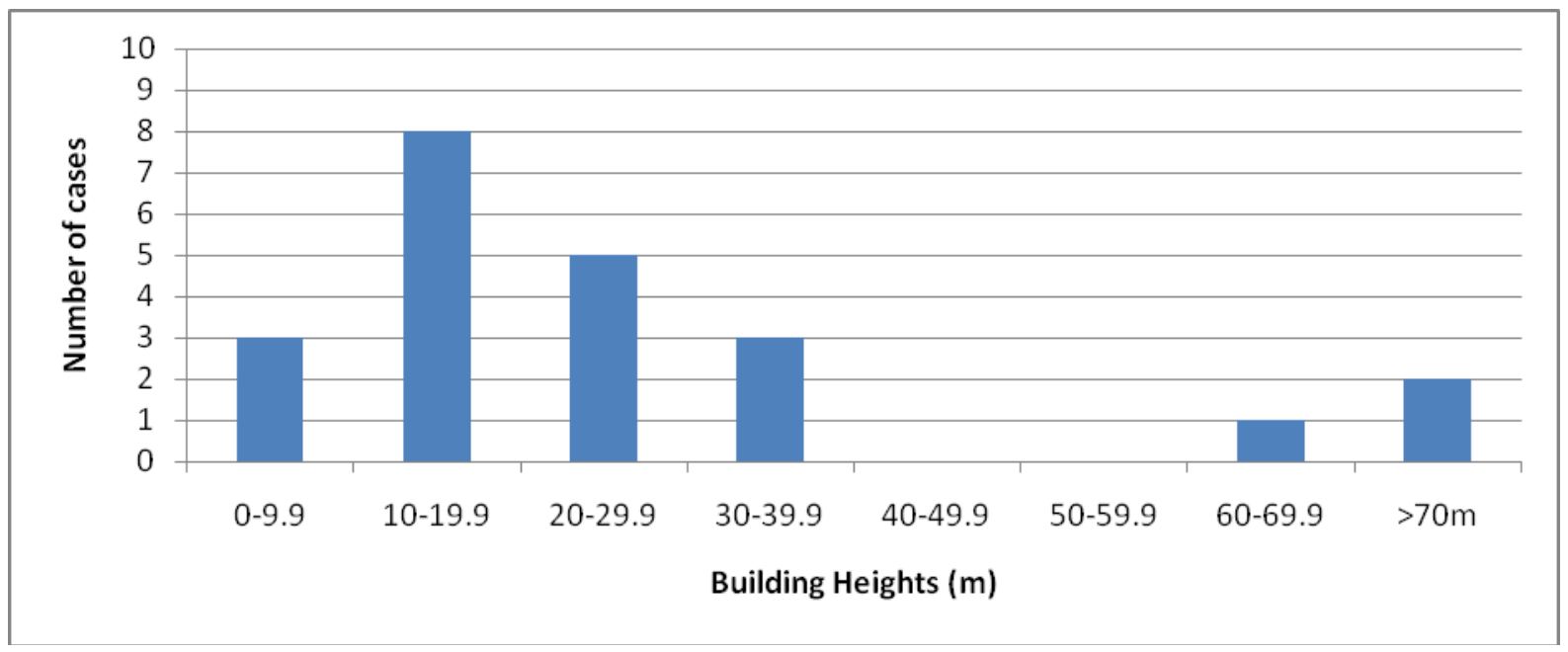

\section{Street Width}

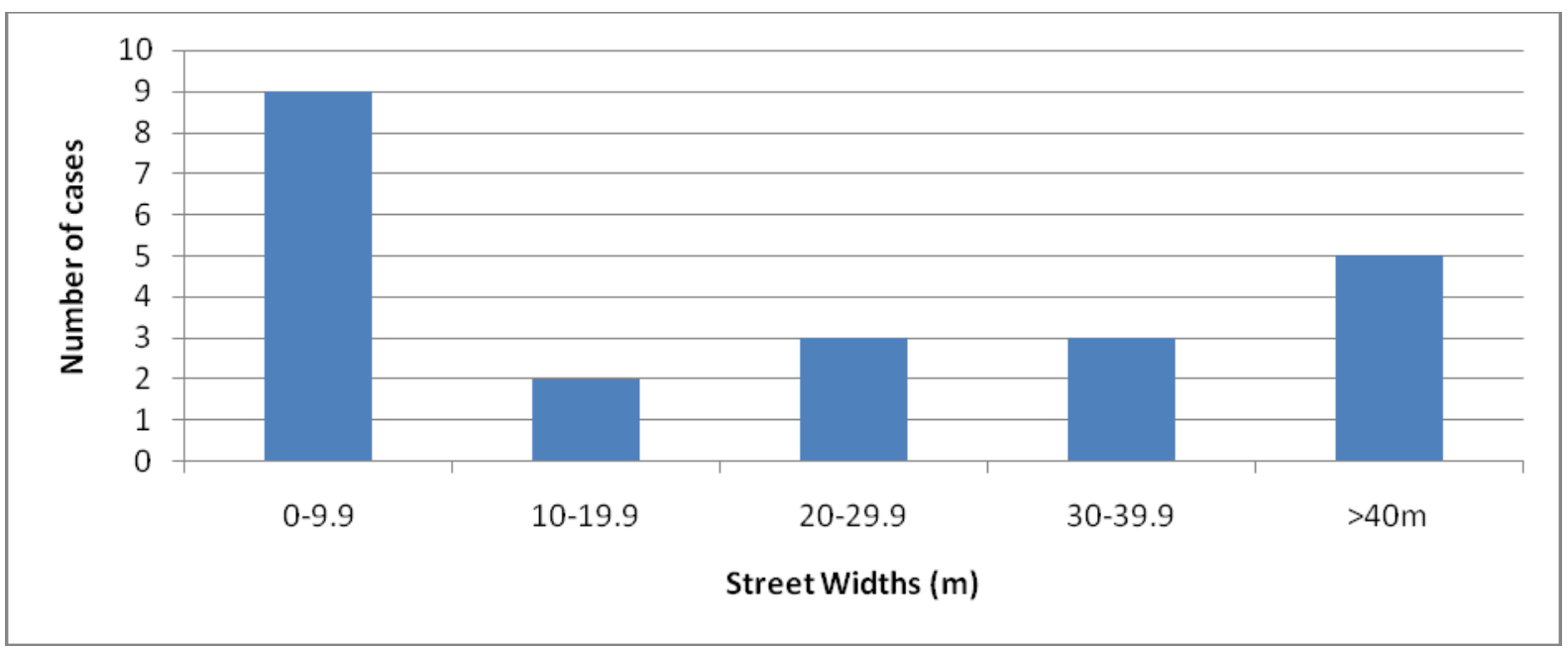

\section{Glazed Area}

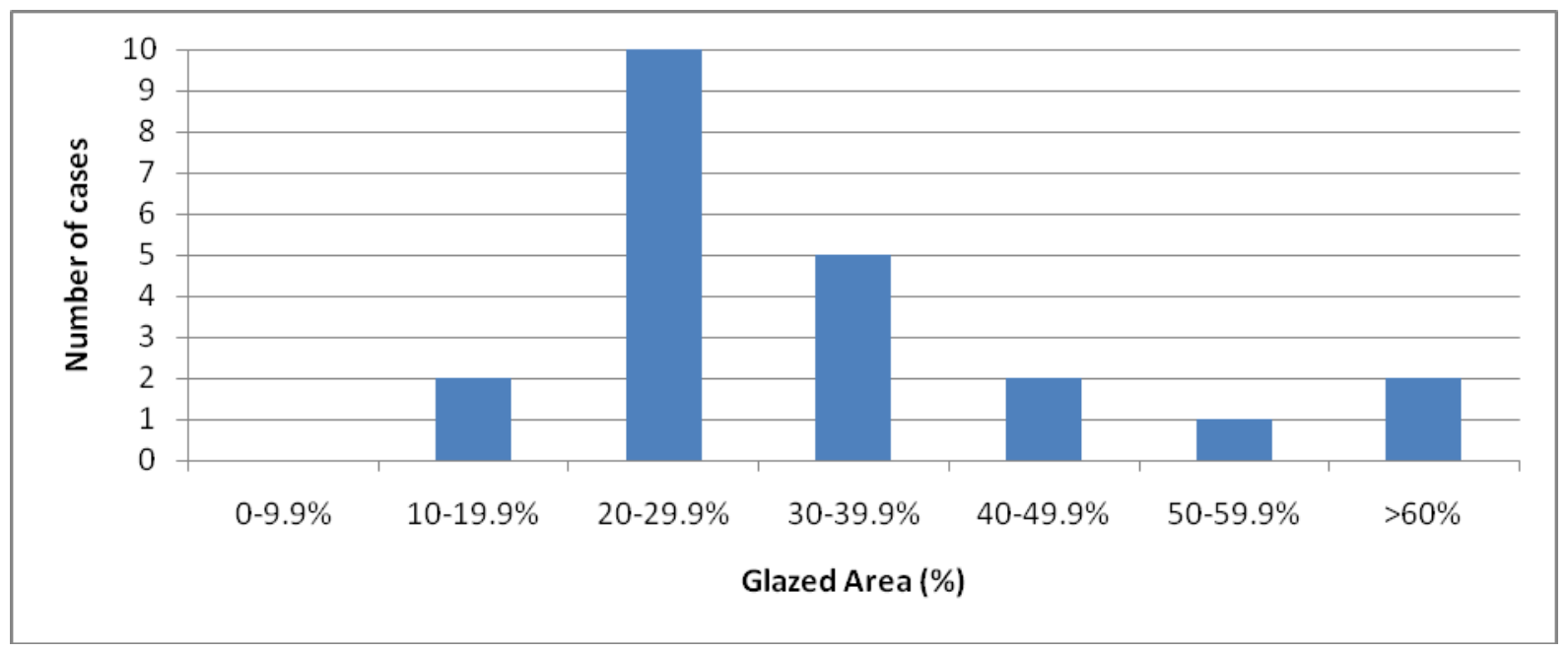




\section{Orientation}

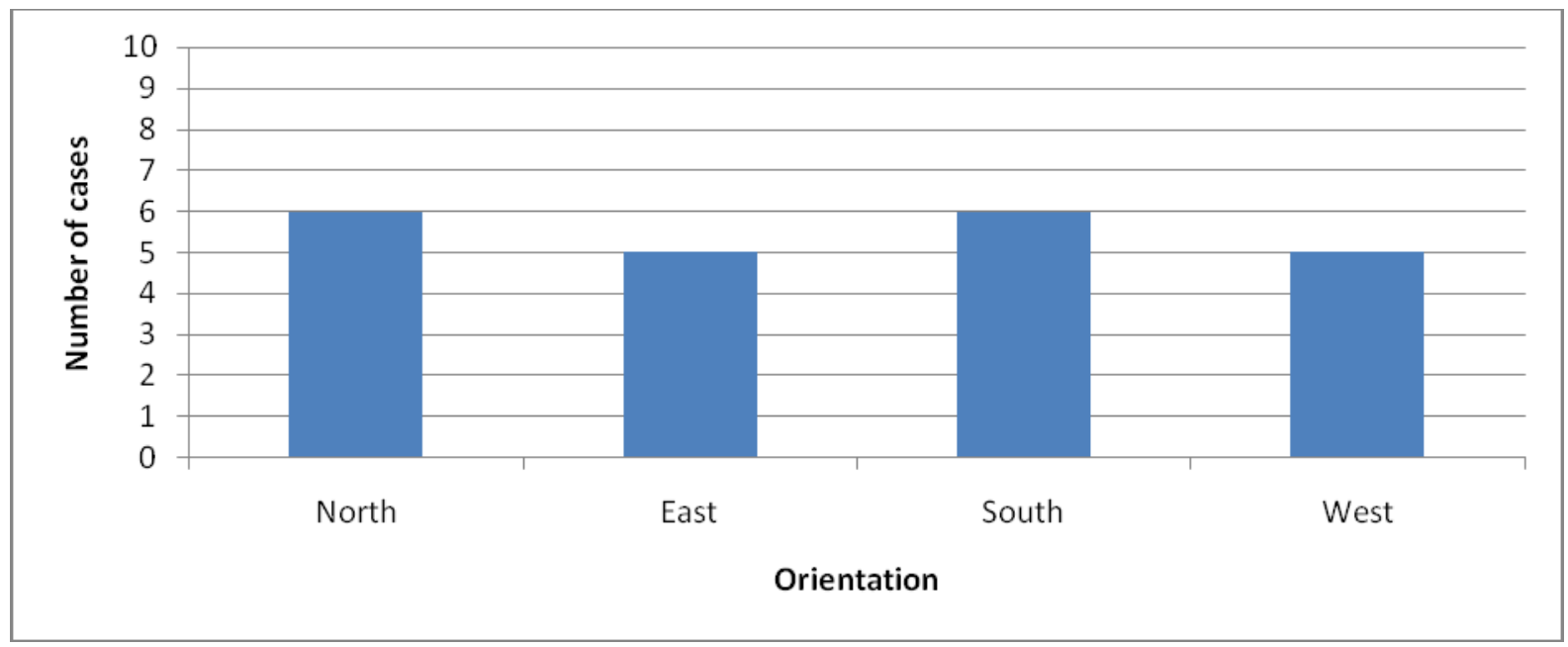

\section{Location}

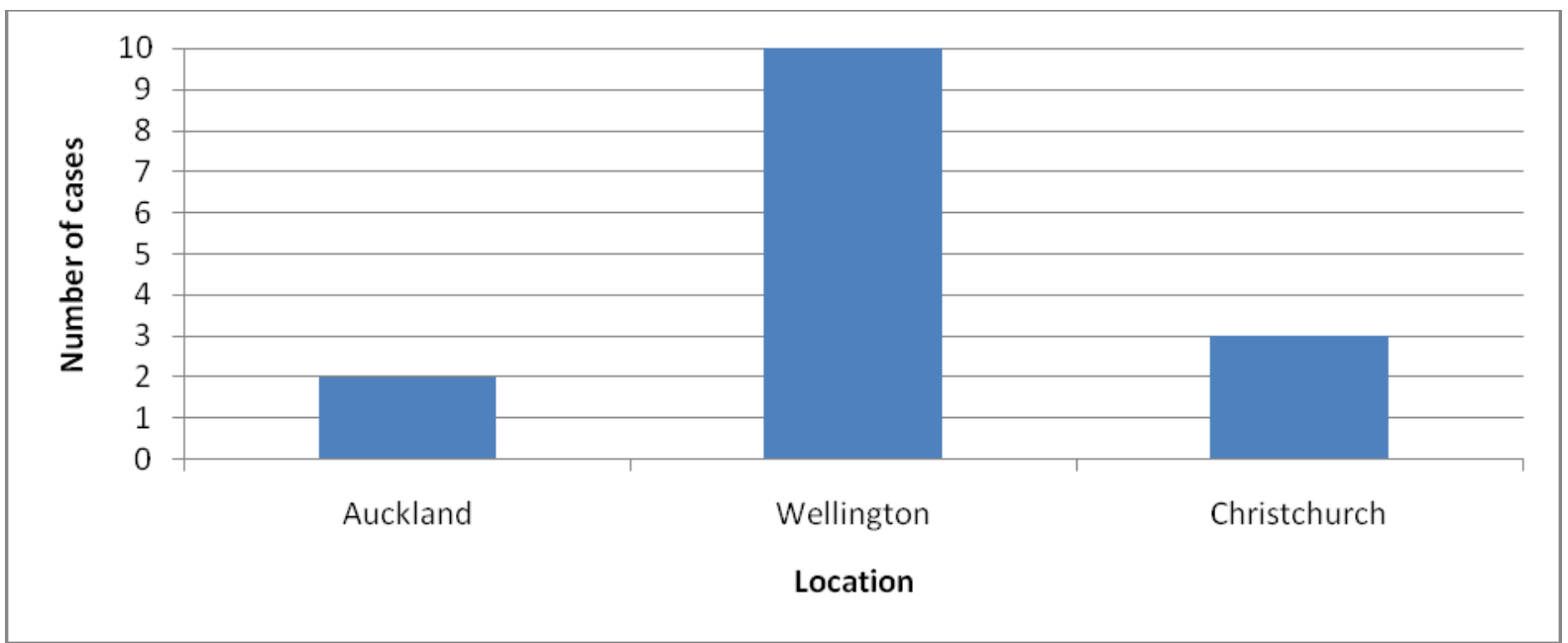

\section{Reflectance}

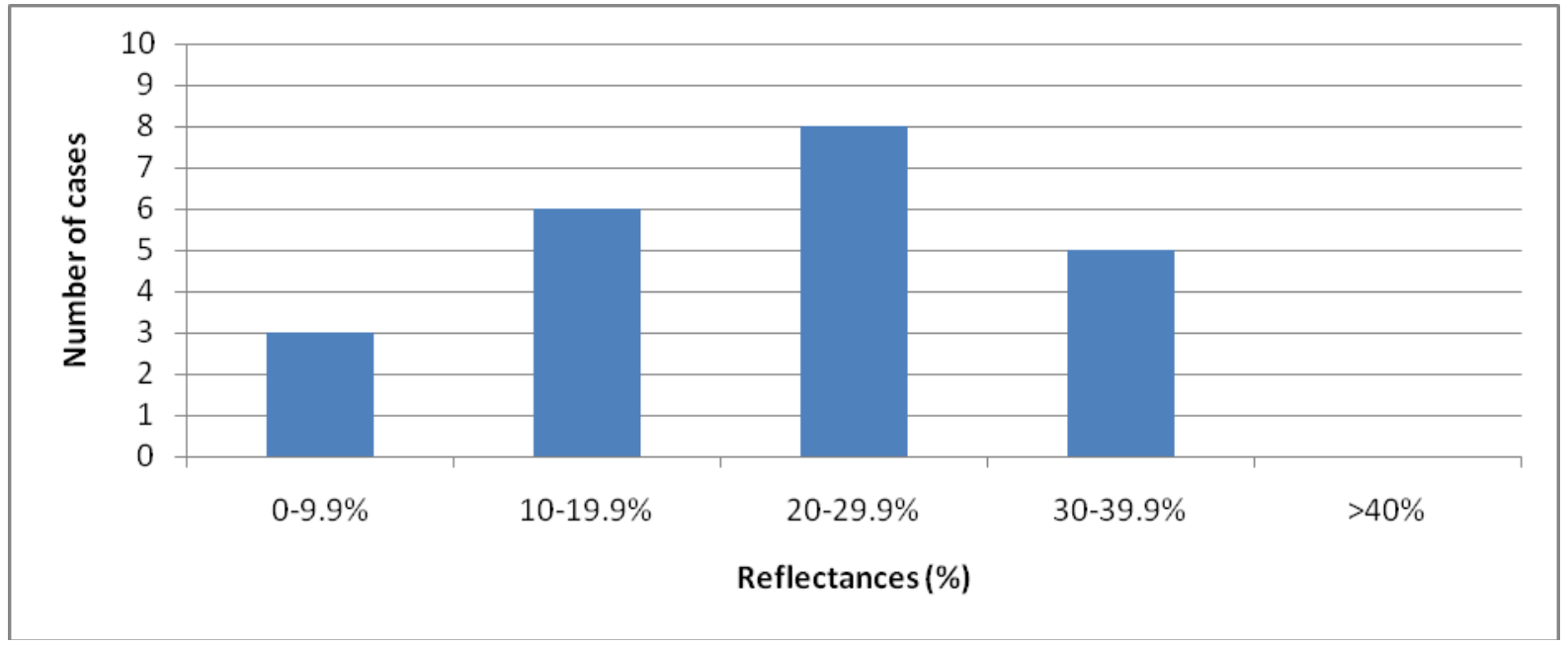

Vertical Location 


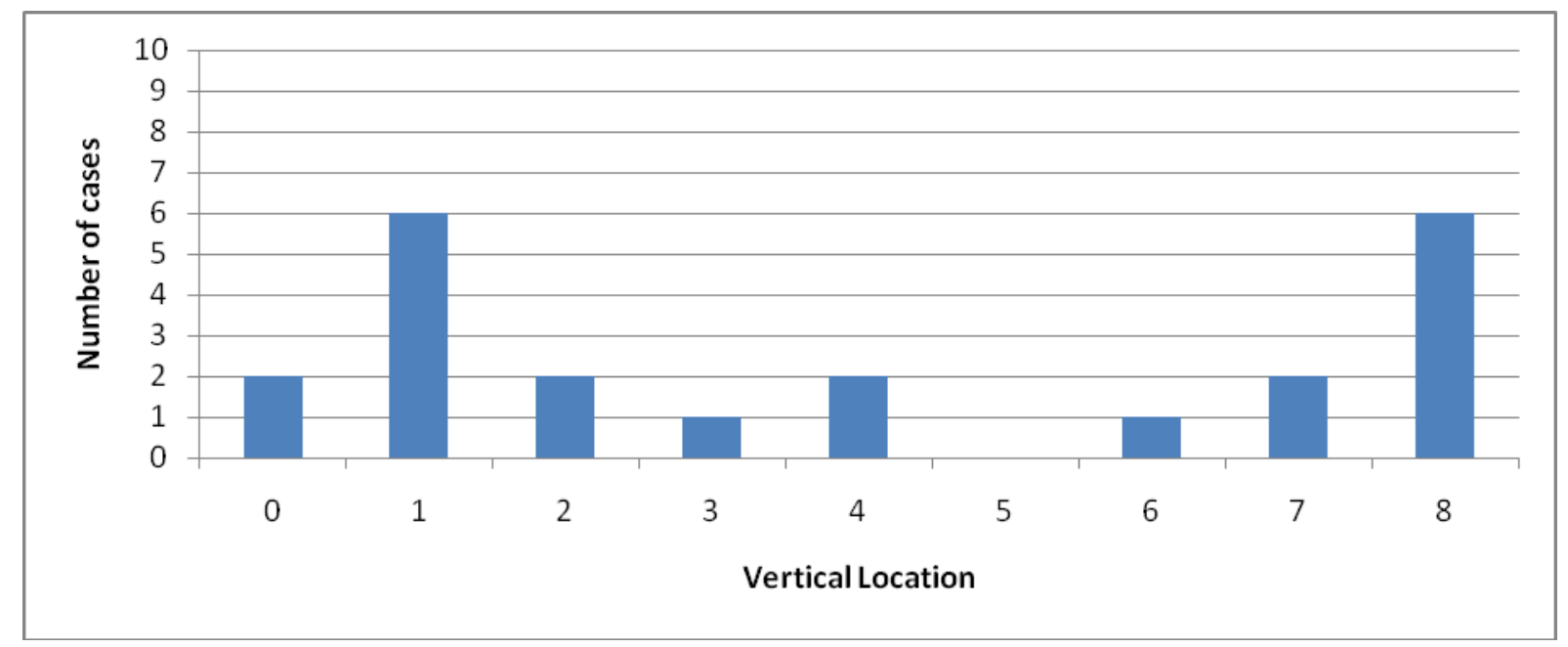




\section{Appendix 9 - Accompanying Document}

\section{Variables:}

\section{Opposite Building Height:}

In situations where there is only one opposite building, use the height of that building. In situations where there is more than one opposite building, this is assessed using an estimated skyline, where a horizontal line is drawn at the average height of the opposite buildings as shown below ${ }^{1}$.

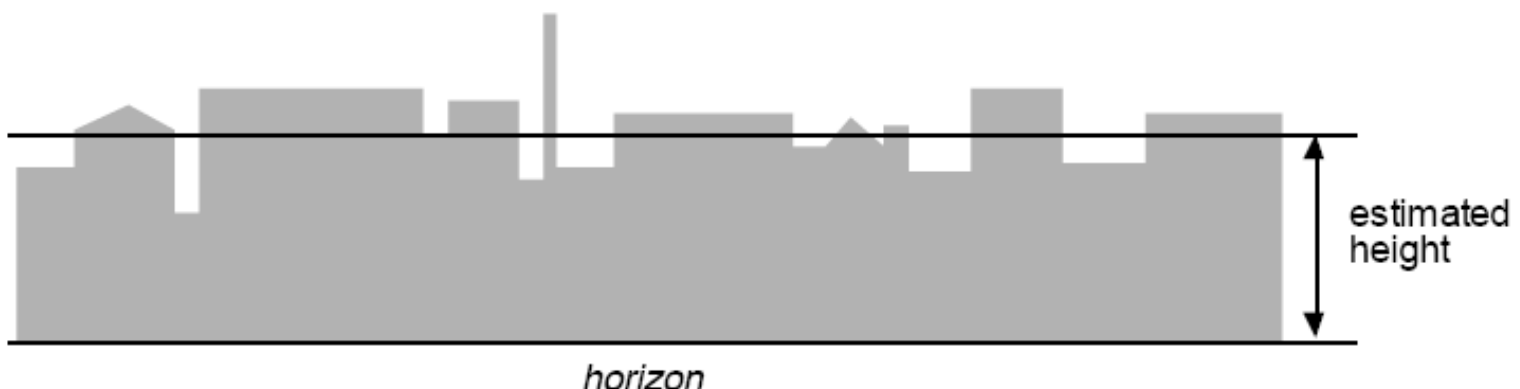

\section{Street Width:}

This is measured in meters as the distance from one façade to the one directly opposite.
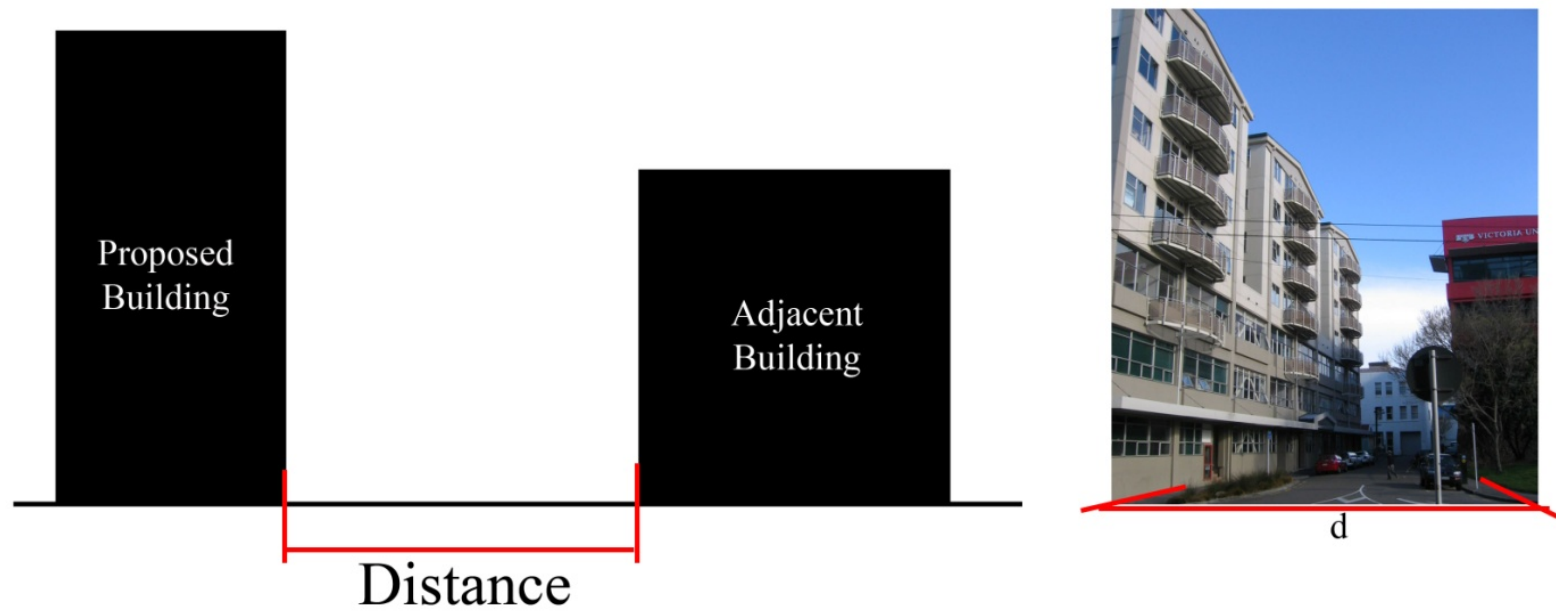

Glazed Area:

Calculate the glazed area and the floor area of a typical apartment on the lowest floor to determine the percentage.

Example:

\footnotetext{
${ }^{1}$ Tregenza, P. (1998). DETR Good Practice Guide 245: Desktop guide to daylight - for architects. Available: http://www.cibse.org/pdfs/GPG245.pdf [10 June 2008]. Pg 6
} 
For a living room with a floor area of $40 \mathrm{~m}^{2}$, a window $2 \mathrm{~m} \times 2 \mathrm{~m}\left(4 \mathrm{~m}^{2}\right)$ will have a glazed area that's $10 \%$ of the floor area, and a window $4 \mathrm{~m} \times 2 \mathrm{~m}\left(8 \mathrm{~m}^{2}\right)$ will have a glazed area that's $20 \%$ of the floor area.

\section{Orientation:}

Currently the only options are "North, "East", "South" and "West", in situations were the orientation does not exactly fit one of these categories, select the closest orientation. For situations were there is more than one orientation, apply the tool for each orientation.

\section{Location:}

There are currently 4 options for location, these are "Auckland", "Wellington", "Christchurch" and "Dunedin", select the most appropriate location. NOTE: The final version of the tool will have all 16 of the NIWA climate zones.

\section{Reflectance of Opposite Building:}

This is the percent of the light reflected from the opposite building surfaces. At this stage an estimate of the total percent of light reflected from all surfaces combined is to be used. The following table gives some examples for typical situations.

\begin{tabular}{|l|c|c|}
\hline Wall Material $^{2}$ & $\begin{array}{c}\text { Percent of Wall that } \\
\text { Glazing }\end{array}$ & Reflectance Value (\%) \\
\hline White Glazed Tiles & $0 \%$ & 80 \\
\hline $\begin{array}{l}\text { Portland cement Smooth finish/ } \\
\text { Light Grey Concrete Block }\end{array}$ & $5 \%$ & 38.5 \\
\hline $\begin{array}{l}\text { Portland cement Smooth finish/ } \\
\text { Light Grey Concrete Block }\end{array}$ & $20 \%$ & 33.3 \\
\hline $\begin{array}{l}\text { Portland cement Smooth finish/ } \\
\text { Light Grey Concrete Block }\end{array}$ & $60 \%$ & 20.8 \\
\hline $\begin{array}{l}\text { Portland cement Rough finish/ } \\
\text { Light Grey Concrete }\end{array}$ & $20 \%$ & 24.0 \\
\hline $\begin{array}{l}\text { Portland cement Rough finish/ } \\
\text { Light Grey Concrete }\end{array}$ & $60 \%$ & 21.4 \\
\hline $\begin{array}{l}\text { Portland cement Rough finish/ } \\
\text { Light Grey Concrete }\end{array}$ & $5 \%$ & 14.8 \\
\hline Dark Concrete & $20 \%$ & 14.6 \\
\hline Dark Concrete & $60 \%$ & 13.5 \\
\hline Dark Concrete & $95 \%$ & 10.8 \\
\hline Glazed Façade & & $25 \%$ \\
\hline
\end{tabular}

\section{Proposed Building Height:}

The maximum height, in meters, of the building being tested.

\section{Vertical location of lowest apartment:}

\footnotetext{
${ }^{2}$ Standards New Zealand. (2006). ASNZS 1680.1:2006 Interior Lighting and workplace lighting: Part 1. General principles and recommendations. Wellington: Standards New Zealand. Pg 122
} 
The lowest floor of apartments in the building. This is to be entered as 0 (for Ground Floor), 1 (for the floor above ground floor) etc, an example follows:

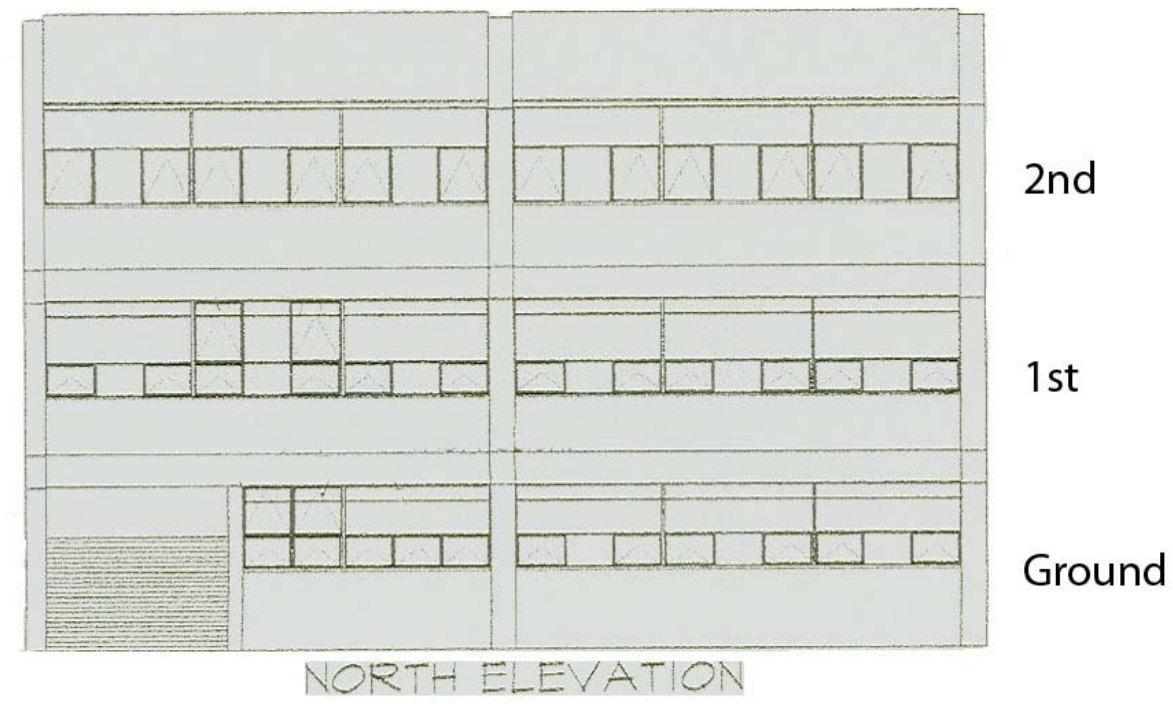

\section{Result:}

The result provided by the tool will be in the form of a 'YES' of 'NO'. An answer or 'YES' means your building is likely to be non-compliant and should therefore have simulations conducted to prove compliance. An answer of 'NO' means your building will exceed the minimum requirement and that simulation are not necessary to prove compliance. 


\section{Appendix 10 - Evaluation Questionnaire}

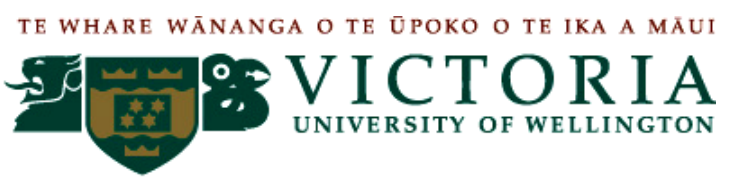

Questionnaire: New Zealand Building Code Clause G7 Compliance Tool:

Development and Implementation.

Researcher: Krystle Stewart, School of Architecture, Victoria University of Wellington

1) How long would you estimate it took you to assess a project using the tool? minutes

2) How do you consider the time it took to use the tool?

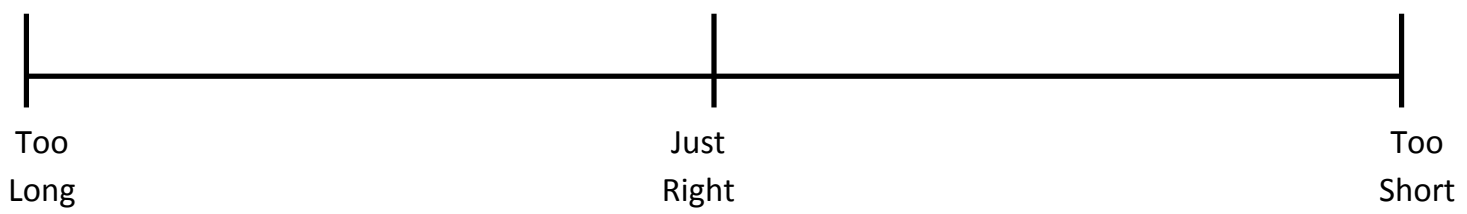

3) How many times did you apply the tool?

4) How difficult was the tool to use?

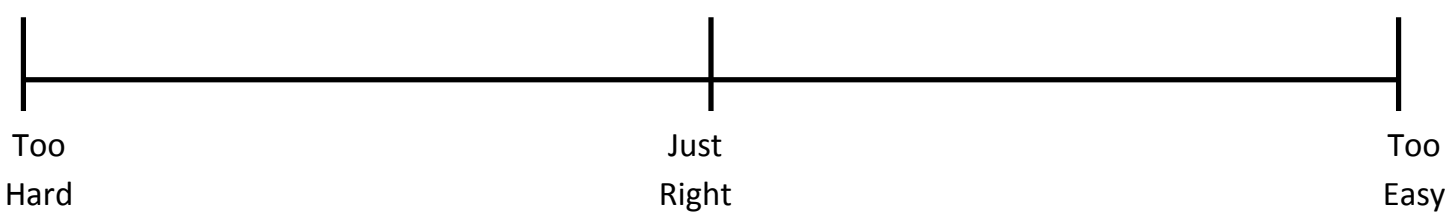

5) What could be done differently to make the tool easier to use?

6) Would you consider the tool to be useful?

$\square$ Yes $\quad \square$ No 
7) Is the result effective?

$\square$ Yes $\quad \square$ No

8) Would you support the implementation of the tool?

$\square$ Yes $\quad \square$ No

9) Any other comments or suggestions relating to the tool?

Thank you for your time! 